\title{
Ernährungsmedizinische Behandlung
}

\author{
M.J. Müller, J.Westenhöfer, Chr. Löser, A. Weimann und H. Przyrembel
}

Zwischen geträumtem Wunsch und ahnendem

Traum schwebt alles Wissen ...

H. Broch (Die Schlafwandler, 1929)

\subsection{Ernährung und Lebensstil}

Da die Ernährung ein zentraler Bestandteil unseres Lebens ist, müssen ernährungsmedizinische Maßnahmen ganzheitlich angelegt sein und die Persönlichkeit in ihren verschiedenen Aspekten berücksichtigen. Ernährungsberatung und -therapie sind immer Teil eines größeren »Konzeptes«, welches verschiedene Bereiche des Lebensstils (wie Aktivität, Inaktivität, Rauchen, etc.) berücksichtigt. Das bewusste Hinwenden zu einer gesunden Lebensweise hilft dem Menschen, auch mit Ernährung verantwortungsbewusster umzugehen.

Der Erfolg von Beratung, Schulung und Erziehung setzt die Eigenverantwortung des Betroffenen voraus. Gesunde wir Kranke weisen die Verantwortung für ihre Gesundheit häufig den Therapeuten zu, was diese wiederum in der Regel akzeptieren. Um Eigenverantwortlichikeit herzustellen, muss dem Patienten und seinem Therapeuten zunächst klar werden, welchen Nutzen der Mensch aus dem ungesunden Lebensstil zieht. Essen, Rauchen, Alkoholgenuss und Inaktivität können durchaus zur Entspannung und zum geselligen Miteinander beitragen und so auch Lebensqualität bedeuten. Die Ambivalenz im Umgang mit Gesundheit und Ernährung muss mit dem Patienten zusammen herausgearbeitet werden. Erst nach dieser Klärung sind die möglichen Hindernisse gegenüber einem gesundheitsförderlichen
Lebensstil zu hinterfragen und die Vorteile einer Lebensstiländerung als Alternative zu »erarbeiten«. Eigenverantwortung wird nur auf einen arbeits- und zeitintensiven Weg erreicht. Ziel dieses Weges ist die Freiheit, die es dem Betroffenen ermöglicht, sich verantwortlich zwischen gesundheitsförderndem und -schädigendem Verhalten zu entscheiden.

\subsubsection{Gesunde Ernährung}

Eine "gesunde Ernährung " ist bedarfsdeckend und berücksichtigt die derzeitigen Kenntnisse hinsichtlich des präventiven Wertes einzelner Nährstoffe (d. h. der Prävention ernährungsabhängiger Erkrankungen). Der Nährstoffbedarf wird mit verschiedenen Methoden ermittelt:

- Bilanzstudien, bei denen Verluste im Vergleich zur Aufnahme einzelner Nährstoffe (Beispiele Eiweiss oder Calcium) erfasst wurde,

- Untersuchungen mit stabilen Isotopen, mit denen der spezifische Bedarf für einzelne Nährstoffe (wie z. B. essentieller Aminosäuren) gemessen wurde,

- Depletions- und nachfolgende Repletionsuntersuchungen, welche mit Diäten, die den jeweiligen Nährstoff in geringen oder hohen Mengen enthalten, durchgeführt wurden (Beispiel: Eiweiss) und

- die Erfassung der Nährstoffaufnahme gesunder Menschen. Dabei wird ausgehend von der Annahme einer Normalverteilung für die einzelnen Nährstoffe (wie z. B. Vitamin C) sichergestellt, dass 97,5\% der Personen ausreichend (d. h. bedarfsdeckend) versorgt werden. 
Bei den Nährstoffempfehlungen der Deutschen Gesellschaft für Ernährung (DGE) und den deutschsprachigen Fachgesellschaften (DACH) »handelt es sich mit Ausnahme der Richtwerte für die Energiezufuhr um Nährstoffmengen, von denen angenommen wird, dass sie nahezu alle Personen der jeweils angegebenen Alters- und Zielgruppen vor ernährungsbedingten Gesundheitsschäden schützen und die Voraussetzungen für volle Leistungsfähigkeit geben.« Der Nährstoffbedarf verschiedener Menschen zeigt aber eine nicht unbeträchtliche inter- aber auch intraindividuelle Varianz. Bei der Energiezufuhr wird in den Empfehlungen der durchschnittliche Bedarf der gesunden Bevölkerung angegeben. Demgegenüber liegt den Empfehlungen zu verschiedenen essentiellen Nährstoffen die Annahme einer Normalverteilung zugrunde. Dabei wird der durchschnittliche Bedarf (Median der Kurve) um 2 Standardabweichungen (oder 20-30\%) erhöht und so der Bedarf von durchschnittlich 98\% aller Personen dieser Population abgedeckt. Mit Ausnahme von Protein ist allerdings der Nährstoffaufnahme nicht normal verteilt. Bei einigen Nährstoffen wie Biotin, Pantothensäure und einigen Spurenelementen ist der genaue Bedarf nicht bekannt. Für diese gelten heute auch Schätzwerte, welche durch Experimente begründet, aber noch nicht abgesichert sind. Die - Tab. 2.1-2.4 zeigen die Nährstoffempfehlungen der DGE für Erwachsene und für Kinder und Jugendliche. - Tab. 2.5 enthält Angaben zur empfehlenswerten Höhe der Wasserzufuhr.

Gesundheitliche Probleme bestehen bei Zufuhr gröBerer, d.h. den Bedarf überschreitenden Mengen z. B. durch toxische (Vitamin A und D) und pharmakologische Wirkungen (Vitamin $B_{6}$, Nikotinsäure, Kochsalz, Fluorid, Selen etc.) einzelner Nährstoffe. Bei hoher Dosierung ist eine Abgrenzung der Nährstoffe von Arzneimitteln notwendig. Diese ergibt sich aus der jeweiligen Zweckbestimmung. Arzneimittel beeinflussen gezielt Körperfunktionen und dienen der Heilung von Erkrankungen, während Lebensmittel zum Zwecke der Ernährung und zum Genuss verzehrt werden. Die Einstufung eines Vitaminpräparates als Arzneimittel erfolgt allerdingd pragmatisch, wenn die Konzentrationen der Inhaltstoffe größer oder gleich dem 3-fachen der gegenwärtigen Empfehlungen sind. Wichtige Einflussgrößen des Nährstoffbedarfs wie die Genetik, die Bioverfügbarkeit von Nährstoffen, der individuelle Lebensstil, der Beruf oder auch Umweltfaktoren (wie z. B. das Klima) werden durch die gegenwärtigen Nährstoffempfehlungen nicht oder nur unzureichend berücksichtigt. Nährstoffempfehlungen werden in Zukunft mehr auf Stoffwechseluntersuchungen, der Pathophysiologie oder auch den genetischen Charakteristika einzelner Menschen und Gruppen basieren.

Die Nährstoffempfehlungen der DGE werden (z. B. durch die Regeln für eine vollwertige Ernährung) in praktisches Handeln umgesetzt.

\section{Vielseitig - aber nicht zuviel}

\section{(- Abwechslungsreiches Essen schmeckt und ist vollwertig.}

Je vielfältiger und sorgfältiger Sie Ihren Speiseplan zusammenstellen, desto besser lässt sich eine mangelhafte Versorgung mit lebensnotwendigen Nährstoffen oder eine Belastung durch unerwünschte Stoffe in der Nahrung vermeiden. Zur Energiezufuhr: Essen Sie gerade soviel, dass Sie kein Über- oder Untergewicht bekommen. Wiegen Sie sich regelmäßig.

\section{Weniger Fett und fettreiche Lebensmittel}

\section{(2) Zuviel Fett mach fett.}

Fett liefert doppelt so viele Kalorien wie die gleiche Menge an Kohlenhydraten oder Eiweiß. Übergewicht und ernährungsabhängige Krankheiten können die Folgen zu fettreicher Ernährung sein. Reduzieren Sie den Verzehr von Streichfetten und bevorzugen Sie fettarme Zubereitungsarten. Achten Sie nicht nur auf sichtbare Fette, sondern insbesondere auch auf die »unsichtbaren« Fette, z. B. in Fleisch, Wurst, Käse, Eiern, Sahne, Nüssen, Kuchen und Schokolade.

\section{Würzig aber nicht salzig}

\section{(8) Kräuter und Gewürze unterstreichen den Eigengeschmack der Speisen.}

Zuviel Salz übertönt hingegen viele Geschmackseindrücke und kann zur Entstehung von Bluthochdruck beitragen. Bevorzugen Sie deshalb Kräuter und Gewürze. Wo sie dennoch auf Salz nicht verzichten können, verwenden Sie Jodsalz, um dem weitverbreiteten Jodmangel vorzubeugen.

\section{Wenig Süßes}

\section{( Zu süß kann schädlich sein!}

Zucker und Süßigkeiten können Karies verursachen. Genießen Sie Süßes zwar ohne Reue, aber nur selten und in kleinen Mengen.

\section{Mehr Vollkornprodukte}

\section{(2) Vollkornprodukte liefern wichtige Nährstoffe und Ballaststoffe.}

Vollkornprodukte, z. B. Vollkornbrot, Naturreis, Getreidegerichte, Vollkornnudeln, Haferflocken oder Müsli enthalten günstige Kohlenhydrate. Neben den für die Verdauung wichtigen Ballaststoffen liefern sie zusätzlich Vitamine, Mineralstoffe und Spurenelemente. 


\section{Reichlich Gemüse, Kartoffeln und Obst}

\section{(8) Diese Lebensmittel gehören in den Mittelpunkt Ihrer Ernährung.}

Essen Sie täglich Frischkost in Form von frischem Obst, Rohkost und Salaten, aber auch Gemüse und Kartoffeln. Wählen Sie auch öfter Hülsenfrüchte. Mit diesen Lebensmitteln erhalten Sie Vitamine, Mineralstoffe, Spurenelemente und Ballaststoffe.

\section{Weniger tierisches Eiweiß}

\section{Pflanzliches Eiweiß ist so wichtig wie tierisches Eiweiß.}

Pflanzliches Eiweiß in Kartoffeln, Hülsenfrüchten und Getreide ist günstig für eine vollwertige Ernährung. Auch Milch, fettarme Milchprodukte und Fisch sind wertvolle Eiweißlieferanten. Es empfiehlt sich, den Verzehr von Fleisch, Wurst und Eier, die relativ viel Fett, Cholesterin und Purine enthalten, auf wenige Mahlzeiten pro Woche zu verringern.

\section{Trinken mit Verstand}

: Ihr Körper braucht Wasser, aber keinen Alkohol.

Mindestens eineinhalb bis zwei Liter Wasser pro Tag benötigt ihr Körper. Löschen Sie Ihren Durst mit Wasser bzw. Mineralwasser, Gemüsesäften, ungesüßtem Früchtetee und verdünnten Obstsäften, in Maßen auch mit ungesüßtem schwarzen Tee und Kaffee. Trinken Sie alkoholische Getränke daher allenfalls zum gelegentlichen Genuss (moderater Alkoholkonsum von $10 \mathrm{~g} / \mathrm{Tag}$ für Frauen und $20 \mathrm{~g} /$ Tag für Männer), aber nicht als alltäglichen Durstlöscher.

\section{Schmackhaft und nährstoffschonend zubereiten}

\section{: Garen Sie kurz mit wenig Wasser und Fett}

Durch zu lange Lagerung, falsche Vorbereitung, zu langes Kochen, Wiederaufwärmen und durch die Verwendung von zuviel Wasser beim Garen werden viele lebensnotwendige Nährstoffe zerstört und ausgelaugt. Garen Sie deshalb so kurz wie möglich und verwenden Sie dazu wenig Wasser oder Fett. So bleiben Nährstoffe und der Eigengeschmack der Speisen erhalten.

Diese Empfehlungen sind allgemein gehalten und sind nicht streng wissenschaftlich $\mathrm{zu}$ verstehen. Im Vergleich zwischen den Ernährungsempfehlungen und dem tatsächlichen Energie- und Nährstoffbedarf zeigen die Ergebnisse grosser Ernährungserhebungen wie der Nationalen Verzehrsstudie oder des Bundesgesundheitssurveys, dass:
- die Energieaufnahme häufig zu hoch ist,

- die Protein- und Purinzufuhr die Empfehlungen in allen Altersgruppen übersteigt,

- die Fett- und Cholesterinaufnahme überhöht ist, während gleichzeitig der Bedarf an essentiellen Fettsäuren trotz niedriger Aufnahme von mehrfach ungesättigten Fettsäuren gesichert erscheint,

- die Ballaststoffaufnahme und die Zufuhr komplexer Kohlenhydrate niedrig ist,

- Männer bis zu 7\% der Energien in Form von Alkohol zu sich nehmen,

- Calcium unter den Mineralstoffen ein »kritischer« Nährstoff ist,

- unter den Spurenelementen Eisen, Zink und Jod »kritisch « sind,

- die Vitamin D-, E-, C- und Carotinoidaufnahme häufig nicht dem Bedarf entspricht und

- die mittleren Zufuhrraten für Folsäure unter den Empfehlungen liegen.

Sog. Risikogruppen sind Gruppen von Personen, bei denen die Empfehlungen häufiger nicht erreicht werden. Dies sind z. B. Menschen mit einem Hauptschulabschluss, Arbeiter(innen) sowie Personen, die in einer fünf- und mehrköpfigen Familie leben und ein niedriges monatliches Pro-Kopf-Einkommen haben. Diese Menschen haben z. B. im Vergleich zu anderen Gruppen eine deutlich niedrigere Aufnahme an Nährstoffen, wie Calcium, Vitamin C und Folsäure.

Für Kinder und Jugendliche wird heute in Deutschland die »Optimierte Mischkost « (Forschungsinstitut für Kinderernährung, Dortmund) empfohlen. Diese berücksichtigt die derzeitigen ernährungsphysiologischen und "präventiven« Erkenntnisse sowie auch die landestypischen Ernährungsweisen und -vorlieben. Für die Ernährung von Kindern und Jugendlichen ist eine höhere Energiedichte (d. h. ein Fettgehalt von 35 Energieprozent) notwendig.

\subsection{2 "Alternative« Ernährung}

Neben der vollwertigen Ernährung nach den Regeln der DGE gibt es verschiedene sog. »alternative Ernährungsformen«. Diese sind wie folgt charakterisiert:

- Bevorzugter oder ausschließlicher Verzehr pflanzlicher Lebensmittel und von Produkten aus dem ökologischem Landbau,

- Vermeidung übermäßig verarbeiteter Lebensmittel,

- Vermeidung von Zusatzstoffen,

- Anspruch auf gesundheitlichen Wert,

- »Einbettung« in einer ganzheitlich orientierten Weltanschauung,

- aber: fraglicher wissenschaftlicher Wert. 


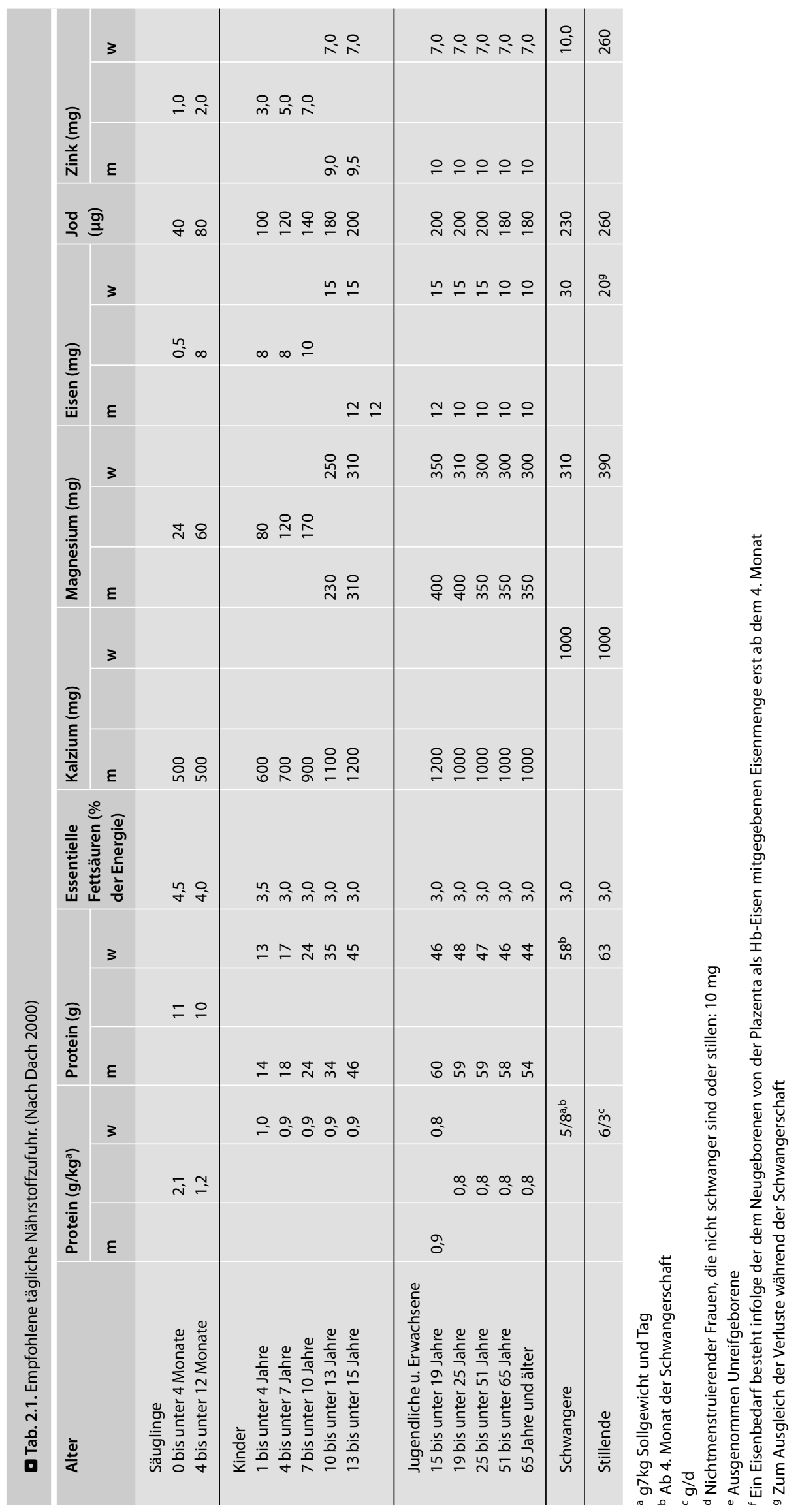




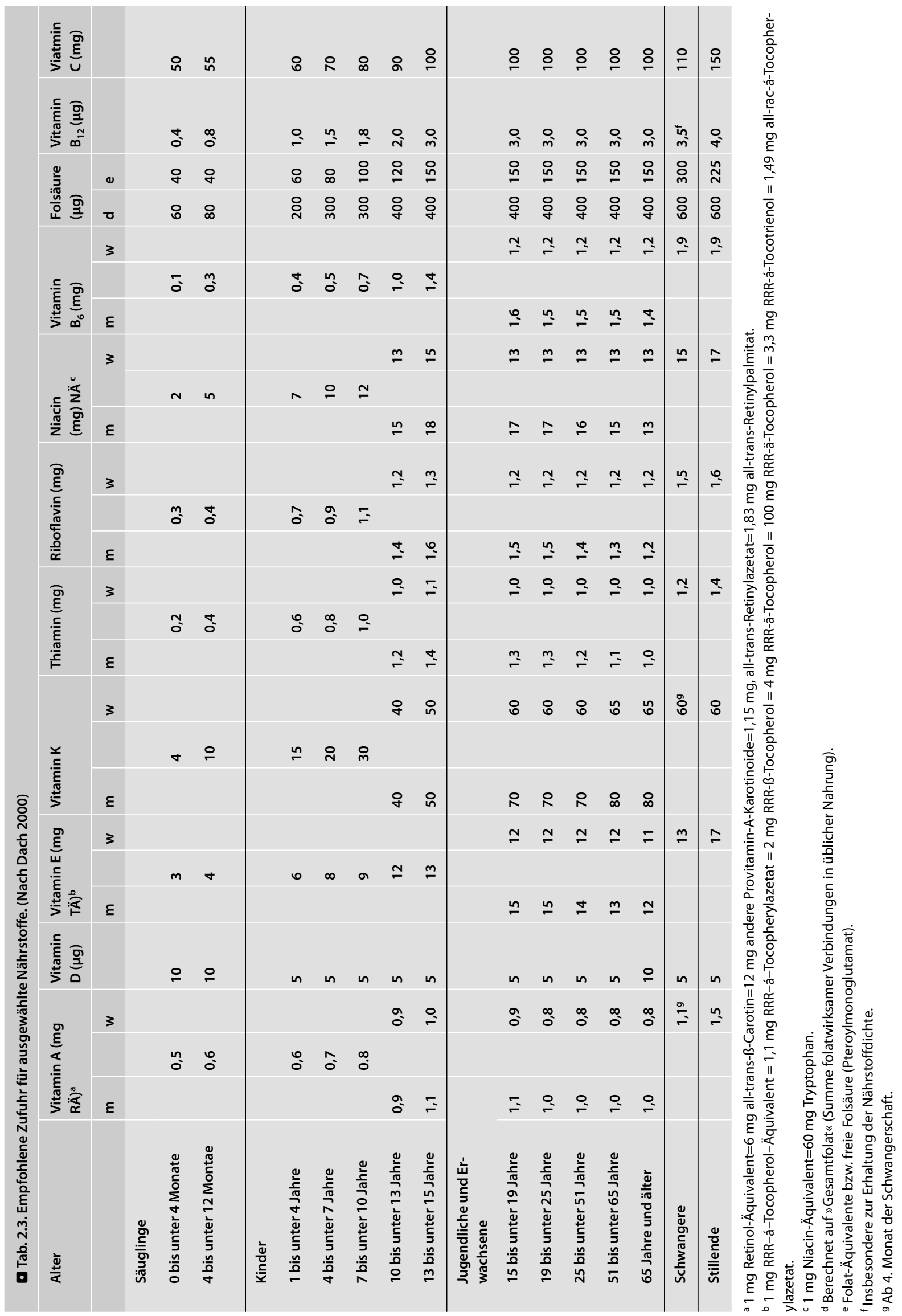


- Tab. 2.2. Richtwerte für die tägliche Fettzufuhr. (Nach Dach 2000)

\begin{tabular}{l|l}
\hline Alter & Fett (\% der Energie) \\
\hline Säuglinge & \\
\hline 1 bis unter 4 Monate & $45-50$ \\
4 bis unter 12 Monate & $35-45$ \\
\hline Kinder & \\
1 bis unter 4 Jahre & $30-40$ \\
4 bis unter 7 Jahre & $30-35$ \\
7 bis unter 10 Jahre & $30-35$ \\
10 bis unter 13 Jahre & $30-35$ \\
13 bis unter 15 Jahre & $30-35$ \\
\hline Jugendliche und Erwachsene & \\
\hline 15 bis unter 19 Jahre & 30 \\
\hline 19 bis unter 25 Jahre & $30^{\mathrm{a}, \mathrm{b}}$ \\
\hline 25 bis unter 51 Jahre & $30^{\mathrm{a}}$ \\
\hline 51 bis unter 65 Jahre & 30 \\
\hline 65 Jahre und älter & 30 \\
\hline Schwangere & \\
\hline Ab 4. Monat & $30-35$ \\
\hline Stillende & $30-35$ \\
\hline
\end{tabular}

a Schlanke Hochleistungssportler oder Schwerstarbeiter können höhere Prozentsätze benötigen.

${ }^{\mathrm{b}}$ Entsprechen $67-80 \mathrm{~g}$ Gesamtfett bei einer Energiezufuhr von $10 \mathrm{MJ}$.

- Tab. 2.4. Schätzwerte für eine angemessene Zufuhr einzelner Nährstoffe. (Nach Dach 2000)

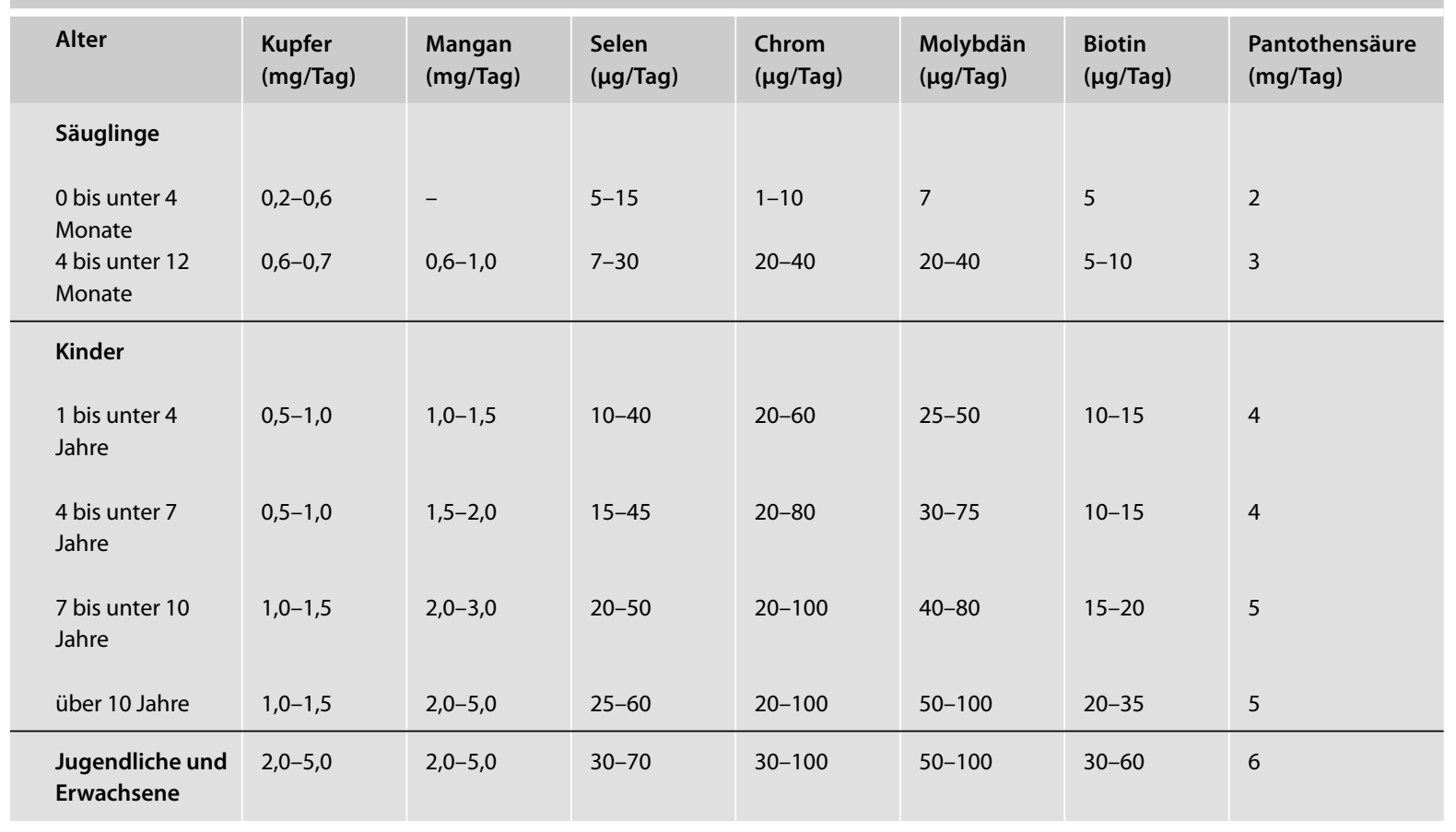


Tab. 2.5. Empfehlendswerte Höhe der Wasserzufuhr. (Nach Dach 2000)

\begin{tabular}{|c|c|c|c|c|c|}
\hline \multirow[t]{2}{*}{ Alter } & \multirow{2}{*}{$\begin{array}{l}\text { Gesamtwasser- } \\
\text { aufnahme } \\
\text { (ml/Tag) }\end{array}$} & \multirow{2}{*}{$\begin{array}{l}\text { Oxidations- } \\
\text { wasser } \\
(\mathrm{ml} / \mathrm{Tag})\end{array}$} & \multicolumn{2}{|c|}{ Wasserzufuhr durch } & \multirow{2}{*}{$\begin{array}{l}\text { Wasserzufuhr } \\
\text { durch Getränke } \\
\text { und feste } \\
\text { Nahrung } \\
\text { (ml/kg KG/Tag) }\end{array}$} \\
\hline & & & $\begin{array}{l}\text { Getränke (ml/ } \\
\text { Tag) }\end{array}$ & $\begin{array}{l}\text { Feste Nahrung } \\
(\mathrm{ml} / \mathrm{Tag})\end{array}$ & \\
\hline $\begin{array}{l}\text { Säuglinge } \\
0 \text { bis unter } 4 \text { Monate } \\
4 \text { bis unter } 12 \text { Monate }\end{array}$ & $\begin{array}{l}680 \\
1000\end{array}$ & $\begin{array}{l}60 \\
100\end{array}$ & $\begin{array}{l}620 \\
400\end{array}$ & $\begin{array}{l}- \\
500\end{array}$ & $\begin{array}{l}130 \\
110\end{array}$ \\
\hline $\begin{array}{l}\text { Kinder } \\
1 \text { bis unter } 4 \text { Jahre } \\
4 \text { bis unter } 7 \text { Jahre } \\
7 \text { bis unter } 10 \text { Jahre } \\
10 \text { bis unter } 13 \text { Jahre } \\
13 \text { bis unter } 15 \text { Jahre }\end{array}$ & $\begin{array}{l}1300 \\
1600 \\
1800 \\
2150 \\
2450\end{array}$ & $\begin{array}{l}130 \\
180 \\
230 \\
270 \\
310\end{array}$ & $\begin{array}{l}820 \\
940 \\
970 \\
1170 \\
1330\end{array}$ & $\begin{array}{l}350 \\
480 \\
600 \\
710 \\
810\end{array}$ & $\begin{array}{l}95 \\
75 \\
60 \\
50 \\
40\end{array}$ \\
\hline $\begin{array}{l}\text { Jugendliche } \\
15 \text { bis unter } 18 \text { Jahre }\end{array}$ & 2800 & 350 & 1530 & 920 & 40 \\
\hline $\begin{array}{l}\text { Erwachsene } \\
19 \text { bis unter } 25 \text { Jahre } \\
25 \text { bis unter } 50 \text { Jahre } \\
51 \text { bis unter } 65 \text { Jahre } \\
65 \text { Jahre und älter }\end{array}$ & $\begin{array}{l}2700 \\
2600 \\
2250 \\
2250\end{array}$ & $\begin{array}{l}340 \\
330 \\
280 \\
260\end{array}$ & $\begin{array}{l}1470 \\
1410 \\
1230 \\
1310\end{array}$ & $\begin{array}{l}890 \\
860 \\
740 \\
680\end{array}$ & $\begin{array}{l}35 \\
35 \\
30 \\
30\end{array}$ \\
\hline Schwangere & 2700 & 340 & 1470 & 890 & 35 \\
\hline Stillende & 3100 & 390 & 1710 & 1000 & 45 \\
\hline
\end{tabular}

${ }^{a}$ gestillte Säuglinge etwa 1,5 ml Wasser/kcal, Kleinkinder etwa 1,2 ml/kcal, Schulkinder und junge Erwachsene etwa 1,0 ml/kcal einschließlich Oxidationswasser (etwa $0,125 \mathrm{ml} / \mathrm{kcal}$ ).

bWasser in fester Nahrung etwa $0,33 \mathrm{ml} / \mathrm{kcal}$.

'Wasserzufuhr durch Getränke und feste Nahrung=Gesamtwasseraufnahme - Oxidationswasser.

Alternative Kostformen sind z. B. die Vollwerternährung nach Körber, Männle und Leitzmann, die Haysche Trennkost, die Makrobiotik und verschiedene vegetarische Ernährungsformen. Prinzipien, Ziele und Lebensmittelauswahl dieser Kostformen sind in $\mathbf{0}$ Tab. 2.6 dargestellt. $\mathrm{Zu}$ den beschriebenen Ernährungsweisen gibt es außerdem verschiedene Varianten: z. B. beim Vegetarismus die Kostformen nach M. Bircher-Benner, J.G. Schnitzer, A. Waerland und R. Steiner.

»Alternative« Kostformen müssen hinterfragt werden im Hinblick auf:

- die Bedarfsdeckung,

- die Zusammensetzung der Kost im Vergleich zu den Nährstoffempfehlungen der Fachgesellschaften,

- mögliche Nebenwirkungen und Risiken und

- die Heilsversprechen.

Tab. 2.7 zeigt die Makronährstoff- und Ballaststoffzufuhr ausgewählter alternativer Kostformen im Vergleich zu den Empfehlungen der DGE. Es ist möglich, sich mit allen dargestellten Ernährungsformen "gesund « zu ernähren. Die Fettzufuhr ist in der Regel eher niedrig (besonders bei Rohkost, bei der eine Fettzufuhr von 30\% ereicht werden kann), die Zufuhr an Kohlenhydraten und Ballaststoffen ist andererseits hoch. Alternative Kostformen bergen aber auch Risiken der Unterversorgung mit einzelnen Nährstoffen, sog. »kritische« Nährstoffe). Sie erfordern genauere Kenntnisse der Ernährung. Bei unsachgemäßer Anwendung kann es zu Mangelzuständen kommen. Alternative Kostformen sind für sog. "vulnerable« Verbrauchergruppen (Kinder, Jugendliche, Schwangere, Stillende, alte Menschen, Menschen mit schwerer Krankheit (d. h. Menschen mit einem besonderen Nährstoffbedarf), nicht oder nur eingeschränkt empfehlenswert.

\subsection{Ernährungsteam}

Ein Ernährungsteam arbeitet interdisziplinär und besteht aus Ärztinnen/en, Krankenschwestern bzw. -pflegern, 
- Tab. 2.6. Lebensmittelauswahl und Ziele ausgewählter alternativer Kostformen

\begin{tabular}{|c|c|c|c|c|}
\hline & Vollwerternährung & Hay-Trennkost & Makrobiotik & Vegetarismus \\
\hline $\begin{array}{l}\text { Lebensmittel- } \\
\text { auswahl }\end{array}$ & $\begin{array}{l}\text { Vorwiegend laktove- } \\
\text { getabil; zur Hälfte als } \\
\text { Rohkost; geringer Verar- } \\
\text { beitungsgrad; Einteilung } \\
\text { der Lebensmittel in } 4 \\
\text { Wertstufen: Unerhitzt, } \\
\text { unverarbeitet, z. B. Voll- } \\
\text { kornprodukte, Gemüse, } \\
\text { Obst, Kartoffeln, Hülsen- } \\
\text { früchte, Milch und Milch- } \\
\text { produkte } \\
\text { Erhitzt, z. B. Fisch, Fleisch } \\
\text { und Eier } \\
\text { Stark verarbeitet, z. B. } \\
\text { Wurstwaren } \\
\text { Isolierte Substanzen, } \\
\text { z. B. Margarine, Limo- } \\
\text { naden } \\
\text { Produkte aus anerkannt } \\
\text { ökologischem Landbau; } \\
\text { keine Zusatzstoffe; regio- } \\
\text { nale Herkunft }\end{array}$ & $\begin{array}{l}\text { Vorwiegend laktovegeta- } \\
\text { bil; reichlich basenbilden- } \\
\text { des Obst und Gemüse; } \\
\text { Einteilung der Lebens- } \\
\text { mittel in } 3 \text { Gruppen: } \\
\text { Konzentriert kohlenhy- } \\
\text { dratreich, z. B. Getreide, } \\
\text { Kartoffeln } \\
\text { Neutral, z. B. Fett } \\
\text { Konzentriert eiweißreich, } \\
\text { z. B. Fleisch, Fisch und } \\
\text { Milch }\end{array}$ & $\begin{array}{l}\text { Vorwiegend vegetabil; } \\
10 \text { Wertestufen; viel Voll- } \\
\text { getreide, Hülsenfrüchte, } \\
\text { Samen, Nüsse, Algen, } \\
\text { Sojaprodukte, kaltge- } \\
\text { presste Öle } \\
\text { Keine: Milch, Milchpro- } \\
\text { dukte, Genussmittel, } \\
\text { Süßstoffe, Zucker, Honig, } \\
\text { Konserven, Tiefkühlkost } \\
\text { Lebensmittel aus der } \\
\text { gleichen Klimazone }\end{array}$ & $\begin{array}{l}\text { Streng vegetarische Kost } \\
\text { (Veganer): } \\
\text { Kein: Fleisch, Fleischpro- } \\
\text { dukte, Fisch, Eier, Milch, } \\
\text { Milchprodukte, Honig, } \\
\text { auch Gebrauchsartikel } \\
\text { tierischer Herkunft } \\
\text { Rohköstler: zusätzlich } \\
\text { keine gekochte Nahrung } \\
\text { Laktovegetarier: } \\
\text { Kein: Fleisch, Fleisch- } \\
\text { produkte, Fisch, Eier } \\
\text { Ovolaktovegetarier: } \\
\text { Kein: Fleisch, Fleisch- } \\
\text { produkte, Fisch }\end{array}$ \\
\hline Ziele & $\begin{array}{l}\text { Optimale Nährstoff- } \\
\text { versorgung; opti-male } \\
\text { körperliche und seelische } \\
\text { Leistungsfähigkeit; } \\
\text { soziale und Umweltver- } \\
\text { träglichkeit; Prävention } \\
\text { ernährungsabhängiger } \\
\text { Erkrankungen; Gesund- } \\
\text { heit; Senkung der Kosten } \\
\text { im Gesundheitswesen }\end{array}$ & $\begin{array}{l}\text { Optimale Verdauung; kei- } \\
\text { ne »Übersäuerung« des } \\
\text { Organismus; Gesundheit; } \\
\text { Heilung von Krankheiten } \\
\text { (wie z. B. Rheuma, Colitis } \\
\text { etc.) }\end{array}$ & $\begin{array}{l}\text { Einklang mit dem Kos- } \\
\text { mos; Bewusstseinserwei- } \\
\text { terung, Gesundheit }\end{array}$ & $\begin{array}{l}\text { Zurück zum gesunden } \\
\text { Menschenverstand; } \\
\text { Gesundheit und Lebens- } \\
\text { freude; Stärkung der kör- } \\
\text { perlichen Abwehr; Verrin- } \\
\text { gerung der Schadstoff- } \\
\text { aufnahme; Schonung der } \\
\text { natürlichen Ressourcen; } \\
\text { Ernährung als Teil der } \\
\text { Körperkultur }\end{array}$ \\
\hline
\end{tabular}

- Tab. 2.7. Makronährstoff- und Ballaststoffzufuhr sowie kritische Nährstoffe alternativer Kostformen auf der Basis von berechneten Kostplänen

\begin{tabular}{|l|l|l|l|l|l}
\hline $\begin{array}{l}\text { Kostform } \\
\text { Vollwert-Ernährung }\end{array}$ & Eiweiß (\%) & Fett (\%) & Kohlenhydrate (\%) & Ballaststoffe (g) & Kritische Nährstoffe \\
\hline Vegetarische Kost & 15 & 31 & 53 & 46 & Eisen, Vitamin D \\
\hline Hay-Trennkost & $13-16$ & $31-35$ & $47-50$ & $42-50$ & $\begin{array}{l}\text { Eiweißa, Jod, Kalzium }{ }^{\text {a }} \text {, Eisen, } \\
\text { Vitamin } \mathrm{B}_{2}{ }^{a}, \mathrm{~B}_{12}, \mathrm{D}\end{array}$ \\
\hline Makrobiotik & 18 & 33 & 48 & 40 & Kalzium, Eisen, Jod, Vitamin D \\
\hline Empfehlungen der DGE & 17 & 32 & 49 & 46 & $\begin{array}{l}\text { Eiweiß, Kalzium, Eisen, } \\
\text { Vitamin } \mathrm{B}_{2}, \mathrm{~B}_{12}, \mathrm{D}\end{array}$ \\
\hline
\end{tabular}

${ }^{\text {a }}$ Nur bei der rein veganen Kost. 
Diätassistentinnen/en sowie ggf. Ökotrophologinnen/ en und Psychologinnen/en (s. - Abb. 2.1). Die interdisziplinäre Zusammenarbeit trägt nachweislich zu einer Verbesserung und Standardisierung der ernährungsmedizinischen Versorgung im Bereich des öffentlichen Gesundheitswesens sowie innerhalb und außerhalb eines Krankenhauses bei. Das Ernährungsteam ist zuständig für die Umsetzung von Konzepten der gesunden Ernährung und der Diätetik (einschließlich der künstlichen Ernährung) im Krankenhaus.

Aufgaben des Ernährungsteams sind:

- die differenzierte Erfassung des Ernährungszustands,

- die Identifikation von ernährungsmedizinischen »Risikopatienten",

- die Indikation für eine diätetische Maßnahme (einschließlich der künstlichen Ernährung),

- das Erstellen spezieller diätetischer Rezepturen,

- die Beratung und Schulung von Patienten und deren Angehörigen,

- Planung und Durchführung einer enteralen bzw. parenteralen Ernährung zu Hause in Zusammenarbeit mit Sozialstationen, der ambulanten Krankenpflege, Apothekern und der Industrie und

- die Entwicklung neuer ernährungsmedizinischer Konzepte sowie die Entwicklung von Pflegestandards.

Die Arbeit eines Ernährungsteams kann schwerpunktmäßig auf Problemgruppen konzentriert sein (z. B. bei der Betreuung von HIV-infizierten Patienten oder auch auf Intensivstationen). Um die Effizienz ernährungsmedizinischen Handelns zu gewährleisten und auch zukünftig eine ausreichende Evaluation der ernährungsmedizinischen Praxis zu sichern, müssen Ernährungsteams an allen größeren Zentren (Gesundheitsämter, ernährungsmedizinische Beratungsstellen, Krankenhäuser, Rehabilitationszentren) eingerichtet werden.

\subsection{Ernährungsberatung}

\subsubsection{Indikation und Zielsetzung}

Eine Ernährungsberatung kann verschiedene Anlässe und Ziele haben:

\section{Klärung der Ernährungssituation}

Es soll geklärt werden, ob es in der Ernährungssituation und im Ernährungsverhalten eines Klienten oder Patienten ${ }^{1}$ Möglichkeiten der Optimierung oder gar die Notwendigkeit einer Veränderung gibt. Der Wunsch für eine solche Klärung kann vom betroffenen Patienten ausgehen, zum Bespiel, weil aufgrund der Medienberichterstattung hinsichtlich der eigenen Ernährung Verunsicherung besteht, weil Unsicherheiten hinsichtlich der Ernährung von Familienangehörigen, insbesondere Kindern, bestehen, oder weil bestimmte Ziele verfolgt werden, wie etwa eine Gewichtsreduktion oder eine Leistungssteigerung im Sport. Der Wunsch für eine solche Klärung kann jedoch auch vom behandelnden Arzt ausgehen, etwa wenn eine Erkrankung festgestellt wurde, die durch die Ernährung beeinflusst werden kann.

\footnotetext{
${ }^{1}$ In diesem Kapitel werden entsprechend den unterschiedlichen Nomenklaturen in Ernährungswissenschaft, Psychologie und Medizin die Begriffe Klient und Patient für den Adressaten der Ernährungsberatung gebraucht. In der Regel wird dieser in Psychologie und z. T. in den Ernährungswissenschaften eher als Klienten bezeichnet, in der Medizin als Patient. Darüber hinaus bezieht sich Patient deutlich auf eine bereits erkrankte Person, während der Begriff Klient auch gesunde Personen umfasst. Im Folgenden wird aus Gründen der sprachlichen Vereinfachung oft der Begriff Patient gebraucht, auch wenn gesunde Personen mit gemeint sind. Gleichfalls wird die männliche Form Patient gebraucht, auch wenn Frauen, die Ernährungsberatung deutlich häufiger in Anspruch nehmen als Männer, mit gemeint sind.
}

\section{Arzt}

Indikation

Patientenauswahl

Überwachung

Problemlösung

\section{Schwester/Pfleger \\ Pflege \\ Schulung \\ Organisation}

\author{
Technischer Assistent \\ Untersuchung/Überwachung \\ Sterile Zubereitung von Infusions- \\ lösungen \\ Datenerfassung
}


Insbesondere bei Stoffwechselerkrankungen wie zum Beispiel Diabetes Mellitus steht dann im Blickpunkt der Beratung weniger eine fakultative Möglichkeit der Ernährungsoptimierung, sondern eher eine als medizinische Notwendigkeit empfundene Anpassung der Ernährung, um Folgeschäden zu vermeiden.

\section{Erarbeiten von Veränderungsmöglichkeiten in der Ernährung}

Sofern die Klärung der Ernährungssituation die Möglichkeit einer Optimierung oder die Notwendigkeit einer Veränderung ergibt, stellt sich die Frage, wie eine optimierte und angepasste Ernährung aussehen kann oder aussehen sollte. Die angestrebten Veränderungen reichen von quantitativen und qualitativen Aspekten der Lebensmittelauswahl (Was und wie viel soll gegessen werden?), über Fragen der Nahrungszubereitung bis zur Gestaltung der Verzehrssituation (Wann und wie oft soll gegessen werden? Was sind günstige Situationen, was ungünstige?).

\section{Unterstützung bei der Veränderung des Ernährungsverhaltens}

Häufig wird es nicht ausreichen, mit dem Patienten Veränderungsmöglichkeiten zu erarbeiten, sondern er wird weitere Unterstützung bei der Verwirklichung dieser Veränderungen benötigen. Eine solche Unterstützung kann notwendig werden, weil die wünschenswerten Veränderungen der Ernährung zur Beeinträchtigung von anderen psychosozialen Bedürfnissen (z. B. Genuss, soziale Einbindung und Anerkennung) führen oder weil seit langem praktizierte Gewohnheiten mit einer hohen Verhaltensstabilität verändert werden sollen.

Kennzeichnend für eine Ernährungsberatung in diesem engeren Sinn ist die Dialogform zwischen Berater einerseits und Patient andererseits. Dieser Dialog kann verschiedene Formen annehmen. Klassisch sind die Einzelberatung, bei der lediglich der Berater und der betroffene Patient beteiligt sind, und die Gruppenberatung, bei der ein, gelegentlich auch zwei Berater mit einer Gruppe von Patienten in Interaktion treten. Daneben ist, insbesondere wenn es um Kinderernährung geht, die Familienberatung gebräuchlich, bei der zum Beispiel die Mutter hinsichtlich der Ernährung ihres Kindes beraten wird. Die Verbreitung des Internet als Kommunikationsmedium hat darüber hinaus die Internet-gestützte Ernährungsberatung als neue Spielart entstehen lassen.

Von dieser dialogorientierten Ernährungsberatung im engeren Sinn sind Ernährungsaufklärung und Ernährungserziehung abzugrenzen. Ziel der Ernährungsaufklärung ist es, beim Verbraucher Interesse für Ernährungsfragen $\mathrm{zu}$ wecken, Problembewusstsein für die eigene Ernährungssituation zu schaffen oder Information über verschiedene Aspekte der Ernährung zu verbreiten. Da- bei richtet sie sich an alle Verbraucher oder zumindest an größere Gruppen von Verbrauchern. Entsprechend dieser Zielsetzung werden für Ernährungsaufklärung vor allem Methoden der Massenkommunikation wie Fernsehen, Internet-Seiten, Plakate, Vertrieb von Informationsschriften und Broschüren eingesetzt. Kennzeichnend für viele Maßnahmen der Ernährungsaufklärung ist, dass sie unabhängig von einer individuellen Nachfrage konzipiert sind und dass die verbreitete Information vielmehr aktiv an die Adressaten herangetragen wird. Ebenso sind solche Maßnahmen in der Regel als "Einweg-»Kommunikation konzipiert: Die Aufklärungsbotschaft wird an die Adressaten übermittelt, es findet jedoch kein Dialog mit dem Adressaten statt. Wenn der Adressat Nachfragen oder weitergehenden Informationsbedarf hat, muss er von sich aus auf aktive Informationssuche gehen.

Als Ernährungserziehung werden pädagogische Maßnahmen zusammengefasst, durch die im Entwicklungsprozess eines Menschen geplant und absichtlich Ernährungswissen vermittelt und Ernährungsverhalten geprägt werden. Ernährungserziehung findet ganz wesentlich in der primären Sozialisationsinstanz Familie statt. Mit der Veränderung sozialer Strukturen bekommen aber auch die sekundären Sozialisationsinstanzen wie Kindergarten und Schule eine immer stärkere Bedeutung für die Ernährungserziehung. Ernährungserziehung sollte darauf abzielen, Menschen zu befähigen, sich zu einem mündigen Verbraucher mit einem gesundheitsförderlichen, sozial verantwortlichen und umweltgerechten Ernährungsverhalten zu entwickeln. Die Ernährungserziehung von Kindern hat eine zentrale Bedeutung in der Entwicklung und Stabilisierung eines günstigen Ernährungsverhaltens "von Anfang an« und ist damit ein zentraler Baustein der Gesundheitsförderung und Primärprävention.

Für die Durchführung einer Ernährungsberatung im engeren Sinn können verschiedene Zielsetzungen systematisch unterschieden werden, die jeweils ein eigenes methodisches Vorgehen erfordern:

Diagnostische Ziele. Sie bestimmen, welche Information benötigt wird, um den Beratungsprozess im weiteren Verlauf zu steuern und zu gestalten. Mögliche diagnostische Ziele sind die Beschreibung der Nahrungsaufnahme oder die Klärung sozialer und psychologischer Faktoren des Essverhaltens. Die verschiedenen Methoden zur Umsetzung diagnostischer Ziele sind im $>$ Kap. 1.2 beschrieben. Beim Einsatz solcher Methoden sollte berücksichtigt werden, dass aus einer diagnostischen Abklärung auch eine praktische therapeutische bzw. Beratungskonsequenz folgen sollte. Sofern die nachfolgenden Beratungsschritte unabhängig vom Ergebnis einer diagnostischen Klärung ablaufen, erscheint der Sinn einer solchen Diagnostik fragwürdig. Im Gegenzug muss jedoch auch berücksichtigt werden, dass viele Patienten eine unzutreffende Selbsteinschätzung ihres eigenen Ernährungsverhaltens 
haben. So konnte in repräsentativen Befragungen festgestellt werden, dass der größte Teil der Bevölkerung zwar weiß, dass reichlich Obst und Gemüse und wenig Fett zentrale Bestandteile einer gesunden Ernährung sind, dass jedoch ebenso viele Bürger der Meinung sind, dass sie sich bereits fettarm mit ausreichenden Mengen Obst und Gemüse ernähren. Somit kann die Vermittlung einer realistischen Selbsteinschätzung des eigenen Ernährungsverhaltens ein wichtiges Teilziel im Rahmen des Beratungsprozesses darstellen.

Kognitive Lernziele. Sie beschreiben, welche Informationen dem Patienten vermittelt werden sollen. In der Beratungspraxis können kognitive Lernziele am einfachsten in der Form "am Ende der Beratung(seinheit) soll der Patient wissen, ... « formuliert werden. Solche Wissens- und Informationsvermittlung ist oft eine wichtige Voraussetzung für den angestrebten Beratungserfolg. So sollte zum Beispiel ein adipöser Patient selbstverständlich wissen, durch welche Lebensmittelauswahl er seine Energiezufuhr reduzieren kann. Oder ein Patient mit Laktoseintoleranz sollte wissen, welche Milchprodukte keinen relevanten Gehalt an Milchzucker haben und daher ohne Beschwerden konsumiert werden können. Allerdings wird die Bedeutung kognitiver Lernziele und damit die Bedeutung von Wissens- und Informationsvermittlung in der Ernährungsberatung aus zweierlei Gründen oft überschätzt. Zum einen ist zu hinterfragen, $o b$ das vermittelte Wissen handlungsrelevant ist oder ob ein günstigeres Ernährungsverhalten nicht auch ohne das vermittelte Wissen erreicht werden kann. Dies gilt besonders für wissenschaftlich geprägte Informationsinhalte. Wenn beispielsweise einem Patienten Wissen über die Empfehlungen zur Zufuhr von gesättigten, mehrfach ungesättigten und einfach ungesättigten Fettsäuren vermittelt werden, dann können diese kaum direkt in praktisches Ernährungsverhalten umgesetzt werden. Die Empfehlung bei der Speisezubereitung bevorzugt Oliven- oder Rapsöl anstelle von anderen Fetten einzusetzen ist dagegen wesentlich weniger kompliziert und leichter umzusetzen. Zum anderen muss festgestellt werden, dass Wissen nicht immer und nicht automatisch in entsprechendes Verhalten umgesetzt wird. Dieses trifft auf nahezu alle Verhaltensbereiche zu (Jeder Autofahrer weiß, dass in geschlossenen Ortschaften die Höchstgeschwindigkeit auf $50 \mathrm{~km} / \mathrm{h}$ begrenzt ist, trotzdem fahren viele Autofahrer schneller), auf Ernährungs- und Essverhalten jedoch im Besonderen. Dabei spielt eine wichtige Rolle, dass Ess- und Ernährungsverhalten eben nicht nur durch Wissen, sondern durch eine Vielzahl von anderen Faktoren mit bestimmt wird.

Sozioemotionale Lernziele. Sie beschreiben, welche gefühlsmäßige Bewertung und/oder soziale Beziehung der Patient durch den Beratungsprozess erwerben soll.
Sozio-emotionale Lernziele können formuliert werden in der Form: »Am Ende der Beratung(seinheit) wird der Patient wünschen, gut finden, mögen, erlebt haben, ..." Eine positive gefühlsmäßige Bewertung von Handlungen oder Situationen kann sehr viel eher verhaltensbestimmend sein, als rein kognitives Wissen um günstige oder ungünstige Verhaltensalternativen. Wenn beispielsweise ein Patient Gemüse mag, wird er es sehr viel eher essen, als wenn er weiß, dass reichlicher Gemüseverzehr gesund ist, er aber gleichzeitig verschiedenste Gemüse nicht mag. Wenn es ein Patient nicht mag, im Stehen und in Hektik nebenbei zu essen, wird er sehr viel weniger zu unkontrolliertem zwischendurch essen neigen, als wenn er lediglich weiß, dass häufiges nebenbei essen eine ausgeglichene Energiebilanz erschwert. Gleichermaßen spielt die Bewertung von Handlungen durch das soziale Umfeld eines Patienten eine wichtige Rolle. Wenn beispielsweise im beruflichen Umfeld oder in einer Freizeitgruppe der Verzicht auf Alkoholkonsum als unmännlich angesehen wird und damit zum Außenseiter in der Gruppe macht, wird es dem Einzelnen sehr schwer fallen, in solchen Situationen entgegen der Erwartung der anderen auf Alkohol zu verzichten.

Pragmatische oder verhaltensorientierte Lernziele. Sie beschreiben, welche praktischen Handlungen der Patient nach der Beratung durchführen kann (Verhaltenspotential) oder tatsächlich durchführen wird (realisiertes Verhalten). Beispiele für Lernziele zum Verhaltenspotential sind: Der Patient ist in der Lage, Gemüse schmackhaft zuzubereiten; der Patient kann sich in Frustrationssituationen anders trösten als durch Essen; der Patient kann dem Impuls, Erdnüsse beim Fernsehen zu knabbern, widerstehen. Beispiele für Lernziele zu realisiertem Verhalten sind: der Patient isst an mindestens fünf Tagen in der Woche eine warme Mahlzeit; der Patient isst pro Tag fünf Portionen Obst und Gemüse; der Patient; der Patient begrenzt seinen Konsum von Schokolade auf zwei Tafeln (je $100 \mathrm{~g}$ ) pro Woche.

Sofern sich die Ernährungsberatung nicht auf die Klärung der Ernährungssituation beschränken kann, ergeben sich zwangsläufig pragmatische und verhaltensorientierte Lernziele als die Ziele, die letztlich für den Beratungserfolg relevant sind. In der Regel werden Diagnostik, Wissensvermittlung und sozio-emotionale Lernziele dem Ziel eines veränderten Ess- und Ernährungsverhaltens untergeordnet sein müssen. Diese Prioritätssetzung kann nochmals am Beispiel des Gemüseverzehrs verdeutlicht werden. Ein typisches kognitives Lernziel wäre: »Der Patient weiß, dass Rohkost einen hohen Gehalt an Vitaminen und Mineralstoffen hat." Ein sozio-emotionales Lernziel wäre: »Der Patient mag Rohkost.« Das letztlich relevante verhaltensorientierte Lernziel hierzu könnte lauten: »Der Patient isst regelmäßig Rohkost." Es wird deutlich, dass das Wissen um die gesundheitlichen Vorteile von Rohkost 
und selbst eine veränderte Präferenz keinen gesundheitlichen Vorteil für den Patienten bringt, sofern dieses nicht dem tatsächlichen Verzehr entspricht. Im Gegenteil: Über den Umweg eines schlechten Gewissens kann Wissen um gesunde Ernährung, das nicht in Verhalten umgesetzt wird, durchaus negative Auswirkungen auf die psychosoziale Befindlichkeit und damit auf die Gesundheit insgesamt haben.

\subsubsection{Ernährungsberatung als Dialog}

Die oben skizzierte Ausgangslage der dialog-orientierten Ernährungsberatung macht deutlich, dass sich Ernährungsberatung nicht auf das » Ratschlag geben « reduzieren lässt. Leider weckt der Begriff »Beratung « im Deutschen häufig die spontane Assoziation an ein asymmetrisches Beratungsverhältnis, bei dem ein Berater dem »Ratsuchenden" hilfreiche und unterstützende "Ratschläge" gibt. Ein solches Verständnis kann an vielen Stellen für den Beratungsprozess hinderlich sein, da es den Patienten in eine passive Empfängerrolle drängt, anstatt ihn $\mathrm{zu}$ aktiver Beteiligung zu motivieren. Dialog-orientierte Ernährungsberatung wird davon profitieren, wenn Beratung weniger im Sinn von »jemanden beraten « als vielmehr im Sinn von "miteinander beraten " verstanden wird. Dies bedeutet auch, dass der Berater nicht über vorgefertigte Lösungskonzepte verfügen muss, die dem Patienten angeboten oder sogar »übergestülpt« werden. Vielmehr stellt der Berater sein inhaltliches und methodisches Expertenwissen zur Verfügung, um mit dem Patienten geeignete Lösungswege zu erarbeiten.

Eine wichtige Grundlage für das Beratungsgespräch ist die so genannte klientenzentrierte Gesprächsführung. Diese Art der Gesprächsführung beruht auf drei Grundvoraussetzungen, die der Berater gegenüber dem Patienten verwirklichen muss:

- Wertschätzung: Der Berater muss dem Patienten glaubhaft vermitteln, dass er ihn als Menschen ernst nimmt und wert schätzt. Wenn dies nicht der Fall ist, wird der Berater dem Patienten zwangsläufig nonverbal vermitteln, dass er ihn nur als "Nummer" wahrnimmt bzw. dass er ihn gering schätzt (»Schon wieder so ein Dicker!«). Eine solche Haltung wird dazu führen, dass der Patient kein Vertrauen zum Berater fassen kann, damit nicht in der Lage ist, sich zu öffnen und dem Berater Schwierigkeiten und Probleme anzuvertrauen. Damit wird ein Veränderungsprozess letztlich blockiert.

- Empathie: Als Empathie wird das einfühlende Verstehen des Beraters in das Erleben des Patienten bezeichnet. Dies bedeutet, dass es dem Berater gelingt, zu verstehen, was und wie der Patient bestimmte Erlebnisse wahrnimmt und verarbeitet und welche Gefühle dies bei ihm auslöst. Wenn ein Patient beispielsweise schil- dert, wie er zwei Tage zuvor eine Heißhungerattacke erlebt hat und der Berater darauf mit dem Hinweis reagiert, dass eine solche Handlung doch völlig unvernünftig ist, dann dokumentiert der Berater lediglich, dass es ihm in nicht gelungen ist, den Patienten zu verstehen. Einfühlendes Verstehen würde hier bedeuten, dass der Berater erfassen und benennen kann, wie der Patient diese Situation erlebt und verarbeitet hat.

- Echtheit, Authentizität: Hiermit ist gemeint, dass der Berater in der Beratungssituation nicht einfach eine professionelle Rolle spielt, die Wertschätzung und einfühlendes Verstehen gegenüber dem Patienten vortäuscht, sondern dass diese Haltungen vom Berater tatsächlich empfunden werden. Echtheit und Authentizität sind wichtig, da lediglich vorgespielte Haltungen und Einstellungen in der Regel durch nonverbale Signale übermittelt werden. Der Patient nimmt solche Signale mehr oder weniger bewusst war und wird darauf reagieren. Dies behindert einen produktiven Beratungsprozess.

Eine empathische Haltung des Beraters kann durch die Techniken der klientenzentrierten Gesprächsführung erleichtert werden. Gleichfalls bewirken diese Techniken, dass der Patient eine Rückmeldung darüber erhält, was der Berater bereits verstanden hat. Die grundlegendste dieser Techniken ist das aktive Zuhören. Dabei ist der Berater dem Patienten aufmerksam zugewandt und signalisiert durch Blickkontakt, Gesichtsausdruck und Körperhaltung, dass er das vom Patienten gesagte interessiert aufnimmt. Durch offene Fragen, die den Patienten nicht auf eine bestimmte, meist knappe Antwort festlegen, wird der Patient ermutigt, Situationen und sein Erleben im Zusammenhang zu schildern. Offene Fragen sind also typischerweise nicht die bekannten W-Fragen (Wer? Was? Wann? Wo? Wie viel?) die lediglich eine knappe Antwort provozieren, sondern solche Fragen und Aufforderungen, die in den Patienten einladen, etwa mehr von sich zu erzählen (z. B. »Wie ging es dann weiter?», »was ist dann passiert?«, »wie ist es Ihnen dabei ergangen?«).

Die Schilderungen des Patienten kann der Berater zusammenfassen und sinngemäß wiederholen. Diese Technik, bei der der Berater lediglich das gesagte mit eigenen Worten umschreibt, wird als Paraphrasieren bezeichnet. Dies dient dazu, dass sich der Berater vergewissern kann, dass er wirklich alle wichtigen Aspekte, die der Patient geschildert hat, richtig aufgefasst hat. Gleichfalls wird sich der Patient, indem der Berater das Gesagte zutreffend paraphrasiert, verstanden fühlen. Dies trägt dazu bei, dass der Patient sich weiter öffnet und dass er weitere relevante Aspekte ansprechen kann. Wenn sich das Paraphrasieren des Beraters nicht auf die in der Patientenaussage enthaltene Sachinformation beschränkt, sondern wenn dabei vor allem auf das subjektive Empfinden des Patienten fokussiert wird, dann 
wird vom »Verbalisieren emotionaler Erlebnisinhalte« gesprochen. Hierbei wiederholt und benennt der Berater also nicht nur, was passiert ist, sondern auch wie sich der Patient dabei gefühlt hat. Solche Berateräußerungen können, gerade zu Beginn, leicht realisiert werden, wenn der Berater seine Äußerung mit den Worten beginnt: "Wenn ich Sie richtig verstanden habe ... dann schildert, was er meint verstanden zu haben, wobei er sich bemühen sollte, zumindest einen emotionalen Aspekt aus dem Erleben des Patienten aufzugreifen.

Eine solche Gesprächsführung legt den Patienten nicht von vornherein auf bestimmte Themen und Aspekte fest, sondern ermöglicht es ihm, dass er nach und nach die Gesichtspunkte benennt, die für ihn selbst wichtig sind. Dies gilt auch und gerade dann, wenn ihm zu Beginn des Gesprächs vielleicht selbst noch nicht klar bewusst ist, welche Aspekte dies letztlich sind. Daher wird diese Art Gesprächsführung auch oft als non-direktiv bezeichnet.

Mit der klienten-zentrierten Gesprächsführung wird der Patient also ermutigt, seine subjektive Sichtweise einer Situation oder Problematik zu schildern und er erhält vom Berater eine Rückmeldung darüber, wie der Berater ihn verstanden hat. Dies wird dazu betragen, dass sich der Patient verstanden fühlt Er kann dann im Gespräch über weitere Aspekte seiner Problematik nachdenken und möglicherweise sogar neue Sichtweise und Problemlösungen entwickeln. Wenn der Berater die Situation und das Erleben des Patienten verstanden hat, bietet dies für ihn die Grundlage zu weiteren Interventionen.

\subsubsection{Ernährungsberatung als Informations- vermittlung}

Informations- und Wissensvermittlung ist in der Ernährungsberatung oft unerlässlich sein. Dabei muss jedoch berücksichtigt werden, dass die Qualität der Informationsvermittlung nicht dadurch bestimmt wird, wie viel Information vom Berater angeboten werden konnte, sondern davon, wie viel Information der Patient aufnehmen konnte bzw. wie viel er davon in ein verbessertes Ernährungsverhalten umsetzen konnte. Wie weit dargebotene Information von einem veränderten Verhalten entfernt sein kann, wird in den folgenden fünf allgemeinen Merksätzen treffend zusammengefasst:

- Gesagt heißt nicht gehört.

- Gehört heißt nicht verstanden.

- Verstanden heißt nicht einverstanden.

- Einverstanden heißt nicht angewendet.

- Angewendet heißt nicht beibehalten.

Wichtige Prozesse der Informationsverarbeitung, die beim Patienten als Empfänger von Informationen ablaufen, können anhand eines Strukturmodells des Gedächtnisses veranschaulicht werden (siehe $\mathbf{D}$ Abb. 2.2).
Alle Umweltreize und Informationen werden durch Sinnesorgane zunächst in Nervenimpulse umgewandet, die wiederum diese Information ans Gehirn übertragen. Dort werden diese Informationen für jede Sinnesmodalität wie »sehen « oder »hören « getrennt in einem sogenannten sensorischen Register oder Ultrakurzzeitgedächtnis zwischengespeichert. In diesem sensorischen Register werden die Umweltreize zunächst nahezu vollständig, allerdings nur für eine sehr kurze Zeit von etwa 0,5-2 s, gespeichert. Ohne weitere Verarbeitung zerfällt dann die gespeicherte Information. Allerdings findet in dieser Zeit bereits eine wichtige Vorverarbeitung der wahrgenommenen Umweltreize statt, die Mustererkennung: Visuelle Reize werden je nach dem als Buchstaben oder gar Wörter erkannt, als Gesichter von Personen oder als die Abbilder von Gegenständen; akustische Reize werden als Wörter oder Musik erkannt. Die solchermaßen aufbereitete Information gelangt dann entweder zur Weiterverarbeitung ins Arbeitsgedächtnis oder wird für immer gelöscht.

Die Übertragung von Information aus dem sensorischen Register ins Arbeitsgedächtnis geschieht nur für einen kleinen, ausgewählten Teil der wahrgenommenen Reize. Dieser Mechanismus wird als selektive Wahrnehmung bezeichnet. Welche Reize und Informationen für die Übertragung ausgewählt werden, wird unter anderem durch Aufmerksamkeitsprozesse des Patienten gesteuert. Dies kann und sollte genutzt werden, um die wesentlichen Beratungsinhalte durch besondere Heraushebung und Betonung von erläuterndem Beiwerk zu unterscheiden und somit eine bevorzugte Weiterverarbeitung zu fördern.

Das Arbeitsgedächtnis ist der Teil des Gedächtnisses in dem die aktive Informationsverarbeitung stattfindet. Dieser Gedächtnissspeicher wird auch häufig als Kurzzeitgedächtnis bezeichnet, da die Information hier nur für kurze Zeit gespeichert werden kann, ca. 5-20 s, wenn keine weitere Verarbeitung stattfindet. Durch aktive Verarbeitungsprozesse wie nachdenken oder wiederholen kann die Information jedoch auch beliebig länger gespeichert werden. Information die danach nicht ins Langzeitgedächtnis übertragen wird, wird einfach wieder vergessen. Neben der zeitlichen Begrenzung ist die

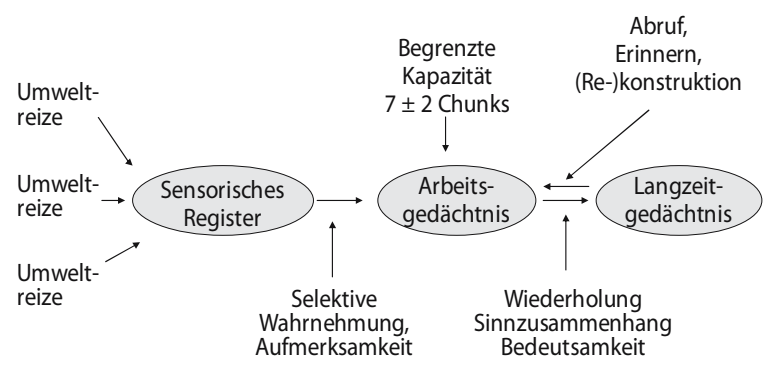

- Abb. 2.2. Strukturmodell des Gedächtnisses. Chunks = Informationsmöglichkeiten 
begrenzte Kapazität hinsichtlich der speicher- und verarbeitbaren Informationsmenge ein herausragendes Merkmal des Arbeitsgedächtnisses. Es hat sich gezeigt, dass das Arbeitsgedächtnis gleichzeitig etwa $7 \pm 2$ also zwischen 5 und 9 Informationseinheiten gleichzeitig verarbeiten und speichern kann. Diese Informationseinheiten wurden Miller, dem Entdecker dieses Prinzips als abstrakt als chunks bezeichnet, weil der Umfang eines solchen chunks je nach den Umständen und Vorerfahrungen sehr unterschiedlich sein kann. Während zum Beispiel für einen erfahrenen Statistiker die Formel für die Berechnung der Varianz möglicherweise eine einzige Informationseinheit darstellt, stellt für den Studierenden in der ersten Statistik-Vorlesung wahrscheinlich jeder einzelne Bestandteil dieser Formel eine eigene Informationseinheit dar. Genauso stellen für den erfahrenen Ernährungsberater auch mittelmäßig komplexe Ernährungszusammenhänge nur eine Informationseinheit dar, während für den Patienten solche Zusammenhänge nur als Verknüpfung mehrerer Informationseinheiten verarbeitbar sind. Hinzu kommt, dass der Berater nicht davon ausgehen kann, dass alle $7 \pm 2$ Informationseinheiten für die Verarbeitung von Sachinformation zur Verfügung stehen. Vielmehr werden auch Kontextinformationen vom Patienten parallel zur Sachinformation verarbeitet, zum Beispiel: Wie sympatisch oder freundlich ist der Berater? Wie fühle ich mich in der Situation (akzeptiert, ausgefragt, angeklagt)? Wie sieht der Berater aus (Gesichtsausdruck, Kleidung)? Wie sind die Räumlichkeiten? Im Allgemeinen sollte nicht davon ausgegangen werden, dass mehr als ca. 3 chunks für die Verarbeitung von Sachinformation zur Verfügung stehen. Sofern die vermittelte Information dann noch nicht ins Langzeitgedächtnis übertragen wurde, wird sie wieder vergessen. Wenn mehr Informationseinheiten dargeboten werden als verarbeitet werden können, dann werden am ehesten die zuletzt angebotenen Informationen erinnert, während frühere Information durch die neue Information verdrängt wird.

Bei der Übertragung von Informationen aus dem Arbeitsgedächtnis in das Langzeitgedächtnis können mehrere Gesichtspunkte berücksichtigt werden. Ein wesentlicher und häufig genutzter Prozess, der die Speicherung von Information im Langzeitgedächtnis erleichtert ist die Wiederholung. Dieser Mechanismus ist im Alltag durch solche Lernprozesse wie Vokabel-Lernen, FormelLernen, Auswendiglernen von Gedichten oder Memorieren von Telefonnummern allgemein bekannt. Auch in der Ernährungsberatung kann durch Wiederholung von Informationen die Chance erhöht werden, dass das vermittelte Wissen langfristig gespeichert wird. Weitere Mechanismen, die für die langfristige Speicherung wichtig sind, sind der Sinnzusammenhang und die persönliche Bedeutsamkeit von Information. Information, die vom Empfänger nicht in einen klaren Sinnzusammenhang eingeordnet werden kann, kann kaum ins Langezeitge- dächtnis übertragen werden. Auch erläuternde Bespiele, die einen neuen Zusammenhang anhand von bekannten Phänomenen darstellen, dienen der Herstellung von Sinnzusammenhängen. Wenn beispielsweise eine Patientin in der Ernährungsberatung darüber berichtet, dass sie immer wieder unter unwiderstehlichem Heißhunger auf Schokolade leidet, und der Berater daraufhin empfiehlt, dass die Patientin mehr Kohlenhydrate essen soll, so wird die Patientin diese Information kaum behalten. Wenn der Berater jedoch kurz erläutert, dass die Kohlenhydrate in der Schokolade möglicherweise den Gehirnstoffwechsel beeinflussen können, dass dies aber durch andere Kohlenhydrate genauso möglich ist, wird die Patientin die Empfehlung des Beraters eher in einen Sinnzusammenhang mit ihrem Problem einordnen können.

\section{Cave \\ - Versteht die Patientin den Begriff Kohlenhydrate richtig? \\ - Kann sie Kohlenhydrate zutreffend entsprechenden Lebensmittel zuordnen?}

Schließlich wird Information umso leichter ins Langzeitgedächtnis übertragen, je stärker ausgeprägt die persönliche Bedeutsamkeit der Information ist. Während sich die meisten Menschen mathematische oder chemische Formeln kaum ohne mehrfache Wiederholung merken können, gelingt dies mit dem Namen eines Menschen, den man kennen lernt und sympathisch findet, meistens auf Anhieb. Daher sollte versucht werden, bei der Vermittlung von Ernährungsinformation und -wissen die persönliche Bedeutung dieser Information für den Patienten herauszuarbeiten, also aufzuzeigen, welche konkreten Vorteile bzw. Nachteile sich aus einem bestimmten Sachverhalt ableiten lassen.

Im Langzeitgedächtnis kann eine große Menge von Information für lange Zeit gespeichert werden. Nach heutigem Wissen wird ein Großteil dieser Information in Form von semantischen Netzwerken gespeichert. Man kann sich das so vorstellen, dass jede gespeicherte Information ein Knoten in einem sehr verstrickten Netz ist. Die Fäden des Netzes sind dann jeweils Verknüpfungen einer Information mit anderen Informationsknoten. Informationen werden also nie isoliert gespeichert, sondern immer in Verknüpfung zu anderer gespeicherter Information. Solche Verknüpfungen können begrifflich-systematisch sein (z. B. Zucker und Stärke [sind] Kohlenhydrate; Brötchen [gehört zu] den Getreideprodukte) aber auch andere Assoziationen beinhalten (z. B. Fett [macht] dick; Fett schmeckt gut; Fett [ist] ungesund). Häufig wird abstrakte Sachinformation auch mit Verknüpfungen zu persönlichen Erlebnissen gespeichert (z. B. Butter - gehört zu - Fett; Knoblauchbutter - ist - Butter; die Knoblauchbutter zu den gegrillten Garnelen im letzten Urlaub war köstlich).

Wenn gespeicherte Information aus dem Langzeitgedächtnis erinnert oder abgerufen werden soll, dann muss 
der zutreffende Informationsknoten in diesem Netzwerk gefunden werden. Dies kann umso leichter gelingen, je vielfältiger eine Information mit anderer Information verknüpft ist. Daher sollte neue Ernährungsinformation anhand von Beispielen und persönlichen Erlebnissen des Patienten so gut wie möglich mit dem bereits vorhanden Wissen und den vorhandenen Erfahrungen verknüpft werden. Wenn gespeicherte Information nicht mehr erinnert oder abgerufen werden kann, liegt dies häufig daran, dass im gespeicherten Netzwerk kein geeigneter Zugangspfad zur Information gefunden werden kann. Manchmal ist dem Betroffen klar, dass er die Information gespeichert hat (»es liegt mir auf der Zunge«, »das war eine Abbildung auf einer Seite rechts oben«), und dennoch kann kein geeigneter Zugangsweg zur Information gefunden werden. Oder der Betroffene findet erst wieder Zugang zur gespeicherten Information, wenn sie ihm erneut präsentiert wird (»Ach ja, stimmt ja!«; Wiedererkennung). Prägnante Merksätze, Eselsbrücken, einprägsame Beispiele oder markante Grafiken können in solchen Fällen sehr gut die Funktion von leicht verfügbaren und auffindbaren Zugangswegen zur gespeicherten Information übernehmen. Letztlich kann aber auch davon ausgegangen werden, dass aufgenommene Information selten vollständig im Langzeitgedächtnis gespeichert ist. Vielmehr sind nur verschiedene Informationsanteile und Verknüpfungen gespeichert. Wenn solchermaßen unvollständig gespeicherte Information abgerufen wird, findet ein aktiver Rekonstruktionsprozess statt. Das bedeutet, dass die Informationslücken aufgefüllt werden, indem die nicht gespeicherte Information durch Inhalte ersetzt wird, die auf dem Hintergrund der eigenen Gesamterfahrung einigermaßen passend erscheinen. Wenn diese "frei erfundenen " Pseudoerinnerungen hinreichend plausibel sind, wird dem Betroffenen nicht einmal auffallen, dass er sich etwas ausgedacht hat, anstatt etwas zu erinnern.

Auf dem Hintergrund dieser gedächtnispsychologischen Überlegungen gibt es die Beobachtung, dass verschiedene Informationskanäle oder die Kombination von Informationskanälen unterschiedliche Erinnerungsleistungen bewirken können (s. - Abb. 2.3). Dabei spielen sicherlich die unterschiedlichen Aufmerksamkeits- und Selektionsprozesse eine wichtige Rolle, aber auch die Anzahl und der Qualität der Verknüpfungen, mit denen die Information im Langzeitgedächtnis gespeichert wird.

Zusammenfassend lassen sich aus den Überlegungen zur Informationsverarbeitung und -speicherung die folgenden Empfehlungen für die Ernährungsberatung geben:

- Beschränken Sie die dargebotene Information pro Beratungssitzung auf zwei bis drei Informationseinheiten.

- Nutzen Sie bei der Informationsvermittlung nach Möglichkeit mehrere Kanäle (zum Beispiel Sprache und Illustration).

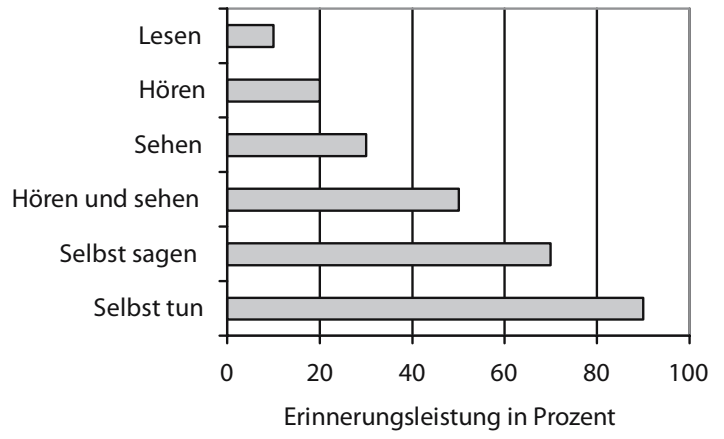

- Abb. 2.3. Erinnerungsleistung in Abhängigkeit vom verwendeten Informationskanal

- Wiederholen Sie die wichtigsten Informationen und machen Sie den Sinnzusammenhang und die persönliche Bedeutsamkeit klar.

- Lassen Sie den Patienten nochmals mit eigenen Worten zusammenfassen, was für ihn die wichtigsten Punkte waren.

- Geben Sie dem Patienten eine knappe, schriftliche Zusammenfassung der wichtigsten Punkte zur Unterstützung der Erinnerung mit.

\subsubsection{Ernährungsberatung als Bedürfnis- modifikation und Motivationsprozess}

Das moderne Ess- und Ernährungsverhalten ist dadurch charakterisiert, dass sowohl hinsichtlich der Lebensmittelauswahl als auch hinsichtlich der Wahl der Verzehrssituation in der Regel eine Fülle von Möglichkeiten bestehen. Welche der jeweils verfügbaren Optionen gewählt wird. ist von einer Vielzahl von Faktoren und Motiven abhängig. Eine Reihe von Motiven, die das Ernährungsverhalten in unterschiedlichen Situationen bestimmen können, ist beispielhaft in - Tab. 2.8 dargestellt.

Die Bedeutung solcher Motive kann von Mensch zu Mensch und von Situation zu Situation unterschiedlich sein. Meistens werden jedoch Menschen ihre Auswahlentscheidung so treffen, dass die für sie in der jeweiligen Situation wichtigsten Motive am besten abgedeckt werden. Sie treffen also eine subjektiv optimierte Ess-Entscheidung. Eine solche subjektiv optimierte Entscheidung muss jedoch keineswegs objektiv optimalen Ernährung entsprechen. Anders ausgedrückt: Psycho-soziale Bedürfnissen können zu einem Essverhalten führen, das nicht im Einklang mit dem physiologischen Bedarf des Organismus steht. Da der Bedarf des Organismus im Rahmen der Ernährungsberatung weitgehend unbeeinflussbar bleibt, muss Ernährungsberatung darauf abzielen die individuellen Bedürfnisse und Entscheidungsmotive zu verändern. 
- Tab. 2.8. Motive in der Steuerung des Ernährungsverhaltens

\begin{tabular}{|c|c|}
\hline Motiv & Beispiel \\
\hline Geschmack, Hedonismus & Erdbeeren mit Schlagsahne sind der höchste Genuss \\
\hline Hunger, Appetit & Ich muss jetzt etwas essen \\
\hline Ökonomische Bedingungen & 10 Tafeln Schokolade im Sonderangebot \\
\hline Kulturelle Einflüsse & Brötchen zum Frühstück \\
\hline Traditionen & Omas Weihnachtsplätzchen \\
\hline Gewohnheiten & Ich esse immer Tomate oder Gurke zum Abendessen \\
\hline Emotionale Wirkung & Bei dem Stress habe ich mir ein Stück Kuchen verdient \\
\hline Geselligkeit, soziale Gründe & Beim Fondue oder Grillabend kann man sich gut unterhalten \\
\hline Angebotslage & Es gibt nichts anderes \\
\hline Resteverwertung & War noch da und musste weg \\
\hline Magische Zuweisungen & Sellerie ist gut für die Potenz \\
\hline Fitnessüberlegungen & Mit einem isotonischen Durstlöscher kann ich länger durchhalten \\
\hline Schönheitsansprüche & Ich will schlanker werden \\
\hline Verträglichkeit & Grünkohl vertrage ich nicht \\
\hline Neugier & Mal sehen, wie das schmeckt \\
\hline Prestige & Da müssen wir etwas Besonderes servieren \\
\hline Angst vor Schaden & Seit BSE esse ich kein Rindfleisch mehr \\
\hline Pseudowissenschaftliche Argumente & Trennkost ist gut zum Abnehmen \\
\hline Pädagogische Gründe & Wenn du dein Zimmer aufgeräumt hast, bekommst du einen Schokoriegel \\
\hline Krankheitserfordernisse & Kein Zucker wegen meines Diabetes \\
\hline Gesundheitsüberlegungen & Gemüse ist gesund \\
\hline
\end{tabular}

Mit der Theorie des geplanten Verhaltens (Theory of planned behavior) steht ein Bezugsrahmen zur Verfügung der aufzeigt, durch welche Faktoren Verhalten beeinflusst und verändert werden kann (s. 0 Abb. 2.4).

Nach diesem Modell kann ein bestimmtes Verhalten am besten durch eine Intention, also eine Verhaltensabsicht vorhergesagt werden. Wenn sich jemand vornimmt, zum Frühstück Müsli zu essen, dann ist es sehr viel wahrscheinlicher, dass er es tut, als wenn er nicht die Absicht dazu hat. Allerdings wird nicht jede Absicht in tatsächliches Verhalten umgesetzt. Zwischen Intention und Verhalten können verschiedene Barrieren und Hindernisse liegen. Das Auftreten solcher Barrieren wird umso wahrscheinlicher, je größer der Zeitabstand zwischen Intentionsbildung und dem geplanten Verhalten liegt. Wenn sich ein Patient vornimmt, zum nächsten Abendessen ein Tomatenbrot anstelle eines Leberwurstbrotes zu essen, dann wird er dies sehr viel wahrscheinlicher tun, als wenn er sich dieselbe Handlung für den Mittwoch in drei Wochen vornimmt. Zum anderen können sich Intentionen im Hinblick auf ihren Allgemeinheitsgrad bzw. auf ihre Spezifität unterscheiden. Die Absicht ein Leberwurstbrot durch ein Tomatenbrot zu ersetzen ist relativ spezifisch. Die Absicht, weniger fettreiche Lebensmittel zu essen oder mehr Gemüse zu essen, wäre sehr viel allgemeiner. Im Allgemeinen können spezifische Verhaltensabsichten sehr viel eher umgesetzt werden als allgemeine Verhaltensabsichten. Letztlich kann der Vorsatz vom Tomatenbrot nur dann umgesetzt werden, wenn beim Abendessen tatsächlich Tomaten vorhanden sind. Abstrakter ausgedrückt: Verhaltensabsichten können nur dann in entsprechendes Verhalten umgesetzt werden, wenn der Betroffene tatsächlich die Kontrolle über das gewünschte Verhalten in der fraglichen Situation hat. Aus diesen 


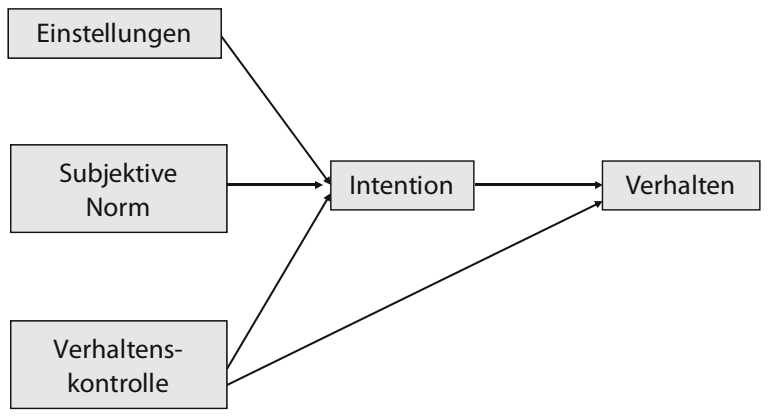

- Abb. 2.4. Grundmodell der Theorie des geplanten Verhaltens

Überlegungen ergibt sich für die Ernährungsberatung die Konsequenz:

- Den Patienten zunächst bei der Bildung von möglichst konkreten, spezifischen und zeitnahen Verhaltensabsichten unterstützen.

- Mit dem Patienten abklären, welche objektiven oder subjektiven Barrieren die Umsetzung einer Absicht in tatsächliches Verhalten erschweren können.

Wie - Abb. 2.4 zeigt, wird die Bildung von Intentionen durch drei weitere Faktorengruppen gefördert oder behindert.

- Einstellungen zu einer Handlung sind eine affektiv-bewertende Komponente bei der Absichtsbildung. Das heißt eine Einstellung bezieht sich darauf, ob der Betroffene das Durchführen einer Handlung für sich selbst als gut und günstig oder als schlecht und ungünstig bewertet. Solche Einstellungen zu einer Handlung setzen sich wiederum aus verschiedenen Teilkomponenten zusammen, so genannten Ergebnisbewertungen mit der jeweils zugehörigen Überzeugungsstärke. Wenn es beispielsweise darum geht, im Rahmen der Übergewichtsbehandlung einen Patienten dazu zu motivieren, mit Walking als Sport $\mathrm{zu}$ beginnen, so könnte der Gedanke an Walking verschiedenste Ergebnisbewertungen beim Patienten auslösen: Walking macht mich fit, das finde ich gut; Walking hilft mir beim abnehmen, das finde ich auch gut; beim Walking komme ich ins Schwitzen, das mag ich nicht; durch Walking wir meine knappe Freizeit noch weiter verplant, das finde ich auch nicht gut ... $\mathrm{Zu}$ jeder dieser Ergebnisbewertungen kommt dann noch eine Überzeugungsstärke, durch die ausgedrückt wird, wie sehr der Patient glaubt, dass das entsprechende Ergebnis tatsächlich eintritt. So kann sich der Patient vielleicht sehr wohl vorstellen, dass er ins Schwitzen kommt, dass seine Freizeit eingeschränkt wird, aber dass seine Fitness erhöht wird und dass das Walken beim Abnehmen hilft, das glaubt er vielleicht doch nicht so ganz. In der Summe überwiegen dann die negativen Ergebnisbewertungen und es ergibt sich eine insgesamt negative Einstellung zur Handlung. Um eine solche Einstellung zu verändern, müssen also mit dem Patienten mögliche positive Konsequenzen der Verhaltensänderung erarbeitet werden. Gleichfalls muss mit dem Patienten besprochen werden, warum es wahrscheinlich ist, dass die positiven Konsequenzen eintreten, damit der Patient eine entsprechende Überzeugung aufbauen kann. Umgekehrt muss erarbeitet werden, in wie fern, die befürchteten negativen Konsequenzen relativiert werden können.

- Subjektive Normen beziehen sich nicht auf die Bewertung einer Handlung durch den Betroffenen selbst, sondern darauf, welche Bewertung der Betroffene in seinem sozialen Umfeld für eine Handlung vermutet. Wenn beispielsweise ein Patient vermutet, dass er in seinem Umfeld auf Unverständnis oder gar Ablehnung stößt, wenn er in Zukunft weniger deftiges Essen bevorzugt, dann ist es eher unwahrscheinlich, dass sich der Patient eine solche Veränderung vornimmt. Daher kann es außerordentlich hilfreich für eine Verhaltensänderung sein, die möglichen Reaktionen wichtiger Bezugspersonen auf eine Verhaltensänderung abzuklären und gegebenenfalls Möglichkeiten $\mathrm{zu}$ erarbeiten, wie der Patient mit ablehnenden Reaktionen umgehen kann bzw. wie er unterstützende Reaktionen in seinem Umfeld fördern kann.

- Verhaltenskontrolle hat nicht nur Auswirkung auf die Durchführung einer Handlung, wie bereits oben diskutiert, sondern auch darauf, ob überhaupt eine entsprechende Verhaltensabsicht gebildet wird. Dabei umfasst Verhaltensabsicht sowohl objektive wie subjektive Aspekte. Mit objektiver Verhaltenskontrolle wird umschrieben, in wie fern eine gewünschte Handlung tatsächlich möglich ist. Wenn sich ein Patient vornimmt, als ersten Schritt zur Verhaltensänderung beim Fernsehen in Zukunft Radieschen anstelle von gerösteten Erdnüssen zu knabbern, dann setzt eine solche Absicht voraus, dass Radieschen im Haus sind bzw. dass Radieschen rechtzeitig eingekauft werden können. Der Vorsatz schwimmen zu gehen, setzt die räumliche und zeitliche Erreichbarkeit eines Schwimmbades voraus. Unter subjektiver Verhaltenskontrolle kann man verstehen, in wie fern sich der Patient die Durchführung einer Handlung zutraut bzw. welche Hemmnisse er bei der Durchführung befürchtet. Oft reichen bereits vermutete oder befürchtete Hindernisse und Schwierigkeiten, dass ein bestimmter Vorsatz gar nicht erst gefasst wird. Dies gilt auch dann, wenn sich bei der Handlung selbst herausstellen würde, dass die befürchteten Schwierigkeiten real gar nicht auftreten. So könnte eine Patientin beispielsweise befürchten, dass es bei einer Geburtstagsfeier im Büro nicht ak- 
zeptiert wird, wenn sie nur ein Stück Kuchen essen möchte. Sie hat Angst, von den Kolleginnen gedrängt zu werden, noch ein zweites oder gar drittes Stück zu essen. Um dieser unangenehmen Situation aus dem Weg zu gehen, nimmt sie sich erst gar nicht vor, es bei einem Stück Kuchen zu belassen. Wenn die Patientin im Rahmen der Ernährungsberatung dann lernt, in der Situation nach einem Stück Kuchen freundlich aber klar zu sagen, »danke, es schmeckt sehr lecker, aber ein Stück Kuchen reicht mir heute wirklich «, wird sie unter Umständen feststellen, dass das von den Kolleginnen problemlos akzeptiert wird und das befürchtete Aufdrängen gar nicht stattfindet. Im Rahmen einer Ernährungsberatung ist es daher sinnvoll, mit dem Patienten zu besprechen, welche möglichen Hindernisse und Schwierigkeiten er bei einer beabsichtigten Verhaltensänderung sieht, und wie diese Schwierigkeiten vermieden oder zumindest verringert werden können.

Die Überzeugung, eine gewünschte Handlung auch tatsächlich durchführen zu können, steht auch im Mittelpunkt zahlreicher anderer Modelle und Theorien der Verhaltenssteuerung. Am deutlichsten wurde dieser Gedanke zunächst in der sozial-kognitiven Lerntheorie von Bandura herausgearbeitet. Nach dieser Theorie ist die Durchführung einer Handlung von zwei Erwartungen

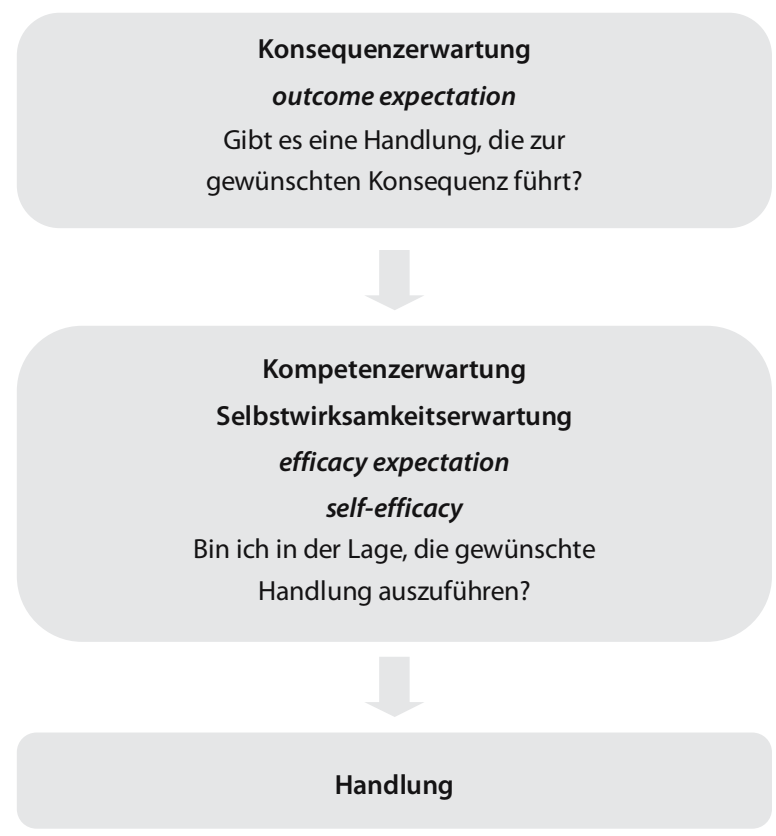

- Abb. 2.5. Grundüberlegungen der sozial-kognitiven Lerntheorie (Bandura) oder Überzeugungen abhängig (s. D Abb. 2.5): Der Konsequenzerwartung und der Kompetenz- oder Selbstwirksamkeitserwartung.

Die Konsequenzerwartung bezieht sich darauf, welche Überzeugung der Betroffene über die Konsequenzen einer Handlung hat. Eine typische Konsequenzerwartung wäre etwa: »Wenn ich meine Kalorienzufuhr einschränke, werde ich abnehmen «. Solche Konsequenzerwartungen können relativ einfach über Wissens- und Informationsvermittlung aufgebaut werden. Entscheidend für die Durchführung einer Handlung sind jedoch die Kompetenz- oder Selbstwirksamkeitserwartungen. Diese beziehen sich darauf, in wie fern sich der Betroffene die Durchführung der gewünschten Handlung auch tatsächlich zutraut. Solche Kompetenzerwartungen wirken sich darauf sowohl darauf aus, in welche Situationen man sich hinein begibt, als auch darauf, welche Mühe und Ausdauer man bei der Durchführung einer Handlung aufbringt. Je stärker ein Patient der Überzeugung ist, dass er es schaffen kann, beim abendlichen Fernsehen auf Bier und Salzstangen zu verzichten, umso höher ist die Wahrscheinlichkeit, dass er es versuchen wird und umso höher ist die Wahrscheinlichkeit, dass er es auch tatsächlich schafft. Daher kommt dem Aufbau von Kompetenz- oder Selbstwirksamkeitserwartungen eine Schlüsselrolle im Beratungsprozess zu. Grundsätzlich können Selbstwirksamkeitserwartungen auf verschiedene Art und Weise aufgebaut werden.

Der wirksamste und nachhaltigste Weg zur Selbstwirksamkeitserwartung ist die eigene, direkte Erfahrung. Wenn jemand die Erfahrung gemacht hat, dass er beim Fernsehen auf Bier und Salzstangen verzichten kann, wird sich dies auch in Zukunft sehr viel eher zutrauen. Direkte Erfahrung muss nicht unbedingt in realen Problemsituationen erworben werden. Auch Verhaltensübungen, Probehandlungen und Rollenspiele bieten die Möglichkeiten, die gewünschte Handlungen durchzuführen und damit durch direkte Erfahrung Kompetenzerwartungen aufzubauen. Ein zweiter, nicht ganz so effektiver Weg zum Aufbau von Kompetenzerwartungen ist die indirekte Erfahrung. Hierbei führt der Betroffene die Handlung nicht selbst durch, sondern er sieht bzw. erlebt, wie die gewünschte Handlung von einem anderen, dem so genannten Modell, durchgeführt wird. Dieser Weg wurde auch als Beobachtungslernen oder Modelllernen beschrieben. Die Wahrscheinlichkeit, dass auf diesem Weg Selbstwirksamkeitserwartung aufgebaut und das Modellverhalten übernommen wird, ist umso größer je stärker die Identifikationsmöglichkeit mit dem Modell ist. Indirekte Erfahrung kann durch das Beobachten von anderen Patienten in der Gruppe, durch das Beobachten von Personen im sozialen Umfeld aber auch durch den Einsatz von Medien wie Filmen vermittelt werden. Der dritte Weg zum Aufbau von Selbstwirksamkeitserwartungen kann als symbolische 
Erfahrung beschrieben werden. Hierbei wird die Handlungsdurchführung weder direkt selbst erlebt, noch indirekt bei anderen beobachtet. Vielmehr wird die erfolgreiche Handlungsdurchführung schriftlich oder mündlich (daher symbolisch) übermittelt; das heißt, man liest davon oder es wird davon erzählt. Dieser Modus dürfte in der typischen Beratungssituation der vorherrschende Modus zum Aufbau von Kompetenzerwartungen sein. Allerdings ist dieser Modus im Vergleich zu direkter und indirekter Erfahrung am wenigsten effektiv und nachhaltig im Aufbau von Selbstwirksamkeitsüberzeugungen.

\subsubsection{Ernährungsberatung als Trainingsprozess zur Verhaltensänderung}

Gelegentlich wird sich eine Ernährungsberatung darauf beschränken können, lediglich die individuelle Ernährungssituation abzuklären oder Information zu Sachfragen zu vermitteln. Der deutlich häufigere Fall wird jedoch sein, dass die Ernährungsberatung auf einen Veränderung des individuellen Ess- und Ernährungsverhaltens abzielt. Eine absichtliche, geplante Verhaltensänderung wiederum ist ein komplexer Prozess, insbesondere bei einem Verhaltensbereich der verschiedenste Handlungen umfasst, wie dies beim Essverhalten der Fall ist. Dieser Prozess kann als Abfolge verschiedener Schritte oder Stufen verstanden werden: Fehlendes Problembewusstsein, Problembewusstsein, Veränderungsbereitschaft, Verhaltensänderung und Verhaltensstabilisierung.

\section{Fehlendes Problembewusstsein}

Auf dieser Stufe ist der Patient mit seinem Verhalten zufrieden und hat daher auch keine Absicht, sein Verhalten $\mathrm{zu}$ ändern. Problembewusstsein setzt voraus, dass der Betroffene sein eigenes Verhalten zumindest teilweise als ungünstig bewertet. Daher können einem fehlenden Problembewusstsein mehrere Faktoren zugrunde liegen. Zum einen hat der Betroffene möglicherweise keinen Anlass, das eigene Verhalten kritisch zu bewerten. Dies wird dann der Fall sein, wenn sein Verhalten im Alltag überwiegend mit positiven Konsequenzen verbunden ist beziehungsweise wenn er mit seinem Verhalten weder auf subjektive Schwierigkeiten noch auf objektive Probleme stößt. Zum Beispiel könnte ein Patient seinen Obst- und Gemüseverzehr unhinterfragt lassen, solange er gesund ist und solange er weder in seinem Umfeld noch in den Medien mit der Einschätzung konfrontiert wird, dass er zu wenig Obst und Gemüse verzehrt. Eine andere Möglichkeit liegt darin, dass der Betroffen keine oder eine unzutreffende Information darüber hat, was eigentlich als günstiges oder wünschenswertes Verhalten anzusehen ist. So haben zwar die meisten Menschen zu gesunder Ernährung die spontane Assoziation »Viel Obst und Gemüse und wenig Fett «, aber Sie wissen nicht genau, was unter »viel Obst und Gemüse« zu verstehen ist. Maßnahmen der Ernährungsaufklärung wie die Kampagne "Fünf am Tag« zielen darauf ab, hier eine konkretere und zutreffende Zielvorstellung über das wünschenswerte Verhalten zu erzeugen. Ähnliches lässt sich oftmals hinsichtlich körperlicher Aktivität und Bewegung beobachten. Zwar weiß und akzeptiert nahezu jeder, dass Bewegung und körperliche Aktivität richtig und wichtig ist. Aber hinsichtlich der Frage, wie viel Bewegung und körperliche Aktivität wünschenswert ist, existieren oft keine richtigen oder genauen Vorstellungen. Eine dritte Möglichkeit, die fehlendem Problembewusstsein zugrunde liegen kann, ist eine unzutreffende Einschätzung und Bewertung des eigenen Verhaltens. In vielen Bereichen, des Gesundheitsverhaltens, ist eine Selbsteinschätzung des Verhaltens relativ einfach. Zum Beispiel bereitet es einem Patienten im Allgemeinen keinerlei Schwierigkeiten, sich selbst als Raucher oder Nichtraucher einzuschätzen. Auch beim Sport ist eine grobe Selbsteinschätzung, »treibe ich überhaupt keinen Sport, einmal die Woche oder mehrfach", noch relativ einfach möglich. Beim Ernährungsverhalten ist eine solche Selbsteinschätzung jedoch häufig schwierig. Zwar wissen die meisten Menschen, dass eine gesunde Ernährung fettarm sein sollte, jedoch bewerten auch die meisten Menschen, wie große Repräsentativstudien zeigen, die eigene Ernährung - im Gegensatz zu Ernährungsepidemiologen - bereits als fettarm. Für eine solche Fehleinschätzung spielt eine wichtige Rolle, dass Ernährungsempfehlungen nicht als »Alles oder Nichts«Empfehlungen formuliert werden können, sondern in der Regel als quantitative Empfehlungen gefasst sind (z. B. mindestens $30 \mathrm{~g}$ Ballaststoffe pro Tag, höchstens 30 Energieprozent Fett). Eine quantitative Selbsteinschätzung ist jedoch deutlich schwieriger zu treffen, als eine einfache Ja-Nein-Entscheidung. Dies trifft erst recht dann zu, wenn sich die quantitative Selbsteinschätzung nicht nur auf die Menge verzehrter Lebensmittel beziehen soll, sondern auf Inhaltsstoffe, die in den verzehrten Lebensmitteln enthalten sind. Während also eine Selbstbewertung hinsichtlich der verzehrten Obstund Gemüseportionen noch einigermaßen zuverlässig erfolgen kann, wird eine zutreffende Selbstbewertung des eigenen Fettkonsums oder der Ballaststoffaufnahme ohne sehr detaillierte Ernährungskenntnisse und entsprechende, zumindest überschlagsweise Berechnungen kaum möglich sein. Daher sollten Ernährungsempfehlungen in der Ernährungsberatung ebenso wie in der Ernährungsaufklärung stets lebensmittelorientiert und nicht nährstofforientiert erfolgen. Nährstofforientierte Ernährungsempfehlungen erschweren eine Selbstbewertung des eigenen Verhaltens beziehungsweise machen diese unmöglich. 


\section{Problembewusstsein}

Problembewusstsein bedeutet, dass der Patient eine Selbstbewertung des eigenen Verhaltens vorgenommen hat und dabei zu dem Ergebnis gekommen ist, dass das eigene Verhalten zumindest in der einen oder anderen Hinsicht als ungünstig einzuschätzen ist. Ein solches Problembewusstsein bedeutet jedoch keineswegs automatisch, dass eine Bereitschaft zur Veränderung des Verhaltens besteht. Sofern der Patient hinsichtlich verschiedener Aspekte seines Ess- und Ernährungsverhaltens noch nicht über ein ausreichendes Problembewusstsein verfügt, kann der Berater den Patienten durch geeignete Interventionen beim Aufbau eines Problembewusstseins unterstützen. Hierbei muss dem Patient eine zutreffende Selbstbewertung des Verhaltens ermöglicht werden. Die wichtigsten Maßnahmen hierzu sind: Analyse des gegenwärtigen Verhaltens, Information über die Nachteile des gegenwärtigen, ungünstigen Verhaltens, Information über verfügbare günstigere Verhaltensalternativen und Information über die Vorteile der möglichen Verhaltensalternativen.

\section{Veränderungsbereitschaft}

Veränderungsbereitschaft ist das Ergebnis eines motivationalen Prozesses, der auf der Stufe des Problembewusstseins einsetzt. Wesentliche Komponenten dieses Motivationsprozesses wurden bereits im $\$ Abschnitt 2.1.4 beschrieben. Nach der Theorie des geplanten Verhaltens sind zunächst eine überwiegend positive Einstellung zum gewünschten Verhalten und eine entsprechende Überzeugung von positiven Reaktionen des sozialen Umfelds (subjektive Norm) notwendig, damit die Absicht zu einer Verhaltensänderung gefasst wird. In der sozial-kognitiven Lerntheorie werden die entsprechenden Überzeugungen von überwiegend positiven Folgen einer Verhaltensänderung als Konsequenzerwartungen zusammengefasst. Somit kommt also der Einschätzung der Konsequenzen eine entscheidende Rolle für eine Veränderungsbereitschaft zu. In der Ernährungsberatung kann dieser Prozess unterstützt werden, indem mit dem Patienten gemeinsam die möglichen positiven wie negativen Folgen des gegenwärtigen Verhaltens sowie die positiven und negativen Folgen eines veränderten Verhaltens herausgearbeitet und abgewogen werden. Erst wenn der Patient für sich ein Überwiegen positiver Konsequenzen der Verhaltensänderung sieht, wird er eine entsprechende Bereitschaft entwickeln. Dabei muss der Berater berücksichtigen, dass die Einschätzung von Verhaltenskonsequenzen von drei Parametern beeinflusst wird:

1. Schweregrad der Konsequenz.

2. Überzeugungsstärke der Konsequenz.

3. Zeitlicher Abstand der Konsequenz.

1. Der Schweregrad der Konsequenz. Damit wird die Überzeugung davon bezeichnet, wie schwerwiegend eine Verhaltenskonsequenz für den Patienten ist. Unter sonst gleichen Bedingungen wird eine Verhaltenskonsequenz selbstverständlich umso größeren Einfluss auf das Verhalten haben, je schwerwiegender und bedeutsamer sie eingeschätzt wird. So wird die Aussicht, aufgrund unzureichenden Obst und Gemüseverzehrs anfälliger für Erkältungskrankheiten zu sein, sicherlich nicht dieselbe motivationale Wirkung haben, wie die Überzeugung, hierdurch anfälliger für Krebserkrankungen zu sein. Dieser Schweregrad spielt auch bei der Abwägung positiver und negativer Konsequenzen eine Rolle. So kann beispielsweise der Vorteil, Kartoffelchips beim Fernsehen ohne große Vorbereitung knappern zu können, von einem Patienten als bedeutsamer und schwerwiegender eingeschätzt werden als die Möglichkeit, durch diesen Konsum zu einer erhöhten Energie-, Fett- und Kochsalzzufuhr beizutragen. Allerdings wird die Bedeutung des Schweregrades durch die beiden anderen Parameter nachhaltig modifiziert.

2. Überzeugungsstärke der Konsequenz. Die Überzeugungsstärke drückt aus, für wie wahrscheinlich ein Patient die Möglichkeit hält, dass eine bestimmte Verhaltenskonsequenz bei ihm selbst tatsächlich eintritt. Hinsichtlich negativer Verhaltenskonsequenzen wird die Überzeugungsstärke auch oft als Vulnerabilität, Verletzbarkeit oder Anfälligkeit bezeichnet. Je geringer die Überzeugungsstärke ausgeprägt ist, umso weniger wird eine Verhaltenskonsequenz von Einfluss auf das Verhalten oder auf die Veränderungsbereitschaft sein. So mag ein adipöser Patient zwar einen Herzinfarkt sehr wohl für eine sehr schwerwiegende Verhaltenskonsequenz halten, aber dennoch davon überzeugt sein, dass er selbst trotz seiner Adipositas kaum gefährdet ist, einen Herzinfarkt zu erleiden. Oder eine Patientin fände es sehr bedeutsam, wenn sie sich nach einer Gewichtsabnahme attraktiver fühlen würde; sie kann sich aber nach vielen erfolglosen Abnahmeversuchen nicht vorstellen, dass ihr dieses tatsächlich gelingt. Die Überzeugungsstärke ist eine subjektive Überzeugung, die von der tatsächlichen Wahrscheinlichkeit zum Teil bedeutsam abweichen kann; zum Teil treten solche Abweichungen systematisch auf. Als optimistischer Fehlschluss (»optimistic bias«) wird das Phänomen bezeichnet, dass Menschen das eigene Gefährdungsrisiko normalerweise als geringer einschätzen als das von anderen Menschen, selbst wenn diese in fast jeder Hinsicht vergleichbar sind. So glauben Übergewichtige durchaus, dass Übergewicht im Allgemeinen zu Herz-KreislaufErkrankungen führen kann. Gleichzeit wird jedoch das Risiko, selbst daran zu erkranken, als geringer eingeschätzt als das von anderen Übergewichtigen. Überzeugungsstärken und Risikoeinschätzungen werden darüber hinaus häufig durch Emotionen beeinflusst. Wenn sich jemand in einer positiven Stimmungslage befindet, wird das Eintreten positiver Konsequenzen für wahrscheinlicher gehalten, wenn sich jemand in einer negativen Stimmung befindet, werden negative Verhaltenskonsequenzen als wahrscheinlicher eingeschätzt. 


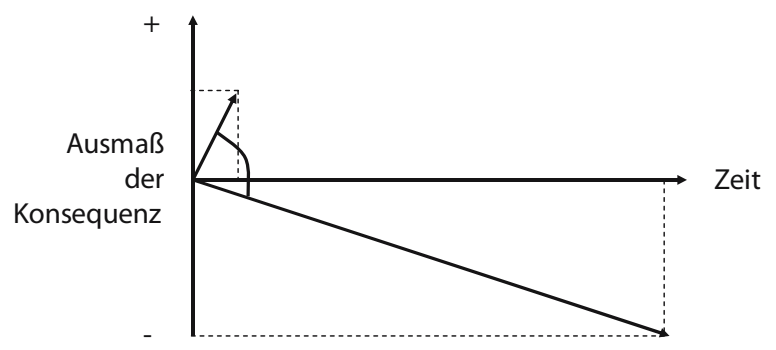

- Abb. 2.6. Einfluss von zeitlichem Abstand und Ausmaß der Konsequenz auf Verhalten: Entscheidend ist weder das absolute Ausmaß der Konsequenz noch der zeitliche Abstand allein, sondern das Produkt von Zeit und Ausmaß, dass durch den Winkel symbolisiert wird

3. Zeitlicher Abstand der Konsequenz. Je schneller eine Konsequenz auf ein Verhalten folgt, desto stärker beeinflusst sie das Auftreten des Verhaltens, je größer der zeitliche Abstand ist, umso geringer ist ihr Einfluss (s. - Abb. 2.6). Dieser Zusammenhang ist nicht nur für das Ess- und Ernährungsverhalten, sondern für viele Bereiche des Gesundheitsverhaltens von ausschlaggebender Bedeutung. Oft haben ungünstige Verhaltensweisen kurzfristig positive Konsequenzen, während die negativen gesundheitlichen Folgen erst mit einer deutlichen Zeitverzögerung auftreten. So mag ein Patient Herz-Kreislauferkrankungen, die aus zu fettreicher Ernährung resultieren können, sehr wohl als ausgesprochen negative Konsequenz bewerten, die jedoch weit in der Zukunft liegt. Andererseits mag der den geschmacklichen Vorteil, den er der fettreichen Ernährung zuschreibt gar nicht so hoch bewerten, jedoch setzt dieser Vorteil sehr schnell bzw. unmittelbar beim Essen ein. Daher kann die schnelle aber vergleichsweise unwichtige Konsequenz das Verhalten nachhaltiger beeinflussen als die bedeutsame aber erst später einsetzende Konsequenz.

Die komplexe Interaktion dieser Einflussfaktoren wird auch am Beispiel des Nikotinkonsums bei jungen Mädchen deutlich, der häufig in engem Zusammenhang mit dem Schlankheitsmotiv steht. Schlank zu sein bzw. schlank zu bleiben, ist eine Verhaltenskonsequenz, die dem Rauchen zugeschrieben wird und die als sehr wichtig und bedeutsam eingeschätzt wird. Selbstverständlich sind auch die gesundheitlichen Gefahren des Zigarettenkonsums bekannt. Aber sofern das entsprechende Gesundheitsrisiko bei der Betreffenden überhaupt eintritt (Überzeugungsstärke), wird dies erst in weiter Zukunft der Fall sein. Der Vorteil, schlank zu sein, wird dagegen sehr viel unmittelbarer erlebt und wird aufgrund von eigenen Erfahrungen und/oder Erfahrungen in der Peer-Group für sehr wahrscheinlich gehalten. Zusammenfassend wird Veränderungsbereitschaft umso eher aufgebaut, je besser es gelingt, herauszuarbeiten, dass eine Veränderung mit überwiegend positiven Konsequenzen einhergeht, die die mit hoher Wahrscheinlichkeit schnell eintreten und die subjektiv bedeutsam sind. Wie bereits im $~$ Kap. 2.5 dargestellt ist eine positive Konsequenzerwartung jedoch häufig nicht ausreichend, um eine Veränderungsbereitschaft zu erreichen. Entscheidend ist vielmehr, dass der Patient zu der Überzeugung gelangt, dass er in der Lage sein wird, die gewünschte Veränderung tatsächlich durchzuführen. Diese Überzeugung wird als Kompetenzerwartung oder Selbstwirksamkeitserwartung bezeichnet. Wie eine solche Überzeugung durch direkte, indirekte oder symbolische Erfahrung aufgebaut werden kann, wurde ebenfalls im $>$ Kap. 2.1.4 erläutert.

\section{Verhaltensänderung}

Wenn der Patient eine ausreichende Veränderungsbereitschaft aufgebaut hat, kann er dazu übergehen, diese Bereitschaft in erste Schritte der Verhaltensänderung zu übersetzen. Am Anfang wird diese Verhaltensänderung oft den Charakter eines Verhaltensversuchs haben, das heißt, der Patient "probiert das mal aus". Der Berater kann diesen Beginn dadurch unterstützen, indem er dem Patienten hilft, entsprechende Verhaltensabsichten $\mathrm{zu}$ formulieren. Wie in $>$ Kap. 2.1.4 dargestellt, wird die Umsetzung einer solchen Absicht umso wahrscheinlicher stattfinden, je konkreter und spezifischer das gewünschte Verhalten formuliert wird und je kurzfristiger die geplante Durchführung stattfinden soll. Zusätzlich kann die Verwirklichung der Verhaltensänderung dadurch erleichtert werden, dass zunächst solche Veränderungen angestrebt werden, die dem Patienten vergleichsweise leicht fallen (anders ausgedrückt: hinsichtlich derer er eine hohe Selbstwirksamkeitserwartung hat). Hierdurch wird insgesamt die Wahrscheinlichkeit für die erfolgreiche Durchführung der angestrebten Verhaltensänderung erhöht. Eine solche erfolgreiche Bemühung wird vom Patienten wiederum als positive Verhaltenskonsequenz und Belohnung erlebt werden und hat daher eine verstärkende Wirkung. Auch die Selbstwirksamkeitserwartung des Patienten wird durch einen solchen Erfolg verstärkt. Beides zusammen wird die zukünftige Durchführung des neuen Verhaltens begünstigen.Nach kurzer Zeit verliert jedoch die erfolgreiche Erprobung und Durchführung einer Verhaltensänderung ihren selbstverstärkenden Charakter. Auch die Selbstwirksamkeits- oder Kompetenzerwartung wird nach mehrfach erfolgreicher Durchführung einer Handlung nicht mehr wesentlich gesteigert.

Spätestens jetzt ist notwendig, dass das neue Verhalten auch konkret erlebbare positive Konsequenzen für den Patienten hat, da es sonst wieder aufgegeben wird. Sofern das neue Verhalten unmittelbar wahrnehmbare positive Konsequenzen hat, kann es in dieser Phase ausreichend sein, wenn der Berater die Aufmerksamkeit des Patienten auf diese positiven Konsequenzen lenkt, also den Patienten in einer positiven Selbstbewertung unterstützt. 
Wenn positive Folgen nicht unmittelbar erlebbar sind, ist es sinnvoll, wenn der Berater den Patienten dabei unterstützt, positive Verhaltenskonsequenzen gezielt herzustellen. Dies kann durch ein gezieltes Selbstbeobachtungsund Selbstverstärkungsprogramm geschehen. Dabei legt der Patient für sich fest, wie häufig er das gewünschte Verhalten in einem bestimmten Zeitraum durchführen will (Zielvereinbarung), protokolliert dann, wie häufig er das Verhalten tatsächlich durchführt (siehe Abschnitt Selbstbeobachtung weiter unten) und belohnt sich selbst mit etwas, was er sich wünscht oder was ihm angenehm ist (siehe Verstärkung weiter unten), wenn er das festgelegte Ziel erreicht hat.

Selbstverständlich kann auch soziale Unterstützung und Anerkennung aus dem Umfeld des Patienten die Aufrechterhaltung der Veränderung unterstützen. Hierzu kann mit dem Patienten überlegt werden, welche Personen aus seinem Umfeld eine solche Unterstützung bieten können. Schließlich hat aber auch der Berater selbst eine wichtige verstärkende Funktion, indem er dem Patienten positive Rückmeldungen zu seinen Bemühungen und Erfolgen gibt. In dieser Phase der Verhaltensänderung muss das neue Verhalten immer wieder durch eine bewusste, kognitive Steuerung eingeleitet und aufrechterhalten werden, da es noch nicht als quasi-automatischer Handlungsablauf verinnerlicht ist.

Erst wenn eine Handlung mehrfach durchgeführt wurde, bildet sich im Gedächtnis ein eigenes Erinnerungsmuster, das den Handlungsablauf zukünftig steuern kann. In der Gedächtnispsychologie wird auf dieses Phänomen mit der Unterscheidung verschiedener Bereiche oder Prozesse im Langzeitgedächtnis Bezug genommen. Auf der einen Seite gibt es das so genannte deklarative Gedächtnis, das für die Speicherung von Wissen und Gedächtnisinhalten zuständig ist, die im weitesten Sinn benannt, erzählt oder erklärt werden können. Hierzu zählen insbesondere das Sachwissen über Begriffe und die Zusammenhänge zwischen diesen Begriffen (semantisches Gedächtnis) als auch die Erinnerung an eigene Erlebnisse mit biographischem Bezug (episodisches Gedächtnis). Das Wissen über gesunde Ernährung, günstiges Essverhalten oder allgemein einen gesunden Lebensstil wäre somit dem deklarativen Gedächtnis bzw. dem Teil des semantischen Gedächtnisses zuzuordnen. Sofern eine Handlung aus dem deklarativen bzw. semantischen Gedächtnis gesteuert werden soll, ist ein erheblicher Denk- und Verhaltensaufwand notwendig. Dies ist zum Beispiel dann der Fall, wenn jemand ein neues, im bislang unvertrautes Elektrogerät anhand der Bedienungsanleitung in Betrieb nehmen will (Beispielsweise erste Programmierung eines neuen Videorekorders). In diesem Fall entspricht die Bedienungsanleitung dem semantischen Wissen, das eine Schritt für Schritt Anweisung hinsichtlich der durchzuführenden Handlungsschritte enthält. Diese werden dann langsam abgearbeitet, indem immer wieder nachgelesen wird, was als nächstes zu tun ist. Ähnlich verhält es sich, wenn jemand ein neues Rezept anhand eines Kochbuchs "nachkocht«.

Ein solcher kognitiver »Steuerungsaufwand « ist jedoch psychologisch außerordentlich unökonomisch und belastend. Daher werden gewohnheitsmäßige Verhaltensmuster normalerweise nicht durch die schrittweise Abarbeitung von deklarativen Gedächtnisinhalten gesteuert. Hierfür ist vielmehr ein Teil des Langzeitgedächtnisses zuständig, der als prozedurales Gedächtnis bezeichnet wird. Im prozeduralen Gedächtnis werden komplette Handlungsabläufe, Verhaltensketten und psycho-motorische Fertigkeiten gespeichert. Der Unterschied zwischen Verhaltenssteuerung durch das deklarative Gedächtnis und das prozedurale Gedächtnis wird beispielsweise in der ersten praktischen Auto-Fahrstunde besonders augenfällig.

In der Regel verfügt der Fahrschüler zu diesem Zeitpunkt bereits über ausreichendes Wissen über Verkehrsregeln, die Bedienelemente und - theoretisch - über das Wissen, wie er mit dem Auto anfahren kann. Oft werden jedoch bei den ersten Versuchen, dieses deklarative Wissen in eine Handlung umzusetzen, der Motor abgewürgt oder der Motor heult laut auf. Erst nach einiger Übungszeit wird das deklarative Wissen in eine prozedurale Fertigkeit übersetzt, die dann ohne bewussten, kognitiven Steuerungsaufwand abrufbar ist. Völlig analoges gilt, wenn es darum geht, eine neue Sportart oder das Spielen eines Musikinstruments zu erlernen. In der Tat gibt es viele Fertigkeiten, die im prozeduralen Gedächtnis gespeichert sind, für die überhaupt kein entsprechender deklarativer Gedächtnisinhalt mehr existiert (z. B: Fahrrad fahren: wie erklärt man jemanden, wie er das Gleichgewicht halten soll? Oder schwimmen: wie kann man das einem Nichtschwimmer erklären?). Praktische Übungsprozesse, also die mehrfache Durchführung einer Handlung, sind der einzige Weg, auf dem solche prozeduralen Steuerungsinhalte erworben werden können (s. $\mathbf{0}$ Abb. 2.7).

Auf diesem Hintergrund kann also Ernährungsberatung in der Phase der Verhaltensänderung als Übungsoder Trainingsprozess beschrieben werden, in der der Ernährungsberater eine ähnliche Funktion hat wie der

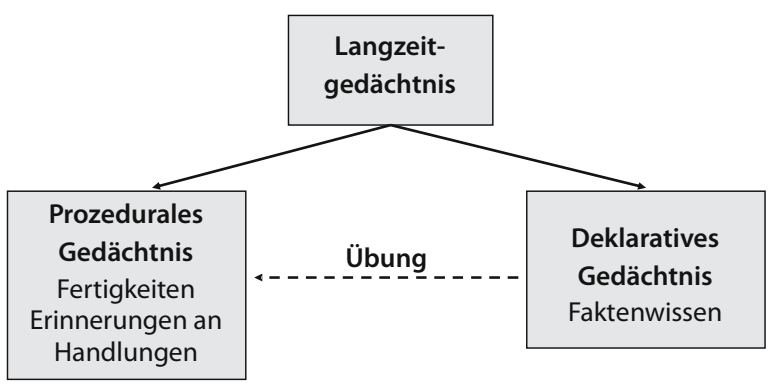

- Abb. 2.7. Prozedurales Gedächtnis und deklaratives Gedächtnis als Teile des Langzeitgedächtnis 
Fahrlehrer, Sporttrainer oder Musiklehrer. Diese Funktion beinhaltet zum einen, die Vermittlung neuer Handlungsabläufe. Vor allem aber kommt es darauf an:

- den Trainingsprozess durch einen geschickten Aufbau und eine geeignete Abfolge von Übungen und Trainingsschritten zu optimieren,

- den Betroffenen in seinem Training zu beobachten,

- um ihm gezielte Rückmeldungen zu den erreichten Fortschritten und zu weiteren Verbesserungsmöglichkeiten zu geben, und

- den Betroffenen durch Vermittlung von Erfolgserlebnissen und durch die soziale Beziehung zum weiteren Training zu motivieren.

\section{Verhaltensstabilisierung}

In der Phase der Verhaltensstabilisierung hat der Patient ein neues Ernährungsverhalten bzw. Elemente davon bereits erlernt und praktiziert es seit einiger Zeit. Die Erfahrung in der Ernährungsberatung allgemein und in der Übergewichtsbehandlung im Besonderen zeigt, dass solche Verhaltensänderungen jedoch oft nur von kurzer Dauer sind. Der Patient fällt im Lauf der Zeit in seine alten Gewohnheiten zurück. Daher besteht eine der größten Herausforderungen in der Beratung darin, erfolgreiche Verhaltensänderungen $\mathrm{zu}$ stabilisieren und Rückfällen vorzubeugen.

Rückfälle in alte Verhaltensmuster geschehen selten unerklärlich und spontan. Sie haben vielmehr in der Regel eine systematische Vorgeschichte (s. Abb. 2.8). Oft beginnt die Kette von Ereignissen, die letztlich zum Rückfall

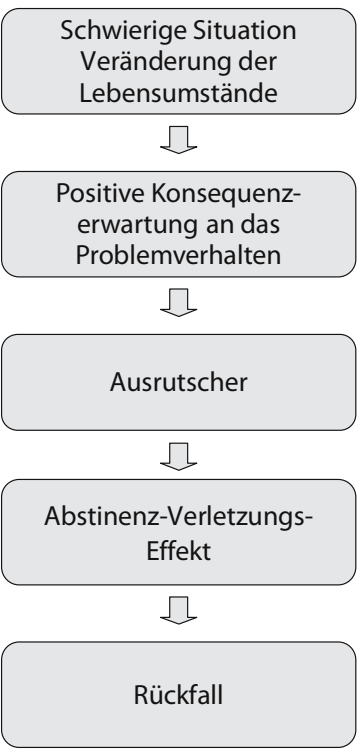

- Abb. 2.8. Ereigniskette, die zum Rückfall in alte Verhaltensmuster führt in altes, ungünstiges Verhalten führt, mit einer Veränderung der Lebensumstände oder mit dem Auftreten von schwierigen Situationen im Leben des Patienten. Dies können sowohl mehr oder weniger einschneidende kritische Lebensereignisse sein als auch die Summierung von alltäglichen kleinen Ärgernissen. Der Patient verfügt nicht über leicht abrufbare und erprobte Handlungsmuster zur Bewältigung dieser Situationen, was eine Stressreaktion zur Folge hat. Die Anforderung, eine solche Situation erfolgreich zu bewältigen, nimmt die Verarbeitungs- und Bewältigungskapazität des Patienten ebenso in Anspruch wie das Bemühen, die erreichte Verhaltensänderung beizubehalten. Da die verfügbare Bewältigungskapazität insgesamt begrenzt ist, wird die Belastung des Patienten in der Situation umso stärker ausfallen, je mehr Bemühungen es von ihm erfordert, das neue Verhalten aufrecht zu erhalten. Diese doppelte Belastung wiederum aktiviert die Erinnerung an frühere Stresssituationen, in denen der Patient noch nicht durch seine Verhaltensänderung zusätzlich belastet war. Das alte Verhalten erscheint ihm jetzt in einem wesentlich positiveren Licht. Es entsteht eine positive Konsequenzerwartung, also die Überzeugung, dass mit dem alten Problemverhalten, beispielsweise mit dem Essen von Süßigkeiten oder fettreichen Lebensmitteln, die derzeitige Situation viel leichter oder besser ertragen werden kann. Gleichfalls hat der Patient aus früherer Erfahrung eine positive Selbstwirksamkeitserwartung im Hinblick auf das Problemverhalten, d. h. er weiß, dass er in der Lage ist, das alte Verhalten durchzuführen, wie er es ja früher oft gemacht hat. Im Gegensatz dazu wird die Selbstwirksamkeitserwartung im Hinblick auf das neue Verhalten aufgrund der geringeren Erfahrung damit noch nicht so stark ausgeprägt sein.

Ein solches Erwartungsmuster führt zu einem »Ausrutscher«. Der Patient gibt das neue Verhalten zunächst einmal auf und realisiert sein altes Problemverhalten. Ein solcher Ausrutscher hat zunächst einen sehr kurzfristigen und einmaligen Charakter. Er wäre damit nicht weiter problematisch, wenn der Patient einen solchen Ausrutscher als Ergebnis einer vorübergehenden Überlastung verarbeiten würde und anschließend sein neues, günstigeres Ernährungsverhalten fortsetzt. Häufig wird ein solcher Ausrutscher jedoch völlig anders verarbeitet. Der Ausrutscher wird von »ich habe es nicht geschafft" als eigenes Versagen verarbeitet und beeinträchtigt in der Folge die Selbstwirksamkeitserwartung des Patienten.

Hinzu kommt, dass Verhaltensänderungen und Fortschritte oft als ein Prozess missverstanden werden, der geradlinig verläuft und bei dem es nur »entweder-oder « gibt. Entweder man hat einen bestimmten Fortschritt erzielt, hat eine bestimmte Hürde genommen und »ist damit durch«, oder eben nicht. Auf dem Hintergrund einer solchen Einstellung wird der Ausrutscher als Indiz dafür gewertet, dass man die Verhaltensänderung eben noch nicht geschafft hat. Diese Bewertung wiederum lässt jede 
weitere Anstrengung, die Verhaltensänderung beizubehalten, sinnlos erscheinen. "Jetzt ist es eh egal«, ist dann die spontane Reaktion auf ein solches Ereignis. Anstatt also mit verstärkten Bemühungen um die Beibehaltung der Verhaltensänderung zu reagieren, werden diese Bemühungen vermindert und aufgegeben. Da ein solcher Effekt zuerst bei Alkoholikern beschrieben wurde, die all ihre Kontrollbemühungen aufgeben, wenn sie die erreichte Abstinenz durch erneuten Alkoholkonsum verletzen, wird dieser Effekt auch als Abstinenz-Verletzungs-Effekt bezeichnet. Erst durch diese Prozesse, verminderte Selbstwirksamkeitserwartung und nachfolgendes Aufgeben der Kontrollbemühungen, kommt es beim Patienten dann schließlich zu einem längeranhaltenden oder dauerhaften Rückfall in alte Gewohnheiten.

\section{Rückfallprophylaxe}

Auf dem Hintergrund der Ereigniskette, die zum Rückfall führt, können in der Phase der Verhaltensstabilisierung Maßnahmen zur Rückfallprophylaxe konzipiert und in die Beratungspraxis integriert werden. Hierzu gehört zunächst einmal, dass der Patient mit dem üblichen Ablauf des Rückfallprozesses, wie beschrieben, vertraut gemacht wird. Schließlich sollten mit ihm für jede Stufe diese Rückfallprozesses geeignete Strategien erarbeitet und eventuell eingeübt werden.

Im Hinblick auf den Beginn der Ereigniskette, Veränderung der Lebensumstände und Auftreten von Schwierigkeiten, ist es hilfreich, wenn der Berater mit dem Patienten erarbeitet, welche Schwierigkeiten und Veränderungen in der nächsten Zukunft erwartet werden können. Falls solche Situationen absehbar sind, sollte mit dem Patienten besprochen werden, wie er mit diesen Situationen am besten umgehen kann und wie er sein neues Ernährungsverhalten trotz der schwierigen Situation am besten beibehalten kann. Möglicherweise wäre es zur Bewältigung solcher Situationen sinnvoll und hilfreich, wenn sich der Patient zusätzliche Ressourcen und Kompetenzen, wie beispielsweise Stressbewältigungstechniken, verbesserte Kommunikationsfähigkeit und soziale Kompetenz oder effektivere Problemlösungsstrategien aneignet. Die Vermittlung solcher Kompetenzen, für die es jeweils erprobte verhaltenstherapeutisch Programme und Standardbausteine gibt, wird jedoch in der Regel die Möglichkeiten in der Ernährungsberatung überschreiten. Hier sollte der Patient auf konkrete, weiterführende Beratungs-, Trainings- oder Therapiemöglichkeiten verwiesen werden. Oft bieten Volkshochschulen oder ähnliche Institutionen vor Ort entsprechende Kurse an. Ergänzend sollte sich der Berater einen Überblick über lokal und regional verfügbare verhaltenstherapeutische Angebote verschaffen.

Ein zweites Element der Rückfallprophylaxe auf dieser Stufe ist es, mit dem Patienten zu besprechen, wie er mer- ken kann, dass er sich in einer Situation befindet, die ihn für Rückfälle anfällig macht. Bei Patienten mit gestörtem Essverhalten und/oder Gewichtsproblemen machen sich solche Situationen oft dadurch bemerkt, dass der Patient anfängt, wieder häufiger über das Thema Essen nachzudenken. Dies kann dann ein solches Ausmaß annehmen, dass seine Gedanken ständig um das Thema Essen kreisen. Ein anderes Warnsignal kann es sein, wenn der Patient anfängt sich zu häufig oder gar nicht mehr zu wiegen. Sowohl ein phobisches Vermeidungsverhalten gegenüber der Waage und dem eigenen Gewicht als auch ein zwanghaft-obsessives Häufig-Wiegen (mehrmals pro Tag) sind Kennzeichen einer unrealistischen und wenig hilfreichen Haltung gegenüber dem eigenen Gewicht.

In Studien hat sich gezeigt, dass die Selbstkontrolle des Körpergewichts durch Wiegen eine der wichtigsten Strategien zur langfristigen Stabilisierung des Körpergewichts ist. Eine bewährte Strategie zur Rückfallprophylaxe ist es daher, dem Patienten zu empfehlen, sich einmal pro Woche, möglichst unter vergleichbaren Umständen, z. B. sonntags vor dem Frühstück, zu wiegen. Wenn das Gewicht des Patienten relative unstabil ist und häufige Schwankungen aufweist, sollte der Patient dieses Gewicht in einer Gewichtskurve eintragen. Wenn das Gewicht relativ stabil ist, muss die nicht unbedingt geschehen. Wenn das Gewicht bedeutsam, z. B. um 2 kg oder mehr, gegenüber einem festgelegten Gewicht, z. B. dem Gewicht am Ende der Beratung oder Therapie angestiegen ist, sollte der Patient versuchen, durch eine verstärkte Selbstkontrolle des Ess- und Bewegungsverhaltens wieder auf das Zielgewicht zurückzukommen. Diese Schwelle kann in einer Gewichtskurve durch eine gelbe Linie (»Alarmstufe Gelb!«) symbolisiert werden. Gleichfalls sollte eine weitere Gewichtsschwelle festgelegt werden, ab der der Patient erneut die Unterstützung durch die Ernährungsberatung in Anspruch nehmen sollte. Diese Gewichtsschwelle, etwa $5 \mathrm{~kg}$ über dem Zielgewicht, kann durch eine rote Linie (»Alarmstufe Rot») in einer Gewichtkurve sichtbar gemacht werden. Die gemeinsame Besprechung solcher Grenzwerte kann dazu beitragen, die Scham- und Schuldgefühle des Patienten zu reduzieren, die nach einer Gewichtszunahme oft eine erneute Inanspruchnahme von Unterstützung verhindern.

Als drittes Element der Rückfallprophylaxe für diese Stufe des Rückfall-Prozesses haben sich schließlich auch regelmäßige Nachbetreuungstermine bewährt. In strukturierten Kurs- und Therapieangeboten wird dies zum Teil dadurch realisiert, dass die Behandlungsfrequenz schrittweise reduziert und die Intervalle verlängert werden. Während der Hauptphase der Behandlung finden Sitzungen oft einmal pro Woche statt, in einer ersten Nachbetreuungsphase dann nur noch einmal alle zwei Woche, dann einmal pro Monat, schließlich einmal pro Viertel- oder Halbjahr. Für die Langzeitbetreuung wären dann jährliche Nachsorgetermine denkbar. Sol- 
che Nachbetreuungstermine müssen nicht unbedingt in Form eines persönlichen Beratungsgesprächs von Angesicht zu Angesicht stattfinden, sondern können zum Teil auch sehr effektiv durch Telekommunikation wie Telefon, Postkartenkontakt oder Internet- bzw. Email-Kontakt ersetzt werden. Neben ihrer immensen Bedeutung für die Rückfallprophylaxe bietet die langfristige Nachbetreuung des Patienten eine gute Möglichkeit die Effektivität der Beratung auch langfristig zu dokumentieren und zu evaluieren.

Für die Stufe der positiven Konsequenzerwartung, wenn also der Patient bereits wieder darüber nachdenkt, wie schön oder unkompliziert sein altes Ernährungsverhalten war, sollte der Patient lernen, solche Gedanken kritisch zu hinterfragen und als irrational zu erkennen. Lebensprobleme und Schwierigkeiten, gleich welcher Art, werden in aller Regel durch ein ungünstiges Ess- und Ernährungsverhalten nicht verbessert sondern eher erschwert. Manche Patienten empfinden es als hilfreich, wenn sie für solche Situationen im Rahmen der Beratung einen »Erste-Hilfe-Brief" an sich selbst schreiben (s. Exkurs »Anleitung für einen Erste-Hilfe-Brief«).

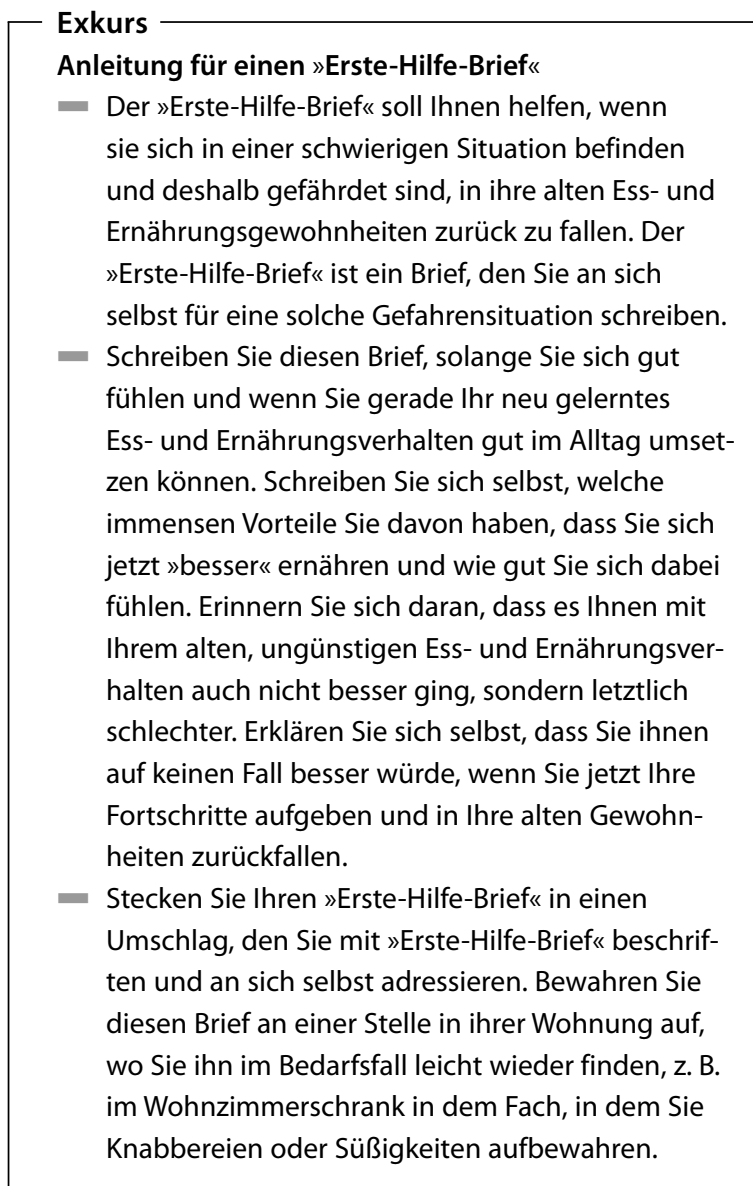

Für den Fall, dass es trotz aller Rückfallprophylaxe zu einem Ausrutscher kommt, sollte der Patient darauf eingestellt sein, dass ein einzelner Ausrutscher für sich genommen kein großes Problem darstellt. Entscheidend ist nicht, was bei einem Ausrutscher geschieht, sondern was nach einem Ausrutscher geschieht, wie also der Ausrutscher verarbeitet wird. Günstig ist es, wenn sich der Patient klar macht, dass der Ausrutscher als ein vorübergehendes Problem verstanden wird, das im Rahmen eines Lernprozesses auftreten kann. Ein solches Problem kann für den Lernprozess förderlich sein, wenn verstanden wird, was den Ausrutscher begünstigt hat und wenn der Patient aus dieser Erfahrung Konsequenzen für zukünftige, ähnliche Ereignisse ziehen kann (»Aus Fehlern lernen«). Wenig hilfreich ist es, wenn der Patient einen Ausrutscher einfach als unbedeutend verharmlost und nicht versucht, Konsequenzen daraus ziehen. Ebenso wenig ist es hilfreich, wenn der Patient stattdessen eine »jetzt ist es eh egal«-Reaktion zeigt. Vielmehr sollte er den Ausrutscher als eindeutigen Hinweis auf Schwierigkeiten verstehen und verarbeiten, die verstärkte Bewältigungsanstrengungen notwendig machen. Wenn es dem Patienten gelingt, Ausrutscher erfolgreich zu bewältigen, wird dies seine Selbstwirksamkeitserwartung bzw. seine Kompentenzerwartung stärken und dazu beitragen, dass er zukünftige Schwierigkeiten leichter meistern kann.

Letztlich sollte mit dem Patienten aber auch angesprochen werden, dass es im Rahmen einer Verhaltensänderung vorkommen kann, dass er das neue Verhalten wieder dauerhaft aufgibt und in alte Gewohnheiten zurückfällt. Der Patient sollte ermutigt werden, in einem solchen Fall nicht mit unproduktiven Schuld- und Schamgefühlen $\mathrm{zu}$ reagieren, sondern stattdessen erneute Hilfe und Unterstützung durch eine erneute Beratung und/oder Therapie in Anspruch nehmen: Eine dauerhafte Verhaltensänderung wird durch die erfolgreiche Bewältigung von Rückfällen letztlich wahrscheinlicher gemacht. Für den Patienten wird der Schritt, erneut Hilfe in Anspruch zu nehmen, dadurch erheblich erleichtert, dass der Berater mit ihm klare Kriterien festlegt, wann eine erneute Beratung in Anspruch genommen werden sollte. Von vornherein vereinbarte Nachsorgetermine können auch dazu dienen, Rückfallsituationen zu erkennen und erneut zu intervenieren, falls der Patient nicht von sich aus mit dem Berater Kontakt aufgenommen hat.

\subsubsection{Prozessmodell der Ernährungsberatung}

Naturgemäß wird eine Ernährungsberatung in Abhängigkeit von der Ausgangssituation des Patienten und von der damit verbundenen Zielsetzung sehr unterschiedlich verlaufen. Dennoch lässt sich ein allgemeines Rahmenmodell skizzieren, das den planmäßigen Aufbau und Ablauf der Ernährungsberatung strukturiert. Sinnvoll ist es dabei, die Ernährungsberatung als Prozess zu verstehen, dessen weiterer Verlauf vom bisherigen Verlauf abhängig ist. Die Grundstruktur für ein solches Prozessmodell ist in - Abb. 2.9 dargestellt. 


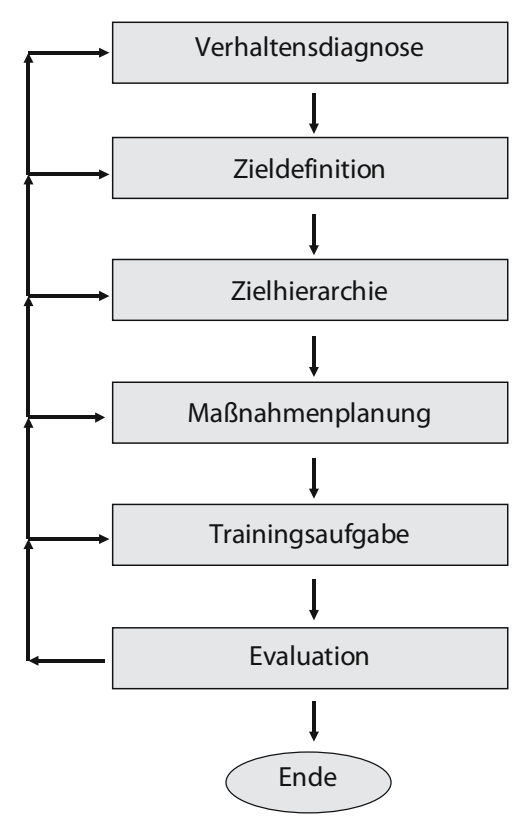

- Abb. 2.9. Prozessmodell der Ernährungsberatung

$\mathrm{Zu}$ Beginn einer Ernährungsberatung muss zunächst die zwischenmenschliche und kommunikative Basis für eine vertrauensvolle Zusammenarbeit zwischen Patient und Berater geschaffen wird. Die Beziehung zwischen Patient und Berater kann als Arbeitsbündnis charakterisiert werden, das zum einen durch gegenseitige Wertschätzung charakterisiert ist, zum andern dadurch, dass es dem Berater gelingt, die Situation und Schwierigkeiten des Patienten einfühlsam zu verstehen und dem Patienten auch das Gefühl zu vermitteln, verstanden zu werden. Die Arbeit an der Aufrechterhaltung und am weiteren Ausbau dieses Arbeitsbündisses wird den weiteren Beratungsprozess begleiten.

\section{Verhaltensdiagnose}

Neben diesem Beziehungsaspekt wird am Beginn der Beratung der erste Schwerpunkt darauf zu legen sein, die Ernährungssituation des Patienten zu klären und Veränderungsmöglichkeiten und -notwendigkeiten zu erfassen. Dieser erste Schritt der Beratung kann als Verhaltensdiagnose umschrieben werden. In der Verhaltensdiagnose wird das relevante Ess- und Ernährungsverhalten beschrieben. Hierzu werden die im $\bullet$ Kap. 1.2 beschriebenen Verfahren der Ernährungserhebung eingesetzt, soweit sie für die Beratungssituation geeignet sind. Beispielsweise wird in einem Explorationsgespräch die Vorerfahrung und subjektive Einschätzung des Patienten hinsichtlich seines Ernährungsverhaltens festgestellt. Anschließend können mit einem Ernährungstagebuch Daten zur Nahrungsaufnahme des Patienten erhoben werden. Je nach
Art des verwendeten Protokolls werden dabei nicht nur die verzehrten Speisen und Getränke notiert, sondern auch die typischen Verzehrssituationen und ihre Begleitumstände. Auf der Basis einer solchen Erhebung kann die Energie- und Nährstoffzufuhr berechnet werden. Im Rahmen der Verhaltensdiagnose kann das Ausmaß des gezügelten Essverhaltens und der Störbarkeit des Essverhaltens mit dem Fragebogen zum Essverhalten festgestellt werden. Zusammenfassend lassen sich diese Elemente der Verhaltensdiagnose als Beschreibung des Ist-Zustandes des Ess- und Ernährungsverhaltens charakterisieren. Diese Beschreibung des Ist-Zustandes wird durch einen Vergleich mit dem Soll-Zustand komplettiert. Hierdurch können Abweichungen von der wünschenswerten Energie- und Nährstoffzufuhr, Abweichungen von einer gesunden Lebensmittelauswahl und Diskrepanzen zu einem günstigen Essverhalten ebenso festgestellt werden, wie die Anteile des Ess- und Ernährungsverhaltens, die dem Patienten als günstig zurückgemeldet werden können. Das Ergebnis der Verhaltensanalyse ist also ein Ist-Soll-Vergleich, der beizubehaltende und zu verändernde Aspekte des Ess- und Ernährungsverhaltens identifiziert.

\section{Zieldefinition und Zielvereinbarung}

Nicht alle Abweichungen des tatsächlichen Ernährungsverhaltens von gesundem und wünschenswertem Ernährungsverhalten müssen jedoch zwangsläufig Gegenstand der weiteren Ernährungsberatung werden. Auf der Grundlage der Verhaltensdiagnose müssen in einem weiteren Schritt der Zieldefinition zwischen Beratung und Patient gemeinsam die Ziele festgelegt werden, die im weiteren Beratungsprozess angestrebt werden. Hierbei sollten, kognitive, sozio-emotionale und pragmatisch-verhaltensorientierte Lernziele, wie in $>$ Kap. 2.1.1 beschrieben, unterschieden werden. Dabei ist es die Verantwortung des Beraters, darauf zu achten, dass diese Ziele geeignet sind und in einer geeigneten Form formuliert werden. Als Checkliste für eine geeignete Zielformulierung bietet es sich die Formel an, "Ziele müssen SMART sein.« Jeder Buchstabe des Wortes SMART symbolisiert eine Anforderung an sinnvolle Ziele: - $\mathrm{S}=$ spezifisch: Ziele müssen in sofern spezifisch sein, dass mit der Formulierung nachvollziehbar wird, ob ein bestimmtes Ziel erreicht ist oder nicht. Das Ziel »ich möchte gesünder essen « ist sehr unspezifisch. Es lässt sich im Einzelfall eigentlich nicht nachvollziehbar feststellen, ob dieses Ziel erreicht ist oder noch nicht. Das Ziel »ich möchte am Tag 5 Portionen Obst oder Gemüse essen « ist hingegen sehr spezifisch. Hier gibt es jedoch auch graduelle Abstufungen. Das Ziel "weniger Fett essen" ist noch relativ unspezifisch, das Ziel »weniger als 30 Prozent der Nahrungsenergie durch Fett essen« ist zwar für den einzelnen Patienten schwer zu überprüfen, aber vergleichsweise spezifisch. 
- $\mathbf{M}=$ messbar: Ziele müssen messbar sein. Jedes Ziel, für das nicht angegeben werden kann, wie der Grad der Zielerreichung gemessen werden kann, ist kein geeignetes Ziel. Das Beratungs- oder Therapieziel, »sich attraktiver fühlen" ist erst dann ein sinnvolles Beratungsziel, wenn angegeben wird, durch welches Verfahren oder Messinstrument die gefühlte Attraktivität gemessen werden kann bzw. soll. Hinsichtlich des Ernährungsverhaltens können die Parameter als Zielkriterium verwendet werden, die durch ein Verfahren der Ernährungserhebung ausreichend valide erfasst werden können. Auch Veränderungen des Körpergewichts als Ziel erfüllen ganz offensichtlich den Anspruch der Messbarkeit.

- A = anspruchsvoll: Ziele, die festgelegt werden sollten in sofern anspruchsvoll sein, dass ihr Erreichen nicht von vornherein feststeht. Ein definiertes Ziel sollte, zumindest eine moderate Herausforderung sein und entsprechende Anstrengungen erfordern. Kaum jemand wird das Erreichen eines Ziels, das absehbar ohne nennenswerten Aufwand erreichbar war, als Selbstbestätigung verarbeiten können.

- $\mathbf{R}=$ realistisch: Auch wenn Ziele anspruchsvoll sein sollen, dürfen sie nicht so anspruchsvoll sein, dass sie eigentlich kaum noch erreicht werden können. Es gilt also immer, die richtige Balance zwischen anspruchsvoller Herausforderung und realistischen Möglichkeiten zu finden. Gerade hinsichtlich Veränderungen von Ernährung und/oder Gewicht besteht eine große Gefahr, dass sich Patienten unrealistisch hohe Ziele setzten, z. B. was das Ausmaß oder die Geschwindigkeit einer angestrebten Gewichtsreduktion anbelangt. Daher ist es wichtig, dass der Berater die realistische Erreichbarkeit von Beratungszielen vor dem Hintergrund seines Expertenwissens beurteilt. Der Patient sollte ermutigt werden, die realistische Erreichbarkeit vor dem Hintergrund seiner Vorerfahrung und im Hinblick auf seine konkreten Möglichkeiten zu beurteilen.

- $\mathbf{T}=$ terminlich festgelegt: Ziele, deren gewünschtes Erreichen nicht zeitlich festgelegt werden, werden oft entweder zu spät und/oder gar nicht mehr erreicht. Eine andere Gefahr besteht gelegentlich darin, dass $\mathrm{zu}$ einem falschen oder auch $\mathrm{zu}$ einem $\mathrm{zu}$ frühen Zeitpunkt zuviel Anstrengung für die Zielerreichung aufgewendet wird.

Die Beratungsziele, die entsprechend dieser Anforderungen formuliert und zwischen Berater und Patient gemeinsam vereinbart werden, sollten schließlich in einer Liste festgehalten werden. Es kann für den Beratungsprozess außerordentlich unterstützend sein, wenn eine solche schriftliche Zielvereinbarung dann von Berater und Patient als Zeichen der Übereinstimmung und Vereinbarung gemeinsam unterschieben werden. Die schriftliche Ziel- vereinbarung sollte nicht nur zur weiteren Gestaltung des Beratungsprozesses zu den Unterlagen des Beraters genommen werden, sondern eine Exemplar bzw. eine Kopie sollte auch dem Patienten mitgegeben werden.

Die schriftliche Zielvereinbarung, die als Ergebnis der Zieldefinition resultiert wird häufig eine ganze Reihe von angestrebten »Veränderungszielen « umfassen. Da es unrealistisch und überfordernd wäre, zu viele verschiedene Ziele gleichzeitig zu verfolgen, müssen die Ziele in eine sinnvolle Reihenfolge gebracht werden. Dieser Beratungsschritt wird als Festlegen einer Zielhierarchie bezeichnet. Hierbei darf sich der Berater nicht von der medizinisch-physiologischen Bedeutsamkeit des Effekts leiten lassen, der mit einer Veränderung erreichbar sein könnte. Das ausschlaggebende Kriterium für die Festlegung dieser Zielhierarchie ist vielmehr der subjektive Verhaltensaufwand, der für den Patienten notwendig ist, um ein Ziel zu erreichen. Zuerst sollten die Ziele angegangen und realisiert werden, die für den Patienten am leichtesten verwirklicht werden können. Es bietet sich dazu an, den Patienten zu bitten, die vermutete Leichtigkeit oder Schwierigkeit der definierten Ziele selbst zu benennen. Der Patient benutzt hierzu die in der Zieldefinition erstellte Zielliste und schreibt hinter jedes festgelegte Ziel eine Prozentzahl, die angibt, wie schwer es ihm vermutlich fallen wird, das Ziel zu erreichen $(0 \%=$ keinerlei Schwierigkeit bis 100\% = unüberwindliche Schwierigkeit). Alternativ könnte eine Fassung der Zielliste mit einer Skala versehen werden (s. 0 Abb. 2.10) und der Patient auf dieser Skala markieren bzw. ankreuzen, wie leicht oder wie schwer ein Ziel für ihn zu erreichen ist. Ziele die insgesamt vom Patienten als schwer erreichbar eingeschätzt werden, sollten in Teil- oder Zwischenziele unterteilt werden, die dann entsprechend leichter zu erreichen sind.

Der verhaltenspsychologische Hintergrund einer solchen Zielhierarchie besteht in folgender Überlegung: Wenn der Patient ein Ziel für sich selbst als leicht erreichbar bezeichnet, bedeutet das, dass er im Hinblick auf dieses Ziel eine hohe Selbstwirksamkeitserwartung bzw. Kompetenzüberzeugung hat. Daher ist es sehr wahrscheinlich (s. \ Kap. 2.1.3), dass er dieses Ziel mit viel Energie und Ausdauer verfolgt, was es umso wahrscheinlicher macht, dass er das Ziel tatsächlich erreicht. Dies wird in positiv verstärken und seine Selbstwirksamkeitserwartung auch im Hinblick auf weitere Ziele der Ernährungsberatung verstärken. Wird hingegen ein Ziel gewählt, das der Patient als zu schwer betrachtet, wird er sich mit einer gewissen »Misserfolgsorientierung « an dieses Ziel heranmachen, daher eher scheitern, was seine Selbstwirksamkeitserwartung und damit Motivation für weitere Ziele negativ beeinflusst. Wenn im Lauf des Beratungsprozesses zunächst die leichten Ziele erfolgreich bearbeitet werden, können dann später die schwierigeren Ziele mit höherer Erfolgsaussicht angegangen werden. Scheitert der Patient hingegen bereits zu Beginn an wichtigen, aber zu schwierigen Zielen, ist der weitere Beratungsprozess blockiert. 


\section{Beratungsprozess}

Nachdem die Beratungsziele gemeinsam mit dem Patienten in eine angemessene Hierarchie gebracht wurden, werden diese durch den Beratungsprozess eines nach dem anderen abgearbeitet. Im nächsten Schritt stellt die Maßnahmenplanung dar. Dabei wird jedes Beratungsziel in eine oder mehrere konkrete Verhaltensmaßnahmen übersetzt, die direkt umgesetzt werden können. Eine solche Maßnahme kann die Vermittlung oder Erarbeitung eines Wissens- oder Informationsinhaltes sein (z. B. Information über den Unterschied zwischen gesättigten und ungesättigten Fettsäuren; Erarbeitung von Wissen über Lebensmittel, die gesättigte Fettsäuren enthalten). Eine solche Maßnahme, kann und wird aber auch häufig der Vorschlag oder die Empfehlung sein, eine bestimmte Veränderung im Ernährungsverhalten vorzunehmen. Wichtig ist in diesem Fall, dass eine solche Maßnahmenempfehlung tatsächlich handlungs- und lebensmittelorientiert ist. Während Beratungsziele häufig nährstofforientiert formuliert werden (z. B. Erhöhen der Ballaststoffzufuhr auf $30 \mathrm{~g}$ pro Tag oder mehr), muss ein Maßnahme die verwendeten Lebensmittel oder Lebensmittelgruppen und die gewünschte Handlung direkt benennen (z. B. Ersetzen Sie eine Scheibe Weiß- oder Mischbrot durch eine
Scheibe Vollkornbrot). "Gute« Maßnahmen zeichnen sich dadurch auf, dass der Aufwand zur Durchführung für den Patienten nicht zu hoch ist, während andererseits eine hohe Erfolgsaussicht für eine erfolgreiche Durchführung besteht. Auch wird es für manche Ziele notwendig sein, diese durch mehrere Maßnahmen umzusetzen. Kreativität und Erfahrung über bewährte Maßnahmen kann helfen, geeignete Maßnahmen zu finden. $\mathrm{Zu}$ dem Ziel, die Zufuhr komplexer Kohlenhydrate zu erhöhen, kann zum Beispiel die Maßnahme beitragen, dass der Patient seine Brotschneidemaschine um ein bis zwei Millimeter breiter stellt. Eine solche Maßnahme verlangt vom Patienten nicht, dass er mehr Brotscheiben isst oder den Brotbelag verändert, was vergleichsweise höheren Verhaltensaufwand bedeuten würde. Für manche Maßnahmen ist es sinnvoll oder gar notwendig, dass ihre Durchführung zuvor im Rahmen der Beratung eingeübt wird. Wenn beispielsweise der Patienten das Ziel erreichen will, bei nachmittäglichen Einladungen oder Feiern Nahrungsaufnahme flexible zu kontrollieren, so könnte er dies unter anderem durch die Maßnahme umsetzen, dass er sich bei einer Feier ein Stück Kuchen gönnt und dann freundlich aber bestimmt sagt: "Der Kuchen hat sehr lecker geschmeckt, aber ich bin jetzt satt und möchte

Variante 1:

\begin{tabular}{|c|c|c|c|c|}
\hline \multirow[t]{2}{*}{ Verhalten, das ich ändern will: } & \multicolumn{3}{|c|}{$\begin{array}{l}\text { Wie schwer wird mir das fallen? } \\
\text { (bitte ankreuzen) }\end{array}$} & \multirow[t]{2}{*}{$\begin{array}{l}\text { Erledigt? } \\
\text { (abhaken } \checkmark \text {, wenn erledigt) }\end{array}$} \\
\hline & sehr schwer & О००००০০ & sehr leicht & \\
\hline & sehr schwer & 0000000 & sehr leicht & \\
\hline & sehr schwer & 0000000 & sehr leicht & \\
\hline
\end{tabular}

Variante 2:

\begin{tabular}{l|l|l} 
Verhalten, das ich ändern will: & Schwierigkeitsgrad & $\begin{array}{c}\text { Erledigt? } \\
\text { (abhaken } \checkmark, \text { wenn erledigt) }\end{array}$ \\
\hline & & \\
\hline
\end{tabular}

Bitte geben Sie bei Schwierigkeitsgrad an, wie schwer Ihnen die Verhaltensänderung fallen wird, von $0 \% /$ überhaupt nicht schwierig) bis $100 \%$ (sehr schwierig) 
nicht mehr «. Wenn der Patient jedoch Schwierigkeiten hat, dies selbstsicher zu sagen, kann es helfen, dies im Rollenspiel zuvor mit dem Patienten zu üben. Schließlich kommen als Maßnahmen alle verhaltenstherapeutischen Techniken in Frage, die im Rahmen der Ernährungsberatung Verwendung finden können.

Verhaltensorientierte Maßnahmen werden schließlich als Trainingsaufgabe formuliert, die der Patient in der nächsten Woche bzw. in den nächsten Wochen zuhause bzw. in seinem Alltag durchführen soll. Eine solche Trainingsaufgabe muss möglichst eindeutig und verständlich formuliert werden, es muss klar sein, wie oft sie in welchem Zeitraum durchgeführt werden soll und woran eine erfolgreiche Durchführung erkannt werden kann. Auch sollte im Sinn einer Erfolgsplanung mit dem Patient besprochen werden, wie die erfolgreiche Durchführung erleichtert werden kann beziehungsweise, durch welche Umstände die Durchführung beeinträchtigt werden könnte und wie diese hinderlichen Umstände abgemildert oder umgangen werden können. Hilfreich ist es, wenn die Trainingsaufgabe schriftlich formuliert und dem Patienten mitgegeben wird. Dies kann sinnvoller Weise auch mit einem Selbstbeobachtungsbogen kombiniert werden, auf dem der Patient die Durchführung der Trainingsaufgabe protokolliert. Die Vereinbarung und Durchführung von solchen Trainingsaufgaben stellt das Kernstück der verhaltensorientierten Ernährungsberatung dar. Verhaltensänderungen finden nicht in den Beratungssitzungen statt sondern zwischen den Beratungssitzungen. Idealerweise sollte eine Beratungssitzung immer damit enden, dass der $\mathrm{Pa}$ tient eine bis maximal drei Trainingsaufgaben hat, die er bis zur nächsten Sitzung üben soll.

\section{Evaluation}

Jede Folgesitzung muss dann - nach der Begrüßung und einleitenden Worten - damit beginnen, dass mit dem Patienten besprochen wird, welche Trainingsaufgaben erfolgreich umgesetzt werden konnten, beziehungsweise wo Schwierigkeiten aufgetreten sind und wie diese gegebenenfalls in Zukunft verringert werden können. Als Ergebnis einer solchen Evaluation (Bewertung) des Trainingserfolges legen Berater und Patient fest, ob ein bestimmter Verhaltensaspekt nochmals weitertrainiert werden soll, ob für ein bestimmtes Ziel eine weitere Verhaltensmaßnahme notwendig ist. Als Ergebnis der Evaluation kann sich aber auch ergeben, dass ein bestimmtes Beratungsziel nunmehr erfolgreich erreicht wurde. Dann wird anhand der früher aufgestellten Zielhierarchie das als nächstes zu erreichende Ziel ausgewählt und durch Maßnahmenplanung umgesetzt. Die Evaluation kann aber auch ergeben, dass eine ganze Reihe von Zielen nunmehr erfolgreich erreicht wurden und dass daher eine erneute Zieldefinition notwendig ist, oder dass eine erneute Verhaltensdiagnose sinnvoll ist, um festzustellen, ob und wo weitere Diskrepanzen zwischen Ist-und-Soll-Zustand bestehen. Auch können Patient und Berater im Rahmen einer solchen Evaluation zu dem Ergebnis kommen, dass keine weiteren Veränderungen mehr angestrebt werden sollen und den Beratungsprozess, eventuell nach der Vereinbarung von Nachsorgeterminen, beenden.

\subsubsection{Verhaltenstherapeutische Maßnahmen in der Ernährungsberatung}

Die Verhaltenstherapie oder Verhaltensmodifikation zielt darauf ab, das Ess- und Ernährungsverhaltens zu verbessern, die körperliche Aktivität zu steigern, sowie die psychischen und sozialen Folgeprobleme von Ernährungsproblemen besser zu bewältigen. Grundlage dieses Ansatzes ist die Überlegung, dass:

- ungünstige Muster des Ess- und Bewegungsverhaltens erlernt wurden und

- das Ess- und Bewegungsverhalten durch Lernprozesse in einer günstigeren Richtung beeinflussbar sind.

Ursprünglich war es ein Kennzeichen der klassischen Verhaltenstherapie, dass lediglich direkt beobachtbares Verhalten als Ziel der therapeutischen Arbeit definiert wurde. Der moderne, auch als kognitive Verhaltenstherapie bezeichnete Ansatz ist wesentlich erweitert und schließt Kognitionen wie Erwartungen, Einstellungen, Überzeugungen und Werte als sinnvolle und wichtige Ziele von Veränderungsprozessen ein.

\section{Selbstbeobachtung}

Durch Selbstbeobachtung wird ein problematisches Verhalten, das vermindert werden soll, oder ein gewünschtes Zielverhalten, das gesteigert werden soll, in den entsprechenden Situationen vom Patienten selbst beobachtet, registriert und protokolliert. Die Selbstbeobachtung ist eine der zentralen verhaltenstherapeutischen Techniken, die als »Basisfähigkeit« für den Prozess der Selbststeuerung angesehen werden kann. Ohne Selbstbeobachtung ist eine planvolle, selbstgesteuerte Verhaltensmodifikation undenkbar. Durch die Selbstbeobachtung werden Informationen erhoben, die für die Steuerung des Veränderungsprozesses unabdingbar sind, wie z. B. die Basishäufigkeit einer Verhaltensweise oder Veränderungen in der Häufigkeit. Häufig werden durch die Selbstbeobachtung des Verhaltens auch bereits erste Verhaltensänderungen in Gang gesetzt.

Zur Beobachtung des Essverhaltens wird oft die Methode des Ernährungstagebuchs gewählt. Dabei soll der Patient festhalten, welche Lebensmittel und Getränke er zu sich nimmt. Zusätzlich kann protokolliert werden, in welchen Situationen gegessen wird und durch wel- 
che Emotionen und Gedanken das Essen ausgelöst oder begleitet wird. Die verhaltenstherapeutischen Selbstbeobachtung des Essverhaltens zielt nicht primär darauf $a b$, anhand des Protokolls eine exakte Berechnung der Energie- und Nährstoffzufuhr zu ermöglichen, sondern darauf, Essverhalten bewusst zu machen und strukturiert $\mathrm{zu}$ erfassen. Bei vielen übergewichtigen und adipösen Patienten wird allein durch die Selbstbeobachtung des Essverhaltens eine erste Gewichtsabnahme erreicht.

Im Rahmen der Verhaltensmodifikation werden oft Selbstbeobachtungstechniken als »Ernährungskontrollsysteme« eingesetzt. Durch solche Ernährungskontrollsysteme werden bestimmte Aspekte der Nahrungsaufnahme quantitativ erfasst. Beispiele hierfür wären das Zählen von Fettpunkten, wobei ein Fettpunkt für eine bestimmte aufgenommene Fettmenge steht, das Zählen von Kalorien oder das Zählen von Obst- und Gemüseportionen, die gegessen wurden. Hierbei wird in der Regel zuerst die Basishäufigkeit als Ausgangspunkt erhoben und darauf aufbauend dann eine sinnvolle Zielhäufigkeit definiert. Durch die Fortführung der Selbstbeobachtung kann der Patient die die Annäherung an die gewünschte Zielhäufigkeit selbst überprüfen.

Selbstbeobachtungstechniken müssen sich jedoch nicht unbedingt auf das Ernährungsverhalten als ganzes beziehen, sondern können sich auch auf einzelne Aspekte konzentrieren. Wenn beispielsweise ein Patient damit Schwierigkeiten hat, dass er zu häufig vor dem Kühlschrank stehend nascht, könnte sich die Selbstbeobachtung lediglich darauf beziehen, wie häufig dieses Verhalten (»vor dem Kühlschrank stehend naschen«) auftritt. Als Instrument für eine solche Selbstbeobachtung böte sich dann eine Strichliste an, die am Kühlschrank befestigt wird. Wesentlich für eine verhaltenstherapeutische Selbstbeobachtung ist es, dass die Häufigkeit oder die Dauer mit der eine Verhaltensweise auftritt quantifiziert, also gezählt oder gemessen wird. Die Aufforderung an den Patienten "achten Sie darauf, wie viel fettreiche Lebensmittel Sie essen « ist in diesem Sinn keine Selbstbeobachtungsaufgabe, da hier keine quantifizierende Protokollierung stattfindet.

Für klar umgrenzte Handlungen stellt die Strichliste die Standardform der Selbstbeobachtung dar. Eine solche Strichliste kann jedoch je nach Umständen und zu beobachtender Handlung durch andere "Protokollierungen" ersetzt werden. Zur Selbstbeobachtung des Süßigkeitenkonsums hat es sich beispielsweise bewährt, dass der Patient morgens eine volle Streichholzschachtel seine Hosentasche steckt und bei jedem süßen Bissen ein Streichholz aus dieser Schachtel in die andere Hosentasche befördert. Dies kann im sozialen Umfeld des Patienten wahrscheinlich wesentlich unauffälliger geschehen als das Führen einer Strichliste, die andere Personen zu Fragen provoziert, die dem Patienten unangenehm oder peinlich sind. Für einen anderen Patienten, der seinen Bierkonsum kontrollieren möchte, könnte es sinnvoll sein, wenn er neben dem Kühlschrank eine Schale aufstellt, in der er die Kronkorken sammelt. Je einfacher und unaufwändiger ein solches Protokoll-System für den Patienten ist, umso eher kann es letztlich die Verhaltenshäufigkeit zuverlässig erfassen.

Schließlich sollten die erhobenen Einzelhäufigkeiten in einer Übersichtsgrafik oder Übersichtstabelle zusammengefasst werden, damit der Patient nicht durch die Vielzahl von Schwankungen und Abweichungen irritiert wird, sondern den Gesamtverlauf der Entwicklung im Auge behält.

\section{Reizkontrolltechniken}

Reizkontrolltechniken oder Stimuluskontrolltechniken setzen an der Überlegung an, dass Verhaltensweisen durch bestimmte Auslösereize (Stimuli) in Gang gesetzt werden können. Interessanterweise spielt dieser Aspekt insbesondere beim Essverhalten eine große Rolle. Dieses Prinzip wurde als klassische Konditionierung zuerst von Iwan Pawlow beschrieben. Dabei kann durch wiederholte Verknüpfung von unkonditionierten Auslösereizen mit beliebigen anderen Reizen eine konditionierte Reaktion auf den ursprünglich neutralen Reiz gelernt werden. Im klassischen Experiment lernten Hunde, mit Speichelfluss auf ein Tonsignal zu reagieren. Es ist wahrscheinlich kein Zufall, dass das Prinzip der klassischen Konditionierung am Beispiel des Appetitverhaltens (Speichelfluss!) entdeckt wurde, da dieses Prinzip sehr zentral das Essverhalten steuert. Im Rahmen der Ernährungsberatung werden Reizkontrolltechniken eingesetzt, um die Vielzahl von Auslösereizen für Nahrungsaufnahme auf eine überschaubare Anzahl einzuschränken. Alle anderen, bisherigen Auslösereize verlieren ihren Signalcharakter und werden gelöscht. $\mathrm{Zu}$ den häufig verwendeten Reizkontrolltechniken gehören Regeln, wie die Anzahl der Essplätze auf einen oder zwei zu beschränken, nur zu bestimmten Uhrzeiten zu essen und keine Nebentätigkeiten wie Fernsehen beim Essen auszuführen. Auch die Empfehlung verlockende Lebensmittel nicht offen sichtbar in der Wohnung herumstehen zu lassen oder nicht hungrig einkaufen zu gehen gehören in diesen Zusammenhang.

\section{Verstärkungstechniken}

Verstärkungstechniken beruhen auf der Gesetzmäßigkeit, dass Verhaltensweisen, auf die eine positive Konsequenz folgt, häufiger ausgeführt werden. Als Verstärker bezeichnet man eine solche positive Verhaltenskonsequenz, die zu einer Erhöhung der Verhaltenshäufigkeit führt. Dabei sind in der Regel kurzfristige Verhaltenskonsequenzen wesentlich wirksamer als langfristige Verhaltenskonsequenzen. Dies stellt gerade bei Veränderungen des Ess- und Bewegungsverhaltens eine Schwierigkeit dar, da solche 
Veränderungen häufig kurzfristig negative Konsequenzen wie Verzicht oder Anstrengung haben und erst langfristig positive Veränderungen wie eine bessere Gesundheit oder Fitness oder ein besseres Aussehen erlebbar werden. Verstärkungstechniken zielen daher darauf, die gewünschten Verhaltensänderungen kurzfristig zu belohnen. Hierzu werden mit dem Patienten Vereinbarungen getroffen, wie er sich beim Erreichen von bestimmten Verhaltenszielen selbst belohnen soll.

Im Rahmen der Ernährungsberatung sollte Essen und Trinken nicht als Verstärker eingesetzt werden, wenngleich manche Patienten Schwierigkeiten haben, andere Dinge oder Tätigkeiten zu benennen, die ihnen Spaß machen oder mit denen sie sich belohnen können. Hier können auch sogenannte token-Systeme (Gutschein-Systeme) eingesetzt werden. Dabei belohnt sich der Patient für die erfolgreiche Durchführung einer Trainingsaufgabe oder für das Erreichen eines Verhaltensziels zunächst "nur" symbolisch, durch einen Punkt oder Gutschein (token). Erst wenn dann eine bestimmte Anzahl von solchen Punkten gesammelt wurde, werden diese gegen etwas mit realem Verstärkungscharakter eingetauscht. Dies können dann durchaus auch etwas teurere oder aufwändigere Dinge oder Tätigkeiten sein (z. B. Fahrrad, Musical-Besuch, Urlaubsreise).

\section{Kognitive Umstrukturierung}

Mit der Technik der kognitiven Umstrukturierung lernen die Patienten, negative und irrationale Gedanken und Einstellungen zu identifizieren, die ihr Verhalten steuern, diese Gedanken und Einstellungen zu hinterfragen und schließlich durch positive und rationalere Kognitionen zu ersetzen. Ein wichtiger Inhalt der kognitiven Umstrukturierung können unrealistische Erwartungen der Patienten an das Ausmaß und die Geschwindigkeit der erreichbaren Gewichtsabnahme sein. Foster und Kollegen fanden z. B. in einer Studie adipöser Patientinnen, dass diese eine Gewichtsabnahme von $17 \mathrm{~kg}$ in einem Jahr, die objektiv gesehen ein großer Erfolg ist, von den Patientinnen als enttäuschend erlebt wird. Es ist notwendig solche unrealistischen Erwartungen zu verändern, da sie sonst eine langfristige Stabilisierung des veränderten Verhaltens behindern.

\section{Computer- und Internetgestützte Ernährungsberatung}

In den letzten Jahren hat das Internet als Kommunikationsmedium eine weite Verbreitung in vielen Bevölkerungsschichten erfahren und es kann damit gerechnet werden, dass sich dies weiter fortsetzen wird. Im Zuge diese Entwicklung haben sich auch eine Vielzahl von Angeboten der Ernährungsinformation und Ernährungsberatung im Internet etabliert. Der Funktionsumfang dieser Angebote kann grob in vier Gruppen gegliedert werden:
Information über Ernährung und Gesundheit. Schriftund Bild-Information zu verschiedenen Ernährungs- und Gesundheitsthemen wird in Form von Artikeln oder ähnlichen Beiträgen angeboten. Vom Charakter her entspricht das Informationsangeboten in Zeitschriften oder Broschüren. Das Internet fungiert hier lediglich anstelle von Papier als Transportmedium.

Interaktive Elemente. Der Benutzer kann individuelle Daten eingeben und erhält als Ergebnis eine daraus resultierende Analyse, Bewertung oder Information. Beispiele hierfür sind BMI-Rechner oder Ernährungsanalysen aufgrund von Lebensmittelhäufigkeitsfragebögen.

Längerfristig angelegte Betreuungsangebote. Hierbei handelt es sich um Konzepte, die als Betreuungsangebot zum Beispiel zur Gewichtsreduktion über mehrere Wochen oder Monate angelegt sind.

Individuelle Kommunikation. Hierzu zählen Foren oder Chats zu Ernährungsthemen oder die individuelle EmailKommunikation mit Fachleuten.

Die Angebote selbst können in drei Kategorien eingeteilt werden:

- Informationssysteme einschließlich interaktiver Elemente

- Gesundheitsportale und

- Beratungsangebote

Aufgrund der teilweise schnellen Veränderungen von Angeboten im Internet kann kein vollständiger und aktueller Überblick über die verschiedenen Angebote gegeben werden. Stattdessen können einige zum Zeitpunkt der Manuskripterstellung aktuelle Angebote exemplarisch beschrieben werden.

Ernährungs-Informationssysteme. Ernährungsinformationssystem der Universität Hohenheim (http://www.unihohenheim.de/ wwwin140/info/info.htm). Unter dem Stichwort Ernährungshinweise finden sich allgemeine Informationen zur Ernährung, die von der vollwertigen Ernährung, über die Ernährung von bestimmten Bevölkerungsgruppen wie Kinder, Schwangere, Sportler und Ältere, über Informationen zu den verschiedensten Nährstoffen bis zur toxikologischen Aspekten und Diäten reichen. Als interaktive Online-Berechnungen werden unter anderem ein Energiebedarfsrechner, ein BMI-Rechner, eine Ernährungsanalyse und eine Lebensmittelanalyse angeboten. Daneben gibt es einen Überblick über Ernährungssoftware und weiterführende Links zu anderen Informationsquellen.

Das Deutsche Ernährungsberatungs- und -informationsnetz (DEBInet) (http://www.ernaehrung.de) bietet unter dem Stichwort Ernährungstipps eine Fülle von Informationen über Ernährung allgemein, in besonderen 
Situationen und über Ernährungstherapie bei verschiedenen Erkrankungen. Auch hier finden sich ein BMI- und Energiebedarfsrechner, sowie ein interaktiver Fragebogen zum Essverhalten. Hilfreich kann auch eine Adressdatenbank mit den Daten von über 1600 Ernährungsberatern sein. Es gibt verschiedene Lexika und weiterführende Links. Zum Angebot gehört auch ein Diskussionforum.

Der aid-Infodienst (http://www.aid.de) des Bundesministeriums für Verbraucherschutz, Ernährung und Landwirtschaft betreibt die Ernährungsinformationsseite http:// www.was-wir-essen.de. Hier finden sich unter anderem Informationen $\mathrm{zu}$ verschiedenen Lebensmitteln, $\mathrm{zu}$ den verschiedenen Stufen der Nahrungskette (Erzeugung, Verarbeitung, Einkauf und Kennzeichnung, Lagerung und Zubereitung, gesundes Essen), zu Schadstoffen sowie Informationen für verschiedene Bevölkerungsgruppen. Neben einem Forum gibt es eine Reihe von Spielen, in denen Ernährungsinformationen spielerisch vermittelt werden.

Die Deutsche Gesellschaft für Ernährung DGE (http://www.dge.de) bietet auf ihrer Homepage unter anderen Informationen für Ernährungsfachkräfte als auch für Verbraucher. Speziell für Jugendliche, aber auch für deren Lehrer und Eltern ist die Website http://www. talking-food.de gedacht. Hier geht es um die Themenbereiche Lebensmittelsicherheit und gesunde Ernährung, die zum Teil in Form von Spielen jugendgerecht aufgearbeitet sind.

\section{Gesundheitsportale}

- www.lifeline.de: Die Themenbereiche dieses Portals gliedern sich in Gesundheit, Ernährung, Wellness, Lust \& Liebe, sowie Baby \& Kind. Im Themenbereich Ernährung gibt es unter anderem Informationen zu Nährstoffen, Diäten, Lebensmitteln und Rezepten. Es gibt einen Expertenrat zu verschiedenen Themenbereichen und eine ganze Reihe von interaktiven Selbsttests. Hinzu kommt eine Suchdatenbank für Ärzte, Krankenhäuser, nichtärztliche Gesundheitsberufe, Kammern und Verbände.

- www.almeda.de: Das Themenspektrum reicht von Körper und Medizin, Ernährung und Diät bis zu Psyche und Lebenshilfe, Recht und Rat. Der Ernährungsbereich. An interaktiven Elementen gibt es einen BMI-Rechner, eine Fett- und Kalorientabelle und eine Rezeptsammlung Was soll ich heute kochen. Auch hier gibt es eine Suchfunktion für Ärzte.

- www.qualimedic.de: Die abgedeckten Themenbereiche umfassen unter anderem Schwangerschaft und Kinderwunsch, Frauen- und Männergesundheit, Herz und Gefäße, Allergien, Zähne und Ernährung. Im Bereich Ernährung finden sich verschiedene Beiträge zu Lebensmitteln und Nährstoffen, Ernährungsformen und Diäten. Neben einem Teilnehmerforum, das dem Austausch der Besucher dient, gibt es eine Expertenforum, in dem Fachleute die Fragen beantworten.
- www.surfmed.de: Die Themenliste beinhaltet medizinische Themen ebenso wie Fitness und Ernährung. Neben kostenlosen Informationen gibt es auch kostenpflichtige Angebote, z. B. einen Lebensbegleiter für $10 €$ pro Jahr, der weitere Ernährungsinformationen, Rezepte und einen individuellen Menuplan beinhaltet. Als kostenpflichtiges Angebot gibt es für $20 €$ auch ein 12-Wochen-Programm »lustvoll schlank« das bei der Gewichtsabnahme und Ernährungsumstellung unterstützt. Alle kostenpflichtigen Programme können in einem Club für $35 €$ pro Jahr genutzt werden.

\section{Beratungsprogramme zur Gewichtsabnahme}

- www.slimnet.de: Das Abnahmeprogramm beruht auf zwei zentralen Elementen, der Verwendung von Fertigprodukten für die Gewichtsreduktion und die Beratung durch Fachleute über das im Rahmen von Chats und Email. Es gibt verschiedene Möglichkeiten der Teilnahme. Ein Intensivtraining, das neben der Beratung die vollständige Ernährung durch Diätprodukte vorsieht, für $248 €$ pro Monat. Das Expertentraining beinhaltet die Beratungsangebote für $12 €$ pro Monat, die Diätprodukte müssen bei Bedarf im Online-Shop zusätzlich erworben werden.

- www.xx-well.com: Dieses Angebot umfasst mehrere Coaches, einen Diätcoach, einen Ernährungscoach, einen Fitnesscoach und einen Non-smoking-Coach. Die Teilnahme kostet beim Diätcoach je nach Abonnementdauer zwischen $12,90 €$ pro Monat und 89,90 pro Jahr. Für persönliche Fragen stehen Experten über eine telefonische Hotline (1,49€ pro Minute) zur Verfügung. Der Diätcoach liefert auf der Basis von persönlichen Fragebogenangaben einen individuellen Rezeptplan und Ernährungs- und Fitnesstips.

- www.lean-and-healthy.de: Dieses Angebot ist ein verhaltenstherapeutisch ausgerichtetes strukturiertes Trainingsprogamm für Menschen mit Gewichtsproblemen. In Form von 52 Wochenprogrammen werden die Teilnehmer bei der Analyse und Veränderung ihres Ess- und Bewegungsverhaltens unterstützt und angeleitet. Die 52 Wochenprogramme beinhalten in der Regel eine Analyse der individuellen Situation, darauf abgestimmte Informationen und konkrete Trainingsaufgaben, mit denen die Teilnehmer zur Verhaltensänderung angeleitet werden. Die Programmteilnahme kostet $50 €$ für das Gesamtprogramm. Daten zur Evaluation dieses Programmes zeigen, dass rund 30\% der Teilnehmer tatsächlich ein Jahr lang aktiv teilnehmen. Teilnehmer, die alle Trainingseinheiten bis zu Ende absolviert haben, erreichen eine Gewichtsreduktion von durchschnittlich $6 \mathrm{~kg}$ (Frauen) bzw. 8 kg (Männer).

\section{Fachgesellschaften und Berufsverbände}

Eine weitere Informationsquelle für Fachkräfte sind die Homepages der Fachgesellschaften und Berufsverbände. 
Eine Auswahl dieser Gesellschaften aus dem Bereich Ernährungswissenschaften und Ernährungsmedizin ist im Folgenden wiedergegeben.

- Deutsche Gesellschaft für Ernährunsmedizin: www.dgem.de

- Deutsche Gesellschaft für Ernährung: www.dge.de

- Deutsche Adipositas-Gesellschaft: www.adipositas-gesellschaft.de

- Deutsche Diabetes-Gesellschaft: www.deutsche-diabetes-gesellschaft.de

- Arbeitsgemeinschaft Adipositas im Kindes und Jugendalter (AGA): www.a-g-a.de

- Berufsverband Deutscher Ernährungsmediziner: www.bdem.de

- Verband der Diplom-Oecotrophologen: www.vdoe.de

\subsection{Patientenschulung}

Patientenschulung ist heute in der Diabetologie, in der Adipositastherapie, für Patienten mit Bluthochdruck, bei Asthma oder auf Zöliakie etabliert. Ziel der Schulung ist es, dem Patienten ein möglichst hohes Maß an Selbständigkeit im Umgang mit seiner Erkrankung zu vermitteln. Dies beinhaltet auch, dass der Patient lernt, seine Behandlung individuell und flexibel an die jeweiligen Gegebenheiten (z. B. eine Änderung der Ernährungsgewohnheiten, Freizeitverhalten, Urlaub, Erkrankung) anzupassen. Die Schulungsprogramme versuchen, den Patienten durch spezielle didaktische und pädagogische Hilfe Fertigkeiten zu vermitteln. Bei jugendlichen Typ I Diabetikern sind dies z. B.: Selbstkontrolle des Stoffwechsels, Diätetik, Insulintherapie Insulininjektionstechniken, Adaptation der Insulindosis, Maßnahmen bei Hypoglykämie, Fußpflege, Folgeschäden, Kontrolluntersuchungen, Schwangerschaft, Vererbung, Kontrazeptiva, psychosoziale Fragen, Führerschein. Bei Typ-II-Diabetikern mittleren Alters geht es z. B. um: Ernährung bei Diabetes, Übergewicht, Hypertonie und Fettstoffwechselstörung, Aufklärung über mikroangiopathische oder arteriosklerotische Folgeerkrankungen, Selbstkontrolle des Blutzuckerspiegels, Harnzucker, Körpergewicht und Blutdruck, körperliche Aktivität, Fußpflege, Nikotinabstinenz. Die Ziele der Schulung sind, das nötige Wissen, die praktischen Fertigkeiten und die Selbstverantwortlichkeit zu vermitteln bzw. zu entwickeln.

Im einzelnen werden im Rahmen der Diabetikerschulung die folgenden Themen behandelt:

- Optimaler und richtiger Einsatz der Insulintherapie.

- Konsequenzen der Insulintherapie für das Leben in Familie, Schule, Beruf und für die eigenen Lebensgewohnheiten.
- Erkennen und Behandeln von Komplikationen der Insulintherapie.

- Messen der Behandlungsergebnisse und entsprechend konsequentes Handeln.

- Ernährung und Bewegung.

- Selbstbehandlung bei interkurrenten Erkrankungen.

- Therapieziele und Strategien, um diese zu erreichen.

- Kommunikation mit den Mitgliedern des Schulungsteams.

- Verständnis und Umgang mit den diabetischen Folgeschäden.

- Unvorhergesehene und neue Probleme der Diabeteseinstellung.

- selbstzerstörerisches Verhalten und Stress.

Der Unterricht wird von Schulungsschwestern für Diabetiker (sog. Diabetesberatern) und Diätassistentinnen erteilt. Es gibt spezielle Konzepte für insulinbehandelte Typ-I- und Typ-II-Diabetiker. Die Gruppengröße ist auf 12 Patienten festgelegt, die Zusammensetzung sollte möglichst homogen sein, die Patienten sollten von Anfang bis Ende teilnehmen. Entscheidend für den Erfolg der Diabetikerbetreuung an Diabeteszentren ist die Bildung von sog. »Diabetesteams«. Sie bestehen aus den Patienten, einem Arzt/einer Ärztin (Diabetologe/in), der Diabetesschulungsschwester (Diabetesberater), einem Fußpfleger und der Diätassistentin. Bei Bedarf werden weitere Spezialisten (Gynäkologe, Geburtshelferin, Augenarzt, Nephrologe, Psychologe) hinzugezogen. Dabei sollte jedes einzelne Mitglied in die Lage versetzt werden, das Diabetesteam zu führen.

Der Erfolg und der Wert von strukturierten Patientenschulungen ist wissenschaftlich gut belegt. Die Programme werden regelmäßig einer Qualitätssicherung unterzogen. Die Qualitätssicherung eines Schulungsprogrammes soll am Beispiel der Pateintenschulungsprogramme für Kinder und Jugendliche mit Adipositas dargestellt werden. Die Programmanbieter müssen hierzu den Kostenträgern folgende Dokumente vorlegen:

- Beschreibung des inhaltlichen und didaktischen Konzepts mit Literaturangaben.

- Darstellung der wesentlichen Prozesse der Durchführung des Schulungsprogramms.

- Beschreibung des Schulungsteams und Nachweis der vorhandenen Qualifikation.

- Beschreibung der verfügbaren Räumlichkeiten.

- Zielgruppenspezifisches Lehrmaterial einschließlich Handbuch/Manual.

- Darstellung der Verlaufs- und Ergebnisdokumentation und Kommunikation mit dem behandelnden Vertragsarzt.

- Nachweis der Ergebnisqualität, ggf. mit Literaturangaben oder Bescheinigung über die Teilnahme an einer bundesweiten Evaluationsstudie. 


\section{Konzeptqualität}

- Das Programmkonzept verfolgt einen Kombinationsansatz.

- Das Programm ist zielgruppenspezifisch konzipiert.

- Einsatz von Medikamenten, Formeldiäten oder Nahrungsergänzungsmittelnn sind kein feststehender Bestandteil des im Schulungsprogramm vermittelten Behandlungskonzepts.

- Ein chirurgischer Eingriff ist nicht feststehender Bestandteil des im Schulungsprogramm vermittelten Behandlungskonzeptes.

- Das Programm strebt die konsentierten Zielvorgaben an bzw. es werden Programmziele formuliert, deren Erreichung sowohl bezüglich des Programms, als auch im Einzelfall messbar ist

\section{Prozessqualität}

- Verbindliche Einschlusskriterien sind angegeben.

- Verbindliche Ausschlusskriterien sind angegeben.

- Verbindliche Abbruchkriterien sind angegeben.

- Die Motivation der Kinder/Jugendlichen bzw. Eltern wird vor Programmbeginn geprüft.

- Die Altershomogenität der Schulungsgruppen ist gewährleistet.

- Individueller Lernfortschritt der Teilnehmer wird berücksichtigt.

- Der behandlender Vertragsarzt ist in den Schulungsablauf seines Patienten eingebunden.

- Es werden Teambesprechungen durchgeführt, in denen die Fortschritte der einzelnen Teilnehmer interdisziplinär reflektiert werden.

\section{Ernährung}

- Die Ernährungsempfehlungen entsprechen den Vorgaben der Fachgesellschaften und Institutionen (AGA, DGE, FKE).

- Eine stark kalorienreduzierte Diät wird nicht empfohlen.

\section{Bewegung}

- Bewegungsprogramm: Schwerpunkt in aktiver Förderung von Eigeninitiative und Eigenverantwortung $\mathrm{zu}$ vermehrter Bewegung.

\section{Verhalten}

- Anwendung verhaltentherapeutischer Methoden in der überwiegenden Zeit der vom Schulungsteam erbrachten Programmstudien für Kinder/Jugendliche.

\section{Elterneinbindung}

- Eltern sind aktiv in Programm für Kinder einbezogen (Angebote an Eltern in Form von Elternabenden, Fortbildungsveranstaltungen, Handbücher und gemeinsamen Aktionen).

- Eltern über Programm für Jugendliche informiert (Minimum: Elternmanual).

- Eltern werden in den Fertigkeiten geschult, die zur Etablierung und Aufrechterhaltung eines geänderten Verhaltens der Kinder notwendig sind.

- Eltern sollen nicht ständig bei Schulungsterminen von Jugendlichen anwesend sein, diese aber bei der Aufrechterhaltung veränderten Verhaltens unterstützen.

\section{Strukturqualität}

- Es wird ein interdisziplinäres Schulungsteam im Sinne der Gemeinsamen Empfehlungen der Spitzenverbände der gesetzlichen Krankenkassen vorgehlaten. Die Teammitglieder weisen spezielle Erfahrungen in der Betreuung von kindern und Jugendlichen auf.

- Das Personal ist für den Einsatz verhaltenstherapeutischer Techniken geschult.

- Es wird ein zielgruppenspezifisches Manual für Kinder/Jugendliche/Therapeuten/Eltern vorgehalten.

- Die Programmdauer liegt unter einem Zeitrahmen von 24 Monaten.

- Die vorgesehene Gruppengröße beträgt bis zu 12 Teilnehmer.

- Anleitung zum Umgang mit Lebensmitteln und deren zubereitung sowie Einkaufübungen umfassen weniger als 30\% der Zeit des Ernährungsmoduls.

- Die verfügbaren Räumlichkeiten genügen den Anforderungen der Gemeinsamen Empfehlungen der Spitzenverbände der gesetzlichen Krankenkassen.

- Die vorgesehene Ablauf- und Ergebnisdokumentation genügt den konsentierten Anforderungen.

\section{Ergebnisqualität}

- Es liegt eine Bestätigung der Teilnahme des Programms an der bundesweiten Evaluationsstudie der BzgA/der AGA vor.

Oder

- Es liegen aussagekräftige Informationen zur Abbrecherquote vor.

- Es liegen Ergebnisse zum Grad der Zielerreichung (Intention-to-treat-Analyse) vor.

- Es liegen systematische Auswertungen von Teilnehmerfragebögen vor. 


\subsection{Diätetik-/Diätkatalog}

Ein Diätkatalog listet, strukturiert und beschreibt die verschiedenen Kostformen und folgt dem Rationalisierungsschema 2004 der Deutschen Arbeitsgemeinschaft für Klinische Ernährung und Diätetik e.V. für die Ernährung und Diätetik in Klinik und Praxis (s. Aktuelle Ernährungsmedizin, 29, 245-253, 2004). Das Rationalisierungsschema ist Grundlage für die Anwendung wichtiger und häufig gebrauchter Diätformen im Krankenhaus. Dieses Schema enthält keine überholten oder pseudowissenschaftlichen Diäten (wie z. B. Ulkusdiät, Gallediät oder Herzdiät). Eine wissenschaftlich begründete, rationelle Diätetik ersetzt eine aus der Erfahrung heraus entwickelte Diätetik. Eine kritische Würdigung der dargestellten Kostformen ergibt, dass viele Diäten pathophysiologisch begründet sind und ihr Wert auch in klinisch-kontrollierten Studien anhand von Zielgrößen wie z. B. Plasmalipidmuster, Ernährungszustand, Beschwerden, Krankheitsverlauf belegt ist. Es fehlen aber häufig Untersuchungen, die den Wert einer speziellen diätetischen Intervention langfristig dokumentieren.

In dem Rationalisierungsschema sind die Kostformen nach Art und Häufigkeit dargestellt.

Das Rationalisierungsschema unterscheidet 4 verschiedene Gruppen von Diäten:

- Vollkost/leichte Vollkost,

- energiedefinierte Diäten,

- protein- und elektrolytdefinierte Diäten,

- gastroenterologische Diäten, Sonderdiäten.

Die künstliche Ernährung wird in einem gesonderten Kapitel (s. Kap. 2.6) behandelt. Ein Diätkatalog ist keine Anleitung für die Lebensmittelauswahl. Er enthält allgemeine Empfehlungen und Richtlinien. Er formuliert die Indikationen, die wissenschaftlich gesicherten Grundlagen sowie die Ziele der einzelnen Kostformen. Er ist eine verbindliche Basis der ernährungsmedizinischen Behandlung und Beratung und für die ernährungsmedizinische Praxis. Die Umsetzung der Empfehlungen im Sinne einer optimalen ernährungsmedizinischen Patientenversorgung obliegt in den einzelnen Krankenhäusern den dort tätigen Experten, d. h. Diätassistentinnen/en, Ernährungsberaterinnen/en, Ökotrophologinnen/en und ernährungsbeauftragten Ärztinnen/en. Etwa 75\% der Krankenhauspatienten essen eine Vollkost, 25\% der ausgegebenen Essen sind spezielle Diäten.

Der folgende Diätkatalog versucht, die Diätetik in den Zusammenhang des jeweiligen Behandlungskonzeptes zu stellen. In diesem Sinne werden auch häufig gebrauchte Medikamente erwähnt, welche wiederum Wechselwirkungen mit der Ernährung haben können.

\subsubsection{Vollkost}

\section{Vollkost}

Indikation. Eine Vollkost ist für alle Patienten geeignet, die keiner speziellen Ernährung bedürfen.

Definition. Die Vollkost wird nach den Regeln einer vollwertigen Ernährung entsprechend den Empfehlungen der Deutschen Arbeitsgemeinschaft für Klinische Ernährung und Diätetik sowie der Deutschen Gesellschaft für Ernährung (Leitsätze für Krankenhausernährung) zusammengestellt. Die Nährstoffrelationen betragen 15-20\% Eiweiß, 30-35\% Fett und 45-55\% Kohlenhydrate. Dieses entspricht bei einer Kalorienmenge von $2200 \mathrm{kcal}$ 80-105 g Eiweiß, 70-80 g Fett sowie 240-270 g Kohlenhydraten. Die Kost enthält etwa $10 \mathrm{~g}$ Linolsäure. Die Vollkost ist eine biologisch vollwertige Kost. Sie deckt den täglichen Bedarf an Makro- und Mikronährstoffen und berücksichtigt die präventivmedizinischen Erkenntnisse der Ernährungsforschung. Der Natriumgehalt übersteigt $100 \mathrm{mmol}$ bzw. der Kochsalzgehalt $6 \mathrm{~g} /$ Tag (•Tab. 2.9).

Ziele. Erhalt oder Verbesserung des Ernährungszustandes, Prävention ernährungsabhängiger Krankheiten.

Anmerkung zur Praxis. Die Ernährung wird auf 4-7 Mahlzeiten verteilt.

\section{Leichte Vollkost}

Indikationen. Unspezifische Intoleranzen gegen bestimmte Speisen und Lebensmittel. Die leichte Vollkost ist auch bei »unkomplizierten" Leber-, Galle-, Magenund Darmerkrankungen sowie im Anschluss an einen Kostaufbau oder bei älteren oder geschwächten Patienten angezeigt.

Definition.Die leichte Vollkost unterscheidet sich von der Vollkost durch Nichtverwendung von Lebensmitteln oder Speisen, die erfahrungsgemäß häufig (bei mehr als 5\% der Patienten) Unverträglichkeiten auslösen (s. - Tab. 2.10).

Ziele.Erhalt oder Verbesserung des Ernährungszustandes, Meiden von Unverträglichkeiten, Prävention ernährungsabhängiger Krankheiten.

Anmerkung. Die Ernährung sollte auf 4-7 Mahlzeiten verteilt werden. Eine leichte Vollkost entspricht der gastroenterologischen Basisdiät oder der Schonkost. Sie hat keinen therapeutischen Effekt. Als leicht verdauliche Kost kann sie Endstufe eines Kostaufbaus sein. Das Konzept der leichten Vollkost ist empirisch. 


\section{Passierte leichte Vollkost}

Indikationen. Beeinträchtigung und Erkrankung im Bereich des oberen Verdauungstrakts (Zahnverlust, Stomatitis, Stenosen, postoperativ).

Definition. Siehe Leichte Vollkost.

Ziele. Erhalt oder Verbesserung des Ernährungszustands, Meiden von Unverträglichkeiten, Prävention ernährungsabhängiger Krankheiten.

\section{Flüssige leichte Vollkost (flüssige Kost oder feinst- homogenisierte, flüssige und säurearme Kost)}

Indikationen. Behinderung der Nahrungsaufnahme und -passage durch Erkrankungen im Mund-, Rachen-, Larynx-, Pharynx- und Ösophagusbereich und möglicherweise nach großen gastrointestinalen Operationen.

Definition. Siehe Leichte Vollkost.
Ziele. Erhalt oder Verbesserung des Ernährungszustandes, Meiden von Unverträglichkeiten, Prävention ernährungsabhängiger Krankheiten.

Anmerkung. Der Nährstoffbedarf wird aufgrund des maximal zumutbaren Flüssigkeitsvolumens und der eingeschränkten Nahrungsmittelauswahl bei dieser Kostform nicht immer "gedeckt«. Formuladiäten sind für eine bedarfsdeckende Ernährung besser geeignet. Flüssige leichte Vollkost ist keine »Sondenkost«.

\section{Ovolaktovegetabile Vollkost}

Indikationen. Abneigung gegen Fleisch, Fisch sowie Fleisch- und Wurstwaren.

Definition. Die Kost ist frei von Fleisch, Fleischwaren, Fisch, Fischwaren. Der Eiweißanteil wird aus Vegetabilien sowie Ei, Milch und Milchprodukten gedeckt, der Puringehalt dieser Kost beträgt $<300$ mg/Tag. Der Fettgehalt

Tab. 2.9. Beispiel einer Vollkost

\begin{tabular}{|c|c|c|c|c|}
\hline Nahrungsmittel & $\begin{array}{l}\text { Menge pro } \\
\text { Person }\end{array}$ & $\begin{array}{l}\text { Beispiele für Änderungs- } \\
\text { möglichkeiten }\end{array}$ & $\begin{array}{l}\text { Menge pro } \\
\text { Person }\end{array}$ & Erläuterungen \\
\hline $\begin{array}{l}\text { Trinkmilch } \\
\text { Speisequark, mager }\end{array}$ & $\begin{array}{l}0,25 \mathrm{I} \\
50 \mathrm{~g}\end{array}$ & $\begin{array}{l}\text { Sauermilchprodukt } \\
\text { Hartkäse, 30\% i. Tr. }\end{array}$ & $\begin{array}{l}250 \mathrm{~g} \\
30 \mathrm{~g}\end{array}$ & $\begin{array}{l}\text { Milch und Milchprodukte sind in } \\
\text { der genannten Menge erforderlich, } \\
\text { damit die Tageskost ausreichend } \\
\text { Kalzium anbietet }\end{array}$ \\
\hline $\begin{array}{l}\text { Fleisch } \\
\text { (einschließlich } 50 \mathrm{~g} \\
\text { Aufschnitt) }\end{array}$ & $150 \mathrm{~g}$ & $\begin{array}{l}\text { Anstelle von Fleisch } \\
\text { Fischfilet } \\
\text { Eier }\end{array}$ & $\begin{array}{l}100 \mathrm{~g} \\
120 \mathrm{~g} \\
2 \text { Stück }\end{array}$ & $\begin{array}{l}\text { Fleischsorten abwechseln, 2-mal } \\
\text { wöchentlich, ein-mal pro Monat } \\
\text { Innereien berücksichtigen }\end{array}$ \\
\hline Koch- und Streichfett & $50 \mathrm{~g}$ & & & $\begin{array}{l}\text { Als Streichfett: Butter oder Margari } \\
\text { ne, Bratfette, Öle mit hohem Anteil } \\
\text { an MUFS verwenden }\end{array}$ \\
\hline Brot & $250 \mathrm{~g}$ & $\begin{array}{l}\text { Anstelle von Brot } \\
\text { Getreideflocken } \\
\text { Trockenmüsli }\end{array}$ & $\begin{array}{l}100 \mathrm{~g} \\
50 \mathrm{~g} \\
50 \mathrm{~g}\end{array}$ & $\begin{array}{l}\text { Vollkornbrot, Vollkornprodukte } \\
\text { bevorzugen }\end{array}$ \\
\hline Zucker & $30 \mathrm{~g}$ & $\begin{array}{l}\text { Anstelle von Zucker } \\
\text { Marmelade oder Honig }\end{array}$ & $\begin{array}{l}10 \mathrm{~g} \\
15 \mathrm{~g}\end{array}$ & \\
\hline Marmelade & $40 \mathrm{~g}$ & Honig in gleicher Menge & $40 \mathrm{~g}$ & \\
\hline Kartoffeln & $200 \mathrm{~g}$ & $\begin{array}{l}\text { Reis ungekocht } \\
\text { Nudeln ungekocht }\end{array}$ & $\begin{array}{l}50 \mathrm{~g} \\
45 \mathrm{~g}\end{array}$ & $\begin{array}{l}\text { Kartoffeln sind ohne Schale } \\
\text { berechnet }\end{array}$ \\
\hline Frisches Gemüse & $300 \mathrm{~g}$ & & & \\
\hline Frisches Obst & $300 \mathrm{~g}$ & $\begin{array}{l}\text { Frischer Obstsaft in gleicher } \\
\text { Menge, Kompott in gleicher } \\
\text { Menge, Trockenobst etwa 1/3 } \\
\text { der Menge }\end{array}$ & & \\
\hline
\end{tabular}


liegt unter 40\%. Eine vegane Kost hat in der Praxis einen niedrigeren Fettanteil. Die Eiweißzufuhr liegt bei einer vegetarischen Ernährung zwischen 0,6 und 0,7 g/kg KG und Tag. Der Phosphatgehalt dieser Kostform ist niedrig. Eine vegetarische Vollkost ist nach den Prinzipien der Vollkost zusammengesetzt. Sie enthält mehr Ballaststoffe als die Vollkost bzw. die leichte Vollkost. D Tab. 2.10 zeigt die Nährstoffrelationen einer vegetarischen Kost im Vergleich zu anderen alternativen Kostformen.

Ziele. Erhalt oder Verbesserung des Ernährungszustands, Prävention von ernährungsabhängiger Krankheiten, Prävention der hepatischen Enzephalopathie bei Patienten mit einer chronischen Lebererkrankung.

Anmerkung. Kann als purinarme Kost bei primärer und sekundärer Gicht bzw. Hyperurikämie und auch als fettarme Kost angewendet werden. Eine ovolaktovegetabile Vollkost ist auch zur Prävention einer hepatischen Enzephalopathie bei klinisch stabilen Patienten mit einer Leberzirrhose geeignet. Da diese Kostform nicht »eiweißdefiniert « ist, ist sie nicht zur Behandlung der hepatischen Enzephalopathie geeignet. Sie kann diagnostische Diät bei fleischfreier Ernährung sein (z. B. zur Diagnose okkulter gastrointestinaler Blutungen). Bei ausreichender und abwechslungsreicher Kost deckt eine ovolaktovegetabile Ernährung den Makro- und Mikronährstoffbedarf vollständig. Abhängig von der Form und Strenge des Vegetarismus können aber die Zufuhr bzw. die Resorption einiger Nährstoffe unterhalb der empfohlenen Werte liegen. Potentielle Gefahren einer streng vegetarischen Kost betreffen die folgenden Nährstoffe: Vitamin $B_{12}$, Eisen (Cave: weitere Verschlechterung der Eisenresorption durch im Tee enthaltene Tannine), Jod, Vitamin D und Kalzium. Mangelzustände werden nur bei einseitiger Lebensmittelauswahl beobachtet und können durch den gleichzeitigen Verzehr verschiedener pflanzlicher Produkte vermieden werden.

In der Praxis ist eine Einteilung in 4 Lebensmittelgruppen (I: Getreide und Cerealien, II: Hülsenfrüchte, III: Nüsse und Samen, IV: Gemüse und Obst) hilfreich. Der Nutzen der Vielfalt soll am Beispiel der pflanzlichen Eiweiße dargestellt werden. In der Gruppe I sind die Aminosäuren Lysin und Threonin, in der Gruppe II Methionin und Tryptophan, in der Gruppe III Lysin und in der Gruppe IV Methionin limitierend. Durch eine richtige "Mischung" verschiedene Gruppen können die "Nachteile« der einzelnen Gruppe ausgeglichen werden. In der Praxis werden für einen Erwachsenen jeweils pro Tag 1 Portion aus den Gruppen II und III, 7 Portionen aus den Gruppen 4 und 5 Portionen aus der Gruppe I empfohlen. Dazu kommen 2 Portionen Milch und Milchprodukte. Für Schwangere/Stillende gelten 4 Portionen aus den Gruppen II und III, 8 Portionen aus der Gruppe IV und

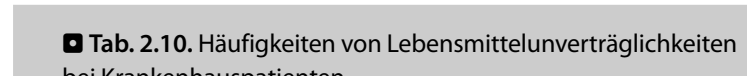
bei Krankenhauspatienten

\begin{tabular}{l|l}
\hline Intoleranzen & $\%$ \\
\hline Hülsenfrüchte & 30,1 \\
\hline Gurkensalat & 28,6 \\
\hline Fritierte Speisen & 22,4 \\
\hline Weißkohl & 20,2 \\
\hline
\end{tabular}

\begin{tabular}{l|l}
\hline $\mathrm{CO}_{2}$-haltige Getränke & 20,1 \\
\hline Grünkohl & 18,1 \\
\hline
\end{tabular}

\begin{tabular}{l|l}
\hline Fette Speisen & 17,2 \\
\hline Paprikagemüse & 16,8 \\
\hline Sauerkraut & 15,8 \\
\hline Rotkraut & 15,8 \\
\hline Süße und fette Backwaren & 15,8 \\
\hline Zwiebeln & 15,8 \\
\hline
\end{tabular}

\begin{tabular}{ll}
\hline Wirsing & 15,6 \\
\hline
\end{tabular}

\begin{tabular}{ll}
\hline Pommes frites & 15,3 \\
\hline
\end{tabular}

\begin{tabular}{l|c|}
\hline Hartgekochte Eier & 14,7 \\
\hline Frisches Brot & 13,6 \\
\hline Bohnenkaffee & 12,5 \\
\hline
\end{tabular}

\begin{tabular}{ll}
\hline Kohlsalat 12, \\
\hline
\end{tabular}

\begin{tabular}{l|l}
\hline Mayonnaise & 11,8 \\
\hline Kartoffelsalat & 11,4 \\
\hline Geräuchertes & 10,7 \\
\hline Eisbein & 9,0 \\
\hline Zu stark gewürzte Speisen & 7,7 \\
\hline $\begin{array}{l}\text { Zu heiße und zu kalte } \\
\text { Speisen }\end{array}$ & 7,6 \\
\hline
\end{tabular}

\begin{tabular}{ll}
\hline Süßigkeiten & 7,6 \\
\hline Weißwein & 7,6 \\
\hline Rohes Stein- und Kernobst & 7,3 \\
\hline Nüsse & 7,1 \\
\hline Sahne & 6,8 \\
\hline Paniert Gebratenes & 6,8 \\
\hline Pilze & 6,1 \\
\hline Rotwein & 6,1 \\
\hline Lauch & 5,9 \\
\hline Spirituosen & 5,8 \\
\hline Birnen & 5,6 \\
\hline
\end{tabular}


6 Portionen aus der Gruppe I sowie 4 Portionen Milch und Milchprodukte. Aufgrund ihres eher niedrigen Fettanteils sowie der (bei naturgemäß hoher Ballaststoffzufuhr) guten Sättigung (die Kost ist in der Praxis eher hypokalorisch) ist eine vegetarische Ernährung auch als Reduktionskost bei leichtem Übergewicht (Adipositas Grad 1) oder bei Hypertriglyceridämie zur Behandlung geeignet.

\subsubsection{Energiedefinierte Diäten}

\section{Reduktionskost}

Indikationen. Übergewicht mit einem hohem gesundheitlichen Risiko für bzw. bei bereits manifestem(r) Diabetes mellitus, Hypertonie, Hyperlipoproteinämie, Atherosklerose und Hyperurikämie. Zur Risikoeinschätzung der Adipositas s. - Tab. 2.11.

Kontraindiktionen. Schwere Erkrankungen (z. B. bis zu 8 Wochen nach einem Herzinfarkt) schließen eine diätetische Gewichtsreduktion aus. Bei Gicht und auch bei bekannten Gallensteinleiden sind keine drastischen Diäten, z. B. keine Diäten mit einem Kaloriengehalt $<1000$ kcal/ Tag erlaubt.

Weitere mögliche Kontraindikationen sind:

- Normalgewicht,

- Kinder und Jugendliche,

- Schwangerschaft (Ausnahme: schwere Schwangerschaftsgestose) und Stillperiode,

- Essstörungen (»binge eating«),

- schwere Allgemeinerkrankungen,

- Porphyrie,

- Alter $>60$ Jahre(?).
Definition. Reduktion der Energie- bzw. Fettzufuhr. Die Reduktionskost wird in 2 Energiestufen mit 1200 und 1500 kcal. angeboten. Die Nährstoffrelation beträgt: 20-25\% Eiweiß, 30-35\% Fett, 40-50\% Kohlenhydrate, 25-35 g Ballaststoffe. Der Mikronährstoff- und Flüssigkeitsbedarf ist gedeckt.

\section{Ziele}

- Verminderung des mit der Adipositas verbundenen gesundheitlichen Risikos,

- langsame, kontinuierliche und dauerhafte (über $>1$ Jahr) Gewichtsabnahme,

- Meidung gesundheitlicher Schäden,

- Verhütung von Essstörungen.

Anmerkung. Die Evidenz-basierten Leitlinien für die Adipositastherapie bei Kindern, Jugendlichen und Erwachsenen finden sich unter http://www.adipositas-gesellschaft.de und http://www.aga.de. Eine Adipositasbehandlung berücksichtigt das Ausmaß der Adipositas und die gesundheitliche Gefährdung. Das Risiko ist wesentlich durch den Taillenumfang bzw. dem Fettverteilungstyp) charakterisiert (s. - Tab. 2.11). Ab dem 65. Lebensjahr relativiert sich der »Risikofaktor Adipositas $«$.

Therapieoptionen. Eine Reduktionskost ist Teil eines ganzheitlichen Konzepts, welches neben der Ernährungsumstellung mehr körperliche Bewegung und eine Stärkung der persönlichen Autonomie durch Stressprophylaxe und Verhaltenstherapie beinhaltet (-Abb. 2.11). Eine isolierte ernährungsmedizinische Maßnahme (sprich z. B. Diät) ist wenig hilfreich. Die Zusammensetzung einer Reduktionskost (- Tab. 2.12) sowie Strategien der indi-

- Tab. 2.11. Algorithmus zur Einschätzung des Adipositasrisikos. BMI body mass index; $\mathrm{kg} / \mathrm{m}^{2}$, wc (waist circumference=Taillenumfang), $\mathrm{M}$ Männer, F Frauen

\begin{tabular}{|c|c|c|c|c|}
\hline Adipositasgrad & $\mathrm{BMI}\left(\mathrm{kg} / \mathrm{m}^{2}\right)$ & wc & Weitere Risikofaktoren ${ }^{a}$ & Risiko $^{b}$ \\
\hline 0 & $<25$ & $\begin{array}{l}\mathrm{M}:>94 \\
\mathrm{~F}:>80\end{array}$ & $\varnothing$ & 0 \\
\hline 1 & $25-29,9$ & $\begin{array}{l}M:>94 \\
F:>80 \\
M:>94 \\
F:>80\end{array}$ & $\begin{array}{l}\varnothing \\
\varnothing /+\end{array}$ & $\begin{array}{l}1 \\
1-2\end{array}$ \\
\hline 2 & $30-39,9$ & $\begin{array}{l}M:>94 \\
F:>80 \\
M:>94 \\
F:>80\end{array}$ & $\begin{array}{l}\varnothing \\
\varnothing /+\end{array}$ & $\begin{array}{l}1-2 \\
2-3\end{array}$ \\
\hline 3 & $\geq 40$ & $\begin{array}{l}M:>94 \\
F:>80\end{array}$ & $\varnothing /+$ & 3 \\
\hline
\end{tabular}

a Hypertonie, Koronare Herzerkrankung, Diabetes mellitus, Nikotinabusus.

${ }^{\mathrm{b}}$ Risiko $=0 \rightarrow 3,0=$ kein Risiko, 3=sehr großes Risiko. 
vidualisierten Adipositastherapie sind in - Tab. 2.12 und - Tab. 2.13 dargestellt.

Das praktische Vorgehen der Adipositastherapie gliedert sich in folgende Abschnitte: Ärztliches Gespräch/ Anamnese (Patient,Angehörige), Eingrenzung der Problemkreise (Ernährung Körperliche Aktivität, Lebensstil,
»Befindlichkeit«, »Umgebung«), Strukturierte Behandlung mit den Themen »Mehr Bewegung «, "Stressprophylaxe/Entspannung«, »Fettarme Ernährung « (Reduktionsdiät). Medikamente und operative/invasive Maßnahmen sind bei einzelnen Patienten (z. B. Gastroplastik bei ausgeprägter Adipositas mit $\mathrm{BMI}>40 \mathrm{~kg} / \mathrm{m}^{2}$ und einem

- Tab. 2.12. Energiegehalt sowie Zufuhr von Makro- und Mikronährstoffen einer Reduktionskost sowie »Niedrigst-Kalorien-Diäten« am Beispiel Modifast ${ }^{\oplus}$ im Vergleich zu Empfehlungen nach Dach für Erwachsene zwischen 25 und 50 Jahren

\begin{tabular}{|c|c|c|c|c|c|}
\hline & \multirow{2}{*}{$\begin{array}{l}\text { Empfehlungen } \\
\text { nach Dach }\end{array}$} & \multirow{2}{*}{$\begin{array}{l}\text { Konventionelle } \\
\text { Reduktionskost } \\
\text { (1200 kcal) }\end{array}$} & \multicolumn{3}{|l|}{ Modifast $^{\oplus}$} \\
\hline & & & 4 B. $(600 \mathrm{kcal})$ & 5 B. $(750 \mathrm{kcal})$ & 6 B. $(900 \mathrm{kcal})$ \\
\hline Kcal & & 1206 & 594 & 742 & 890 \\
\hline Protein(g) & $0,8 \mathrm{~g} / \mathrm{kg} \mathrm{KG}$ & 67 & 67 & 83 & 100 \\
\hline Protein (\%) & $12-13$ & 23 & 46 & 46 & 46 \\
\hline Fett (g) & & 44 & 10 & 12 & 120 \\
\hline Fett (\%) & $25-30$ & 34 & 14 & 14 & 14 \\
\hline Kohlenhydrate (g) & & 127 & 60 & 75 & 90 \\
\hline Kohlenhydrate (\%) & 50 & 43 & 39 & 39 & 39 \\
\hline Ballaststoffe (g) & $\begin{array}{l}\geq 30 / \text { Tag bzw. } \\
12,5 / 1000 \mathrm{kcal}\end{array}$ & 26 & - & - & - \\
\hline Natrium (mg) & 550 & 1342 & 1328 & 1660 & 1992 \\
\hline Kalium (mg) & 2000 & 3233 & 2688 & 3600 & 4132 \\
\hline Magnesium (mg) & $350 / 300$ & 284 & 368 & 460 & 552 \\
\hline Kalzium (mg) & 900 & 695 & 1088 & 1360 & 1632 \\
\hline Phosphor (mg) & 1400 & 1138 & 1088 & 1360 & 1632 \\
\hline Eisen (mg) & $10 / 15$ & 14 & 33 & 42 & 50 \\
\hline $\operatorname{lod}(\mu \mathrm{g})$ & 200 & 27 & - & - & - \\
\hline Zink (mg) & $15 / 20$ & 3 & - & - & - \\
\hline $\begin{array}{l}\text { Vitamin A/Retinol- } \\
\text { Äquivalent (mg) }\end{array}$ & $1,0 / 0,8$ & 0,4 & 0,6 & 0,8 & 0,9 \\
\hline Vitamin D $(\mu \mathrm{g})$ & 5 & 0,6 & 1,8 & 2,2 & 2,6 \\
\hline Vitamin E (mg) & 12 & 18 & 16 & 20 & 24 \\
\hline Folsäure $(\mu \mathrm{g})$ & $300(150)$ & 244 & 531 & 664 & 797 \\
\hline Vitamin $B_{1}(\mathrm{mg})$ & $1,3 / 1,1$ & 1,1 & 2,1 & 3,4 & 4,0 \\
\hline Vitamin $B_{2}(\mathrm{mg})$ & $1,7 / 1,5$ & 1,5 & 2,7 & 3,4 & 3,6 \\
\hline Vitamin $B_{6}(\mathrm{mg})$ & $1,8 / 1,6$ & 2,4 & 2,4 & 3,0 & 3,6 \\
\hline Vitamin C (mg) & 75 & 183 & 100 & 125 & 150 \\
\hline
\end{tabular}


hohen gesundheitlichen Risiko, z. B. bei Schlaf-ApnoeSyndrom.

\section{"Binge eating disorder"}

Nach wiederholten und häufigen drastischen Diäten können sich bei Adipösen Essstörungen (sog. Fressanfälle oder »binge eating disorder «) entwickeln. Diagnostische Kriterien des »binge eating «-s) sind nach DSM IV, 1993:

- Essen unverhältnismäßig großer Mengen in kurzer Zeit;

- Verlust der Kontrolle während des Essens;

- Drei der folgenden Kriterien:
- sehr schnell essen,

- essen bis zum Unwohlsein,

- essen großer Mengen auch ohne Hungergefühl und ohne geplante Mahlzeiten,

- alleine essen,

- Ängstlichkeit, Langeweile, Depression als Auslöser der Essattacke,

- Schuldgefühle, Abscheu oder Depressionen nach einer Essattacke;

- ständiger Kampf gegen die Eßstörung;

- Eßattacken mindestens 2-mal/Woche über 6 Monate;

- die Kriterien einer Bulimia nervosa werden nicht erfüllt.

- Tab. 2.13. »Individualisierte« Maßnahmen und angestrebte Erfolge der Adipositastherapie

\begin{tabular}{l|l|l}
\hline Maßnahme & Praxis & Angestrebter Erfolg \\
\hline Diät & $\begin{array}{l}600 \mathrm{kcal} / \mathrm{Tag} \\
\text { Basis: gemessener REE »re-adjustment « alle 2 Mo. }\end{array}$ & 3-5\% KG/3 Monate \\
\hline Aktivität & PAL: 1,75 & \\
& $=60-80 \mathrm{~min} / \mathrm{Tag}$ »walken«, »joggen«, schwimmen & Langfristige Stabilisierung des KG's \\
\hline Motivation & Ernährungsberatung: alle 1-2 Wo & Langfristige Stabilisierung des Körpergewichts \\
\hline Medikamente? & Sibutramin: $10(-20) \mathrm{mg} / \mathrm{d}$ & \\
& $\begin{array}{l}\text { Orlistat: } 120 \mathrm{mg} / \mathrm{d} \\
\text { Rimonabaut } 5(-20) \mathrm{mg} / \mathrm{d}\end{array}$ & $\begin{array}{l}\text { Langfristige Stabilisierung des KG's } \\
\text { (Cave: Behandlungsdauer) }\end{array}$ \\
\hline Operation & Magenverkleinerung (Gastroplastik) & \\
\hline
\end{tabular}

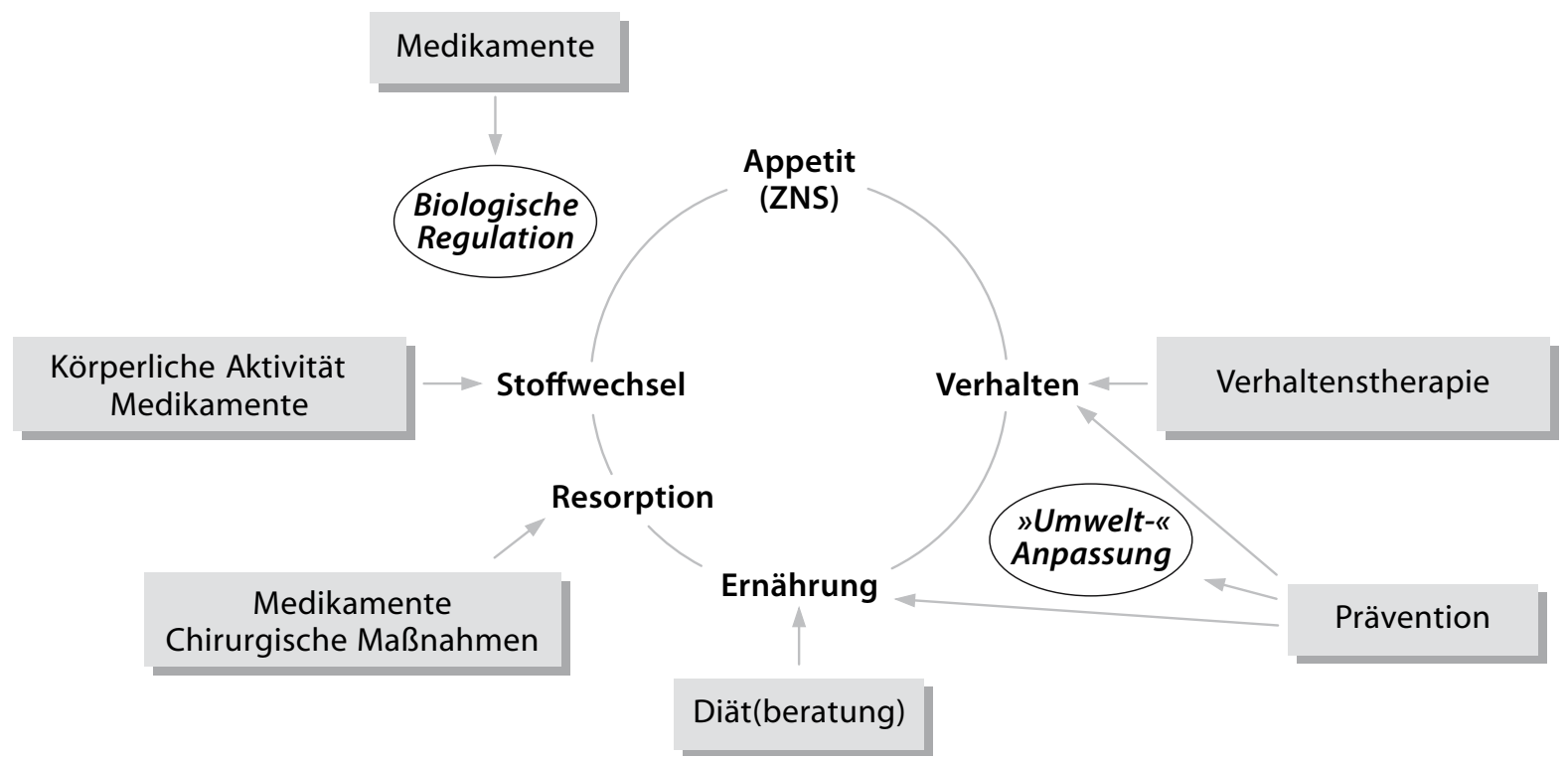

- Abb. 2.11. Therapeutische Interventionen bei Adiposita 


\section{"Crash-Diäten«}

Für einen nachhaltigen (d.h. über 2-5 Jahre) Erfolg ist eine Weiterbetreuung der Patienten unerlässlich. Die Adipositastherapie ist für den Betroffenen und seinen Arzt meist eine lebenslange Behandlung. "Crash-Diäten « sind obsolet. Im Vergleich erscheinen kohlenhydratreiche und extrem fettarme Reduktionsdiäten (z. B. Ornish-Diät, s. unten) und fettreiche und kohlenhydratarme Reduktionsdiäten (»low carb diets«, z. B. Atkins-Diät) zumindest mittelfristig (d. h.bis maximal zu 1 Jahr nachbeobachtet) im Hinblick auf den Erfolg ( $=$ Gewichtsabnahme) vergleichbar. Bei »low carb diets « ist der Anstieg der Serum-Harnsäure-, Ketonkörper- und Serum-Harnstoffspiegel zu beachten und ein mögliches Abbruchkriterium. Bei Kalorienmengen unter $1200 \mathrm{kcal} /$ Tag ist eine bedarfsdeckende Ernährung unter Verwendung "normaler« Lebensmittel nicht mehr gewährleistet. »Crash-Diäten« dienen auch nicht dem notwendigen »Umlernprozess« der Patienten. Niedrigstkaloriendiäten (=»very low caloric diets $=$ VLCD oder $»$ very low energy diets «=VLED) enthalten weniger Kalorien als eine konventionelle Reduktionskost (i.e. 400-800 kcal/Tag). Die Zusammensetzung der VLED’s ist im $\$ 14 \mathrm{a}$ der Diätverordnung sowie gemäß Richtlinie 96/8/EG festgelegt (s. - Tab. 2.12). Die Zufuhr an Mikronährstoffen und Mineralstoffen entspricht den Empfehlungen der Deutschen Gesellschaft für Ernährung für eine ausgewogene und isokalorische Ernährung. Genaue Bedarfszahlen für eine hypokalorische Ernährung sind nicht bekannt. VLED's dürfen nur unter ärztlicher Aufsicht und nicht länger als 12 Wochen angewendet werden. Vor wiederholtem und unkontrolliertem Gebrauch wird gewarnt. Es ist auf eine ausreichende und kalorienfreie Flüssigkeitszufuhr (Kaffee, Tee, Mineralwasser) zu achten ( $>1,5 / \mathrm{Tag})$. Obsolet sind Obst- und Reistage sowie eine Nulldiät oder das modifizierte Fasten. Für die Langzeitbehandlung ist eine fettarme Ernährung anzuraten.

\section{Evaluation}

Für die Evaluation von Programmen zur Gewichtsreduktion gelten heute strenge Kriterien:

- Langfristiger Gewichtsverlust: 1 Jahr und länger, $\geq 5 \%$ des Körpergewichts, Reduktion um 1 BMI-Einheit und mehr.

- Verbesserung der mit dem Übergewicht assoziierten Erkrankungen bzw. Risikofaktoren wie Bluthochdruck, Hypercholesterinämie, Hypertriglyzeridämie, Hyperglykämie, Diabetes mellitus Typ II(b).

- Verbessertes Gesundheitsverhalten: regelmäßige Ernährungsprotokolle, Verzehr gemäß den Empfehlungen zur "gesunden « Ernährung; regelmäßige körperliche Aktivität, d. h. tgl. mindestens $1 / 2$ Stunde spazieren gehen, aerobe Belastung (=Ausdauersport) an $4 \mathrm{Ta}$ gen/Woche.
- Regelmäßige ärztliche Konsultationen.

- Überwachung und Erfassung gegenteiliger Effekte wie z. B. Essstörungen, Gallensteinleiden usw.

Gemessen an diesem "Kriterium « sind die "Erfolge» der Adipositasbehandlung begrenzt. Nachhaltig (über 1-5 Jahre) können nur 5-30\% der behandleten Patienten ihr reduziertes Gewicht "halten« (Ausnahme: Gastroplastik).

\section{Ernährung bei Diabetes mellitus}

Indikationen. Diabetes mellitus Typ I und II.

Definition. Die Diabeteskost entspricht der Vollkost.

\section{Ziele}

- " Einstellung " bzw. Verbesserung des Glukosestoffwechsels unter Berücksichtigung der übrigen Risikofaktoren (Lipidstoffwechsel, Blutdruck).

- Verhinderung bzw. Verzögerung des Auftretens diabetischer Spätschäden und damit Senkung der Mortalität.

- Gewichtskonstanz bei Typ-I-Diabetikern und normalgewichtigen Typ-II-Diabetikern. Gewichtsreduktion bei übergewichtigen Typ-II-Diabetikern.

- Normales Wachstum und normale Entwicklung bei Kindern und Jugendlichen.

- Verbesserung der Lebensqualität.

Anmerkung. Die Diagnosekriterien sind in -Tab.2.14 dargestellt. "Gesunde« Ernährung ist ein Teil der Diabetestherapie. Angesichts der Fortschritte der Insulintherapie und Diabetikerschulung ist die Bedeutung der Ernährung und der Diät für den Diabetiker relativiert worden. Die Behandlungsziele sind in $\mathbf{0}$ Tab. 2.15 formuliert. Die Wertigkeit einzelner diätetischer Prinzipien ist bei Typ-I- und Typ-II-Diabetikern unterschiedlich (•Tab. 2.16). Während für den übergewichtigen Typ II(b)-Diabetiker Energie- und Fettreduktion im Vordergrund stehen, bleiben für den Typ-1-Diabetiker die Regeln einer "gesunden« Ernährung. Modifikationen sind bei Komplikationen (z. B. Nephropathie) möglich und sinnvoll. Die Ernährungstherapie muss das Gesamtkonzept der Diabetestherapie berücksichtigen, eine »isolierte« Ernährungsberatung ist obsolet. Eine »konventionelle« Diabetesdiät wird bei Typ-I-Diabetikern individuell berechnet und verordnet, sie hat lediglich bei »starrem « Insulinbehandlungskonzept eine Bedeutung (z. B. bei 2 festen Insulininjektionen pro Tag). Ein Beispiel ist in - Tab. 2.17 und - Tab. 2.18 dargestellt. Die Ernährung wird flexibel wie möglich an die Wünsche und Bedürfnisse der Patienten angepasst.

KHE. Für die Schätzung des Kohlenhydratanteils in der Diät des Typ-I-Diabetikers werden im deutschsprachigen 
Raum Broteinheiten (=BE) oder Kohlenhydrataustauscheinheiten $(=\mathrm{KHE})$ benutzt, die einer Kohlenhydratmenge von 10-12 g entsprechen (z. B. 1 Brötchen von 25 g, 20 g Teigwaren, 80 g Kartoffeln, $250 \mathrm{ml}$ Milch, $100 \mathrm{~g}$ Melone oder Apfel, $100 \mathrm{ml}$ Apfelsaft). Diese Einheiten sind Schätzgrößen, welche insulinbehandelten Diabetikern die Portionierung von kohlenhydrathaltigen Lebensmitteln sowie die Berechnung der Insulindosis erleichtern. Da die Varianz des Kohlenhydratgehalts in Lebensmitteln hoch ist (bis zu 30\%), dienen Kohlenhydrataustauscheinheiten lediglich der Orientierung. Im Rahmen der Diätetik für übergewichtige und nicht mit Insulin behandelten TypII-Diabetiker sind sie ohne Wert.

GI, GL. Der sog. »Glykämische Index « charakterisiert die "Blutzuckerwirksamkeit" des Kohlenhydratteils in den Lebensmitteln im Vergleich zu einem Refernzlebensmittel (=100 g Weißbrot) (s. - Tab. 2.19). Die Blutzuckerwirkung der Nahrungskohlenhydrate wird nicht allein durch die Art der Kohlenhydrate sondern auch z. B. durch den
»Kontext " des Lebensmittels (d. h. z. B. durch seinen Fettanteil) bestimmt. Um den glykämischen Index (GI) in eine den tatsächlich verzehrten Lebensmittelmenge entsprechende Größe umzurechnen, wird die »Glykämische Last« (GL) angegeben (s. - Tab. 2.19). - Tab. 2.20 zeigt Beispiele für GI und GL.

Die Höhe von GI und GL zeigt eine Beziehung zum Ernährungszustand zum mittleren Blutzuckerspiegel und zum Plasma-HDL-Spiegel. Die Ergebnisse epidemiologischer Studien lassen vermuten, dass ein niedriger GI/GL "präventiv« gegenüber Übergewicht, Typ-2-Diabetes und koronare Herzerkrankung ist. In der Praxis der Behandlung von Patienten mit einem Typ-2-Diabetes mellitus zeigt eine Diät mit niedrigem GI im Vergleich zu einer Diät mit einem hohen GI Effekte auf den Fruktosaminspiegel $(-0,1 \mathrm{mmol} / \mathrm{T})$, dem HbA1C-Wert $(-0,27 \%)$ und dem Cholesterinspiegel im Plasma $(-0,15 \mathrm{mmol} / \mathrm{T})$. Demgegenüber fanden sich in einer iner Metaanalyse keine signifikanten Effekte auf LDLc, HDLc und Triglyceridwerte.

\begin{tabular}{|l|l|}
\hline - Tab. 2.14. Diagnose eines Diabetes mellitus & \\
\hline Symptome des Diabetes mellitus & $\begin{array}{l}\text { Polyurie } \\
\text { Polydipsie } \\
\text { Unerklärter Gewichtsverlust }\end{array}$ \\
\hline Erhöhter Plasmaglukosespiegel & $\begin{array}{l}\text { "zufällige« Messung } \\
>200 \mathrm{mg} / \mathrm{dl} \text { oder }>11,1 \mathrm{mmol} / \mathrm{l}\end{array}$ \\
\hline Erhöhter Nüchternplasmaglukosespiegel & $\begin{array}{l}>126 \mathrm{mg} / \mathrm{dl}(>7,0 \mathrm{mmol} / \mathrm{l}) \\
\text { nüchtern=mind. } 8 \mathrm{~h} \text { Nahrungskarenz }\end{array}$ \\
\hline Erhöhter 2-h-Plasmaglukosespiegel & $>200 \mathrm{mg} / \mathrm{dl}$ in einem Standart oGGT $75 \mathrm{~g}$ Glukose \\
\hline
\end{tabular}

Quelle: ADA, 1997; Diabetes Care 20, 1183, 1997

- Tab. 2.15. Therapieziele bei Diabetes mellitus. (Aus Toeller 2002)

\begin{tabular}{l|l}
\hline Blutglukose $(\mathrm{mg} / \mathrm{dl})$ & $\begin{array}{l}\text { Nüchtern/prandial } 90-120 \\
1-2 \mathrm{~h} \text { postprandial } 130-160 \\
\text { vor dem Schlafengehen 110-140 }\end{array}$ \\
\hline Hämoglobin A1c $(\%)$ & $6-7,5$ \\
\hline Gesamt-Cholesterin $(\mathrm{mg} / \mathrm{dl})$ & $<200(<170)^{*}$ \\
\hline LDL-Cholesterin $(\mathrm{mg} / \mathrm{dl})$ & $<130(<100)^{*}$ \\
\hline HDL-Cholesterin $(\mathrm{mg} / \mathrm{dl})$ & $<150$ \\
\hline Blutdruck systolisch $(\mathrm{mm} \mathrm{Hg})$ & $\leq 140(\leq 130)^{* *}$ \\
\hline Blutdruck diastolisch $(\mathrm{mm} \mathrm{Hg})$ & $\leq 85(\leq 80)^{* *}$ \\
\hline Body Mass-Index $\left(\mathrm{kg} / \mathrm{m}^{2}\right)$ & $18,5-25$
\end{tabular}

* Diabetiker mit mikro- bzw. makrovaskulären Erkrankungen

** Diabetiker mit Mikroalbuminurie und /oder manifester Nephropathie 
- Tab. 2.16. Prinzipien der Diätetik bei Patienten mit einem Diabetes mellitus

\begin{tabular}{|c|c|c|}
\hline Diätische Maßnahme & $\begin{array}{l}\text { Diabetes mellitus Typ II } \\
\text { Übergewicht }\end{array}$ & $\begin{array}{l}\text { Diabetes mellitus Typ I } \\
\text { Diabetes }\end{array}$ \\
\hline Energierestrikition & Sehr wichtig & Obsolet \\
\hline Fettrestriktion & Sehr wichtig & $\begin{array}{l}\text { Im Rahmen der Empfehlungen für eine } \\
\text { gesunde Ernährung wichtig }\end{array}$ \\
\hline Eiweißzufuhr von $0,8 \mathrm{~g} / \mathrm{kg} \times \mathrm{Tag}$ & Bei beginnender Nephropathie & Bei beginnender Nephropathie \\
\hline Eiweißrestriktion & Bei manifester Nephropathie & Bei manifester Nephropathie \\
\hline Kochsalzbeschränkung & Bei Hypertonie & Bei Hypertonie \\
\hline Häufige Mahlzeiten & & Wichtig \\
\hline Tagesplan & & $\begin{array}{l}\text { Relativ wichtig abhängig vom Behand- } \\
\text { lungsregime, bei intensivierter Insulin- } \\
\text { therapie unwichtig, bei konventioneller } \\
\text { Insulintherapie wichtig }\end{array}$ \\
\hline $\begin{array}{l}\text { Zusätzliche Ernährung bei } \\
\text { Muskeltätigkeit }\end{array}$ & & Relativ wichtig \\
\hline $\begin{array}{l}\text { Wechselwirkung von Medikamenten } \\
\text { und Ernährung }\end{array}$ & Wichtig & Relativ wichtig \\
\hline
\end{tabular}

- Tab. 2.17. Konventioneller Diätplan für Diabetiker. E Eiweiß, F Fett, KH Kohlenhydrate, KHE Kohlenhydrataustauscheinheiten

\begin{tabular}{|c|c|c|c|c|c|c|c|c|}
\hline & $10 \mathrm{KHE}$ & $12 \mathrm{KHE}$ & $15 \mathrm{KHE}$ & $18 \mathrm{KHE}$ & 20KHE & $22 \mathrm{KHE}$ & $24 \mathrm{KHE}$ & $26 \mathrm{KHE}$ \\
\hline $\begin{array}{l}\text { Energiegehalt } \\
\text { (kcal) }\end{array}$ & 1200 & 1400 & 1600 & 1800 & 2000 & 2200 & 2400 & 2600 \\
\hline Makronährstoffe & $\begin{array}{l}60 \mathrm{gE} \\
45 \mathrm{gF} \\
130 \mathrm{~g} \mathrm{KH}\end{array}$ & $\begin{array}{l}65 \mathrm{gE} \\
50 \mathrm{gF} \\
155 \mathrm{~g} \mathrm{KH}\end{array}$ & $\begin{array}{l}70 \mathrm{gE} \\
50 \mathrm{gF} \\
190 \mathrm{~g} \mathrm{KH}\end{array}$ & $\begin{array}{l}75 \mathrm{gE} \\
65 \mathrm{gF} \\
230 \mathrm{~g} \mathrm{KH}\end{array}$ & $\begin{array}{l}80 \mathrm{~g} \mathrm{E} \\
75 \mathrm{~g} \mathrm{~F} \\
250 \mathrm{~g} \mathrm{KH}\end{array}$ & $\begin{array}{l}80 \mathrm{gE} \\
80 \mathrm{gF} \\
275 \mathrm{~g} \mathrm{KH}\end{array}$ & $\begin{array}{l}90 \mathrm{gE} \\
80 \mathrm{gF} \\
300 \mathrm{~g} \mathrm{KH}\end{array}$ & $\begin{array}{l}100 \mathrm{~g} \mathrm{E} \\
90 \mathrm{~g} \mathrm{~F} \\
325 \mathrm{~g} \mathrm{KH}\end{array}$ \\
\hline \multicolumn{9}{|l|}{ KHE-Verteilung } \\
\hline 1. Frühstück & $2 \mathrm{~K} \mathrm{KHE}$ & $2 \frac{1}{2} \mathrm{KHE}$ & $3 \frac{1}{2} \mathrm{KHE}$ & $31 \frac{1}{2} \mathrm{KHE}$ & $41 \frac{1}{2} \mathrm{KHE}$ & $4 \frac{1}{2} \mathrm{KHE}$ & $4 \frac{1}{2} \mathrm{KHE}$ & $41 \frac{1}{2} \mathrm{KHE}$ \\
\hline 2. Frühstück & $1 \frac{1}{2} \mathrm{KHE}$ & $2 \mathrm{KHE}$ & $2 \mathrm{KHE}$ & $3 \mathrm{KHE}$ & $3 \mathrm{KHE}$ & $3 \mathrm{KHE}$ & $4 \mathrm{KHE}$ & $4 \mathrm{KHE}$ \\
\hline Zwischenmahlzeit & - & - & - & - & - & - & - & $2 \mathrm{KHE}$ \\
\hline Mittagessen & $2 \mathrm{KHE}$ & $3 \mathrm{KHE}$ & $3 \mathrm{KHE}$ & $4 \mathrm{KHE}$ & $4 \mathrm{KHE}$ & $5 \mathrm{KHE}$ & $5 \mathrm{KHE}$ & $5 \mathrm{KHE}$ \\
\hline Zwischenmahlzeit & $1 \frac{1}{2} \mathrm{KHE}$ & $1 \frac{1}{2} \mathrm{KHE}$ & $2 \frac{1}{2} \mathrm{KHE}$ & $2^{1} \frac{1}{2} \mathrm{KHE}$ & $2 \frac{1}{2} \mathrm{KHE}$ & $3 \frac{1}{2} \mathrm{KHE}$ & $3 \frac{1}{2} \mathrm{KHE}$ & $3 \frac{1}{2} \mathrm{KHE}$ \\
\hline Abendessen & $2 \mathrm{KHE}$ & $2 \mathrm{KHE}$ & $3 \mathrm{KHE}$ & $3 \mathrm{KHE}$ & $4 \mathrm{KHE}$ & $4 \mathrm{KHE}$ & $4 \mathrm{KHE}$ & $4 \mathrm{KHE}$ \\
\hline Spätmahlzeit & $1 \mathrm{KHE}$ & $1 \mathrm{KHE}$ & $1 \mathrm{KHE}$ & $2 \mathrm{KHE}$ & $2 \mathrm{KHE}$ & $2 \mathrm{KHE}$ & $2 \mathrm{KHE}$ & $3 \mathrm{KHE}$ \\
\hline
\end{tabular}


- Tab. 2.18. Beispiel eines Tageskostplanes für eine konventionelle Diabetesdiät.

\begin{tabular}{|c|c|}
\hline \multicolumn{2}{|l|}{ Tagesbeispiel } \\
\hline $\begin{array}{l}\text { Frühstück } \\
4 \mathrm{KHE}\end{array}$ & $\begin{array}{l}90 \mathrm{~g} \text { Roggenbrot } \\
10 \mathrm{~g} \text { Streichfett } \\
25 \mathrm{~g} \text { Konfitüre mit Zuckeraus- } \\
\text { tauschstoff } \\
\text { Kaffee/Tee }\end{array}$ \\
\hline $\begin{array}{l}\text { Zwischenmahlzeit } \\
3 \mathrm{KHE}\end{array}$ & $\begin{array}{l}60 \mathrm{~g} \text { Mischbrot } \\
5 \mathrm{~g} \text { Streichfett } \\
100 \mathrm{~g} \text { Tomatenscheiben } \\
250 \mathrm{~g} \text { Milch, 1,5\% Fettgehalt }\end{array}$ \\
\hline $\begin{array}{l}\text { Mittag } \\
5 \mathrm{KHE}\end{array}$ & $\begin{array}{l}320 \mathrm{~g} \text { Kartoffeln } \\
200 \mathrm{~g} \text { Bohnengemüse } \\
100 \mathrm{~g} \text { Rostbeaf } \\
15 \mathrm{~g} \text { Kochfett } \\
100 \mathrm{~g} \mathrm{Apfel}\end{array}$ \\
\hline $\begin{array}{l}\text { Zwischenmahlzeit } \\
2 \mathrm{KHE}\end{array}$ & $\begin{array}{l}45 \mathrm{~g} \text { Roggenbrot } \\
5 \mathrm{~g} \text { Streichfett } \\
15 \mathrm{~g} \text { Konfitüre mit Zuckeraus- } \\
\text { tauschstoff } \\
\text { Kaffee/Tee }\end{array}$ \\
\hline $\begin{array}{l}\text { Abend } \\
4 \mathrm{KHE}\end{array}$ & $\begin{array}{l}70 \mathrm{~g} \text { Vollkornbrot } \\
30 \mathrm{~g} \text { Mischbrot } \\
10 \mathrm{~g} \text { Streichfett } \\
20 \mathrm{~g} \text { Edamerkäse, 30\% Fett i. Tr. } \\
20 \mathrm{~g} \text { Schinken } \\
200 \mathrm{~g} \text { Gurkensalat } \\
\text { Tee/Mineralwasser }\end{array}$ \\
\hline $\begin{array}{l}\text { Zwischenmahlzeit } \\
2 \mathrm{KHE}\end{array}$ & $\begin{array}{l}30 \mathrm{~g} \text { Roggenbrot } \\
5 \mathrm{~g} \text { Streichfett } \\
50 \mathrm{~g} \text { Paprikastreifen } \\
120 \mathrm{~g} \text { Birne }\end{array}$ \\
\hline
\end{tabular}

\section{Körperliche Aktivität, Alkohol}

Bei Umstellung der körperlichen Aktivität eines Diabetikers sind die Dosierung des Insulins und gleichermaßen der Nährstoffbedarf neu zu berechnen. Kommt es bei Diabetikern trotz Einstellung des Glukosestoffwechsels zu keiner Besserung des Plasmalipidmusters, muss eine möglicherweise gleichzeitig bestehende primäre Hyperlipoproteinämie ausgeschlossen werden. Die Alkoholmenge wird in der Berechnung der Diät berücksichtigt, sie sollte $20 \mathrm{~g} /$ Tag nicht überschreiten. Eine Alkoholkarenz ist $\mathrm{zu}$ empfehlen bei medikamentöser Therapie (Cave: Hypoglykämie bei gleichzeitiger Therapie mit Sulfonyl-

- Tab. 2.19. Glykämischer Index (Gl) und Glykämische Last (GL).

\section{GI}

Der GI beschreibt die

Fläche unter der Blutzuckerkurve nach dem Verzehr von $50 \mathrm{~g}$ Kohlenhydraten in Form eines Lebensmittels im Vergleich zur Fläche nach dem Verzehr von $50 \mathrm{~g}$ Glukose (bzw. $100 \mathrm{~g}$ Weißbrot).
GL

Die GL ergibt sich aus dem Gl und dem Kohlenhydratgehalt eines Lebensmittels

Beispiel:

Möhren (roh), Gl=71

$100 \mathrm{~g}$ Möhren enthalten

7,5 g Kohlenhydrate

$\mathrm{GL}$ von $100 \mathrm{~g}$ Möhren=

$\frac{71 \times 7,5}{100}=5,3$

- Tab. 2.20. Gi- und GL-Werte ausgewählter kohlenhydrathaltiger Lebensmittel, bezogen auf eine Verzehrmenge von $50 \mathrm{~g}$ verwertbaren Kohlenhydraten (50 g Glukose als Referenzgröße)

\begin{tabular}{|c|c|c|c|c|}
\hline Lebensmittel & GI & Portionsgröße (g) & KH-Menge (g/Portion) & GL (pro Portion) \\
\hline $\begin{array}{l}\text { Getreideprodukte } \\
\text { Weißbrot } \\
\text { Reis (gekocht) } \\
\text { Spaghetti } \\
\text { Cornflakes }\end{array}$ & $\begin{array}{l}70 \\
55 \\
45 \\
81\end{array}$ & $\begin{array}{l}30 \\
150 \\
180 \\
30\end{array}$ & $\begin{array}{l}14 \\
40 \\
48 \\
26\end{array}$ & $\begin{array}{l}10 \\
22 \\
21 \\
21\end{array}$ \\
\hline $\begin{array}{l}\text { Obst } \\
\text { Apfel } \\
\text { Weintrauben } \\
\text { Banane }\end{array}$ & $\begin{array}{l}38 \\
46 \\
46\end{array}$ & $\begin{array}{l}120 \\
120 \\
120\end{array}$ & $\begin{array}{l}15 \\
18 \\
24\end{array}$ & $\begin{array}{l}6 \\
8 \\
12\end{array}$ \\
\hline $\begin{array}{l}\text { Gemüse } \\
\text { Kartoffeln (gekocht) } \\
\text { Kidneybohnen } \\
\text { Karotten }\end{array}$ & $\begin{array}{l}56-100 \\
52 \\
47\end{array}$ & $\begin{array}{l}150 \\
150 \\
80\end{array}$ & $\begin{array}{l}17-26 \\
17 \\
6\end{array}$ & $\begin{array}{l}11-18 \\
9 \\
3\end{array}$ \\
\hline $\begin{array}{l}\text { Milchprodukte } \\
\text { Milch } \\
\text { Joghurt }\end{array}$ & $\begin{array}{l}27 \\
38\end{array}$ & $\begin{array}{l}250 \\
200\end{array}$ & $\begin{array}{l}12 \\
29\end{array}$ & $\begin{array}{l}3 \\
11\end{array}$ \\
\hline $\begin{array}{l}\text { Sonstiges } \\
\text { Schoko-Riegel } \\
\text { Kartoffelchips }\end{array}$ & $\begin{array}{l}65 \\
54\end{array}$ & $\begin{array}{l}60 \\
50\end{array}$ & $\begin{array}{l}40 \\
21\end{array}$ & $\begin{array}{l}26 \\
11\end{array}$ \\
\hline
\end{tabular}


harnstoffen), bei Hypertriglyzeridämie, Hypertonie und bei schlecht eingestellten Diabetikern während der Neueinstellung. Alkoholkarenz ist darüber hinaus bei übergewichtigen Diabetikern wünschenswert.

\section{Hypertonus, Fettstoffwechselstörung}

Bei Blutdruckwerten $>130 / 80 \mathrm{mmHg}$ und Hypertonie wird die Diabetesdiät als Reduktions- und natriumarme Kost $(<6 \mathrm{~g} \mathrm{NaCl} / \mathrm{Tag})$, bei Mikroalbuminurie und Nephropathie beträgt die Eiweißmenge $0,8 \mathrm{~g} / \mathrm{kgxd}$, sie sollte nicht unter $0,6 \mathrm{~g} / \mathrm{kg} \times \mathrm{d}$ gesenkt werden. Bei Diabetes mellitus und Fettstoffwechselstörungen (Typ IV oder IIb nach Frederikson, s.unten) ist ausgehend vom Plasmalipidmuster, der Fettanteil zu begrenzen (s.u.) (z. B. Plasmacholsteringehalt $>170 \mathrm{mg} \%$ bzw. LDLc $>150 \mathrm{mg} \%$ ). Bei übergewichtigen Diabetikern und Patienten mit Hyperlipidämie (Typ IIb, Typ IV) ist zunächst eine Reduktionskost indiziert. Bei hohen Triglyzeridspiegeln und gleichzeitig niedrigem HDL-Cholesterin wird eine Mediterrane Ernährung mit einem hohen Anteil an Omega-3Fettsäuren ( $>6 \mathrm{~g} / \mathrm{Tag}$ ) empfohlen. Bei Chylomikronämie sind eine sehr fettarme Ernährung (10-20\% der Energiezufuhr) und ein vorübergehender LCT/MCT-Austausch sinnvoll.

\section{Schwangerschaft, Stillperiode}

Bei schwangeren Diabetikerinnen ist auf eine adäquate Gewichtszunahme zu achten. Diese beträgt bei einem "Ausgangs-BMI « $<20 \mathrm{~kg} / \mathrm{m}^{2}, 12-18 \mathrm{~kg}$, bei einem BMI von $20-25 \mathrm{~kg} / \mathrm{m}^{2} 11,5-16 \mathrm{~kg}$, bei einem BMI von $26-$ $30 \mathrm{~kg} / \mathrm{m}^{2} 7-11,5 \mathrm{~kg}$ und bei einem $\mathrm{BMI}>30 \mathrm{~kg} / \mathrm{m}^{2}<6 \mathrm{~kg}$. In der Stillperiode besteht ein Mehrbedarf von $+500 \mathrm{kcal} /$ Tag (zum Gestationsdiabetes s. $>$ Kap. 1).

\section{Diätetische LM für Diabetiker}

Die Ernährung des Diabetikers wird aus Lebensmitteln des allgemeinen Verzehrs hergestellt. Kalorienfreie Süßstoffe können benutzt werden. Diätetische Lebensmittel für Diabetiker (nach $\$ 12$ der Diätverordnung) sind kein notwendiger Bestandteil einer Diabetesdiät. Bedenken bestehen besonders gegenüber energie- und fettreichen

Tab. 2.21. Therapeutische Effekte verschiedener Monotherapien bei Patienten mit einem Typ-II-Diabetes

\begin{tabular}{|c|c|c|c|c|}
\hline & Insulin & Sulfonylharnstoffe & Metformin & Acarbose \\
\hline Übergewicht & $\uparrow \downarrow$ & $\uparrow$ & $\downarrow$ & $=$ \\
\hline Hyperglykämie & $\downarrow \downarrow$ & $\downarrow$ & $\downarrow$ & $\downarrow$ \\
\hline Insulinresistenz & $\uparrow-$ & $\uparrow-$ & $\downarrow$ & $\downarrow ?$ \\
\hline Hyperinsulinismus & $\uparrow$ & $\uparrow$ & $\downarrow$ & $\downarrow$ \\
\hline Triglyzeride & $\downarrow \uparrow$ & - & $\downarrow$ & $\downarrow_{-}$ \\
\hline Cholesterin & $=\downarrow$ & $=$ & $\downarrow$ & $=\downarrow$ \\
\hline HDL-Cholesterin & $\uparrow$ & - & $=\uparrow$ & $=$ \\
\hline Hypertonie & $=\uparrow$ & $=$ & $=$ & $=$ \\
\hline Arteriosklerose & $\uparrow ?$ & $\uparrow ?$ & $\downarrow ?$ & $\downarrow ?$ \\
\hline
\end{tabular}

Tab. 2.22. Unerwünschte Wirkungen der medikamentösen Behandlung bei Typ-II-Diabetes

\begin{tabular}{l|l|l|l|l} 
& Insulin & Sulfonylharnstoffe & Metformin & Acarbose \\
\hline Hypoglykämie & ++ & + & - & ++ \\
\hline $\begin{array}{l}\text { Gastrointestinale } \\
\text { Störungen }\end{array}$ & - & - & +- & - \\
\hline $\begin{array}{l}\text { Blutbild- } \\
\text { veränderungen }\end{array}$ & - & $(+)$ & $(-)$ & + \\
\hline Laktatazidosen & - & - & $(+)$ & - \\
\hline $\begin{array}{l}\text { Tödliche Komplika- } \\
\text { tionen }\end{array}$ & $(+)$ & $(+)$ & &
\end{tabular}


- Tab. 2.23. Klinische Indikatoren für die Kapazität der Insulinsekretion bei Typ-II-Diabetikern

\begin{tabular}{|c|c|c|}
\hline & \multicolumn{2}{|l|}{ »Residuale« Insulinsekretion } \\
\hline & Ausreichend & Erschöpft \\
\hline Anamnese & $\begin{array}{l}\text { Diabetesdauer }<5 \text { Jahre } \\
\text { Gewichtszunahme } \\
\text { Keine Symptome (Durst etc.) }\end{array}$ & $\begin{array}{l}\text { Diabetesdauer }>5 \text { Jahre } \\
\text { Gewichtsabnahme } \\
\text { Zunehmende Symptome }\end{array}$ \\
\hline Status & Keine oder nur leichte diabetesspezifische Komplikationen & $\begin{array}{l}\text { Neuropathie } \\
\text { Albuminurie } \\
\text { Proliferative Retinopathie }\end{array}$ \\
\hline Labor & $\begin{array}{l}\text { „Base excess« } \uparrow \text {, } \\
\text { Laktat }= \\
\text { C-Peptid }>1,1 \mathrm{nmol} / \mathrm{I} \text { (nach Stimulation) } \\
\text { Serum-Insulin } \uparrow \\
\text { (basal oder stimuliert) }\end{array}$ & $\begin{array}{l}\text { Azidose } \\
\text { Laktat } \uparrow \\
\text { C-Peptid }<1,1 \mathrm{nmol} / \mathrm{l} \text { (nach Stimulation) } \\
\text { Serum-Insulin } \downarrow \text {, } \\
\text { (basal oder stimuliert) }\end{array}$ \\
\hline
\end{tabular}

Lebensmittelprodukten für Diabetiker (z. B. Diabetikerschokolade), deren Nachteile durch die Verwendung von Zuckeraustauschstoffen nicht aufgewogen werden.

Bei »intraktablen" Einstellungsproblemen wurden früher sog. "Hafertage« bzw. eine »Haferdiät« durchgeführt. Diese Kostform enthält eine definierte Kohlenhydratmenge (z. B. $120-200 \mathrm{~g} \mathrm{KH}, 10-17 \mathrm{BE}$ oder KHE) überwiegend in Form von Hafergrütze, Haferflocken, Hafermehl (mit Obst (z. B. mit ungezuckertem Kompott) "gesüßt» oder "pikant (z. B. mit Tomate gewürzt«). Diese Kost ist nicht bedarfsdeckend.

\section{Medikamente}

Diabetiker müssen gezielt im Hinblick auf ihre medikamentöse Behandlung beraten werden. Die - Tab. 2.21 und - Tab. 2.22 zeigen mögliche Nebenwirkungen der medikamentösen Diabetesbehandlung. Für die Insulinbehandlung gibt es verschiedene Präparate. Für die Ernährungsberatung ist besonders die Kenntnis des Wirkungseintritts $(=\mathrm{WE})$ sowie der Wirkungsdauer $(=\mathrm{WD})$ von Insulin wichtig, um die Mahlzeit zeitgerecht $\mathrm{zu}$ planen. Sehr kurz wirksame Insulinanaloga wirken bereits $10 \mathrm{~min}$ nach ihrer Gabe $(\mathrm{WD}=4 \mathrm{~h})$. Konventionelle Normalinsuline (Beispiele: Huminsulin normal, Antrapid HM, Berlinsulin Rapid, B. Braun ratiopharm Rapid oder Insuman Rapid) haben eine WE von 20 min bei einer WD von bis zu 8 Stunden. Sog. NPH Insuline (Bieispiele: Huminsulin Basal, Insulatard Human, Berlinsulin H Basal, B.Braun ratiopharm Basal, Insuman Basal) haben eine WE von $45 \mathrm{~min}$ bei einer WD von $24 \mathrm{~h}$. Normal- und NPH-Insuline gibt es in verschiedenen Mischungen (zwischen 10/90 und 40/60; Beispiel: Antraphane 30/70 HM). Insulin-Zinksuspension (Beipiele Monotard HM, Ultratard HM) haben eine WE von 120 bzw. 180 min, WD beträgt zwischen 24 und $28 \mathrm{~h}$. Einen Überblick der auf dem Markt befindlichen Insulinpräparate geben die Insulintabellen von v. Kriegstein (s. z. B. Diabetes und Stoffwechsel 12/2003). Indikationen für eine Insulintherapie sind Insulinabhängiger Diabetes mellitus (Typ I), Coma diabeticum, Kontraindikationen der oralen Antidiabetika wie »Sekundärversagen «, Schwangerschaft, diabetische Polyneuropathie, mikroangiopathisches Spätsyndrom, diabetische Nephropathie. Bei Typ-II-Diabetikern ist die Abschätzung der residualen Insulinsekretionskapazität Grundlage der Therapieentscheidung (s. \ Tab. 2.23).

\section{Ernährung bei Fettstoffwechselstörungen}

Indikationen. Fettstoffwechselstörungen, Atherosklerose.

Definition. Fettarme ( $<30 \%$ der Energiezufuhr, Cholesterin $<300 \mathrm{mg} / \mathrm{Tag}$ ) oder fettmodifizierte (hoher Anteil an mehrfach ungesättigten Fettsäuren), ballaststoffreiche Diät.

Ziele. Normalisierung der Plasmalipide (Gesamtcholesterin, LDL-Cholesterin, HDL-Cholesterin, Triglyzeride), Senkung des kardiovaskulären Risikos als Primär- oder Sekundärprävention der Atherosklerose.

Anmerkung. - Abb. 2.12 gibt einen Überblick über die Nahrungsfette, - Tab. 2.24 zeigt schematisch die Beeinflussung von Plasmalipiden durch verschiedene Ernährungsformen. Grundzüge einer "lipidsenkenden" Kost sind die Begrenzung der Fett- und Cholesterinzufuhr, die qualitative Änderung der Nahrungsfette (z. B. Erhöhung des Anteils einfach- und mehrfach ungesättigter bei gleichzeitiger Senkung des Anteils gesättigter Fettsäuren), 
- Tab. 2.24. Diätische Beeinflussung verschiedener Plas-malipidfraktionen

\begin{tabular}{|c|c|c|c|c|}
\hline \multirow[t]{2}{*}{ Kostform } & \multicolumn{4}{|l|}{ Lipidfraktionen } \\
\hline & Chylomikronen & VLDL & LDL & HDL \\
\hline Reduktionskost & $\downarrow \downarrow$ & $\downarrow \downarrow$ & $=$ & $(\downarrow)$ \\
\hline Fettarme Kost & $\downarrow$ & $(\uparrow)$ & $\downarrow \downarrow$ & $\downarrow$ \\
\hline Cholesterinarm & $=$ & $=$ & $(\downarrow)$ & $(\downarrow)$ \\
\hline $\begin{array}{l}\text { Fettaustausch } \\
\text { - Linolsäurereich } \\
\text { - Ölsäurereich } \\
\text { - LCT-MCT }\end{array}$ & $\begin{array}{l}= \\
= \\
\downarrow\end{array}$ & $\begin{array}{l}= \\
= \\
=\end{array}$ & $\begin{array}{l}\downarrow \\
\downarrow \\
=\end{array}$ & $\begin{array}{l}(\downarrow) \\
= \\
(\uparrow)\end{array}$ \\
\hline$\omega_{3}$-Fettsäuren $>5 \mathrm{~g} / \mathrm{Tag}$ & $=$ & $\downarrow$ & $(\downarrow)$ & $=$ \\
\hline Ballaststoffreich & $=$ & $\downarrow$ & $\downarrow$ & $=$ \\
\hline $\begin{array}{l}\text { Komplexe } \\
\text { Kohlenhydrate } \uparrow\end{array}$ & $=$ & $(\downarrow)$ & $=$ & $=$ \\
\hline Alkoholkarenz & $\downarrow$ & $\downarrow$ & $=$ & $(\downarrow)$ \\
\hline
\end{tabular}

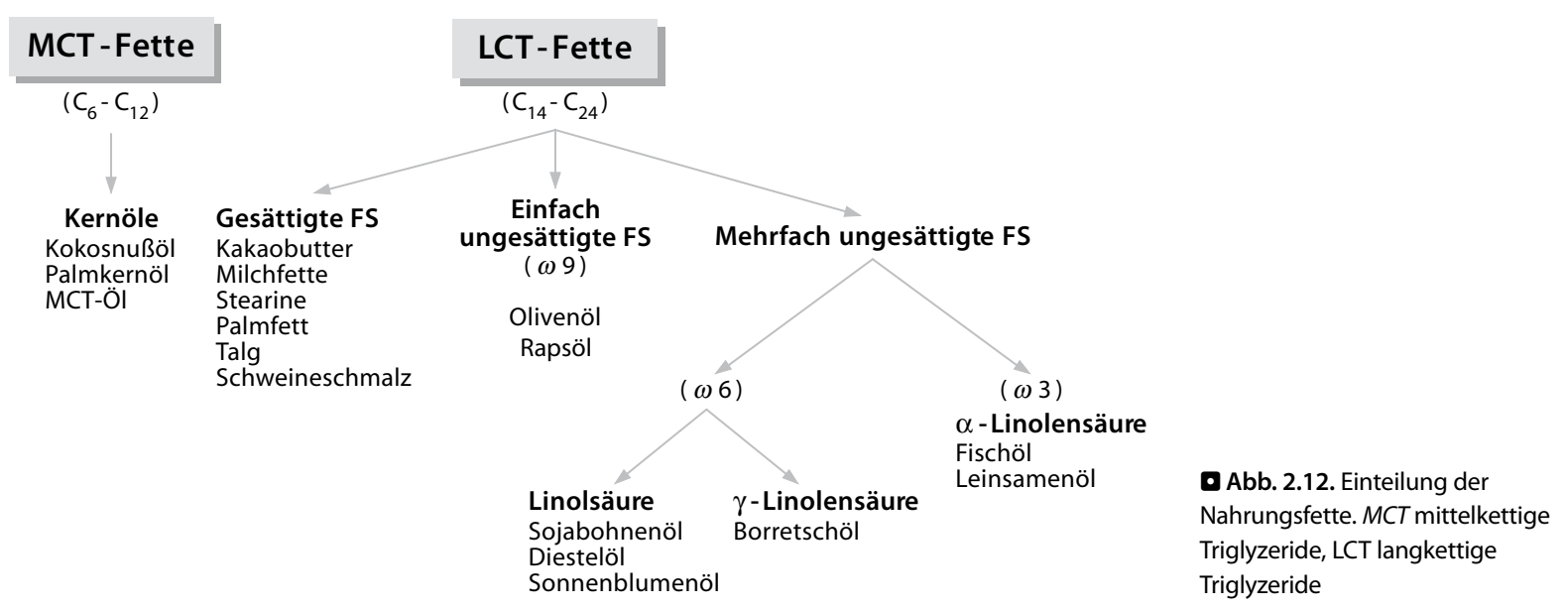

die Erhöhung des Anteils komplexer Kohlenhydrate und der Ballaststoffzufuhr (lösliche Ballaststoffe: Pektin, Guar, Haferkleie, Hülsenfrüchte), der Austausch tierischer gegen pflanzliche Eiweiß sowie bei Hypertriglyzeridämie die Alkoholkarenz (vgl. - Tab. 2.25). Die »American Heart Association « (AHA) hat für die Prävention der koronaren Herzerkrankungen die folgenden Richtlinien herausgegeben:

- Die Gesamtfettzufuhr beträgt $<30 \%$ der Energiezufuhr.

- Dabei ist der Anteil der gesättigten Fettsäuren $<10 \%$, die mehrfach ungesättigten Fettsäuren sind auf maximal $10 \%$ beschränkt, während die einfach ungesättigten Fettsäuren bis zu 15\% der Energiezufuhr betragen.

- Die Cholesterinaufnahme ist $<300 \mathrm{mg} / \mathrm{Tag}$.
- Kohlenhydrate decken $>50 \%$ der Energiezufuhr, komplexe Kohlenhydrate werden bevorzugt.

- Die Kochsalzmenge wird auf 6 g/Tag begrenzt.

- Ein Glas Wein oder Bier pro Tag sind erlaubt.

- »Normales« Körpergewicht.

Bei erhöhten Plasmacholesterinspiegeln werden eine Stufe-1- oder eine Stufe-2-Diät empfohlen. Die Stufen unterscheiden sich im Anteil der "gesättigten " Fette (Stufe 1: 8-10\% der Energiezufuhr, Stufe 2: $<7 \%$ der Energiezufuhr) und der Cholesterinmenge (Stufe 1: <300 mg/Tag; Stufe 2: <200 mg/Tag).

Zur Lebensmittelauswahl einer fettarmen Ernährung nach den Empfehlungen der AHA und den Empfehlungen der Europäischen Atherosklerosegesellschaf s. - Tab. 2.25. Bei der diätetischen Behandlung von Fett- 
stoffwechselstörungen sind Art und Menge der Nahrungsfette und des -cholesterins bzw. der Fettgehalt von Lebensmitteln $^{2}$ (vgl. - Tab. 2.26-2.29), Kohlenhydrate, Energiebilanz und Körpergewicht zu berücksichtigen.

\footnotetext{
${ }^{2}$ Dem Patienten ist eine Hilfestellung im Hinblick auf die Einschätzung des Fettgehaltes von Lebensmitteln zu geben.

Beispiel Käse: Man unterscheidet 8 Fettgehaltsstufen:

- Doppelrahmstufe 68-87\% Fett,

- Rahmstufe 50-59\% Fett,

- Vollfettstufe $45-49,5 \%$ Fett,

- Fettstufe $40-44,9 \%$ Fett,

- Dreiviertelfettstufe 30-39,9\% Fett,

- Halbfettstufe $20-29,9 \%$ Fett,

- Viertelfettstufe 10-19,9\% Fett,

- Magerstufe 0-9,9\% Fett.

Der absolute Fettgehalt beträgt bei einem Emmentaler Käse (=Hartkäse) mit 45\% Fett i. Tr. 31,5\%, bei einem Gouda (=Schnittkäse) mit 45\% Fett i. Tr. 27\%, bei einem Camembert mit 60\% Fett i. Tr. 30\% und Speisequark mit 40\% Fett i. Tr. 12\%. »Light«-Käse enthält maximal 32,5\% Fett i. Tr. (Dreiviertelfettstufe).
}

\section{Ballaststoffe}

Die lipidsenkende Kost sollte ballaststoffreich sein und insbesondere lösliche Ballaststoffe enthalten. Nichtlösliche Ballaststoffe wie Lignin, Zellulose, Weizen- und Maiskleie haben keinen Effekt auf den Fettstoffwechsel. Demgegenüber haben Pektine, Guar und z. T. auch Haferkleie in großen Mengen einen deutlichen Effekt auf das Plasmalipidmuster: $10 \mathrm{~g}$ Pektine (enthalten in $2,5 \mathrm{~kg}$ Äpfeln) senken den Cholesterinspiegel um etwa 10\%.

\section{Vitamine}

Im Hinblick auf die Senkung des kardiovaskulären Risikos enthält die Kostform immer auch reichlich Antioxidantien (Vitamin E, C, und $\beta$-Carotin) durch hohen Verzehr pflanzlicher Fette, Obst und Frischgemüse.

\section{Risiko- und Schutzfaktoren}

- Tab. 2.30 zeigt die heute bekannten Schutz- und Risikofaktoren der Ernährung. Eine lipidsenkende Kost

- Tab. 2.25. Prinzipien einer fettarmen Ernährung zur Prävention und Behandlung der koronaren Herzerkrankung. SFA gesättigte Fettsäuren, MUFA einfach ungesättigte Fettsäuren, PUFA mehrfach ungesättigte Fettsäuren, KH Kohlenhydrate, BST Ballaststoffe

\begin{tabular}{|c|c|c|}
\hline Prinzip & Menge & Lebensmittel \\
\hline $\begin{array}{l}\text { Gesamtfett } \downarrow \\
\text { SFA } \downarrow\end{array}$ & $\begin{array}{l}<30 \% \text { Energie } \\
7-10 \%\end{array}$ & $\begin{array}{l}\text { Reduzieren oder meiden: } \\
\text { Butter, gehärtete Margarine, Sahne, Eis, fetter Käse, fettes Fleisch, Würste, Torten, } \\
\text { Pasteten, Kondensmilch, fettreiche Milchprodukte, (Spirituosen) } \\
\text { Erlaubt: Fisch, Geflügel }\end{array}$ \\
\hline MUFA $\uparrow$ & $10-15 \%$ & Erlaubt: Pflanzliche Öle, Olivenöl \\
\hline PUFA $\uparrow$ & $7-10 \%$ & \\
\hline Cholesterin $\downarrow$ & $<300 \mathrm{mg} / \mathrm{Tag}$ & Erlaubt: 2 Eier/Woche, Innereiern 2-mal/Monat \\
\hline Komplexe KH $\uparrow$ & $>40 \%$ Energie & Erlaubt: Obst, Gemüse, Bohnen, Linsen, Cerealien \\
\hline BST $\uparrow$ & $35 \mathrm{~g} / \mathrm{Tag}$ & \\
\hline
\end{tabular}

- Tab. 2.26. Lebensmittel mit hohem Fettgehalt

\begin{tabular}{l|l} 
& Gehalt pro $100 \mathrm{~g}^{\mathrm{a}}$ \\
\hline Schmalz & $90 \mathrm{~g}$ \\
\hline Speck & $60-80 \mathrm{~g}$ \\
\hline Salami & $50 \mathrm{~g}$ \\
\hline Leberwurst & $40 \mathrm{~g}$ \\
\hline Camembert (35\% F.i. Tr.) & $34 \mathrm{~g}$ \\
\hline Eigelb & $32 \mathrm{~g}$ \\
\hline Gans & $31 \mathrm{~g}$ \\
\hline Huhn & $20 \mathrm{~g}$ \\
\hline Gouda (45\% F.i.Tr.) & $20 \mathrm{~g}$
\end{tabular}

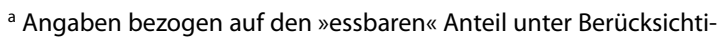
gung der Verluste vor der Zubereitung
- Tab. 2.27. Lebensmittel mit hohem Cholesteringehalt

\begin{tabular}{l|l} 
& Gehalt pro $100 \mathrm{~g}^{\mathrm{a}}$ \\
\hline Hirn & $\sim 2000 \mathrm{mg}$ \\
\hline $\begin{array}{l}\text { Hühnerei insgesamt } \\
\text { Eigelb }\end{array}$ & $\begin{array}{l}\sim 600 \mathrm{mg} \\
1650 \mathrm{mg}\end{array}$ \\
\hline Leber & $250-360 \mathrm{mg}$ \\
\hline Hummer & $180 \mathrm{mg}$ \\
\hline Muscheln & $150 \mathrm{mg}$ \\
\hline Garnelen & $140 \mathrm{mg}$
\end{tabular}

${ }^{a}$ Angaben bezogen auf den »essbaren« Anteil unter Berücksichtigung der Verluste vor der Zubereitung. 
wird individuell entsprechend der zugrundeliegenden Fettstoffwechselstörung konzipiert. Eine differenzierte Diagnostik des Lipidstoffwechsels ist deshalb Voraussetzung einer ernährungsmedizinischen Behandlung. Die Sinnhaftigkeit der »Diät« ergibt sich aus der Einschätzung des koronaren Risikos (s. - Tab. 2.31). Die Empfehlungen gelten speziell für Risikogruppen. Das diagnostische Vorgehen und die gezielte Überwachung des Patienten mit einem erhöhten LDL-Spiegel ist in - Abb. 2.13 dargestellt. Bei bestehender KHK und erhöhten LDLc ist der Anteil der gesättigten Fettsäuren auf unter 7\% der Energieaufnahme, die Cholesterinmenge in der Nahrung auf unter $300 \mathrm{mg} / \mathrm{d}$ und das Gewicht auf Normalwerte zu reduzieren. Bei niedrigen HDLc, erhöhten Triglycerid-
- Tab. 2.28. Trans-fettsäuregehalt ausgewählter Lebensmittel in $\%$ des Gesamtfettgehalts

\begin{tabular}{l|l}
\hline & (\%) \\
\hline Kekse & 48 \\
\hline Salzgebäck & 43 \\
\hline Lutschbonbons & 39 \\
\hline Pommes Frites & 37 \\
\hline Pudding & 36 \\
\hline Margarine & 36 \\
\hline Kuchen & 35 \\
\hline Kartoffelchips & 30
\end{tabular}

Tab. 2.29. Fettsäurezusammensetzung einiger Nahrungsfette

\begin{tabular}{|c|c|c|c|c|}
\hline & \multicolumn{3}{|l|}{ Fettsäuren (in\%) } & \multirow{2}{*}{$\begin{array}{l}\text { P/S- } \\
\text { Quotient }\end{array}$} \\
\hline & Gesättigt (SFA) & Einfach ungesättigt (MUFA) & Mehrfach ungesättigt (PUFA) & \\
\hline Butter & 65 & 31 & 4 & 0,06 \\
\hline Schweineschmalz & 42 & 48 & 10 & 0,23 \\
\hline Gänseschmalz & 27 & 57 & 11 & 0,41 \\
\hline Erdnussöl & 16 & 56 & 28 & 1,75 \\
\hline Olivenöl & 14 & 77 & 9 & 0,64 \\
\hline Maiskeimöl & 13 & 27 & 60 & 4,61 \\
\hline Sojaöl & 13 & 23 & 64 & 4,92 \\
\hline Leinöl & 10 & 18 & 72 & 7,20 \\
\hline Sonnenblumenöl & 11 & 25 & 64 & 5,81 \\
\hline Distelöl & 9 & 13 & 78 & 8,66 \\
\hline Kokosfett & 91 & 7 & 2 & 0,02 \\
\hline Palmkernfett & 83 & 14 & 3 & 0,04 \\
\hline Margarine & $12-50$ & $22-36$ & $16-32$ & 0,77 \\
\hline
\end{tabular}

- Tab. 2.30. Ernährung und Koronare Herzerkrankung: Risiko- und Schutzfaktoren des Krankheitsprozesses

\begin{tabular}{|c|c|c|}
\hline & Risikofaktoren & Schutzfaktoren \\
\hline Atherosklerose & $\begin{array}{l}\text { Gesättigte Fettsäuren } \\
\text { Cholesterin } \\
\text { Transfettsäuren }\end{array}$ & $\begin{array}{l}\text { Ungesättigte Fettsäuren } \\
\text { Antioxidantien } \\
\text { Alkohol } \\
\text { (Folsäure) } \\
\text { (Ballaststoffe) }\end{array}$ \\
\hline Endothelentzündung & $\begin{array}{l}\text { Homocystein } \\
\text { Ox-LDL }\end{array}$ & Folsäure \\
\hline Thrombose & $\begin{array}{l}\text { Homocystein } \\
\text { Gesättigte Fettsäuren, } \\
\text { Transfettsäuren } \\
\text { Linolsäure }\end{array}$ & $\begin{array}{l}\Omega 3 \text { Fettsäuren } \\
\text { Alkohol } \\
\text { Antioxidantien? } \\
\text { Folsäure }\end{array}$ \\
\hline Herzrhythmusstörung & $\begin{array}{l}\text { Gesättigte Fettsäuren, } \\
\text { Linolsäure }\end{array}$ & $\Omega 3$ Fettsäuren \\
\hline
\end{tabular}


spiegeln und hohen Mengen kleiner und dichter LDLPartikel (sog. »small-dense $L D L$ «) sind gesättigte Fettsäuren weitestgehend durch einfach ungesättigte Fettsäuren zu ersetzen. Die Kohlenhydrataufnahme wird begrenzt $(<50 \%)$, Gewichtsreduktion und eine regelmäßige körperliche Aktivität sind anzustreben. Bei Diabetes mellitus und Insulinresistenz sind komplexe Kohlenhydrate zu bevorzugen und der Ballaststoffanteil in der Ernährung zu erhöhen. Die Empfehlungen der American Heart Association und auch des National Cholesterin Education Panel (NCEP) werden fortlaufend aktualisiert (vgl. z. B. Nutr. Rev 59, 298-306, 2001)

- Tab. 2.31. Koronare Risikofaktoren neben LDL-Cholesterin

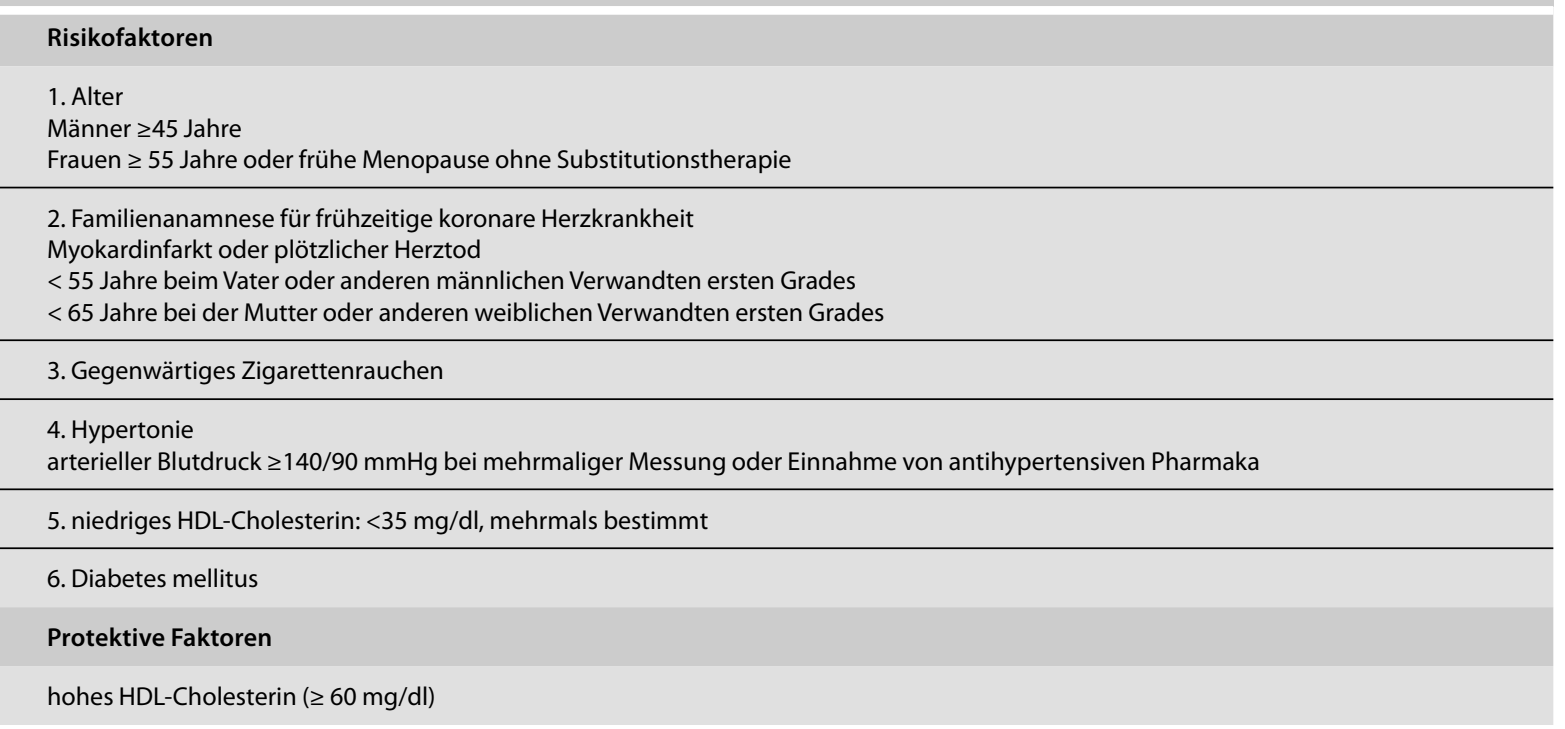

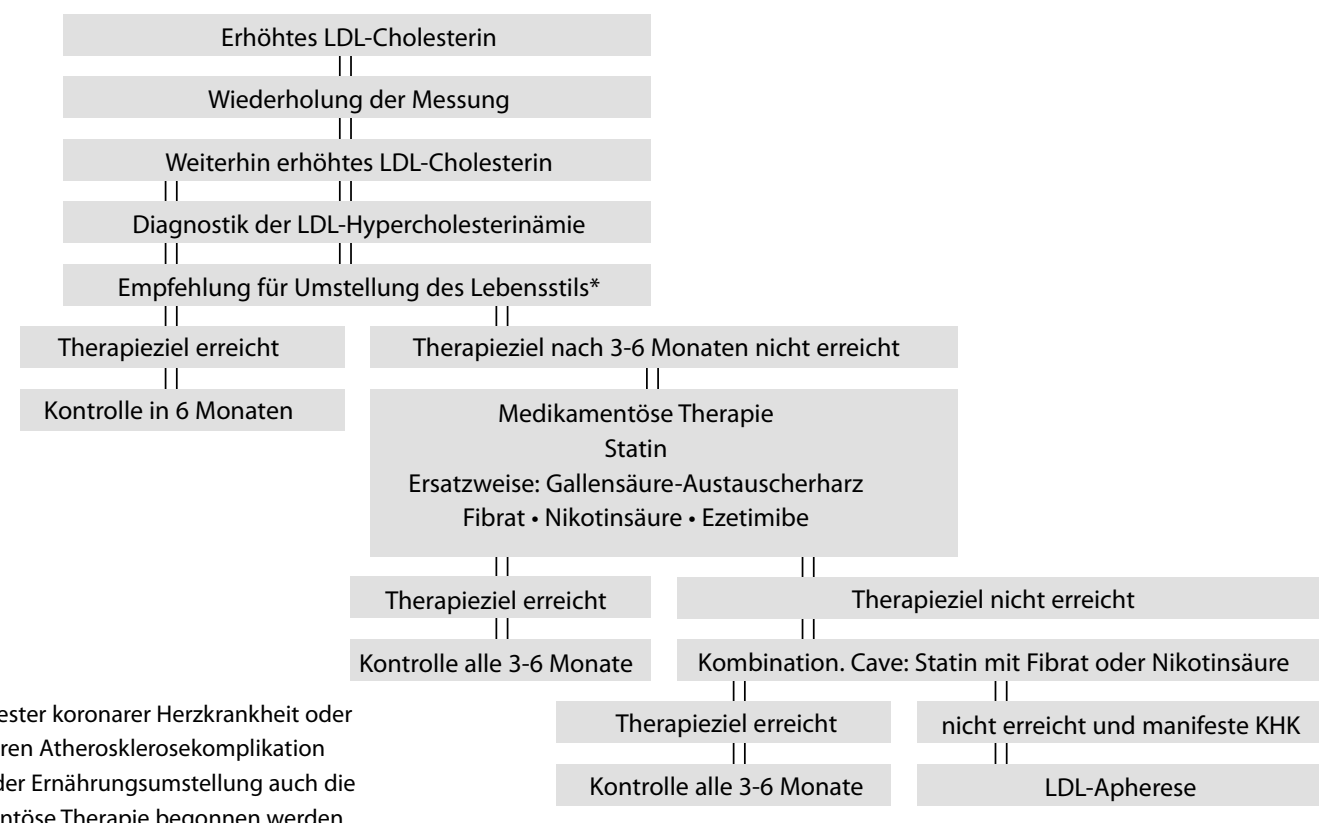

*Bei manifester koronarer Herzkrankheit oder einer anderen Atherosklerosekomplikation sollte mit der Ernährungsumstellung auch die medikamentöse Therapie begonnen werden. Vierwöchentliche Kontrollen bis zum Erreichen des Therapieziels. 


\section{Therapieerfolg}

Der Erfolg einer diätetischen Behandlung ist abhängig von dem Ausmaß der Fettstoffwechselstörung (=Höhe der Plasmalidspiegel), der jeweiligen diätetischen Einschränkung bzw. Modifikation und dem Gesamtkonzept der Behandlung. Eine 1\%ige Senkung des Plasmacholesterinspiegels bewirkt eine 2\%ige Senkung des kardiovaskulären Risikos. In einer unausgewählten Gruppe von Patienten mit einer Fettstoffwechselstörung kann z. B. mit einer fettarmen Diät maximal eine Senkung der Plasmalipidparameter um 15-20\% der Ausgangswerte erreicht werden. Im Einzelfall können diese Veränderungen des LDL-Cholesterin durch Diät aber variabel sein und zwischen 0 und 50\% liegen. Möglicherweise profitiert eine bisher weder phäno- noch genotypisch charakterisierte Untergruppe von Patienten besonders von einer diätetischen Behandlung. Dies gilt z. B. für Apo E4 homozygote Patineten. Patienten mit hohen CRP-Spiegel (=hohe entzündliche Aktivität, s. Kap. 1) zeigen einen geringen lipidsenkenden Effekt von einer fettarmen Diät. Männer mit einem Apo E 3,4-Phänotyp zeigen eine deutlichere LDL-Cholesterinsenkung unter Diät.

\section{Medikamente}

Die Diät ist immer Teil eines Gesamtbehandlungskonzeptes, welches in jedem Fall den Lebensstil (Stressprophylaxe, regelmäßige körperliche Belastung, Nikotinkarenz, mäßiger Alkoholkonsum), im Einzelfall aber auch eine medikamentöse Behandlung umfassen muss. Die Diät muss einer möglichen medikamentösen Behandlung vorhergehen und sollte auch unter medikamentöser Behandlung fortgeführt werden (vgl. - Tab. 2.32-2.34). Ein Medikament kann nie die Diät ersetzen. Es sollte gegeben werden, wenn die Diät alleine nicht ausreicht, das Ziel (i.e. die Senkung des Cholesterin- bzw. Triglyceridspiegels) zu erreichen (s. - Tab. 2.35). Der Erfolg der Maßnahme wird frühestens nach 6-8 Wochen beurteilt. - Abb. 2.13 beschreibt einen Entscheidungsalgorithmus bei Vorliegen einer Hypercholesterinämie. - Abb. 2.14 bietet analog eine Entscheidungshilfe für die Behandlung einer Hypertriglyzeridämie.

\section{Spezielle Diätetik Fettarme Diät bei Hypercholesterinämie}

Indikationen. Familiäre Hypercholesterinämien, Hyperlipoproteinämie Typ IIa nach Frederikson.

Definition. Isokalorische und kohlenhydratreiche Kost mit Einschränkung des Fettanteils auf maximal 25\% der Gesamtkalorien, cholesterinarm ( $<200 \mathrm{mg} / \mathrm{Tag})$, hoher Linolsäureanteil (etwa $8 \mathrm{kal} . \%$ ) sowie ballaststoffreich (>30 g/Tag).

Ziele. Senkung des Cholesterin- bzw. LDL-Cholesterinspiegels, Senkung des atherogenen Risikos.

Anmerkung. Der Bedarf an fettlöslichen Vitaminen wird in dieser Kost u.U. nur knapp gedeckt. Für Patienten ist es nicht immer leicht, die relativ großen Mengen ballaststoffreicher, vegetabiler Nahrungsmittel aufzuessen. Diätmargarine (wie Becel pro aktiv enthält Phytosterole) senken den LDLc-Spiegel. Die Vorgehensweisen bei LDL-Hypercholesterinämie ist in Abb. 2.12 dargestellt.

\section{Diät bei kombinierte Fettstoffwechselstörungen}

Indikationen. Kombinierte Hyperlipidämien Typ IIb und Typ III n. Fredrickson.

Definition. Bevorzugung komplexer Kohlenhydrate und Begrenzung der Oligosaccharide wie bei Diabetesdiät. Fettantei: 30-35 Energieprozent, der Anteil der mehrfach ungesättigten Fettsäuren (hauptsächlich Linolsäure) sowie von Monoensäuren (z. B. Ölsäure) und gesättigten Fettsäuren (hauptsächlich Palmitinsäure) beträgt je etwa ein Drittel. Der P/S-Quotient ist 1,0 (wünschenswert $>0,5$ ). Cholesteringehalt: $<300 \mathrm{mg} / \mathrm{Tag}(100 \mathrm{mg} / 1000 \mathrm{kcal})$, Ballaststoffanteil: $30 \mathrm{~g} / \mathrm{Tag}$.

Ziele. Normalisierung der Triglyzerid- oder Cholesterinbzw. LDL-Cholesterinspiegel, Senkung des atherogenen Risikos.

\begin{tabular}{|c|c|c|c|c|}
\hline & LDL & VLDL & HDL & Glukose \\
\hline Fibrate & $\downarrow$ & $\downarrow$ & $\uparrow$ & $\leftrightarrow$ \\
\hline HMG-CoA-Reduktasehemmer & $\downarrow$ & $\leftrightarrow$ & $\leftrightarrow, \uparrow$ & $\leftrightarrow$ \\
\hline Resine & $\downarrow$ & $\uparrow$ & $\leftrightarrow, \uparrow$ & $\leftrightarrow$ \\
\hline Nikotinsäure & $\downarrow$ & $\downarrow$ & $\uparrow$ & $(\downarrow), \uparrow$ \\
\hline Etetimib & $\downarrow$ & $\leftrightarrow$ & $\leftrightarrow$ & $\leftrightarrow$ \\
\hline
\end{tabular}


Anmerkung. Die Kost ist reich an pflanzlichen und arm an tierischen Fetten. Leicht resorbierbare Zucker werden gemieden. Der Einsatz von Omega-3-Fettsäuren trägt zur Senkung erhöhter Triglyzerid- (VLDL-) Spiegel bei. Hierzu bieten sich Omega-Fettsäuren-reiche natürliche Lebensmittel, Lebertran und Fischölkonzentrate an. Wirksame Dosierungen sind $2 \mathrm{~g}$ Eikosapentaensäure und 1,4 g Docosahexaensäure. Dies entspricht beispielsweise $170 \mathrm{~g}$ Makrele bzw. $155 \mathrm{~g}$ Hering/Tag, 2,3 l (!) Lebertran bzw. $10 \mathrm{~g}$ eines Fischölkonzentrats (z. B. Omacor, Eicosan). Für die Hemmung der Plättchenaggregation ist die Einnahme größerer Mengen von Omeg-3-Fettsäuren notwendig.

\section{Diät bei Hyperchylomikronämie}

Indikationen. Familiärer Apo- $\mathrm{C}_{2}$-Mangel, Hyperlipoproteinämie Typ I.

Definition. Sehr fettarme Diät (Nahrungsfettmenge $<20 \%$ der Kalorien), MCT-Kost unter weitgehender Vermei- dung von LCT-Fetten (Austausch der Koch- und Streichfette durch MCT).

Ziele. Normalisierung der Plasmatriglyzeridspiegel, Senkung des Risikos einer akuten Pankreatitis (Cave: Plasmatriglyzeridspiegeln $>10 \mathrm{mmol} / \mathrm{l}$ ), Senkung des atherogenen Risikos.

Anmerkung. Bei einer Hyperlipoproteinämie vom Typ I und Übergewicht sollten zunächst eine Reduktionskost, eine vollständige Alkoholkarenz sowie dann eine linolsäurereiche Kost durchgeführt werden. Bei schlechtem Ansprechen sind die Gewichtsreduktion und eine weitere Reduzierung der Fettmenge angezeigt. Bei schweren Entgleisungen des Lipidstoffwechsels (Plasmatriglyzeridspiegel $>10 \mathrm{mmol} / \mathrm{l}$ ) ist eine stationäre Aufnahme zur Durchführung einer vorübergehenden parenteralen Ernährung und der Gabe von Heparin wegen der Gefahr der akuten Pankreatitis notwendig.

- Tab. 2.33. Wirkstoffgruppen, Effekte und Nebenwirkungen von Lipostatika

\begin{tabular}{|c|c|c|c|}
\hline Wirkstoffgruppe & Wirkprinzip & Effekt auf Plasmalipoproteine & $\begin{array}{l}\text { Unerwünschte Begleit- } \\
\text { erscheinungen }\end{array}$ \\
\hline $\begin{array}{l}\text { HMG-CoA-Reduktase- } \\
\text { hemmer (= Statin) }\end{array}$ & $\begin{array}{l}\text { Aktivierung der LDL-Rezeptoren durch } \\
\text { Drosselung der zelleigenen Choleste- } \\
\text { rinsynthese }\end{array}$ & $\begin{array}{l}\text { 1. } \downarrow \text { LDL-Cholesterin um } 20-40 \% \\
\text { 2. } \downarrow \text { Triglyzeride um } 5-10 \% \\
\text { 3. } \uparrow \mathrm{HDL} \text {-Cholesterin um } 5-10 \%\end{array}$ & Selten CPK-Anstieg \\
\hline Anionenaustauscher & $\begin{array}{l}\text { Reduktion der Gallensäurerückresorp- } \\
\text { tion aus dem Darm. Die Leber muss } \\
\text { nun mehr Gallensäure produzierenund } \\
\text { verbraucht mehr LDL- Cholesterin }\end{array}$ & $\begin{array}{l}\text { 1. } \downarrow \text { LDL-Cholesterin um } 10-35 \% \\
\text { 2. } \uparrow \text { (!) Triglyzeride um } 5-20 \% \\
\text { 3. } \uparrow \mathrm{HDL} \text {-Cholesterin um } 5-10 \%\end{array}$ & Obstipation \\
\hline $\begin{array}{l}\text { Kombination von HMG- } \\
\text { CoA-Reduktasehem- } \\
\text { mern mit Anionenaus- } \\
\text { tauschern }\end{array}$ & $\begin{array}{l}\text { Höchstmögliche Aktivierung der hepa- } \\
\text { tischen LDL-Rezeptoren }\end{array}$ & 1. $\downarrow$ LDL-Cholesterin $40-60 \%$ & Siehe oben \\
\hline Fibrate & $\begin{array}{l}\text { Steigerung des intravasalen Triglyze- } \\
\text { ridabbaus durch die Aktivierung der } \\
\text { Lipoproteinlipase } \\
\text { Senkung der freien Fettsäuren } \\
\text { Fragliche Aktivierung der LDL-Rezep- } \\
\text { toren }\end{array}$ & $\begin{array}{l}\text { 1. } \downarrow \text { Triglyzeride um } 40-60 \% \\
\text { 2. } \downarrow \text { LDL-Cholesterin um } 10-20 \% \\
\text { 3. } \uparrow \text { HDL-Cholesterin um } 10-20 \%\end{array}$ & $\begin{array}{l}\text { Selten »Magendrücken« } \\
\text { Gallensteinrisiko erhöht } \\
\text { bei Medikamenten von } \\
\text { Clofibratyp } \\
\text { Diarrhoen }\end{array}$ \\
\hline Nikotinsäure & $\begin{array}{l}\text { Hemmung der Lipohyse im Fettgewe- } \\
\text { be mit vermindertem Ausstrom freier } \\
\text { Fettsäuren in das Plasma } \\
\text { Verminderte VLDL-Synthese der Leber } \\
\text { aufgrund des niedrigeren Angebots } \\
\text { freier Fettsäuren } \\
\text { Fragliche Aktivierung der LDL-Rezep- } \\
\text { toren }\end{array}$ & $\begin{array}{l}\text { 1. } \downarrow \text { Triglyzeride um } 40-60 \% \\
\text { 2. } \downarrow \text { LDL-Cholesterin um } 10-20 \% \\
\text { 3. } \uparrow \mathrm{HDL} \text {-Cholesterin um } 10-40 \%\end{array}$ & $\begin{array}{l}\text { 1. »Flush« mit Tachy- } \\
\text { phylaxie } \\
\text { 2. Gelegentlich erhöhte } \\
\text { Plasmaspiegel der Gluko- } \\
\text { se und Harnsäure }\end{array}$ \\
\hline$\omega_{3}$-Fettsäuren & $\begin{array}{l}\text { Inhibition der hepatischen Triglyzerid- } \\
\text { synthese }\end{array}$ & $\begin{array}{l}\text { 1. } \downarrow \text { Triglyzeride um } 20-60 \% \text { (stark } \\
\text { dosisabhängig) }\end{array}$ & $\begin{array}{l}\text { Nicht selten aufstoßen } \\
\text { mit Fischgeschmack }\end{array}$ \\
\hline Ezetimib & Hemmung der Cholesterinresorption & 1. $\downarrow$ LDL-Cholesterin um $18 \%$ & Kopfschmerzen \\
\hline
\end{tabular}




\section{Diät bei Hypertriglyceridämie (VLDL $\uparrow$ )}

Indikation. Erhöhte VLDL-Spiegel, z. B. bei Metabolischen Syndrom, Hyperlipoproteinämie Typ IV n. Frederikson.

Definition. Moderat fettreduzierte, Mediterrane Ernährung.

Ziele. Senkung des VLDL-Spiegels.

Anmerkung. Die Richtlinien der Behandlung sind in - Tab. 2.36 dargestellt. Reduktionsdiät, Alkoholkarenz, die
Meidung einfacher Zucker bzw. Zuckeraustauschstoffen sowie mehr körperliche Aktivität sind die Grundzüge der Behandlung.

\section{Extrem fettarme Diäten bei Atherosklerose}

Indikationen. Fortgeschrittene Arteriosklerose, z. B. nach wiederholter Bypassoperation.

Definition. Fettgehalt $<20 \%(<10 \%)$ der Energiezufuhr, Cholesterin $<100$ (10) mg/Tag, ballaststoffreiche Kost ( $\geq 30 \mathrm{~g} / \mathrm{Tag}$ ).

- Tab. 2.34. Auflistung und Dosierung von Lipostatika

\begin{tabular}{|c|c|c|c|}
\hline Gruppe & Wirkstoff & Handelsname (Beispiele) & Dosierung (pro Tag) \\
\hline 1. Fibrate & $\begin{array}{l}\text { Bezafibrat } \\
\text { Clofibrat } \\
\text { Fenofibrat } \\
\text { Gemfibrozil } \\
\text { Etofibrat }\end{array}$ & $\begin{array}{l}\text { Bezacur } \\
\text { Bezafibrat } \\
\text { Cedur } \\
\text { Clofibrat } \\
500 \text { Stada } \\
\text { Duolip } 500 \\
\text { Regelan } \\
\text { Durafenat }^{\circledast} \\
\text { Fenofibrat }^{\ominus} \\
\text { Fenofibratratiopharm } \\
\text { Lipanthyl } \\
\text { Gevilon } \\
\text { Lipo-Merzretard }\end{array}$ & $\begin{array}{l}\text { 3-mal } 200 \mathrm{mg} \\
\text { 3-mal } 500 \mathrm{mg} \\
\text { 2-mal } 100 \mathrm{mg} \\
\text { 2-mal } 450 \mathrm{mg} \\
\text { 1-mal } 500 \mathrm{mg}\end{array}$ \\
\hline $\begin{array}{l}\text { 2. HMG-CoA-Reduktasehem- } \\
\text { mer (Statine) }\end{array}$ & $\begin{array}{l}\text { Pravastatin } \\
\text { Lovastatin } \\
\text { Simvastatin } \\
\text { Fluvastatin }\end{array}$ & $\begin{array}{l}\text { Pravasin } \\
\text { Liprevil } \\
\text { Mevinacor } \\
\text { Zocor } \\
\text { Denan } \\
\text { Locol } \\
\text { Cranoc }\end{array}$ & $\begin{array}{l}10-40 \mathrm{mg} \\
10-80 \mathrm{mg} \\
5-40 \mathrm{mg} \\
40-(80) \mathrm{mg}\end{array}$ \\
\hline 3. Resine & $\begin{array}{l}\text { Colestyramin } \\
\text { Colestipol }\end{array}$ & $\begin{array}{l}\text { Quantalan } \\
\text { Colestyramin-Stada } \\
\text { Colestyrucent } \\
\text { Colestabyl }^{\circledR} \\
\text { Colestid }^{\oplus}\end{array}$ & $\begin{array}{l}8-32 \mathrm{~g} \\
10-40 \mathrm{mg}\end{array}$ \\
\hline 4. Nikotinsäure & $\begin{array}{l}\text { Xantinolnicotinat } \\
\text { Inositolnicotinat } \\
\text { Acipimox }\end{array}$ & $\begin{array}{l}\text { Complamin spez. } \\
\text { Nicolip } \\
\text { Olbemox }\end{array}$ & $\begin{array}{l}\text { Bis } 3 \mathrm{~g} \\
\text { 2-mal } 250 \mathrm{~g}\end{array}$ \\
\hline 5. Ezetimib & & Ezetrol & $10 \mathrm{mg}$ \\
\hline
\end{tabular}

- Tab. 2.35. Algorithmus für die kombinierte Behandlung einer Hypercholesterinämie. KHK, koronare Herzerkrankung

\begin{tabular}{|c|c|c|c|c|}
\hline & $\begin{array}{l}\text { Plasmacholesterin } \\
\text { (mmol/l) }\end{array}$ & Diät & Medikament & $\begin{array}{l}\text { Behandlungsziel } \\
(\mathrm{mmol} / \mathrm{l})\end{array}$ \\
\hline \multirow[t]{2}{*}{$<2$ Risikofaktoren } & $\geq 4,1$ & + & 0 & $<4,1$ \\
\hline & $\geq 4,9$ & + & + & $<4,1$ \\
\hline \multirow[t]{2}{*}{$\geq 2$ Risikofaktoren } & $\geq 3,4$ & + & 0 & $<3,4$ \\
\hline & $\geq 4,1$ & + & + & $<3,4$ \\
\hline \multirow[t]{2}{*}{ Manifeste KHK } & $>2,6$ & + & 0 & $\geq 2,6$ \\
\hline & $\geq 3,4$ & + & + & \\
\hline
\end{tabular}




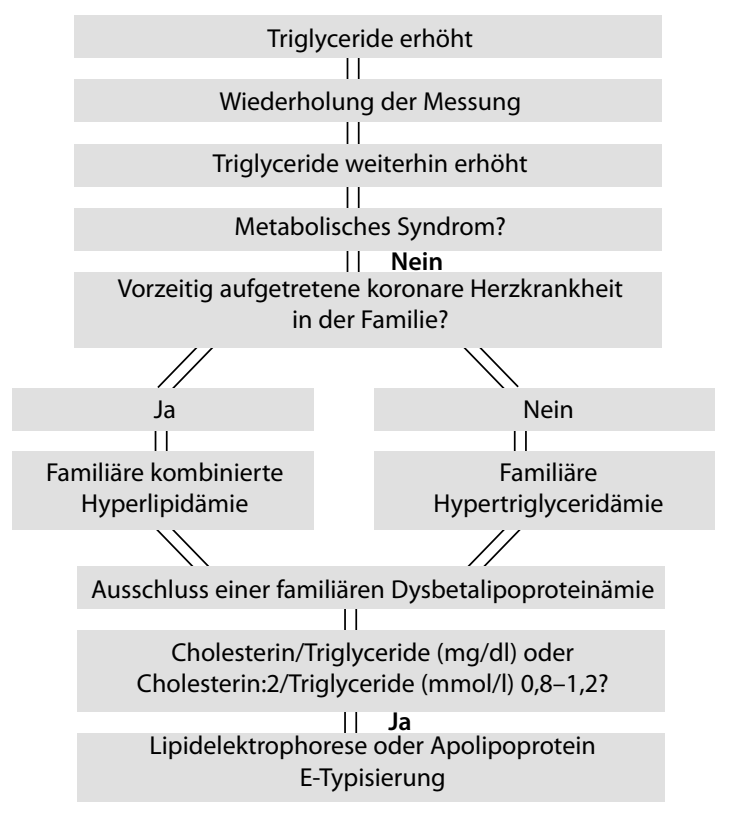

D Abb. 2.14. Diagnostikschema bei Hypertriglyzeridämie
Ziele. Diätetische Senkung des Plasmacholesterins bzw. LDL-Cholesterin <180 bzw. 100 mg/dl, Regression bzw. »Nicht-Progression« bei fortgeschrittener Arteriosklerose.

Anmerkung. Nährstoffzufuhr und ein beispielhafter Tageskostplan einer sehr fettarmen Diät sind in den - Tab. 2.37 und - Tab. 2.38 dargestellt. Eine derart extreme Kostform ist als Dauerkost nur im Rahmen eines ganzheitlichen Behandlungskonzeptes, welches Bewegungsprogramme, Stressbewältigungstechniken und einen verhaltensmedizinischen Ansatz beinhaltet, durchführbar. Praktisch bedeutet dies für den Patienten eine vollständige Umstellung der Lebensweise. Diese Kostform beinhaltet einen Verzicht auf Wurstwaren, Eier und Fleisch, es sind nur geringe Mengen fettarmer Käsesorten und Magerquark erlaubt. Vegetarische Brotaufstriche, Cerealien, Obst und Gemüse sind Hauptbestandteile dieser Kost. Als Getränke werden Mineralwässer und Früchtetees empfohlen. Kaffee, Tee (außer Früchtetees) und Alkohol sollten, wenn möglich, gemieden werden. Durch die drastische Senkung der Fettzufuhr kann das Plasmacholesterin um $21 \mathrm{mg} / \mathrm{dl}$, durch die Reduktion

- Tab. 2.36. Richtlinien für die Behandlung einer Hypertriglyzeridämie

\begin{tabular}{|c|c|c|}
\hline & Konservativ & Medikamentös \\
\hline $\begin{array}{l}\text { Triglyzeride } 2,3-4,6 \mathrm{mmol} / \mathrm{I} \\
\mathrm{LDL} \text {-Cholesterin }<3,5 \mathrm{mmol} / \mathrm{I}\end{array}$ & \multirow{2}{*}{$\begin{array}{l}\text { Reduktionsdiät bei Übergewicht, } \\
\text { Alkoholkarenz, fettarme Diät, } \\
\text { Bewegung, Behandlung der } \\
\text { Ursache }\end{array}$} & Bei $\mathrm{HDL}<0,9 \mathrm{mmol} / \mathrm{l}$ und koronarem Risiko \\
\hline $\begin{array}{l}\text { Triglyzeride }>4,6 \mathrm{mmol} / \mathrm{I} \\
\mathrm{LDL} \text {-Cholesterin }<3,5 \mathrm{mmol} / \mathrm{I}\end{array}$ & & Bei persistierender Hypertriglyzeridämie \\
\hline
\end{tabular}

- Tab. 2.37. Diätetische Empfehlungen für eine sehr fettarme Ernährung nach D. Ornish bei Patienten mit einer fortgeschrittenen Koronaren Herzerkrankung

\begin{tabular}{l|l}
\hline Gesamtkalorien & Nur beschränken bei Übergewicht/ Adipositas sowie erhöhten Gesamttriglyzeridspiegeln \\
\hline Gesamtfett & $<10 \%$ der Kalorienzufuhr (Nüsse, Oliven, Avocados etc. meiden) \\
\hline Cholesterin & $5-8 \mathrm{mg} / \mathrm{Tag}$ \\
\hline Natrium & $<2 \mathrm{~g} / \mathrm{Tag}$ \\
\hline Zucker & Nicht empfohlen \\
\hline Süßstoffe & Meiden \\
\hline Alkohol & $25 \mathrm{ml}$ Spirituosen oder \\
& $\begin{array}{l}125 \mathrm{ml} \text { Wein oder } \\
\text { 300 ml Bier (bei alkoholfreiem Bier max. 1 Flasche/Tag) }\end{array}$ \\
\hline Supplemente/ & $\begin{array}{l}\text { Vermeiden:Lecithin } \\
\text { Vitamine }\end{array}$ \\
$\begin{array}{l}\text { Weizenkeime } \\
\text { Fischölkapseln } \\
\text { Cholin } \\
\text { Empfohlen: Multivitaminpräparate } \\
\text { (Vitamin B })\end{array}$ \\
\hline Kaffee & Nicht empfohlen \\
\hline
\end{tabular}


der Cholesterinzufuhr noch einmal um $8 \mathrm{mg} / \mathrm{dl}$ gesenkt werden. Aufgrund der hohen Ballaststoffzufuhr und der mit dieser Kostform verbundene Gewichtsreduktion ist im Verlauf eine weitere Reduktion des Plasmacholesterins um $8 \mathrm{mg} / \mathrm{dl}$ möglich. Weitere $-8 \mathrm{mg} / \mathrm{dl}$ können durch den Austausch tierischen gegen pflanzlichen Eiweißes erreicht werden. Insgesamt kann der Plasmacholesterinspiegel durch eine sehr fettarme Diät um $45 \mathrm{mg} / \mathrm{dl}$ oder $5-15 \%$ des Ausgangswerts gesenkt werden. Eine Vitaminsupplementierung ist notwendig. Diese Diät ist bisher als Ornish-Diät oder auch als Piritkin-Diät mit einer Nachbeobachtungszeit von 4 Jahren und guten Behandlungserfolgen (d. h. Regression der Atherosklerose gemessen an den Befunden der Koronarangiographie und PET bzw. auch den Symptomen der KHK) durchgeführt worden.

\section{Ernährung bei Hyperurikämie und Gicht}

Indikationen. Hyperurikämie, Gicht, Harnsäuresteine.

Definition. Puringehalt $<300$ bzw. $<200 \mathrm{mg} / \mathrm{Tag}$ oder $<2000$ bzw. $<1000$ mg Harnsäure/Woche; Eiweißzufuhr 0,8-1,0 g/kg KG/Tag, hoher Anteil komplexer Kohlenhydrate, begrenzte Zufuhr von Saccharose und Fruchtzucker und Vermeidung von Alkohol.

Ziele. Normalisierung der Harnsäurespiegel $(<5,5 \mathrm{mg} \%=$ $325 \mu \mathrm{mol} / \mathrm{l})$, Linderung der Symptome.

\section{Anmerkung}

Pathophysiologie. Ein körpereigener Abbau der Harnsäure ist nicht möglich: 75\% der Harnsäure werden renal, 25\% enteral ausgeschieden. Zwei Drittel der Purine im Blut stammen aus der Nahrung. Eine purinarme Diät ist deshalb Grundlage der Gichtbehandlung. - Tab. 2.39 zeigt die Ursachen einer Hyperurikämie. Harnsäurebildung und -ausscheidung sind nicht durch einen Regelkreis miteinander verbunden.

Ernährung/Diät. Als purinarme Kost eignet sich die ovolaktovegetabile Vollkost. Wird diese nicht akzeptiert, werden purinreiche Nahrungsmittel (z. B. Leber, Niere, Bries, Herz, Fischsorten wie Ölsardinen, Sardellen, Kabeljau, Schalen- und Krustentiere, Hülsenfrüchte wie Linsen, Erbsen, weiße Bohnen, Sojabohnen) ausgeschlossen und der Genuss von Fleisch und Fleischwaren eingeschränkt. Der Eiweißbedarf kann durch Milch, Milchprodukte, Eier sowie pflanzliche Proteine gedeckt werden. Purinarme Nahrungsmittel wie Milch, Obst, Gemüse, Kartoffeln, Brot, Teigwaren (s. - Tab. 2.40) sind $\mathrm{zu}$ bevorzugen. Vorsicht bei großen Verzehrsmengen nur mäßig purinreicher Gemüse wie Kohl, Spinat, Spargel (Harnsäuregehalt 30-70 mg/100 g). Die in Tee, Kaffee und Kakao enthaltenden Xanthinbasen führen in den üblichen Verzehrsmengen nicht zu einer Erhöhung der Plasmaharnsäurespiegel. Absolute Alkoholkarenz
- Tab. 2.38. Tageskostplan einer sehr fettarmen (Fettgehalt $10 \%$ der Nahrungsenergien)Ernährung bei einem Patienten mit einer fortgeschritten koronaren Herzerkrankung

\begin{tabular}{|c|c|}
\hline $\begin{array}{l}\text { 1. Frühstück } \\
\text { Müsli mit Joghurt }\end{array}$ & $\begin{array}{l}200 \mathrm{~g} \text { Magerjoghurt, max. 0,3\% Fett } \\
100 \mathrm{~g} \text { Apfel } \\
30 \mathrm{~g} \text { Haferflocken } \\
20 \mathrm{~g} \text { Honig (Blütenhonig) } \\
20 \mathrm{~g} \text { Weinbeere (Weintraube) } \\
\text { getrocknet (Rosine) } \\
50 \mathrm{~g} \text { Banane } \\
1 \text { Becher (3 g) Zichorienkaffee }\end{array}$ \\
\hline $\begin{array}{l}\text { 2. Frühstück } \\
\text { Brötchen mit } \\
\text { Konfitüre } \\
\text { Tee }\end{array}$ & $\begin{array}{l}45 \mathrm{~g} \text { Brötchen (Semmeln) } \\
10 \mathrm{~g} \text { Margarine (Diätmargarine) } \\
25 \mathrm{~g} \text { Erdbeerkonfitüre } \\
8 \mathrm{~g} \text { Zucker (Rohrzucker, Rüben- } \\
\text { zucker) } \\
2 \text { Tassen ( } 3 \mathrm{~g}) \text { Tee (Schwarzer Tee) }\end{array}$ \\
\hline $\begin{array}{l}\text { Mittag } \\
\text { Chili con Carne }\end{array}$ & $\begin{array}{l}40 \mathrm{~g} \text { Zwiebeln } \\
2 \mathrm{~g} \text { Knoblauch } \\
100 \mathrm{~g} \text { Tomaten } \\
200 \mathrm{~g} \text { Bohnen, rot gekocht (Kidney- } \\
\text { Bohne) } \\
5 \mathrm{~g} \text { Rohrzucker aus Zuckerrüben } \\
\text { (brauner Zucker) } \\
60 \mathrm{~g} \text { Reis poliert } \\
10 \mathrm{~g} \text { Weizenmehl }\end{array}$ \\
\hline $\begin{array}{l}\text { Dessert } \\
\text { Obstsalat }\end{array}$ & $\begin{array}{l}30 \mathrm{~g} \text { Pfirsich } \\
50 \mathrm{~g} \text { Apfel } \\
30 \mathrm{~g} \text { Banane } \\
50 \mathrm{~g} \text { Weintrauben } \\
10 \mathrm{~g} \text { Zucker (Rohrzucker, Rüben- } \\
\quad \text { zucker) }\end{array}$ \\
\hline $\begin{array}{l}\text { "Kaffeestück" } \\
\text { Brioches }\end{array}$ & $\begin{array}{l}31,2 \mathrm{~g} \text { Weizenmehl } \\
\text { 1,2 g Bäckerhefe gepresst } \\
5 \mathrm{~g} \text { Margarine (Diätmargarine) } \\
\text { 3,8 g Zucker (Rohrzucker, Rüben- } \\
\text { zucker) } \\
\text { 3,8 g Hühnereiweiß, Flüssig- } \\
\text { eiweiß, Eiklar } \\
8 \mathrm{~g} \text { Zucker (Rohrzucker, Rüben- } \\
\text { zucker) } \\
10 \mathrm{~g} \text { Erdbeerkonfitüre } \\
2 \text { Tassen }(6 \mathrm{~g}) \text { Zichorienkaffee }\end{array}$ \\
\hline $\begin{array}{l}\text { Abendessen } \\
\text { Brot und } \\
\text { Tomatensalat }\end{array}$ & $\begin{array}{l}50 \mathrm{~g} \text { Roggenvollkornbrot } \\
40 \mathrm{~g} \text { Weizenvollkornbrot } \\
20 \mathrm{~g} \text { Speisequark mager } \\
20 \mathrm{~g} \text { Pflaumenkonfitüre (Zwetsch- } \\
\text { genkonfitüre) } \\
30 \mathrm{~g} \text { Cottagekäse (Hüttenkäse) } \\
150 \mathrm{~g} \text { Tomaten } \\
3 \mathrm{~g} \text { Safloröl (Distelöl) gereinigt } \\
3 \text { Tassen }(6 \mathrm{~g}) \text { Tee (Schwarzer Tee) }\end{array}$ \\
\hline $\begin{array}{l}\text { Spätmahlzeit } \\
\text { Erdbeermilch }\end{array}$ & $\begin{array}{l}180 \mathrm{~g} \text { Buttermilch } \\
100 \mathrm{~g} \text { Erdbeeren } \\
\text { kcal: } 1969,27, \mathrm{KJ} 8246,50 \\
\text { Eiweiß } 68 \mathrm{~g}(14 \%) \\
\text { Fett } 26 \mathrm{~g}(12 \%) \\
\text { Gesättigte Fettsäuren (SFA) } 3,3 \mathrm{~g} \\
\text { Einfach ungesättigte Fettsäuren } \\
\text { (MUFA) } 2,1 \mathrm{~g} \\
\text { Mehrfach ungesättigte Fettsäuren } \\
\text { (PUFA) 9,6 g } \\
\text { Kohlenhydrate } 357 \mathrm{~g}(74 \%) \\
\text { Ballaststoffe } 39,23 \mathrm{~g}\end{array}$ \\
\hline
\end{tabular}


- Tab. 2.39. Ursachen einer Hyperurikämie

\begin{tabular}{|c|c|}
\hline Gesteigerte Harnsäureproduktion & Verminderte Harnsäureausscheidung \\
\hline $\begin{array}{l}\text { Genetische Ursachen } \\
\text { Enzymdefekt }\end{array}$ & $\begin{array}{l}\text { Genetische Ursachen } \\
\text { Reduzierte Klärrate }\end{array}$ \\
\hline $\begin{array}{l}\text { „Erworbene Ursachen« } \\
\text { Myeloproliferative Erkrankungen } \\
\text { Hoher Purinverzehr } \\
\text { Adipositas »metabolisches Syndrom« } \\
\text { Alkoholkonsum } \\
\text { Fruktose } \\
\text { (Körperliche Belastung) }\end{array}$ & $\begin{array}{l}\text { Erworbene Ursachen } \\
\text { Niereninsuffizienz } \\
\text { Medikamente } \\
\text { (Thiazide, Salizylate niedrig dosiert) } \\
\text { Metabolite } \\
\text { (Laktat, Ketonkörper) }\end{array}$ \\
\hline
\end{tabular}

ist notwendig. Verschiedene Biersorten enthalten darüber hinaus unterschiedliche Mengen von Purinen (Pils-, Exportbier: 45 mg/l, Alt-, Kölschbier: 39 mg/l, Hefeweizenbier: $27 \mathrm{mg} / \mathrm{l}$, Diätbier: $33 \mathrm{mg} / \mathrm{l}$, alkoholfreies Bier: 27mg/l). Da eine Hyperurikämie häufig im Rahmen eines »Metabolischen Syndroms « auftritt, werden in der Beratungspraxis die Nahrungsfette mit einem hohen Anteil an gesättigten Fettsäuren begrenzt $(<35 \%)$. Auf eine ausreichende Trinkmenge ist zu achten. Bei einer Hyperurikämie $<8 \mathrm{mg} \%(=475 \mu \mathrm{mol} / \mathrm{l})$ kann eine alleinige diätetische Behandlung versucht werden. In der Praxis sind bei einer purinarmen Dauerkost einmal täglich $100 \mathrm{~g}$ Fleisch, Wurst und Fisch, Milch und Milchprodukte sowie 1 Glas Wein/Tag erlaubt. Tee-,Kaffee- und Wasserkonsum sind nicht beschränkt. Verboten sind Innereien, einige Fischsorten (z. B. Salzhering) und Hülsenfrüchte. Bei einer »streng" purinarmen Kost $(<200$ mg/Tag oder bis zu $1000 \mathrm{mg}$ Purine/Woche) werden Fleisch, Wurst und Fisch allenfalls 1- bis 2-mal/Woche eingeplant. Eine "strenge« Reduktionskost ist bei sehr hohen Harnsäurespiegeln und beim akuten Gichtanfall kontraindiziert, da die renale Harnsäureausscheidung durch Fasten aufgrund der hohen Ketonkörper- und Laktosespiegel im Plasma vermindert ist. Die Kalorienmenge darf in diesem Fall $1000 \mathrm{kcal} /$ Tag nicht unterschreiten.

Fruchtzucker erhöht den Umsatz von Nukleotiden und ist bei Gicht kontraindiziert.

Medikamente. Die Hemmung des letzten Schrittes der Harnsäuresynthese, des Enzyms Xanthinoxidase, durch Allopurinol (=Zyloric, Dosierung 100-300 mg/Tag, initial $100 \mathrm{mg} / \mathrm{Tag}$, bei Kindern: $15 \mathrm{mg} / \mathrm{kg} \mathrm{KG} / \mathrm{Tag}$; Cave: eingeschränkte Kreatininclearance, Wechselwirkung mit den Enzymsystemen der oxidativen Biotransformation und damit dem Metabolismus anderer Medikamente, z. B. Phenytoin, Interaktion mit zytostatisch wirksamen Medikamenten, Azathioprim und Mercaptopurin sowie dem immunsuppressiv wirksamen Cyclosporin durch eine Hemmung des Abbaus) die medikamentöse Behandlung der Wahl. Gleichzeitig wird die Ausscheidung von Harnsäure im Urin verbessert (durch ein sog. Urikosuri- akum wie Probenecid, Dosierung 2-mal 250-500 mg/Tag, Dosierung nach Urin-pH, Ziel: um 8,5; Cave: eingeschränkte Nierenfunktion, Disposition zur Urolithiasis, Wechselwirkung mit anderen Medikamenten wie Allopurinol, nicht-steroidalen Antiphlogistika, Rifampicin, Sulfonamide, Cephalosporin. Dieser Effekt kann durch eine gezielte Lebensmittelauswahl unter Berücksichtigung urinansäuernder und alkalisierender Lebensmittel unterstützt werden (s. - Tab. 2.41). Bei fortgeschrittener Niereninsuffizienz sind Urikostatika und Urikosurika kontraindiziert. Bei Gichtniere und Harnsäuresteinen ist eine Diurese von mindestens 2 1/Tag (d. h. je nach Schwitzen und körperlicher Belastung 2-41 Tee und Mineralwässer trinken, möglicherweise Gabe eines Schleifendiuretikums wie Furosemid z. B. Lasix) sowie eine Alkalisierung des Urins durch Kalium-Natrium-Hydrogenzitrat (z. B. Uralyt-U, Dosierung bis zu 4 Messlöffel am Tag nach Urin$\mathrm{pH}$ von $6,5-6,8$ ) anzustreben. (Uralyt-U enthält pro g $44 \mathrm{mmol}$ Natrium und $44 \mathrm{mmol}$ Kalium; Cave: Niereninsuffizienz, metabolische Alkalose, Harnwegsinfekte). Die Alkalisierung begünstigt die Umwandlung von Harnsäure zu ihrem löslichen Natriumsalz. Die Behandlung der »asymptomatischen « Hyperurikämie ist kontrovers. Sie sollte bei familiärer Disposition für Gicht, dem Vorliegen von Nierensteinen oder Nierenversagen sowie bei einer Harnsäureausscheidung von $>6,5 \mathrm{mmol}$ oder $>1100 \mathrm{mg} /$ Tag begonnen werden.

\section{Sekundäre Hyperurikämie}

Bei sekundärer Hyperurikämie (z. B. bei vermehrter Harnsäurebildung, bei chronisch myeloischer Leukämie, Osteomyelosklerose, Polycythämia vera, im Rahmen einer Zytostatikabehandlung) kann die Dosierung von Allopurinol vorübergehend erhöht werden. Wenn unklar ist, ob eine Hyperurikämie exogen oder endogen zu erklären ist, muss eine Diagnostik unter kontrollierten Ernährungsbedingungen durchgeführt werden. Bei gleichzeitig bestehendem Diabetes mellitus und/oder Fettstoffwechselstörung ist auf eine optimale Stoffwechselführung zu achten. 
- Tab. 2.40. Puringehalt von Lebensmitteln. (Nach Wolfram und Colling und Wolfram 1987)

\begin{tabular}{|l|l|}
\hline Lebensmittel & $\mathbf{m g} / \mathbf{1 0 0 g}$ \\
\hline Innereien & \\
\hline Kalbsbries & 918 \\
\hline Kalbsleber & 221 \\
Kalbslunge & 147 \\
Kalbslunge & 218 \\
\hline Fleisch & \\
\hline Kalbslende & 164 \\
Rinderfilet & 154 \\
Schweinefilet & 152 \\
\hline Schweinebraten & 182 \\
\hline
\end{tabular}

\begin{tabular}{l|r} 
Wurstwaren & \\
Bierschinken & 85 \\
Corned beef & 57 \\
Schinken, gekocht, mager & 131 \\
Frankfurter & 69 \\
\hline
\end{tabular}

\begin{tabular}{l|l}
\hline Fisch & \\
Forelle ohne Haut & 153 \\
Forelle mit Haut & 311 \\
Karpfen ohne Haut & 104 \\
Karpfen mit Haut & 149 \\
Anchovis, Sardellen & 160 \\
Thunfisch in Öl & 198 \\
\hline
\end{tabular}

\begin{tabular}{l|l}
\hline Gemüse & \\
Spinat & 64 \\
Bohnen, grün, frisch & 43 \\
Brokkoli & 47 \\
Blumenkohl & 45 \\
Feldsalat & 34 \\
Spargel & 26 \\
\hline
\end{tabular}

\begin{tabular}{l|l}
\hline Milch & \\
Vollmilch & 0 \\
Quark & 0 \\
\hline Brot & \\
Mischbrot & 84 \\
Weißbrot & 73 \\
Grahambrot & 63 \\
\hline
\end{tabular}

\begin{tabular}{l|r|}
\hline Verschiedenes & \\
\hline Reis, natur, gekocht & 69 \\
\hline Reis, poliert, gekocht & 54 \\
\hline Nudeln, trocken & 185 \\
\hline Nudeln, gekocht & 42 \\
\hline Kartoffeln, gekocht & 18 \\
\hline Vollei & 16 \\
\hline Butter & 0 \\
\hline Margarine & 0 \\
\hline Vollbier, hell & 13 \\
\hline Weißbier & 15 \\
\hline Weißwein & 0 \\
\hline Kaffee $(100 \mathrm{ml})$ & 4 \\
\hline Tee $(100 \mathrm{ml})$ & 2 \\
\hline
\end{tabular}

- Tab. 2.41. Urinansäuernde und -alkalisierende. Lebensmittel

\begin{tabular}{l|l}
$\begin{array}{l}\mathrm{pH}-\text { Wert } \\
\downarrow\end{array}$ & $\begin{array}{l}\text { Fleisch, Fisch, Geflügel, Eier, Käse, Walnüsse, } \\
\text { Vollkornbrot, Cerealien, Nudeln, Reis, Pflau- } \\
\text { men, Preiselbeeren, Kuchen }\end{array}$ \\
\hline $\begin{array}{l}\text { pH-Wert } \\
\uparrow\end{array}$ & $\begin{array}{l}\text { Milch, Milchprodukte, rotes und grünes } \\
\text { Gemüse, Früchte }\end{array}$ \\
\hline Neutral & Streichfette, Süßigkeiten, Kaffee, Tee
\end{tabular}

\section{Akuter Gichtanfall}

Der akute Gichtanfall (Auslöser z. B. ein Diätfehler und Alkoholexcess, schwere katabole Belastungen wie Erkrankungen, längere Nahrungskarenz, körperliche Belastung) wird medikamentös mit Colchicin (z. B. Colchicum Dispert initial $2 \mathrm{mg}$, dann alle $3 \mathrm{~h} 1 \mathrm{mg}$ Colchicin, maximal $9 \mathrm{mg} /$ Tag behandelt. Cave: Nebenwirkungen sind Durchfälle, Übelkeit, Knochenmarksdepression, Alopezie.

80\% der Patienten tolerieren die maximale Dosierung nicht. Colchicin ist ein Alkaloid und hemmt die Zellteilung. Es wirkt nicht analgetisch und hat auch keinen Effekt auf die Harnsäureausscheidung im Urin. Bei häufigen Gichtattacken kann Colchicin niedrig dosiert (z. B. $0,5 \mathrm{mg}$ jeden 2. Tag) und z. B. zusammen mit Allopurinol als Prophylaktikum gegeben werden. Andere entzündungshemmende Medikamente wie Indomethacin (z. B. Amuno, Dosierung initial $75 \mathrm{mg}$, dann $50 \mathrm{mg}$ alle $6 \mathrm{~h}$ ) sind bei Gicht ebenfalls wirksam. Dazu kommt eine ausreichende Flüssigkeitszufuhr (3-5 1/Tag, Tee und Mineralwässer), zunächst sehr purinarme (Puringehalt $<120 \mathrm{mg} / \mathrm{Tag}$ ) und leicht verdauliche Kost, später dann im Verlauf eine purinarme Diät.

\section{Energiereiche Kost bei Malnutrition}

Indikationen. Schlechter Ernährungszustand (BMI $<18,5 \mathrm{~kg} / \mathrm{m}^{2}$ ), Appetitlosigkeit, frühzeitiges Sättigungsgefühl, Chemotherapie, Immunschwäche.

Definition. Energiereiche Kost mit weitgehend «normaler « Nährstoffrelation, der Fettanteil darf $40 \%$ der Gesamtkalorien betragen. Die Eiweißzufuhr beträgt 1,0$1,5 \mathrm{~g} / \mathrm{kgKG} / \mathrm{Tag}$.

Ziele. Erhalt oder Verbesserung des Ernährungszustandes und der mit der Malnutrition assoziierten Probleme.

Anmerkung. Bei hoher Eiweißzufuhr ist auch die $\mathrm{Zu}$ fuhr von Phosphat erhöht (80g Eiweiß entspricht einer Phosphatzufuhr von etwa $1200 \mathrm{mg}$ ), was bei Patienten mit eingeschränkter Nierenfunktion zu berücksichtigen ist. Die Kost hat eine hohe Nährstoffdichte und ist volumenreduziert. Sie enthält keine blähenden Nahrungs- 
bestandteile, der Ballaststoffanteil beträgt $<20 \mathrm{~g} /$ Tag. Es werden 6-8 Mahlzeiten pro Tag eingeplant.Bei ausgeprägter Anorexie und Appetitlosigkeit bzw. bereits manifester Malnutrition sind übertriebene Erwartungen im Hinblick auf den Behandlungserfolg zu vermeiden. In der Regel wird eine manifeste Malnutrition durch diese Kostform nicht wesentlich verbessert. Meist bedeutet sie einen Zeitverlust im Hinblick auf die Durchführung einer Künstlichen Ernährung, vergl. $>$ Kap. 2.6.

\subsubsection{Ernährung bei Hochdruck, Oedemen und Nierenerkrankungen}

\section{Natriumarme Kost}

Indikationen. Essentielle Hypertonie. Sekundäre Hypertonie bei chronischer Niereninsuffizienz im Stadium der kompensierten Retention, Hyperaldosteronismus, präterminale Niereninsuffizienz (mit und ohne Eiweißbeschränkung; Cave: polyurisches Nierenversagen), alle Formen von Ödemen, Aszites, Schwangerschaftsgestose.

Definition. Der Natriumgehalt beträgt bei »natriumarmer Kost « $50 \mathrm{mmol}$ (=3 g NaCl) pro Tag, die übrige $\mathrm{Zu}$ sammensetzung entspricht einer leichten Vollkost.

Ziele. Normalisierung des Blutdrucks, Behandlung von Ödemen und Aszites

Anmerkung. Bei Umrechnung von $\mathrm{Na}^{+}$auf Kochsalz gilt $\mathrm{Na}^{+} \times 2,5$ oder z. B. $400 \mathrm{mg} \mathrm{Na}{ }^{+}=1000 \mathrm{mg} \mathrm{NaCl}$.

Lebensstil. Eine nichtmedikamentöse Behandlung des Bluthochdrucks wird bei allen Patienten mit Hochdruck und insbesondere bei Patienten mit einem labilen Hypertonus durchgeführt. Bei übergewichtigen Patienten wird zunächst das Körpergewicht reduziert und der Alkohol gemieden. Das Konzept der Hypertonusbehandlung umfasst eine Änderung des Lebensstils, welcher Stressprophylaxe, Diät, regelmäßige körperliche Bewegung und Sport (aerobe Belastungen wie Joggen und Schwimmen) sowie die Kontrolle weiterer kardiovaskulärer Risikofaktoren umfasst. Bei der Herstellung einer natriumarmen Diät werden das Zusalzen und stark gesalzene Lebensmittel (z. B. Brot, Wurst, Käse) gemieden und z. T. durch spezielle diätetische Lebensmittel ersetzt.

Natrium in Lebensmitteln. Der Natriumgehalt (in mg) gebräuchlicher Lebensmittel (jeweils bezogen auf $100 \mathrm{~g}$ ) beträgt bei Getreide-/Getreideerzeugnisse: Getreide, Mehl, Haferflocken, Kleie <15 mg, Corn Flakes $91 \mathrm{mg}$, Brot und Brötchen etwa $500 \mathrm{mg}$, Salzstangen etwa $1800 \mathrm{mg}$, Hülsenfrüchte $<30 \mathrm{mg}$, Obst und Gemüse: Frischgemüse bis $80 \mathrm{mg}$, Gemüsekonserven 60-280 mg, Sauerkraut etwa $350 \mathrm{mg}$, Tomatenmark etwa $560 \mathrm{mg}$, Ketchup $1300 \mathrm{mg}$,
Oliven zwischen 2000 und $3000 \mathrm{mg}$. Natriumreich sind besonders häufig Fertigmenüs, Backmischungen, Maggiwürze, Tomatenketchup, Wurstwaren und geräucherte Lebensmittel (s. - Tab. 2.42). Der Natriumgehalt in natriumarmen Mineralwässern beträgt $1 .<20 \mathrm{mg} / \mathrm{l} \mathrm{z}$. B. in Contrex, Perrier; (s. • Tab. 2.43).

Kalium. Anstelle von Kochsalz und Würzmischungen mit Kochsalz werden Gewürze und Kräuter aller Art verwendet. Die Kaliumzufuhr wird auf 6 g/Tag erhöht. Der Kaliumgehalt von Kochsalzersatzmitteln ist zu berücksichtigen $\left(1 / 2\right.$ Teelöffel=387 $\left.\mathrm{mg} \mathrm{K}^{+}\right)$. Eine hohe Kaliumzufuhr und kaliumhaltige Kochsalzersatzmittel sind bei renaler Hypertonie, Niereninsuffizienz, bei Behandlung mit Aldosteronantagonisten oder ACE (»angiotensin converting enzyme)-Hemmer« obsolet.

Flüssigkeit. Die tägliche Flüssigkeitszufuhr darf 2 1/Tag nicht übersteigen. Geeignete Getränke sind Kaffee, Tee, Mineralwasser mit einem Natriumgehalt $<20 \mathrm{mg} / \mathrm{l}$. Wegen der blutdrucksenkenden Wirkung von essentiellen Fettsäuren sollte der P/S-Quotient $>1,0$ liegen.

\section{DASH-Diät}

Als Kombination einzelner diätetischer Maßnahmen empfiehlt sich die sog. DASH-Diät (DASH=Diatary Approach to Stopp Hypotension). Es handelt sich um eine in kontrollierten wissenschaftlichen Untersuchungen (DASH = Dietary Approach to Stopp Hypertension) als wirksam (Blutdrucksenkung ca. $10 \mathrm{mmHg}$ ) charakterisierte Ernährungsform, welche in ihrer Zusammensetzung der Mediterranen Ernährung entspricht. Aufgrund der hohen Obst- und Gemüseaufnahme ist die Kaliumzufuhr hoch, dies erklärt wesentlich die Wirksamkeit der DASH-Diät. - Abb. 2.15 zeigt die Lebensmittelpyramide der DASHDiät im Vergleich zur Mediterranen Ernährung. DASHDiät wird idealerweise im Rahmen eines Lebensstilprogramms durchgeführt.

Medikamente. Der Einfluss der Kochsalzbeschränkung auf den Blutdruck ist additiv zu der Wirkung von Medikamenten wie z. B. Thiaziddiuretika und auch $\beta$-Blockern. Zur medikamentösen Behandlung des Bluthochdrucks sind die Empfehlungen der Fachgesellschaften zu beachten. Bei systolischen Blutdruckwerten von 120-139 mmHg und diastolischen Werten von $80-89 \mathrm{mmHg}$ ist eine Lebensstilumstellung notwendig, Medikamente werden nicht verordnet. Bei systolischen Werten zwischen 140$159 \mathrm{mmHg}$ und einem diastolischen Blutdruck zwischen 90 und $99 \mathrm{mmHg}$ erhalten die meisten Patienten Thiazid-oder Schleifen-Diuretika (Beispiel: Chlorthiazid bzw. Furosemid) sog. $\beta$-Blocker (Beispiel: Atenolol oder Metropolol) oder Calciumkanalantagonisten (Beispiel: Verapamil oder Diltiazem). Sog. Angiotensin-Konversions 
- Tab. 2.42. Lebensmittel mit hohem Kochsalzgehalt

\begin{tabular}{|c|c|}
\hline $\begin{array}{l}250-400 \text { mg Natrium } \\
(0,6-1,0 \mathrm{~g} \mathrm{Kochsalz})^{\mathrm{a}}\end{array}$ & $\begin{array}{l}\text { Dosengemüse } \\
\text { Fischkonserven } \\
\text { Fischsalate } \\
\text { Graubrot, Pumpernickel } \\
\text { Brötchen }\end{array}$ \\
\hline $\begin{array}{l}>400 \text { mg Natrium } \\
(>1,0 \mathrm{~g} \mathrm{Kochsalz})^{\mathrm{a}}\end{array}$ & $\begin{array}{l}\text { Schinken } \\
\text { Wurst } \\
\text { Salzheringe, Matjes } \\
\text { Fertiggerichte } \\
\text { Salzgurken } \\
\text { Oliven } \\
\text { Ketchup } \\
\text { Salatgebäck } \\
\text { Chips }\end{array}$ \\
\hline
\end{tabular}

${ }^{a}$ Angaben bezogen auf den »essbaren« Anteil unter Berücksichtigung der Verluste vor der Zubereitung.

- Tab. 2.43. Natriumgehalt verschiedener Mineral-, Quell-, und Tafelwässer

\begin{tabular}{|c|c|}
\hline Natriumarm (<20 mg Na/l) & \\
\hline Bertolds Quelle & 43 mg/l \\
\hline Bismark Quelle & $10,6 \mathrm{mg} / \mathrm{l}$ \\
\hline Contrex & $6,2 \mathrm{mg} / \mathrm{l}$ \\
\hline Dürrheimer Johannisquelle stilles Wasser & $4,1 \mathrm{mg} / \mathrm{l}$ \\
\hline Dürrheimer Johannisquelle Mineralwasser & $12,8 \mathrm{mg} / \mathrm{l}$ \\
\hline Perrier & $14,0 \mathrm{mg} / \mathrm{l}$ \\
\hline St. Margareten Heilwasser & $19,1 \mathrm{mg} / \mathrm{l}$ \\
\hline Volvic & $8,0 \mathrm{mg} / \mathrm{l}$ \\
\hline Vilsa & $14,3 \mathrm{mg} / \mathrm{l}$ \\
\hline Wildunger Reinhards Quelle & $16,4 \mathrm{mg} / \mathrm{l}$ \\
\hline Wittmannsthal Quelle & $4,3 \mathrm{mg} / \mathrm{l}$ \\
\hline Mäßig natriumarm (20-200 Na/l) & \\
\hline Driburger Caspar Heinrich Quelle & $32,2 \mathrm{mg} / \mathrm{l}$ \\
\hline Driburger Grafenquelle & $137,7 \mathrm{mg} / \mathrm{l}$ \\
\hline Geroldsteiner Sprudel Dolomit Quelle & $121,8 \mathrm{mg} / \mathrm{l}$ \\
\hline Göppinger Sauerbrunn & $26,9 \mathrm{mg} / \mathrm{l}$ \\
\hline Harzer Grauhof & $25,3 \mathrm{mg} / \mathrm{l}$ \\
\hline Leopoldsquelle (Bad Rippoldsau) & $147,2 \mathrm{mg} / \mathrm{l}$ \\
\hline Schloßgarten Quelle & $20,9 \mathrm{mg} / \mathrm{l}$ \\
\hline St. Gero Heilwasser & $174,7 \mathrm{mg} / \mathrm{l}$ \\
\hline St. Martin (Wattenscheid) & $164,7 \mathrm{mg} / \mathrm{l}$ \\
\hline Stark natriumhaltig (>200 mg Na/l) & \\
\hline Appolinaris & $747,0 \mathrm{mg} / \mathrm{l}$ \\
\hline Staatl. Fachingen & $602,5 \mathrm{mg} / \mathrm{l}$ \\
\hline Göppinger Christophquelle & $340,8 \mathrm{mg} / \mathrm{l}$ \\
\hline Grenzacher Heilwasser & $1809,0 \mathrm{mg} / \mathrm{l}$ \\
\hline Heppinger Heilwasser & $856,0 \mathrm{mg} / \mathrm{l}$ \\
\hline Mergentheimer Albertquelle & $12650,0 \mathrm{mg} / \mathrm{l}$ \\
\hline Staatl. Selters & $1031 \mathrm{mg} / \mathrm{l}$ \\
\hline
\end{tabular}

Hemmer (angiotensin converting enzyme inhibitors, ACEHemmer (Beispiel: Captopril, Enalapril)) oder auch Angiotensin II-Rezeptorinhibitoren (Beispiel: Losartan, Valsartan) senken ebenfalls den Blutdruck und werden typischerweise auch in Kombination mit anderen Antihypertensive systolischen Blutdruck $>160 \mathrm{mmHg}$ und diastolischen Blutdruck > $100 \mathrm{mmHg}$ »eingesetzt «. D Tab. 2.44 gibt Hinweise für eine differenzierte medikamentöse Behandlung der Hypertonie. Eine medikamentöse Behandlung des Bluthochdrucks wird als Monotherapie oder als Kombinationsbehandlung mit zwei Arzneimitteln durchgeführt. Bei »Versagen« einer Monotherapie erfolgt zunächst ein Wechsel auf eine Substanz einer anderen Gruppe. Ist ein Medikament differentialtherapeutisch eindeutig indiziert und ein deutlicher Effekt vorhanden, welcher allerdings noch nicht dem Therapieziel entspricht, so wird eine Verstärkung des Effekts durch Kombination zweier Medikamente versucht. Dabei können alle genannten Medikamente mit einem Diuretikum kombiniert werden. Calciumantagonisten werden zusammen mit einem $\beta$-Blocker oder einem ACE-Hemmer verordnet. Beta-Blocker können mit einem Calciumantagonisten, Angiotensin II Rezeptorinhibitoren mit einem Diuretikum kombiniert werden. Bei ACE-Hemmern bietet sich die Kombination mit einem Diuretikum oder einem Calciumantagonisten an.

Die Auswahl des Medikamentes erfolgt aufgrund der zugrundeliegenden Erkrankung. Bei koronarer Herzerkrankung und Bluthochdruck wird zunächst mit einem beta Blocker behandelt. Bei akutem Koronarsyndrom und hohem Blutdruck wird der beta Blocker mit einem ACE-Hemmer kombiniert. Nach Infarkt kann auch eine zusätzliche Gabe eines Diuretikums induziert sein. Bei Herzversagen wird ebenfalls diese Kombination empfohlen. Bei Diabetes mellitus und Hypertonus werden Diuretika, ACE-Hemmer oder auch Calciumantagonisten empfohlen. Bei diabetischer Nephropathie werden ACEHemmer oder Angiotensin II-Rezeptorantagonisten zur Verhinderung der Progression der Nierenerkrankung und zur Senkung der Albuminurie »eingesetzt «. Nach Schlaganfall empfiehlt sich die Kombination von ACE-Hemmer und Diuretikum.

Wirksamkeit. Die Wirksamkeit der genannten Maßnahmen ist unterschiedlich: Gewichtsreduktion $(5-20 \mathrm{mmHg} / 10 \mathrm{~kg})$, DASH-Diät (8-14 mmHg), Kochsalzeinschränkung (2$8 \mathrm{mmHg}$ ), regelmäßige, körperliche Aktivität (4-9 mmHg) und Alkoholkarenz $(5 \mathrm{mmHg})$ sind additiv wirksam. Alle Patienten müssen langfristig betreut werden. Der »Zielblutdruck« liegt unabhängig vom Alter bei <140/90 mmHg. Bei Patienten mit Diabetes mellitus oder Hypertonus und chronischer Nierenerkrankung und einer Protenurie $<1 \mathrm{~g} /$ Tag beträgt das Therapieziel $<130 / 80 \mathrm{mmHg}$. Übersteigt ie Eiweißausscheidung im Urin 1g/Tag, so ist der »Zielblut- 


\section{A. Mediterrane Ernährung}

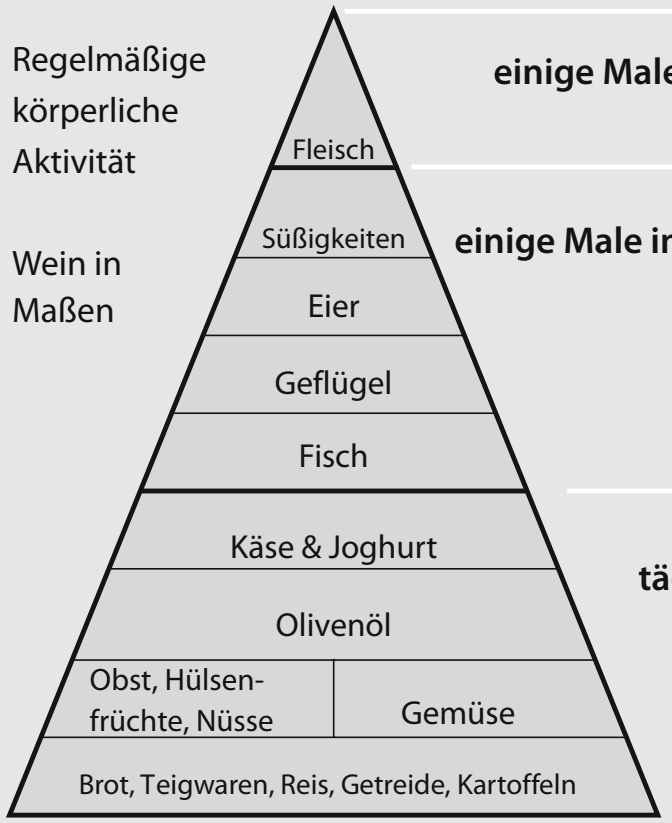

\section{B. DASH-Diät}

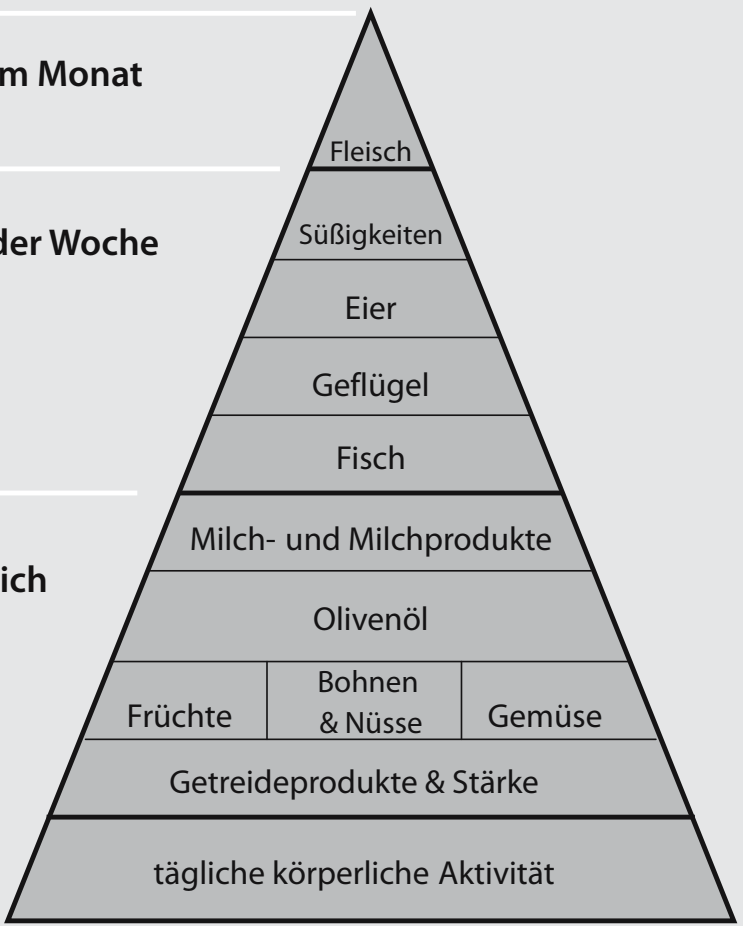

- Abb. 2.15. Lebensmittelpyramiden für Mediterrane Ernährung (links) und DASH-Diät (rechts)

- Tab. 2.44. Differenzierte medikamentöse Behandlung bei Patienten mit einem Bluthochdruck

\begin{tabular}{|c|c|}
\hline Ältere Patienten (>65 Jahre) & z. B. Diuretika \\
\hline Linksherzhypertrophie & z. B. ACE'-Hemmer, zentralenAntisympathotonika \\
\hline Koronare Herzerkrankung & $\beta$-Blocker und Kalziumantagonisten \\
\hline Vorrausgegangener Myokardinfarkt & z. B. ACE ${ }^{1}$-Hemmer und $\beta$-Blocker \\
\hline Herzinsuffizienz & Diuretika und $\mathrm{ACE}^{1}$-Hemmer \\
\hline Niereninsuffizienz & $\begin{array}{l}\text { Bei Serumkreatinin }>2,0 \mathrm{mg} / \mathrm{dl} \text { in der Regel Gabe von Schleifendiuretika, kaliumsparende } \\
\text { Diuretika können besonders in Kombination mit ACE'-Hemmern zur Hyperkaliämie füh- } \\
\text { ren. Verzögerte Elimination mancher Antihypertensiva (Dosisanpassung!) beachten }\end{array}$ \\
\hline Obstruktive Ventilationsstörungen & Kalziumantagonisten, $\mathrm{ACE}^{1}$-Hemmer und $\mathrm{a}_{1}$-Blocker, $\beta$-Blocker kontraindiziert \\
\hline Diabetes mellitus & $\begin{array}{l}\text { Bei jüngeren Patienten mit Typ-I- und Typ-II-Diabetes mellitus: ACE'-Hemmer } \\
\text { Bei älteren Patienten mit Typ-II-Diabetes: Orientierung der Therapie an den Begleit- } \\
\text { erkrankungen } \\
\text { Bei Diabetes allgemein: Zurückhaltung mit nicht-selektiven } \beta \text {-Blockern }\end{array}$ \\
\hline Gicht/Hyperurikämie & Zurückhaltung mit Diuretika \\
\hline Gravidität & $\beta_{1}$-selektive Blocker, deren Unschädlichkeit für die fötale Entwicklung nachgewiesen ist \\
\hline
\end{tabular}


druck « 125/75 mmHg. Ein »optimaler Blutdruck« liegt bei Werten von $120 / 80 \mathrm{mmHg}$ vor.

Ödeme. Bei Ödemen ist die Flüssigkeitszufuhr auf zunächst $1 \mathrm{l} / \mathrm{Tag} \mathrm{zu}$ beschränken. Bei »idiopathischen" und auch bei diuretikainduzierten Ödemen ist eine Natriumrestriktion nicht angezeigt. Eine noch weitere Einschränkung der Natriumzufuhr $(<50 \mathrm{mmol} / \mathrm{Tag}=3 \mathrm{~g} / \mathrm{Tag}$, z. B. als Reistag) kann in Einzelfällen bei Patienten mit einem Aszites und einer 24-h-Natriumausscheidung im Urin von $>25 \mathrm{mmol} /$ Tag als Initialbehandlung sinnvoll sein. Eine »Reis-Obst-Diät« enthält 250-300 g Reis (Trockengewicht) und 750-1000 g Obst (Trockengewicht) in geeigneter Zubereitung (d. h. ohne Zusatz von Salz, Milch, Fett). Dieses entspricht etwa $300 \mathrm{~g}$ Kohlenhydrate, $10 \mathrm{~g}$ Fett, $(=1250-1500 \mathrm{kcal} / \mathrm{Tag})$ und $1,5-2,0 \mathrm{mmol}$ Natrium/Tag. Diese Kostform ist ohne Zusätze (z. B. Quarkspeisen, Pflanzenöle, Vitamine) nicht bedarfsdeckend und deshalb nur kurzfristig (d. h. für 3-5 Tage) durchführbar. Zudem ist sie dem Patienten über längere Zeit nicht zumutbar.

Leberzirrhose. Eine diätetische Natriumbeschränkung ist bei Patienten mit einer Leberzirrhose und Aszites sowie einer Natriumausscheidung von $<25 \mathrm{mmol} / \mathrm{Tag}$ nicht geeignet. Streng natriumarme Diäten enthalten $<20 \mathrm{mmol}$ Natrium bzw. $<1 \mathrm{~g} \mathrm{NaCl}$. Der Mindestbedarf liegt bei $0,5 \mathrm{~g}$ Natrium/Tag.

Compliance. Die »Compliance« für eine natriumarme Diät kann anhand der Natriumausscheidung im 24-hUrin überprüft werden.

Probleme. Bei einer natriumarmen Ernährung ist die Versorgung mit Kalzium und Magnesium zu beachten. Eine streng natriumarme Diät geht mit einer Abnahme des Blutvolumens einher. Dieses kann bei älteren Menschen mit arteriosklerotisch veränderten Blutgefäßen oder bei starken Salzverlusten (z. B. Schwitzen oder Erbrechen) zu Kreislaufproblemen führen.

\section{Protein-reduzierte Diät}

Indikationen. Krankheiten und Zustände mit eingeschränkter Eiweißtoleranz bei Nieren- oder Leberinsuffizienz.

Definition. 0,6 g Eiweiß pro Kilogramm Körpergewicht und Tag).Reduzierte Eiweißzufuhr unter Bevorzugung biologisch hochwertiger Eiweiße (75\% der Nahrungseiweiße) bei ausreichender Energiezufuhr (35-40 kcal/ kg KG und Tag).

Ziele. Prävention und Therapie urämischer Symptome. Verzögerung des Fortschreitens der Nierenerkrankung.
Verbesserung der Bewusstseinslage bei hepatischer Encephalopathie.

Kontraindikationen. Rasch progrediente Niereninsuffizienz, terminale Niereninsuffizienz, Manifestation urämischer Syndrome (Gastroenteritis, Perikarditis, Polyneuropathie), schwere Erkrankungen (z. B. ein Tumorleiden), Schwangerschaft und Stillperiode sowie Alter $(<18$ bzw. $>60$ Jahre).

Anmerkungen. Die Grundzüge der ernährungsmedizinischen Behandlung akuter und chronischer Nierenerkrankungen sind in den - Tab. 2.45 und $\mathbf{0}$ Tab. 2.46 dargestellt.

Praxis. Die Einschränkung der Eiweiß- und Kaliumzufuhr ergibt sich aus dem jeweiligem Grad der Dekompensation der Nierenfunktion. Bei vollständiger Kompensation ist die Eiweißzufuhr nicht eingeschränkt, die Kaliumzufuhr beträgt 50-75 mmol/Tag. Im Stadium der kompensierten Retention (Serumharnstoff $<17 \mathrm{mmol} / \mathrm{l}$, Kreatinin $<350 \mu \mathrm{mol} / \mathrm{l}$ ) wird die Eiweißzufuhr nicht, die Kaliumzufuhr abhängig von der Neigung zur Hyperkaliämie individuell begrenzt. Liegen die Harnstoffkonzentrationen im Serum bei $25 \mathrm{mmol} / \mathrm{l}$ und das Kreatinin $<530 \mu \mathrm{mol} / \mathrm{l}$, so wird die Eiweißzufuhr auf $30-40 \mathrm{~g} / \mathrm{Tag}$, bei einem Harnstoff $>25 \mathrm{mmol} / \mathrm{l}$ und einem Kreatinin bis $630 \mu \mathrm{mol} / \mathrm{l}$ auf $25-30 \mathrm{~g} / \mathrm{Tag}$ reduziert. Eine eiweißarme Diät sollte im Hinblick auf eine Senkung der glomerulären Filtration, eine Verzögerung der Progression der Nierenerkrankung und die Protektion der verbliebenen Nierenfunktion möglichst frühzeitig begonnen werden. Die Progression der Niereninsuffizienz kann mittels einer frühzeitig begonnenen eiweißdefinierten oder -armen Diät um die Hälfte verzögert werden. Neben der Eiweißbeschränkung verzögern die folgenden Faktoren die Progression einer chronischen Nierenerkrankung: Kochsalzbeschränkung $(<6 \mathrm{~g} / \mathrm{d})$, Blutdruck $<130 / 80 \mathrm{mmHg}$ bei Proteinurie $<1 \mathrm{~g} /$ Tag bzw. $<125 / 75 \mathrm{mmHg}$ bei Proteinurie $>2 \mathrm{~g} / \mathrm{Tag}$, medikamentöse Blutdrucksenkung mit z. B. ACE-Inhibitoren, Stoffwechselkontrolle bei Diabetes mellitus (HbAlc 7\%), normales Plasmalipidmuster (Gesamtcholesterin $<5,2 \mathrm{mmol} / \mathrm{l}$, LDLc $<3,1 \mathrm{mmol} / \mathrm{l})$ und Nichtrauchen. Der Hämoglobinwert sollte $>11 \mathrm{~g} / \mathrm{dl}$, der Serumcalciumwert $>$ $2,2 \mathrm{mmol} / \mathrm{l}$ und der Serumphophatspiegel $<1,8 \mathrm{mmol} / \mathrm{l}$ liegen. Eine eiweißarme Diät ist vorzugsweise vegetarisch. Dieses gilt besonders bei Patienten mit einer Neigung zur Azidose. In der Praxis beeinflusst eine proteinarme Diät nicht nur die glomeruläre Filtration, sondern hat darüber hinaus weitere günstige Effekt auf den Blutdruck (eiweißarme Diäten sind in der Regel natriumarm), die Proteinurie, die Hyperlipidämie (durch den günstigen Einfluss auf die Proteinurie), den Hyperparthyroidismus bzw. die Hyperphosphatämie (die Diäten sind phosphatarm) und die Azidose (die Diäten haben einen geringen Gehalt an schwefelhaltigen Aminosäuren). Eine Einschränkung der Kaliumzufuhr erfolgt anhand der Laborwerte. 
Tab. 2.45. Diätetik bei akutem Nierenversagen. (Nach Kluthe u. Quirin 1996)

\begin{tabular}{|c|c|c|c|}
\hline & Prädialytisches Stadium & Oligo-/Anurie & Polyurische Phase \\
\hline Trinkmenge/Wasser & Nach Bilanz & Nach Bilanz & Nach Bilanz \\
\hline $\begin{array}{l}\text { Energie } \\
\text { (kcal/kg KG/Tag) }\end{array}$ & 35 & 35 & 35 \\
\hline $\begin{array}{l}\text { Eiweiß } \\
\text { (g/kg KG/Tag) }\end{array}$ & 0,4 & $\begin{array}{l}0,5-0,6 \\
\text { (bei Dialyse }-1,2 \text { ) }\end{array}$ & $0,5-0,6$ \\
\hline $\begin{array}{l}\text { Kalium } \\
\text { (mmol/Tag) }\end{array}$ & $<20$ & $<30-60$ & $\begin{array}{l}50 \\
\text { Nach Bilanz }\end{array}$ \\
\hline $\begin{array}{l}\text { Natrium } \\
\text { (mmol/Tag) }\end{array}$ & $<50$ & $<50$ & Nach Bilanz \\
\hline
\end{tabular}

- Tab. 2.46. Diätetik bei chronischem Nierenversagen. (Nach Kluthe u. Quirin, 1996)

\begin{tabular}{|c|c|c|c|}
\hline & Vor Dialyse & Hämodialyse & Peritonealdialyse \\
\hline $\begin{array}{l}\text { Trinkmenge/Wasser } \\
(1 / \text { Tag) }\end{array}$ & Nach Bilanz & $\begin{array}{l}-0,5 \\
\text { (Nach Bilanz) }\end{array}$ & $\begin{array}{l}-0,5 \\
\text { Nach Bilanz }\end{array}$ \\
\hline $\begin{array}{l}\text { Energie } \\
\text { (kcal/kg KG/Tag) }\end{array}$ & 35 & 35 & $\begin{array}{l}35 \\
\text { Cave: Peritoneal-spüllösung }\end{array}$ \\
\hline $\begin{array}{l}\text { Eiweiß } \\
\text { (g/kg KG/Tag) }\end{array}$ & 0,4 & 1,2 & $\begin{array}{l}1,2 \\
\text { Cave: Peritoneal-spüllösung }\end{array}$ \\
\hline $\begin{array}{l}\text { Kalium } \\
\text { (mmol/Tag) }\end{array}$ & $20-40$ & -50 & $\begin{array}{l}-50 \\
\text { Nach Bilanz }\end{array}$ \\
\hline $\begin{array}{l}\text { Natrium } \\
\text { (mmol/Tag) }\end{array}$ & -51 & -51 & -51 \\
\hline
\end{tabular}

Substitution. Die Diätetik bei Nierenkranken umfasst ferner die Phosphateinschränkung (bei fortgeschrittener Niereninsuffizienz auf $<1 \mathrm{~g} / \mathrm{Tag}$ ) sowie die Vitamin- (z. B. als Dreisavit sowie Gabe von 1,25-Dihydroxy-Vitamin $\mathrm{D}_{3}$ $0,25 \mu \mathrm{g}$ Rocaltrol/Tag) und Eisensubstitution. Die Notwendigkeit zur Eisengabe (orale Dosierung 10,0 mg/ Tag) besteht bei Dialysebehandlung auch während einer Behandlung mit Erythropoietin. Bei starker Eiweißbeschränkung werden zusätzlich Pyridoxin und Folsäure gegeben. Unter Erythropoietintherapie kann es zu einem Anstieg der Kreatininspiegel kommen.

Glomerulonephritis. Bei akuter Glomerulonephritis stehen die Störung der Flüssigkeitsbilanz und die Ödembildung im Vordergrund. Empfehlung ist die Begrenzung der Flüssigkeitszufuhr (=24-h-Urinausscheidung+500 ml) und die Kochsalzeinschränkung. Besteht eine ausgeprägte Retention der harnpflichtigen Substanzen und zusätzlich eine Neigung zu einer Hyperkaliämie, wird vorübergehend eine eiweiß-, natrium- und kaliumarme Kost empfohlen. Bei chronischer Glomerulonephritis mit Hypertonie und geringer Proteinurie (=3 g/Tag) erfolgt vorrangig eine Natriumbeschränkung. Liegt die Proteinurie bei $>3 \mathrm{~g} / \mathrm{Tag}$ (=nephrotische Verlaufsform einer chronischen Glomerulonephritis) oder bestehen eine Hypoalbuminämie und Ödeme, kann die Eiweißzufuhr maximal 1,3 g/kg KG täglich betragen. Eine Kochsalzbeschränkung ist meistens angezeigt. Bei höheren Zufuhrraten kommt es zu einer schnelleren Progredienz des Nierenfunktionsverlustes.

Interstitielle Nephritis. Bei interstitieller Nephritis (z. B. bei Analgetikanephropathie) wird auf eine ausreichende Diurese geachtet. Dabei kann es zu Kaliumverlusten kommen.

Diabetische Nephropathie. Bei diabetischer Nephropathie und Hypertonie sollte die Eiweißzufuhr nicht über $0,8 \mathrm{~g} / \mathrm{kg} \mathrm{KG}$ täglich liegen.

Phosphat. Eine eiweißarme Diät ist in der Regel natriumund phosphatarm. Sie ist so auch bei Hyperphosphatämie und sekundärem Hyperparathyroidismus geeignet. Phos- 
phatreiche Lebensmittel wie Milch und Milchprodukte sind zu meiden. Der Phosphatgehalt der Kost sollte nicht über $800-900 \mathrm{mg} /$ Tag betragen. Dieses bedeutet in der Praxis eine Begrenzung der Milch- und Vollkornprodukte. Eine streng phosphatarme Ernährung $(<600 \mathrm{mg} / \mathrm{Tag})$ könnte durch Elimination phosphatreicher Nahrungsmittel (Milch, Käse, Eier, Fleisch) erreicht werden. Dies ist aber praktisch nicht möglich, da diese Nahrungseiweiße wegen ihrer hohen biologischen Wertigkeit im Rahmen einer eiweißarmen Diät verwendet werden müssen. Schmelzkäse, Colagetränke etc. sind wegen ihres hohen Phosphatgehalts verboten. Die diätetische Restriktion reicht häufig nicht aus, um den Phosphatspiegel zu normalisieren. Es empfiehlt sich die Gabe von Phosphatbindern (z. B. Aludrox, Cave: Resorption und Akkumulation von Aluminium bei längerer Gabe, aluminiuminduzierte Osteomalazie, mikrozytäre Anämie und Enzephalopathie) zur Hemmung der Phosphatresorption mit dem Ziel, die Serumphosphatspiegel auf Werte $<1,78 \mathrm{mmol} / \mathrm{l}$ zu senken. Bei der Behandlung von Störungen des Kalzium-Phosphat-Haushaltes bei Nierenkranken hilft das Kalzium-Phosphat-Löslichkeitsprodukt (Serumkalzium mal Serumphosphat jeweils in $\mathrm{mg} / \mathrm{dl}$, normal $<60$ ). Wird der Wert überschritten, ist mit Verkalkungen und Atherosklerose $\mathrm{zu}$ rechnen. Liegt das Löslichkeitsprodukt darunter, werden kalziumhaltige Phosphatbinder (z. B. Kalziumkarbonat, Cave: Hyperkalzämie, besonders bei gleichzeitiger Vitamin-D-Gabe) gegeben. Kalziumkarbonat dient auch der Korrektur der bei chronischer Niereninsuffizienz häufigen metabolischen Azidose.

Eiweißqualität. Eiweißdefinierte und mäßig proteinarme Diäten können "gemischt« oder »selektiv« zusammengestellt sein. Als "gemischt proteinarm" enthält diese Diätform mindestens 50\% hochwertiges Eiweiß, welche im Rahmen des Diätplanes in Form von kleinen Fleisch-, Fisch- oder Geflügelportionen gegeben werden. Als »selektiv proteinarme« Diät werden biologisch hochwertige Eiweißgemische (z. B. die Kartoffel-Ei-Diät) mit mindestens $50 \%$ des Eiweißanteils als biologisch hochwertiges Protein verwendet. Dabei wird Eiweiß in verschiedenen Stufen $(20-60 \mathrm{~g})$ zugesetzt. Eine Energieanreicherung erfolgt mit Maltodextrin. Bei heute nur noch ausnahmensweise angewandten "selektiv sehr proteinarmen" Diäten wird neben der Kartoffel-Ei-Diät auch die "Schwedendiät» mit freier Lebensmittelauswahl und zusätzlicher Gabe von essentiellen Aminosäuren angewendet. Bei allen eiweißdefinierten Diäten ist auf eine ausreichende Energiezufuhr $(25-40 \mathrm{kcal} / \mathrm{kg} \mathrm{KG} \times \mathrm{d}) \mathrm{zu}$ achten und der Ernährungszustand differenziert zu untersuchen. Für die praktische Gestaltung proteindefinierter Diäten ist die Verwendung von Eiweißaustauschtabellen hilfreich (s. $\mathbf{0}$ Abb. 2.16).

Diäten mit einem Eiweißgehalt $<40 \mathrm{~g} / \mathrm{Tag}$ werden heute aufgrund der frühzeitigen Indikation für eine Dialysebehandlung nicht mehr angewendet.
Kalium. Eine katabole Stoffwechsellage und eine Azidose begünstigen die Hyperkaliämie. Diese wird diätetisch durch Verzicht auf bestimmte Gemüse (getrocknete Früchte, Linsen, Tomaten), Obst (Nüsse, Zitrusfrüchte, Bananen), Süßigkeiten (Schokolade), Fruchtsäfte, Instantkaffee, Kakao-, Vollkorn- und Hefeprodukte behandelt. Zum Kaliumgehalt ausgewählter Lebensmittel s. auch - Tab. 2.47. Die niedrige Kaliumzufuhr bedeutet bei höheren Kalorienstufen einen Qualitätsverlust der Nahrung.

\section{Dialyseadaptierte Kost}

Indikation. Hämodialyse, Peritonealdialyse.

Definition. Die Eiweißzufuhr beträgt bei einer Frequenz von 3 Dialysen pro Woche zwischen 1 und 1,2 g/kg KG und Tag. Die Kalorienzufuhr wird individuell berechnet (30-35 kcal $/ \mathrm{kg} \mathrm{KG} \times \mathrm{d}$ bei Hämodialyse, $25-30 \mathrm{kcal} /$ $\mathrm{kg}$ KGxd bei Peritonealdialyse). Der Natriumzufuhr ist $100 \mathrm{mmol} / \mathrm{Tag}(3 \mathrm{~g} \mathrm{NaCl})$, der Kaliumgehalt $60-80 \mathrm{mmol} /$ Tag. Die Kalziumzufuhr beträgt 1,5-2,0 g/Tag, der Phosphatgehalt ist $<2,0 \mathrm{~g} /$ Tag. Die in der Tageskost enthaltene Flüssigkeitsmenge beträgt (bei einer Kalorienzufuhr von etwa $2000 \mathrm{kcal}$ ) $800-1000 \mathrm{ml}$. Die Trinkflüssigkeit muss zwischen den »Dialysetagen « individuell nach Restdiurese festgelegt werden (=Restdiurese $+500 \mathrm{ml} / \mathrm{Tag}$ ).

Ziele. Unterstützung der Dialysebehandlung, ausgeglichene Flüssigkeitsbilanz, maximale Gewichtszunahme von $1,5 \mathrm{~kg}$ zwischen zwei Dialysen. Verhütung einer Hyperkaliämie, Kompensation des Säure-Basen-Haushaltes, Substitution von Verlusten, Vermeidung metabolischer Komplikationen, Erhalt des Ernährungszustandes.

\begin{tabular}{l|l}
\hline \multicolumn{2}{|l}{ - Tab. 2.47. Kaliumgehalt verschiedener Lebensmittel } \\
\hline Weiße Bohnen & $1300 \mathrm{mg}$ \\
\hline Erbsen & $930 \mathrm{mg}$ \\
\hline Linsen & $810 \mathrm{mg}$ \\
\hline Kartoffeln & $400 \mathrm{mg}$ \\
\hline Bananen & $382 \mathrm{mg}$ \\
\hline Spinat & $324 \mathrm{mg}$ \\
\hline Tomaten & $206 \mathrm{mg}$ \\
\hline Orangen & $185 \mathrm{mg}$ \\
\hline Trauben & $183 \mathrm{mg}$ \\
\hline
\end{tabular}

a Angaben bezogen auf den »essbaren« Anteil unter Berücksichtigung der Verluste durch dir Zubereitung. 
$\sim 3$ g Eiweiß oder Protein sind enthalten in:

\section{Milchprodukte}

$90 \mathrm{~g} \mathrm{Milch,} \mathrm{3,5 \%} \mathrm{Fett}$

$75 \mathrm{~g}$ Joghurt, 3,5\% Fett

Käse

25 g Doppelrahmfrischkäse

$20 \mathrm{~g}$ Rahmfrischkäse, $50 \%$ F.i.Tr.

$30 \mathrm{~g}$ Speisequark, $40 \%$ F.i.Tr.

$25 \mathrm{~g}$ Speisequark, 20\% F.i.Tr.

$20 \mathrm{~g}$ Speisequark, mager

30 g Schichtkäse, $40 \%$ F.i.Tr.

$25 \mathrm{~g}$ Schichtkäse, 20\% F.i.Tr.

$20 \mathrm{~g}$ Schichtkäse, $10 \%$ F.i.Tr.

$25 \mathrm{~g}$ Hüttenkäse

$25 \mathrm{~g}$ Schmelzkäse, $60 \%$ F.i.Tr.

$20 \mathrm{~g}$ Schmelzkäse, $45 \%$ F.i.Tr.

Fleischwaren

20 g Cervelatwurst

$269 \mathrm{~g}$ Fleischkäse

$20 \mathrm{~g}$ Leberpastete

$25 \mathrm{~g}$ Leberwurst

$20 \mathrm{~g}$ Luncheon meat

\section{Fischwaren}

Brot

Obst
25 g Mettwurst

$35 \mathrm{~g}$ Speck, durchwachsen

20 g Kaviar, Ersatz

Vegetabile Pasten

35 g Tartex Delikatess

35 g Tartex Kräuter

$40 \mathrm{~g}$ Tartex Equisit

35 g Tartex Paprika

$50 \mathrm{~g}$ Tartex ung. Art

35 g Tartex, natriumarm, Kräuter

$35 \mathrm{~g}$ Tartex, natriumarm Delikatess

$50 \mathrm{~g}$ Roggenbrot

$35 \mathrm{~g}$ Brötchen, hell

$160 \mathrm{~g}$ Avocado

230 g Himbeeren, Johannisbeeren

$\sim 5 \mathrm{~g}$ Eiweiß oder Protein sind enthalten in:

Wurst

$35 \mathrm{~g}$ Leberwurst

$40 \mathrm{~g}$ Lyoner

$40 \mathrm{~g}$ Mettwurst

$40 \mathrm{~g}$ Mortadella

$45 \mathrm{~g}$ Münchner Weißwurst

$25 \mathrm{~g}$ Plockwurst

$30 \mathrm{~g}$ Salami

$180 \mathrm{~g}$ Speck, fett

$55 \mathrm{~g}$ Speck, durchwachsen

$25 \mathrm{~g}$ Schweineschinken, gekocht

$30 \mathrm{~g}$ Schweinschinken, roh

$35 \mathrm{~g}$ Wiener Würstchen

Fische und Fischwaren

$25 \mathrm{~g}$ Barsch

$25 \mathrm{~g}$ Heilbutt

$30 \mathrm{~g}$ Hering

$30 \mathrm{~g}$ Kabeljau

$30 \mathrm{~g}$ Rotbarsch

$30 \mathrm{~g}$ Schellfisch

$30 \mathrm{~g}$ Scholle

$30 \mathrm{~g}$ Seezunge

$25 \mathrm{~g}$ Thunfisch

$30 \mathrm{~g}$ Tintenfisch

$25 \mathrm{~g}$ Forelle

30 g Karpfen

$50 \mathrm{~g}$ Miesmuscheln

$25 \mathrm{~g} \mathrm{Aal}$, geräuchert

$30 \mathrm{~g}$ Brathering

$25 \mathrm{~g}$ Bückling
$40 \mathrm{~g}$ Hering in Gelee

$35 \mathrm{~g}$ Hering in Tomatensauce

$30 \mathrm{~g}$ Hering, mariniert

$35 \mathrm{~g}$ Kaviarersatz

$30 \mathrm{~g}$ Krebsfleisch in Dosen

$25 \mathrm{~g}$ Makrele, geräuchert

$30 \mathrm{~g}$ Matjeshering

$20 \mathrm{~g}$ Ölsardinen

$20 \mathrm{~g}$ Thunfisch in Öl

Milchprodukte

$140 \mathrm{~g}$ Buttermilch

$115 \mathrm{~g}$ Früchtejoghurt

$130 \mathrm{~g}$ Vollmilchjoghurt

$140 \mathrm{~g}$ Joghurt, fettarm, $1,5 \%$ Fett

$150 \mathrm{~g}$ Trinkmilch

$150 \mathrm{~g}$ Trinkmilch, 1,5\% Fett

Käse

$35 \mathrm{~g}$ Rahmfrischkäse, $50 \%$ F.i.Tr.

$45 \mathrm{~g}$ Doppelrahmfrischkäse, $60 \%$ F.i.Tr.

$40 \mathrm{~g}$ Hüttenkäse

$35 \mathrm{~g}$ Speisequark, mager

$45 \mathrm{~g}$ Speisequark, $40 \%$ F.i.Tr.

40 g Schichtkäse, $10 \%$ F.i.Tr.

50 g Schichtkäse, $40 \%$ F.i.Tr.

$20 \mathrm{~g}$ Chester, $50 \%$ F.i.Tr.

Innereien

$30 \mathrm{~g} \mathrm{Herz}$ i.D.

$50 \mathrm{~g}$ Hirn (Kalb)

$25 \mathrm{~g}$ Leber i.D.

$15 \mathrm{~g}$ Emmentaler, 45\%

$15 \mathrm{~g}$ Parmesan

$35 \mathrm{~g}$ Schmelzkäse, $45 \%$ F.i.Tr. Wild und Geflügel

$40 \mathrm{~g}$ Schmelzkäse, $60 \%$ F.i.Tr. 25 g Rehrücken

$25 \mathrm{~g}$ Butterkäse, $50 \%$ F.i.Tr. $30 \mathrm{~g}$ Ente

$20 \mathrm{~g}$ Edamer, $45 \%$ F.i.Tr. $\quad 35 \mathrm{~g}$ Gans

$25 \mathrm{~g}$ Edelpilzkäse, $50 \%$ F.i.Tr. $25 \mathrm{~g}$ Huhn

$20 \mathrm{~g}$ Gouda, 45\% F.i.Tr. $\quad 25 \mathrm{~g}$ Truthahn

$20 \mathrm{~g}$ Tilister, $45 \%$ F.i.Tr.

$25 \mathrm{~g}$ Briekäse, $50 \%$ F.i.Tr. Wurst

$20 \mathrm{~g}$ Camembert, $30 \%$ F.i.Tr. $30 \mathrm{~g}$ Bierschinken

25 g Camembert, 45\% Fi.Tr. 40 g Blutwurst

$30 \mathrm{~g}$ Camembert, $60 \%$ F.i.Tr. $\quad 40 \mathrm{~g}$ Bockwurst

$25 \mathrm{~g}$ Münsterkäse, 50\% F.i.Tr. $40 \mathrm{~g}$ Bratwurst

$20 \mathrm{~g}$ Limburger, $20 \%$ F.i.Tr. $\quad 30 \mathrm{~g}$ Cervelatwurst

$20 \mathrm{~g}$ Limburger, $40 \%$ F.i.Tr. $20 \mathrm{~g}$ Corned Beef,

$20 \mathrm{~g}$ Romadur, $20 \%$ F.i.Tr.

25 g Romadur, 50\% F.i.Tr.

amerik.

Eier

$40 \mathrm{~g}$ Hühnerei

Fleisch

$45 \mathrm{~g}$ Schweinebauch

$30 \mathrm{~g}$ Schweinefleisch, mittelfett

$35 \mathrm{~g}$ Schweinefleisch, fett

$25 \mathrm{~g}$ Schweinehackfleisch

$25 \mathrm{~g}$ Rindfleisch, mager

$30 \mathrm{~g}$ Rindfleisch, fett

$20 \mathrm{~g}$ Rinderhackfleisch

$25 \mathrm{~g}$ Kalbfleisch i.D.

$30 \mathrm{~g}$ Hammelfleisch i.D.

$25 \mathrm{~g}$ Kaninchenfleisch

$25 \mathrm{~g}$ Corned Beef, dtsch.

$25 \mathrm{~g}$ Dosenschinken

$40 \mathrm{~g}$ Dosenwürstchen

$45 \mathrm{~g}$ Fleischkäse

$40 \mathrm{~g}$ Fleischwurst

$40 \mathrm{~g}$ Frankfurter

Würstchen

$45 \mathrm{~g}$ Gelbwurst

$40 \mathrm{~g}$ Jagdwurst

$25 \mathrm{~g}$ Kasseler

$35 \mathrm{~g}$ Leberpastate

Brot

$60 \mathrm{~g}$ Weizenbrot (Vollkorn)

$65 \mathrm{~g}$ Mehrkornbrot

$\sim 7 \mathrm{~g}$ Eiweiß oder Protein sind enthalten in:

Milchprodukte

$200 \mathrm{~g}$ Buttermilch

$160 \mathrm{~g}$ Früchtejoghurt

$100 \mathrm{~g}$ Vollmilchjoghurt

$200 \mathrm{~g}$ Joghurt, fettarm, $1,5 \%$ Fett

$210 \mathrm{~g}$ Trinkmilch

$210 \mathrm{~g}$ Trinkmilch, 1,5\% Fett

Käse

50 g Rahmfrischkäse, $50 \%$ F.i.Tr.

$60 \mathrm{~g}$ Doppelrahmfrischkäse, $60 \%$ F.i. Tr.

$50 \mathrm{~g}$ Speisequark, mager

$60 \mathrm{~g}$ Speisequark, $40 \%$ F.i. Tr.

55 g Hüttenkäse

$55 \mathrm{~g}$ Schichtkäse, 10\% F. i. Tr.

$65 \mathrm{~g} \mathrm{Schichtkäse,} 40 \%$ F.i. Tr.

$30 \mathrm{~g}$ Chester, $50 \%$ F. i. Tr.

$20 \mathrm{~g}$ Parmesan
$35 \mathrm{~g}$ Gorgonzolakäse

50 g Schmelzkäse, $45 \%$ F.i. Tr.

55 g Schmelzkäse, $60 \%$ F.i. Tr.

35 g Butterkäse, $50 \%$ F.i. Tr.

30 g Edamer, $45 \%$ F.i. Tr.

35 g Edelpilzkäse, $50 \%$ F.i. Tr.

30 g Gouda, $45 \%$ F.i. Tr.

$25 \mathrm{~g}$ Tilsiter, $45 \%$ F.i. Tr.

$30 \mathrm{~g}$ Briekäse, $50 \%$ F.i. Tr.

30 g Camembert, $30 \%$ F.i. Tr.

35 g Camembert, $45 \%$ F.i. Tr.

$40 \mathrm{~g}$ Camembert, $60 \%$ F.i. Tr.

Münsterkäse, $50 \%$ F.i. Tr. 


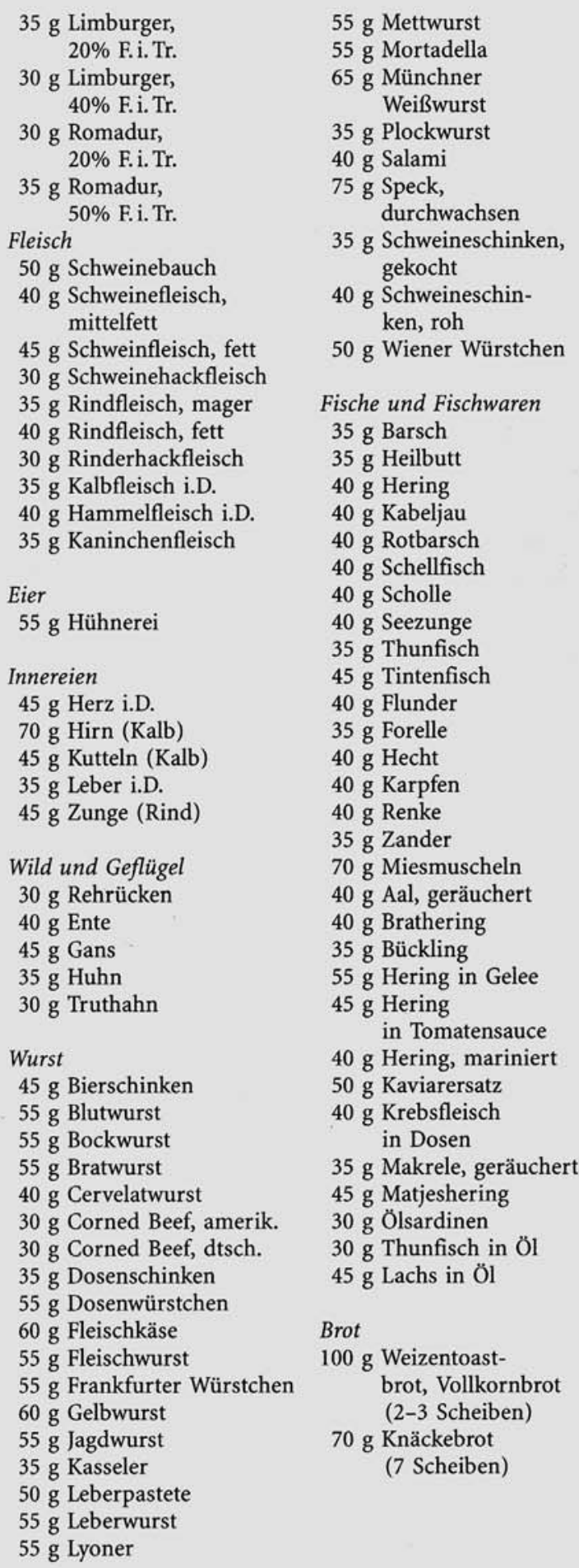

$55 \mathrm{~g}$ Mettwurst

$55 \mathrm{~g}$ Mortadella

$65 \mathrm{~g}$ Münchner Weißwurst

35 g Plockwurst

$40 \mathrm{~g}$ Salami

75 g Speck, durchwachsen

35 g Schweineschinken, gekocht

$40 \mathrm{~g}$ Schweineschinken, roh

$50 \mathrm{~g}$ Wiener Würstchen

Fische und Fischwaren

$35 \mathrm{~g}$ Barsch

$35 \mathrm{~g}$ Heilbutt

$40 \mathrm{~g}$ Hering

$40 \mathrm{~g} \mathrm{Kabeljau}$

$40 \mathrm{~g}$ Rotbarsch

$40 \mathrm{~g}$ Schellfisch

$40 \mathrm{~g}$ Scholle

$40 \mathrm{~g}$ Seezunge

$35 \mathrm{~g}$ Thunfisch

$45 \mathrm{~g}$ Tintenfisch

$40 \mathrm{~g}$ Flunder

$35 \mathrm{~g}$ Forelle

$40 \mathrm{~g}$ Hecht

$40 \mathrm{~g}$ Karpfen

$40 \mathrm{~g}$ Renke

$35 \mathrm{~g}$ Zander

$70 \mathrm{~g}$ Miesmuscheln

$40 \mathrm{~g} \mathrm{Aal}$, geräuchert

$40 \mathrm{~g}$ Brathering

$35 \mathrm{~g}$ Bückling

$55 \mathrm{~g}$ Hering in Gelee

$45 \mathrm{~g}$ Hering in Tomatensauce

$40 \mathrm{~g}$ Hering, mariniert

$50 \mathrm{~g}$ Kaviarersatz

$40 \mathrm{~g}$ Krebsfleisch in Dosen

$35 \mathrm{~g}$ Makrele, geräuchert

$45 \mathrm{~g}$ Matjeshering

$30 \mathrm{~g}$ Ölsardinen

$30 \mathrm{~g}$ Thunfisch in Öl

$45 \mathrm{~g}$ Lachs in Öl

Brot

$100 \mathrm{~g}$ Weizentoastbrot, Vollkornbrot (2-3 Scheiben)

$70 \mathrm{~g}$ Knäckebrot

(7 Scheiben)

Die Werte beziehen sich auf den eßbaren Anteil und das Rohgewicht. Berechnungsunterlagen: Prodi (Kluthe, B. 1988, Wissenschaftl. Verlagsgesellschaft Stuttgart)

Werte aus: Große GU Nährwerttabelle 1996/97.

\section{Anmerkung}

Spezielle Probleme. Die diätetischen Empfehlungen berücksichtigen die speziellen Probleme des Dialysepatienten, die Häufigkeit der Dialyse und die Restfunktion der Niere. Während einer Hämodialyse werden bis zu $10 \mathrm{~g}$ Aminosäuren in das Dialysat verloren. Die Ernährung sollte also eher eiweißreich sein. Dagegen muss eine Akkumulation von Harnstoff zwischen den Dialysetagen vermieden werden. Bei hoher Harnstoffproduktionsrate wird zwischen »exogenem « und »endogenem « Eiweiß-(Aminosäure-) »Katabolismus" unterschieden. Eine Abgrenzung ist anhand des klinischen Verlaufs sowie nach Auswertung eines Ernährungsprotokolls möglich. Kochsalzersatzmittel oder Diätsalze sind in der Praxis nicht notwendige Bestandteile der Diät. Phosphatreiche Nahrungsmittel (z. B. Schmelzkäse, Hartkäse, Erdnüsse, geräucherte Lebensmittel, Colagetränke, Konservierungsstoffe) sind zu meiden. Bei Hyperphosphatämie beträgt die Phosphatzufuhr $<1000 \mathrm{mg} / \mathrm{Tag}$. Die Cholesterinzufuhr liegt bei $<300 \mathrm{mg} /$ Tag, linolsäurereiche Fette werden bevorzugt. Wasserlösliche Vitamine (Dreisavit N, 1-mal 1 Tbl./Tag), 1,25-Dihydroxy-Vitamin D (z. B. 0,25 lg Rocaltrol/Tag), Kalzium und Eisen $(100 \mathrm{mg} / \mathrm{Tag})$ müssen im Einzelfall substituiert werden. Keine Substitution von Vitamin A!

Peritonealdialyse. Bei chronischer Peritonealdialyse besteht keine Indikation für eine Dialysekost. Während der Peritonealdialyse ist die »Kalorienzufuhr « über das Dialysat (monomere Glukose bis zu $150 \mathrm{~g}$ am Tag entsprechend $25 \%$ der Gesamtkalorien) sowie ein möglicher Eiweißverlust in das Dialysat (bis zu $15 \mathrm{~g} / \mathrm{Tag}$ ) zu berücksichtigen. Der Kalorienbedarf ist bei Patienten unter chronischer Peritonealdialyse niedriger, während ihr Eiweißbedarf gleichzeitig erhöht ist. Die Zufuhr an Nahrungskohlenhydraten ist auf $35-40 \%$ des Energiebedarfes zu beschränken, die Eiweißzufuhr auf 1,2-1,5 g/kg KG am Tag zu erhöhen. Komplexe Kohlenhydrate sind zu bevorzugen. Extrem phosphatreiche Lebensmittel müssen gemieden und wasserlösliche Vitamine substituiert werden. Bei hohen Triglyzeridspiegeln im Plasma ist die Zufuhr von Monound Disacchariden zu begrenzen. Die Flüssigkeitszufuhr liegt bis zu $800 \mathrm{ml}$ über der Restdiurese.

Flüssigkeit. Bei der Berechnung der Flüssigkeitszufuhr sind die Getränke, der Wassergehalt von Lebensmitteln und das bei der Oxidation der Nährstoffe entstehende Wasser zu berücksichtigen. Der Flüssigkeitsanteil beträgt bei Suppen oder reiner Trinkflüssigkeit 100\% des Gesamtgewichts des Lebensmittels bzw. des Gerichtes, bei Kartoffelbrei, Quarkspeisen und Joghurt 75\%, bei gekochten Kartoffeln, Reis, Müsli, Eis, Getreide etc. 50\%, bei Bratkartoffeln, Pizza, Aufläufen etc. $25 \%$ und bei Brot, Backwaren (ohne Obstbelag), Fleischgerichten (ohne Sauce), Fetten, Ölen und Wurstwaren 0\%. Bei Obst wird ein Flüssigkeitsgehalt von $80-90 \%$ angenommen. 
Für den Patienten ist die Überwachung der Flüssigkeitszufuhr anhand einer Maßtabelle hilfreich:

- 1 Teelöffel $=5 \mathrm{ml}$,

- 1 Esslöffel $=15 \mathrm{ml}$,

- 1 Wasserglas oder

- 1 Tasse $=80-100 \mathrm{ml}$,

- 1 Becher/1 Suppentasse = bis zu $200 \mathrm{ml}$,

- 1 Suppenteller $=250 \mathrm{ml}$.

Bei Obst und Gemüse ist der Wassergehalt anzurechnen. Der "unsichtbare" Wassergehalt anderer Lebensmittel bleibt dagegen in der Praxis weitgehend unberücksichtigt. Im Mittel wird ein Wert von 0,7-1,0 1/Tag aufgenommen. Die dialyseadaptierte Kost kann salzarm oder kaliumarm gestaltet werden. In der Praxis kann der Anteil des "Oxidationswassers" (etwa 0,31/Tag) mit den Flüssigkeitsverlusten durch Atmung, Schwitzen und mit dem Stuhl gleichgesetzt werden. Bei hochkalorischer Ernährung oder bei ausgeprägtem »Katabolismus« ist die Menge des »Oxidationswassers« erhöht.

Hypertriglyzeridämie. Bei Hypertriglyzeridämie wird primär auf die Vermeidung von Zuckern (und Zuckeraustauschstoffen) sowie Alkoholkarenz geachtet. Eine Restriktion der Nahrungsfette ist angesichts der angestrebten Nährstoffrelation, des häufig schlechten Appetits der Patienten und ihres Ernährungszustands nicht praxisgerecht.

Supplement. Bei inadäquater Eiweiß- und Kalorienzufuhr werden Supplemente (z. B. Sonana Renamil oder Survimed renal) empfohlen.

Nierentransplantation. Nach Nierentransplantation gibt es keine eigenständige Kostform. Ziel ist es, den Ernährungszustand zu verbessern und die mit der immunsuppressiven Behandlung assoziierten Stoffwechselprobleme (z. B. eine katabole Stoffwechsellage, Hypertriglyzeridämie, Kohlenhydratverwertungsstörung) zu kompensieren. Bei sekundärem Hyperparathyreoidismus sind eine Phosphatbegrenzung sowie die zusätzliche Gabe von Kalzium und Vitamin D notwendig.

\section{Protein- und elektrolytdefinierte Kost bei Leberzirrhose}

Indikation. Eingeschränkte Leberfunktion mit Encephalopathie.

Ziele. Verbesserung der Symptome und Folgen der Leberfunktionsstörung.

Definition. Festlegung der Eiweißmenge $(0,6 \mathrm{~g} / \mathrm{kg} \times \mathrm{d})$ und Qualität (lacto-vegetablie Eiweiße).

\section{Anmerkungen}

Praxis. Die Indikation zu einer diätetischen Behandlung eines Patienten mit einer chronischen Lebererkrankung ergibt sich aus den mit der Leberzirrhose assoziierten Komplikationen wie Aszites, Encephalopathie; Cholestase und Malnutrition. Im Hinblick auf die bei Leberzirrhose häufige Malnutrition sind besonders Patienten mit einer spontanen Energiezufuhr unter $2000 \mathrm{kcal} / \mathrm{Tag}$ und einer Eiweißaufnahme unter $60 \mathrm{~g} /$ Tag gefährdet. $\mathrm{NH}_{3}{ }^{+}$wird im Dünndarm und der Niere "produziert" und durch Muskel- und Leberstoffwechsel »entgiftet«. Zur Vermeidung der intestinalen Ammoniakbildung wird Lactulose gegeben. Nicht-resorbierbare Antibiotika (Neomycin), Ornitinaspartat und/oder die Gabe von verzweigtkettigen Aminosäuren sind weitere im Einzelfall Möglichkeiten, die zur Verbesserung der encephalopathischen Symptome beitragen können. In kontrollierten Studien sind z. T. weder Lactulose noch eine Eiweißrestriktion wirksam, in der Placebogruppe kommt es bei $20-40 \%$ der Patienten auch ohne »entgiftende« Behandlung zu einer Verbesserung der Symptome.

Mikronährstoffe. Vitamine sind pragmatisch zu substituieren. Bei chronischer Enzephalopathie sind die Plasmazinkspiegel häufig niedrig. Eine Substitution z. B. mit Zinkaspartat (z. B. Unizink 50) oder Bis- (L-Histidin) Zink (z. B. Zinkamin-Falk) ist in unkontrollierten Studien auf die Zeichen der Encephalopathie wirksam. Die Dosierung beträgt bis zu $50 \mathrm{mg} / \mathrm{Tag}$, bei ausgeprägtem Zinkmangel (=Acrodermatitis enterohepatica) werden kurzfristig bis zu 100 mg/Tag gegeben. Vorsicht bei der Gabe von Zinksulfat, bei hohen Dosen von Zinksulfat besteht die Gefahr von Diarrhoe).

\section{Diäten bei Urolithiasis}

Indikation. Urolithiasis.

Definition. Je nach Steinart kalzium-, oxalsäure-, bzw. purindefiniert.

Ziele. Prävention oder Behandlung einer Urolithiasis, Vermeidung exzessiver Zufuhrraten der genannten Nährstoffe.

Anmerkung. Risikofaktoren für Nierensteine sind ein »familiäres Risiko« (z. B. Nierensteine in der Familie), Insulinresistenz, Bluthochdruck, primärer Hyperparathyroidismus, Gicht, frühzeitige Menopause und anatomische Faktoren wie Obstruktionen oder Strikturen im Bereich der ableitenden Harnwege. Eine Hypercalciurie ( $>200 \mathrm{mg} / 24 \mathrm{~h}$ oder $>4 \mathrm{mg} / \mathrm{kg}$ Körpergewicht $/ 24 \mathrm{~h}$ ), eine Hyperuricosurie und eine Hyperoxalurie $(>45 \mathrm{mg} / 24 \mathrm{~h}$ ) sind die häufigsten Ursachen von Nierensteinen. In allen Fällen von Urolithiasis ist eine hohe Flüssigkeitszufuhr zu 
empfehlen (>3 1/Tag, Ziel: tagsüber $200-300 \mathrm{ml} / \mathrm{h}$ !, mindestens einmal trinken pro Nacht). Höhere Flüssigkeitsmengen sind nur begrenzt wirksam. (Cave: Eine höhere Flüssigkeitszufuhr ist notwendig bei z. B. hoher Außentemperatur, Schwitzen, starker körperlicher Aktivität).

Das Urinvolumen muss über 2,5 l/Tag liegen. Die Menge an tierischem Eiweiß wird auf etwa $50 \mathrm{~g} / \mathrm{Tag}$ beschränkt. Dieses dient der Präventione eines sauren $\mathrm{pH}$ Wertes sowie einer Steigerung der Citratausscheidung im Urin. Die Kochsalzzufuhr soll $<50 \mathrm{mmol} /$ Tag betragen. Die Oxalsäureaufnahme ist zu begrenzen $(<50 \mathrm{mg} / \mathrm{Tag})$. Die Modifikation der Diät ergibt sich aus der chemischen Analyse der Steine. Die wichtigsten Bestandteile der Nierensteine sind Kalzium, Oxalsäure, Harnsäure, Phosphat und Glycin.

- Kalziumsteine. In 50\% der Fälle finden sich eine normale Urinkalziumausscheidung, nur bei 50\% der Patienten besteht eine Hyperkalziurie. Diese wird in 90\% der Fälle durch eine gesteigerte Kalziumresorption und nur bei $10 \%$ der Patienten renal erklärt. Nur $50 \%$ der Fälle von "resorptiver « Hyperkalziurie sind durch eine Umstellung der Ernährung im Sinne einer »kalziumdefinierten« Diät beeinflussbar. Die Kalziumzufuhr wird durch Begrenzung von Milch und Milchprodukten beschränkt, sie sollte bei Männern $800 \mathrm{mg}$ und bei Frauen 1000 mg/Tag (nach der Menopause $1200 \mathrm{mg} / \mathrm{Tag}$ ) nicht unterschreiten. Kalciumarme Diäten sind unwirksam und obsolet, bei niedriger Kalciumzufuhr stiegt die Oxalsäureresorption. Eine kalziumarme Ernährung (bei Männern $<800 \mathrm{mg} / \mathrm{Tag}$, bei Frauen $<1000 \mathrm{mg}$ bzw. $<1200 \mathrm{mg} / \mathrm{Tag}$ ) verursacht eine negative Kalziumbilanz (Cave: Osteoporose) und erhöht die Gefahr von Oxalatsteinen. "Exzesse" an kalziumreichen Lebensmitteln (Spinat, Rhabarber, Endivien, Okra, getrocknete Früchte) wurden vermieden. Eine Hyperkalziurie kann durch eine hohe Zufuhr von Natrium, tierischem Eiweiß und Zucker verstärkt werden. Eine Begrenzung dieser Nährstoffe auf die Empfehlungen einer Vollkost ist deshalb sinnvoll. Eine ballaststoffreiche Ernährung steigert die fäkale Kalziumausscheidung und kann deshalb unterstützend wirken. Bei Kalziumphosphatsteinen sollte ein sulfathaltiges Mineralwasser mit wenig Hydrogenkarbonat getrunken werden. Bei bettlägerigen Patienten besteht häufig eine Hyperkalziurie, welche durch körperliche Aktivität (d. h. physikalisch-therapeutische Maßnahmen) und nicht diätetisch behandelt wird.

- Kalziumoxalatsteine. Eine oxalsäurearme Ernährung enthält $<50 \mathrm{mg}$ Oxalsäure/Tag. Da nur 10\% der Oxalsäureausscheidung im Urin aus Lebensmitteln stammt, ist der Erfolg der Diät begrenzt. Dennoch hat sie bei Patienten mit Oxalatsteinen auf Dauer einen nachgewiesenen therapeutischen Wert. Da Oxalsäure auch Endprodukt des Vitamin-C-Stoffwechsels ist und die
Kalzium- und Oxalsäureresorption im Darm sich gegenseitig beeinflussen, werden die Vitamin-C-Zufuhr $(<1 \mathrm{~g} / \mathrm{Tag})$ und die Kalziumzufuhr bei idiopathischer (=adäquate Kalziumzufuhr) und absorptiver Hyperkalziurie (s. oben) ebenfalls begrenzt. Oxalsäurereiche Lebensmittel (>10 mg/Portion) sind z. B. Stark- oder dunkle Biere, Ovomaltine, Tee, Kakao, Tofu, Bohnen, rote Bete, Sellerie, Okra, Rhabarber, Spinat, Blaubeeren, Trauben, Erdbeeren, Stachelbeeren, Nüsse, Sesam, Tomaten. Bei Malassimilation und Steatorrhoe wird die Hyperoxalurie durch die Bindung des Kalziums und der Fettsäuren im Dünndarm und die dadurch erhöhte Resorption der freien Oxalsäure im Kolon erklärt. In diesem Fall müssen die Malassimilation behandelt bzw. Kalziumsupplemente ( $1 \mathrm{~g} / \mathrm{Tag})$ gegeben werden. Hier wird ein hydrogenkarbonatreiches Mineralwasser empfohlen.

- Phosphatsteine. Phosphatarme Ernährung oder die Gabe von Phosphatbindern therapeutisch sind nicht sehr wirksam. Wichtig ist die Alkalisierung des Urins $(\mathrm{pH}<7,0)$.

- Cystinurie. Alkalisierung des Urins. Eine gleichzeitige Reduktion der Methioninzufuhr kann duch Eiweißbegrenzung und die Vermeidung von "Proteinexzessen « (nicht mehr als $100 \mathrm{~g}$ Eiweiß/Tag) erreicht werden. Eine natriumarme Diät trägt ebenfalls zu einer Senkung der Cystinausscheidung im Urin bei.

\section{- Harnsäuresteine}

Zur Begrenzung der Purinzufuhr s. $\triangleright$ Kap. 2.5.2.

\subsubsection{Sonderdiäten}

\section{Gastroenterologische Diäten Diät bei Malassimilation/Kost mit mittelkettigen Triglyzeriden (MCT)}

Indikationen. Nachgewiesene Steatorrhoe von $>15 \mathrm{~g} / \mathrm{Tag}$ (Analyse der Gesamtstuhlmenge von 3 Tagen, bei normaler Fettzufuhr mindestens 70-100 g/Tag) oder auch niedrige $\beta$-Carotinspiegel $(<45 \mu \mathrm{g} / \mathrm{l})$ bei adäquater $\mathrm{Er}$ nährung, z. B. bei exokriner Pankreasinsuffizienz (unter Enzymsubstitution und einer Stuhlfrequenz von $>3-\mathrm{mal} /$ Tag), bei Malassimilation, Morbus Whipple, chologener Diarrhoe, A-b-Lipoproteinämie, Endstufe des Kostaufbaus bei Malabsorption, exsudativer Enteropathie, $\mathrm{Mu}$ koviszidose, Hyperchylomikronämie, evtl. bei Vorliegen eines Chylothorax.

Definition. Leichte Vollkost mit weitgehendem Ersatz der LCT-Fette durch definierte Mengen von MCT-Fetten (=Fette mit mittelkettigen gesättigten Fettsäuren, MCTAnteil $60-80 \%$ der Gesamtfette). Laktosegehalt $<20 \mathrm{~g}$, ballaststoffarm (15-20 g). MCT-Fette sollten entsprechend dem Kostaufbau bei Malabsorption stufenweise eingefügt werden. 
Ziele. Sicherung der Energiezufuhr, Verminderung der Steatorrhoe, Erhalt oder Besserung des Ernährungszustands.

Anmerkung. LCT-Fette in der Nahrung werden durch Verzicht auf Lebensmittel mit hohem Anteil versteckter Fette vermindert. Streich- und Kochfette werden durch MCT ersetzt. MCT-Fette enthalten Fettsäuren mit einer Kettenlänge von 8-10 C-Atomen. Sie haben einen Energiegehalt von $8,3 \mathrm{kcal} / \mathrm{g}$. MCT-Fette befinden sich als Margarine (z. B. ceres MCT Diät-Margarine, $80 \mathrm{~g}$ Fett, davon 90\% MCT, 3\% Linolsäure, 7\% sonstige Fettsäuren) oder als Öl im Handel (z. B. ceres MCT Diät-Speiseöl, $100 \mathrm{~g}$ Fett, 95\% MCT, 5\% sonstige Fettsäuren).

Anwendung. Bei Raumtemperatur ist MCT-Öl dünnflüssig, klar und geruchlos. MCT-Fette werden in der Regel gut toleriert und akzeptiert. Wenn kein Kostaufbau vorangegangen ist, müssen MCT-Fette stufenweise gegeben werden, beginnend mit $10 \mathrm{~g} / \mathrm{Tag}$. In den folgenden Tagen wird die Fettmenge je nach subjektiver Verträglichkeit um jeweils $10 \mathrm{~g}$ bis auf die »Endstufe» gesteigert. Im Einzelfall können nach einem adäquaten Kostaufbau die Tagesmengen zwischen 100 und $120 \mathrm{~g} / \mathrm{Tag}$ (bis zu 40\% der Energiezufuhr) liegen. Als Dauerkostform werden zusätzlich linolsäurereiche Fette gegeben, um einen Mangel an essentiellen Fettsäuren zu vermeiden. Fettlösliche Vitamine werden individuell, z. B. nach Auswertung eines Diätprotokolls substituiert. MCT-Fette haben einen niedrigen Schmelzpunkt, MCT-Speiseöle können nicht so hoch erhitzt werden wie normale Speiseöle.

Warmhalten muss vermieden werden, weil dadurch andere Bestandteile der Speisen einen bitteren Geschmack bekommen. Mit MCT zubereitete Gerichte werden deshalb sofort nach dem Garen verzehrt.

Probleme. Mögliche Zeichen der Unverträglichkeit sind bei hoher und nicht stufenweise adaptierter Zufuhr von MCT-Fetten (>80-100 g/Tag): Übelkeit, abdominelle Distension, Durchfälle, Bauch- und Kopfschmerzen. Diese Kostform ist eine Dauerkostform. Bei exsudativer Enteropathie wird die MCT-Kost mit einer hohen Eiweißzufuhr (100-120 g/Tag) kombiniert, um die intestinalen Proteinverluste zu kompensieren. MCT-Fette sollten bei Patienten mit einer Neigung zur Ketonämie oder metabolischer Azidose gemieden werden. Bei Patienten mit einer Leberzirrhose und einem hohen portokavalen Shuntvolumen sind bei hoher MCT-Zufuhr enzephalopathieähnliche Symptome, Hyperventilation, eine Hyperammonämie und eine Laktatazidose beschrieben worden. Die Möglichkeit dieser Symptome lässt eine vorsichtige und eher niedrige Dosierung von MCT-Fetten bei Patienten mit Lebererkrankungen sinnvoll erscheinen.

\section{Laktosearme Diät}

Indikationen. Primäre oder sekundäre Laktoseintoleranz. Bei sekundärer Laktoseintoleranz (z. B. im Rahmen einer Sprue oder bei Morbus Crohn) wird nach Behandlung der Grunderkrankung ein Kostaufbau, welcher auch laktosehaltige Produkte berücksichtigt, durchgeführt.

Definition. Eine mäßig laktosearme Kost enthält 8-10 g Laktose/Tag, eine laktosefreie Kost $<1$ g Laktose/Tag.

Ziele. Linderung der Beschwerden, Prävention einer Malnutrition.

Anmerkung. Die Diagnose einer Laktoseintoleranz beruht auf der klinischen Symptomatik, deren Beeinflussung durch laktosefreie Ernährung, den Ergebnissen des $\mathrm{H}_{2}$-Atemtests, einem oralen Laktosetoleranztest und dem fehlenden Enzymnachweis in der Dünndarmbiopsie (s. - Kap. 1). Die Prävalenz eines primären Laktasemangels beträgt in Nordeuropa zwischen 5 und 15\% der Bevölkerung. Sie ist außerhalb Nordeuropas hoch. Sie betrifft besonders Menschen aus Mittelmeerländern (60-85\%), Asiaten (90-100\%), Schwarze (85-100\%) und Indianer (50-95\%). Aufgrund der hohen inter- individuellen Varianz der Beschwerden muss die Laktosetoleranz individuell ermittelt werden. Die Kost ist milchfrei (1 1 Milch enthalten $50 \mathrm{~g}$ Laktose) und vermeidet Milchprodukte sowie Lebensmittel mit hohem Laktosegehalt (z. B. Frischkäse, Eiscreme, Fertigsaucen, Schokolade. Nichtpasteurisierter Joghurt (laktaseaktiv) und Sauermilchprodukte werden meist gut vertragen. Einige Patienten tolerieren geringe Mengen an Laktose $(<10 \mathrm{~g} / \mathrm{Tag}=1 / 2$ Tasse Milch, z. B. als Milch im Kaffee, 1/2 Becher Joghurt, 1 kleine Portion Eis; zum Laktosegehalt von Milch und Milcherzeugnissen s. - Tab. 2.48). Laktobazillen-enthaltende Milchprodukte (»Probiotika«) werden nicht besser toleriert als »normale» Produkte. Der Laktosegehalt von Säuglingsnahrung und Formuladiäten ist zu beachten. Bei Laktoseintoleranz im Säuglingsalter muss eine laktosefreie (z. B. Pregestimil, Humana SL) oder laktosearme (Humana HS oder Aledin) Säuglingsnahrung gewählt werden. Der Bedarf an Kalzium (bei einer milchfreien Ernährung werden nur etwa $300 \mathrm{mg}$ Kalzium/Tag zugeführt), Vitamin $\mathrm{B}_{2}$ und D wird durch eine laktosefreie Ernährung nicht gedeckt. Eine Supplementierung wird für Kinder, Jugendliche, Schwangere und Stillende sowie bei bestehendem Osteoporoserisiko, unbedingt empfohlen. Die Mehrzahl der Patienten ist unter einer laktosearmen Ernährung beschwerdefrei. Die zu Beschwerden führende Menge ist andererseits häufig gering (3-5g Laktose).

\section{Kostaufbau}

Die verschiedenen Formen des Kostaufbaus sind empirisch und wissenschaftlich nicht begründet. 
Kostaufbau nach größeren Operationen. Nach größeren Operationen und/oder längeren Phasen (>7 Tage) einer künstlichen Ernährung und wenn der Zustand des Patienten eine Ernährung mit Vollkost in fester Form nicht sofort zulässt, ist eine flüssige und/oder eine passierte leichte Vollkost indiziert. Eine flüssige Vollkost deckt nicht den Energie- und Eiweißbedarf des Patienten. Der Kostaufbau wird über z. B. 4 Stufen (von 600-2400 kcal/ Tag) über jeweils 3 Tage durchgeführt. Er kann nach Auftreten von Symptomen oder Toleranz verlängert bzw. verkürzt werden. In den ersten Stufen ist die Kost kohlenhydrat-reich $(>60 \%)$ und fettarm $(\approx 20 \%)$. Während des Kostaufbaus wird ergänzende künstlich ernährt.

Kostaufbau nach akuter Pankreatitis. Stufenweiser qualitativer und quantitativer Aufbau der oralen Ernährung mit kleinen Mahlzeiten im Anschluss an eine Nahrungskarenz und parenterale Ernährung. Der Kostaufbau erfolgt über insgesamt 4 in der Regel 3tägige Stufen. Die Dauer der einzelnen Stufen kann angesichts des subjektiven oder objektiven Beschwerdebildes der einzelnen Patienten variiert werden. Stufe 1 hat eine Nährstoffrelation von $83 \%$ Kohlenhydraten, 10\% Fett und 7\% Eiweiß (ca. $1400 \mathrm{kcal} / \mathrm{Tag}$ ).Auf Stufe 2 sind es $73 \%$ Kohlenhydrate, $12 \%$ Fett, 15\% Eiweiß (ca. $1600 \mathrm{kcal} / \mathrm{Tag}$ ).Stufe 3 enthält $65 \%$ Kohlenhydrate, 16\% Fett, 19\% Eiweiß (ca. 2000 kcal/ Tag).Stufe 4 hat 58\% Kohlenhydrate, 21\% Fett und 21\% Eiweiß (ca. $2250 \mathrm{kcal} / \mathrm{Tag}$ ). An Stufe 4 schließt sich eine leicht verdauliche Kost an.

Gabe von Pankreasfermenten. Bei chronischer Pankreatitis und auch nach totaler Pankreatektomie (z. B. bei Z.n. Pankreaskarzinom) ist die diätetische Versorgung bei gleichzeitiger und ausreichender Substitution mit Pankreasfermenten (Richtdosis 25-50000 IU Lipase zu den Hauptmahlzeiten und 20000 IU Lipase zu den Zwischenmahlzeiten; zeitgerechte Einnahme, d. h. vor dem Essen und fraktioniert während der Mahlzeiten; Ziel: Stuhlfrequenz $<3-\mathrm{mal} / \mathrm{Tag}$ ) und ggf. gleichzeitiger Insulinsubstitution häufig problemlos. - Tab. 2.49 zeigt die heute am häufigsten verschriebenen Pankreasenzympräparate. Die Indikation zur Fermentsubstitution besteht bei klinischen Beschwerden (wie z. B. Gewichtsverlust, Dyspepsie, Diarrhoen) und/oder einer Steatorrhoe ( $>15 \mathrm{~g}$ Fettausscheidung/Tag) bei bekannter Ursache (z. B. eine chronische Pankreatitis). »Therapiestandard « ist die Enzymsubstitution mit säuregeschützten und mikrophärisch verkapselten Enzympräperaten (Partikelgröße $<1,4 \mathrm{~mm}$ ). Bei ausgeprägter Steatorrhoe (z. B. bei zystischer Fibrose) werden die Enzympräperate zusammen mit einem Hemmer der Magensäuresekretion gegeben. (Cave: niedriger intraduodenaler $\mathrm{pH}$ bei normaler Magensäure-, aber niedriger Bikarbonatsekretion durch die Bauchspeicheldrüse).

Bei einer exokrinen Pankreasinsuffizienz ist eine adäqute (Richtdosis 25-50000 IU Lipase zu den Haupt-
- Tab. 2.48. Laktosegehalt von Milch und Milcherzeugnissen. (Aus Rennes 1994)

\begin{tabular}{|c|c|}
\hline & $g / 100 g$ \\
\hline Konsummilch (Frischmilch, H-Milch) & $4,8-5,0$ \\
\hline $\begin{array}{l}\text { Milchmixgetränke (Schoko, Mokka, Vanille, } \\
\text { Erdbeer, Banane, Himbeer, Nuss) }\end{array}$ & $4,4-5,4$ \\
\hline Dickmilch & $3,7-5,3$ \\
\hline Frucht-Dickmilch & $3,2-4,4$ \\
\hline Joghurt & $3,7-5,6$ \\
\hline $\begin{array}{l}\text { Joghurtzubereitungen (Schoko, Nuss, Müsli, } \\
\text { Mokka, Vanille) }\end{array}$ & $3,5-6,0$ \\
\hline Kefir & $3,5-6,0$ \\
\hline Buttermilch & $3,5-4,0$ \\
\hline Sahne, Rahm (süß, sauer) & $2,8-3,6$ \\
\hline Creme fraîche & $2,0-3,6$ \\
\hline Creme double & $2,6-4,5$ \\
\hline Kaffeesahne $10-15 \%$ Fett & $3,8-4,0$ \\
\hline Kondensmilch 4-10\% Fett & $9,3-12,5$ \\
\hline Butter & $0,6-0,7$ \\
\hline Butterschmalz & - \\
\hline Milchpulver & $38,0-51,5$ \\
\hline Molke, Molkegetränke & $2,0-5,2$ \\
\hline $\begin{array}{l}\text { Desserts (fertigprodukte: Cremes, Pudding, } \\
\text { Milchreis, Grießbrei) }\end{array}$ & $3,3-6,3$ \\
\hline Eiscreme (Milch-, Frucht-, Joghurteis) & $5,1-6,9$ \\
\hline Sahneeis & 1,9 \\
\hline Magerquark & 4,1 \\
\hline Rahm-, Doppelrahmfrischkäse & $3,4-4,0$ \\
\hline Speisequark 10-70\% Fett i. Tr. & $2,0-3,8$ \\
\hline Schichtkäse $10-50 \%$ Fett i. Tr. & $2,9-3,8$ \\
\hline Hüttenkäse 20\% Fett i. Tr. & 2,6 \\
\hline Frischkäsezubereitungen $10-70 \%$ Fett i. Tr. & $2,0-3,8$ \\
\hline Schmelzkäse $10-70 \%$ Fett i. Tr. & $2,8-6,3$ \\
\hline Käsefondue (Fertigprodukt) & 1,8 \\
\hline Käsepastete $60-70 \%$ Fett i. Tr. & 1,9 \\
\hline Kochkäse 0-45\% Fett i. Tr. & $3,2-3,9$ \\
\hline $\begin{array}{l}\text { Hart-, Schnitt-, Weichkäse: } \\
\text { Emmentaler, Bergkäse, Berghofkäse, Reibkäse, } \\
\text { Parmesan, Alpkäse, Edamer, Gouda, Tilsiter, } \\
\text { Stauferkäse, Steppenkäse, Trappistenkäse, } \\
\text { Appenzeller, Backsteiner, Brie, Camembert, } \\
\text { Weichkäse, Weinkäse, Weißlacker, Chester, } \\
\text { Edelpilzkäse, Schafskäse, Havarti, Jerome, } \\
\text { Limburger, Romadur, Mozzarella, Münsterkäse, } \\
\text { Raclette, Räucherkäse, Sandwich-Käsepastete, } \\
\text { Bad Aiblinger Rahmkäse, Butterkäse, Esrom, } \\
\text { Sauermilchkäse (Harzer, Mainzer, Handkäse) }\end{array}$ & laktosefrei \\
\hline
\end{tabular}


und $20000 \mathrm{IU}$ Lipase zu den Zwischenmahlzeiten oder $1000-2500 \mathrm{IU} / \mathrm{kg} \mathrm{KG} \times \mathrm{d}$ oder 1000-1500 IU/g Fett) und zeitgerechte Einnahme (direkt und möglichst fraktioniert während der Mahlzeit) notwendig. Fettlösliche Vitamine sind zu substituieren. Die Dosierung von Pankreasfermenten erfolgt nach klinischem Befund, maximal können bis zu 200000 Einheiten pro Tag gegeben werden. Zu der Auswahl von Pankreasenzympräparaten s. - Tab. 2.49. Bei Magenoperation oder Hypo- bzw. Anazidität empfielt sich die Darreichungsform als Granulat. Ist die exokrine Pankreasinsuffizienz nicht vollständig durch Enzymsubstitution zu kompensieren, ist eine Kost mit mittelkettigen Triglyzeriden sinnvoll.

Untergewicht. Einer angesichts des häufig schlechten Ernährungszustandes vieler dieser Patienten wünschenswerte hochkalorische Ernährung sind durch den gelegentlich schlechten Appetit oder bei Z.n. Pankreatektomie den Diabetes mellitus Grenzen gesetzt. Eine vollständige Kompensation des Ernährungszustands wird deshalb in der Praxis häufig nicht erreicht.

Kostaufbau bei Malassimilation. Beispielsweise bei chronische Diarrhoen, nachgewiesene Malassimilation (z. B. nach ausgedehnter Dünndarmresektion, bei
Kurzdarmsyndrom, bei chronischer Pankreatitis, nach vollständiger Pankreatektomie, nach Whipple-Operation, Morbus Whipple). Der Kostaufbau erfolgt über 4-5 Stufen, eine adäquate Nährstoffzufuhr wird erst in den beiden letzten Stufen erreicht. Die Flüssigkeitsbilanz muss (z. T. parenteral) auf allen Stufen individuell ausgeglichen werden. In Stufe 1 wird eine nahezu fettfreie und laktosefreie Kost angeboten. Die gastrointestinal »tolerierte« Gesamtkalorienmenge beträgt zwischen 1000 und $1200 \mathrm{kcal} /$ Tag, der Kohlenhydratanteil beträgt 85\%, der Fettanteil 6\%, der Eiweißanteil 7\% der Energiezufuhr. In Stufe 2 werden die Zufuhr tierischer Eiweiße und der stufenweise Einbau von MCT-Fetten (maximal $20 \mathrm{~g}$ ) eingeleitet. Die Kalorienzufuhr beträgt zwischen 1200 und $1600 \mathrm{kcal} / \mathrm{Tag}$. Die Kohlenhydratzufuhr liegt bei 57\%, der Fettanteil bei 28\% (der MCT-Anteil bei 16\% Gesamtkalorien) und der Eiweißanteil bei $16 \%$. Stufe 2 enthält etwa $5 \mathrm{~g}$ Laktose sowie 5-10 g Ballaststoffe. Auf der Stufe 3 wird die Kost quantitativ durch die Zulage von Milch (in zubereiteter Form) und Weizenmischbrot erweitert. Kalorienzufuhr (bis ca. $1900 \mathrm{kcal} / \mathrm{Tag}$ ). Die Zusammensetzung ist wie folgt: ca. 53\% Kohlenhydrate, ca. 29\% Fett und ca. 17\% Eiweiß). Der Laktoseanteil wird auf 15 g gesteigert. Die Ballaststoffmenge beträgt etwa $15 \mathrm{~g}$. Auf Stufe 4 werden zusätzlich ballaststoffarme Gemüsesorten eingebaut, rohes

- Tab. 2.49. Liste der am häufigsten in Deutschland verschriebenen Pankreasenzympräparate

\begin{tabular}{|c|c|c|c|c|c|}
\hline $\begin{array}{l}\text { Pankreasenzym- } \\
\text { präparate }\end{array}$ & & Firma & Lipase & Amylase & Proteasen \\
\hline Kreon & $\begin{array}{l}10.000 \\
25.000\end{array}$ & $\begin{array}{l}\text { Solvay } \\
\text { Solvay }\end{array}$ & $\begin{array}{l}10.000 \\
25.000\end{array}$ & $\begin{array}{l}8.000 \\
18.000\end{array}$ & $\begin{array}{l}600 \\
1.000\end{array}$ \\
\hline Enzym Lefax & & Asche & $\begin{array}{l}2.200+\text { Simethi- } \\
\text { con } 41,2 \mathrm{mg}\end{array}$ & 1.800 & 100 \\
\hline $\begin{array}{l}\text { Enzym Lefax } \\
\text { forte }\end{array}$ & & Asche & $\begin{array}{l}\text { 10.500+Dimeti- } \\
\text { con } 40 \mathrm{mg}\end{array}$ & 6.000 & 600 \\
\hline Panzytrat & $\begin{array}{l}10.000 \\
25.000 \\
40.000\end{array}$ & $\begin{array}{l}\text { Knoll } \\
\text { Knoll } \\
\text { Knoll }\end{array}$ & $\begin{array}{l}10.000 \\
25.000 \\
40.000\end{array}$ & $\begin{array}{l}9.000 \\
12.000 \\
15.000\end{array}$ & $\begin{array}{l}500 \\
800 \\
900\end{array}$ \\
\hline Pankreon forte & 28.000 & Solvay & 28.000 & 22.000 & 1.500 \\
\hline Pangrol & 25.000 & Berlin-Chemie & 25.000 & 22.500 & 1.250 \\
\hline Meteozym & & Novartis & $\begin{array}{l}\text { 15.000+Dimeti- } \\
\text { con-1000-silici- } \\
\text { um-dioxid } 100 \\
\mathrm{mg}\end{array}$ & 11.000 & 900 \\
\hline Pancreatan & $\begin{array}{l}25.000 \\
36.000\end{array}$ & $\begin{array}{l}\text { Novartis } \\
\text { Novartis }\end{array}$ & $\begin{array}{l}25.000 \\
36.000\end{array}$ & $\begin{array}{l}22.500 \\
18.00\end{array}$ & $\begin{array}{l}1.250 \\
1.200\end{array}$ \\
\hline Ozym & $\begin{array}{l}10.000 \\
20.000\end{array}$ & $\begin{array}{l}\text { Trommsdorff } \\
\text { Trommsdorff }\end{array}$ & $\begin{array}{l}10.000 \\
20.000\end{array}$ & $\begin{array}{l}9.000 \\
18.000\end{array}$ & $\begin{array}{l}1.000 \\
1.000\end{array}$ \\
\hline Pankreatin Mikro & 20.000 & Ratiopharm & 20.000 & 18.000 & 1.000 \\
\hline
\end{tabular}


Gemüse wird vermieden. Die Ballaststoffmenge beträgt jetzt etwa 20 g. Der Kaloriengehalt sowie die Nährstoffrelation entsprechen der Stufe 3. Der Laktosegehalt beträgt bis $\mathrm{zu} 20 \mathrm{~g}$. Auf Stufe 5 werden ballaststoffarmes Obst und bis zu $25 \mathrm{~g}$ Ballaststoffe eingeplant. Kalorienzufuhr und Nährstoffrelation sind gegenüber den Vorstufen nicht verändert. Die Gesamtenergiezufuhr auf Stufe 5 kann individuell entsprechend der Nahrungsausnutzung bzw. der Akzeptanz durch den Patienten bis auf 3000-3200 kcal gesteigert werden. Der Fettanteil beträgt bis zu 40\% der Gesamtenergiemenge, der MCT-Anteil kann bis auf 50\% (maximal 70\%) der gesamten Fettmenge gesteigert werden. Die letzte Stufe entspricht der Dauerkostform. Ziele der Massnahme sind die Adaptation des Dünndarms und optimale Ausnutzung der Nahrung, Ausgleich der Flüssigkeitsbilanz, Erhalt oder Verbesserung des Ernährungszustands. Alle Nahrungsmittel liegen in leicht resorbierbarer Form vor.

\section{Kostaufbau und Ernährung bei Patienten mit chro-} nisch entzündlichen Darmerkrankungen. Siehe auch ballaststoffarme Kost indiziert bei subakuter Phase bei Morbus Crohn bzw. Colitis ulcerosa und toxischen Megagacolon, im Anschluss an eine künstliche Ernährung. Der Kostaufbau schließt sich unmittelbar an eine parenterale Ernährung beginnend mit Stufe 1 über 4 Stufen oder aber in »abgekürzter « Form in Anschluss an eine enterale künstliche Ernährung beginnend mit Stufe 3 an. Die Diät ist abhängig von den Komplikationen der Erkrankung (Stenosen, Ausdehnung des intestinalen Befundes, Fisteln) und der Schwere des klinischen Bildes bei abklingender Entzündungsaktivität und Befundbesserung wird eine ballaststoffarme Kost oder eine leichte (leicht verdauliche) Vollkost verordnet. Die Ballaststoffmenge beträgt bei einer ballaststoffarmen Kost weniger als $15 \mathrm{~g} / \mathrm{Tag}$. Der Laktosegehalt wird bei sekundärer Laktoseintoleranz auf $<10 \mathrm{~g} /$ Tag begrenzt. Stufe 1: Überwiegende Zufuhr von Kohlenhydraten, Kalorienzufuhr um 2000 kcal/Tag. Nährstoffrelation 77\% Kohlenhydrate, 16\% Fett, 7\% Eiweiß. Stufe 2: Angestrebt werden $2200 \mathrm{kcal} / \mathrm{Tag}$ bei einer Nährstoffrelation von 51\% Kohlenhydrate, 34\% Fett und 16\% Eiweiß.Stufe 3: Angestrebte Kalorienmenge zwischen 2200 und $2500 \mathrm{kcal} / \mathrm{Tag}$, Nährstoffrelation $49 \%$ Kohlenhydrate, 37\% Fett, $14 \%$ Eiweiß und Stufe 4: mit einer Gesamtkalorienmenge zwischen 2300 und $2500 \mathrm{kcal} / \mathrm{Tag}$, mit einer Nährstoffrelation von $50 \%$ Kohlenhydraten, 35\% Fett und 15\% Eiweiß. Ballaststoffe werden erst ab Stufe 3 hinzugefügt. Die Nahrungsmittelauswahl ist ab Stufe 3 erweitert. Ziele der Diät sind die Adaptation von Dünn- und Dickdarm an eine »normale« Ernährung, Erhalt des Ernährungszustands, Linderung der Symptome. Die Dauer des Kostaufbaus wird individuell bestimmt und ist auch abhängig von den Komplikationen der Erkrankung. In der Regel wird jede "Stufe« über 3 Tage durchgeführt.
Dauerkostform. Im Anschluss an den Kostaufbau und in der Remissionsphase wird eine leichte (leicht verdauliche) oder ballaststoffarme Vollkost empfohlen. Die Dauerkostform wird für Patienten mit chronisch-entzündlichen Darmerkrankungen individuell und unter Berücksichtigung der Toleranz gestaltet. Neben der Vermeidung von Beschwerden und dem Wunsch nach einer möglichst langfristigen Remission bestimmen der Ernährungszustand sowie die Vermeidung möglicher nutritiver Defizite (Cave: z. B. Anämien, Wachstumsstörungen bei Kindern und Jugendlichen, vgl. - Tab. 2.50) die jeweilige Kostform. Diätetische Empfehlungen sind besonders bei Patienten mit einem Morbus Crohn des Dünndarms angezeigt. Mit Ausnahme von Stenosen und "hochsitzenden" Fisteln besteht bei dieser Patientengruppe keine zwingende Indikation für eine Begrenzung der Ballaststoffzufuhr. In schweren Fällen, welche diätetisch im Kostaufbau nicht kompensiert werden können, oder bei erneutem Schub ist eine Eliminationsdiät angezeigt.

Medikamente. Bei medikamentöser/systemischer Behandlung mit 5-Aminosalizylsäure (z. B. Azulfidine, Salofalk, Claversal, Dipentum) muss Folsäure, nach Ileumresektion Vitamin- $B_{12}$ substituiert werden. Bei Ileumresektion und Gallensäureverlust wird ein »Ionenaustauscher « Cholestyramin (z. B. Quantalan, Lipocol-Merz) gegeben. Um eine mögliche Interferenz mit essentiellen Nahrungsbestandteilen zu vermeiden, ist auf eine zu den Mahlzeiten zeitversetzte Einnahme des Ionenaustauschers zu achten. Bei Colitis ulcerosa sind Omega-3-Fettsäuren bzw. Fischöl-supplementierte Diäten (in einer Dosierung von 2,7 g Eicosapentaensäure) und die topische Anwendung von Nährsubstraten für den Colonozyten (=kurzkettige Fettsäuren: Azetat, Butyrat, Propionat als Einlauf, z. B. 2-mal täglich $50 \mathrm{ml} \mathrm{mit} 60 \mathrm{mmol} / \mathrm{l}$ Natriumacetat, $30 \mathrm{mmol} / \mathrm{l}$ Natriumpropionat, $40 \mathrm{mmol} / \mathrm{l}$ Natriumbutyrat und $20 \mathrm{mmol} / \mathrm{l}$ Natriumchlorid; Osmolalität : 280 $290 \mathrm{mosmol} / \mathrm{l}, \mathrm{pH} 7,0$; die Lösung ist bei $4^{\circ} \mathrm{C} 4$ Monate stabil; Behandlungsdauer zunächst 4 Wochen), mögliche Behandlungstherapien.

\section{Glutenfreie Kost (Kost bei Zöliakie)}

Indikationen. Glutensensitive Enteropathie (einheimische Sprue, Zöliakie), Dermatitis herpetiformis Duhring mit Zottenatrophie, Stomatitis aphthosa mit Zottenatrophie.

Definition. Elimination aller glutenhaltigen Lebensmittel (Weizen, Gerste, Roggen, Hafer, Hirse, Dinkel, Grünkern und daraus hergestellte Produkte; zum Glutengehalt in Lebensmitteln s. - Tab. 2.51). Die Nährstoffrelation sowie der Ballaststoffgehalt der Nahrungsmittel entsprechen der Vollkost.

Ziele. Vermeidung der Gliadinexposition sowie die Regeneration der Dünndarmschleimhaut. 
- Tab. 2.50. Prävalenz ernährungsmedizinischer Probleme bei Patienten mit chronisch entzündlichen Darmerkrankungen

\begin{tabular}{l|l|l} 
& \multicolumn{2}{|c}{ Prävalenz (\%) } \\
& $\begin{array}{l}\text { Morbus } \\
\text { Crohn }\end{array}$ & $\begin{array}{l}\text { Colitis } \\
\text { ulcerosa }\end{array}$ \\
\hline Gewichtsverlust & $67-75$ & $18-62$ \\
\hline Hypoalbuminämie & $25-80$ & $25-50$ \\
\hline Intestinaler Eiweißverlust & 75 & $?$ \\
\hline Negative Stickstoffbilanz & 69 & $?$ \\
\hline Anämie & $25-85$ & 66 \\
\hline Eisenmangel & 39 & 81 \\
\hline Vitamin-B ${ }_{12}$-Mangel & 48 & 5 \\
\hline Folsäuremangel & 67 & $30-40$ \\
\hline Kalziummangel & 13 & $?$ \\
\hline Magnesiummangel & $14-33$ & $?$ \\
\hline Kaliummangel & $5-20$ & $?$ \\
\hline Vitamin-A-Mangel & 11 & $?$ \\
\hline Vitamin-C-Mangel & $?$ & $?$ \\
\hline Vitamin-D-Mangel & 75 & 35 \\
\hline Vitamin-K-Mangel & $?$ & $?$ \\
\hline Zinkmangel & 50 & $?$ \\
\hline Kupfermangel & $?$ & $?$ \\
\hline & & $?$ \\
\hline
\end{tabular}

Anmerkung. Pragmatisch gesehen sind glutenfreie Lebensmittel, welche von Zöliakiepatienten sicher und ohne Beschwerden toleriert werden. Die Patienten vermeiden vorgefertigte Nahrungsmittel ohne genaue Definition des Bindemittels. Es ist auch auf Trägermittel von Medikamenten zu achten. Idealerweise enthält ein glutenfreies Lebensmittel in keinem seiner Bestandteile Weizen, Hafer, Gerste, Roggen und Titricale. Analytisch bedeutet glutenfrei einen Glutengehalt von $0,05 \mathrm{~g}$ pro $100 \mathrm{~g}$ Stickstoff oder von $0,3 \%$ Eiweiß aus glutenhaltigen Cerealien. Aufgrund methodischer Probleme bei der Bestimmung von Gluten wird heute ein Gliadin- bzw. Prolamingehalt (i.e. der antigenwirksame Bestandteil des Glutens) von $<10 \mathrm{mg}$ pro $100 \mathrm{~g}$ Weizenstärke als glutenfrei akzeptiert. Im Codex Alimentarius enthalten glutenfreie Lebensmittel $<200 \mathrm{mg} / \mathrm{kg} \mathrm{Glu}-$ ten. Etwa 6\% der Lebensmittel, welche als glutenfrei deklariert werden, enthalten mehr als $300 \mathrm{mg} / \mathrm{kg}$. Schon geringe Mengen (=Spuren) von Gliadin (z. B. 0,5 mg) können die Symptome der Sprue bewirken und die Heilung verhindern. Wünschenswert wäre ein Grenzwert $<1 \mathrm{mg} / 100 \mathrm{~g}$ Weizenstärke. Erlaubt sind Mais, Reis, Kartoffeln, Tapioka bzw. aus den o.g. Getreiden hergestellte reine Stärkepräparate. Bei alkoholischen Getränken müssen Korn und auch einige Biersorten gemieden werden. Die Zufuhr von Laktose wird auf maximal $20 \mathrm{~g} / \mathrm{Tag}$ begrenzt, da bei Patienten mit ausgedehnter Sprue eine sekundäre Laktoseintoleranz bestehen kann. Der Austausch von LCT- gegen MCT-Fette ist vom Nachweis einer Steatorrhoe abhängig. Initial wird eine glutenfreie Kost wegen der ausgeprägten Zottenatrophie als leichte Vollkost konzipiert. Evtl. ist ein Kostaufbau

- Tab. 2.51. Gluten in Lebensmitteln. (Aus Hammermühle Diät)

\section{Leicht erkennbar}

Lebensmittel, die Anteile der Getreidearten Weizen (Dinkeln, Grünkern), Roggen und Hafer enthalten, z. B.

- Brot, Brötchen, Pizza

- Teigwaren

- Mehl in gebundenen Soßen, Suppen, Fertiggerichten

- Flocken in Müsli-Mischungen und Milchprodukten

- Kleiezusätze

- Panierte Fleischmahlzeiten oder Frittiertes im Teigmantel

Noch unklar:Wildreis

\section{Maskiert}

Lebensmittelzutaten, die nicht direkt auf Getreide schließen lassen, aber in der

Zutatenliste erscheinen, $z$. B.

- Malz in Bier, Bonbons, Schokoerzeugnissen, Caro-Kaffee, Ovomaltine

- Pflanzeneiweiß als Zutaten in Fertiggerichten, Wurstprodukten; »NovelFood"

- Bindemittel z. B. in Milchprodukten oder Fruchtzubereitungen

- Stabilisator oder Verdickungsmittel/Backhilfsmittel werden oft als Mischungen verwendet, worin auch Gluten enthalten sein kann

- Mehl in Dragee-Überzügen, z. B. von Smarties, dragierten Nüssen, auch in Medikamenten, jedoch hier ohne Kennzeichnung

- Stärke als Zutat; es könnte glutenhaltige Weizenstärke verwendet werden

\section{Nicht erkennbar}

- Gluten-Zusätze als Trägerstoff von Aromen und Gewürzmischungen (z. B. in Wurst oder Chips)

- Gluten-Zusätze als emulgierendes Bindemittel ohne Deklaration in Schmelzkäsezubereitungen oder Milchprodukten (speziell: »LightProdukte«)

- Gluten als Bestandteileiner zusammengesetzten Zutat in einem Fertigprodukt, deren Anteil unter $25 \%$ liegt (z. B. Fruchtzubereitungen in einem Joghurt)

- Kontamination von Lebensmitteln, die aus glutenfreien Zutaten zusammengesetzt sind bei Produktion auf »verunreinigten« Anlagen oder Geräten 
unter Verwendung glutenfreier Lebensmittel notwendig. Bei Nichtansprechen auf die Diät innerhalb eines Zeitraums von 8 Wochen liegt häufig ein Diätfehler, selten eine schwere Verlaufsform vor, welche möglicherweise zusätzlich medikamentös (z. B. mit Steroiden) behandelt werden muss. In diesen schweren Fällen ist eine (unter Beachtung der Prinzipien einer glutenfreien Kost) vorübergehend durchgeführte Eliminationsdiät gerechtfertigt. Aufgrund des mit einer Zöliakie assoziierten erhöhten Malignomrisikos wird eine glutenfreie Kost von den betroffenen Patienten lebenslang beibehalten. Informationen und aktuelle Nahrungslisten über die Zöliakie-Gesellschaft.

\section{Ballaststoffreiche Kost}

Indikationen. Obstipation (Stuhlfrequenz $<2$-mal/Woche), Divertikulose, chologene Diarrhoe, Hypercholesterinämie, Diabetes mellitus.

Definition. Die Kost hat einen hohen Ballaststoffanteil von über $30 \mathrm{~g} / \mathrm{Tag}(>15 \mathrm{~g} / 1000 \mathrm{kcal})$ unter Bevorzugung zellulose- und hemizellulosehaltiger Nahrungsmittel (Vollkornerzeugnisse). Gemüse überwiegend als Rohkost, Frischobst, Trockenfrüchte. Sie vermeidet stark blähende (Hülsenfrüchte, frisches Brot) sowie stopfenden Nahrungsmitteln (z. B. Banane, Schokolade, Rotwein). Die Nährstoffrelation entspricht der Vollkost.

Ziele. Linderung der Beschwerden, Stuhlfrequenz mindestens 3- bis 4-mal/Woche, Verbesserung der Stoffwechsellage.

Anmerkung. Obstipation ist durch anstrengende/schwierige Entleerung (bei $>25 \%$ der Stuhlgänge), festem Stuhl $(>25 \%)$, das Gefühl der unvollständigen Entleerung $(>25 \%)$, digitale Versuche der Entleerung $(>25 \%)$ und $<3$ Stuhlgängen pro definiert. Vor Einleitung einer diätetischen Maßnahme ist eine Abklärung (bei Patienten <50 Jahre: Rektosigmoidoskopie; >50 Jahre Coloskopie) absolut notwendig. Ballaststoffe sind die Anteile pflanzlicher Zellen, welche nicht verdaut werden können. Ballaststoffkomponenten sind Zellulose, Hemizellulose, Pektine, Lignin, Schleimstoffe. Zur Wirksamkeit verschiedener Ballaststoffe s. - Tab. 2.52, zum Ballaststoffquellen und- gehalt ausgewählter Lebensmittel s. - Tab. 2.53-2.56). Bei einer ausgewogenen Ernährung werden bis zu 1/3 der Ballaststoffe als lösliche Substanzen zugeführt. Brot und Backwaren sind ballaststoffreich, wenn sie $>6 \mathrm{~g} / 100 \mathrm{~g}$ enthalten. Die Ballaststoffe sollten je zur Hälfte in Form von Getreide sowie Obst und Gemüse eingerechnet werden. Ballaststoffzusätze in Brot und Müsli sind möglich. Nicht vorgesehen sind Zusätze von Kleie und Ballaststoffpräparaten sowie Leinsamen und Laxantien.

Ballaststoffpräparate. Ballaststoffpräparate dürfen nur bei medizinischen Indikationen und unter ärztlicher Überwachung gegeben werden. Bei Gabe von Ballaststoffpräparaten ist besonders auf eine ausgewogene Ernährung zu achten. Der Ballaststoffanteil kann bei individueller Toleranz weiter gesteigert werden. Der Zusatz von grob gemahlener Weizenkleie ist stufenweise, beginnend mit $5 \mathrm{~g} /$ Tag auf maximal $20-30 \mathrm{~g} / \mathrm{Tag}$ zu steigern. Weizenkleie enthält $40 \mathrm{~g}$ Ballaststoffe $/ 100 \mathrm{~g}$, wovon $40-50 \%$ bakteriell abgebaut werden. Mucofalk enthält Ballaststoffe aus indischer Flohsamenschale. $5 \mathrm{~g}$ Granulat entsprechen $3,25 \mathrm{~g}$ Ballaststoffe.

Probleme. Bei Einnahme von Ballaststoffen bzw. Ballaststoffpräparaten ist auf eine reichliche Flüssigkeitszufuhr (>2 1/Tag) zu achten. Eine sehr ballaststoffreiche Kost kann mit Verlusten von Mineralstoffen und Spurenelementen (Cave: Kalzium, Eisen, Zink) im Stuhl einhergehen.

Eine Ballaststoffzufuhr in einer Höhe $>50 \mathrm{~g} / \mathrm{Tag}$ ist ohne nachweisbaren Effekt und wird von den Patienten nicht toleriert (Übelkeit, Flatulenz, Blähung). Probleme werden häufig zu Beginn der Umstellung auf eine ballaststoffreiche Ernährung beobachtet. Praktische Empfehlungen für die Kostumstellung sind, zuerst Vollkornbrot und ungeschälten Reis vermehrt zu verzehren, danach stufenweise Rohkost, Obst, und Hülsenfrüchte einzuführen, bei Kuchen nach und nach Vollkornmehl verwenden, Kümmel, Anis, und Fenchelsamen als Streuwürze und zusätzlich als Tee erhöhen die Bekömmlichkeit. Die Kombination von stark zuckerhaltigen Lebensmitteln und Vollkornspeisen wird von manchen Menschen schlecht vertragen. Deshalb sollte zunächst auf Süßigkeiten, stark gesüßte Getränke, usw. verzichtet werden.

- Tab. 2.52. Wirkung und gezielter Einsatz von Ballaststoffen in der Ernährung

\begin{tabular}{l|l|l}
\hline Ballaststoffe & Effekt & Indikation \\
\hline $\begin{array}{l}\text { Unlöslich } \\
\text { z. B. Zellulose, Hemicellulose (Lignin) }\end{array}$ & $\begin{array}{l}\text { Stuhlvolumen } \uparrow \\
\text { Kurzkettige Fettsäuren } \uparrow\end{array}$ & $\begin{array}{l}\text { Verstopfung, Divertikulitis, } \\
\text { Hämorrhoiden }\end{array}$ \\
\hline Löslich & $\begin{array}{l}\text { Dünndarmpassage } \downarrow \text {, Resorption ver- } \\
\text { z. B. Hemizellulose, Pektine, } \beta \text {-Glukane }\end{array}$ & $\begin{array}{l}\text { Diabetes mellitus, }{ }^{1} \text { Fettstoffwechsel- } \\
\text { zögert, kurzkettige Fettsäuren } \uparrow\end{array}$ \\
\hline
\end{tabular}

${ }^{1}$ Belegt für Guar und isoliertes Pektin. 
- Tab. 2.53. Ballaststoffquellen in der Nahrung

\begin{tabular}{l|l}
\hline Zellulose & $\begin{array}{l}\text { Kohl } \\
\text { Bohnen } \\
\text { Wurzelgemüse } \\
\text { Vollkornprodukte } \\
\text { Kleie }\end{array}$ \\
\hline Hemizellulose & $\begin{array}{l}\text { Kleie } \\
\text { Cerealien } \\
\text { Vollkornprodukte }\end{array}$ \\
\hline Lignin & Kleie \\
\hline Pektin & $\begin{array}{l}\text { Äpfel } \\
\text { Zitrusfrüchte } \\
\end{array}$ \\
& $\begin{array}{l}\text { Erdbeeren } \\
\text { Gemüse }\end{array}$ \\
\hline B-Glukane & Hafermehl \\
& getrocknete Bohnen
\end{tabular}

Tab. 2.55. Ballaststoffgehalt ausgewählter Lebensmittel pro Portionsgröße

\begin{tabular}{c|l}
\hline$\leq 0,5 \mathrm{~g}$ & $\begin{array}{l}\text { Äpfel (ohne Schale), Aprikosen, Tomaten, } \\
\text { Reis, Spaghetti, Cornflakes }\end{array}$ \\
\hline $0,6-1,0 \mathrm{~g}$ & $\begin{array}{l}\text { Orangen, Pflaumen, Blumenkohl, Kohlrabi, } \\
\text { Sojasprossen (gekocht) }\end{array}$ \\
\hline $1,1-2,0 \mathrm{~g}$ & $\begin{array}{l}\text { Äpfel, getrocknete Pflaumen, Chicoree, } \\
\text { Sojasprossen (roh) }\end{array}$ \\
\hline $2,1-3,0 \mathrm{~g}$ & Bohnen, Erbsen, Vollkornnudeln, Birnen \\
\hline$>3,1 \mathrm{~g}$ & Stachelbeeren, Graupen, Linsen, Kleie \\
\hline
\end{tabular}

Medikamente. Wirkgruppen und Prinzipien von Abführmitteln (Laxantien) sind in - Tab. 2.57 dargestellt. Die Medikamente wirken unterschiedlich und erhöhen das Stuhlvolumen (Ballaststoffpräparate) bzw. wirken osmotisch (nicht resorbierbare Kohlenhydrate oder z. B. Magnesiumhydroxid) oder stimulierend (wie z. B. Anthrachinone). Praktische Hinweise zur Abklärung und Behandlung eines obstipierten Patienten - Tab. 2.58 zu entnehmen.

\section{Ballaststoffarme Kost}

Indikationen. Stenosen und Strikturen im Intestinaltrakt, Fisteln, akute Divertikulitis, während der 1. Woche nach Anlage eines Stomas (Anus praeter), Gastroparese, Strahlenenteritis, Vorbereitung auf abdominelle Operationen, nach Oberbaucheingriffen, Kurzdarmsyndrom.

Definition. Es handelt sich um eine Abwandlung der leichten Vollkost unter Reduktion des Anteils an faserrei-

Tab. 2.54. Ballaststoffgehalt verschiedener Lebensmittel.

\begin{tabular}{l|l} 
& Gehalt pro $100 \mathrm{~g}^{\mathrm{a}}$ \\
\hline Hülsenfrüchte & $3-12 \mathrm{~g}$ \\
\hline Vollkornbrot & $5-10 \mathrm{~g}$ \\
\hline Knäckebrot & $4-12 \mathrm{~g}$ \\
\hline Müsli & $5-9 \mathrm{~g}$ \\
\hline Teigwaren & $3-8 \mathrm{~g}$ \\
\hline Gemüse & $3-10 \mathrm{~g}$ \\
\hline Obst & $4-7 \mathrm{~g}$ \\
\hline
\end{tabular}

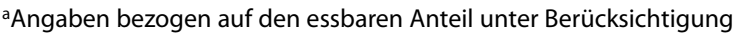
der Verluste durch die Zubereitung. Datenbasis: Bundeslebensmittelschlüssel. Die Daten wurden aufgerundet.

- Tab. 2.56. Ballaststoffarme und ballaststoffreiche Ernährung (eine Auswahl)

\begin{tabular}{|l|l|l|l}
\hline Ballaststoffarm & Ballaststoffe $(\mathbf{g} / \mathbf{d})$ & Ballaststoffreich & Ballaststoffe (g/d) \\
\hline $100 \mathrm{~g}$ Weißbrot & 2,2 & $200 \mathrm{~g}$ Vollkornbrot & 15,2 \\
\hline $100 \mathrm{~g}$ Mischbrot & 4,2 & $100 \mathrm{~g}$ Kartoffeln & 1,7 \\
\hline $100 \mathrm{~g}$ Kartoffeln & 1,7 & $200 \mathrm{~g}$ Kartoffeln & 5,2 \\
\hline $200 \mathrm{~g}$ Kartoffeln & 5,2 & $60 \mathrm{~g}$ Tomaten & 0,8 \\
\hline $60 \mathrm{~g}$ Tomaten & 0,8 & $200 \mathrm{~g}$ Orangen & 5,8 \\
\hline $150 \mathrm{~g}$ Orangen & 4,4 & $200 \mathrm{~g}$ Äpfel & 4,6 \\
\hline $60 \mathrm{~g}$ Kuchen & 0,5 & $200 \mathrm{~g}$ Rosenkohl & 13,0 \\
\hline & & $200 \mathrm{~g}$ Dicke Bohnen & 13,0 \\
\hline Summe & 19,0 & Summe & 53,7 \\
\hline
\end{tabular}


chen Lebensmitteln sowie Einbau von leicht verdaulichen Fetten. Ballaststoffgehalt $<15 \mathrm{~g} /$ Tag.

Ziele. Minimierung des Stuhlvolumens, Verzögerung der Darmpassage, Vermeiden von Beschwerden und Komplikationen.

Anmerkung. Eine Begrenzung der Ballaststoffmenge und deren Ausmaß sind abhängig von der Schwere des Krankheitsbildes. Sie kann vorübergehend (z. B. zur OPVorbereitung) oder als Dauerkost verabreicht werden. Eine »ballaststoffbegrenzte" Diät vermeidet Lebensmittel mit einem sehr hohen Ballaststoffgehalt (i.e. $>0,5 \mathrm{~g}$ Ballaststoffe/Portion, d. h. z. B. Äpfel, Aprikosen, Feigen, Nektarinen, Pflaumen, Wassermelonen, Brokkoli, Kohl, Chicoree, Kohlrabi, Bohnen). In weniger schweren Fällen können auch Lebensmittel mit einem Ballaststoffgehalt bis $1,0 \mathrm{~g} /$ Portion zugelassen werden. Geeignete Lebensmittel sind $z$. B.:

- Cerealien: Weißbrot, Reis, Nudeln, Obst/Gemüse: Obst-/Gemüsesäfte (ohne Pflaumen),

- Milch/Milchprodukte: Pudding, Eiscreme, Milchsuppen,

- Fleisch/Fisch/Eier: Mageres Fleisch, Fisch.

Bei einer streng »ballaststoffarmen« Diät werden alle Vollkornprodukte, Cerealien, Reis, Früchte, Gemüse, Kartoffeln, Nüsse und Samen gemieden. Milch und Milchprodukte werden auf 2 Tassen/Tag begrenzt.
Probleme. Wird eine ballaststoffarme Ernährung als Dauerkost verwendet, besteht aufgrund der eingeschränkten Lebensmittelauswahl die Gefahr eines Nährstoffmangels. In diesen Fällen ist die Substitution von Vitaminen, Mineralstoffen und Spurenelementen notwendig. Evtl. Begrenzung des Laktosegehalt auf $15 \mathrm{~g} / \mathrm{Tag}$, bei nachgewiesener Steatorrhoe, evtl. Austausch der LCT- gegen MCT-Fette.

Stoma. Der durchschnittliche Flüssigkeitsverlust aus einem Ileostoma beträgt bis zu $1000 \mathrm{ml} /$ Tag. Diese Wasserund Elektrolytverluste müssen im Diätplan berücksichtigt werden. Die Ernährung sollte kaliumreich (mindestens $3 \mathrm{~g} / \mathrm{Tag}$ ) sein und etwa $6 \mathrm{~g} \mathrm{NaCl}$ enthalten. Vermeiden von oxalsäurereichen Lebensmitteln (z. B. Rote Rüben, Spinat, Mangold, Rhabarber, Kakao). Bei einem Kolostoma erfolgt z. T. eine weitgehende Wasseresorption und damit die Stuhleindickung je nach Kolonrestlänge. Blähende (z. B. Kohlarten, Zwiebeln, Hülsenfrüchte, frisches Brot) und geruchserzeugende Speisen (z. B. Fisch, Zwiebeln, Eier) sind für Stomaträger nicht geeignet.

\section{Kost bei »Dumping-Syndrom «}

Indikationen. Beschwerden im Sinne eines Dumping Syndroms (=gastrointestinale und vasomotorische Symptome als Folge der raschen Magenentleerung) bei Zustand nach Gastrektomie, Billroth-II-Operation bzw. gestörter Pylorusfunktion. Dumping Syndrome wurden bei bis zu $16 \%$ nach Billroth-II-Resektion und bis zu $4 \%$ nach Billroth-I-Resektion beobachtet.

- Tab. 2.57. Wirkprinzip und Übersicht der Laxantien (Abführmittel). (Aus Mutschler 1996)

\begin{tabular}{|c|c|c|}
\hline & & Handelsname (Beispiele) \\
\hline Quellstoffe & $\begin{array}{l}\text { Leinsamen, Flohsamen, Weizenkleie, } \\
\text { Bassorin }\end{array}$ & Agiocur, Metamucil \\
\hline Osmotisch wirksame Laxantien & $\begin{array}{l}\text { Salinische Abführmittel } \\
\text { Magnesium } \\
\text { Natriumsulfat } \\
\text { Zuckeralkohole, Zucker } \\
\text { Mannit } \\
\text { Sorbit } \\
\text { Laktilol } \\
\text { Laktose } \\
\text { Laktulose }\end{array}$ & $\begin{array}{l}\text { Bitter- und Glaubersalz } \\
\text { Importal } \\
\text { Bifiteral, Lactuflor }\end{array}$ \\
\hline $\begin{array}{l}\text { Antiresorptiv und hydragog wirkende } \\
\text { Laxantien }\end{array}$ & $\begin{array}{l}\text { Rizinusöl } \\
\text { Anthraglykoside } \\
\text { Anthraglykoside und Quellstoffe } \\
\text { Bisacodyl, Natriumpicosulfat }\end{array}$ & $\begin{array}{l}\text { Laxopol } \\
\text { Colonorm, Liquidepur, Neda, } \\
\text { Kräuterlax } \\
\text { Agiolax, Normacol } \\
\text { Dulcolax, Florisan, Dulcolax NP }\end{array}$ \\
\hline Gleitmittel & $\begin{array}{l}\text { Paraffinum subliquidum } \\
\text { Docusat-Natrium }\end{array}$ & In Tirgon enthalten \\
\hline $\begin{array}{l}\text { Substanzen mit Wirkung auf den } \\
\text { Defäkationsreflex }\end{array}$ & $\begin{array}{l}\text { Sorbit } \\
\text { Glyzerin }\end{array}$ & Babylax, Glycilax, Mikroklist \\
\hline
\end{tabular}


- Tab. 2.58. Charakterisierung und Behandlung von Obstipation

\begin{tabular}{|c|c|c|c|}
\hline $\begin{array}{l}\text { Definition und Diagnostik } \\
\text { der chronischen Obstipation }\end{array}$ & Behandlung der Obstipation & $\begin{array}{l}\text { Geeignete Lebensmittel bei } \\
\text { Obstipation }\end{array}$ & $\begin{array}{l}\text { Ungeeignete Lebens- } \\
\text { mittel bei Obstipation }\end{array}$ \\
\hline $\begin{array}{l}\text { - Ausschluss stenosierender } \\
\text { Prozesse sowie einer syste- } \\
\text { mischen Erkrankung } \\
\text { - Definition der habituellen } \\
\text { bzw. funktionellen Obsti- } \\
\text { pation: } \\
\text { - Defäkation nur durch } \\
\text { Pressen möglich } \\
\text { - Gefühl der unvollständi- } \\
\text { gen Darmentleerung } \\
\text { - Stuhlgang zu hart, zu } \\
\text { wenig, zu selten } \\
\text { (<2-3mal/Woche) } \\
\text { - Objektivierung/Abklärung: } \\
\text { - Stuhlgewicht } \\
\text { - Stuhlfrequenz } \\
\text { - Transitzeit } \\
\text { - (Rekto-/Sigmoido- } \\
\text { skopie?, Koloskopie?) }\end{array}$ & $\begin{array}{l}\text { Ernährung: Ballaststoffreiche } \\
\text { Kost (mind. } 30 \mathrm{~g} \text {, Cave: }<50 \\
\text { g/Tag sind ohne weiteren } \\
\text { Effekt), Sauermilchprodukte } \\
\text { mit laxierender Wirkung. } \\
\text { Bei Laxantienabusus stufen- } \\
\text { weiise Reduktion der Medi- } \\
\text { kamente und Steigerung des } \\
\text { Ballaststoffanteils. } \\
\text { Wenn keine Ernährungs- } \\
\text { umstellung möglich ist: } \\
\text { Ballaststoffpräparate bei } \\
\text { gleichzeitig hoher Flüssig- } \\
\text { keitszufuhr (>2 I/Tag), z. B. } \\
20-30 \mathrm{~g} \text { Weizenkleie (=2-3 } \\
\text { gehäufte Esslöffel) morgens } \\
\text { in Fruchtsaft evtl. mit } 1 \text { Ess- } \\
\text { löffel Milchzucker gesüßt } \\
\text { oder } 3 \text { mal } 10 \mathrm{~g} \text { Weizenkleie/ } \\
\text { Tag } 25 \mathrm{~g} \text { Leinsamen (=Ess- } \\
\text { löffel) als Schleimsuppe }\end{array}$ & $\begin{array}{l}\text { - Getränke: Kaffee, Butter- } \\
\text { milch, Kefir, Obstsäfte } \\
\text { - Cerealien: Haferflocken, } \\
\text { Müsli, Cornflakes, Vollkorn- } \\
\text { brot, Schrotbrot } \\
\text { - Kartoffeln } \\
\text { - Gemüse: Als Rohkost, wenig- } \\
\text { Zwiebeln und Hülsenfrüchte, } \\
\text { Sauerkraut } \\
\text { - Obst: Alle Sorten } \\
\text { (außer Bananen) } \\
\text { - Fisch, Fleisch, Wurstwaren, } \\
\text { Eier: Nicht so reichlich }\end{array}$ & $\begin{array}{l}\text { - Weißbrot, helle Brotsor- } \\
\text { ten, Feingebäck, Kuchen } \\
\text { - Teigwaren, Mehlspeisen, } \\
\text { Süßwaren } \\
\text { - Hülsenfrüchte, Grünkohl, } \\
\text { Weißkohl } \\
\text { - Fleisch, Fisch, Wurst- } \\
\text { waren, Eier in großen } \\
\text { Mengen } \\
\text { - Schwarzer Tee, Kakao, } \\
\text { Rotwein }\end{array}$ \\
\hline
\end{tabular}

Definition. Die Nahrungszusammensetzung entspricht der leichten Vollkost mit einer hohen Mahlzeitenfrequenz (über den Tag verteilt) unter Vermeidung von Nahrungsbestandteilen mit hoher Osmolarität (Vermeidung von Oligosacchariden bzw. Zuckeraustauschstoffen =»zuckerarme Kost $)$.

Ziele. Vermeiden des »Früh-« (kurz, d. h. bis zu 2 Stunden postprandial auftretende Symptome wie Übelkeit, Brechreiz, Völlegefühl, Stuhldrang, Diarrhoe, Schwindel, Blässe, Tachycardie, Schwitzen, Synkope) und "Spätdumpings « (ca. 2-3 h postprandial auftretende Symptome wie Hungergefühl, Schweißausbruch, Blutdruckabfall, Hypoglykämie, Hypokaliämie), Prävention der Malnutrition, Verbesserung des Ernährungszustands.

Anmerkungen. Bei Patienten nach einer Magenresektion haben der Erhalt bzw. die Verbesserung des Ernährungszustands und die Linderung der Symptome höxhste Priorität. Dies wird durch eine bedarfdeckende Ernährung, das Meiden leicht resorbierbarer und Bevorzugung komplexer Kohlenhydrate und die Akzeptanz der Ernährung durch den Patienten erreicht. Die Kost sollte gering hyperkalorisch sein, die Verteilung erfolgt auf 8-10 kleine Mahlzeiten mit fester Konsistenz. Der Patient sollte langsam essen und keine Flüssigkeit zu den Mahlzeiten zu sich nehmen. Getränke werden nur in kleineren Portionen (ca $150 \mathrm{ml}$ ) zwischen den Mahlzeiten (frühestens $1 \mathrm{~h}$ nach dem Essen) aufgenommen, sie sind weder sehr heiß noch sehr kalt. Suppen und Breie werden meist schlecht vertragen. Vorsicht bei Trinkmilch und Milchprodukten. Der Laktosegehalt der Diät liegt $<20$ g/Tag. Zur Vermeidung eines hohen Kohlenhydratanteils kann nach Akzeptanz des Patienten und klinischem Verlauf der Fettanteil erhöht werden. Dies ist insbesondere bei untergewichtigen Patienten mit einem Dumping-Syndrom angezeigt.

Fettmalassimilation. Liegt zusätzlich eine Störung der Fettmalassimilation vor (Steatorrhoe über $>15 \mathrm{~g} / \mathrm{Tag}$ ), ist ein MCT-Austausch zu erwägen. Ein probatorischer Behandlungsversuch mit Pankreasfermenten ist möglich. Quellförmige Ballaststoffe (Pektine) führen häufig zu einer deutlichen Verbesserung der subjektiven Beschwerden. Eventuell ist auch die zusätzliche Gabe von Pektinen als Medikament (Guarmehl, z. B. Glucotard, $5 \mathrm{~g}$ zu jeder Hauptmahlzeit, die gleichzeitige Gabe anderer Medikamente ist zu vermeiden) hilfreich.

Medikamente. Alternativ kann die Spaltung der Zucker durch einen Disaccharidasehemmer (Acarbose, z. B. Glucobay, 3-mal 50- bis 3-mal $100 \mathrm{mg} / \mathrm{Tag}$, maximal 3-mal $200 \mathrm{mg} / \mathrm{Tag}$ ) gehemmt werden. Die Toleranz ist durch Flatulenz, Meteorismus eingeschränkt. (Cave: Die 
durch Flatulenz, Meteorismus eingeschränkte Wechselwirkung von Acarbose mit anderen Medikamenten wie Antazida, Cholestyramin). Eine Besserung der »Dumping-Symptome « wird auch nach Gabe des synthetischen Somatostatinanalogons, Octreotid (z. B. Sandostatin) berichtet.

Malnutrition. Bei Malnutrition und geplanter künstlicher Ernährung sollte eine modifiziert nährstoffdefinierte Diät für Diabetiker mit Ballaststoffen als Supplement gegeben werden. Ein 30-minütiges Liegen nach dem Essen ist erfahrungsgemäß hilfreich. Die Mahlzeiten können im Liegen verzehrt werden. Die Verwendung einer Leibbinde ist im Einzelfall zu überlegen.

Substitution. Bei Zustand nach totaler Gastrektomie müssen Vitamin- $B_{12}$ substituiert (z. B. Vitamin- $B_{12}$ As 1000, jeden 3. Monat $1000 \mathrm{lg}$ i.m.) und die Eisenspeicher des Körpers kontrolliert werden. Häufig sind auch Probleme der Vitamin-D- und Proteinversorgung. In jedem Fall ist eine individuelle Beratung notwendig.

\section{Kost für Stomaträger, bei Kurzdarmsyndrom}

Indikationen. Ileostoma, Ileal-Pouch, Kolostoma, Kurzdarmsyndrom.

Definition. Siehe ballaststoffarme Diät, es handelt sich um keine eigenständige Kostform, eine individuelle Festlegung der Ernährung ist notwendig.

Ziele. Begrenzung der Stomaverluste und Beschwerden (»Darmkontrolle«), Erhalt der Flüssigkeitsbilanz.

\section{Anmerkungen}

Stoma. Bei einem Kolostoma im Bereich des Colon descendens oder transversum bestehen meist keine Probleme. Bei einem Stoma im Bereich des Zökums oder des Colon ascendens sind die Flüssigkeitsverluste größer. Die meisten Patienten mit einem Kolostoma tolerieren eine Vollkost. Modifikationen sind nur bei vermehrter Gasbildung (Bohnen, Brokkoli, Blumenkohl, Zwiebeln), sehr hohem Stuhlvolumen (zum Eindicken: Bananen, Brot, Pasta, stärkehaltige Lebensmittel) oder Geruchsbelästigung (geruchsverursachende Lebensmittel: Eier, Fisch, Knoblauch, Zwiebeln; geruchsvermindernde Lebensmittel: Buttermilch, Joghurt) notwendig. Wie beim Ileostoma betragen die Flüssigkeitsverluste im Mittel $500 \mathrm{ml} /$ Tag. Bei Verstopfung haben frisches Obst und Obstsäfte häufig einen laxierenden Effekt. Bei Obstipation ist unbedingt die Flüssigkeitsbilanz zu überprüfen. Allgemeine Regeln sind: Regelmäßig über den Tag verteilt essen, ausgiebig kauen, adäquat trinken, abends weniger essen, bei »neuen " Lebensmitteln vorsichtig die Toleranz "antesten«. Für Patienten mit einem ilealen Pouch gelten initial ähnliche Regeln.
Kurzdarm. Die diätetische Versorgung von Patienten mit einem Kurzdarmsyndrom ist abhängig vom Ausmaß der Resektion, dem betroffenen (d. h. den "fehlenden« sowie den verbleibenden) Dünndarmabschnitt sowie dem Erhalt oder Nicht-Erhalt der Ileozökalklappe. Nach Ileumresektion kommt es häufig zur Malassimilation von Gallensäuren und entsprechend zu sekretorischen Diarrhoen des Kolons. Therapeutisch ist ein Ionenaustauscher (Cholestyramin, z. B. Quantalan) wirksam. Unter dieser Medikation kann eine Steatorrhoe verstärkt werden. Ausgedehnte Ileumresektionen führen zu Fettstühlen (i.e. eine Steatorrhoe $>15 \mathrm{~g} / \mathrm{Tag}$ bei einer Fettzufuhr von 70-100 g/Tag). Diese gehen mit Kalzium- und Magnesiumverlusten einher. Sind gleichzeitig große Abschnitte des Jejunums reseziert worden, kann es bei signifikanter Kohlenhydratmalassimilation auch zu osmotischen Diarrhoen kommen. Da das Ileum die Funktion des oberen Dünndarms, dieser aber nicht die Aufgabe des Ileums übernehmen kann, werden proximale Dünndarmresektionen in der Regel besser toleriert (d. h. kompensiert) als distale Resektionen. Nach Dünndarmresektion und bei erhaltener Kolonpassage sind Oxalatsteine häufig. Die Supplementierung von Mineralstoffen (Kalzium: 500 mg/ Tag, Eisen besonders beachten!) und Vitaminen (fettlösliche Vitamine, Vitamin- $B_{12}$ ) erfolgt empirisch. Wichtig ist der Ausgleich der Flüssigkeits- und Elektrolytbilanz. Dabei können orale Rehydrationslösungen (z. B. Elotrans, Cave: Niereninsuffizienz, Elektrolytentgleisungen) helfen. Das synthetische Somatostatinanalogon (Octreotid, z. B. Sandostatin) hilft, die Flüssigkeits- und Elektrolytverluste zu mindern.

\section{Kost bei Patienten mit einem gastroösophagealen Reflux}

Keine eigenständige Kostform. Ernährungsprinzipien bei Patienten mit einem gastroösophagealen Reflux sind die Normalisierung des Körpergewichts; postprandial Oberkörperhochlage (d. h. für etwa 3 Stunden nicht liegen!); Meiden von Fetten, Schokolade, Alkohol, Pfefferminz, Zigaretten, Medikamenten, welche den Druck auf den unteren Ösophagussphinkter senken, sind (z. B. Theophyllin, Anticholinergika, Kalziumantagonisten, Nitrate). Symptomatisch wird mit Antazida therapiert.

\section{Kost bei Flatulenz}

Keine eigene Kostform. Das Konzept der Ernährung erfolgt entsprechend der individuellen Toleranz. Üblicherweise handelt es sich um eine leichte Vollkost unter Vermeidung von empirisch vermehrt wasserstoffproduzierenden (Gassynthese, Kohlenmonoxidsynthese) Lebensmitteln (wie Bohnen, Brokkoli, Sauerkraut, Kohlrabi, Zwiebeln, Pflaumen, Süßstoffe; diätetische Lebensmittel, die Zuckeraustauschstoffe wie Sorbit und Mannit enthalten, fettreiche Speisen). Von den möglichen Ernährungsursachen sind die Aerophagie (Essgewohnheiten hinter- 
fragen) und Motilitätsstörungen des Darms abzugrenzen. Eine exzessive Produktion von Gas im Kolon kann auch Ausdruck einer Malassimilation sein und muss entsprechend abgeklärt werden.

\section{Kost bei gestörter Magenentleerung, "Gastroparese»}

Keine eigene Kostform. Eine diagnostische Abklärung ist notwendig. Bei der Differentialdiagnose sind kurzfristige (z. B. postoperativ, virale Gastroenteritis, Hypokaliämie, Medikamente wie Anticholinergika, trizyklische Antidepressiva, Opiate, Aluminium enthaltende Antazida) und chronische Beschwerden (z. B. autonome Neuropathie bei Diabetes mellitus, Sklerodermie, Ulkuskrankheit, atrophische Gastritis, neurologische Erkrankungen, z. B. nach Vagotomie oder Magenresektion) zu unterscheiden. Anamnestisch muss geklärt werden, ob die Symptome bei flüssigen und/oder festen Speisen auftreten. Motilitätsstörungen bzw. Erkrankungen im distalen Bereich des Magens beeinflussen regelhaft die Entleerung fester Speisen, während Flüssigkeiten normal passieren. Ballaststoffe haben die längste Verweilzeit im Magen. Fette und eine hohe Osmolarität der Nahrung hemmen die Magenentleerung. Bei einem vermehrten Anfall von nicht verdauten Nahrungsbestandteilen und fehlender Motilitäts- und Pylorusfunktion (z. B. nach Vagotomie und Pyloroplastik oder nach Roux-Y-OP) kann es zur Bildung von Bezoaren (im Magen liegendes Konvolent aus Nahrungsresten, Fasern oder auch verschluckten Haaren) kommen. Therapeutisch muss die der Gastroparese zugrundeliegende Erkrankung behandelt werden. Bei milden Beschwerden kann eine ballaststoff- und fettarme Ernährung hilfreich sein. Viele kleine Mahlzeiten sowie - je nach Toleranz - eine flüssige oder passierte leichte Vollkost werden empfohlen.

\section{Kost bei Enteritis/Gastroenteritis/Reisediarrhoen/ antibiotikaassoziierten Durchfällen}

Keine eigene Kostform. Ursachen und Charakterisierung infektiöser Durchfallerkrankungen sind in den 0 Tab. 2.59 und - Tab. 2.60 sowie - Abb. 2.17 angegeben. Akute Diarrhoen schwinden in der Regel spontan innerhalb von 1-2 Tagen oder spätestens nach Identifikation der infektiösen Ursache und deren Behandlung. Eine akute Enteritis oder Gastroenteritis tritt nach Verzehr von Lebensmitteln auf, welche pathogene Keime oder toxische Produkte von Mikroorganismen enthalten (s. - Tab. 2.60). - Abb. 2.17 gibt einen Überblick über Lebensmittelvergiftungen. Die Ernährung hat bei diesen Erkrankungen zunächst eine präventive Bedeutung (i.e. Lebensmittelhygiene). Lebensmittelinfektionen ohne Durchfallserkrankung werden durch Listerien (aus Weichkäse, Milch, Rohwurst, Räucherfisch), Clostridium botulinum (z. B. aus hausgemachten Konserven, das Toxin entsteht im Lebensmittel), Hepatitis-AVirus (über Verzehr von Muscheln oder unsauberem Wasser) erklärt. Bei chronischen Diarrhoen, d. h. länger als 3 Wochen dauernden Durchfällen, erfolgt eine Abklärung und ist ggf. eine spezielle diätetische Intervention notwendig (s. z. B. Diät bei Malassimilation). Diätetische Maßnahmen bei akuter Diarrhoe sind allgemeiner Natur und dienen der Flüssigkeits- und Elektrolytsubstitution, einer Steigerung der Wasser- und Elektrolytresorption und möglicherweise der Reduktion der Motilität (=Verzögerung der Darmpassage).

Substitution. Für die Flüssigkeits- und Elektrolytsubstitution stehen industriell gefertigte Glukose- und Elektrolytlösungen (Verhältnis von Glukose zu Natrium 1:1, für Erwachsene z. B. Elotrans, für Kinder z. B. Oralpädon) zur Verfügung. Empirische Diätformen zur Behandlung der akuten Diarrhoe sind die Rohapfeldiät (geriebener Apfel), Karottensuppe nach Maro und das Teefasten. Die Behandlung der Enteritis/Gastroenteritis besteht in der Elimination der Noxe sowie dem Ausgleich der Wasserund Elektrolytbilanz, in leichteren Fällen in der Gabe von Tee unter Zusatz von Kochsalz. Die Rehydratationsphase dauert 2-3 Tage. Die WHO-Empfehlung zur oralen Rehydratation (Oralyt) besteht aus 3,5 g Kochsalz, 2,9 g Trinatriumzitrat, 1,5 g Kaliumchlorid, 20,0 g Glukose auf $1000 \mathrm{ml} \mathrm{H}_{2} \mathrm{O}$. Ein damit zu vergleichendes Handelspräperat ist z. B. Elotrans. Für die Praxis empfiehlt sich: 3,5 g Tafelsalz+2,5 g Backpulver $\left(\mathrm{NaHCO}_{3}\right)+1,5 \mathrm{~g} \mathrm{KCl}+20 \mathrm{~g}$ Glukose, auf 1,01 Wasser auffüllen. Bei hohen Wasserverlusten (>10 l/Tag) oder unstillbarem Erbrechen wird der Flüssigkeitsverlust parenteral ausgeglichen (z. B. 1000

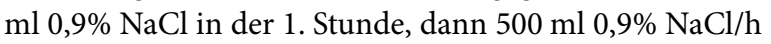
unter Bilanzierung).

Medikamente. Eine medikamentöse Behandlung akuter Diarrhoen ist selten erforderlich. Die - Tab. 2.61 zeigt in der Übersicht Probleme und Einsatzbereiche verschiedener Medikamente, welche zur Behandlung der akuten Diarrhoe eingesetzt werden. Bei Gabe von Antibiotika (z. B. Cephalosporine, Breitspektrumpenizilline, besonders Ampicillin, Amoxycillin und Lincamycine wie Clindamycin) kann es als Nebenwirkung zu schweren Durchfällen kommen (sog. Antibiotika-assoziierte Kolitis).

Pseudomembranöse Kolitis. Diese wird als pseudomembranöse Kolitis durch Clostridien (Clostridium difficile) ausgelöst. Das Krankheitsbild tritt z. B. bei Patienten mit bei Malignomen unter Chemotherapie oder bei Patienten mit dialysepflichtigen Nierenerkrankungen auf. Im Einzelfall kann sich eine clostridienbedingte Kolitis auch ohne den Einfluss von Antibiotika entwickeln. Klinisch sind die Patienten trotz der Durchfälle meist in einem guten Allgemeinzustand. Bei schwerem Verlauf einer pseudomembranösen Kolitis sind die Durchfälle allerdings wässrig, schleimig und blutig. Der Zustand der Patienten ist dann regelhaft schlecht: Volumenmangel, Elektrolytverlust, Hypalbuminämie und Fieber be- 
stimmen die klinischen Zeichen der Erkrankung. Ernährungsmedizinisch besteht regelhaft eine Indikation für eine künstliche Ernährung. Die Therapie der Erkrankung ist das Absetzen des in Frage kommenden Antibiotikums sowie bei schwerer Kolitis die Gabe des Antibiotikums Vancomycin (4-mal $500 \mathrm{~g} / \mathrm{Tag}$ für 7-10 Tage. Cave: Bei parenteraler und hochdosierter Gabe können eine Minderung des Hörvermögens und eine Verschlechterung der Nierenfunktion auftreten).

\section{Kost bei Colon irritabile}

Keine eigene oder wissenschaftlich gesicherte Kostform. Entgegen einer weit verbreiteten Annahme bestehen keine Beziehungen zwischen den Beschwerden eines Colon irritabile und der Ballaststoffzufuhr. Bei Colon irritabile handelt es sich um eine häufige, ernst zu nehmende und die Patienten sehr belastende Symptomatik, deren Ursachen (z. B. eine Motilitätsstörung?) letztlich unklar sind.
- Tab. 2.59. Reisediarrhoe durch kontaminierte Lebensmittel oder Wasser

\begin{tabular}{|l|l|}
\hline Bakterien & Escherichia coli (toxisch) \\
\hline Shigella \\
Salmonella \\
\hline Staphylococcus aureus \\
Campylobacter jejuni \\
Aeromonas \\
Pseudomonas \\
Vibrionen \\
Clostridien \\
\hline Viren & Norwalkvirus \\
& Rotaviren \\
\hline Parasiten & Giardia lamblia \\
& Entamoeba histolytica \\
& Kryptosporidium \\
& Isospora belli \\
& Blastocystis hominis \\
& Strongyloides stercoralis \\
\hline
\end{tabular}

- Tab. 2.60. Ursachen und Symptome von Lebensmittelinfektionen

\begin{tabular}{|c|c|c|c|}
\hline Erreger & Lebensmittel & Symptome & $\begin{array}{l}\text { Intervall bis zum } \\
\text { Auftreten der } \\
\text { Symptome }\end{array}$ \\
\hline Clostridum perfringens & $\begin{array}{l}\text { Warmgehaltene Fleisch- } \\
\text { speisen, Saucen }\end{array}$ & $\begin{array}{l}\text { Durchfall, Bauchschmerzen, Blähun- } \\
\text { gen, selten: Übelkeit, Erbrechen }\end{array}$ & 9-15 Stunden \\
\hline Staphylococcus aureus & $\begin{array}{l}\text { Fleisch, Geflügel, proteinreiche } \\
\text { Lebensmittel }\end{array}$ & $\begin{array}{l}\text { Durchfälle, Erbrechen, Übelkeit, Bauch- } \\
\text { schmerzen, Krämpfe }\end{array}$ & $0,5-8$ Stunden \\
\hline Chlostridium botulinum & $\begin{array}{l}\text { Dosenfleisch und -gemüse, } \\
\text { vakuumverpackte Lebens- } \\
\text { mittel }\end{array}$ & $\begin{array}{l}\text { Neurotoxische Symptome wie: Doppel- } \\
\text { bilder, Sprechstörungen, Schluckbe- } \\
\text { schwerden, Atembeschwerden }\end{array}$ & 4-36 Stunden \\
\hline Salmonellen & $\begin{array}{l}\text { Nicht pasteurisierte Milch, } \\
\text { rohes Fleisch, Eier }\end{array}$ & $\begin{array}{l}\text { Übelkeit, Bauchschmerzen, Durchfall, } \\
\text { Fieber, Schüttelfrost, Kopfschmerzen }\end{array}$ & 6-8 Stunden \\
\hline Listerien & $\begin{array}{l}\text { Rohmilch (-produkte), Fisch, } \\
\text { Blattgemüse }\end{array}$ & $\begin{array}{l}\text { Fieber, Kopfschmerzen, Übelkeit, Er- } \\
\text { brechen, Komplikationen: Meningitis, } \\
\text { Sepsis, Abort, Totgeburt }\end{array}$ & 7-30 Tage \\
\hline Campylobacter jejuni & $\begin{array}{l}\text { Rohes Fleisch, nicht-pasteuri- } \\
\text { sierte Milch }\end{array}$ & Durchfälle, Bauchschmerzen, Krämpfe & 2-5 Tage \\
\hline Escherichia coli & $\begin{array}{l}\text { Rohes Fleisch, nicht-pasteuri- } \\
\text { sierte Milch }\end{array}$ & $\begin{array}{l}\text { Hämorrhagische Kolitis, blutige Durch- } \\
\text { fälle, Krämpfe, Übelkeit, Erbrechen, } \\
\text { Temperaturerhöhung (gering) }\end{array}$ & 3-4 Tage \\
\hline Kryptosporidien & $\begin{array}{l}\text { Verunreinigtes Wasser, } \\
\text { verunreinigte Lebensmittel }\end{array}$ & $\begin{array}{l}\text { Grippeähnliche Symptome, wässrige } \\
\text { Durchfälle, Übelkeit, Erbrechen, Fieber, } \\
\text { Abgeschlagenheit }\end{array}$ & 2-14 Tage \\
\hline Giardia lamblia & $\begin{array}{l}\text { Verunreinigte und ungekochte } \\
\text { Lebensmittel von infizierten } \\
\text { Händlern, verunreinigtes } \\
\text { Wasser }\end{array}$ & $\begin{array}{l}\text { Explosive, wässrige Diarrhoe, Bauch- } \\
\text { krämpfe, Übelkeit, Erbrechen }\end{array}$ & 1-3 Tage \\
\hline Hepatitis A & $\begin{array}{l}\text { Rohe Fisch- und Seefrüchte } \\
\text { aus verunreinigtem Wasser } \\
\text { (Kloake), Verbreitung auch } \\
\text { über infizierte Händler }\end{array}$ & $\begin{array}{l}\text { Abgeschlagenheit, Inappetenz, Übel- } \\
\text { keit, Erbrechen, Durchfälle, Ikterus }\end{array}$ & 10-50 Tage \\
\hline Entamoeba histolytica & $\begin{array}{l}\text { Verunreinigtes Wasser und } \\
\text { Gemüse }\end{array}$ & $\begin{array}{l}\text { Bauchschmerzen, Krämpfe, Durchfall, } \\
\text { Gewichtsverlust, Müdigkeit, Anämie }\end{array}$ & 3-10 Tage \\
\hline
\end{tabular}


Die Diagnose wird nach Ausschlussdiagnostik (=Ausschluss anderer möglicher Ursachen der Symptomatik, z. B. eine Tumorerkrankung) gestellt. Ein Colon irritabile ist wie folgt charakterisiert: Bauchschmerzen (nach Nahrungsaufnahme, Besserung im Zusammenhang mit intestinaler Motilität bzw. nach Stuhlgang), Diarrhoe, Obstipation und/oder veränderte Stuhlbeschaffenheit, Schleimabgang, inkomplette Darmentleerung bei Defäkation, Blähungen, abdominelle Distension. Die Symptome treten ständig oder immer wiederkehrend während wenigstens 3 Monaten auf.

Differentialdiagnose. Mögliche Differentialdiagnosen des Colon irritabile sind: Tumorerkrankungen, chronisch-entzündliche Darmerkrankungen, Laxantienabusus, Laktoseintoleranz, intestinale Parasiten (Giardien?), gynäkologische Erkrankungen (Endometriose?), psychiatrische Erkrankungen (Depressionen?, Panikattacken?).
Zur Abklärung dieser Differentialdiagnosen sind eine ausführliche Anamnese und Diagnostik notwendig.

Behandlung. Ziele einer ernährungsmedizinischen Behandlung von Patienten mit einem Colon irritabile sind Erhalt des Ernährungszustands, Linderung der Symptome sowie Identifikation von Unverträglichkeiten. Diese Ziele werden erfahrungsgemäß am ehesten durch Einhaltung folgender Regeln erreicht: adäquate Nährstoffzufuhr, Vermeidung unverträglicher Nahrungsbestandteile (z. B. Milchprodukte, Gasbildner, Zuckeraustauschstoffe), schrittweise Steigerung der Ballaststoffzufuhr, Coffein und Alkohol begrenzen, Spannungslösung, Stressprophylaxe.

\section{Diät bei Candidainfektion (»Anti-Pilz-Diät«)}

Keine wissenschaftlich abgesicherte Kostform. Bei Vorliegen einer klinisch signifikanten Candidainfektion (Candi-

\section{Lebensmittelvergiftungen}

\begin{tabular}{|c|c|}
\hline $\begin{array}{l}\text { Parasiten, } \\
\text { Helminthen: }\end{array}$ & $\begin{array}{l}\text { Bakterien, Viren, } \\
\text { Rickettsien: }\end{array}$ \\
\hline $\begin{array}{l}\text { Toxoplasmose } \\
\text { Zystizerkose } \\
\text { Trichellinose } \\
\text { Amoebiasis } \\
\text { u.a. }\end{array}$ & $\begin{array}{l}\text { Salmonellose } \\
\text { Camphylobacteriose } \\
\text { Yersiniose } \\
\text { "Reisediarrhoe" } \\
\text { Shighellose } \\
\text { Listeriose } \\
\text { Tuberkulose } \\
\text { Brucellose } \\
\text { Hepatitis A } \\
\text { Rotaviren } \\
\text { u.a. }\end{array}$ \\
\hline
\end{tabular}

- Abb. 2.17. Ursachen von Lebensmittelinfektionen und -intoxikationen

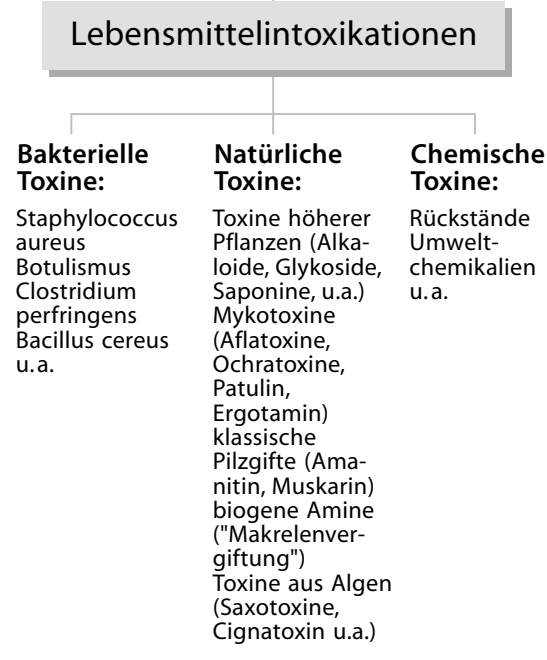

- Tab. 2.61. Medikamentöse Behandlung bei Diarrhoe. (Nach Mutschler 1996)

\begin{tabular}{l|l} 
Opioide & $\begin{array}{l}\text { z. B. Loperamid, Reasec } \\
\text { Cave: bei enteroinvasiven Bakterien wegen der Gefahr einer erhöhten Toxinbildung nach Ruhigstel- } \\
\text { lung kontrainduziert }\end{array}$ \\
\hline Adsorbentien/ Adstringentien & z. B. Aktivkohle oder gerbstoffhaltige Präparate wie Tannalbin; auch Pektine \\
\hline Antibiotika & $\begin{array}{l}\text { Grundsätzlich bei Reisediarrhoe nicht indiziert. Auch bei Salmonellosen nicht routinemäßig geben! } \\
\text { Ausnahmen: Bei Shighellosen, Yersiniosen und schwerer klinischer Symptomatik kann antiinfektiös } \\
\text { (z. B. mit Cotrimoxasol, Gyrasehemmer oder Erythromycin) behandelt werden }\end{array}$ \\
\hline lonenaustauscherharze & Colestyramin bei chologener Diarrhoe \\
\hline
\end{tabular}


dose) wird medikamentös z. B. mit oral nicht resorbierbaren Antimykotika wie Amphotericin B (Amphomoronal Suspension oder Lutschtabletten) behandelt. Bei schweren symptomatischen Verlaufsformen (z. B. Candidose der Speiseröhre) empfiehlt sich Fluconazol (Diflucan; 200 mg/Tag für 14 Tage). Bei Fluconazol-resistenten Candidastämmen wird eine Kombinationsbehandlung mit Amphotericin B und Ancotil durchgeführt. Ketoconazol (Nystatin) wird heute nicht mehr gegeben.

Eine zusätzliche diätetische Behandlung ist weder inhaltlich noch empirisch zu begründen. Eine sog. "AntiPilz-Diät« ist nicht tatsächlich "pilzfrei«, sie soll aber ungünstige Ernährungsbedingungen für die Candidaspezies schaffen. "Anti-Pilz-Diäten « bestehen in dem Meiden von hefe- und schimmelpilzähnlichen Lebensmitteln wie Edelpilzkäse, Camenbert, Harzerkäse, einer optimalen Lebensmittelhygiene sowie der Einschränkung von leicht resorbierbaren Kohlenhydraten wie Zucker, Limonaden, Honig, Süßigkeiten, Weißmehl, Weißmehlprodukte, Alkohol (Bier, Wein, Sekt) und Früchten mit hohem Zuckergehalt (z. B. Weintrauben, Bananen, Honigmelonen). Milch (Cave: Fruchtjoghurt) und Milchprodukte werden nicht eingeschränkt, Sauermilchprodukte sogar empfohlen.

Die eingeschränkte Lebensmittelauswahl wird über einen Zeitraum von 6 Monaten eingehalten, nach etwa einem Monat soll eine Besserung der Beschwerden eintreten. "Kritische« Nährstoffe dieser wissenschaftlich ungeprüften kohlenhydratarmen Kostform sind Eisen, Jod und Vitamin D. Neben dieser Ernährungsform werden auch Eliminationsdiäten und die Supplementierung von Vitaminen zur Behandlung von Patienten mit einer Candidainfektion vorgeschlagen. Diese Vorgehensweisen sind wissenschaftlich ungesichert.

\section{Diäten bei speziellen Systemerkrankungen Ernährungstherapie bei Rheumaerkrankungen}

Indikationen. Rheuma, entzündliche Gelenkerkrankungen (chronische Polyarthritis, M. Bechterew).

Definition. Reduktion der Arachidonsäuremenge in der Ernährung auf $<50 \mathrm{mg} / \mathrm{d}$.

Ziele. Hemmung der Entzündung, Linderung der Symptome, Reduzierung der Medikamente (Zahl, Dosis), Behandlung / Prävention der Osteoporose.

\section{Anmerkung}

Arachidonsäure. Arachidonsäure wird über tierische Lebensmittel aufgenommen und im Körper selbst gebildet. Sie ist Vorstufe von Entzündungsmediatoren. Der Arachidonsäuregehalt (in $\mathrm{mg} / 100 \mathrm{~g}$ ) tierischer Lebensmittel beträgt z. B. 870 (Schweineleber), 230 (Leberwurst), 62 (Hühnerfleisch), 297 (Eigelb) und 4 bzw. 2 (Milch mit 3,5 bzw. 1,5\% Fettgehalt) oder 1700 (Schmalz). Durch
Einschränkung des Verzehrs an tierischen Lebensmitteln (max. 2 Fleischmahlzeiten/Woche, Wurst und Innerein meiden, Verzicht auf tierische Fette), werden weniger Entzündungsmediatoren gebildet, die Gelenkbeschwerden werden gelindert. In der Vollkost sind etwa 200-400mg Arachidonsäure/Tag enthalten. Bei lactovegetabiler Ernährung wird diese Menge auf 50-80 mg reduziert.

Omega-3-Fettsäuren, Antioxidatien. Die hohe Zufuhr von Omega-3-Fettsäuren (2 Seefischmahlzeiten pro Woche, Verwendung von Leinöl, Rapsöl und Walnussöl) sowie eine adäquate Zufuhr von Antioxidatien (Vitamin C, Vitamin E; 5 Portionen Obst und Gemüse pro Tag) haben einen günstigen Einfluss auf die Symptome und wirken präventiv. Die Zufuhr von alpha-Linolensäure wird auf 6g/Tag von Eicopentaensäure auf 2 (-6) g/Tag erhöht. Die Vitamin C-Menge beträgt $200 \mathrm{mg} / \mathrm{Tag}$, die Vitamin E-Zufuhr wird auf $400 \mathrm{mg} / \mathrm{Tag}$ gesteigert.

Calcium. Eine Osteoporoseprophylaxe besteht in einer ausreichenden Calciumaufnahme (0,5 1 Milch bzw. Milchprodukte, calciumreiche Minerlwasser). Bei medikamentöser Behandlung mit Cortison werden 1000 mg Calcium als Supplemente empfohlen. In schweren Fällen kann kurzfristiges Fasten (max. 3 Tage) die Symptome der Rheumaerkrankung deutlich lindern.

Wechselwirkung mit Medikamenten. Bei der medikamentösen Behandlung des Rheumakranken ist an Wechselwirkungen zwischen Nährstoffen (Folsäure) und einzelnen Medikamenten (Methotrexat) zu denken. Methotrexat hemmt die Bildung von Tetrahydrofolsäure. In diesem Fall muss Folsäure (bis zu $5 \mathrm{mg} / \mathrm{d}$ ) supplementiert werden.

Malnutrition. Bei vielen Rheumakranken bestehen Zeichen der Malnutrition (Untergewicht), die eine Ernährungsintervention notwendig machen. Bei Immobilität müssen die negativen Stickstoff- und Calciumbilanzen korrigiert werden. Dazu wird die Eiweißaufnahme auf bis $\mathrm{zu} 1,2 \mathrm{~g} / \mathrm{kg} / \mathrm{d}$ und die Calciumzufuhr um $500 \mathrm{mg} / \mathrm{d}$ erhöht. Eine »Substitutionstherapie« mit $25 \mu \mathrm{g}$ Vitamin D wird empfohlen.

\section{Ketogene Diäten}

Indikationen. Pharmakoresistente cerebrale Anfallsleiden im Kindesalter (Epilepsie), Glu T1-Defekt ${ }^{3}$, Pyruvatdehydrogenasemangel.

Definiton. Kohlenhydratarme Ernährung mit einem Fett (g)/Kohlenhydrat(g)-Verhältnis von 3-4:1.

\footnotetext{
${ }^{3}$ Glu T1 = Glukosetransporter 1, Haupttransportprotein für Glukose
} an der Bluthirnschranke. 
Ziele. Linderung der Beschwerdesymptomatik, Reduktion der Zahl der Anfälle.

Kontraindikationen. Kontraindikationen bestehen bei Störungen der Synthese und der Verwertung von Ketonkörpern (z. B. Störungen der Fettsäureoxidation und Ketonkörperbildung, Defekte des Ketonkörperabbaus). Einzelne Antiepileptika (wie Valproat) hemmen die $\beta$ Oxidation und sind deshalb nicht in Kombination mit ketogenen Diäten anzuwenden. Ketogene Diäten bewirken eine Senkung des Urin-pH's und sind deshalb bei Steinleiden relativ kontrainduiziert.

Flüssigkeit. Die Flüssigkeitsaufnahme muss hoch sein (Kinder mit einem Gewicht von $1-10 \mathrm{~kg}, 80 \mathrm{ml} / \mathrm{kg} / \mathrm{Tag}$, bei $10-20 \mathrm{~kg}, 800 \mathrm{ml}+40 \mathrm{ml} / \mathrm{kg} / \mathrm{Tag}$, bei $>20 \mathrm{~kg}, 1200 \mathrm{ml}+$ $20 \mathrm{ml} / \mathrm{kg} / \mathrm{Tag}$ ).

Durchführung. Die Diät wird nach einer Einleitungsphase (=1 Fastentag) stufenweise über 3-5 Tage eingeführt. Langfristig wird der "Erfolg" an der klinischen Symptomatik sowie der Ketonkörperausscheidung im Urin kontrolliert. Eine aus Sicht dieser Diät bzw. der Therapie »optimale« Ketonurie liegt zwischen 80 und $160 \mathrm{mmol}$ $\beta$-Hydroxybutyrat im Spontanurin. Bei »Erfolg « wird die Diät über 2 Jahre durchgeführt. Ein »Ausschleichen« der Diät (z. B. Veränderung des Fett/Kohlenhydrat-Verhältnisses von 4:1 auf 3:1 bzw. 2:1) kann versucht werden. Modifikation einer ketogenen Diät ist die MCT-Diät. Dabei wird der MCT-Anteil auf z. B. 40\% der Fettmenge erhöht. Nebeneffekte sind Unwohlsein und Übelkeit und Hyperurikämie. Eine ketogene Diät muss mit allen wasserlöslichen Vitaminen sowie Mengen-und Spurenelemente ergänzt werden. Die Aufnahme von Vitamin A, $\mathrm{E}$ und $\mathrm{K}$ ist demgegenüber hoch.

Erfolg. Die Wahrscheinlichkeit mit einer solchen Diät eine vollständige Anfallsfreiheit zu erreichen liegt zwischen 6 und 30\%. Eine Reduktion der Anfallshäufigkeit wurde demgegenüber bei bis zu $90 \%$ der Patienten beobachtet. Die Akzeptanz der Diät ist bei den Patienten und ihren Familien hoch, der Aufwand ist allerdings beträchlich.

\section{Seltene und spezielle Diätformen Keimreduzierte Kost}

Indikationen. Ernährung bei immunsupprimierten $\mathrm{Pa}$ tienten (z. B. bei Patienten mit HIV-Infektion, unter medikamentöser Immunsuppresion nach Organtransplantation).

Definition. Eine keimreduzierte Kost entspricht in ihrer Zusammensetzung einer leichten Vollkost, welche sterilisiert werden muss. Nach Aufhebung der Isolation muss das Essen nicht mehr sterilisiert werden. Die Patienten bekommen dann eine keimarme leichte Vollkost.

Ziele. Verminderung der Gefahr einer Lebensmittelinfektion.

Anmerkung. - Tab. 2.62 gibt einen Überblick über die für eine keimreduzierte Kost geeignete und ungeeignete Lebensmittel. Der Wert dieser Kostform ist wissenschaftlich nicht geprüft, erscheint aber klinisch plausibel. Da manche Patienten aufgrund ihrer Beschwerden (Übelkeit, Erbrechen, Ösophagitis, GVH (=»graft versus host«)Reaktion am Darm) über längere Zeit nicht genügend Nahrung zu sich nehmen können, wird zusätzlich parenteral ernährt. Bei Nachlassen der Beschwerden wird ein Kostaufbau versucht. Dieser entspricht dem Kostaufbau nach akutem Schub einer chronisch-entzündlichen Darmerkrankung. Der Kostaufbau kann steril durchgeführt werden. Bei Patienten mit einer HIV-Infektion sind besonders phasenhaft Ernährungsprobleme zu erwarten. Deshalb sind Schweregrad und Verlauf der Erkrankung zu berücksichtigen.

\section{Eisenarme Kost}

Indikationen. Hämosiderose, primäre oder sekundäre Hämochromatose.

Definition. Vollkost mit einen Eisengehalt von $<12 \mathrm{mg} /$ Tag.

Ziel. Verminderung der Eisenspeicher.

Anmerkung. Eine eisenarme Kost ist keine Therapie der Hämochromatose (sic: Aderlass). Eisen ist ubiquitär in Lebensmitteln enthalten. Ein hoher Eisengehalt findet sich in Innereien $(10-20 \mathrm{mg} / 100 \mathrm{~g})$, Vollkornbrot $(4,6 \mathrm{mg} / 100 \mathrm{~g})$, Spinat $(4 \mathrm{mg} / 100 \mathrm{~g})$, Hülsenfrüchte $(6 \mathrm{mg} / 100 \mathrm{~g})$ und Fleisch $(2,5 \mathrm{mg} / 100 \mathrm{~g})$. Eine eisenarme Kost ist laktovegetabil und vermeidet eisenreiche Lebensmittel wie Hülsenfrüchte, Blattgemüse und Trockenfrüchte. Die Kost enthält Ballaststoffe in Form von phytat- (Vollkornerzeugnisse, Kleie) und pektinhaltigen (Äpfel, Karotten, Steinobst) Lebensmitteln. Schwarzer Tee (Tannine hemmen die Eisenresorption) sollte reichlich getrunken werden. Aufgrund der eingeschränkten Lebensmittelauswahl muss anhand des Kostplans und evtl. eines Ernährungsprotokolls die Nährstoffversorgung überprüft und ggf. korrigiert werden. Alkoholexzess steigert die Eisenresorption.

\section{Kupferarme Kost}

Indikation. Morbus Wilson (hepatozerebrale Degeneration).

Definition. Der Kupferanteil der Kost ist $<1-1,5$ mg/Tag. 
- Tab. 2.62. Lebensmittelauswahl bei einer keimreduzierten Kost für immunsupprimierte Patienten

\begin{tabular}{|c|c|c|}
\hline & Geeignet & Ungeeignet \\
\hline Fleisch und Fisch & Gur durchgegart & Roh/halbroh, Schalen und Krustentiere, Salate \\
\hline Käsesorten & $\begin{array}{l}\text { Hart- und Schnittkäse, Quark, Schichtkäse, } \\
\text { Harzer }\end{array}$ & Edelpilzkäse, Schafskäse, Weichkäse, Käsesalate \\
\hline Milch und Milchprodukte & $\begin{array}{l}\text { Pasteurisierte Trinkmilch, Kondensmilch, } \\
\text { abgepacktes Speiseeis, Joghurt }\end{array}$ & Lose Milch und Milchprodukte, offenes Eis, Softeis \\
\hline Fette & Butter, Pflanzenmargarine, Pflanzenöle & $\begin{array}{l}\text { Kräuterbutter, Mayonnaise, Remoulade, Fer- } \\
\text { tigsaucen }\end{array}$ \\
\hline Eier & Harte Eier, Eierpfannkuchen & Rohe Eier, Rühr- und Spiegeleier \\
\hline Getreideerzeugnisse & $\begin{array}{l}\text { Gegarte Zubereitung, alle Brotsorten, } \\
\text { gebackene Kuchen, Hefegebäck }\end{array}$ & $\begin{array}{l}\text { Getreidekörner, Müslis, Obstkuchen mit rohem } \\
\text { Obst, industriell hergestellte Torten }\end{array}$ \\
\hline Kartoffeln & Salzkartoffeln, Pellkartoffeln, Kartoffelbrei & $\begin{array}{l}\text { Kartoffelsalat mit Mayonnaise, industriell } \\
\text { hergestellte Salate }\end{array}$ \\
\hline Gemüse & Nur in gegarter Form, schälbare Gemüse & $\begin{array}{l}\text { Rohes Gemüse, Trockengemüse, industriell } \\
\text { hergestellte Salate }\end{array}$ \\
\hline Getränke & $\begin{array}{l}\text { Pasteurisierte Getränke, möglichst frisch } \\
\text { trinken }\end{array}$ & Tee aus getrockneten Früchten \\
\hline
\end{tabular}

Ziel. Verminderung der Kupferspeicher.

Anmerkung. In der Praxis handelt es sich um eine eher laktovegetabile Kost unter Ausschaltung kupferreicher Lebensmittel wie Vollkornprodukte, Trockenobst, Nüsse, Emmentaler- und Edamerkäse, Innereien, Pilze, Kakao und Schokolade. Der Kupfergehalt einzelner Nahrungsmittel beträgt z. B. für Emmentaler Käse (bis zu $750 \mathrm{mg} / 100 \mathrm{~g}$ ), Innereien (bis zu $6 \mathrm{~g} / 100 \mathrm{~g}$ ), Schalen- und Krustentiere $(2,5 \mathrm{mg} / 100 \mathrm{~g})$, Champignons $(0,4 \mathrm{mg} / 100 \mathrm{~g})$, Haferflocken $(530 \mathrm{mg} / 100 \mathrm{~g})$, eingelegte Gurken $(90 \mathrm{mg} / 100 \mathrm{~g})$, Grünkohl $(90 \mathrm{mg} / 100 \mathrm{~g})$, Nüsse $(1,3 \mathrm{~g} / 100 \mathrm{~g})$, Schokolade (bis zu 2,5g/100 g), Kaffee (bis zu $3 \mathrm{~g} / 100 \mathrm{~g}$ ). Kritische Nährstoffe sind die Vitamine der B-Gruppe, Eisen und Zink. Die Diät muss lebenslang durchgeführt werden. Es darf kein Kupfergeschirr verwendt werden. Bei Kupferrohren ist eine Überprüfung des Leitungswassers notwendig. Bei einem Kupfergehalt des Trinkwassers von $>0,1 \mathrm{mg} \%$ muss demineralisiertes Wasser benutzt werden. Speisesalz, Pfeffer, Kaffee und schwarzer Tee können ebenfalls größere Mengen Kupfer enthalten und sollten gemieden werden. Zubereitung der Gerichte in Glas-, Porzellan- oder Chromagangeschirr.

\section{(?) Cave}

\section{Weichspüler.}

Da Zink mit Kupfer um den intestinalen Transport konkurriert, kann eine Behandlung mit einem oralen Zinksupplement zur Verringerung der Kupferresorption ver- sucht werden. Dieses ist eine Option für die Patienten, die die Behandlung mit Chelatbildnern nicht vertragen. Bei medikamentöser Therapie mit D-Penicillamin ist die zusätzliche Gabe von Zink nicht sinnvoll. D-Penicillamin ist ein Pyridoxin (Vitamin-B $\mathrm{B}_{6}$ Antagonist, entsprechend müssen gleichzeitig $25 \mathrm{mg}$ Pyridoxin (z. B. Hexobion)/d substituiert werden.

\section{Kalziumarme (Oxalsäurearme) Kost}

Indikationen. Primärer Hyperparathyreoidismus, Hyperkalziämie bei paraneoplastischem Syndrom, Hyperkalziurie, Kalziumoxalatsteine.

Definition. Die Kost entspricht einer Vollkost unter Begrenzung der Kalziummenge unter $400 \mathrm{mg}$ oder $10 \mathrm{mmol} /$ Tag. Kalziumreiche Nahrungsmittel (Milch, Milchprodukte, Eier) und Mineralwässer sollten gemieden werden. Die Menge kalziumarmer Getränke sollte $>2,5 \mathrm{l} / \mathrm{Tag}$ liegen. Bei Kalziumoxalatsteinen werden oxalsäurereiche Nahrungsmittel (Fruchtsäfte, süße Limonade, Spinat, Mangold, Nüsse, Schokolade) gemieden.

Ziele. Normalisierung des Kalziumspiegels, Prävention der Nephrolithiasis.

Anmerkung. Eine Kalziumarme Kost ist wegen der Gefahr einer Osteoporose eigentlich obsolet und nur vorübergehend erlaubt, sie ist nach Beseitigung der Ursache der Hyperkalziämie überflüssig. 


\section{Serotoninarme Diät}

Indikationen. Karzinoid-Syndrom, vorübergehend als "Suchdiät« bei Untersuchung der 5-Hydroxyindolessigsäureausscheidung.

Definition. Vollkost unter Meidung von serotoninhaltigen Lebensmitteln.

Ziele. Senkung der Serotoninbildung und der 5-Hydroxyindolessigsäureausscheidung im Urin (normal $<10 \mathrm{mg}$ / Tag).

Anmerkung. Lebensmittel mit einem hohen Serotoningehalt sind z. B. Walnüsse 170-340 lg/g, Bananen 20-30 lg/g, Ananas $10 \mathrm{lg} / \mathrm{g}$, Tomaten $6 \mathrm{lg} / \mathrm{g}$. Bei sehr hoher Eiweißzufuhr ist der Tryptophangehalt der Nahrung zu beachten. Da Serotonin aus Tryptophan gebildet wird, beEinflusst eine sehr hohe Tryptophanzufuhr ( $>5 \mathrm{~g} / \mathrm{Tag})$ auch die Serotoninbildung. Tryptophanreiche Lebensmittel sind z. B. Sojamehl (560 mg/100 g), Parmesankäse ( $490 \mathrm{mg} / 100 \mathrm{~g})$, Emmentaler ( $370 \mathrm{mg} / 100 \mathrm{~g}$ ), Leber (um $300 \mathrm{~g} / 100 \mathrm{~g}$ ).

\section{Allergenarme Kost, Eliminationsdiät}

Indikationen. Verdacht auf bzw. nachgewiesene Nahrungsmittelallergie, chronische Urtikaria, Kostaufbau und »Kostfindung«.

Definition. Als diagnostische Diät: Auslasskost oder Eliminationsdiät. Als langfristige Diät: Vollkost oder leichte Vollkost unter Ausschaltung objektivierter, allergen wirksamer Nahrungsbestandteile.

Ziele. Beschwerdefreiheit, Diagnostik und Behandlung der Nahrungsmittelallergie unter Elimination des allergen wirksamen Nahrungsbestandteils bzw. im Einzelfall möglicherweise erfolgreicher oraler Hyposensibilisierung.

Anmerkung. Die Nahrungsmittelallergie ist von anderen Formen der Nahrungsmittelunverträglichkeit zu unterscheiden (- Abb. 2.18). Die Diagnose einer Nahrungsmittelallergie gilt erst dann als gesichert, wenn die Symptome nach Elimination verschwinden und diese nach erneuter Exposition wieder erscheinen. Die Durchführung einer Eliminationsdiät ist nur bei begründetem Verdacht auf eine Nahrungsmittelallergie und zur Identifizierung der die Symptome auslösenden Lebensmittel sinnvoll. Eine allergenfreie Diät besteht ausschließlich aus Reis, Kartoffeln, Salz, Zucker und Mineralwasser. Bei Allergien gegen Kartoffeln oder Reis müssen andere Grundnahrungsmittel gewählt werden. Eine Eliminationsdiät im engeren Sinne eliminiert nacheinander und schrittweise potentiell allergen wirksame Nahrungsbestandteile wie z. B. konservierungsstoffeenthaltende Lebensmittel, Lebensmittelfarbstoffe, Gewürze, Nüsse, Ei, Fisch oder Getreideprodukte. Im weiteren Sinne kann als »Eliminationsdiät» auch ein von einer Grunddiät ausgehender, stufenweiser und kontrollierter Kostaufbau von einer milchfreien Kartoffel-Reis-Diät (ohne Gewürze, ohne Zulagen) bis zur gezielten Zugabe (Addition) potentieller Allergene im Sinne eines Provokationstests durchgeführt werden. Die Symptome der Nahrungsmittelallergie müssen auf der ersten Stufe der »Eliminationsdiät«, d. h. vor einem Kostaufbau abgeklungen sein.

- Stufe 1: Die Grunddiät besteht aus schwarzem Tee, gekochten Kartoffeln und Reis sowie Maiskeimöl, Salz und Traubenzucker. Nach 7-10 Tagen schließt sich z. B. der Kostaufbau mit Milch, Käse, Quark, Joghurt,

- Stufe 2: Getreideprodukten wie Brötchen, Graubrot, Gries, Hartweizennudeln (keine Konservierungsstoffe),

- Stufe 3: schwarzem Tee, kaltgepresstem Planzenöle,

- Stufe 4: Gemüse, Obst, Obstsalat,

- Stufe 5: Ei, Eiernudeln, Omelett, Fisch,

- Stufe 6: Fleisch und Wurst,

- Stufe 7: Blumenkohl und Paprika und

- Stufe 8: Gewürze, Kaffee und Alkohol an.

Während einer Eliminationsdiät wird von Patienten ein genaues Ernährungsprotokoll unter Angabe möglicher Beschwerden geführt. In der anschließenden Dauerkost wird das objektivierte Allergen eliminiert, z. B. als »milcheiweißfreie Kost« (Vollkost unter Ausschluss jeglicher Milch und Milchprodukte sowie Milchzusätze enthaltender Lebensmittel) oder »nickelfreie Kost« (Vollkost unter Herabsetzung des Nickelgehalts auf $<2 \mathrm{mg} /$ Tag unter Vermeidung von Getreideerzeugnissen, Hülsenfrüchten, Pilzen, Nüssen, Mandeln, Kakao, schwarzen Tees) (- Tab. 2.63).

\section{Kohlenhydratreiche Kost bei hepatischer Porphyrie}

Indikation. Akut intermittierende Porphyrie.

Definition. Kohlenhydratreiche Ernährung (während der akuten Krankheitsphase bis zu 600 g/Tag, Dauerbehandlung mit ca $400 \mathrm{~g} / \mathrm{Tag}$ ) unter Kontrolle des Flüssigkeitund Elektrolythaushalts.

Ziele. Besserung der Symptome, Senkung der Bildung von d-Aminolävulinsäure und der Ausscheidung von Porphyrin und Porphyrinvorläufern (Porphobilinogen) im Urin.

Anmerkung. Empirische (d. h. wissenschaftlich nicht geprüfte) und rein symptomatische Diät, welche früh begonnen werden muss und nicht bei allen Patienten gleich wirksam ist. Bei längerfristiger Anwendung müssen BVitamine substituiert werden (z. B. BVK Roche). (Cave: Längere Nüchternperioden, Alkohol und zahlreiche Medikamente wie Barbiturate provozieren die Symptome. Bei erythropoetischer Porphyrie werden zur Senkung der Photosensibilität oral Karotinoide gegeben. 


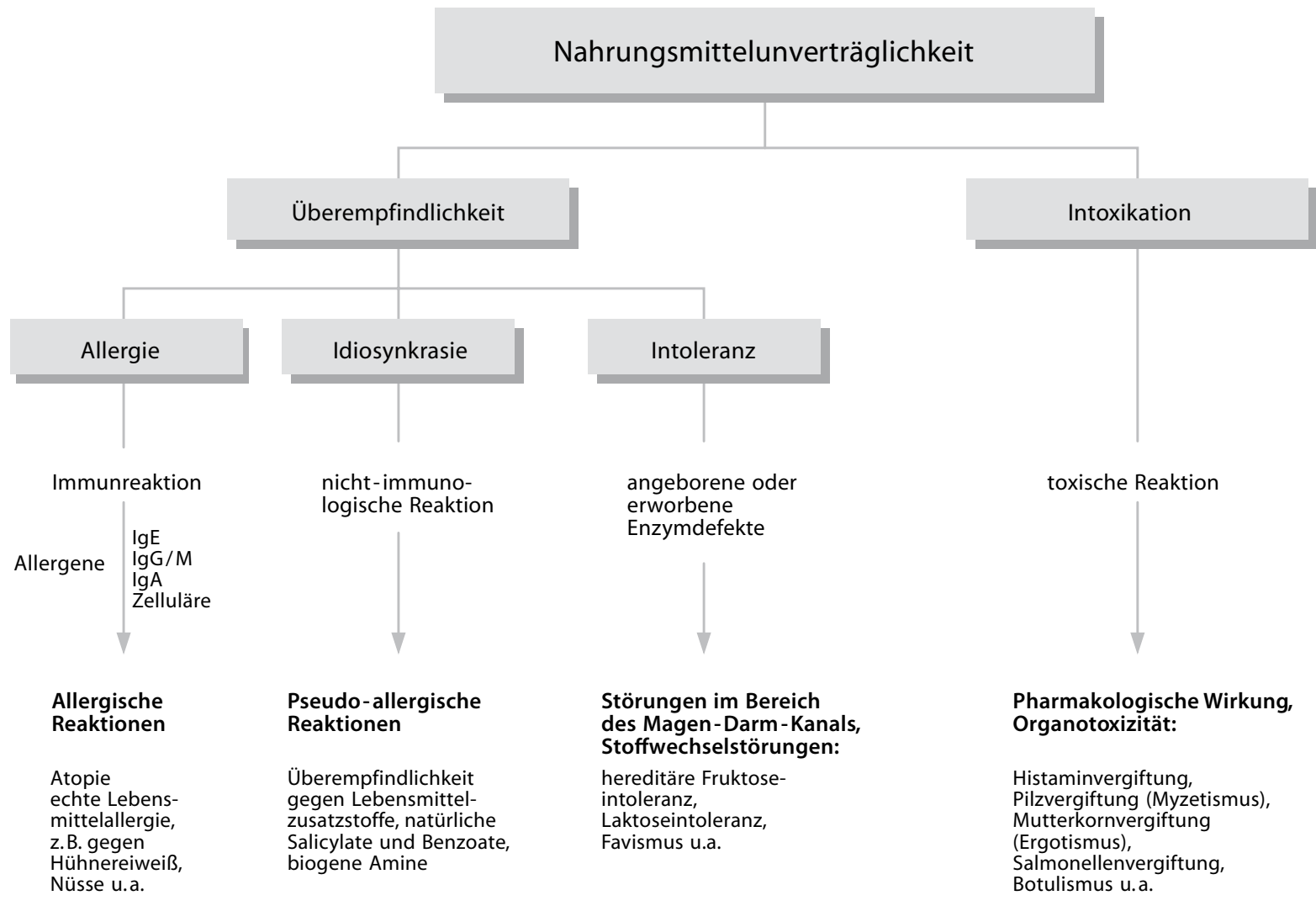

- Abb. 2.18. Verschiedene Formen der Nahrungsmittelunverträglichkeit

\section{Hydroxyprolinarme Kost}

Indikation. Diagnostik von Knochen- und Bindegewebserkrankungen.

Definition. Kost unter Ausschluss kollagenhaltiger Lebensmittel wie Fleisch, Fleischwaren und -produkten (z. B. Brühen) und gelatinehaltige Lebensmittel (z. B. Saucen, Marmeladen, Eis etc.).

Ziel. Diagnostik des Kollagenstoffwechsels.

Anmerkung. Die Kost wird über insgesamt 4 Tage gegeben. Die Hydroxyprolinausscheidung im 24-h-Sammelurin wird am 3. und 4. Tag gemessen. Durch gleichzeitige Bestimmung der Serum- und Urinkreatininkonzentrationen ist eine Korrektur von Sammelfehlern möglich.

\section{Kost bei Phäochromozytomdiagnostik}

Indikationen. Verdacht auf ein Phäochromozytom, Hypertonus, Neuroblastom oder Ganglioneurom, Multiple Endokrine Neoplasie (MEN).
Definition. Kost unter Vermeidung von Lebensmitteln, welche die Katecholaminsekretion anregen oder Vanillinmandelsäure enthalten.

Ziel. Diagnostik eines Phäochromozytoms.

Anmerkung. »Kritische« Lebensmittel in der Phäochromozytomdiagnostik sind z. B. Mandeln, Nüsse, Bananen, Vanille, Käse, Tee und Kaffee. Die Konzentrationen der Katecholamine (Adrenalin, Noradrenalin) und der Vanillinmandelsäure (=VMS) werden über 3 Tage im angesäuerten 24-h-Sammelurin bestimmt. Die Diät wird 3 Tage vor und während der Sammelperiode eingehalten werden. Gleichzeitig werden Medikamente, welche die Katecholaminsekretion beeinflussen (z. B. Antidepressiva, Antihypertensiva, Diuretika, Sympathiko- und Parasympathikomimetika, a- und b-Blocker), für mindestens 5 Tage vor und während der Sammelperiode abgesetzt. Verschiedene Antibiotika wie Tetrazykline, Ampicillin oder Erythromycin stören die fluorimetrische Bestimmung der Katecholamine. Nikotinkarenz ist obligat. Körperliche Belastungen werden gemieden. 
- Tab. 2.63. Beispiel verschiedener Stufen (Stufe 1-8) einer Eliminationsdiät

\begin{tabular}{|c|c|}
\hline \multicolumn{2}{|c|}{ Stufe 1: Grunddiät } \\
\hline \multicolumn{2}{|l|}{ 1. Tag } \\
\hline \multicolumn{2}{|l|}{ Frühstück } \\
\hline $\begin{array}{l}1 \text { Becher } / 200 \mathrm{ml} \\
300 \mathrm{~g} \\
0,5 \mathrm{TL} / 2 \mathrm{~g}\end{array}$ & $\begin{array}{l}\text { Tee schwarz fermeniert } \\
\text { Reis gegart } \\
\text { Jodiertes Salz }\end{array}$ \\
\hline \multicolumn{2}{|l|}{ Mittagessen } \\
\hline $\begin{array}{l}300 \mathrm{~g} \\
0,5 \mathrm{TL} / 2 \mathrm{~g} \\
0,2 \mathrm{l}\end{array}$ & $\begin{array}{l}\text { Reis gegart } \\
\text { Jodiertes Salz } \\
\text { Natürliches Mineralwasser }\end{array}$ \\
\hline \multicolumn{2}{|l|}{ Abendessen } \\
\hline $\begin{array}{l}300 \mathrm{~g} \\
0,5 \mathrm{TL} / 2 \mathrm{~g} \\
1 \mathrm{Becher} / 200 \mathrm{ml}\end{array}$ & $\begin{array}{l}\text { Reis parboiled gegart } \\
\text { Jodiertes Salz } \\
\text { Tee schwarz fermentiert }\end{array}$ \\
\hline \multicolumn{2}{|l|}{ 2. Tag } \\
\hline \multicolumn{2}{|l|}{ Frühstück } \\
\hline $\begin{array}{l}1 \mathrm{Becher} / 200 \mathrm{ml} \\
300 \mathrm{~g} \\
0,5 \mathrm{TL} / 2 \mathrm{~g}\end{array}$ & $\begin{array}{l}\text { Tee schwarz fermentiert } \\
\text { Reis gegart } \\
\text { Jodiertes Salz }\end{array}$ \\
\hline \multicolumn{2}{|l|}{ Mittagessen } \\
\hline $\begin{array}{l}300 \mathrm{~g} \\
0,5 \mathrm{TL} / 2 \mathrm{~g} \\
0,2 \mathrm{I}\end{array}$ & $\begin{array}{l}\text { Pellkartoffeln (4) } \\
\text { Jodiertes Salz } \\
\text { Natürliches Mineralwasser }\end{array}$ \\
\hline \multicolumn{2}{|l|}{ Abendessen } \\
\hline $\begin{array}{l}300 \mathrm{~g} \\
0,5 \mathrm{TL} / 2 \mathrm{~g} \\
1 \text { Becher } / 200 \mathrm{ml}\end{array}$ & $\begin{array}{l}\text { Reis parboiled gegart } \\
\text { Jodiertes Salz } \\
\text { Tee schwarz fermentiert }\end{array}$ \\
\hline \multicolumn{2}{|c|}{ Gesamtsumme für beide Tage: $1833 \mathrm{kcal}$} \\
\hline $\begin{array}{l}\text { Eiweiß } \\
\text { Fett } \\
\text { Kohlenhydrate }\end{array}$ & $\begin{array}{l}40 \mathrm{~g}(9 \%) \\
3 \mathrm{~g}(1 \%) \\
404 \mathrm{~g}(90 \%)\end{array}$ \\
\hline \multicolumn{2}{|l|}{ Stufe 2: Milch } \\
\hline \multicolumn{2}{|l|}{ Frühstück } \\
\hline $\begin{array}{l}1 \mathrm{Becher} / 200 \mathrm{~g} \\
100 \mathrm{~g} \\
50 \mathrm{~g} \\
150 \mathrm{~g}\end{array}$ & $\begin{array}{l}\text { Tee schwarz fermentiert } \\
\text { Quellreis } \\
\text { Sahne 30\% Fett } \\
\text { Trinkwasser }\end{array}$ \\
\hline \multicolumn{2}{|c|}{ Mittagessen (Kartoffelgratin) } \\
\hline $\begin{array}{l}250 \mathrm{~g} \\
50 \mathrm{~g} \\
50 \mathrm{~g} \\
0,5 \mathrm{TL} / 2 \mathrm{~g} \\
0,21\end{array}$ & $\begin{array}{l}\text { Pellkartoffeln (4) } \\
\text { Gouda Doppelrahmstufe } \\
\text { Sahne 30\% Fett } \\
\text { Jodiertes Salz } \\
\text { Natürliches Mineralwasser }\end{array}$ \\
\hline \multicolumn{2}{|l|}{ Zwischenmahlzeit } \\
\hline $100 \mathrm{~g}$ & Joghurt vollfett \\
\hline \multicolumn{2}{|l|}{ Abendessen } \\
\hline $\begin{array}{l}250 \mathrm{~g} \\
80 \mathrm{~g} \\
0,1 \mathrm{l} \\
0,5 \mathrm{TL} / 2 \mathrm{~g} \\
1 \mathrm{Becher} / 200 \mathrm{~g}\end{array}$ & $\begin{array}{l}\text { Pellkartoffeln (4) } \\
\text { Quark Doppelrahmstufe } \\
\text { Natürliches Mineralwasser } \\
\text { Jodiertes Salz } \\
\text { Tee schwarz fermentiert }\end{array}$ \\
\hline
\end{tabular}

Am 2. Tag statt Quark evtl. geschmolzenen Käse mit den angegebenen Käsesorten

Gesamtsumme für beide Tage: $1265 \mathrm{kcal}$

\begin{tabular}{l|l}
\hline Eiweiß & $34 \mathrm{~g}(11 \%)$ \\
Fett & $78 \mathrm{~g}(56 \%)$ \\
\hline Kohlenhydrate & $104 \mathrm{~g}(33 \%)$
\end{tabular}

\section{Stufe 3: Getreide}

1. Tag

\section{Frühstück}

\begin{tabular}{l|l}
\hline 1 Becher $/ 200 \mathrm{ml}$ & Tee schwarz fermentiert \\
1 Scheibe/60 g & Vollkornbrot \\
$30 \mathrm{~g}$ & Brie 70\% F. i. Tr. \\
$10 \mathrm{~g}$ & Honig (Blütenhonig) \\
$15 \mathrm{~g}$ & Butter \\
\hline Mittagessen & \\
\hline $100 \mathrm{~g}$ & Teigwaren aus Hartgrieß \\
$80 \mathrm{~g}$ & Gouda Doppelrahmstufe \\
$20 \mathrm{~g}$ & Sahne 30\% Fett \\
$50 \mathrm{~g}$ & Trinkwasser \\
$0,5 \mathrm{TL} / 2 \mathrm{~g}$ & Jodiertes Salz \\
$0,2 \mathrm{I}$ & Natürliches Mineralwasser \\
\hline
\end{tabular}

\section{Zwischenmahlzeit}

\begin{tabular}{ll}
\hline 5 Stücke $/ 40 \mathrm{~g}$ & Butterkeks \\
\hline Abendessen & \\
\hline 2 Scheiben $/ 120 \mathrm{~g}$ & Vollkornbrot \\
$20 \mathrm{~g}$ & Butter \\
$2 \mathrm{Scheiben} / 50 \mathrm{~g}$ & Gouda Doppelrahmstufe \\
$30 \mathrm{~g}$ & Quark Doppelrahmstufe \\
1 Becher $/ 200 \mathrm{ml}$ & Tee schwarz fermentiert \\
\hline Kcal: 1968 & \\
\hline Eiweiß & \\
Fett & $61 \mathrm{~g}(13 \%)$ \\
Kohlenhydrate & $114 \mathrm{~g}(52 \%)$ \\
\hline
\end{tabular}

\section{Tag}

\section{Frühstück}

1 Stück/55 g

1 Scheibe/60 g

$20 \mathrm{~g}$

$30 \mathrm{~g}$

$15 \mathrm{~g}$

$114 \mathrm{~g}(52 \%)$

Mittagessen

\begin{tabular}{l|l}
\hline $200 \mathrm{~g}$ & Mais gegart \\
$3 \mathrm{Stücke} / 180 \mathrm{~g}$ & Kartoffeln geschält frisch gegart \\
$20 \mathrm{~g}$ & Butter
\end{tabular}

$20 \mathrm{~g}$ Butter

\begin{tabular}{ll}
\hline Abendessen & \\
\hline 1 Becher $/ 200 \mathrm{~g}$ & Tee schwarz fermentiert (Getränk) \\
1 Scheibe $60 \mathrm{~g}$ & Vollkornbrot \\
1 Scheibe $/ 50 \mathrm{~g}$ & Graubrot-Weizenmischbrot \\
2 Scheiben $/ 50 \mathrm{~g}$ & Gouda Doppelrahmstufe \\
$20 \mathrm{~g}$ & Butter \\
\hline kcal: 1634 & \\
\hline Eiweiß & $40 \mathrm{~g}(10 \%)$ \\
Fett & $86 \mathrm{~g}(48 \%)$ \\
Kohlenhydrate & $168 \mathrm{~g}(42 \%)$ \\
& \\
\end{tabular}




\begin{tabular}{|c|c|}
\hline \multicolumn{2}{|l|}{ Stufe 4: Ei } \\
\hline \multicolumn{2}{|l|}{ 1. Tag } \\
\hline \multicolumn{2}{|l|}{ Frühstück } \\
\hline 1 Becher $/ 200 \mathrm{ml}$ & Tee schwarz fermentiert (Getränk) \\
\hline 1 Scheibe/60 g & Vollkornbrot \\
\hline 1 Stück/55 g & Vollkornbrötchen \\
\hline 1 Stück/50 g & Hühnerei \\
\hline $20 \mathrm{~g}$ & Erdbeerkonfitüre \\
\hline $20 \mathrm{~g}$ & Butter \\
\hline
\end{tabular}

\begin{tabular}{l|l}
\hline Mittagessen (Käsespätzle) \\
\hline $100 \mathrm{~g}$ & Teigwaren (allgemein) Spätzle \\
$80 \mathrm{~g}$ & Gouda Doppelrahmstufe \\
$0,5 \mathrm{TL} / 2 \mathrm{~g}$ & Jodiertes Salz \\
$0,2 \mathrm{l}$ & Nätüliches Mineralwasser \\
\hline
\end{tabular}

\begin{tabular}{|c|c|}
\hline \multicolumn{2}{|c|}{ Zwischenmahlzeit } \\
\hline 5 Stücke $/ 40 \mathrm{~g}$ & Butterkeks \\
\hline \multicolumn{2}{|c|}{ Abendessen (Kartoffelsalat) } \\
\hline $\begin{array}{l}5 \text { Stücke/300 g } \\
0,5 \mathrm{TL} / 2 \mathrm{~g} \\
1 \mathrm{EL} / 10 \mathrm{~g}\end{array}$ & $\begin{array}{l}\text { Kartoffeln geschält frisch gegart } \\
\text { Jodiertes Salz } \\
\text { Sonnenblumenöl }\end{array}$ \\
\hline \multicolumn{2}{|l|}{ kcal 1691} \\
\hline $\begin{array}{l}\text { Eiweiß } \\
\text { Fett } \\
\text { Kohlenhydrate }\end{array}$ & $\begin{array}{l}54 \mathrm{~g}(13 \%) \\
76 \mathrm{~g} \mathrm{(40 \% )} \\
195 \mathrm{~g}(47 \%)\end{array}$ \\
\hline
\end{tabular}

\begin{tabular}{|l|l|}
\hline 2. Tag & \\
\hline Frühstück & \\
\hline 1 Stück/55 g & Vollkornbrötchen \\
$1 \mathrm{Scheibe} / 60 \mathrm{~g}$ & Vollkornbrot \\
$20 \mathrm{~g}$ & Butter \\
$30 \mathrm{~g}$ & Camembert 70\% F. i. Tr. \\
$20 \mathrm{~g}$ & Erdbeerkonfitüre \\
\hline
\end{tabular}

Mittagessen (Kartoffelpüree)

\begin{tabular}{l|l}
5 Stücke $/ 300 \mathrm{~g}$ & Kartoffeln geschält frisch gegart \\
$80 \mathrm{~g}$ & Kuhmilch vollfett \\
$0,5 \mathrm{TL} / 2 \mathrm{~g}$ & Jodiertes Salz \\
\hline
\end{tabular}

\begin{tabular}{ll}
\hline Abendessen & \\
\hline 1 Becher $/ 200 \mathrm{ml}$ & $\begin{array}{l}\text { Tee schwarz fermentiert (Getränk) } \\
\text { Vollkornbrot } \\
\text { Graubrot-Weizenmischbrot }\end{array}$ \\
1 Scheibe $/ 50 \mathrm{~g}$ & $\begin{array}{l}\text { Gouda Fettstufe } \\
\text { Butter }\end{array}$ \\
2 Scheiben $/ 50 \mathrm{~g}$ & \\
$20 \mathrm{~g}$ & \\
\hline Kcal 1406 & $39 \mathrm{~g} \mathrm{(11 \% )}$ \\
\hline Eiweiß & $70 \mathrm{~g}(45 \%)$ \\
Fett & $151 \mathrm{~g}(44 \%)$ \\
Kohlenhydrate & \\
\hline Stufe $5:$ Gemüse & \\
\hline 1. Tag & \\
\hline Frühstück & \\
\hline 1 Scheibe $/ 60 \mathrm{~g}$ & Vollkornbrot \\
1 Stück55 & Vollkornbrötchen \\
1 Stück50 g & Hühnerei \\
1 Scheibe $25 \mathrm{~g}$ & Gouda Fettstufe \\
$20 \mathrm{~g}$ & Erdebeerkonfitüre \\
$20 \mathrm{~g}$ & Margarine \\
\hline
\end{tabular}

\section{Mittagessen (Reispfanne mit Gemüse)}

\begin{tabular}{l|l}
\hline $150 \mathrm{~g}$ & Reis gegart \\
$50 \mathrm{~g}$ & Paprikaschoten frisch gegart \\
$50 \mathrm{~g}$ & Porree frisch gegart \\
$30 \mathrm{~g}$ & Zwiebeln frisch gegart \\
$50 \mathrm{~g}$ & Champignon frisch gegart \\
$0,2 \mathrm{l}$ & Natürliches Mineralwasser \\
$0,5 \mathrm{TL} / 2 \mathrm{~g}$ & Jodiertes Salz \\
$1,5 \mathrm{EL} / 15 \mathrm{ml}$ & Sonnenblumenöl \\
\hline Zwischenmahlzeit & \\
\hline $100 \mathrm{~g}$ & Birnenkompott gebunden \\
\hline $10 \mathrm{~g}$ & Zucker weiß \\
\hline
\end{tabular}

\section{Abendessen (Brot mit Salat aus gegartem Gemüse)}

\begin{tabular}{l|l}
\hline 1 Scheibe $/ 60 \mathrm{~g}$ & Vollkornbrot \\
$1 \mathrm{Scheibe} / 45 \mathrm{~g}$ & Graubrot-Roggenmischbrot \\
$20 \mathrm{~g}$ & Margarine \\
$30 \mathrm{~g}$ & Camembert Fettstufe \\
$80 \mathrm{~g}$ & Blumenkohl frisch gegart \\
$80 \mathrm{~g}$ & Mohrrüben frisch gegart \\
$0,5 \mathrm{TL} / 2 \mathrm{~g}$ & Jodiertes Salz \\
$1 \mathrm{EL} / 10 \mathrm{ml}$ & Sonnenblumenöl \\
\hline kcal 1590 & \\
\hline Eiweiß & \\
Fett & $44 \mathrm{~g}(11 \%)$ \\
Kohlenhydrate & $78 \mathrm{~g}(44 \%)$ \\
\hline
\end{tabular}

\section{Tag}

\section{Frühstück}

\begin{tabular}{l|l}
\hline 1 Stück/55 g & Vollkornbrötchen \\
1 Scheibe/60 g & Vollkornbrot \\
$20 \mathrm{~g}$ & Margarine \\
$30 \mathrm{~g}$ & Camembert Fettstufe \\
$20 \mathrm{~g}$ & Erdbeerkonfitüre \\
\hline
\end{tabular}

\section{Zwischenmahlzeit}

\begin{tabular}{|c|c|}
\hline $\begin{array}{l}100 \mathrm{~g} \\
10 \mathrm{~g}\end{array}$ & $\begin{array}{l}\text { Apfel frisch gegart } \\
\text { Zucker weiß }\end{array}$ \\
\hline \multicolumn{2}{|c|}{ Mittagessen (Gemüseeintopf) } \\
\hline $\begin{array}{l}3 \text { Stücke/180 g } \\
80 \mathrm{~g} \\
50 \mathrm{~g} \\
0,5 \mathrm{TL} / 2 \mathrm{~g}\end{array}$ & $\begin{array}{l}\text { Kartoffeln geschält frisch gegart } \\
\text { Broccoli frisch gegart } \\
\text { Kohlrabi frisch gegart } \\
\text { Jodiertes Salz }\end{array}$ \\
\hline \multicolumn{2}{|l|}{ Abendessen } \\
\hline $\begin{array}{l}1 \text { Becher } / 200 \mathrm{ml} \\
1 \mathrm{Scheibe} / 60 \mathrm{~g} \\
1 \text { Scheibe } / 50 \mathrm{~g} \\
50 \mathrm{~g} \\
2 \mathrm{Scheiben} / 50 \mathrm{~g} \\
20 \mathrm{~g} \\
1 \text { Stück } / 130 \mathrm{~g}\end{array}$ & $\begin{array}{l}\text { Tee schwarz fermentiert } \\
\text { Vollkornbrot } \\
\text { Graubrot-Weizenmischbrot } \\
\text { Avocado frisch } \\
\text { Gouda Fettstufe } \\
\text { Butter } \\
\text { Banane frisch }\end{array}$ \\
\hline \multicolumn{2}{|l|}{ kcal 1515} \\
\hline $\begin{array}{l}\text { Eiweiß } \\
\text { Fett } \\
\text { Kohlenhydrate }\end{array}$ & $\begin{array}{l}45 \mathrm{~g}(12 \%) \\
65 \mathrm{~g}(39 \%) \\
181 \mathrm{~g}(49 \%)\end{array}$ \\
\hline
\end{tabular}




\begin{tabular}{|c|c|c|c|}
\hline \multicolumn{2}{|l|}{ Stufe 6: Fisch } & \multicolumn{2}{|l|}{ Stufe 7: Fleisch } \\
\hline \multicolumn{2}{|l|}{ 1. Tag } & \multicolumn{2}{|l|}{ 1. Tag } \\
\hline \multicolumn{2}{|l|}{ Frühstück } & \multicolumn{2}{|l|}{ Frühstück } \\
\hline $\begin{array}{l}1 \text { Scheibe } / 60 \mathrm{~g} \\
1 \text { Stück/ } 55 \mathrm{~g} \\
1 \text { Scheibe } 25 \mathrm{~g} \\
20 \mathrm{~g}\end{array}$ & $\begin{array}{l}\text { Vollkornbrot } \\
\text { Vollkornbrötchen } \\
\text { Gouda Fettstufe } \\
\text { Erdbeerkonfitüre }\end{array}$ & $\begin{array}{l}2 \text { Stücke/ } 110 \mathrm{~g} \\
1 \text { Scheibe/30 g } \\
20 \mathrm{~g} \\
20 \mathrm{~g}\end{array}$ & $\begin{array}{l}\text { Vollkornbrötchen } \\
\text { Bratenfleisch } \\
\text { Erdbeerkonitüre } \\
\text { Margarine Linolsäurereich }\end{array}$ \\
\hline $20 \mathrm{~g}$ & Margarine & \multicolumn{2}{|c|}{ Mittagessen Huhn in Ananassoße } \\
\hline $\begin{array}{l}\text { Mittagessen } \\
150 \mathrm{~g} \\
150 \mathrm{~g} \\
150 \mathrm{~g} \\
30 \mathrm{~g} \\
0,2 \mathrm{I} \\
0,5 \mathrm{TL} / 2 \mathrm{~g} \\
1,5 \mathrm{EL} / 15 \mathrm{~g} \\
\end{array}$ & $\begin{array}{l}\text { Reis gegart } \\
\text { Fisch mittelfett } \\
\text { Mohrrüben frisch gegart } \\
\text { Zwiebeln frisch gegart } \\
\text { Natürliches Mineralwasser } \\
\text { Jodiertes Salz } \\
\text { Sonnenblumenöl } \\
\end{array}$ & $\begin{array}{l}150 \mathrm{~g} \\
120 \mathrm{~g} \\
50 \mathrm{~g} \\
\\
30 \mathrm{~g} \\
0,5 \mathrm{TL} \\
1 \mathrm{EL} / 2 \mathrm{~g} \\
0,21\end{array}$ & $\begin{array}{l}\text { Reis gegart } \\
\text { Geflügel gegart } \\
\text { Ananas Konserve abgetropft (1 } \\
\text { Scheibe) } \\
\text { Zwiebeln frisch gegart } \\
\text { Jodiertes Salz } \\
\text { Sonnenblumenöl } \\
\text { Natürliches Mineralwasser }\end{array}$ \\
\hline \multicolumn{2}{|c|}{ Zwischenmahlzeit } & \multicolumn{2}{|l|}{ Zwischenmahlzeit } \\
\hline 1 Stück/130 g & Birne frisch & 1 Becher/150 g & Fruchtjoghurt fettarm \\
\hline \multicolumn{2}{|l|}{ Abendessen } & \multicolumn{2}{|l|}{ Abendessen } \\
\hline $\begin{array}{l}1 \text { Scheibe/60 g } \\
1 \text { Scheibe/45 g } \\
20 \mathrm{~g} \\
30 \mathrm{~g} \\
80 \mathrm{~g} \\
80 \mathrm{~g} \\
0,5 \mathrm{TL} / 2 \mathrm{~g}\end{array}$ & $\begin{array}{l}\text { Vollkornbrot } \\
\text { Graubrot-Roggenmischbrot } \\
\text { Margarine } \\
\text { Camembert Fettstufe } \\
\text { Blumenkohl frisch gegart } \\
\text { Mohrrüben frisch gegart } \\
\text { Jodiertes Salz }\end{array}$ & $\begin{array}{l}1 \text { Scheibe/60 g } \\
1 \text { Scheibe } / 45 \mathrm{~g} \\
20 \mathrm{~g} \\
1 \text { Scheibe } / 30 \mathrm{~g} \\
1 \text { Stück } / 50 \mathrm{~g} \\
30 \mathrm{~g}\end{array}$ & $\begin{array}{l}\text { Vollkornbrot } \\
\text { Graubrot-Roggenmischbrot } \\
\text { Margarine } \\
\text { Schwein Bratenaufschnitt } \\
\text { Tomate frisch } \\
\text { Schafskäse 40\% F.i.Tr. }\end{array}$ \\
\hline $1 \mathrm{EL} / 10 \mathrm{~g}$ & Sonnenblumenöl & \multicolumn{2}{|l|}{ kcal 1645} \\
\hline \multicolumn{2}{|l|}{ kcal 1646} & \multirow{2}{*}{$\begin{array}{l}\text { Eiweiß } \\
\text { Fett } \\
\text { Kohlenhydrate }\end{array}$} & $79 \mathrm{~g}(19 \%)$ \\
\hline $\begin{array}{l}\text { Eiweiß } \\
\text { Fett }\end{array}$ & $\begin{array}{l}64 \mathrm{~g} \mathrm{(16 \% )} \\
78 \mathrm{~g}(78 \mathrm{~g}(43 \%)\end{array}$ & & $\begin{array}{l}69 \mathrm{~g}(37 \%) \\
177 \mathrm{~g}(43 \%)\end{array}$ \\
\hline Kohlenhydrate & $164 \mathrm{~g}(41 \%)$ & \multicolumn{2}{|l|}{ 2. Tag } \\
\hline \multicolumn{2}{|l|}{ 2. Tag } & \multicolumn{2}{|l|}{ Frühstück } \\
\hline \multicolumn{2}{|l|}{ Frühstück } & \multirow{2}{*}{$\begin{array}{l}1 \text { Stück/55 g } \\
1 \text { Scheibe/35 g } \\
20 \mathrm{~g} \\
25 \mathrm{~g} \\
20 \mathrm{~g}\end{array}$} & Vollkornbrötchen \\
\hline $\begin{array}{l}1 \text { Stück/55 g } \\
1 \text { Scheibe/60 g } \\
20 \mathrm{~g} \\
1 \text { Stück/90 g } \\
30 \mathrm{q}\end{array}$ & $\begin{array}{l}\text { Vollkornbrötchen } \\
\text { Vollkornbrot } \\
\text { Margarine } \\
\text { Kiwi frisch } \\
\text { Camembert Fettstufe }\end{array}$ & & $\begin{array}{l}\text { Weißbrot } \\
\text { Margarine } \\
\text { Frischkäse } \\
\text { Pflaumenmus }\end{array}$ \\
\hline $20 \mathrm{~g}$ & Erdbeerkonfitüre & \multicolumn{2}{|l|}{ Zwischenmahlzeit } \\
\hline \multicolumn{2}{|c|}{ Zwischenmahlzeit } & 1 Stück/150 g & Birne frisch \\
\hline 1 Stück/80 g & Apfel frisch & \multicolumn{2}{|c|}{ Mittagessen (Gulasch mit Kartoffeln und Bohnen) } \\
\hline \multicolumn{2}{|c|}{ Mittagessen (Gemüseeintopf) } & \multirow[b]{2}{*}{$\begin{array}{l}3 \text { Stücke/ } 180 \mathrm{~g} \\
120 \mathrm{~g} \\
30 \mathrm{~g} \\
0,5 \mathrm{TL} / 2 \mathrm{~g} \\
0,5 \mathrm{EL} / 5 \mathrm{~g} \\
150 \mathrm{~g}\end{array}$} & Kartoffeln geschält frisch gegart \\
\hline $\begin{array}{l}3 \text { Stücke/ } 180 \mathrm{~g} \\
80 \mathrm{~g} \\
50 \mathrm{~g} \\
0,5 \mathrm{TL} / 2 \mathrm{~g} \\
1 \mathrm{EL} / 10 \mathrm{~g}\end{array}$ & $\begin{array}{l}\text { Kartoffeln geschält frisch gegart } \\
\text { Broccoli frisch gegart } \\
\text { Kohlrabi frisch gegart } \\
\text { Jodiertes Salz } \\
\text { Sonnenblumenöl }\end{array}$ & & $\begin{array}{l}\text { Bratenfleisch } \\
\text { Zwiebeln frisch gegart } \\
\text { Jodiertes Salz } \\
\text { Sonnenblumenöl } \\
\text { Bohnen grün frisch gegart }\end{array}$ \\
\hline \multicolumn{2}{|c|}{ Zwischenmahlzeit } & \multicolumn{2}{|l|}{ Zwischenmahlzeit } \\
\hline 5 Stücke $/ 40 \mathrm{~g}$ & Butterkeks & 1 Stück $/ 80 \mathrm{~g}$ & Apfel frisch \\
\hline \multicolumn{2}{|l|}{ Abendessen } & \multicolumn{2}{|c|}{ Abendessen (Fleischsalat) } \\
\hline $\begin{array}{l}3 \text { Stücke/180 g } \\
200 \mathrm{~g} \\
20 \mathrm{~g} \\
1 \text { Stück } / 130 \mathrm{~g} \\
\end{array}$ & $\begin{array}{l}\text { Kartoffeln geschält frisch gegart } \\
\text { Fischfilet neapolitanisch, } \\
\text { in Soße (1) } \\
\text { Butter } \\
\text { Banane frisch } \\
\end{array}$ & $\begin{array}{l}2 \text { Scheiben } / 120 \mathrm{~g} \\
20 \mathrm{~g} \\
1 \mathrm{Sch} \text { heibe } / 25 \mathrm{~g} \\
80 \mathrm{~g} \\
20 \mathrm{~g} \\
20 \mathrm{~g}\end{array}$ & $\begin{array}{l}\text { Vollkornbrot } \\
\text { Margarine } \\
\text { Emmentaler } \\
\text { Rindfleisch gegart } \\
\text { Mayonnaise } \\
\text { Gewürzqurken Sauerkonserve }\end{array}$ \\
\hline \multicolumn{2}{|l|}{ kcal 1672} & \multicolumn{2}{|l|}{ kcal 1801} \\
\hline $\begin{array}{l}\text { Eiweiß } \\
\text { Fett } \\
\text { Kohlenhydrate }\end{array}$ & $\begin{array}{l}60 \mathrm{~g}(15 \%) \\
68 \mathrm{~g}(37 \%) \\
195 \mathrm{~g}(48 \%)\end{array}$ & $\begin{array}{l}\text { Eiweiß } \\
\text { Fett } \\
\text { Kohlenhydrate }\end{array}$ & $\begin{array}{l}90 \mathrm{~g}(20 \%) \\
85 \mathrm{~g}(43 \%) \\
163 \mathrm{~g}(37 \%)\end{array}$ \\
\hline
\end{tabular}




\subsection{Ernährung bei angeborenen Stoffwechselerkrankungen}

Aufgrund der speziellen Probleme angeborener Stoffwechselerkrankungen enthält dieses Kapitel über diätetisch beeinflussbare angeborene Stoffwechselerkrankungen neben kurzen Beschreibungen der klinischen Symptomatik und der biochemischen Folgen, die sich aus dem jeweiligen Stoffwechseldefekt ergeben, die Prinzipien der Behandlungsmöglichkeiten und deren praktische Umsetzung.

Alle Nährstoffgruppen - Eiweiß, Kohlenhydrate, Fette, Mineralstoffe/Spurenelemente und Vitamine - können von Stoffwechselstörungen betroffen sein. In diesem Buch interessieren nur die Störungen, die einer Ernährungsbehandlung zugänglich sind bzw. diese erforderlich machen. Sie lassen sich in Störungen des Transports (- Tab. 2.64), Störungen der Synthese und in Störungen des Abbaus (s. - Tab. 2.65) einteilen. Enzymdefekte können durch Veränderungen der katalytischen Eigenschaften des Enzyms bzw. der Synthese der Koenzyme bedingt sein. Schon der »Vater" der »Inborn errors of metabolism «, Sir Archibald Garrod, beschrieb 1908 in seinen Vorlesungen zu diesem Thema sowohl Störungen des Transports (Beispiel: Cystinurie) als auch Störungen des Abbaus (Beispiele: Pentosurie, Albinismus und Alkaptonurie).
- Abb. 2.19 veranschaulicht das allgemeine Prinzip der Pathogenese angeborener Stoffwechselerkrankungen und gibt Beispiele für die jeweilige Störung, die zur Krankheit führt:

- Beim angeborene Laktasemangel kommt es durch Verbleiben der nicht gespaltenen Laktose im Darm $\mathrm{zu}$ deren mikrobiologischer Spaltung mit schwersten osmotischen Durchfällen (Mechanismus a).

- Beim Hartnup-Syndrom kommt es bei schweren Verlaufsformen durch den Transportdefekt für neutrale Aminosäuren zu einem Mangel an Tryptophan (Mechanismus b), dem Vorläufer der Niacinsynthese, gleichzeitig durch die Resorption Endprodukte des bakterieller Tryptophanabbaus zur Indolurie (Mechanismus a).

- Die verminderte Umwandlung von Phenylalanin in Tyrosin führt zu einer Erhöhung des Phenylalanins in Blut und Geweben (Mechanismus c) mit Störungen der Myelinisierung ${ }^{4}$, gleichzeitig werden vermehrt sog. abnormale Metabolite gebildet, wie Phenylessigsäure (verantwortlich für den auffälligen Körpergeruch) (=Mechanismus e) bzw. wird vermindert Melanin gebildet (blond, blauäugig) (=Mechanismus d). ${ }^{4}$ Myelin, am Sphingmyelin und Cerebrosiden bestehende markhaltige
Nervenfasern.

- Tab. 2.64. Einteilung angeborener Stoffwechselerkrankungen: Störungen des Transports

\begin{tabular}{|c|c|}
\hline A & $\begin{array}{l}\text { Aminosäuren } \\
\text { Cystinurie } \\
\text { Diabasische Hyperaminoazidurie Typ I, Typ II (lysinurische Proteinintoleranz) } \\
\text { Hartnup-Syndrom } \\
\text { Cystinose (lysosomaler Transportdefekt) }\end{array}$ \\
\hline B & $\begin{array}{l}\text { Kohlenhydrate } \\
\text { Glukose-Galaktose-Malabsorption } \\
\text { Disaccharidintoleranz (Laktasemangel) }\end{array}$ \\
\hline$C$ & $\begin{array}{l}\text { Lipide } \\
\text { A- } \beta \text {-Lipoproteinämie } \\
\text { Hypo- } \beta \text {-Lipoproteinämie } \\
\text { Apoliprotein-C II-Mangel } \\
\text { Lipoproteinlipasemangel }\end{array}$ \\
\hline D & $\begin{array}{l}\text { lonen/Mineralien/Spurenelemente } \\
\text { Chloriddiarrhoe } \\
\text { Zystische Fibrose } \\
\text { Hyperkalzurie } \\
\text { Hypophosphatämische Rachitis } \\
\text { Primäre Hypomagnesiämie } \\
\text { Akrodermatitis enteropathica (Zink) } \\
\text { Menkes-Syndrom (Kupfer) } \\
\text { Wilson-Kranlheit (Kupfer) }\end{array}$ \\
\hline $\mathrm{E}$ & $\begin{array}{l}\text { Vitamine } \\
\text { Folatmalabsorption } \\
\text { Vitamin- } \text { B }_{12} \text {-Malabsorption (Imerslund-Gräsbeck) } \\
\text { Transcobalamin-II-Mangel } \\
\text { Vitamin-D-dependente Rachitis Typ II }\end{array}$ \\
\hline
\end{tabular}


- Tab. 2.65. Einteilung angeborener Stoffwechselerkrankungen.Störungen des Abbaus

\begin{tabular}{|c|c|}
\hline \multicolumn{2}{|l|}{ A Aminosäuren } \\
\hline $\begin{array}{l}\text { Enzymdefekte } \\
\text { Phenylketonurie/Hyperphenylalaninämie } \\
\text { Maternale Hyperphenylalaninämie } \\
\text { Tyrosinämie Typ I (Fumarylazetoazetase) } \\
\text { Tyrosinämie Typ II (Tyrosinaminotransferase) } \\
\text { Tyrosinämie Typ III (4-Hydroxyphenylpyruvatdioxigenase) } \\
\text { Ahornsirupkrankheit (MSUD) } \\
\text { Isovalerianazidurie } \\
\text { Methylglutaconazidurie } \\
\text { 3-hydroxy-3-Methylglutarazidurie } \\
\text { 3-Ketothiolase } \\
\text { Propionazidämie } \\
\text { Multipler Carboxylasemangel (Holocarboxylasesynthetase) } \\
\text { Methylmalonazidurie mut } \\
\text { Methylmalonazidurie mut } \\
\text { Homocystinurie (Cystathionin-ß-Synthase) } \\
\text { Hypermethioninämie (Methioninadensyltransferase) } \\
\text { Hyperlysinämie Typ I } \\
\text { Hyperlysinämie Typ II } \\
\text { Glutarazidurie I } \\
\text { Nicht-ketotische Hyperglycinämie } \\
\text { N-Acetylglutamatsynthetasedefekt } \\
\text { Carbamylphosphatsynthetasedefekt } \\
\text { Ornithinstranscarbamylasedefekt } \\
\text { Argininosuccinatsynthetasedefekt (Citrullinämie) } \\
\text { Argininosuccinatlyasedefekt (Argininbernsteinsäurekrankheit) } \\
\text { Arginasedefekt } \\
\text { Hyperornithinämie (Ornithin-5-Aminotransferase) } \\
\text { Hyperornithinämie-Hyperammon inämie-Homocitrullinurie (HHH- } \\
\text { Syndrom) }\end{array}$ & $\begin{array}{l}\text { Kofaktordefekte } \\
\text { Pterin-4a-Carbinolamindehydratase } \\
\text { Dehydropteridinreduktasemangel } \\
\text { GTP-Zyklohydrolasemangel } \\
\text { 6-Pyruvoyltetrahydropterinsynthase (PTPS)-Mangel } \\
\text { Thiaminabhängige MSUD } \\
\text { Biotinidasedefekt } \\
\text { Cbl A (Cobalamin-II-Reduktase) }^{\mathrm{a}} \\
\text { Cbl B (Adenosylcobalamintransferase) } \\
\text { Cbl C (Mangel an Adenosyl- und } \\
\text { Cbl D Methylcobalanin)c } \\
\text { Cbl E (Methylcobalaminmangel) } \\
\text { Cbl F (Mangel an Adenosyl- und Methylcobalamin) } \\
\text { Gbl G (Methioninsynthasemangel) }^{\mathrm{b}} \\
\text { Methylentetrahydrofolatreduktasemangel }\end{array}$ \\
\hline B Kohlenhydrate & \\
\hline
\end{tabular}

Pyruvatdehydrogenasemangel Glykogensynthetasemangel Glukose-6-Phosphatasemangel Phosphorylasemangel Phosphorylase-b-Kinasemangel Amylo-1,6-Glukosidasemangel Fruktose-1,6-Bisphosphatasemangel

Fruktose-1-Phosphataldolasemangel (hereditäre Fruktoseintoleranz)

Galaktokinasemangel

Galaktose-1-Phosphaturidyltransferasemangel)

UDP-Galaktose-4-Epimerasemangel

\section{Lipide}

Familiäre Hypertriglyzeridämie

Familiäre HyperCholesterinämie

Familiäre- $\beta$-Lipoproteinämie

Familiäre kombinierte Hyperlipoproteinämie

Carnitintransportdefekt

Carnitinpalmitoyltransferase-I-Mangel

Carnitinpalmitoyltransferase-II-Mangel

Acylcarnitintranslokasemangel

Kurzketten-Acyl-CoA-Dehydrogenasemangel

Mittelketten-Acyl-CoA-Dehydrogenasemangel

Langketten-Acyl-CoA-Dehydrogenasemangel

Sehr-Langketten-Acyl-CoA-Dehydrogenasemangel

Langketten-3-Hydroxyacyl-CoA-Dehydrogenasemangel

Langketten-3-Oxoacyl-CoA-Thiolasemangel

Mangel an trifunktionellem Protein

Multipler Acyl-CoA-Dehydrogenasemangel (Glutarazidurie Typ II)

- Mangel an elektronentransferierendem Faktor (ETF)

- ETF-Dehydrogenasemangel

- Riboflavinresponsiv

2,4-Dienoyl-CoA-Reduktasemangel

X-chromosomale Adrenoleukodystrophie

Refsum-Krankheit

${ }^{a}$ Methylmalonazidurie ${ }^{b}$ Homocystinurie und Hypomethioniinämie ${ }^{c}$ Methylmalonazidurie und Homocystinurie 
- Die Unfähigkeit, den Blutglukosespiegel durch Glukoneogenese bzw. Glykogenabbau aufrecht zu erhalten, führt bei der Glykogenose Typ I zu Hypoglykämie (Mechanismus d).

- Störungen der mitochondrialen $\beta$-Oxidation führen $\mathrm{zu}$ vermehrter mikrosomaler $\omega$ - und $\omega$-1-Oxidation von Fettsäuren (Mechanismus e). Bei den sog. "abnormalen" Metaboliten handelt es sich in aller Regel um normale Metaboliten, die wegen der Blockierung eines Stoffwechselweges jetzt im Übermaß gebildet werden. Sie dienen umgekehrt als diagnostische Marker und spielen pathogenetisch meist keine Rolle.

- Die verminderte Hämsynthese durch den Mangel an Porphobilinogendeaminase führt zu einer Aufhebung der negativen Kontrolle der Aktivität der $\delta$-Aminolävulinsäuresynthetase. Bei erhöhtem Bedarf an Häm kommt es über die Stimulation der $\delta$-Aminolävulinsäuresynthetase zu vermehrter Bildung von $\delta$-Aminolävulinsäure (Mechanismus $\mathrm{f}$ ) und (wegen des angeborenen Enzymdefekts) von Porphobilinogen, beide Metaboliten sind für die Symptomatik der akuten intermittierenden Porphyrie verantwortlich.

In allen Fällen ist aus der Kenntnis der Pathogenese bereits das therapeutische Vorgehen abzuleiten. Praktisch heißt das, nachdem die Diagnose aufgrund von Anamnese, Klinik, biochemischen Daten, evtl. Belastungstests gestellt und möglichst bestätigt durch Bestimmung des Defekts in in-vitro-Untersuchungen bestätigt ist, gibt der biochemisch bewanderte Arzt die Grundzüge der

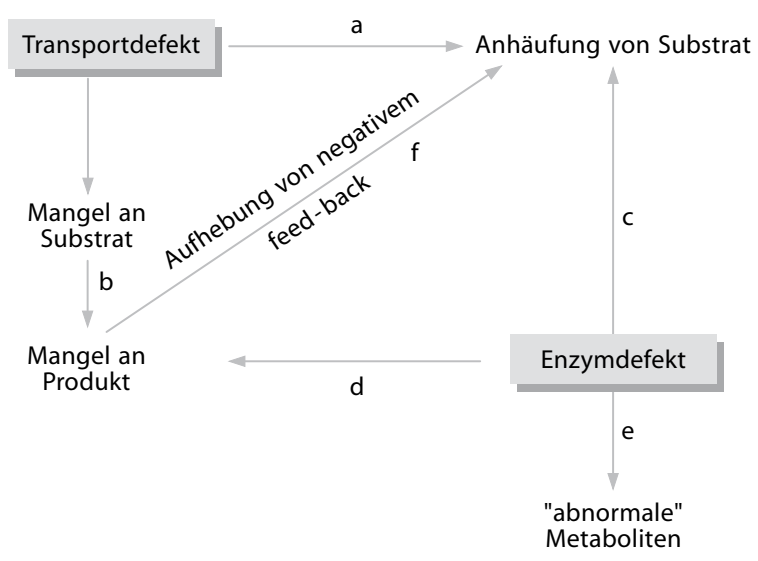

Beispiele:

a) Laktasemangel

b) Hartnup-Syndrom

c) Phenylketonurie (Phenylalaninhydroxylase)

d) Glykogenose Typ I (Glukose-6-Phosphatase)

e) Dicarbonsäuren (Störungen der mitochondrialen $\beta$-Oxidation)

f) akute intermittierende Porphyrie

- Abb. 2.19. Schematische Darstellung und Charakterisierung verschiedener angeborener Stoffwechseldefekte
Behandlung vor und überwacht deren Umsetzung in ein ernährungstherapeutisches Regime durch eine erfahrene Diätassistentin sowie im Verlauf den Erfolg der Behandlung anhand von klinischen (Wachstum, Gedeihen, psychische, intellektuelle, neurologische und motorische Entwicklung) und biochemischen Parametern (in Abhängigkeit vom jeweils vorliegenden Defekt). Ein ernährungstherapeutisches Regime kann das psychologische und soziale »Klima« in einer Familie schwerwiegend beeinflussen. Die kontinuierliche Unterstützung durch Psychologen, Sozialarbeiter und speziell geschulte Pflegekräfte ist daher erforderlich. Da die Diagnose in der Regel im Säuglings- oder Kindesalter gestellt wird und die Behandlung meist lebenslang erfolgen muss, sind eine Einbeziehung der ganzen Familie in die Betreuung notwendig, eine frühzeitige Schulung des Patienten zur Selbständigkeit durch Krankheitseinsicht, angepasst an das Verständnis des jeweiligen Alters und im Idealfall eine sorgfältig vorbereitete Übergabe an die »Erwachsenenmedizin.

\subsubsection{Aminosäuretransportstörungen}

Aminosäurentransportstörungen können sowohl die Darmschleimhaut wie den Nierentubulus betreffen. Im letzteren Falle kommt es zu einer Hyperaminoazidurie mit spezifischem Muster je nachdem, welches Transportsystem betroffen ist.

\section{Cystinurie}

Autosomal-rezessiv vererbte Störung des tubulären und intestinalen Transports von Cystin, Lysin, Arginin und Ornithin mit mindestens 3 allelen Mutationen. Die vermehrte Cystinkonzentration im Urin $(\leq 100 \mathrm{mg} / \mathrm{l})$ führt wegen dessen geringer Löslichkeit zur Bildung von Cystinsteinen. Abgesehen von einer hohen Wasserzufuhr, die sich nach der Cystinkonzentration in Tag- und Nachturinen richten muss, sind in der Regel keine ernährungstherapeutischen Maßnahmen notwendig. Eine Alkalisierung des Urins zur Verbesserung der Cystinlöslichkeit erfordert hohe Pufferdosen (Urin-pH >7,5). Nur bei Säuglingen kann evtl. der intestinale Transportdefekt eine erhöhte Eiweißzufuhr rechtfertigen.

Medikamentöse Behandlung. D-Penicillamin, N-Azetylpenicillamin, Mercaptopropionylglycin (Nebenwirkungen!) nur bei Versagen der konservativen Behandlung (s.oben).

\section{Dibasische Hyperaminoazidurie}

Die autosomal-rezessiv vererbte vermehrte Ausscheidung von den basischen Aminosäuren Lysin, Ornithin 
und Arginin bei normaler oder gering erhöhter Ausscheidung von Cystin kommt in 2 unterschiedlichen Formen vor:

- Typ I: Störung des luminalen Transportes von den genannten Aminosäuren, die keiner Behandlung bedarf.

- Typ II (lysinurische Proteinintoleranz): Störung des Aminosäurentransports an der basolateralen Zellmembran in Dünndarm und Nierentubulus. Es kommt zu einer Erniedrigung dieser Aminosäuren im Plasma und, auf bisher ungeklärte Weise, zu einer Funktionsstörung des Harnstoffzyklus mit Proteinintoleranz, Hyperammonämie und Orotazidurie.

Während gestillte Säuglinge in der Regel symptomfrei sind, führt eine höhere Eiweißzufuhr zu Nahrungsverweigerung, Erbrechen, Gedeihstörungen, Bewusstseinseintrübung und im chronischen Verlauf $\mathrm{zu}$ Minderwuchs, Osteoporose, Anfälligkeit für interstitielle Pneumonien. Die geistige Entwicklung kann normal sein, wenn schwere und langdauernde hyperammonämische Perioden vermieden werden. Die Behandlung besteht in einer Einschränkung der Eiweißzufuhr und der Substitution von Citrullin, das normal transportiert wird und die Funktion des Harnstoffzyklus verbessern kann. Die vertragene Eiweiß- und notwendige Citrullinmenge, verteilt auf mindestens 5 Mahlzeiten pro Tag, muss sorgfältig austitriert werden anhand von postprandialen Ammoniakblutspiegeln und Orotsäureausscheidung im Urin. Der Transportdefekt kann nicht durch die Gabe von Aminosäurenpeptiden überspielt werden.

\section{Hartnup Syndrom}

Autosomal-rezessiv vererbte Transportstörung für neutrale Aminosäuren im Intestinum und Nierentubulus, die einhergeht mit dem typischen Aminosäurenausscheidungsmuster, einer Indolurie und Zeichen eines Niacinmangels. Die beiden letzteren sind Folge der Malabsorption von Tryptophan, das im Körper nicht für eine Synthese von Niacin zur Verfügung steht. Nur bei gleichzeitiger mangelnder Versorgung mit Niacin aus der Nahrung kommt es zu pellagraähnlichen Symptomen mit lichtabhängigen Hauterscheinungen sowie Ataxie und gelegentlich Entwicklungsrückstand.

Medikamentöse Behandlung. Nikotinamid in pharmakologischen Dosen.

\section{Cystinose}

Sie ist eine lysosomale Speicherkrankheit, die autosomalrezessiv vererbt wird und auf einer Störung im Transport von intralysosomal durch Hydrolyse entstandenem Cystin beruht. Sie kann daher grundsätzlich fast alle Zellen und Organe betreffen. 3 klinische Verlaufsformen werden unterschieden:

- Infantile Form: Mit frühzeitiger Störung aller renalen tubulären Transportsysteme (DeToni-Debré-Fanconi-Syndrom), mit Minderwuchs, renaler Rachitis, Cystinspeicherung in Kornea, Knochenmark, endokrinen Organen und frühzeitigem Tod durch Niereninsuffizienz;

- Adulte Form: Ohne Nierenfunktionsstörung und mit Cystinspeicherung in Kornea und Knochenmark;

- Juvenile Form: Späterer Beginn und langsamerer Verlauf der Nierenfunktionsstörung.

Die Behandlung der infantilen Form besteht in adäquatem Ersatz von Wasser, Elektrolyt- und Basenverlusten, Supplementierung mit Thyroxin, 1,25- Dihydroxycholecalciferol und ggf. Carnitin und Insulin. Die Dialyse dient als Überbrückung bis zur Nierentransplantation. Cysteamin bzw. Phosphocysteamin können intralysosomales Cystin durch Bildung von Cysteincysteamindisulfiden entspeichern. Eine diätetische Behandlung durch Verringerung der Zufuhr von schwefelhaltigen Aminosäuren ist unwirksam.

\subsubsection{Kohlenhydrattransportstörungen}

Ein Mangel des Glukosetransporters (GLUT1), der für den Transport von Glucose über die Bluthirnschranke verantwortlich ist, wird autosomal dominant vererbt. Er manifestiert sich im frühen Säuglingsalter in Form von Krampfanfällen, Entwicklungsrückstand und zunehmender Mikrozephalie. Typisch ist eine Erniedrigung des Quotienten aus den Glukosekonzentrationen in Liquor und Blut $(<0,4)$. Die Patienten sprechen gut auf eine ketogene Diät an (Fett zu Kohlenhydraten/Eiweiß gewichtsbezogen im Verhältnis von 4 bis 2:1) (s. - Kap. 2.5.4).

Bei den folgenden angeborenen Störungen des Kohlenhydrattransports handelt es sich entweder um Störungen des natriumabhängigen Transports von Monosacchariden oder um Störungen der im intestinalen Bürstensaum lokalisierten Disaccharidhydrolasen. Allen Störungen gemeinsam sind osmotische Durchfälle bei Zufuhr der betroffenen Zucker und niedrige $\mathrm{pH}$-Werte im Stuhl, durch bakterielle Vergärung der nicht resorbierten Zucker im Dickdarm.

\section{Glukose-Galaktose-Malabsorption}

Die Glukose-Galaktose-Malabsorption ist sehr selten. Neben dem Dünndarm kann auch der Nierentubulus betroffen sein (renale Glukosurie). Alle glukose- oder galaktosehaltigen Zucker werden vom 1. Lebenstag an nicht vertragen, so dass als einziges mögliches Kohlenhydrat 
Fruktose übrigbleibt. Die hiervon tolerierte Menge wird individuell ausgetestet. Mit zunehmendem Alter nimmt in der Regel die Verträglichkeit von Stärke zu.

\section{Saccharase-Isomaltasemangel}

Autosomal-rezessiv und genetisch heterogen, geht er mit einer Intoleranz für Saccharose und isomaltosehaltige Stärken einher, die aus der Nahrung eliminiert werden müssen. Die Verträglichkeit von Stärke muss ausgetestet werden.

\section{Laktasemangel (Laktoseintoleranz) (s. Kap. 2.5.4)}

Dieser tritt primär genetisch bedingt in 2 Formen auf:

- Angeborener Laktasemangel: Autosomal-rezessiv und sehr selten, mit heftigen Durchfällen von der ersten Milchfütterung an. Der Milchzucker in der Nahrung muss weggelassen und durch Glukose, Saccharose, Maltodextrin oder Stärke ersetzt werden;

- »Erworbener« Laktasemangel: Er führt genetisch bedingt und mit geographisch sehr unterschiedlicher Inzidenz (Europa ca. 5\%, Afrika, Asien, Alaska bis 90\%), im 2. Lebensjahr zunehmend zu einer Abnahme der Laktaseaktivität und Intoleranz für Laktose mit Durchfällen und Bauchschmerzen. Die individuell vertragene Laktosemenge muss ausgetestet werden. Yoghurt und Käse werden in der Regel vertragen. Wie bei allen Ernährungsformen mit Verzicht auf Milch und ggf. Milchprodukte muss auf eine ausreichende Kalziumzufuhr geachtet werden.

Ein sekundärer Laktasemangel ist in seiner Symptomatik der primären Form ähnlich. Er wird in der Folge von entzündlichen Darmerkrankungen und z. B. bei der Zöliakie beobachtet und bildet sich mit Behandlung der Grundkrankheit zurück.

\subsubsection{Lipidtransportstörungen}

Als genetische Störungen des Transports (exogener sowie endogener Lipide) sollen hier Defekte vom Apolipoprotein B (A-b- sowie Hypo-b-lipoproteinämie) und der Lipoproteinlipase genannt sein (Lipoproteinlipasemangel und Apolipoprotein-CII-Mangel).

\section{Apolipoprotein-B-Defekte}

Diese führen zu Hypocholesterinämie, Fettmalabsorption, neuromuskulären Störungen, Störungen der Hämatopoese mit Akanthozytose und Retinitis pigmentosa (Bassen-Kornzweig-Syndrom, autosomal-rezessiv, bzw. Hypo-b-Lipoproteinämie, autosomal-dominant). Die Behandlung besteht in Einschränkung der Fettzufuhr bei ausreichender Energiezufuhr und Supplementierung der fettlöslichen Vitamine, insbesondere von Vitamin E in pharmakologischen Dosen.

\section{Defekt der Lipoproteinlipase}

Er manifestiert sich in der Regel im Säuglings- und Kindesalter als Hyperchylomikronämie mit massiver Hypertriglyzeridämie, Hepatosplenomegalie, Lipaemia retinalis und eruptiven Xanthomen. Vor einer diätetischen Behandlung mit Beschränkung der Fettzufuhr auf 10-20\% der Gesamtenergiezufuhr unter teilweisem Ersatz des Fettes durch mittelkettige Triglyzeride (MCT, s. - Kap. 2.5.4) kann das SerumCholesterin deutlich erhöht sein. Der Apolipoprotein-CII-Mangel und der Defekt der hepatischen Lipase zeigen ähnliche Symptome, allerdings treten diese erst in späteren Alter auf und erfordern eine ähnliche Behandlung.

\subsubsection{Transportstörungen für Mineralstoffe bzw. Spurenelemente}

(Magnesium, Zink, Phosphat, Kupfer, Kalzium und Chlorid).

Eine genetisch bedingte autosomal rezessiv vererbte Hypomagnesiämie kann Folge eines intestinalen Magnesiumabsorptionsdefekts sein (Hypomagnesiämie mit sekundärer Hypokalzämie) oder Folge eines renalen tubulären Reabsorptionsdefekts (Familiäre Hypomagnesiämie mit Hyperkaziurie und Nephrokalzinose).

\section{Intestinale Hypomagnesiämie}

Sie geht mit einer Hypokalzämie einher und wird bereits in den ersten Lebenswochen symptomatisch mit Übererregbarkeit, Nahrungsverweigerung, Tetanie und generalisierten Krampfanfällen. Typisch ist eine niedrige Magnesiumausscheidung im Urin. Bei frühzeitiger Diagnose und Behandlung ist die Prognose gut. Nach anfänglicher i.v.-Magnesiumzufuhr wird die orale $\mathrm{Zu}$ fuhr verteilt auf 3-6 Dosen eingeführt (bis zu $5 \mathrm{mmol}$ Magnesium/kgKG/Tag) unter Vermeidung von Durchfällen. Die Behandlung ist lebenslang notwendig. Kalzium, Vitamin D oder Parathormon beeinflussen die Hypokalzämie und die Symptome nicht.

\section{Familiäre Hypomagnesieämie}

Familiäre Hypomagnesieämie als Folge eines genetischen Defekts in der renalen Magnesiumrückresorption kann im Kindesalter zu Tetanie führen, sie wird aber häufig erst im Erwachsenenalter erkannt. In 50\% der Fälle besteht dann bereits eine Nephrokalzinose, seltener eine Chondrokalzinose oder Osteochondrose. 
Die erhöhte Magnesiumausscheidung bei Hypomagnesiämie kann von anderen tubulären Funktionsstörungen begleitet sein: tubuläre Azidose, Glukosurie, Hyperaminoazidurie, Hyperkalziurie und Hyperkaliurie. Eine Magnesiumsubstitution ist nur in der Hälfte der Fälle erfolgreich.

\section{Akrodermatitis enteropathica}

Ursache ist eine intestinale Zinkabsorptionsstörung mit niedrigen Plasmazinkspiegeln und erniedrigter Aktivität zinkhaltiger Metalloenzyme. Typisch sind periorale und akrale Hautveränderungen sowie Schleimhautveränderungen mit Anorexie, Durchfällen und Gedeihstörungen, beginnend im frühen Säuglingsalter bei Formulafütterung bzw. nach Abstillen. Bei frühzeitiger und ausreichender Behandlung mit Zinksalzen (Anpassung während $\mathrm{Pu}$ bertät und insbesondere während Schwangerschaft und Stillzeit) ist die Prognose gut.

\section{Wilsonschen-Krankheit}

Die Ursache dieser autosomal-rezessiven Störung der Kupferhomöostase mit Kupferablagerung in verschiedenen Organen, sind unterschiedliche Mutationen im ATP7B-Gen, das für eine kupfertransportierende ATPase kodiert. Typisch sind erhöhte Kupferkonzentrationen in der Leber, eine erhöhte Kupferausscheidung im Urin und erniedrigte Plasmacoeruloplasminspiegel (s. $\triangleright$ Kap. 1.6.11). Die Symptomatologie im Kindesalter ( $>7$ Jahre) betreffen vorwiegend die Leberfunktion oder eine akute hämolytische Anämie. Die für das Erwachsenenalter typischen neurologischen Symptome (extrapyramidale Bewegungsstörungen, Verhaltensstörungen bzw. Psychosen) werden in der Regel vor der Pubertät nicht gesehen oder aber als Folge einer hepatischen Enzephalopathie (mit sehr schlechter Prognose, falls akut keine Lebertransplantation möglich ist). Ein Kayser-Fleischer-Ring wurde frühestens im Schulalter beobachtet. Das Behandlungsziel ist die Verringerung des Körpergesamtkupfers, entweder durch D-Penicillamin unter gleichzeitiger Supplementierung von Vitamin $B_{6}$, durch Zinksulfat bzw. -azetat oder durch Trientindihydrochlorid. Unter der medikamentösen Behandlung nimmt zunächst die Kupferausscheidung stark zu. Anfangs sollte eine kupferarme Diät $(<1,5-2 \mathrm{mg}$ Kupfer/Tag) durch Auswahl geeigneter kupferarmer Lebensmittel und unter Berücksichtigung der Kupferkonzentration im Trinkwasser (anstreben $<0,1 \mathrm{mg} / \mathrm{l}$ ) gegeben werden.

\section{Menkes-Syndrom}

Diese Krankheit wird X-chromosomal vererbt und beruht auf verschiedenen Mutationen im ATP7A-Gen, das für ein Protein kodiert, das Kupfer zu kupferabhängigen
Enzymen transportiert.Es kommt zu einer Kupferverteilungsstörung im Körper mit zunehmend niedrigen Kupferspiegeln in Serum und Leber, niedrigen Coeruloplasminspiegeln und erhöhten Kupfergehalten in z. B. Darm und Niere. Die betroffenen Jungen zeigen frühzeitig eine Regression, neurologische Symptome, Krampfanfälle, Osteoporose, brüchiges Haar und einen typischen Gesichtsausdruck und sterben meist vor Ende des 3. Lebensjahres. Frühzeitige, i.e. vor Auftreten von neurologischen Symptomen, einsetzende Behandlung mit Kupferhistidininjektionen kann offenbar neurologische Symptome verhindern, eine fast normale mentale Entwicklung ermöglichen und die Lebenszeit verlängern. Wahrscheinlich ist unter der Behandlung eine jährliche Kontrolle des Leberkupfergehalts erforderlich.

\section{Chloriddiarrhoe}

Die autosomal rezessiv vererbte Erkrankung beruht auf Mutationen in einem für ein Transportprotein des Darmes kodierenden Gen mit einer Störung der Chloridabsorption im unteren Ileum und Kolon, Frühgeburtlichkeit bei Hydramnion und wässrigen Durchfällen (Chloridgehalt typisch höher als die Summe aus Natrium und Kalium) mit metabolischer Alkalose, Hypochlorämie, Dehydration und sekundärem Hyperaldosteronismus. Die Behandlung besteht in Substitution (parenteral, später oral) von Natrium- und Kaliumchlorid bis zur Erzielung normaler Serumelektrolytwerte und einer geringen Chloridausscheidung im Urin.

\section{Hyperkalziurie}

Sie ist entweder Folge einer vermehrten Kalziumaufnahme im Darm auf Grund verschiedener Defekte in verschiedenen Genen (1,25-Dihydroxycholecalciferol im Serum erhöht) oder einer verminderten Kalzium- und Phosphatrückresorption im Nierentubulus mit hypophosphatämischer Rachitis. Im ersteren Falle sind eine kalziumarme Ernährung sowie ggf. Kationenaustauscher oder Phosphatsalze angebracht.

\section{Hypophosphatämische X-chromosomale Vitamin-D-resistente Rachitis}

Hypophosphatämische X-chromosomale Vitamin-Dresistente Rachitis (sog. Phosphatdiabetes). Sie beruht auf einer verminderten Phosphatrückresorption im proximalen Nierentubulus und führt bei den betroffenen Jungen (heterozygote Mädchen zeigen mildere Symptome) trotz üblicher Rachitisprophylaxe zu einer Symptomatik mit dysproportioniertem Minderwuchs, Hypophosphatämie, Hyperphosphaturie und Hyperphosphatasie. Die Behandlung besteht in der Anhebung des Serumphosphats auf annähernd normale Werte 
durch Phosphatsubstitution, möglichst auf 6 Dosen gleichmäßig über Tag und Nacht verteilt, der Gabe von 1,25-Dihydroxycholecalciferol bis zu $1 \mathrm{mg} / \mathrm{Tag}$ und einer reduzierten Natriumzufuhr mit der Nahrung. Die Calcitriolbehandlung kann nach vollendetem Wachstum abgesetzt werden.

\section{Zystische Fibrose (Mukoviszidose)}

Es handelt sich um eine Transportstörung für Chloridionen aufgrund eines autosomal-rezessiv vererbten Defektes des "Zystische«-Fibrose-Transmembran-Conduction-Regulators (CFTR). Die häufigste Mutation $\Delta \mathrm{F} 508$ resultiert in einem abnormalen Eiweiß, das seinen Wirkungsort in der apikalen Plasmamembran nicht erreicht. Betroffen sind die Ausführungsgänge aller exokrinen Drüsen, die durch zähes Sekret verstopft sind (Atemwege, Pankreas). Die Chloridkonzentration im Schweiß ist erhöht $(>60$; normal $<25 \mathrm{mmol} / \mathrm{l})$. Die Krankheit führt zu chronischen Atemwegserkrankungen, Durchfällen im Säuglingsalter, Malabsorption durch Pankreasinsuffizienz, Leberzirrhose und Diabetes mellitus durch Pankreasfibrose. In der Behandlung sind neben Kochsalzsupplementierung, insbesondere im Säuglingsalter, eine ausreichend hohe Substitution mit Pankreasenzymen und eine energiereiche (ca. $120 \%$ des altersentsprechenden Bedarfs) und eiweißreiche Kost sowie eine konsequente medikamentöse und physiotherapeutische Behandlung von Atemwegsinfektionen entscheidend. Bei ausreichender Pankreasenzymsubstitution besteht kein Grund zu einer Fettreduktion in der Ernährung. Auf die ausreichende Versorgung mit essentiellen Fettsäuren, fettlöslichen Vitaminen (auch wasserlösliche Vitamine ggf. substituieren!), Mineralstoffen und Spurenelementen ist sorgfältig zu achten. Für anorektische Patienten hat sich eine nächtliche unterstützende Ernährungsbehandlung mit hochkalorischen bilanzierten Diäten (eiweißreich, nicht fettarm) über eine perkutan gelegte, endoskopisch geführt platzierte Gastrostomiesonde (=PEG) bewährt.

\subsubsection{Vitamintransportstörungen}

\section{Hereditäre Folatmalabsorption}

Sie ist ein seltenes Krankheitsbild und Folge eines Transportdefekts für Monoglutamatfolsäure an der Dünndarmschleimhaut und am choroidalen Plexus. Frühzeitige Gedeihstörungen mit Durchfällen, Mundschleimhautläsionen, eine megaloblastäre Anämie, geistige Retardierung und neurologische Auffälligkeiten sind die Folge. Behandlung mit Folsäure, Folinsäure (oral oder parenteral) und Methyltetrahydrofolsäure (bis $100 \mathrm{mg} /$ Tag) heilt die Anämie und normalisiert die Folatspiegel im Serum, nicht jedoch in allen Fällen die Folatspiegel im Liquor.

\section{Vitamin- $\mathbf{B}_{12}$-Malabsorption}

Mit erniedrigten Vitamin- $B_{12}$-Spiegeln im Serum, megaloblastärer Anämie und neurodegenerativen Symptomen kann sie die Folge eines genetisch bedingten Mangels an Intrinsic-factor sein (ohne Magenschleimhauterkrankung) oder Folge eines intrinsic factor-Rezeptor (Cubilin)-Mangels (Imerslund-Gräsbeck-Syndrom), bei dem typischerweise eine Proteinurie beobachtet wird. Beide Arten von Defekten sprechen in bezug auf die Anämie gut auf parenterale Vitamin- $\mathrm{B}_{12}$-Gaben an (1-5 lg/Tag). Das Manifestationsalter ist das 2. Lebensjahr bis Ende des 4. Lebensjahres.

\section{Transcobalamin-II-Mangel}

Dieser wird autosomal-rezessiv vererbt und führt wegen mangelnden Transports und demzufolge rezeptorvermittelter zellulärer Vitamin- $B_{12}$-Aufnahme zu einem intrazellulären Cobalaminmangel mit megaloblastärer Anämie oder Panzytopenie, schweren neurodegenerativen Störungen und Immunschwäche, manifest bereits im 1. und 2. Lebensmonat. Diese Patienten benötigen sehr viel höhere Vitamin- $B_{12}$-Dosen (1 mg Hydroxocobalamin/Tag initial). Alle Formen des Vitamin- $\mathrm{B}_{12}$-Mangels können mit einer mäßigen Methylmalonazidurie und (seltener) Homocystinurie einhergehen.

\section{Vitamin D-abhängige Rachitis Typ II}

Autosomal rezessiv vererbt beruht sie auf einem Rezeptordefekt für 1,25-Dihydroxycholecalciferol, dessen Serumspiegel erhöht sind. Die Krankheit zeigt alle Symptome der Vitamin-D-Mangelrachitis plus typisch eine Alopezie und kann sich im Säuglings- bzw. Erwachsenenalter erstmals manifestieren. Die Behandlung erfolgt mit hohen Dosen Calcitriol (10-50 mg/Tag) und Kalziumsupplementierung.

\subsubsection{Biosynthesestörungen}

\section{Serin}

Obwohl Serin keine unverzichtbare Aminosäure ist und im menschlichen Körper aus Glyzin gebildet werden kann, scheint die normale fetale Entwicklung und das Funktionieren bestimmter Organe, insbesondere des Gehirns von einer unbeeinträchtigten de-novo Biosynthese abzuhängen. Ein Defekt des ersten Enzyms der Serinsynthese, der Phosphoglyzerat-Dehydrogenase führt zu neurologischen Symptomen, angeborener Mikrozephalie, Tetraspastik, Krampfanfällen und schwerem Entwicklungsrückstand. Charakteristisch ist eine Erniedrigung der Konzentration von Serin, manchmal auch Glyzin, in Plasma und Liquor, wobei die Liquoranalyse aussagefräftiger ist. Eine frühzeitige Behandlung mit Gabe von 
hohen Dosen Serin $(600 \mathrm{mg} / \mathrm{kgKG} / \mathrm{Tag})$ allein oder in Kombination mit Glyzin ist erfolgreich in Bezug auf die Spastik, führt aber nicht in allen Fällen zur Anfallsfreiheit. Nur die jüngste bisher behandelte Patientin (7 Monate) zeigte unter der Behandlung ein Aufholwachstum und ein Fortschreiten der psychomotorischen Entwicklung auf niedrigem Niveau. Ein Defekt des dritten Enzyms der Serinsynthese, der Phosphoserinphosphatase wurde bisher nur bei einem Patienten gefunden und führte ebenfalls zu niedrigen Serinkonzentrationen im Liquor, jedoch zu einem milderen Krankheitsbild, das gut auf Seringaben anspricht. Da Serin in der Niere nur mit geringer Affinität rückresorbiert wird, ist eine Verteilung der Serindosis auf viele kleine Einzelgaben notwendig. Beide Serinbiosynthesedefekte sind selten und können übersehen werden, wenn erniedrigte Aminosäurenspiegel übersehen werden.

\section{Mannosephosphatisomerase-Mangel (Angebo- rene Störung der Glykosylierung Typ Ib, CDG lb)}

Angeborene Störungen der Glykosylierung sind erbliche Multisystemerkrankungen, die wegen der verkürzten oder fehlenden Kohlenhydratseitenketten von Glykoproteinen und -lipiden in der isoelektrischen Fokussierung von z. B. Serumtransferrin abnormale Muster aufweisen. Der Typ Ib unterscheidet sich von den zehn anderen bisher bekannten Typen, die alle mit schwerem geistigen und motorischen Rückstand, Blutgerinnungsstörungen und schlaganfallähnlichen Episoden einhergehen, durch die fehlende Retardierung und die Manifestation als Eiweißverlustenteropathie mit Durchfall, Erbrechen, Hypoglykämie und Leberfibrose. Durch den Enzymdefekt ist die Umsetzung von Fruktose-6-Phosphat in Mannose-6-Phosphat gestört, das aber für die meisten Glykosylierungsreaktionen im Körper benötigt wird. Da die Mannoseaufnahme mit der Nahrung nur gering ist und bei Störung der endogenen Synthese unzureichend ist, bot sich eine orale Supplementierung mit Mannose an $(0,5-1,0 \mathrm{~g} / \mathrm{kgKG} / \mathrm{Tag}$, verteilt auf fünf Dosen). Unter der Behandlung verschwinden die Symptome und normalisieren sich die Serumproteine, die Blutgerinnung, die Blutglukose und langfristig das Muster des Transferrins in der isoelektrischen Fokussierung. Bei Überdosierung treten osmotische Durchfälle und erhöhte $\mathrm{HbA}_{1} \mathrm{C}$-Spiegel auf.

\subsubsection{Aminosäurenabbaustörungen}

Dazu werden in diesem Kapitel sowohl die Störungen gezählt, die zu spezifischen Hyperaminoazidämien führen, weil der Defekt die ersten Stufen des Ab- oder Umbaus betrifft, als auch diejenigen, die mit einer typischen Metabolitenausscheidung im Urin (organische Azidurien) und nicht oder unregelmäßig einer Hyperaminoazidämie einhergehen, weil der Defekt späte Stufen des Abbaus der jeweiligen Aminosäure betrifft. Störungen des Abbaus unverzichtbarer Aminosäuren lassen sich, durch Reduktion ihrer Zufuhr bzw. Förderung der Ausscheidung ihrer Metaboliten, grundsätzlich erfolgreicher behandeln als Störungen im Stoffwechsel verzichtbarer (=nicht essentieller) Aminosäuren, die bei letzteren die endogenen Synthese (Beispiel: nicht-ketotische Hyperglyzinämie) nicht kontrollierbar ist. In der Behandlung von Abbaustörungen essentieller Aminosäuren muss selbstverständlich der individuelle Bedarf für die jeweils betroffene(n) Aminosäure(n) gedeckt werden, der sich zusammensetzt aus dem Erhaltungsbedarf, ggf. dem Wachstumsbedarf und dem Ersatz obligatorischer Verluste über Urin, Stuhl und Schweiß. Diese sog. »Toleranz« für eine essentielle Aminosäure, deren früher Abbau vollständig gestört ist, entspricht dem minimalen Bedarf, der eine ausgeglichene bzw. positive Stickstoffbilanz ermöglicht. Diese Aminosäurenstoffwechseldefekte bieten so auch eine Möglichkeit, die mit anderen Methoden ermittelten Bedarfsberechnungen für unverzichtbare und bedingt unverzichtbare Aminosäuren zu validieren. Daraus ergibt sich, dass eine sog. »eiweißreduzierte« Kost in der Behandlung von Aminosäurenstoffwechseldefekten nur gegenüber unkontrollierten Ernährungsformen »reduziert« ist, jedoch immer den Bedarf an Gesamtstickstoff und einzelnen essentiellen Aminosäuren decken muss. Dabei kann man sich an den Werten für die sog. »sichere" Eiweißaufnahme orientieren. Die errechnete Eiweißmenge muss aber immer korrigiert werden unter Verwendung des errechneten Aminosäurenscore im Vergleich zum sog. Referenzprotein (Frauenmilchprotein für das 1. Lebensjahr, danach Referenzprotein für das 2.-5. Lebensjahr der FAO/WHO) und der Verdaulichkeit des verwendeten Proteins. Diese Korrektur ist unerlässlich, da in sog. »eiweißreduzierten " Diäten oft pflanzliche Proteine niedriger biologischer Qualität und geringer Verdaulichkeit benutzt werden.Defekte im Abbau der aus Aminosäuren entstandenen organischen Säuren sind grundsätzlich weniger durch eine Reduktion der Zufuhr der betroffenen Aminosäure(n) bzw. des Eiweißes in der Nahrung zu beeinflussen als die "frühen" Defekte mit spezifischer Hyperaminoazidämie. Während bei den letzteren eine Titration der Zufuhr gegen den Blutspiegel der Aminosäure erfolgen kann, ist bei den ersteren die Höhe der Metabolite in Plasma und Urin meist kein guter Parameter bzw. nicht der entscheidende Parameter, an dem die Qualität der diätetischen Behandlung gemessen werden kann. Wenn mit der o.g. "eiweißreduzierten" Kost keine ausreichende Versorgung mit Stickstoff und allen essentiellen Aminosäuren sicher erreicht werden kann, müssen Eiweißersatzprodukte eingesetzt werden, die alle (bis auf die betroffene(n)), Aminosäure(n) in sinnvoller Kombination (d. h. z. B. angeglichen an Frau- 
enmilch- oder Eiprotein) enthalten. Diese Eiweißersatzprodukte sind entweder Hydrolysate von Rinderserum oder Kasein oder Mischungen reiner L-Aminosäuren. Letztere schmecken besser und enthalten mehr Aminosäuren in geringerem Volumen. Da die zur Bedarfsdeckung verwendeten, vorwiegend pflanzlichen Proteine in Kombination mit den notwendigen Kohlenhydraten und Fetten in der Regel keine ausreichende Zufuhr von allen Vitaminen, Mineralstoffen und Spurenelementen ermöglichen, sind die Eiweißersatzpräparate entweder mit diesen angereichert oder es müssen Supplemente gegeben werden. Regelmäßige rechnerische Überprüfung der Zufuhr aller Nährstoffe sowie die Suche nach klinischen und chemischen Zeichen der Unterversorgung sind unverzichtbar und müssen die Kontrolle von Längenwachstum, Kopfwachstum, Gewichtszunahme, des Zustands von Haut und Hautanhangsgebilden (Fingernägel, Haare) sowie der psychomotorischen und neurologischen Entwicklung ergänzen.

\subsubsection{Störungen des Phenylalaninstoffwechsels}

Phenylalanin ist eine essentielle Aminosäure, die mit praktisch jedem Nahrungseiweiß aufgenommen wird. Der minimale Bedarf beträgt, unabhängig vom Alter, zwischen 300 und $400 \mathrm{mg} / \mathrm{Tag}$, wobei abhängig vom Wachstum bis zu 50\% für die Synthese von Körpereiweiß verbraucht und der Rest zu Tyrosin hydroxyliert wird. Eine Umwandlung in andere Metaboliten (durch Transaminierung zu Phenylbrenztraubensäure und Decarboxylierung zu Phenylethylamin) spielt quantitativ eine zu geringe Rolle, um einen Defekt der Phenylalaninhydroxylase kompensieren zu können. Der Phenylalaninpool des Körpers wird bestimmt durch die Zufuhr mit der Nahrung, die Zufuhr aus dem Abbau körpereigenen Proteins, die Proteinsynthese und die Oxidation zu Harnstoff und $\mathrm{CO}_{2}$ nach vorheriger Hydroxylierung $\mathrm{zu}$ Tyrosin (s. - Abb. 2.20). Ein Fehlen bzw. eine verminderte Aktivität der Phenylalaninhydroxylase würde also bei nicht entsprechender Drosselung der Zufuhr auf das für die Proteinsynthese benötigte Maß zu einem Anwachsen des Phenylalaninpools, messbar an der Erhöhung des Plasmaspiegels, führen. Da für die Phenylalaninhydroxylierung neben dem Leberenzym der im Körper synthetisierte Kofaktor Tetrahydrobiopterin (BH4) erforderlich ist, können Defekte in dessen Synthese (Nr. 7 in - Abb. 2.21) bzw. Reaktivierung (Nr. 6 in - Abb. 2.21) ebenfalls die Reaktion hemmen. BH4 ist gleichzeitig Kofaktor für die Hydroxylierung von Tyrosin zu Dihydroxyphenylalanin (DOPA) und von Tryptophan zu 5-Hydroxytryptophan. Beide sind Vorläufer von Neurotransmittern, so dass es bei Störungen der BH4-Synthese bzw. -reaktivierung zusätzlich zur Störung des Phenylalaninstoffwechsels zu einem Mangel an Neurotransmittern

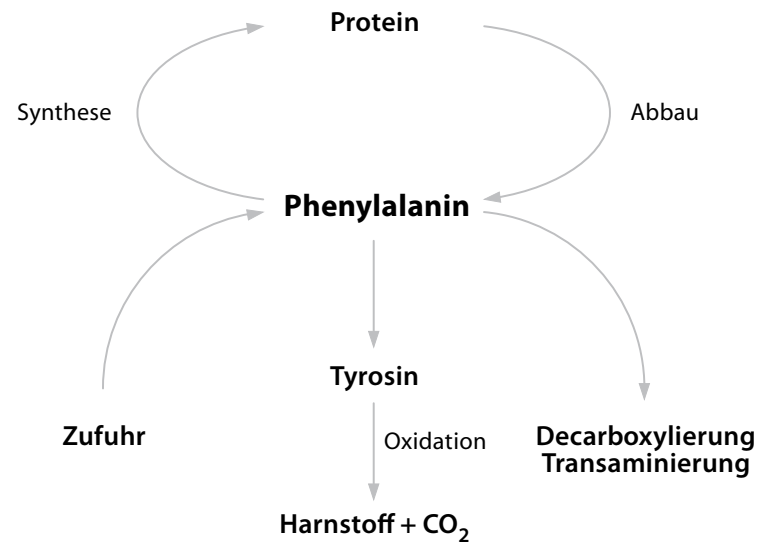

- Abb. 2.20. Schematische Darstellung der den Phenylalaninpool beeinflussenden Stellgrößen

kommt. Eine Behandlung dieser Defekte muss also außer der angestrebten näherungsweisen Normalisierung des Phenylalaninspiegels eine Normalisierung der Neurotransmitterspiegel, gemessen an deren Abbauprodukten im Liquor, zum Ziel haben.

\section{Phenylketonurie/Hyperphenylalaninämie}

Die klassische Phenylketonurie (PKU) mit praktisch fehlender Aktivität der Phenylalaninhydroxylase und Phenylalaninspiegeln vor bzw. ohne Behandlung $>1200 \mu \mathrm{mol} / \mathrm{l}$ führt unbehandelt zu irreversiblen Entwicklungsstörungen (Debilität bis Idiotie), deren sichtbares Korrelat eine gestörte Myelinisierung ist, zu Pigmentarmut, häufig auch zu Ekzem und Psychosen mit Autoaggression. Im Urin wird vermehrt Phenylbrenztraubensäure ausgeschieden (»Phenylbrenztraubensäureschwachsinn", "Imbecillitas phenylpyruvica«). Eine Vielzahl von verschiedenen Genmutationen ist als Ursache beschrieben worden. Die exakte molekulargenetische Diagnose lässt Aussagen über die Schwere des Enzymdefekts zu, neuerdings auch eine Voraussage, ob das mutierte Enzym in seiner Aktivität durch $\mathrm{BH}_{4}$ stimuliert werden kann. Wegen der Vielzahl der möglichen $\mathrm{Mu}$ tationen sind die meisten Patienten gemischt heterozygot, da sie von den beiden Elternteilen unterschiedliche Mutationen vererbt bekommen haben. Mildere Formen der Krankheit, milde Phenylketonurie (Phenylalanin im Plasma 600-1200 mmol/l) und Hyperphenylalaninämie (HPA) (Nicht-PKU-HPA) (Phenylalanin $<600 \mathrm{mmol} / \mathrm{l}$ unter normaler Ernährung)) zeichnen sich durch höhere Restaktivität der Phenylalaninhydroxylase und dementsprechend höhere (bis »normale«) Toleranz für Nahrungsphenylalanin aus. 


\section{Diagnostik}

Neugeborenenscreening entweder mit semiquantitativer Bestimmung des Phenylalanins (aus Fersenblut auf Filterpapier am 3.-7. Lebenstag) oder quantitativ mittels Tandem-Massenspektrometrie. Spiegel von über $120 \mu \mathrm{mol} / \mathrm{l}$ erfordern in jedem Falle eine kurzfristige Kontrolle bzw. eine Bestätigung durch eine quantitative Bestimmung. Zur Abgrenzung der Hydroxylasedefekte von $\mathrm{BH}_{4}$-Mangelkrankheiten sind in allen Fällen vor einer diätetischen Behandlung folgende Untersuchungen anzusetzen: Pterinmuster im Urin, Messung der Dihydropteridinreduktaseaktivität im Blut (Filterpapier) und Bestimmung der Plasmaspiegel von Phenylalanin und Tyrosin vor und nach Gabe von $20 \mathrm{mg} \mathrm{BH}_{4} / \mathrm{kg}$ : nur bei $\mathrm{BH}_{4}$-Synthesedefekten und bei den meisten Patienten mit $\mathrm{BH}_{4}$-Reaktivierungsdefekten kommt es zu einem raschen Absinken des Phenylalanin- und Ansteigen des Tyrosinspiegels. Bei Patienten mit milder PKU oder HPA empfiehlt sich eine mehrtägige Testphase mit $\mathrm{BH}_{4}(5-10 \mathrm{mg} / \mathrm{kgKG} / \mathrm{Tag})$ unter normaler Phenylalaninzufuhr, um gegebenenfals ein Ansprechen auf den Kofaktor zu demonstrieren, was die diätetische Behandlung vereinfachen würde.

\section{Behandlung}

Alle Patienten mit Phenylalaninspiegeln von $>600 \mu \mathrm{mol} /$ 1 unter freier Kost müssen durch Reduktion der Phenylalaninzufuhr behandelt werden, gegebenenfalls ersatzweise oder zusätzlich durch Gabe von $\mathrm{BH}_{4}$. Behandlungsziel sind Phenylalaninspiegel zwischen 40 und $240 \mu \mathrm{mol} / \mathrm{l}$, die eine normale Entwicklung gewährleisten. Die Behandlung muss so früh wie möglich begonnen und lebensbegleitend sein. In Deutschland wird empfohlen, bis zum Alter von 10 Jahren die o.g. Spiegel anzustreben, zwischen 11 und 15 Jahren den Phenylalaninspiegel unter $900 \mu \mathrm{mol} / \mathrm{l}$ und danach unter $1200 \mu \mathrm{mol} / \mathrm{l} \mathrm{zu}$ halten, da sich gezeigt hat, dass auch nach guter Diätführung in den ersten 10 Lebensjahren und trotz normaler Intelligenz erhöhte Phenylalaninspiegel zu reversiblen neuropsychologischen Störungen führen können. Bei der genannten geringen Toleranz für Phenylalanin müssen Patienten mit klassischer Phenylketonurie 60-90\% ihres Eiweißbedarfs durch phenylalaninfreie Eiweißersatzpräparate decken, der Prozentsatz steigt mit zunehmendem Alter. Zur Deckung des Phenylalaninbedarfs wird in der frühen Säuglingszeit Muttermilch oder

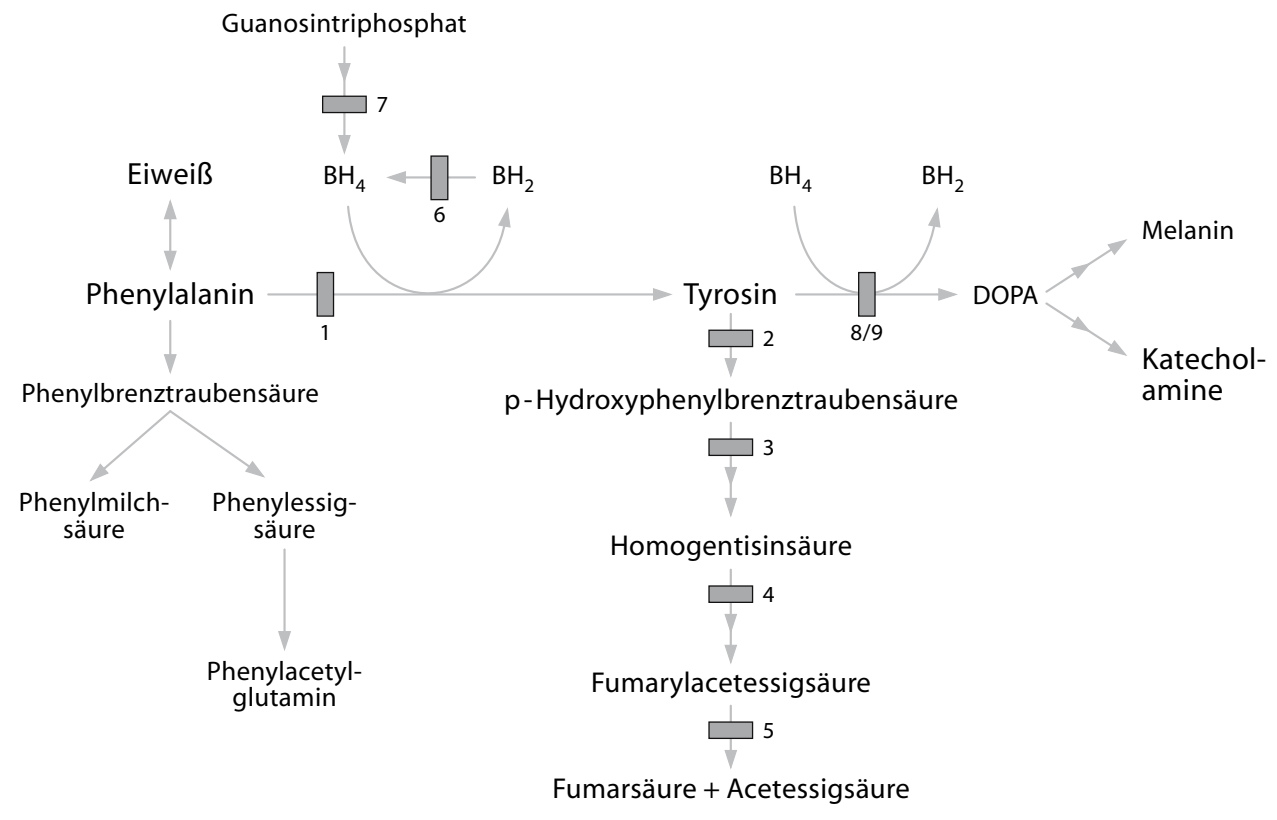

1 Phenylalaninhydroxylase: Phenylketonurie, Hyperphenylalaninämie

2 Tyrosinaminotransferase: Tyrosinämie II

3 p-Hydroxyphenylpyruvatdioxygenase: Tyrosinämie III

4 Homogentisinatdioxygenase: Alkaptonurie

5 Fumarylacetoacetase:Tyrosinämie I

6 Tetrahydrobiopterinregeneration: verschiedene Defekte

7 Tetrahydrobiopterinsynthese: verschiedene Defekte

8 Tyrosinhydroxylase $\left(\mathrm{BH}_{4}\right.$-Mangel)

9 Tyrosinase (Melanocyten): bestimmte Albinismusformen

$\mathrm{BH}_{4}=$ Tetrahydrobiopterin 
Säuglingsanfangsnahrung in nach Phenylalaningehalt geschätzter bzw. berechneter Menge gegeben. Die diätetische Einstellung des Neugeborenen bereitet in der Regel wenig praktische Probleme: Nach einer kurzen Phase phenylalaninfreier Ernährung (phenylalaninfreies Eiweißersatzpräparat, Fett und Kohlenhydrate nach Bedarf) unter 2-tägiger Kontrolle des Phenylalaninspiegels wird phenylalaninhaltige Milch in berechneter Menge, z. B. $40 \mathrm{mg}$ Phenylalanin $/ \mathrm{kg}$, zugefügt, sobald der Phenylalaninspiegel sich dem gewünschten Bereich nähert. Die Mengen werden dem Phenylalaninspiegel angepasst, der zunächst zwei Mal pro Woche, dann wöchentlich, später alle 2-4 Wochen zu kontrollieren ist (s. - Tab. 2.66).

Im Säuglings- und Kleinkindesalter sind häufige Berechnungen der Energie- und Nährstoffzufuhr erforderlich. Beikost wird wie üblich frühestens ab dem 5. Lebensmonat eingeführt, wobei eiweißarme Gemüse- und Obstsorten gegeben werden und von eiweißarmen Getreide- und Reisprodukten Gebrauch gemacht wird. Die Eltern, später die Patienten, sind von Anfang an in der selbständigen Berechnung der Phenylalaninzufuhr anhand von Nährstofftabellen und der Herstellung der Diät $\mathrm{zu}$ unterweisen. Ihre Beratung durch eine (erfahrene) Diätassistentin ist Voraussetzung. Besondere Beachtung muss folgenden Tatsachen geschenkt werden:

- Tyrosin: Patienten mit Phenylketonurie sind auf eine ausreichende Tyrosinzufuhr mit der Nahrung angewiesen. Phenylalaninfreie Eiweißersatzpräparate enthalten ausreichend Tyrosin. Im Zweifelsfall müssen die Plasmaspiegel kontrolliert werden, wobei eine einmalige Bestimmung nicht ausreicht.

- Länge, Gewicht, Kopfumfang: Sie sollen sich altersgerecht entwickeln und müssen kontrolliert werden. Übermäßiges Gewicht darf nicht durch Einschränkung der Energiezufuhr verringert werden, da die dadurch erzeugte katabole Stoffwechsellage zu Phenylalaninspiegelerhöhungen führen würde. Besser ist ein langfristiges Konstanthalten von Energiezufuhr und Gewicht, bis letzteres dem Sollgewicht entspricht.

- Verteilung von Eiweißersatzpräparat: Da freie Aminosäuren und Peptide rascher resorbiert werden als intaktes Eiweiß, die Eiweißsynthese jedoch vom Vorhandensein aller benötigten Aminosäuren abhängt, ist nach Gabe von größeren Mengen Eiweißersatz mit einer beschleunigten Oxidation der im Überschuss zugeführten Aminosäuren zu rechnen. Die Eiweißersatzprodukte müssen daher auf mindestens 3 oder besser 5 Dosen am Tag verteilt werden. Ebenso sollte das »erlaubte" Phenylalanin nach Möglichkeit gleichmäßig über die Mahlzeiten verteilt werden.

- Fett: Wegen des weitgehenden Verzichts auf tierische Lebensmittel und der vorwiegenden Verwendung von
Pflanzenölen ist die Diät in der Regel reich an mehrfach ungesättigten Fettsäuren und arm an gesättigten und einfach ungesättigten sowie an $\omega$-3-Fettsäuren und Cholesterin. Durch die Wahl geeigneter Fettmischungen (Butter, Schmalz, Fischöl) kann einem Mangel entgegengewirkt werden. Ob dieses erforderlich ist, ist z.Z. noch zu wenig untersucht. Auf eine hohe Vitamin-E-Zufuhr ist zu achten.

- Ballaststoffe: Sie sind in den eiweißarmen Brot- und Teigwaren nur in geringem Maße vorhanden.

- Mikronährstoffe: Diese sind in den phenylalaninfreien Eiweißersatzprodukten enthalten. Trotzdem muss ihre Zufuhr regelmäßig rechnerisch überprüft werden, insbesondere in bezug auf Kalzium, Eisen und Vitamine. Trotz guter diätetischer Einstellung weisen PKU-Patienten in einem hohen Prozentsatz eine verminderte Knochendichte auf. Carnitin, dessen Zufuhr vorwiegend über tierische Lebensmittel erfolgt, wurde häufig erniedrigt gefunden, möglicherweise aufgrund einer durch Kofaktormangel (Vitamin $\mathrm{B}_{6}$, Vitamin $\mathrm{C}$, Eisen)-bedingten verminderten Eigensynthese. Viele PKU-Patienten weisen erniedrigte Selenspiegel und erniedrigte Aktivitäten von Selenoenzymen auf, weil die phenylalaninfreien Eiweißersatzprodukte zum Teil selenfrei sind. Die klinische Bedeutung ist noch nicht bekannt. Neurologische Störungen mit spastischer Diplegie bei älteren Patienten sind möglicherweise die Folge von Vitamin- $\mathrm{B}_{12}$-Mangel. Es hat sich gezeigt, dass insbesondere ältere Patienten, deren diätetische Einstellung gelockert wurde und die deshalb meinten, auf die schlechtschmeckenden phenylalaninfreien Eiweißersatzprodukte verzichten zu können, unter einseitiger veganer Ernährungsweise durch Mikronährstoffmangel hochgradig gefährdet sind. Wahrscheinlich ist es "gesünder", denjenigen Patienten, die eine Diät mit phenylalaninfreien Ersatzprodukten nicht mehr einhalten können oder wollen, zu einer "normalen « Ernährung zu raten unter Inkaufnahme erhöhter Phenylalaninspiegel, als einseitige eiweißarme Ernährungsformen zu tolerieren.

- Kombiniert mit Nahrungsverweigerung und/oder Erbrechen führen Infektionen wegen des entstehenden Katabolismus zu Erhöhungen des Phenylalaninspiegels. Sie sollten nach Möglichkeit bereits im Initialstadium durch eine hohe Energiezufuhr, Streichung oder Halbierung der Phenylalaninmenge bei Fortsetzung des phenylalaninfreien Eiweißersatzproduktes behandelt werden.

- Psychosoziale Betreuung ist neben der ärztlichen und biochemischen Kontrolle erforderlich. Da das Behandlungsziel die normale Entwicklung des Patienten ist, die Krankheitseinsicht und Selbständigkeit erst ermöglicht, ergeben sich Probleme des »Bedingt-Gesundseins". 


\begin{tabular}{l|l|l}
$\begin{array}{l}\text { - Tab. 2.66. Empfohlene Phenylalaninzufuhr gemäß der deut- } \\
\text { schen PKO-Verbandstudie }\end{array}$ \\
\hline \begin{tabular}{l|l} 
Alter in \\
Monaten
\end{tabular} & $\begin{array}{l}\text { Anzahl der } \\
\text { Probanden }\end{array}$ & $\begin{array}{l}\text { Phenylalaninzufuhr } \\
\text { (mg/kg/Tag) }\end{array}$ \\
\hline 6 & 137 & $34 \pm 7$ \\
12 & 138 & $28 \pm 7$ \\
18 & 135 & $26 \pm 6$ \\
\hline 24 & 137 & $23 \pm 5$ \\
\hline 30 & 137 & $22 \pm 5$ \\
\hline 36 & 132 & $20 \pm 5$ \\
42 & 133 & $19 \pm 5$ \\
48 & 128 & $18 \pm 5$ \\
\hline 54 & 123 & $17 \pm 5$ \\
60 & 108 & $17 \pm 5$ \\
66 & 94 & $16 \pm 4$ \\
72 & 85 & $15 \pm 5$ \\
\hline
\end{tabular}

\section{Tetrahydrobiopterin $\left(\mathrm{BH}_{4}\right)$-Mangel}

Hierunter werden die 4 bisher bekannten Defekte der $\mathrm{BH}_{4}$-Synthese und Reaktivierung zusammengefasst, die ca. $2 \%$ der entdeckten PKU- bzw. HPA-Fälle zugrunde liegen.Trotz diätetischer Behandlung kommt es bei 3 von diesen Störungen zu schwersten neurologischen Störungen und z. T. frühem Tod. Die Ausnahme ist der Pterin$4 \alpha$-Karbinolamindehydratasemangel (Primapterinurie), bei dem eine diätetische Behandlung zu normaler neurologischer Entwicklung führte bzw. eine Behandlung mit BH4 nur in den ersten Lebensmonaten erforderlich war.

Die Behandlung besteht grundsätzlich in einer Normalisierung des Plasmaphenylalaninspiegels durch Diät: (Dihydropteridinreduktase(DHPR)-Mangel) oderdurch BH4 (GTP-Zyklohydrolasemangel, PTPS-Mangel=partieller DHPR-Mangel) und in einer Substitution von Neurotransmittervorstufen L-DOPA und 5-Hydroxytryptophan plus dem peripheren Decarboxylasehemmer Carbidopa (GTP-Zyklohydrolasemangel, PTPS-Mangel, DHPRMangel, Folinsäure: DHPR-Mangel).

\section{Maternale Hyperphenylalaninämie}

Sie bezeichnet die Embryofetopathie, die Kinder von Müttern betrifft, deren Phenylalaninspiegel während der Schwangerschaft $>400 \mu \mathrm{mol} / \mathrm{l}$ betrug. Die hauptsächlichen Symptome, die mit der Höhe des mütterlichen Phenylalaninspiegels an Häufigkeit und Schwere zunehmen, sind intrauteriner Wachstumsrückstand, Mikrozephalie, Fehlbildungen, insbesondere Herzfehler und spätere geistige Retardierung. Wegen des aktiven Transports von Phenylalanin über die Plazenta liegen die kindlichen Phenylalaninspiegel um ca. 2/3 höher als die mütterlichen. Die diätetische Behandlung der Mütter, deren Blutspiegel zwischen 120 und $360 \mu \mathrm{mol} / \mathrm{l}$ liegen sollten, kann die intrauterine

Fruchtschädigung verhindern (-Tab. 2.67). Sie entspricht im Prinzip der üblichen diätetischen Behandlung, ist aber ungleich schwieriger praktisch durchzuführen: Wiedereinführen oder erstmaliges Zuführen von phenylalaninfreiem Eiweißersatzprodukt; viele nach den o.g. Kriterien behandlungsbedürftige Patientinnen sind von der Diät abgesetzt oder sind früher nicht als behandlungsbedürftig eingestuft worden, so dass sie einerseits nicht über das Risiko für ihr Kind informiert sind und andererseits keinen Kontakt mehr mit einem Behandlungszentrum haben; Schwangerschaftserbrechen; rasche Zunahme der Phenylalanintoleranz während des 2 . Trimenon ( $400 \mathrm{mg}$ auf $>1400 \mathrm{mg}$ Phenylalanin/Tag); rasche Zunahme des Bedarfs an Tyrosin im 2. Trimenon erfordert Tyrosinsupplementierung im Grammbereich; Gewährleistung einer ausreichenden Energie- und Nährstoffzufuhr trotz strenger Diätkontrolle.

Die Resultate der Behandlung von Schwangeren mit Phenylketonurie oder Hyperphenylalaninämie in bezug auf ihre Kinder sind abhängig:vom Zeitpunkt des Eintretens der diätetischen Kontrolle, d. h. Phenylalaninspiegel bleibend unter $<360 \mu \mathrm{mol} / \mathrm{l}$ : optimal liegt dieser Zeitpunkt vor der Konzeption; positive Effekte sind nachgewiesen bei Erreichen bis zur 10. Schwangerschaftswoche; von der Höhe der Blutspiegel: optimal $<360 \mu \mathrm{mol} / \mathrm{l}$, nicht $<60 \mu \mathrm{mol} / \mathrm{l}$; von der Energie- und Nährstoffversorgung der Schwangeren: keine Gewichtsabnahme im 1. Trimenon; ausreichende Zufuhr von Eiweiß, Fett, Cholesterin, Kalzium, Phosphor, Vitamin A und Folat. Die Schwierigkeiten sollten mit den potentiellen Müttern frühzeitig besprochen werden, die Notwendigkeit einer eventuellen stationären Aufnahme sollte bekannt sein. Mütter mit Phenylketonurie können ihre heterozygoten Kinder unbesorgt stillen, der Phenylalaningehalt der Muttermilch ist nur in Hinsicht auf das freie Phenylalanin erhöht (20fach) und quantitativ daher bedeutungslos.

\subsubsection{Störungen des Tyrosinstoffwechsels}

Die drei diätetisch behandelbaren angeborenen Tyrosinstoffwechselstörungen sind in Abb. 2.21 als Defekte 3-5 eingezeichnet.

\section{Tyrosinämie Typ I}

Sie beruht auf einem Defekt der Fumarylazetoazetase. Sie geht mit einer erhöhten Ausscheidung von Succzinylazeton und 5-Aminolaevulinsäure einher, gelegentlich, insbesondere bei jungen Kindern, mit einer Hypermethioninämie durch sekundäre Hemmung der Methioninadenosyltransferase. Klinisch kommt es zu einer progredienten Leberschädigung mit Zirrhose und Hepatombildung (Erhöhung von $\alpha$-Fetoprotein), zu einer Tubulopathie mit allen Zeichen des DeToniDebré-Fanconi-Syndroms, bei einzelnen Patienten zu 
- Tab. 2.67. Mütterlicher Phenylalaninspiegel ohne Diät ( $\mu \mathrm{mol} / \mathrm{L})$. (Koch et al. 2003)

Auswirkungen Maternaler PKU oder Hyperphenylalaninämie auf das Schwangerschaftsergebnis (in\%). Vergleich der Daten von Lenke und Levy (1980): unbehandelt mit den Daten der Kollaborativen MPKU-Studie (2003): behandelt

\begin{tabular}{|c|c|c|c|c|c|}
\hline & $>1200$ & 901-1199 & $601-900$ & $180-600$ & Ohne PKU \\
\hline $\begin{array}{l}\text { Geistige Retardierung } \\
\text { unbehandelt } \\
\text { behandelt }\end{array}$ & $\begin{array}{l}92 \\
28\end{array}$ & $\begin{array}{l}73 \\
8\end{array}$ & $\begin{array}{l}22 \\
15\end{array}$ & $\begin{array}{l}21 \\
2\end{array}$ & $\begin{array}{l}5 \\
4,3\end{array}$ \\
\hline $\begin{array}{l}\text { Mikrozephalie } \\
\text { unbehandelt } \\
\text { behandelt }^{*}\end{array}$ & $\begin{array}{l}73 \\
23\end{array}$ & $\begin{array}{l}68 \\
8\end{array}$ & $\begin{array}{l}35 \\
13\end{array}$ & $\begin{array}{l}24 \\
5\end{array}$ & $\begin{array}{l}4,8 \\
1,3\end{array}$ \\
\hline $\begin{array}{l}\text { Herzfehler } \\
- \text { unbehandelt } \\
- \text { behandelt }\end{array}$ & $\begin{array}{l}12 \\
11\end{array}$ & $\begin{array}{l}15 \\
5\end{array}$ & $\begin{array}{l}6 \\
3\end{array}$ & $\begin{array}{l}0 \\
2\end{array}$ & $\begin{array}{l}0,8 \\
1,0\end{array}$ \\
\hline $\begin{array}{l}\text { Niedriges Geburtsge- } \\
\text { wicht }(<2500 \mathrm{~g}) \\
\text { - unbehandelt } \\
\text { - behandelt }\end{array}$ & $\begin{array}{l}40 \\
21\end{array}$ & $\begin{array}{l}52 \\
6\end{array}$ & $\begin{array}{l}56 \\
9\end{array}$ & $\begin{array}{l}13 \\
3\end{array}$ & $\begin{array}{l}9,6 \\
5,7\end{array}$ \\
\hline $\begin{array}{l}\text { Spontanabort } \\
\text { - unbehandelt } \\
\text { - behandelt }\end{array}$ & $\begin{array}{l}24 \\
17\end{array}$ & $\begin{array}{l}30 \\
10\end{array}$ & $\begin{array}{l}0 \\
18\end{array}$ & $\begin{array}{l}8 \\
5\end{array}$ & $\begin{array}{l}5-20 \\
\text { keine Angabe }\end{array}$ \\
\hline
\end{tabular}

* Ohne Zwillingsgeburten

Symptomen wie bei akuter intermittierender Porphyrie und bei akuten Verlaufsformen beinahe gesetzmäßig zu hypertrophischen linksventrikulären Kardiomyopathien. Chronische Verläufe mit ersten Symptomen jenseits des 1. Lebensjahrs, wobei die Tubulopathie und Rachitis im Vordergrund stehen, sind möglich.Eine kurative Behandlung ist zur Zeit nur durch eine Lebertransplantation möglich. Deren Zeitpunkt sollte vor dem Auftreten von Hepatomen liegen und ist daher frühzeitig zu planen. Alternativ zu der diätetischen Behandlung (s. unten), die zwar die Nieren- und Leberfunktion günstig beeinflusst, die Hepatombildung jedoch nicht verhindert, und zur Transplantation können mit dem 4-Hydroxyphenylpyruvatdioxygenase-Hemmer 2-(2-Nitro-4-Trifluoromethylbenzoyl)-1,3-Zyklohexandion (NTBC) $(0,1-0,6 \mathrm{mg} /$ $\mathrm{kg} / \mathrm{d}$ oral) sowohl die biochemischen Veränderungen als auch die Leber- und Nierenfunktion günstig beeinflusst und computertomographisch sichtbare Leberveränderungen (nicht jedoch bereits maligne Hepatome) zur Rückbildung gebracht werden.

\section{Tyrosinämie Typ II (Richner-Hanhart-Syndrom)}

Diese beruht auf einem Defekt der zytosolischen Tyrosinaminotransferase und führt zu einer Keratitis, später Korneatrübung und Glaukom. Hautveränderungen in Form von schmerzhaften Blasen oder Erosionen an Handflächen und Fußsohlen mit nachfolgender Hy- perkeratose treten nach den Augenveränderungen auf. Einzelne Patienten waren geistig retardiert. Die Tyrosinspiegel sind anfangs höher als beim Typ I und höher als bei der transienten Neugeborenentyrosinämie. Die Störung spricht gut auf eine diätetische Behandlung an (s. unten).

\section{Tyrosinämie Typ III}

Sie beruht auf einem Defekt der 4-Hydroxyphenylpyruvatdioxygenase in der Leber mit gelegentlich intermittierender Ataxie oder geringer geistiger Retardierung, jedoch ohne Leberfunktionsstörungen.

Diätetik. Die diätetische Behandlung aller 3 Störungen besteht in der Reduktion der Zufuhr von Phenylalanin und Tyrosin, so dass der Tyrosinspiegel zwischen 100 und $220 \mu \mathrm{mol} / 1$ liegt, der Phenylalaninspiegel nicht unter $60 \mu \mathrm{mol} / \mathrm{l}$ absinkt. Der altersentsprechende Eiweißbedarf wird teilweise durch ein phenylalanin- und tyrosinfreies Eiweißersatzprodukt gedeckt. Bei zusätzlicher Hypermethioninämie kann ein zusätzlich methioninfreies Eiweißersatzprodukt verwendet werden. Ein bestehendes DeToni-Debré-Fanconi-Syndom wird symptomatisch, wie bei Cystinose bzw. hypophosphatämischer Rachitis beschrieben, behandelt. Es gelten dieselben allgemeinen Regeln für die Durchführung der Diät wie bei der Phenylketonurie beschrieben. 


\subsubsection{Abbaustörungen der verzweigtkettigen Aminosäuren}

Diese essentiellen Aminosäuren werden in der Leber transaminiert und in die entsprechenden Oxosäuren umgewandelt, die durch einen gemeinsamen Oxosäurendehydrogenasekomplex decarboxyliert werden. Nach diesem Schritt werden die entstandenen organischen Säuren auf getrennten Wegen verstoffwechselt (s. - Abb. 2.22). Im Leucinabbau sind für jeden der Schritte Defekte beschrieben. Der Abbau von Valin und Isoleucin führt ebenso wie der von Threonin und Methionin über Propionyl-CoA und Methylmalonyl-CoA. Auch die in geringen Mengen vorkommenden ungeradzahligen Fettsäuren sowie die Seitenkette des Cholesterins und ganz besonders Darmbakterien tragen zur Produktion von Propionyl-CoA bzw. Propionat bei. Nicht eingetragen in das Abbauschema sind die vielfältigen Nebenabbauwege, die bei einem Enzymblock vermehrt benutzt und zum Auftreten bestimmter in der Differentialdiagnose hilfreicher Nebenmetabolite führen. Besprochen werden sollen nur die Ahornsirupkrankheit (Defekt 3 in - Abb.2.22), die Isovalerianacidämie (Defekt 5 in 0 Abb. 2.22), die Propionazidämie (Defekt 9 in $\mathbf{A b b}$.2.22), die verschiedenen Methylmalonazidurien (Defekt 10 in - Abb. 2.22 sowie verschiedene Defekte des intrazellulären Cobalaminstoffwechsels) und die zwei Formen des multiplen Carboxylase-Mangels.

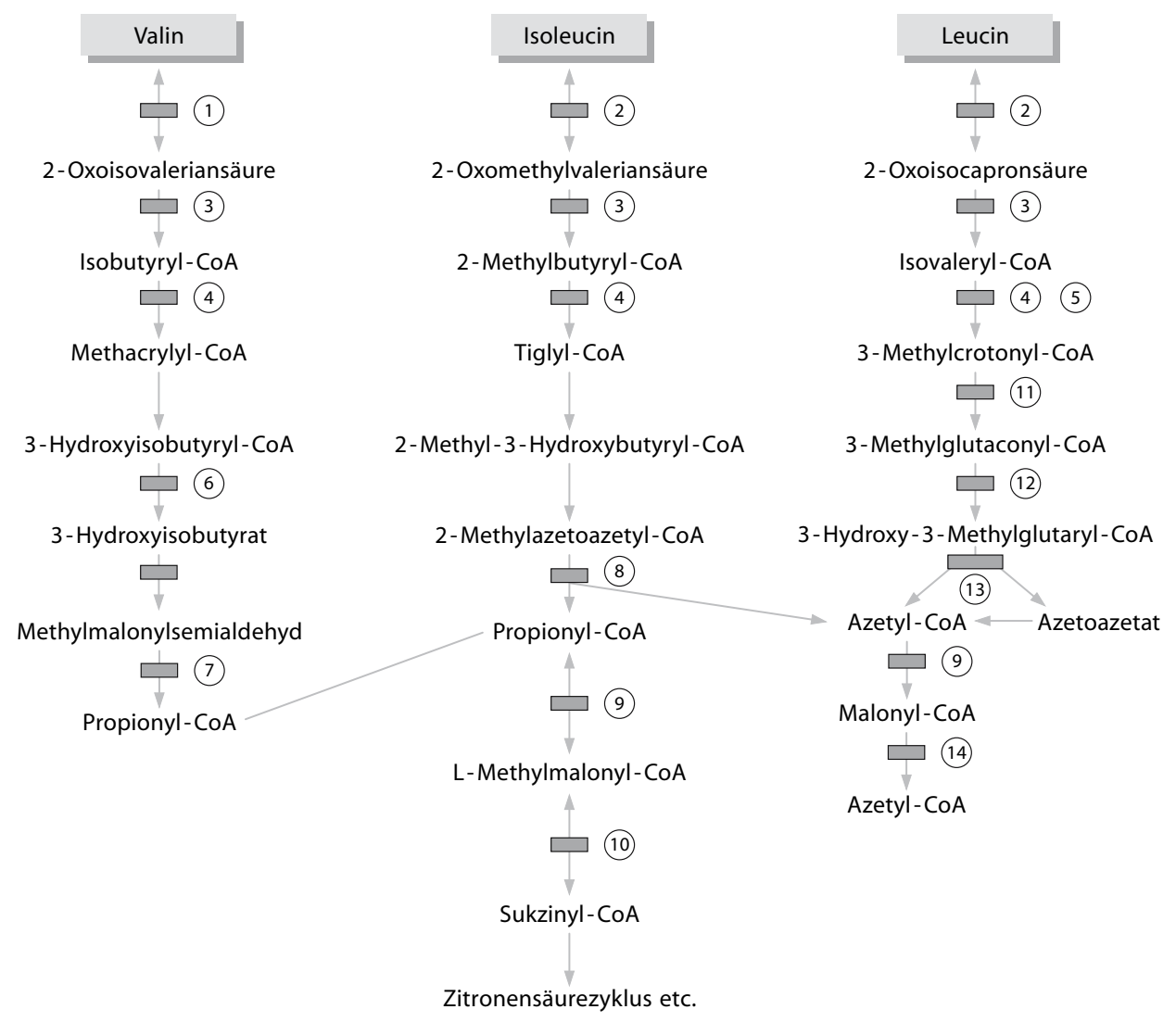

\footnotetext{
1 Aminotransferase: Hypervalinämie

2 Aminotransferase: Leucin-Isoleucinämie

3 Oxosäurendehydrogenase: Ahornsirupkrankheit

4 Acyl-CoA-Dehydrogenasen: Multipler Acyl-CoA-Dehydrogenasenmangel (Glutarazidurie II)

5 Isovaleryl-COA-Dehydrogenase: Isovalerianazidämie

6 3-Hydroxyisobutyryl-CoA-Deacylase

7 Methylmalonylsemialdehyddehydrogenase

8 2-Methylazetoazety-CoA-Thiolase

9 Propionyl-CoA-Carboxylase: Propionazidämie, multipler Carboxylasemangel, Biotinidasemangel

10 Methylmalonyl-CoA-Mutase: Methylmalonazidurie, Vitamin- $\mathrm{B}_{12}$-Mangel

1 3-Methylcrotonyl-CoA-Carboxylase

2 3-Methylglutaconyl-CoA-Hydratase

13 3-Hydroxy-3-Methylglutaryl-CoA-Lyase

14 Malonyl-CoA-Decarboxylase
}

- Abb. 2.22. Reaktionen und Defekte des Stoffwechsels verzweigtkettiger Aminosäuren 


\section{Ahornsirupkrankheit}

Der Enzymdefekt führt zu einer Erhöhung der verzweigtkettigen Aminosäuren sowie deren Oxosäuren in Plasma und Urin. Letztere sind für den typischen Geruch dieser Patienten nach Ahornsirup oder Maggi verantwortlich. Die Erhöhung von Leucin und 2-Oxoisocapronsäure steht im Vordergrund, letztere scheint verantwortlich zu sein für die akute und chronische Symptomatologie. Je nach Restaktivität des Enzymkomplexes kommt es zu akuter neonataler (nach freiem Intervall Trinkschwäche, Hypotonie, Bewusstseinseintrübung, Atemdepression, Koma mit Ketoazidose, Hypo-, Normo- oder Hyperglykämie) oder chronischer Manifestation (Attacken von Ketoazidose, psychomotorischer Entwicklungsrückstand, Ataxie). Die Prognose hängt vom Zeitpunkt der Diagnose, dem Beginn und Effekt der therapeutischen Maßnahmen ab. Das gilt auch für spätere ketoazidotische Attacken im Rahmen von z. B. Infekten.

Behandlung. Bei akuten Erstmanifestationen oder Entgleisungen ist sie auf die rasche Erniedrigung der Leucin- und 2-Oxoisocapronsäurespiegel ausgerichtet durch wiederholte oder Langzeit-Blut-Austauschtransfusionen bzw. durch Peritonealdialyse. Gleichzeitig muss durch hohe Energiezufuhr (Kohlenhydrate und Fett parenteral oder oral) und evtl. Insulin versucht werden, eine anabole Stoffwechsellage zu erzeugen. Dem dient auch die frühzeitige Gabe eines Eiweißersatzprodukts, das frei von verzweigtkettigen Aminosäuren ist. Deren Plasmaspiegel sollten keineswegs auf subnormale Werte absinken, was u.U. eine frühzeitige Substitution von Valin und Isoleucin erforderlich macht. In der Langzeitbehandlung spielt eine reduzierte Zufuhr an den verzweigtkettigen Aminosäuren in Abhängigkeit von deren Plasmaspiegeln (Leucin nicht unter 100 bzw. nicht über $500 \mu \mathrm{mol} / \mathrm{l}$ ) die Hauptrolle. Patienten mit der schweren Form der Ahornsirupkrankheit tolerieren in den ersten 2 Lebensmonaten zwischen 60 und $100 \mathrm{mg}$ Leucin/kgKG/Tag, mit $1 \mathrm{Jahr}$ $45-55 \mathrm{mg}$ und mit 3 Jahren $30-35 \mathrm{mg} / \mathrm{kgKG}$. Später beträgt die tolerierte Leucinmenge pro Tag immer weniger als $600 \mathrm{mg}$. Die Zufuhr von Valin und von Isoleucin, die mit gering erhöhten Plasmaspiegeln einhergeht, liegt niedriger als die für Leucin. Natürliches Eiweiß, das dieser Toleranz für verzweigtkettige Aminosäuren entspricht, wird in Form von Säuglingsanfangsnahrung, später in Form von eiweißarmen, vorwiegend pflanzlichen Lebensmitteln gegeben. Die Menge reicht in der Regel nicht aus, um den Eiweißbedarf zu decken, weshalb ein spezielles Eiweißersatzpräparat zusätzlich gegeben werden muss. Für die Durchführung der Diät siehe Phenylketonurie. Mildere Verlaufsformen haben eine höhere Toleranz für verzweigtkettige Aminosäuren und u.U. die altersgerechten empfohlenen Eiweißmengen. Einige wenige Patienten haben sich als "Thiamin-sensibel« erwiesen, d. h. pharmakologische Dosen von Vitamin $B_{1}(10-200 \mathrm{mg} / \mathrm{Tag})$ haben zu einer deutlichen Erhöhung oder sogar Normalisierung der Toleranz für verzweigtkettige Aminosäuren geführt.

\section{Isovalerianazidämie}

Sie ist eine vergleichsweise gut behandelbare Stoffwechselstörung im Abbau des Leucins. Die Klinik ähnelt der der Ahornsirupkrankheit mit dem Unterschied, dass die Patienten und ihre Körperflüssigkeiten nach Schweißfüßen riechen. Leucin-freies Eiweißersatzpräparat zur Verfügung. Oft lässt sich jedoch durch Gabe von Glycin (250-600 mg/kgKG in akuten Situationen, $150-200 \mathrm{mg} /$ $\mathrm{kgKG}$ auf Dauer, verteilt auf 4 bis 8 Einzelgaben) und Carnitin (bis $400 \mathrm{mg} / \mathrm{kgKG}$ in akuten Situationen, $100 \mathrm{mg} /$ kgKG als Dauerbehandlung) durch vermehrte Ausscheidung von Isovalerylglycin bzw. Isovalerylcarnitin eine Toleranz für niedrig-normale Leucin- und Proteinmengen erzeugen. Die Spiegel von Carnitin sollten darunter normal, die von Glycin im Plasma hoch-normal sein. Von großer Wichtigkeit in der Behandlung aller organischer Azidurien ist eine ausreichende, besser reichliche Flüssigkeitszufuhr.

\section{Propionazidämie/Methylmalonazidurie}

Die Propionazidämie mit Defekt der Propionyl-CoACarboxylase und die verschiedenen Formen der Methylmalonazidurie sind die häufigsten organischen Azidurien. Auch sie können neonatal akut verlaufen (nach kurzzeitiger symptomfreier Periode) und bei fehlender Diagnose und Behandlung rasch zum Tode führen. Typisch sind eine rasche Dehydration, eine Ketoazidose, eine Hyperammoniämie (Verwechslung mit Harnstoffzyklusdefekten möglich!), eine Neutro- und Thrombopenie, makrozytäre Anämie, evtl. Panzytopenie (häufigste Fehldiagnose: Sepsis!). Daneben gibt es intermittierende, spät mit akuten Attacken einhergehende und chronisch-progressive (Anorexie, Erbrechen, Gedeihstörung, Osteoporose, Muskelhypotonie oder -schwäche, Entwicklungsrückstand, Epilepsie) Verlaufsformen. Akute Attacken können mit Symptomen eines zerebrovaskulären Insults und mit Kleinhirnblutungen einhergehen (wie auch die Isovalerianazidämie, Harnstoffzyklusdefekte und Defekte der mitochondrialen Atmungskette). Kardiomyopathien kommen vor. Es besteht eine Neigung zu rezidivierenden Infektionen, insbesondere mit Candida. Generalisierte Epidermiolysen sind beobachtet worden.Patienten mit Methylmalonazidurie entwickeln in einem hohen Prozentsatz tubulointerstitielle Nephritiden mit Einschränkung der glomerulären Filtration und Tubulopathien. Es ist bisher nicht deutlich, ob deren Entstehung mit der Höhe der Methylmalonatausscheidung korreliert.Eine Methylmalonazidurie kann Folge eines Fehlens der Methylmalonyl-CoA-Mutase (mut), 
einer Mutation der Methylmalonyl-CoA-Mutase (mut-) und Folge von intrazellulären Cobalaminstoffwechselstörungen sein:

- Fehlen des Methylmalonyl-CoA-Mutase-Cofaktors Adenosylcobalamin: $\mathrm{Cbl} \mathrm{A}$ und B. Verlauf ähnlich wie bei Mutasedefekten, in der Regel milder. Vitamin$\mathrm{B}_{12}$-responsiv

- Fehlen oder Mangel an Adenosylcobalamin und Methylcobalamin (Kofaktor der Methioninsynthase): Cbl C, D und F. Gleichzeitiges Auftreten von Methylmalonazidurie und Homocystinurie mit Hypomethioninämie. Verlauf wie Mutasedefekte, zusätzlich neurologische und psychotische Symptome, megaloblastäre Anämien möglich (nur Cbl C). Vitamin- $\mathrm{B}_{12^{-}}$ responsiv.

- Absorptions- und Transportdefekte für Vitamin $\mathrm{B}_{12}$ ).

- Vitamin- $\mathrm{B}_{12}$-Mangel, ernährungsbedingt, nicht genetisch.

Behandlung. Die Behandlung von Propionazidämie und Methylmalonazidämie strebt in der akuten Entgleisung nach rascher Entfernung toxischer Metabolite durch Austauschtransfusionen, Peritoneal- oder Hämodialyse (Propionazidämie) oder forcierte Diurese (Methylmalonazidurie) und Herstellung einer anabolen Stoffwechsellage durch hohe Energiezufuhr (Kohlenhydrate und Fett oral oder parenteral; nach Normalisierung des Blutammoniaks auch Zufuhr eines valin-, isoleucin-, methionin-, threoninfreien Eiweißersatzprodukts zur Deckung des altersgerechten Bedarfs an Eiweiß). Die betroffenen Aminosäuren können in Form von natürlichem Eiweiß nach Normalisierung/Verbesserung der Spiegel an organischen Säuren in steigenden Mengen hinzugefügt werden. Für die Langzeitbehandlung muss unter Kontrolle von Wachstum und biochemischen Parametern ein Weg zwischen Eiweißmangelernährung und tolerierter Eiweißzufuhr gefunden werden. Spezielle Eiweißersatzpräparate können dabei eine Hilfe sein. Es ist aber unbekannt, welche Zielgrößen der Metabolitspiegel bzw. -ausscheidung im einzelnen anzustreben sind: für die Methylmalonazidurie sollte Methylmalonsäure im Serum nicht nachweisbar und die Methylmalonatausscheidung im Urin in der GröBenordnung von $2-3 \mathrm{mmol} / \mathrm{mmol}$ Kreatinin liegen. Die Toleranz für Valin bei schweren Verlaufsformen liegt in den ersten 2-3 Lebensjahren zwischen 250 und $500 \mathrm{mg} /$ Tag, um danach auf etwa $600-800 \mathrm{mg} /$ Tag langsam anzusteigen. In diesen Fällen resultiert eine ungenügende Eiweißzufuhr, wenn auf Eiweißersatzpräparate verzichtet wird. In allen Fällen von Propionazidämie sollte extra Biotin (bis zu $10 \mathrm{mg} / \mathrm{Tag}$ ) gegeben werden, obwohl eine Biotinabhängigkeit des isolierten Propionyl-CoA-Carboxylasemangels bisher nicht beschrieben wurde. In allen Fällen von Methylmalonazidurie sollte genügend lange und in genügend hoher Dosis (1 mg Hydroxocobalamin/ Tag i.m.) nach einer evtl. »Vitamin- $\mathrm{B}_{12}$-Responsivität« ge- sucht werden, diese wird kenntlich an deutlicher Verringerung der Methylmalonatausscheidung. Carnitin (bis zu 100 mg/kgKG/Tag) kann die Ausscheidung von Propionat verbessern. Eine Langzeitbehandlung mit dem Antibiotikum Metronidazol (10-20 mg/kgKG/Tag) führt bei vielen Patienten zu deutlicher Verminderung der Ausscheidung von Propionatmetaboliten. Viele Patienten benötigen wegen schwerer Anorexie über Jahre eine (nächtliche) kontinuierliche Sondenernährung.

\section{Multipler Carboxylasedefekt}

Betroffen sind die 3 mitochondrialen Carboxylasen (Propionyl-CoA-Carboxylase - Defekt 9 in - Abb. 2.22, 3-Methylcrotonyl-CoA-Carboxylase -Defekt 11 in - Abb. 2.22, Pyruvatcarboxylase - Defekt 11 in - Abb. 2.23) und die zytosolische Azetyl-CoA-Carboxylase, was zu einer Beeinträchtigung des Stoffwechsels der verzweigtkettigen Aminosäuren, der Glukoneogenese und der Fettsäuresynthese mit einem sehr charakteristischen Metabolitenmuster im Urin führt. Ursache ist entweder ein Defekt der Holocarboxylasesynthetase, die die Apoenzyme durch Bindung von Biotin aktiviert (Neugeborenenform), oder der Biotinidase, die Biotin aus Biocytin und Biotinylpeptiden freisetzt und verfügbar macht (infantile Form). Die Neugeborenenform führt regelmäßig zu lebensbedrohlichen ketoazidotischen Attacken mit Hyperlaktatämie und Hyperammoniämie, während bei der infantilen Form neurologische Störungen mehr im Vordergrund stehen (Ataxie, Gehörverlust, Optikusatrophie, Atemstörungen). Beiden gemeinsam ist eine Neigung zu Hautausschlägen (generalisiert bei der Neugeborenenform, periorifiziell bei der infantilen Form) und Haarausfall.

Behandlung. Sie besteht in beiden Fällen in der lebenslangen Gabe von Biotin in hohen Dosen: $10-40 \mathrm{mg} /$ Tag bei Patienten mit Holocarboxylasesynthetasedefekt; 1-10 mg/Tag bei Patienten mit Biotinidasedefekt. In seltenen Fällen von schwerem Holocarboxylasesynthetasemangel kann zusätzlich eine Reduktion der Eiweißzufuhr erforderlich werden. Die Behandlung akuter Stoffwechselkrisen erfordert neben Biotin intensivmedizinische symptomatische Maßnahmen.

\subsubsection{Defekte im Stoffwechsel schwefelhaltiger Aminosäuren}

Der Methioninstoffwechsel führt unter Abgabe einer Methylgruppe zur Bildung von Homozystein, das entweder über die Zystathionin- $\beta$-Synthetase (Reaktion 2 in - Abb. 2.24) irreversibel (Kofaktor ist Pyridoxalphosphat) zu Zystein und Sulfat umgesetzt oder aber zu Methionin remethyliert werden kann. Zwei Remethylierungswege sind möglich: 
- Betain: Homocystein-Methyltransferase mit Betain als Methylgruppenlieferant oder

- 5-Methyltetrahydrofolat: Homozysteinmethyltransferase (Methioninsynthase) mit 5-Methyltetrahydrofolat als Methylgruppenlieferant und Methylcobalamin als Kofaktor (Reaktion 4 in $\mathbf{D}$ Abb. 2.24).

Störungen der Bildung von Methyltetrahydrofolat durch die Methylentetrahydrofolatreductase (Reaktion 5 in - Abb. 2.24) und Störungen der Bildung von Methylcobalamin (Cbl C, D, E, F, G) führen ebenso wie Defekte der Zystathionin- $\beta$-Synthetase zu Homozystinurie. Nur die verschiedenen Formen der Homozystinurie sollen besprochen werden. Andere Defekte im Stoffwechsel schwefelhaltiger Aminosäuren sind entweder ohne sicheren Krankheitswert oder aber sie sind nicht behandelbar.

\section{Zystathionin- $\beta$-Synthetasemangel}

Er führt zu Homozysteinämie und -urie, Hypermethioninämie und niedrigen zystin- und Serinspiegeln im Plasma. Zystathionin ist nicht nachweisbar.

Symptomatologie unbehandelter Patienten:

- Augen Myopie und Linsenluxation mit den entsprechenden Komplikationen;

- Skelett: Osteoporose, Dolichostenomelie, Arachnodaktylie (50\%), Kontrakturen;

- zentrales Nervensystem: mentale Retardierung (50\%), Epilepsie, psychiatrische Störungen (50\%), Herdsymptome;

- Gefäßsystem: Endothelläsionen und sklerotische Veränderungen der Gefäßwände mit thrombembolischen Komplikationen in Arterien und Venen. Sie werden für neurologische Herdsymptome und möglicherweise für die Retardierung verantwortlich gemacht.
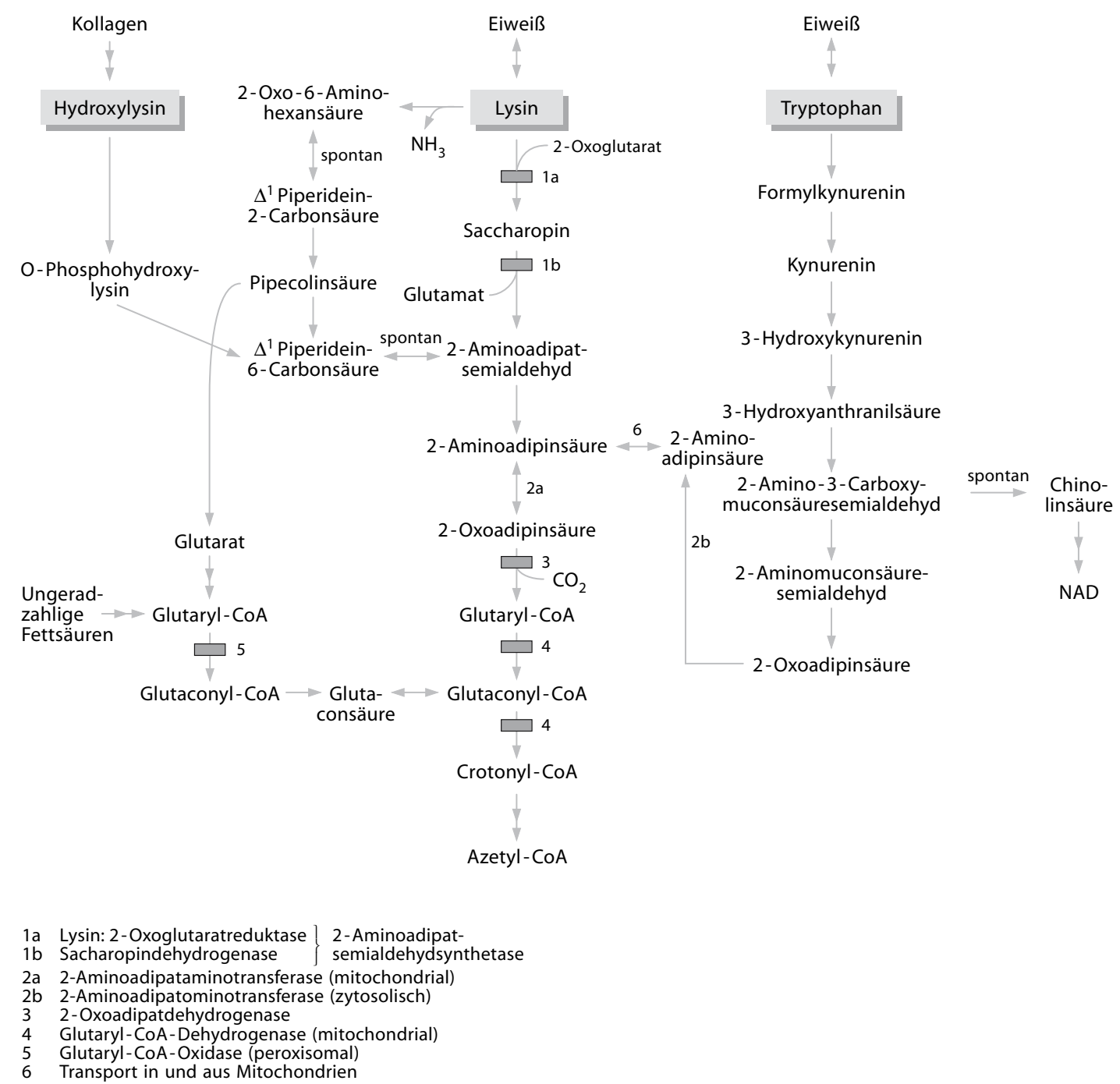

- Abb. 2.23. Reaktionen und Defekte des Lysin-, Tryptophan- und Hydrolysinstoffwechsels 
Behandlung. Etwa die Hälfte der Patienten reagiert auf hohe Vitamin- $\mathrm{B}_{6}$-Dosen $(10-1000 \mathrm{mg} / \mathrm{Tag})$ mit einer Normalisierung der biochemischen Abnormalitäten. Ziel ist eine Normalisierung des Gesamthomozyst(e)ins im Plasma. Da es zu einem Folsäuremangel unter Vitamin- $\mathrm{B}_{6}$-Gabe kommen kann, sollte zusätzlich Folsäure gegeben werden (bis zu 2-5 mg/Tag).Tritt keine Normalisierung der biochemischen Abnormalitäten ein, ist eine diätetische Behandlung mit Reduktion der Methioninzufuhr, ggf. unter Verwendung eines methioninfreien Eiweißersatzprodukts, erforderlich. Gleichzeitig ist eine erhöhte Zystinzufuhr (bis $200 \mathrm{mg} / \mathrm{kg} \mathrm{KG/Tag)} \mathrm{erforder-}$ lich, das bei diesen Patienten zu einer unverzichtbaren Aminosäure wird. Angestrebt werden normale Homozystin-, Methionin- und Zystinspiegel im Plasma und das Fehlen von Homozystin im Urin. Zur Erreichung dieses Ziels kann zusätzlich Betain (100-200 mg/kg KG/Tag) gegeben werden. Es ist nicht gut untersucht, ob eine medikamentöse Hemmung der Thrombozytenaggregation mit Azetylsalizylsäure und/oder Dipyridamol thrombembolische Ereignisse verhüten hilft. Unter Vitamin- $\mathrm{B}_{6}$ - bzw. diätetischer Behandlung, die in der Neugeborenenzeit begonnen worden ist, können die Retardierung verhütet und eine Linsenluxation hinausgezögert werden, ebenso wie das Auftreten von Thromboembolien. Die Osteoporoseentstehung wird oft nicht beeinflusst.

\section{Methylentetrahydrofolatreduktasemangel}

Die dadurch verursachte Homozystinurie geht mit niedrig-normalen Methionin- und erniedrigten Methyltetrahydrofolatspiegeln einher. Die Homozystinausscheidung ist sehr viel geringer als beim Zystathionin- $\beta$-Synthetasemangel. Im Gegensatz zu Vitamin- $B_{12}$-Stoffwechselstörungen mit Homozystinurie ( $\mathrm{Cbl} \mathrm{E}$ und $\mathrm{Cbl} \mathrm{G}$ ) durch Methylcobalaminmangel bzw. mit kombinierter Homozystinurie und Methylmalonazidurie $(\mathrm{Cbl} \mathrm{C}, \mathrm{D}$ und F) tritt keine megaloblastäre Anämie auf. Das klinische Bild ist außerordentlich variabel mit ersten Symptomen im Neugeborenenalter bis in die Adoleszenz: Entwicklungsverzögerung, Gangstörungen, Epilepsie und psychiatrische Störungen. In der Behandlung ist die Gabe von Betain (bis $20 \mathrm{~g} / \mathrm{Tag}$ ) am erfolgversprechendsten. Sie kann kombiniert werden mit Folsäure (Folin- oder Methyltetrahydrofolsäure), Vitamin $B_{6}, B_{12}$ und Carnitin.

\section{Homozystinurie}

Durch gestörte Synthese von sowohl Adenosylcobalamin als Methylcobalamin (Cbl C, D und F). Sie geht zusätzlich mit einer Methylmalonazidurie einher. Die Prognose ist abhängig vom Zeitpunkt des Beginns der Hydroxocobalaminbehandlung. Betain kann zusätzlich

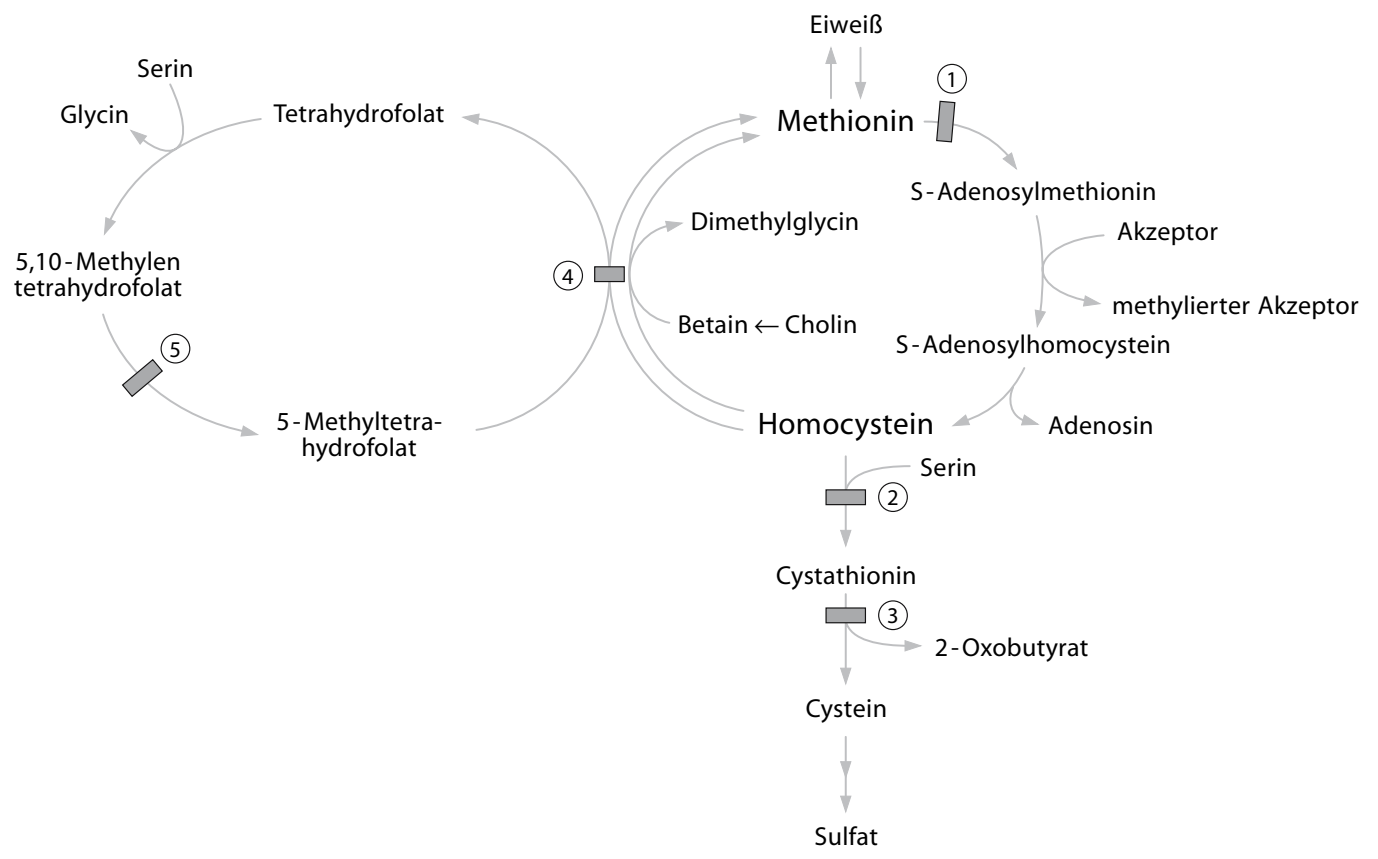

\footnotetext{
Methioninadenosyltransferase: Hypermethinioninämie

Cystathioninsynthase: Homocystinurie

Cystathionase: Cystathioninurie

Methyltetrahydrofolat: Homocysteinmethyltransferase: Homocystinurie

5 Methylentetrahydrofolatreduktase: Homocystinurie
}

- Abb. 2.24. Reaktionen und Defekte des Methioninstoffwechsels 
nützlich sein. Durch gestörte Synthese von reduziertem Methylcobalamin bzw. dessen Verwendung in der Methioninsynthasereaktion (Cbl E und G). Sie geht mit Hypomethioninämie, Entwicklungsrückstand und schwerer megaloblastärer Anämie einher. Die Prognose ist abhängig vom Zeitpunkt des Beginns der Behandlung mit Hydroxocobalamin ( $1 \mathrm{mg}$ i.m. täglich bis $2 \mathrm{mal}$ pro Woche. Während die biochemischen Veränderungen sich rasch normalisieren, sind eingetretene neurologische Defekte irreversibel.

\section{Störungen des Lysin- und Tryptophanstoffwechsels}

Von den vielen Störungen im Stoffwechsel des Lysins und Tryptophans sowie des aus dem Kollagenabbau stammenden Hydroxylysins soll nur auf die Hyperlysinämie und die Glutarazidurie I eingegangen werden. Lysin und Tryptophan sind unverzichtbare (=essentielle) Aminosäuren. Der 1. Schritt des hauptsächlichen Lysinabbaus in Leber und Niere wird durch 2 funktionell unabhängige Teile eines gemeinsamen Enzymproteins (2-Aminoadipatsemialdehydsynthetase) katalysiert. Verlust oder Verminderung beider Teilenzymaktivitäten führt zu Hyperlysinämie Typ I, Reduktion der Aktivität der Lysin:2-Oxoglutaratreductase bei gleichzeitig praktisch fehlender Aktivität der Saccharopindehydrogenase führt zu Hyperlysinämie Typ II mit Überwiegen der Erhöhung von Saccharopin in Plasma und Urin (Reaktionen 1a und $1 \mathrm{~b}$ in - Abb. 2.23). Hyperlysinämie Typ I und Typ II: Ein Drittel der bekannten Patienten ist symptomlos. Die anderen wurden auffällig durch Retardierung, Hypotonie oder Spastik, Verhaltensstörungen, einzelne zeigten Augensymptome. Eine strenge Reduktion der Lysinzufuhr mit der Nahrung (40 mg/kg KG/Tag) unter Verwendung eines lysinfreien Eiweißersatzprodukts kann die Lysinspiegel normalisieren. Das gilt auch für die Defekte der 2-Aminoadipataminotransferase und der 2-Oxoadipinsäuredehydrogenase (Reaktionen 2 und 3 in $\mathbf{0}$ Abb. 2.23).

\section{Glutarazidurie Typ I}

Sie ist die Folge eines Defekts der Glutaryl-CoA-Dehydrogenase, die Glutaryl-CoA zu Glutakonyl-CoA und Glutakonyl-CoA zu Crotonyl-CoA umsetzt (Reaktion 4 in - Abb. 2.23). Der Abbau von sowohl Lysin, Tryptophan und Hydroxylysin ist betroffen, diese Aminosäuren sind jedoch nicht erhöht in Körperflüssigkeiten zu finden, im Gegensatz zu Glutarat und seinen Metaboliten. Die Höhe der Ausscheidung korreliert jedoch nicht mit der Schwere des Krankheitsbildes. Dieses ist charakterisiert durch extrapyramidale Bewegungsstörungen (Dystonie, Choreoathetose), die nach normaler Neugeborenenperiode und verlangsamter motorischer Entwicklung mit Hypotonie progredient oder akut enzephalopathisch im
Rahmen von Infekten auftreten können. Der Verlust motorischer Fähigkeiten einschließlich des Sprechens führt zu weitgehender Hilflosigkeit bei erhaltener Intelligenz. Makrozephalie im Säuglingsalter ist ein richtungsweisendes Symptom. Bildgebende Verfahren zeigen typisch zerebrale, insbesondere temporale Atrophie, Hypodensität der weißen Substanz, Atrophie und Gliose von Basalganglien.

Behandlung. Sie kann durch Zufuhrbeschränkung von Lysin und Tryptophan mit oder ohne Verwendung eines lysin-, tryptophanfreien Eiweißersatzpräparats trachten, die Glutaratausscheidung zu mindern. Da diese aber nicht mit der Klinik korreliert, sind die Ergebnisse, zumindest bei symptomatischen Patienten, bescheiden. Einige Patienten reagieren günstig auf hohe Dosen Riboflavin (100-400 mg/Tag). L-Carnitin (100 mg/kgKG/ Tag) normalisiert das erniedrigte Plasmacarnitin und erhöht die Ausscheidung von Glutarylcarnitin. Baclofen $(1,5-2 \mathrm{mg} / \mathrm{kgKG} / \mathrm{Tag})$ wirkt sich bei einzelnen Patienten günstig auf die Bewegungsstörung aus.Es besteht der Eindruck, dass eine eiweißarme Diät, die den Stickstoffund Energiebedarf deckt, in Kombination mit Carnitin und Riboflavin die Patienten weniger anfällig für krisenhafte Entgleisungen macht. Ob sie bei a-/präsymptomatischen Patienten die Manifestation verhindern kann, ist nicht bewiesen.

\section{Nicht-ketotische Hyperglyzinämie}

Glyzin wird dem Körper mit allen eiweißhaltigen Lebensmitteln zugeführt. Endogen entsteht es aus Serin und Betain (s. - Abb. 2.24). Über das "glyzinspaltende System «, das aus 4 Komponenten besteht, existiert eine Verbindung zum Folatstoffwechsel. Unterschiedliche Defekte in den verschiedenen Komponenten dieses Systems von unterschiedlichem Schweregrad führen zur nicht-ketotischen Hyperglyzinämie mit neonataler oder spät-manifestierender Verlaufsform. Glyzin ist in Plasma, Liquor und Urin stark erhöht. Neugeborene entwickeln eine extreme Hypotonie, Krampfanfälle, Atemstörungen, Koma.

Behandlung. Diätetisch, d. h. durch Einschränkung der Eiweißzufuhr, evtl. unter Verwendung serin-glyzinfreier Eiweißersatzprodukte, und durch eine Stimulierung der Glyzinausscheidung mit Benzoat lassen sich die Plasma-, nicht jedoch die Liquorspiegel von Glyzin senken. Ein klinischer Effekt ist nicht nachweisbar. Ebensowenig erfolgreich ist die Gabe von potentiellen Methylgruppendonatoren (Methionin, Cholin, Folinsäure). In einzelnen Fällen sind günstige Effekte von Strychnin (Verbesserung der Hypotonie) und Diazepam gesehen worden, die aber nie zu einer normalen Entwicklung der Patienten führten. 


\subsubsection{Harnstoffzyklusdefekte}

Der vollständige Harnstoffzyklus zur Entgiftung des aus dem Aminosäurenabbau anfallenden Ammoniaks läuft nur in der Leber ab. Für seine intramitochondrial ablaufenden Reaktionen (- Abb. 2.25) ist das Vorhandensein von ATP, Azetyl-CoA und Arginin (Stimulation der NAzetylglutamatsynthetase - NAGS) Voraussetzung. Daher gehen alle Störungen in deren Produktion (z. B. Atmungskettendefekte, organische Azidurien, Fettsäureoxidation) mit sekundären Hyperammoniämien einher. Für den im Zytosol ablaufenden Teil müssen Zitrullin und Ornithin über die Mitochondrienmembran transportiert werden. Eine Störung dieses Transports (HHH-Syndrom) führt ebenfalls zu Hyperammoniämie. Auch die im Zytosol ablaufenden Reaktionen benötigen ATP und darüber hinaus Aspartat bzw. dessen Vorläufer. Arginin ist nicht nur Lieferant für Harnstoff, sondern auch Ausgangsstoff für die Kreatininsynthese. Mit 1 mol Harnstoff werden 2 mol Stickstoff ausgeschieden, eines von Ammoniak, eines von Aspartat stammend, während der Bestand an Ornithin in der Zelle derselbe bleibt. Carbamylphosphat, das wegen Mangel an Ornithin oder wegen ungenügender Kondensation mit Ornithin (Defekt 3 in - Abb. 2.25) nicht intramitochondrial verstoffwechselt werden kann, wird extramitochondrial in der Pyrimidinsynthese verwandt (erhöhte Ausscheidung von Orotsäure). Bis auf die Hyperargininämie gehen alle Defekte des Harnstoffzyklus gesetzmäßig mit Hyperammoniämie einher. Alle Defekte können sich bereits neonatal (70\%) manifestieren (Nahrungsverweigerung, Erbrechen, Lethargie, Koma, Krampfanfälle, Hypotonie bzw. Tonuserhöhung bei der Hyperargininämie, Hepatomegalie und Hepatopathie, Neigung zu Hypothermie) oder aber später (ca. $20 \%$ im 1. Lebensjahr) bis zum Erwachsenenalter bzw. symptomlos bleiben. Chronische Symptome sind geistige Retardierung, Ataxie, Epilepsie, episodische Enzephalopathie. 50\% der Patienten mit chronisch verlaufender Argininbernsteinsäurekrankheit haben Trichorrhexis nodosa. Patienten mit Hyperargininämie zeigen einen langsam progredienten Verlust geistiger und motorischer Fähigkeiten und Spastik der Beine, Epilepsie, Ataxie, Athetose.

Nur der Ornithincarbamolytransferase (OCT)-Mangel (Defekt 3 in - Abb. 2.26) wird wird X-chromosomal gebunden vererbt, d.h. männliche Patienten zeigen schwere Verlaufsformen mit neonatalem Beginn und hoher Letalität, weibliche Heterozygote ganz unterschiedliche, u.U. symptomlose Verläufe. Alle anderen Harnstoffzyklusdefekte werden autosomal rezessiv vererbt. Die

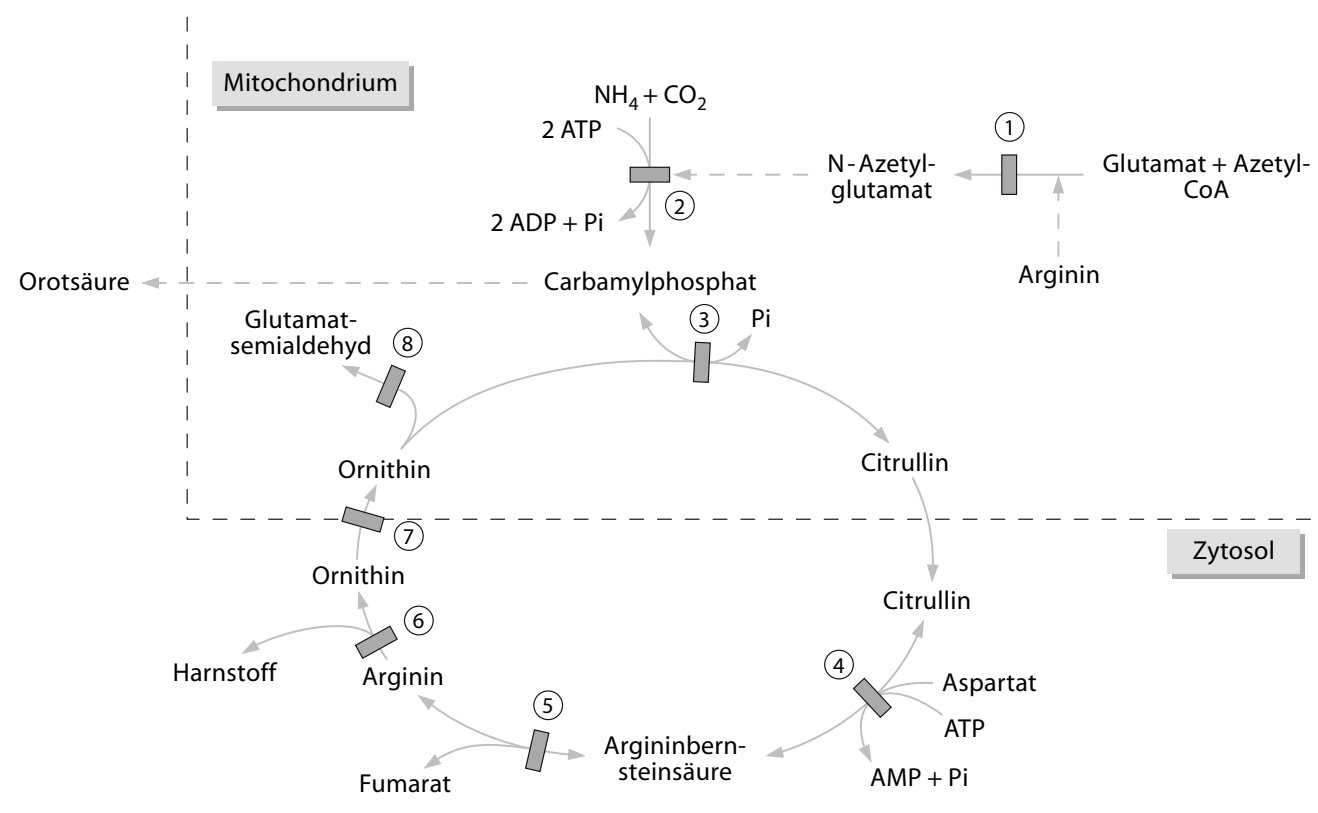

1 N-Azetylglutamatsynthetase: Defekt mit Hyperammonämie

2 Carbamylphosphatsynthetase: Defekt mit Hyperammonämie

3 Ornithincarbamyltransferase: Defekt mit Hyperammonämie

4 Argininosuccinatsynthetase: Citrullinämie mit Hyperammonämie

5 Argininsoccinatylase: Argininbernsteinsäurekrankheit mit Hyperammonämie

6 Arginase: Hyperargininämie

7 Ornithintransport: $\mathrm{HHH}$-Syndrom = Hyperammonämie, Hyperornithinämie, Homocitrullinurie

8 Ornithinaminotransferase: Hyperornithinämie, keine Hyperammonämie

- Abb. 2.25. Reaktionen und Defekte des Harnstoffzyklus 
Prognose der Patienten mit Harnstoffzyklusdefekten ist abhängig vom Ausmaß des Enzymdefekts, von der Schwere und Dauer der Symptome bis zur korrekten Diagnose und zum Therapiebeginn und selbstverständlich von der Qualität der Therapie.Deshalb sollten auch bei jeder, insbesondere jeder neonatalen, Hyperammoniämie (Neugeborene $>150 \mu \mathrm{mol} / \mathrm{l}$, sonst $>80 \mu \mathrm{mol} / \mathrm{l})$ unspezifische Behandlung und Diagnostik gleichzeitig ablaufen:

- Notfallmäßige Diagnostik: Die Resultate sollten innerhalb von $24 \mathrm{~h}$ bekannt sein:

- Aminosäuren in Plasma und einer Urinportion,

- Orotsäure und organische Säuren im Urin,

- freies und Gesamtcarnitin im Plasma,

- kurz-/mittelkettige Fettsäuren im Urin,

- Bestimmung von Anion-gaps, Glukose, Kreatinin, Harnstoff, Laktat, Säurebasenstatus im Blut.

\section{Unspezifische Behandlung}

Nach Abnahme der entsprechenden Körperflüssigkeiten und bei Blutammoniakerhöhungen auf das Dreifache des Normalen sind folgende Maßnahmen angezeigt:

- Stoppen der Eiweißzufuhr,

- Hohe Energiezufuhr durch Infusion von Glukose 6$8 \mathrm{mg} / \mathrm{kgKG} / \mathrm{min}$, evtl. mit Insulin, und Infusion von Lipiden, wenn keine Kontraindikation besteht

- Infusion von Argininhydrochlorid $(2 \mathrm{mmol} / \mathrm{kg}$ in $2 \mathrm{~h}$, gefolgt von $1-3 \mathrm{mmol} / \mathrm{kg} / \mathrm{d} ; 1 \mathrm{mmol}$ Arginin$\mathrm{HCl}=210 \mathrm{mg} ; 1 \mathrm{mmol}$ Arginin=174 mg),

- Förderung der renalen Stickstoffausscheidung durch Infusion von Natriumbenzoat $250 \mathrm{mg} / \mathrm{kgKG}$ in $2 \mathrm{~h}$, gefolgt von $250 \mathrm{mg} / \mathrm{kgKG} / \mathrm{Tag}$ und/oder von Natriumphenylbutyrat oder Natriumphenylazetat $250 \mathrm{mg} /$ $\mathrm{kgKG}$ in $2 \mathrm{~h}$, gefolgt von $250 \mathrm{mg} / \mathrm{kg} \mathrm{KG} / \mathrm{Tag}$.

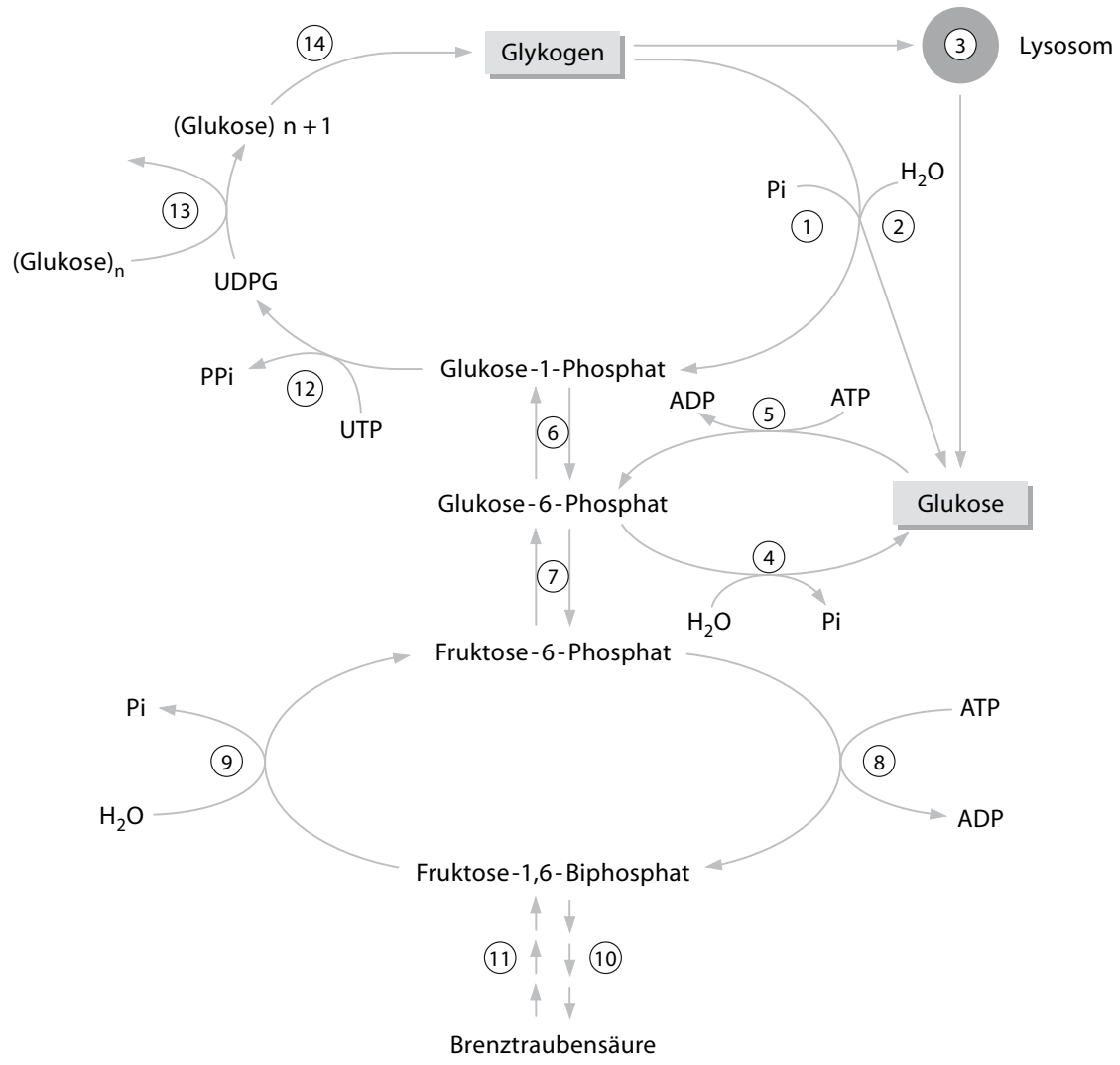

1 Phosphorylase: Glykogenose VI (Muskelphosphorylase: Glykogenose V)

2 Amylo-1,6-Glukosidase: Glykogenose III

saure $\alpha$-Glukosidase: Glykogenose II

4 Glukose-6-Phosphatase: Glykogenose I

Glukokinase

Phosphoglukomutase

Phosphoglukoisomerase

Phosphofruktokinase (Muskel: Glykogenose VII)

Fruktose-1,6-Biphosphatase

Glykolyse

Glukoneogenese

2 UDPG-Pyrophosphorylase

Glykogensynthase: "Glykogenose O"

4 1,4 1,6 Transglukosidase: Glykogenose IV

- Abb. 2.26. Reaktionen und Defekte des hepatischen Glykogen- und Glukosestoffwechsels 


\section{Behandlungsprinzipien nach Diagnose}

- Beschränkung des endogenen Eiweißabbaus durch ausreichend hohe Energiezufuhr, insbesondere während Infekten, bei Anorexie unter Einsatz von nasogastraler Sondenernährung. Verminderung der bakteriellen Ammoniakproduktion im Darm durch Antibiotika und Laktulose.

- Beschränkung der exogenen Stickstoffzufuhr. Wenn die tolerierte Menge an natürlichem Eiweiß nicht ausreicht, um den Bedarf an unverzichtbaren Aminosäuren zu decken, wird ein Teil der tolerierten natürlichen Eiweißmenge ersetzt durch eine Aminosäuremischung mit einem besonders hohen Anteil an unverzichtbaren Aminosäuren (hoher Gehalt an verzweigtkettigen Aminosäuren mit relativ weniger aromatischen Aminosäuren). Während man für ausbalanzierte Mischungen aller Aminosäuren mit einem Äquivalenzfaktor von 1,2 rechnet $(1,2 \mathrm{~g}$ Aminosäuren entsprechen $1 \mathrm{~g}$ Protein), kann für diese Mischungen angenommen werden, dass ihre Äquivalenz doppelt so hoch ist $(0,6 \mathrm{~g}$ spezielle Aminosäuremischung entsprechen $1 \mathrm{~g}$ Protein). Die Summe aus natürlichem Eiweiß und auf diese Weise berechnetem Proteinäquivalent sollte dem altersgerechten Eiweißminimalbedarf entsprechen (Beispiel: 0,6 g natürliches Eiweiß plus 0,6 g Aminosäuremischung ergäben 1,6 g Eiweißäquivalent pro kg, d. h. ausreichend für einen Säugling zwischen 7 und 9 Monaten). Theoretisch könnte die Stickstoffzufuhr durch Ersatz der essentiellen Aminosäuren durch ihre entsprechenden Oxosäuren weiter vermindert werden. In der Praxis sind diese Mischungen aber wenig erfolgreich, insbesondere kommt es eher zu Aminosäurendysbalancen im Plasma. Bei ausgeprägter Anorexie, wie sie häufig vorkommt, kann der Appetit durch eine zusätzliche Beschränkung der Tryptophanzufuhr gesteigert werden. Allerdings ist hier Vorsicht geboten wegen der Möglichkeit von Tryptophanmangelerscheinungen (Haarausfall, Optikusatrophie!). Sowohl Eiweiß wie Aminosäuren als auch die weiter unten genannten Supplemente sollten auf viele Mahlzeiten gleichmäßig verteilt werden (bis zu 12 in der Neugeborenenzeit, 5 bis 6 später). Längerdauernde Phasen eiweißfreier Ernährung sind unbedingt zu vermeiden. Während der Behandlung hyperammonämischer Krisen sollte darum bereits bei Absinken der Ammoniakspiegel unter $200 \mu \mathrm{mol} / \mathrm{l}$ mit der Zufuhr von Eiweiß in langsam ansteigender Menge begonnen werden. Wie immer bei Verzicht auf eiweißreiche Lebensmittel insbesondere tierischer Herkunft ist auf eine ausreichende Zufuhr von Mineralstoffen, Vitaminen und Spurenelementen sorgfältig zu achten. Carnitin (30$50 \mathrm{mg} / \mathrm{kgKG})$ und auch Folsäure $(1 \mathrm{mg} / \mathrm{Tag})$ sollten grundsätzlich substituiert werden.

- Ersatz von Stoffen, die aufgrund des Enzymdefekts unzureichend gebildet werden: Zitrullin für mito- chondriale und Transportdefekte bzw. Arginin für zytosolische Defekte. In der Erstversorgung erhalten alle Neugeborenen mit Hyperammoniämie Arginin: Carbamylphosphatsynthetase (CPS)-, OCT-, NAGSMangel: $2 \mathrm{mmol} / \mathrm{kgKG} / \mathrm{Tag}$; Zitrullinämie (AS-Mangel), Argininbernsteinsäurekrankheit (AL-Mangel): (2-) $4 \mathrm{mmol} / \mathrm{kgKG} / \mathrm{Tag}$; (Hyperargininämie: Stoppen der Notfallbehandlung mit Arginin). Für die Dauerbehandlung wird bei OCT- und CPS-Mangel Arginin durch Zitrullin ersetzt. Die Dosierung von Arginin und Zitrullin sollte ein Plasmaarginin (nüchtern) von $100-150 \mu \mathrm{mol} / \mathrm{l}$ gewährleisten und richtet sich ansonsten nach dem Ammoniakspiegel. Sowohl Arginin wie Argininhydrochlorid können verwendet werden. Letzteres erzeugt in hohen Dosen bei manchen $\mathrm{Pa}$ tienten metabolische Azidosen, die durch die Gabe von Zitrat (äquimolar mit der Arginindosis) verhütet werden können. Bei NAGS-Mangel kann CPS durch Carbamylglutamat (1500-1800 mg/Tag) erfolgreich stimuliert werden.

- Förderung der Ammoniak- bzw. Stickstoffausscheidung: In akuten Krisen mit Ammoniakspiegeln $>400 \mu \mathrm{mol} / \mathrm{l}$, die nicht oder nicht ausreichend auf die Notfallbehandlung mit i.v.-Arginin- und Phenylbutyrat-/Phenylazetat-/Benzoatzufuhr ansprechen, ist eine Hämodialyse die effektivste Maßnahme zur raschen Senkung des Ammoniakspiegels. Der Effekt einer Peritonealdialyse tritt viel zu langsam ein. Blutaustauschtransfusionen führen nur vorübergehend zu einer Verminderung des Blutammoniaks und sollten nicht durchgeführt werden.

- Zur Förderung der Stickstoffausscheidung im Urin kann Natriumbenzoat gegeben werden (akut $250 \mathrm{mg}$ / $\mathrm{kgKG}$ in $2 \mathrm{~h}$ gefolgt durch $250-500 \mathrm{mg} / \mathrm{kgKG} / \mathrm{Tag}$ i.v.; chronisch $250-350 \mathrm{mg} / \mathrm{kg} / \mathrm{Tag}$ oral). Der Benzoatspiegel im Plasma sollte unter i.v.-Gabe kontrolliert werden $(<2000 \mu \mathrm{mol} / \mathrm{l})$. Benzoat wird nach Aktivierung in der Leber zu Hippurat umgesetzt, das rasch im Urin ausgeschieden wird. Statt Benzoat können besser Phenylazetat oder Phenylbutyrat verwandt werden. Diese werden in der Niere zu rasch ausgeschiedenem Phenylazetylglutamin umgesetzt. Ihr Vorteil gegenüber Benzoat liegt darin, dass sie pro Mol $2 \mathrm{~mol}$ Stickstoff binden. Phenylbutyrat steht für die orale Verabreichung in Tablettenform zur Verfügung.

Durch die geschilderten Maßnahmen ist die Prognose von Patienten mit Harnstoffzyklusdefekten in Bezug auf Überleben und Entwicklung besser geworden. Sie hängt nicht von der Höhe des initialen Ammoniakspiegels ab. Wird bei Neugeborenen eine Hyperammonämie diagnostiziert, sollte so rasch und so intensiv wie möglich gehandelt werden. Besonders in den ersten Tagen der intensiven Behandlung muss über die Fortsetzung der Behandlung regelmäßig beschlossen werden. 


\subsubsection{Hyperornithinämie-, Hyperammoniämie-, Homozitrullinurie- Syndrom (HHH-Syndrom)}

Diese Krankheit wird im Anschluss an die Harnstoffzyklusdefekte besprochen, da die Symptomatologie und die Behandlung vergleichbar sind. Es handelt sich jedoch um eine Transportstörung (Defekt 7 in - Abb. 2.25) von Ornithin in das Mitochondrium, wodurch es zu einem funktionellen Defekt der OTC (und des gesamten Harnstoffzyklus) sowie der Ornithinaminotransferase kommt. Das erklärt die Hyperammoniämie und Ornithinerhöhung. Homozitrullin entsteht durch Ersatz von Ornithin durch Lysin in der OTC-Reaktion. Behandlung: Sie besteht in Eiweißbeschränkung und Gabe von Zitrullin oder Arginin.

\section{Hyperornithinämie}

Es handelt sich um einen Defekt der Ornithinaminotransferase (Defekt 8 in $\mathbf{D}$ Abb. 2.25), über die Ornithin in Prolin und Glutamat umgesetzt werden kann.Der Defekt führt zu einer spezifischen Retinadegeneration (gyrierte Atrophie von Retina und Chorioidea) mit Nachtblindheit und Myopie in der Kindheit, Gesichtsfeldeinschränkung, gestörter Dunkeladaptation und ausgelöschtem Elektroretinogramm im Erwachsenenalter. Jugendliche entwickeln subkapsuläre Katarakte. In der 5. Dekade besteht beinahe vollständige Blindheit zusätzlich zeigen die Patienten typische Muskelfaserveränderungen, jedoch nur in der Minderzahl eine Muskelschwäche, und Haarveränderungen (dünn, spärlich, gerade).

Behandlung. Einige Patienten sind Vitamin- $\mathrm{B}_{6}$-responsiv, d. h. 15-600 mg/Tag führten zu Plasmaornithinerniedrigungen um $25-60 \%$. Ein mindestens zweiwöchiger Versuch mit Vitamin $B_{6}$ sollte bei allen neu diagnostizierten Patienten unternommen werden. Eine argininbeschränkte Diät (Eiweißrestriktion ggf. mit argininfreien Aminosäuremischungen, wie sie in der Behandlung von Harnstoffzyklusdefekten benutzt werden) kann die Ornithinspiegel senken bis normalisieren. Allerdings ist ein Argininmangel zu vermeiden, der Ursache einer Hyperammonämie werden könnte. Eine derartige Behandlung ist bisher nur bei Patienten mit bereits bestehenden $\mathrm{Au}$ gensymptomen eingesetzt worden. Bei jüngeren Patienten schien sie deren Progredienz zu stoppen oder zu verlangsamen. $\mathrm{Ob}$ sie die Entstehung von Augenveränderungen bei präsymptomatischem Beginn verhüten kann, ist unbekannt. Eine Supplementierung der Patienten mit Prolin $(2-10 \mathrm{~g} / \mathrm{Tag})$ oder Kreatin $(1,5 \mathrm{~g} / \mathrm{Tag})$ unter der Annahme, dass ein Mangel dieser beiden Stoffe pathogenetisch für die Symptome verantwortlich ist, hat zu nicht eindeutigen Ergebnissen geführt. Unter Kreatin trat eine Verbesserung der Muskelhistopathologie ein.

\subsubsection{Störungen im Kohlenhydratstoffwechsel}

Hier sollen Störungen des Glykogen-, Fruktose- und Galaktosestoffwechsels besprochen werden. Von den Störungen des Pyruvatstoffwechsels wird nur der Defekt der Pyruvatdehydrogenase als möglicherweise diätetisch beEinflussbar erwähnt.

\section{Glykogenstoffwechselstörungen}

In der Regel führen sie zu Glykogenspeicherung in verschiedenen Organen, von manchen wird jedoch auch der Glykogensynthasemangel als »Glykogenose $\mathrm{O}$ « bezeichnet. Da die Durchnumerierung der Glykogenosen unterschiedlich gehandhabt wird, ist es der Deutlichkeit wegen besser, den Enzymdefekt anzugeben (s. - Abb. 2.26). Von den in - Tab. 2.68 aufgeführten Glykogenosen betreffen 2 (Typ V und VII) ausschließlich die Muskulatur. Sie sollen hier nicht besprochen werden, da sie nicht eigentlich über die Ernährung zu beeinflussen sind.Ebenso soll die Pompe-Krankheit, Typ II, Defekt der lysosomalen sauren a-Glukosidase, hier nicht besprochen werden, obwohl eine eiweißreiche, mit verzweigtkettigen Aminosäuren angereicherte Ernährung angeblich die Muskelkraft verbessern und die Progredienz verlangsamen soll. Eine »Enzymersatzbehandlung" für diese Krankheit befindet sich noch in der Erprobungsphase.Für den Typ IV mit Defekt des branching enzyme und progredienter Hepatosplenomegalie, Leberzirrhose, Gedeihstörung und Muskelatrophie existiert zur Zeit außer der Lebertransplantation keine erfolgversprechende Behandlung. Der Glykogenvorrat der normalen Leber dient der Aufrechterhaltung des Blutglukosespiegels, unabhängig von der exogenen Glukosezufuhr bzw. Glukoneogenese. Glykogen besteht aus Glukoseketten in $1 \rightarrow 4$-Verknüpfungen $1 \rightarrow 6$-Verknüpfung etwa alle 12-18 Glukoseeinheiten. Für die Synthese aus Glukose-1-Phosphat sind u.a. die Enzyme Glykogensynthase (Nr. 13 in - Abb. 2.26) und (1-4):(1-6) Transglukosidase (Nr. 14 in - Abb. 2.26) (branching enzyme) und ein Glykogenmolekül erforderlich. Der Abbau der $1 \rightarrow$ 4 glukosidischen Bindungen geschieht durch die VitaminB-abhängige Phosphorylase (Nr. 1 in - Abb. 2.26), deren Aktivität durch ein kompliziertes System und das Enzym Phosphorylase-b-Kinase geregelt wird. Es entsteht Glukose-1-Phosphat. Dieses Enzym kann jedoch Glykogen nur bis 4 Glukoseeinheiten vor einer Verzweigungsstelle spalten, dann wird die Amylo- $1 \rightarrow 6$-Glukosidase (Nr. 2 in - Abb. 2.26) (debranching enzyme) nötig, die beschränkt Glukose in freier Form abspaltet, nachdem 3 Glukoseeinheiten auf die Hauptkette des Glykogens transferiert worden sind. Das erklärt, warum es bei einem Defekt des debranching enzyme erst nach längerem Fasten, wenn überhaupt, zu einer Hypoglykämie kommt. Ein Defekt der Glukose-6-Phosphatase dagegen führt frühzeitig, manchmal schon nach 2 stündigem Fasten, zu einer Hy- 
poglykämie, da phosphorylierte Glukose aus dem hauptsächlichen Glykogenabbau und aus der Glukoneogenese nur durch dieses Enzym als freie Glukose an das Blut abgegeben werden kann. Die Glukose-6-Phosphatase (Nr. 4 in - Abb. 2.26) stellt ein mikrosomales komplexes System dar, dessen 5 Komponenten isoliert defekt sein können: Bei Glykogenose Typ Ia ist die Gesamtphosphatase-Aktivität in Leber, Niere, Darmschleimhaut vermindert. Beim Typ Ib ist die Glukose-6-Phosphattranslokase und beim Typ Ic die Pyrophosphattranslokase defekt.

\section{Glykogensynthasedefekt (Glykogenose 0)}

Sehr selten und eigentlich keine Glykogenose. Die Patienten weisen eine schwere Fastenhypoglykämie und Ketose sowie eine postprandiale Hyperlaktatämie auf. Behandlung: Häufige eiweißreiche Mahlzeiten sollen diese Störungen vermeiden.

\section{Glukose-6-Phosphatasemangel (Glykogenose I)}

Dieses ist die häufigste und schwerwiegendste Störung des Glykogenstoffwechsels. Da sich die Typen Ia, Ib, Ic klinisch ähneln, sollen sie gemeinsam besprochen werden. Charakteristisch für Typ Ib ist eine erhöhte Infektanfälligkeit bei Neutropenie und Granulozytenfunktionsstörung, die sich durch eine diätetische Behandlung und Gabe von Lithium nicht, wohl aber durch den Granulozytenkolonie-stimulierenden Faktor günstig beeinflussen lassen. Typisch sind eine Nüchternhypoglykämie und Hyperlaktatämie, da der Abbau von Glykogen über die Glykolyse ungestört ist, eine Hypertriglyzeridämie und Hyperurikämie. Leber und Nieren sind frühzeitig vergrößert durch zunehmende Glykogen- und (in der Leber) Fettspeicherung. Im 2.-3. Lebensjahrzehnt haben die meisten Patienten Leberadenome gebildet, die aber selten maligne entarten. Auch bei diätetisch behandelten Patienten kommt es nach einem Stadium von Hyperfiltration und Mikroalbuminurie zu fokaler oder globaler segmentärer Glomerulosklerose mit Niereninsuffizienz. Die Patienten sind minderwüchsig, zeigen spärliche Muskulatur, Stammfettsucht und haben ein »Puppengesicht«. Trotz wiederholter schwerster Hypoglykämieattacken sind die Patienten in der Regel geistig normal. Es besteht eine Neigung zu Diarrhoe, Blutungen und Osteoporose.

Behandlung. Das Ziel ist die Aufrechterhaltung eines normalen Blutglukosespiegels und die Normalisierung des Laktatspiegels durch kontinuierliche Zufuhr von Glukose in Höhe der Glukoseproduktionsrate für das jeweilige Alter ausgedrückt in $\mathrm{mg} / \mathrm{kgKG} / \mathrm{min}$. Laktose und Saccharose sollten weitgehend vermieden werden (laktosefreie Säuglingsanfangsnahrung z. B. auf Sojaeiweißbasis), da sie nicht in Glukose umgewandelt werden können und durch glykolytischen Abbau die Laktatproduktion erhöhen. Die Nahrung sollte $60-70 \%$ der Energiezufuhr in Form von komplexen Kohlenhydraten, $15-20 \%$ Fett (reichlich mehrfach ungesättigte Fettsäuren) und $12-15 \%$ Eiweiß enthalten. Der Abstand zwischen den Mahlzeiten muss individuell ausgetestet werden. Da auch nachts 2-4 Mahlzeiten erforderlich sind, kann stattdessen über eine Magensonde kontinuierlich eine Lösung von Glukosepolymeren oder vollständige Flüssignahrung infundiert werden. Bei letzterem ist die Aspirationsgefahr größer. Die nächtliche Ernährung sollte

Tab. 2.68. Systematik der Glykogenspeicherkrankheiten

\begin{tabular}{|c|c|c|c|c|}
\hline Typ & Eigenname & Enzymdefekt & Enzymdiagnostik in & Vererbungsmodus \\
\hline $\begin{array}{l}\text { la } \\
\text { lb } \\
\text { lc }\end{array}$ & v. Gierke & $\begin{array}{l}\text { Glukose-6-Phosphatase } \\
\text { Glukose-6-Phosphattranslokase } \\
\text { Pi/Ppi-Translokase }\end{array}$ & $\begin{array}{l}\text { Leber } \\
\text { Leber (frisch) } \\
\text { Leber (frisch) }\end{array}$ & Autosomal-rezessiv \\
\hline II & Pompe & $\begin{array}{l}\text { Lysosomale saure } \\
\text { a-Glukosidase }\end{array}$ & Leukozyten, Fibroblasten & Autosomal-rezessiv \\
\hline III & Cori & $\begin{array}{l}\text { Amylo-(1,6)-Glukosidase } \\
\text { (Debranching enzyme) }\end{array}$ & $\begin{array}{l}\text { Leukozyten, Fibroblasten } \\
\text { Leber, Erythrozyten }\end{array}$ & Autosomal-rezessiv \\
\hline IV & Amylopektinosis & Branching enzyme & Leber & Autosomal-rezessiv \\
\hline V & McArdle & Muskel-Phosphorylase & Muskel & Autosomal-rezessiv \\
\hline VI & Hers & $\begin{array}{l}\text { Phosphorylase } \\
\text { Phosphorylasekinase }\end{array}$ & $\begin{array}{l}\text { Leukozyten, Leber } \\
\text { Leukozyten, Leber, Erythrozyten }\end{array}$ & $\begin{array}{l}\text { Autosomal-rezessiv } \\
\text { X-chromosomal bzw. } \\
\text { autosomal-rezessiv }\end{array}$ \\
\hline VII & Tarui & Muskel-Phosphofruktokinase & Erythrozyten, Muskel, Fibrolasten & Autosomal-rezessiv \\
\hline
\end{tabular}


abends unmittelbar auf die letzte Mahlzeit folgen und darf morgens erst gleichzeitig mit der ersten Mahlzeit beendet werden. Bei älteren Patienten kann ungekochte Maisstärke in Wasser oder Yoghurt verwendet werden, da die langsame Freisetzung von Glukose die »tolerierte » Nüchternphase auf 6-8 h verlängern kann. Ungekochte Stärke kann auch tagsüber, mit den Mahlzeiten eingenommen, den Blutzucker stabilisieren helfen. Normoglykämie und normale Laktatspiegel korrigieren in der Regel die Lipidämie und Hyperurikämie. Bei anhaltend erhöhten Harnsäurespiegeln sollte Allopurinol gegeben und eine chronische metabolische Azidose durch die Gabe von Natriumbikarbonat ausgeglichen werden. Es muss auf eine ausreichende Zufuhr von Kalzium und anderen Nährstoffen geachtet werden. Während banaler Infekte sind die Patienten durch Erbrechen und Appetitlosigkeit gefährdet. Auch kleine operative Eingriffe erfordern in der Vorbereitungs- und Aufwachphase eine adäquate parenterale Glukosezufuhr. Ein Notfallausweis sollte getragen werden. Lebertransplantationen sind erfolgreich mit Korrektur aller biochemischen Abnormalitäten durchgeführt worden.

\section{Debranching-enzyme-Mangel (Glykogenose III)}

In der Regel stehen die Symptome von seiten der Leber im Vordergrund (Hepato(spleno)megalie, Hyperlipidämie, Ikterus, Hepatopathie bis Zirrhose, Minderwuchs, unterschiedlich stark ausgeprägte Hypoglykämieneigung und Ketose), einzelne Patienten jedoch zeigen bei geringer hepatischer Beteiligung eine langsam progrediente Myopathie mit Atrophie, manchmal linksventrikuläre Hypertrophie und Kardiomyopathie.

Behandlung. Sie folgt denselben Prinzipien wie die der Glykogenose I, nur dass auf die Beschränkung der Laktose- und Saccharose-(Fruktose-) Zufuhr verzichtet werden und reichlich Eiweiß gegeben werden kann. Ob eine enterale Ernährung über Nacht oder ungekochte Stärke erforderlich sind, muss individuell entschieden werden. Mit Eintritt der Pubertät oder kurz danach normalisieren sich die biochemische und klinische Abnormalitäten in der Regel spontan.

\section{Defekte des Phosporylasesystems (Glykogenose Typ VI)}

Die Symptome (Hepatomegalie, Hypotonie, Minderwuchs, Hypercholesterinämie, Hypoglykämie und Ketose) ähneln denen der Glykogenose III. Der Verlauf ist jedoch insgesamt milder.

Behandlung. Eine diätetische Behandlung ist nur bei kleinen Kindern erforderlich und kann sich in vielen Fällen auf eine abendliche Spätmahlzeit beschränken. Lange
Fastenperioden sollten vermieden werden. Zusammensetzung der Diät wie bei Typ III. Der häufig ausgeprägte Minderwuchs junger Kinder wird mit der Pubertät aufgeholt.

\subsubsection{Fruktosestoffwechseldefekte}

\section{Hereditäre Fruktoseintoleranz}

Ein Defekt der Fruktose-1-Phosphataldolase (Nr. 5 in - Abb. 2.27) führt sowohl zu einer Hemmung der Glukoneogenese als auch der Glykogenolyse nach Zufuhr von Fruktose (Saccharose) durch Anhäufung von Fruktose-1Phosphat bzw. durch Hemmung der Phosphorylase und damit zu Hypoglykämie. Die Symptome sind abhängig von der Zufuhr von Fruktose mit der Nahrung oder mit Medikamenten: Erbrechen, Durchfall, Nahrungsverweigerung, Gedeihstörung. Je jünger der Patient ist, desto schwerer sind die hepatischen und renalen Störungen: Hepatomegalie, Blutungsneigung, Ikterus, Ödeme und/ oder Aszites, proximale Tubulopathie im Sinne eines DeToni-Debré-Fanconi-Syndroms. Ältere Patienten haben in der Regel ein kariesfreies Gebiss.

Behandlung. Fruktose, Saccharose und Sorbitol müssen möglichst vollständig aus Nahrung und Medikamenten eliminiert werden. Darunter normalisieren sich die Leber- und Nierenfunktionsstörungen innerhalb von 2 Wochen. Nur bei schweren Leberfunktionsstörungen ist anfangs eine Eiweißbeschränkung angezeigt. Da alle Obst- und auch einige Gemüsesorten verboten sind, muss mindestens Vitamin C substituiert werden. Vorsicht ist geboten bei allen Fertiglebensmitteln, deren Zusammensetzung nicht bekannt ist. Ständige Diätfehler können zu Wachstumsretardierung als einzigem Symptom führen. Grobe Diätfehler vermeiden die Patienten wegen der direkt folgenden unangenehmen Symptome selbst. Säuglinge können bei fehlender Diagnose und Behandlung sterben. Ältere Patienten mit/ohne Diagnose sind durch sorbithaltige Infusionslösungen vital gefähr$\operatorname{det}$ (Notfallausweis!).

\section{Fruktose-1,6-Biphosphatasemangel}

Das Fehlen dieses Schlüsselenzyms der Glukoneogenese (Nr. 8 in - Abb. 2.27) führt bei Neugeborenen (mit geringen Glykogenvorräten und geringer Nahrungszufuhr) und nach längerem Fasten (bei weitgehend verbrauchtem Glykogenvorrat) zu Hypoglykämie, Laktat- und Ketoazidose. Zufuhr von Fruktose und Sorbitol in größeren Mengen bewirkt über den gleichen Mechanismus wie bei der hereditären Fruktoseintoleranz zu Hypoglykämie. Neugeborene oder junge Säuglinge zeigen episodische Hyperventilation, Bewusstseinsstörungen, Atemstillstand, Hypotonie und mäßige Hepatomegalie. Diese Attacken 
sind lebensbedrohlich. Mit zunehmendem Alter nehmen Frequenz und Schwere dieser Episoden ab und die Fastentoleranz zu.

Behandlung. Durch häufige Mahlzeiten (evtl. kontinuierliche Zufuhr über eine Sonde), Gebrauch von langsam absorbierbaren Kohlenhydraten, Vermeiden von Fruktose, Saccharose und Sorbitol muss das Auftreten von Hypoglykämien verhindert werden. Der Fettgehalt der Nahrung sollte auf 20-25\%, der Eiweißgehalt auf 10\% der Energiezufuhr begrenzt werden (auch Glyzerol und manche Aminosäuren sind Vorläufer für die Glukoneogenese).

\subsubsection{Störungen des Galaktosestoffwechsels}

Galaktose aus der Nahrung wird in 3 enzymatischen Schritten in Glukose ungewandelt (Nr. 1, 2 und 3 in - Abb. 2.27). Defekte sind bei allen drei Enzymen bekannt und führen zu einer Galaktoseerhöhung in Blut und Urin: "Galaktosämie«. Üblicherweise wird jedoch unter »Galaktosämie« die mit einem Defekt der Galaktose-1-Phosphaturidyltransferase einhergehende Krankheit bezeichnet. Die Entdeckung aller 3 Defekte im Neugeborenenscreening mit einem Test, der Galaktose nachweist, ist nur möglich, wenn das Kind mit der Nahrung Laktose (Galaktose) erhält. Für Galaktosämiepati-

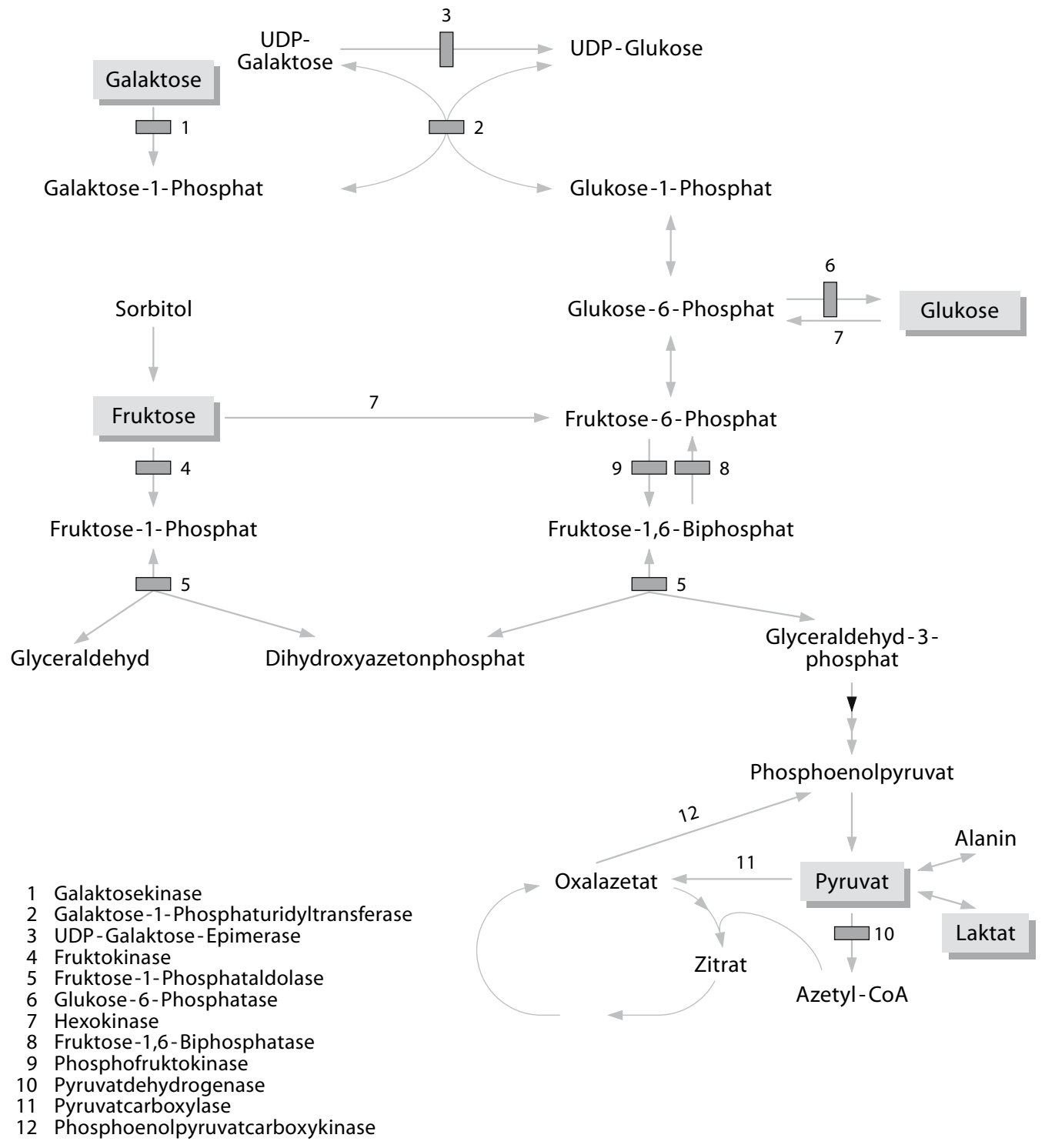

- Abb. 2.27. Reaktionen und Defekte des Stoffwechsels von Galaktose, Fruktose und Pyruvat 
enten besteht das Ziel im Vermeiden jeglicher Zufuhr an Galaktose (Nahrung, Medikamente, Zahnpasta etc.). Dieses ist allerdings unerreichbar. Galaktose kommt als Komponente von Galaktolipiden und Polysacchariden in vielen Gemüsesorten vor, außerdem in dem Disaccharid Melibiose und den Tri-/Tetrasacchariden Raffinose und Stachyose (Bohnen, Erbsen, Linsen). Raffinose und Stachyose können von den menschlichen Verdauungsenzymen nicht gespalten werden, allerdings ist ungeklärt, ob nicht bestimmte Darmbakterien in der Lage sind, Galaktose abzuspalten. Die Patienten sind überdies in der Lage, aus Glukose Galaktose selbst zu synthetisieren, so dass eine Normalisierung der Galaktose-1-Phosphatwerte auch unter strengster Diät nicht erreicht wird. Dieser Prozess beginnt offenbar bereits intrauterin. Trotzdem wird Schwangeren, die ein möglicherweise galaktosämisches Kind erwarten, eine Reduktion der Galaktosezufuhr empfohlen (kein vollständiger Verzicht!). Das Weglassen der Milch aus der Ernährung macht eine Kalziumsubstitution erforderlich. Die Gabe von Uridinsupplementen an Galaktosämiepatienten befindet sich noch im experimentellen Stadium. Ovarialinsuffiziente Patientinnen mit nachgewiesen erhöhten Gonadotropinspiegeln müssen zum gegebenen Zeitpunkt $( \pm 16$ Jahre) mit Östrogenen und Gestagenen substituiert werden.

\section{Galaktokinasemangel (Nr. 1 in $\bullet$ Abb. 2.27)}

Das Hauptsymptom ist eine Katarakt, die bereits bei der Geburt bestehen kann. Galaktose-1-Phosphat in Blutzellen ist nicht erhöht.

\section{Galaktose-1-Phosphaturidyltransferasemangel (Galaktosämie) (Nr. 2 in 0 Abb. 2.27)}

Laktosezufuhr führt bereits in den ersten Lebenstagen zu Erbrechen, Hepatomegalie, schwerer Hepatopathie mit Ikterus. Eine Katarakt ist Folge einer Linsenquellung durch eingelagerten Galaktitol, den Alkohol der Galaktose. Mit zunehmendem Alter werden eine Gedeihstörung, Minderwuchs und mentale Retardierung deutlich. Frauen sind in hohem Prozentsatz unfruchtbar wegen primärer Ovarialinsuffizienz (hypergonadotroper Hypogonadismus). Nierenfunktionsstörungen im Sinne eines DeToni-Debré-Fanconi-Syndroms treten auf. Galaktose1-Phosphat ist in Erythrozyten erhöht.

\section{Epimerasemangel (Nr. 3 in $\mathbf{D}$ Abb. 2.27)}

Wenn dieser Defekt nur die Erythrozyten betrifft, bleibt er symptomlos. Generalisiert entspricht er in seiner Symptomatologie der klassischen Galaktosämie. In Erythrozyten sind sowohl Galaktose-1-Phosphat als auch UDPGalaktose erhöht.
Behandlung. Sie ist für alle 3 Defekte diätetisch und besteht im Weglassen von Galaktose/Laktose aus der Nahrung, sobald der Verdacht auf die Erkrankung auftritt. Patienten mit generalisiertem Epimerase-defekt benötigen dagegen kleine Mengen Galaktose $(<2 \mathrm{~g} / \mathrm{Tag})$ für die Synthese von galaktosylierten Strukturproteinen und -lipiden. Es ist allerdings sehr schwierig, herauszufinden, welche Galaktosemengen gegeben werden können, ohne zur Akkumulation toxischer Mengen von Galaktose-1Phosphat zu führen.

\section{Pyruvatdehydrogenasemangel (Nr. 10 in 0 Abb. 2.27)}

Unterschiedliche Defekte der 5 verschiedenen Komponenten des PDH-Komplexes sind möglich. Das spiegelt sich in der variablen Symptomatologie wider. Pathogenetisch wichtig ist der Mangel an Azetyl-CoA (Zitronensäurezyklus, Fettsäuresynthese, Azetylcholinsynthese). Junge Säuglinge zeigen eine lebensbedrohliche metabolische Azidose mit Hypotonie, Gedeihstörung, Krampfanfällen, Dysmorphien. Neurologische Störungen nehmen mit dem Alter zu. Ältere Patienten mit milderen Defekten zeigen Ataxie und Schwindelanfälle. Blutlaktat kann, muss aber nicht deutlich erhöht sein. Gelegentlich ist der Laktatspiegel nur im Liquor hoch. Typisch ist die Kohlenhydratintoleranz mit Zunahme von Laktat und dem Auftreten von Symptomen.

Behandlung. Eine diätetische Behandlung mit einer ketogenen Diät ( $>60 \%$ der Energiezufuhr in Form von Fett, das z. T. aus MCT bestehen kann, s. - Kap. 2.5.4) sollte so früh wie möglich begonnen werden. Dabei erfolgt eine Kontrolle der Konzentrationen von Laktat und Ketonkörpern im Blut. Das Ziel ist eine hohe Produktion von Azetyl-CoA als Substart des Zitratzyklus und eine Hemmung der Glykolyse. In einzelnen Fällen meist milderer Verlaufsformen hat sich die Gabe von Thiamin (bis zu $500 \mathrm{mg} / \mathrm{kgKG} / \mathrm{Tag}$ ) als günstig erwiesen. Dagegen sind Liponsäure und Biotin offenbar ohne Effekt. Eine Aktivierung des PDH-Komplexes durch Dichloroazetat kann versucht werden. Durch Hämodialyse oder -filtration mit azetathaltigen Spüllösungen kann die bei Diätfehler auftretende Laktatazidose akut behandelt werden.

\subsubsection{Störungen des Lipidstoffwechsels/ Genetische Hyperlipidämien}

Es handelt sich um Störungen des Abbaus von Lipiden aufgrund von Veränderungen im Lipoproteinstoffwechsel oder aufgrund von Veränderungen in der Synthese oder Zusammensetzung dieser Lipoproteine. Die endogene Lipidsynthese findet vorzugsweise in der Leber statt mit de-novo-Synthese von Triglyzeriden und Cholesterin, die zusammen mit Anteilen von Nahrungscholesterin als tri- 
glyzeridreiche Lipoproteinpartikel (Very low density lipoprotein, VLDL) in die Blutbahn abgegeben werden mit einer spezifischen Ausstattung an Apoproteinen (Apo B100, Apo CII und CIII, Apo E). Durch die endotheliale Lipoproteinlipase wird der Triglyzeridgehalt verringert und Apo CII entfernt. Die resultierenden Intermediate-density-Lipoproteine (IDL) werden entweder Rezeptor-abhängig von der Leber aufgenommen oder weiter lipolytisch verändert zu Low-density-Lipoproteinen (LDL), reich an Cholesterin und relativ arm an Triglyzeriden und mit Apo B100 als einzig verbleibendem Apoprotein. LDL werden vermittelt durch den LDL-Rezeptor endozytotisch in Zellen aufgenommen, wobei die Leber die größte Rolle spielt. Cholesterinester werden hydrolysiert und hemmen die Cholesterinneusynthese über 3-Hydroxy-3-Methylglutaryl-CoA(HMGCoA)-Reduktase, die Rezeptorsynthese wird stimuliert, ebenso wie die Aktivität der AcylCholesterin-Acyltransferase (ACAT).

\section{Familiäre Hypertriglyzeridämie (Hyperlipoproteinämie Typ IV)}

Ein ursächlicher genetischer Defekt ist nicht bekannt, aber mehrere Genloci wurden identifiziert. Die Vererbung ist autosomal-dominant. Die Triglyzeridsynthese in der Leber ist erhöht, so dass der Lipidanteil in den VLDL relativ gegenüber dem Apo-B-Gehalt erhöht ist. Gleichzeitig ist der Abbau vermindert. Häufig bestehen keinerlei Symptome und wird die Triglyzeriderhöhung zufällig erkannt. Nur in Einzelfällen mit schwerer Triglyzeridämie und zusätzlicher Chylomikronämie kann es zu Bauchschmerzattacken und Pankreatitis kommen, außerdem treten eruptiven Xanthomen, Xanthelasmen, Arcus corneae und Lipaemia retinalis auf. Ursachen einer sekundären Hypertriglyzeridämie wie Diabetes mellitus, Hypothyreose, Alkoholismus etc. müssen ausgeschlossen werden. Die Behandlung ist dieselbe wie bei Lipoproteinlipase- und Apolipoprotein C II-Mangel.

\section{Familiäre Hypercholesterinämie}

Genetisch unterschiedliche LDL-Rezeptordefekte existieren in homozygoter und heterozygoter Form. Bei der homozygoten Form wird unterschieden zwischen »Rezeptornegativen « ( $<2 \%$ der normalen Aktivität) und "Rezeptordefekten " (zwischen 2 und 30\% der normalen Aktivität) Formen.Die Diagnose wird in der Regel bereits im Kleinkindesalter gestellt wegen kutaner Xanthome, zunächst am Gesäß, später über großen Gelenken. Ein Arcus corneae tritt oft vor dem Alter von 10 Jahren auf. Rezeptornegative Patienten haben zu $60 \%$ bereits vor Erreichen des 10. Lebensjahres Zeichen einer koronaren Herzkrankheit, 26\% sterben daran im Alter von 10-12 Jahren. Bei Rezeptordefekten ist der Verlauf etwas günstiger. Das Plasmacholesterin ist in der Regel höher als $16 \mathrm{mmol} / \mathrm{l}$, praktisch ausschließlich LDL-Cholesterin. Dem entspricht auch eine massive Apo-B100Erhöhung. Definitionsgemäß müssen beide Elternteile heterozygot sein.

Behandlung. Eine diätetische Behandlung ist erfolglos. Eine medikamentöse Behandlung bei Rezeptor-negativen Patienten ebenfalls. Trotzdem sollte sie zusammen mit LDL-Apherese eingesetzt werden. Medikamente in der Reihenfolge ihres Effekts sind:

- HMG-CoA-Reduktasehemmer,

- Gallensäurebindende Harze,

- Nikotinsäure in sehr hohen Dosen. Sie können in Kombination günstiger sein als einzeln.

Lebertransplantationen führten zu praktisch normalen LDL-Konzentrationen. Heterozygote für die familiäre Hypercholesterinämie haben ungefähr $50 \%$ der normalen LDL-Rezeptoraktivität mit 2- bis 3fach höheren LDLCholesteringehalten $(8-12 \mathrm{mmol} / \mathrm{l})$ im Plasma als normal.

In der Regel sind sie bis ins Erwachsenenalter symptomlos, nur $10-15 \%$ der Patienten zwischen 10 und 19 Jahren zeigen Achillessehnenverdickungen, Tendinitis oder Xanthome. Das erste Symptom in den anderen Fällen ist die koronare Herzkrankheit, die in 5\% im Alter von 30 Jahren, in $20 \%$ mit 40 Jahren und $50-75 \%$ mit 50 bzw. 60 Jahren besteht.

Die diätetische Behandlung sollte sowohl Kindern (über 2 Jahren) als auch Erwachsenen verordnet werden: Für die medikamentöse Behandlung gilt:

- Ionenaustauscherharze: bei Kindern etwa ab 6 Jahren einzusetzen, wenn durch Diät eine LDL-Erniedrigung unter $4,3 \mathrm{mmol} / \mathrm{l}$ nicht zu erreichen ist,

- HMG-CoA-Reduktasehemmer: sind in ihrem Nutzen bei Kindern nicht gut untersucht.

\section{Familiäre Dys- $\beta$-lipoproteinämie}

Der Abbau von IDL ist abhängig von Apo $E$ in seinen verschiedenen Phänotypen. Homozygotie für Apo E2/2 führt $\mathrm{zu}$ vermindertem IDL-Abbau und verminderter LDL-Produktion. Für die klinische Manifestation mit tuberösen und eruptiven Xanthomen über großen Gelenken und den Handflächen, Arcus corneae, Xanthelasmen, koronare Herzkrankheit sowie periphere und zerebrale Gefäßkrankheit sind fördernde Faktoren (Hypothyreose, Diabetes mellitus, Adipositas) verantwortlich. Nüchtern sind sowohl Triglyzeride als auch Cholesterin in etwa gleichem Ausmaß erhöht.

Behandlung. Die diätetische Behandlung entspricht der für die Heterozygotie der familiären Hypercholesterinämie. Zusätzlich sollten Zucker durch komplexe Kohlenhydrate ersetzt werden. 


\section{Familiäre kombinierte Hyperlipoproteinämie}

Diese wahrscheinlich autosomal-dominant vererbte und genetisch heterogene Krankheit hat drei chemische Phänotypen: Erhöhung von VLDL allein, von LDL allein und von LDL und VLDL. Der zugrundeliegende Defekt ist unbekannt. Typisch ist das Vorliegen unterschiedlicher Phänotypen innerhalb einer Familie, alle mit einer Reduktion von HDL-Cholesterin. Die Krankheit geht v.a. mit einem erhöhten Risiko für die koronare Herzkrankheit einher: 15-20\% der Patienten vor dem 60. Lebensjahr.

Behandlung. Die diätetische Behandlung entspricht im wesentlichen der der familiären Dys- $\beta$-lipoproteinämie. Die Diagnose einer genetisch bedingten Hyperlipidämie bei einem Patienten sollte in allen Fällen Anlass für eine Untersuchung der Familienmitglieder sein, einschließlich der Kinder.

\section{Störungen der mitochondrialen Fettsäureoxidation}

Es gibt eine Vielzahl von Defekten (s. - Tab. 2.69 und - Abb. 2.28). Fettsäuren, die von Zellen aufgenommen bzw. in ihnen synthetisiert wurden, müssen zunächst zu Koenzym-A-Fettsäuren aktiviert (kein Defekt bekannt), dann zu Carnitinestern umgewandelt (Carnitinpalmitoyltransferase, Defekt beschrieben), durch die Mitochondrienmembran transportiert (Acylcarnitintranslokase, Defekt beschrieben) und auf Koenzym A übertragen werden, um dann in Zyklen der $\beta$-Oxidation jeweils um 2 C-Atome verkürzt zu werden. Die Enzyme der $\beta$-Oxidation sind kettenlängenspezifisch, was für die diätetische Behandlung von Bedeutung sein kann (Patienten mit Defekten im Abbau von langkettigen Fettsäuren können z. B. mit mittelkettigen Triglyzeriden in ihrer Nahrung behandelt werden, s. Kap. 2.5.4). Da einerseits die Oxidation von Fettsäuren Azetyl-CoA für die Ketonkörpersynthese liefert, andererseits bei den Dehydrierungsschritten anfallende reduzierte Flavin- und Nicotinamidnukleotide über die Atmungskette reoxidiert und dabei energiereiche Verbindungen produziert werden, und da erhöhte Fettsäuren intrazellulär die Glukoneogenese hemmen, gehen grundsätzlich alle Störungen der mitochondrialen Fettsäureoxidation (Ausnahme kurzkettiger Acyl-CoADehydrogenasemangel), einschließlich ihres Transports in die Mitochondrien, mit einer hypoketotischen Hypoglykämie sowie mit Beeinträchtigung der Funktion von Organen einher, insbesondere Muskel, Herz, Leber. Das Ausmaß dieser Symptome und das Manifestationsalter hängen davon ab, ob der Defekt komplett ist, ob er systemisch alle Organe oder nur spezifisch ein oder wenige Organe betrifft. Dazu kommen äußere Faktoren wie Fasten, körperlicher Belastung und hypokalorische und auch sehr fettreiche Ernährung (z. B. bei Atkins-Diät). Je später sich die Defekte manifestieren, desto eher ist ein milder Verlauf, z. B. mit Myalgie, Muskelschwäche, insbesondere während Fasten bzw. unter Belastung, als alleinigen Symptomen zu beobachten. Andererseits kann der Verlauf letal sein bei Nichterkennen und gelegentlich trotz Behandlung. Postmortal wird typischerweise eine Verfettung von Muskel, Myokard, Leber etc. gefunden; Leberfibrosen werden bei manchen Defekten beinahe gesetzmäßig beobachtet, ebenso wie hypertrophische oder dilatative Kardiomyopathien (s. - Tab. 2.69). Für die Differentialdiagnose der einzelnen Defekte sind Bestimmungen des Ausscheidungsmusters von Fettsäuren und Fettsäurenmetaboliten, einschließlich Dicarbonsäuren, Carnitinestern und Glycinkonjugaten, im Urin unerlässlich. Die Untersuchung findet am besten während einer spontanen Stoffwechselkrise statt mit gleichzeitiger Bestimmung von Blutglukose, Ketonkörpern, freien Fettsäuren und Carnitin im Serum. Nur in Ausnahmefällen sollten Fastentests oder Fettbelastungstests erforderlich sein. Fastentests dürfen nur unter ständiger Überwachung mit der Möglichkeit einer intensiv-medizinischen Behandlung durchgeführt werden!

\section{Defekt der mittelkettigen Acyl-CoA- Dehydrogenase}

Er kommt am häufigsten vor und kann in den meisten Fällen auf eine bestimmte Mutation des Gens auf Chromosom 1 zurückgeführt werden. Die Patienten werden regelmäßig erst auffällig, wenn von der häufigen Fütterung im frühen Säuglingsalter auf weniger Mahlzeiten

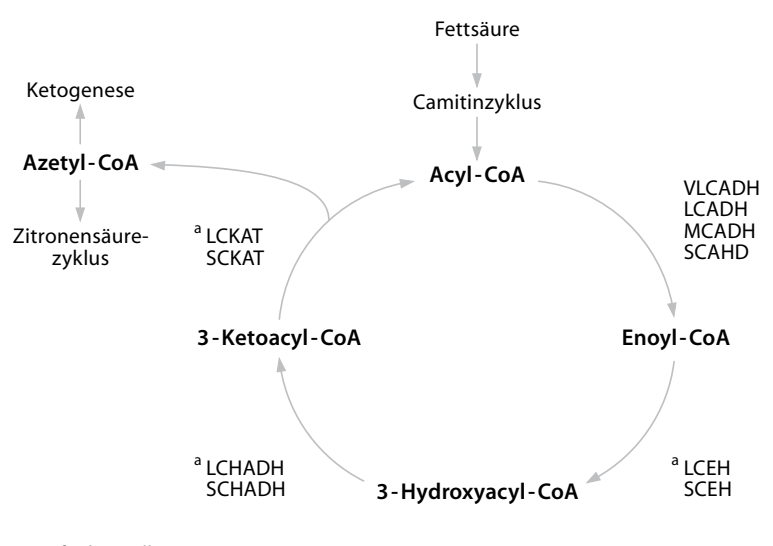

a Trifunktionelles Protein

- Abb. 2.28. Substrate und Reaktionsfolgen der mitochondrialen Fettsäureoxidation. $V L C A D H$ sehr langkettige Acyl-CoA-Dehydrogenase, $\angle C A D H$ langkettige Acyl-CoA-Dehydrogenase, $M C A D H$ mittelkettige Acyl-CoA-Dehydrogenase, SCADH kurzkettige Acyl-CoA-Dehydrogenase, $L C E H$ langkettige Enoyl-CoA-Hydratase, SCEH kurzkettige EnoylCoA-Hydratase, $\mathrm{LCHADH}$ langkettige 3-Hydroxyacryl-CoA-Dehydrogenase, SCHADH kurzkettige 3-Hydroxyacryl-CoA-Dehydrogenase, LCKAT langkettige 3-Ketothiolase, SCKAT kurzkettige 3-Ketothiolase 
- Tab. 2.69. Charakterisierung von Defekten der mitochondrialen $\beta$-Oxidation der Fettsäuren

\begin{tabular}{|c|c|c|c|c|c|c|c|c|}
\hline Enzymdefekt & $\begin{array}{l}\text { Manifestations- } \\
\text { alter }\end{array}$ & $\begin{array}{l}\text { Hyptonie/ } \\
\text { Myopathie }\end{array}$ & $\begin{array}{l}\text { Hepato- } \\
\text { megalie }\end{array}$ & $\begin{array}{l}\text { Kardio- } \\
\text { megalie }\end{array}$ & $\begin{array}{l}\text { Hypoketotische } \\
\text { Hypoglykämie }\end{array}$ & Myalgie & $\begin{array}{l}\text { Myoglobin- } \\
\text { urie }\end{array}$ & Therapie \\
\hline Carnitintransport & $\begin{array}{l}6 \text { Monate- } \\
4 \text { Jahre }\end{array}$ & + & + & + & + & & & Carnitin \\
\hline $\begin{array}{l}\text { Carnitinpal mityl- } \\
\text { transferase I }\end{array}$ & $<1 \mathrm{Jahr}$ & & $(+)$ & & + & & & $\begin{array}{l}\text { Carnitin; kohlenhyd- } \\
\text { ratreich; fettarm }\end{array}$ \\
\hline $\begin{array}{l}\text { Acylcarnitintrans- } \\
\text { lokase }\end{array}$ & Neugeborene & + & + & AV-Block & + & & & $\begin{array}{l}\text { Wahrscheinlich } \\
\text { erfolglos }\end{array}$ \\
\hline $\begin{array}{l}\text { Carnitinpalmityl- } \\
\text { transferase II } \\
\text { - Muskuläre } \\
\text { Form } \\
\text { - Infantile Form }\end{array}$ & $\begin{array}{l}\text { 5.-30. } \\
\text { Lebensjahr } \\
<6 \text { Monate }\end{array}$ & & & AV-Block + & $\begin{array}{l}(+) \\
+\end{array}$ & & & \\
\hline $\begin{array}{l}\text { Kurzkettige } \\
\text { Acyl-CoA- } \\
\text { Dehydrogenase }\end{array}$ & $>1 \mathrm{Jahr}$ & + & + & & & & & $\begin{array}{l}\text { Wahrscheinlich } \\
\text { erfolglos }\end{array}$ \\
\hline $\begin{array}{l}\text { Mittelkettige } \\
\text { Acyl-CoA- } \\
\text { Dehydrogenase }\end{array}$ & $\begin{array}{l}6 \text { Monate- } \\
4 \text { Jahre }\end{array}$ & & $(+)$ & $(+)$ & + & & & $\begin{array}{l}\text { Vermeiden von } \\
\text { Fasten; fettarm; } \\
\text { kohlenhydratreich; } \\
\text { Carnitingabe }\end{array}$ \\
\hline $\begin{array}{l}\text { Langkettige } \\
\text { Acyl-CoA- } \\
\text { Dehydrogenase }\end{array}$ & $\begin{array}{l}\text { Neugeborene- } \\
10 \text { Jahre }\end{array}$ & + & + & + & + & + & + & $\begin{array}{l}\text { Vermeiden von } \\
\text { Fasten; fettarm; } \\
\text { kohlenhydratreich; } \\
\text { Carnitingabe }\end{array}$ \\
\hline $\begin{array}{l}\text { Sehr langkettige } \\
\text { Acyl-CoA- } \\
\text { Dehydrogenase }\end{array}$ & Neugeborene & & + & $\begin{array}{l}\text { Kammer- } \\
\text { flimmern }\end{array}$ & & & & $\begin{array}{l}\text { Vermeiden von } \\
\text { Fasten; fettarm; } \\
\text { Carnitingabe }\end{array}$ \\
\hline $\begin{array}{l}\text { Langkettige } \\
\text { 3-Hydroxy- } \\
\text { Acyl-CoA- } \\
\text { Dehydrogenase }\end{array}$ & $\begin{array}{l}\text { Neugeborene- } \\
1 \mathrm{Jahr}\end{array}$ & + & + & + & + & + & $\begin{array}{l}\text { + Retinitis } \\
\text { pigmentosa } \\
\text { + periphere } \\
\text { Neuropathie }\end{array}$ & $\begin{array}{l}\text { Vermeiden von } \\
\text { Fasten; fettarm; } \\
\text { kohlenhydratreich; } \\
\text { MCT; Carnitingabe }\end{array}$ \\
\hline $\begin{array}{l}\text { Langkettige } \\
\text { 3-Oxoacyl- } \\
\text { CoA-Thiolase }\end{array}$ & $<2$ Jahre & + & + & & & & & $\begin{array}{l}\text { Vermeiden von } \\
\text { Fasten }\end{array}$ \\
\hline $\begin{array}{l}\text { Trifunktionelles } \\
\text { Protein }\end{array}$ & Neugeborene & + & & + & + & & & $\begin{array}{l}\text { Wahrscheinlich } \\
\text { erfolglos }\end{array}$ \\
\hline 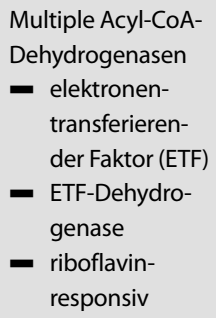 & $\begin{array}{l}\text { Neugeborene- } \\
19 \text { Jahre }\end{array}$ & & & & & & & \\
\hline $\begin{array}{l}\text { 2,4-Dienoyl- } \\
\text { CoA-Reduktase }\end{array}$ & Neugeborene & + & & + & & & & $\begin{array}{l}\text { MCT-Carnitingaben; } \\
\text { wahrscheinlich } \\
\text { erfolglos }\end{array}$ \\
\hline
\end{tabular}


pro Tagübergegangen wird. Weitere Auslöser sind banale Gastroenteritiden, Nahrungsverweigerung, Erbrechen, Bewusstseinstrübung bis zu Koma und Krampfanfälle. Die Mortalität während der Erstmanifestation ist hoch (bis 25\%). Eine Hypotonie oder Muskelschwäche wird in der Regel nicht gesehen.

Behandlung. In der akuten Krise ist eine hochdosierte parenterale Glukosezufuhr erforderlich (10 mg/kgKG/ $\mathrm{min}$ ), um die Insulinausschüttung zu stimulieren und damit sowohl die Fettsäureoxidation als auch die Lipolyse zu hemmen. Trotz dieser Maßnahmen kann das Koma 1-2 Tage bestehen bleiben. In der langfristigen Behandlung ist das Vermeiden von längeren Nüchternpausen entscheidend, es spllten mindestens 6 Mahlzeiten pro Tag, die letzte spätabends oder nachts verzehrt werden. In Einzelfällen kann eine nächtliche Magendauersondenernährung erforderlich sein. Es muss individuell ausgetestet werden, ob der Patient von ungekochter Stärke spätabends oder per nächtlicher Sonde profitiert. Eine Einschränkung der Fettzufuhr ist in der Regel nicht erforderlich, in Einzelfällen ist eine Reduktion auf 10-20\% der Energiezufuhr jedoch hilfreich. Über den Nutzen einer Carnitinsupplementierung sind die Meinungen geteilt: Einerseits ist von einem verbesserten Transport der Carnitinfettsäureester zum Ort des mitochondrialen Enzymdefekts kein positiver Effekt zu erwarten, andererseits jedoch verlieren die Patienten vermehrt Carnitin in Form von Estern im Urin und haben in der Regel erniedrigte oder niedrig normale Carnitinspiegel mit erhöhten Acylcarnitin/Carnitin-Quotienten. Zusätzliche Zufuhr von Carnitin könnte zu einem erleichterten Abtransport von Fettsäuremetaboliten aus den Mitochondrien bzw. aus den Zellen führen und den Quotienten freies CoA/Acyl-CoA normalisieren.

\section{Störungen der peroxisomalen Fettsäureoxidation}

Peroxisomen erfüllen wichtige Funktionen im Stoffwechsel: u.a. Biosynthese von Etherphospholipiden, $\beta$-Oxidation von Fettsäuren, insbesondere von großer Kettenlänge (>24 C-Atome), Gallensäuresynthese, Pipecolinsäureoxidation, Glyoxalatabbau. Bei Störungen der Biogenese von Peroxisomen sind alle oder mehrere dieser Funktionen gleichzeitig gestört, was sich u.a. in einer Erhöhung der Plasmaspiegel an sehr langkettigen Fettsäuren und von Phytansäure äußert.

Behandlung. Eine diätetische Behandlung solcher komplexer Defekte (z. B. Zellweger-Syndrom oder infantile Refsum-Krankheit) ist zwar versucht worden, ist aber von vornherein zum Scheitern verurteilt, da diese Kinder bereits mit schweren morphologischen Defekten und funktionellen Störungen zur Welt kommen.

\section{X-chromosomale Adrenoleukodystrophie}

Heterozygote Frauen zeigen nur außerordentlich selten Symptome. Mehr als $40 \%$ der betroffenen Jungen bekommen um das 7. Lebensjahr neurologische Symptome mit Verhaltensauffälligkeiten, Seh- und Hörstörungen, Gangstörungen. Innerhalb von wenigen Jahren sterben diese Patienten blind, quadriplegisch und dement. Eine Addison-Symptomatik (=Nebennierenrindeninsuffizienz) mit Hautpigmentierung kann den neurologischen Symptomen vorausgehen. Bei $10 \%$ der erwachsenen Patienten ist die Nebennierenrindeninsuffizienz das einzige Symptom. Der Rest entwickelt in Adoleszenz oder Erwachsenenalter eine Adrenomyeloneuropathie. Der Erkrankung liegt ein Defekt in einem peroxisomalen Membranprotein, das Substrate wie sehr langkettige Fettsäuren über Membranen hinweg transportieren kann, zu Grunde. Als Folge akkumulieren sehr langkettige Fettsäuren im Plasma; typisch ist eine Erhöhung des Verhältnisses C26/C22-Fettsäuren im Plasma.

Behandlung. Die diätetische Behandlung trachtet durch Beschränkung der Zufuhr an sehr/langkettigen Fettsäuren (enthalten in Fruchtschalen, Samen, Nüssen) auf weniger als $10 \mathrm{mg} / \mathrm{Tag}$ (normal 12-40 mg/Tag) und Hemmung der endogenen Synthese von sehr langkettigen Fettsäuren durch Ölsäure und Erucasäure, deren Plasmaspiegel zu normalisieren. Die Fettzufuhr wird auf ca. 20\% der Energiezufuhr beschränkt; weitere $20 \%$ der Energiezufuhr werden als eine Mischung aus 75\% Erucasäure und 18\% Ölsäuretriglyzeriden (»Lorenzo’s oil«) gegeben. Die Diät ist sehr kompliziert und aufwendig. Es existieren Listen von C26-Gehalten von Lebensmitteln und Rezeptbücher. Die klinischen Erfolge bei symptomatischen und besonders jungen Patienten sind zweifelhaft. Man ist heute eher geneigt, den genetischen Defekt im präsymptomatischen Stadium durch eine Knochenmarktransplantation zu korrigieren.

\section{Refsum-Krankheit}

Bei dieser autosomal-rezessiven Krankheit wird mit der Nahrung zugeführte Phytansäure (eine verzweigtkettige Fettsäure mit 20 Kohlenstoffatomen) gespeichert. Ursache ist ein Defekt der peroxisomalen Phytanoyl-CoAHydroxylase oder ein verändertes »Importprotein« für peroxisomale Enzyme. Die Phytansäurespeicherung ist nahrungs- und altersabhängig. Phytansäure findet sich z. B. in Milchfett von Tieren, die chlorophyllhaltiges Futter bekommen haben und wird vom Menschen möglicherweise auch aus freiem Nahrungsphytol gebildet. Die hauptsächlichen Symptome sind Retinitis pigmentosa, periphere Neuropathie, zerebelläre Ataxie, sensorische Taubheit, Anosmie, Ichthyosis, Skelettveränderungen, Arrhythmien und AV-Blocks des Herzens.

Behandlung. Die diätetische Behandlung sollte die Phytansäurezufuhr auf unter $10-15 \mathrm{mg} / \mathrm{Tag}$ senken. Weil der 
Phytansäuregehalt in Lebensmitteln von der Jahreszeit und regionalen Gegebenheiten abhängt, sind Nährstoff-Tabellen unzuverlässig. Es wird daher die Gesamtfettzufuhr auf etwa $15 \mathrm{~g} /$ Tag beschränkt bei gleichzeitiger Supplementierung der benötigten essentiellen Fettsäuren in Form von pflanzlichen Ölen mit bekanntem Phytansäuregehalt. Die fehlenden "Fettkalorien" sind durch Kohlenhydrate zu ersetzen. Verboten ist Fleisch von Wiederkäuern, während mageres Schweine-, Pferde- und Geflügelfleisch erlaubt ist. Verboten sind alle grünen Gemüse und Früchte. Der eigentlichen diätetischen Behandlung kann eine Plasmapherese und ein stationärer Aufenthalt mit praktisch phytansäurefreier Flüssignahrung vorangestellt werden. Während der Diät ist auf ausreichende Zufuhr an Vitaminen, Mineralstoffen und Spurenelementen zu achten. Eine ausreichende Energiezufuhr ist notwendig, eine Gewichtsabnahme muss vermieden werden, da dadurch aus den Fettgewebedepots große Mengen Phytansäure frei werden, die die Symptome vorübergehend verschlimmern.

\subsection{Diätkatalog bei angeborenen Stoffwechselerkrankungen}

\section{Diät bei Phenylketonurie (PKU) \\ Indikation. Phenylketonurie}

Definition. Kost unter Anpassung an den Plasmaphenylalaninspiegel und Beschränkung phenylalaninhaltiger Lebensmittel (selektive Aminosäurebegrenzung).

Ziele. Kompensation der Stoffwechselstörung durch Senkung des Plasmaphenylalaninspiegels auf Werte zwischen 0,7 und $4 \mathrm{mg} / \mathrm{dl}(0,04-0,24 \mu \mathrm{mol} / \mathrm{l})$, um Symptomfreiheit und eine normale geistige Entwicklung zu gewährleisten.

Anmerkung. Die Kost wird individuell und nach Alter des Patienten berechnet. Nahrungsmittel, welche einen Proteinanteil mit hohem Phenylalaningehalt enthalten, sind zu meiden. Da praktisch alle Nahrungseiweiße 5\% Phenylalanin enthalten, sind in der Regel ein phenylalaninfreies und tyrosinangereichertes Aminosäure- bzw. Proteinsupplement (z. B. SHS P-AM, Milupa PKU) sowie eine schrittweise Zulage von Phenylalanin zur Deckung des Eiweißbedarfs bzw. des Bedarfs an essentiellen Aminosäuren notwendig. Da Tyrosin bei PKU eine essentielle Aminosäure ist, muss der Tyrosinbedarf gedeckt werden. Der Phenylalaninbedarf beträgt im Säuglingsalter 60$30 \mathrm{mg} / \mathrm{kgKG}$, im 2.-3. Lebensjahr $30-20 \mathrm{mg} / \mathrm{kgKG}$, im 4.-6. Lebensjahr $20-15 \mathrm{mg} / \mathrm{kgKG}$, im 7.-12. Lebensjahr $15-10 \mathrm{mg} / \mathrm{kgKG}$ und bei Erwachsenen $10-5 \mathrm{mg} / \mathrm{kgKG}$. Die Basis der Diät sind eiweißfreie Kalorienträger wie Maisstärke, Zucker, Maltodextrine, Pflanzenöle, Obst. Ab dem 11. Lebensjahr können die diätetischen Restriktionen gelockert werden (Phenylalaninspiegel $0,7-15 \mathrm{mg}$ / dl). Auch im Erwachsenenalter sollten die Phenylalaninspiegel nicht über $20 \mathrm{mg} / \mathrm{dl}$ ansteigen, was eine überwiegend vegetarische Ernährung (Eiweißzufuhr: 0,5-0,6 g/kg $\times$ Tag) erfordert. Das Vermeiden von aspartamhaltigen Süßstoffen und Getränken bzw. Lebensmitteln wird empfohlen. Der Zufuhr von Kalzium ist kritisch, die Versorgung mit B-Vitaminen (einschließlich Vitamin- $\mathrm{B}_{12}$ und Folsäure) ist zu überwachen. Bei PKU durch Tetrahydrobiopterinmangel besteht keine Indikation für eine diätetische Behandlung. Therapie ist die medikamentöse Gabe von Tetrahydrobiopterin und Neurotransmittervorstufen (L-DOPA, 5-Hydroxytryptophan).

\section{Diät bei Ahornsirupkrankheit (Verzweigtkettenketonurie)}

Indikation. Ahornsiruperkrankung.

Definition. Kost unter Anpassung an die Plasmakonzentrationen und Beschränkung der Leucin-, Isoleuzin- und Valinzufuhr auf die lebensnotwendige Menge.

Ziele. Kompensation der Stoffwechselstörung durch eine verminderte Nahrungszufuhr von verzweigtkettigen Aminsäuren, Ziel ist ein Plasmaleuzinspiegel von 0,1$0,5 \mathrm{mmol} / \mathrm{l}$.

Anmerkung. Da die Relationen der verzweigtkettigen Aminosäuren in natürlichen Eiweißen vom Bedarf des Menschen abweichen, ist ein teilweiser Ersatz von normalem Nahrungseiweiß durch Aminosäuregemische, welche keine verzweigtkettigen Aminosäuren enthalten (z. B. SHS ILV-AM oder Milupa MSUD), notwendig. Bei intermittierender Form ist eine Therapie nur in den akuten Stadien notwendig. Allerdings sollte die Eiweißzufuhr isonitrogene Werte nicht überschreiten. Bei der thiaminabhängigen Form ist keine Diät, sondern die hochdosierte Gabe von Thiamin (10-200 mg/Tag) angezeigt.

\section{Diät bei Homocystinurie

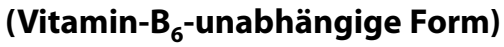

Indikation. Homocystinurie.

Definition. Eiweiß- und methioninarme und cystinangereicherte Diät.

Ziele. Kompensation der Abbaustörung des Methionins zu Cystin bei Deckung des Methionin- und Cystinbedarfs und Vermeidung von Methioninmangelschäden (Leberzirrhose); nicht messbare Homocystinausscheidung im Urin, normale Plasmamethioninspiegel $(<0,04 \mu \mathrm{mol} / \mathrm{ml})$.

Anmerkung. Die Diät ist eiweißarm. Aminosäuren werden als methioninfreies Aminosäuregemisch gegeben 
(z. B. M-AM SHS, Milupa HOM). Der Cystingehalt des letztgenannten Präparats ist niedrig, der Cystinbedarf (100-150 mg/kgKG/Tag) ist durch Cystinsupplemente zu decken. Die untere Methioninzufuhrgrenze beträgt bei Säuglingen $20-40 \mathrm{mg} / \mathrm{kgKG} / \mathrm{Tag}$, bei Kindern $10-20 \mathrm{mg} /$ $\mathrm{kgKG} / \mathrm{Tag}$ und bei Erwachsenen $<10 \mathrm{mg} / \mathrm{kgKG} / \mathrm{Tag}$. Bei der pyridoxinabhängigen Form müssen Vitamin- $B_{6}$ (z. B. Hexobion, Dosierung individuell) sowie auch Folsäure (z. B. Folsan, $400 \mu \mathrm{g} / \mathrm{Tag}$ ) gegeben werden. Die Diät sollte unter strenger Kontrolle der Plasmaaminosäurespiegel durchgeführt werden.

\section{Diät bei Hypertyrosinämie}

Indikation. Hypertyrosinämie.

Definition. Eine eiweißarme Kost, welche besonders arm an aromatischen Aminosäuren ist. Begrenzung der Phenylalaninzufuhr auf den Bedarf.

Ziele. Senkung der Plasmatyrosinspiegel $<110 \mu \mathrm{mol} / \mathrm{l}$ unter gleichzeitiger Deckung des altersstufenentsprechenden Phenylalaninbedarfs (10-90 mg/Tag)

Anmerkung. Substitution des Aminosäurebedarfs durch phenylalanin- und tyrosinfreie Supplemente (z. B. SHS PT-AM, Milupa Tyr). Kostaufbau analog der PKU-Diät. Bei gleichzeitiger Hypermethioninämie muss die Kost auch methioninarm sein, bei gleichzeitiger Fruktoseintoleranz.

\section{Diät bei Galaktoseintoleranz}

Indikationen. Galaktoseinteroleranz, Galaktosämie.

Definition. Vollkost unter Verzicht auf laktose- und raffinosehaltige Lebensmittel. Ausschaltung der Galaktose.

Ziele. Kompensation der Stoffwechselstörung, Senkung des Blutzellgalaktose- und Galaktose-1-Phosphatgehalts auf Werte $<4 \mathrm{mg} \%$, um eine verzögerte geistige Entwicklung, Kataraktbildung, Leberversagen und Tod zu verhindern.

Anmerkung. Milchfreie Ernährung (auch keine Muttermilch!) mit Sojanahrung (z. B. Milupa SOM, Humana SL, Sojaval G). Galaktosefreie Breikost von z. B. den Firmen Hipp, Humana und Milupa. Galaktose ist auch in bestimmten Gemüsen (Linsen, Erbsen, Spinat, rote Beete) und Innereien (Leber) enthalten. Vorsicht bei Teigwaren, Kartoffelprodukten, Fertiggerichten, Soßen, Dessertspeisen. Kalzium, Vitamin- $\mathrm{B}_{2}$ und fettlösliche Vitamine müssen in der Regel supplementiert werden. Milchsäure (!) und Laktalbumin enthalten keine Galaktose. Patienten mit Galaktokinasemangel müssen zur Prävention der Ka- taraktbildung ebenfalls eine galaktosefreie Diät einhalten. Patienten mit einem UDP-Galaktose-4-Epimerasemangel, welcher nicht in jedem Fall generalisiert auftritt, brauchen ggf. keine Galaktose-freie Diät. (Cave: Auf Milchzuckerhaltige Arzneimittel, laktosehaltige Zahnpasta, Laktulose ist zu achten. Die Diät ist lebenslang durchzuführen).

\section{Diät bei Fruktoseintoleranz}

Indikationen. Fruktoseintoleranz, Fruktosämie.

Definition. Anfangsnahrung oder Vollkost unter Verzicht auf fruktosehaltige Lebensmittel und Saccharose, Begrenzung der Fruktosezufuhr auf 0.5-1 g Fruktose/Tag.

Ziel. Kompensation eines Enzymdefekts.

Anmerkung. Alle Obstsorten mit Ausnahme von Zitronen enthalten Fruktose. Fruktosearme (Fruktoseanteil $<2 \mathrm{~g} / 100 \mathrm{~g}$ essbares Lebensmittel) Gemüsesorten sind Spinat, Feldsalat und Kartoffeln. Vorsicht bei sorbithaltigen Arzneimittelzusätzen (Säften, Dragees), fruktose- bzw. sorbithaltigen Süßstoffen, Formuladiäten und bei parenteraler Ernährung mit Zuckeraustauschstoffen. Fruktose- und sorbithaltige Zuckeraustauschstoffe sollten in der parenteralen Ernährung generell gemieden werden. Bei Säuglingen und Kleinkindern sind Fertigbreie nicht geeignet. Statt diesen müssen fruktose- und saccharosefreie Säuglingsmilch oder fruktosefreie Spezialnahrung verwendet werden. Vorsicht bei gesüßten Säuglingstees. Aufgrund der eingeschränkten Nahrungsmittelauswahl müssen wasserlösliche Vitamine substituiert werden.

\section{Diät bei Glykogenspeicherkrankheiten}

Indikationen. Glykogenspeicherkrankheit Typ I, VI.

Definition. Relativ kohlenhydratreiche Kost (60-70\% der Energiezufuhr) unter Elimination von Fruktose, Saccharose, Galaktose und Laktose. Glukose, Oligosaccharide und auch Stärke werden nicht beschränkt. Die Fettzufuhr sollte $20 \%$ der Gesamtenergie nicht überschreiten, die relative Eiweißmenge liegt bei $10-20 \%$.

Ziele. Hemmung der Glykogensynthese durch Elimination von Vorstufen, Vermeidung von Hypoglykämie und Laktatazidose, Normalisierung von Plasmatriglyzeridund Harnsäurespiegel, Verminderung der Lebergröße, normales Wachstum und Entwicklung.

Anmerkung. Kohlenhydratquellen sind langsam resorbierbare Polymere z. B. Maltodextrine, Stärkeabbauprodukte oder Traubenzucker. Wegen der Gefahr einer Hypoglykämie ist die Nahrungszufuhr auf viele kleine 
Mahlzeiten (alle 3-4 h oder häufiger) zu verteilen. Nachts ist eine niedrigdosierte und kontinuierliche Nahrungszufuhr über eine gastrale Sonde sinnvoll (z. B. Maltodextrin, $4 \mathrm{~g} / \mathrm{kg}$ KG über $12 \mathrm{~h}$ ). Bei Säuglingen darf keine Muttermilch gegeben werden. Alternativ sind maltodextrinangereicherte Ersatznahrungen (z. B. Humana SL oder Pregestimil) geeignet. Aufgrund der Vermeidung von Milchprodukten und Obst sowie der eher niedrigen Fettzufuhr sind der Bedarf an Kalzium, essentiellen Fettsäuren, Vitamin A, D, $B_{2}$ und $C$ nicht adäquat gedeckt. Die Kost ist ballaststoffarm. Eine sekundäre Hyperlipidämie und auch eine meist asymptomatische Hyperurikämie sind häufig. Bei Glukogenose Typ V sind die genannten diätetischen Maßnahmen nicht notwendig. Vor und während körperlicher Anstrengungen ist zusätzlich Glukose zu verzehren.

\section{Diät bei Refsum-Syndrom}

Indikation. Refsum-Syndrom (hereditäre Abbaustörung für Phytansäure).

Definition. Restriktion von Phytansäure in der Ernährung von $60-90 \mathrm{mg} / \mathrm{Tag}$ auf $<20 \mathrm{mg} /$ Tag.

Ziel. Senkung des Plasmaphytansäurespiegels $(<10 \mathrm{mg} / \mathrm{dl})$.

Anmerkung. Phytansäure und Phytane sind in fettem Rindfleisch, Milchfett, Obst, grünem Gemüse, Nüssen und Kaffee enthalten. Fetthaltige Molkereiprodukte, Pflanzenmargarine, Rind-, Kalb- und Wildfleisch sind zu meiden. Praktisch bedeutet eine solche Einschränkung der Lebensmittelauswahl eine drastische Reduktion der Fett- und damit auch der Energiezufuhr. Eventuell Austausch der LCT- gegen MCT-Fette. Eine Substitution von Kalzium, wasser- und fettlöslichen Vitaminen ist notwendig. Diese Kostform muss lebenslang beibehalten werden.

\subsection{Künstliche Ernährung}

Eine künstliche Ernährung unterscheidet sich von einer normalen Ernährung durch:

- die Wahl des Zugangsweges (enteral über Sonden, Gastrostomien oder Jejunostomien und parenteral über i.v.-Kathetersysteme),

- den Applikationsmodus (in der Regel kontinuierliche Infusion über einen Zeitraum von 8-24 h) und

- die Nährlösung (hoch- und niedermolekulare Formuladiäten als diätetische Lebensmittel und Infusionslösungen als Arzneimittel).

Eine Indikation für eine künstliche Ernährung besteht immer dann, wenn ein Patient nicht essen kann, darf oder will. Sie kann ernährungsmedizinisch (z. B. zur Verbesserung des Ernährungszustands bzw. zum Erhalt der Stoffwechsel-, Flüssigkeits- und Elektrolyt-Homoöstase), aber auch ernährungstherapeutisch (z. B. bei akutem Schub einer chronisch-entzündlichen Darmerkrankung) wirken. Künstliche Ernährung kann präventiv zur Verhütung einer Malnutrition oder bei bereits manifesten Ernährungsdefiziten zu deren Behandlung und Kompensation wirksam sein.

Die Indikation für eine künstliche Ernährung muss frühzeitig gestellt werden. Eine künstliche Ernährung beeinflusst den Stoffwechsel und den Ernährungszustand und auch die Krankheitsaktivität. Variablen der Wirksamkeit sind der Ernährungszustand, der Stoffwechsel, der klinische outcome, der Krankheitsverlauf (z. B. die Wundheilung, postoperative Komplikationen), die Dauer des Krankenhausaufenthalts und das Überleben der Patienten. Bei Schwerkranken kann die Ernährung (i.e. »nutritional support«) eine Schadensbegrenzung oder im besten Fall den Erhalt des Ernährungszustands bewirken. Eine deutliche anabole Stoffwechsellage und eine Verbesserung der Körperzellmasse wird in dieser Situation nur durch wirksame Behandlung bzw. Heilung der Grunderkrankung oder aber durch die zusätzliche Gabe von pharmakologisch, d. h. anabol-wirksamen Faktoren (z. B. Wachstumsfaktoren, Wachstumshormon; i.e. »metabolic support«) erreicht. Eine künstliche Ernährung kann zu einer Senkung der Anzahl von Komplikationen (z. B. bei onkologischen Patienten während einer Chemotherapie) und zu einer Verbesserung der Lebensqualität der Patienten beitragen. Der Wert einer künstlichen Ernährung steigt mit deren frühem Begiin, der Dauer und konsequenten Anwendung.

Die Entscheidung für oder gegen eine künstliche Ernährung berücksichtigt auch die Prognose des Patienten und seine persönliche Situation:

- Ist die Grunderkrankung behandelbar?

- Welchen Wunsch haben der Patient und seine Angehörigen?

- Ist ein Gewinn an Lebensqualität zu erwarten?

Die künstliche Ernährung wird vorzugsweise enteral durchgeführt. Spezielle Indikationen für eine parenterale Ernährung ergeben sich, wenn:

- eine enterale Ernährung das Krankheitsbild verschlechtert (z. B. im Verlauf einer akuten Pankreatitis mit Komplikationen),

- eine absolute Kontraindikation gegen eine enterale Ernährung besteht (z. B. bei Ileus, diffuser Peritonitis, schwersten und intraktablen Diarrhoen, schweren Stoffwechselentgleisungen),

- eine enterale Ernährung nicht möglich ist (z. B. wegen unstillbaren Erbrechens im Rahmen einer Chemotherapie, bei hochsitzenden intestinalen Fisteln, initial bei der Behandlung eines Kurzdarmsyndroms), 
- eine enterale Ernährung den Nährstoffbedarf nicht ausreichend decken kann (z. B. bei einem Kurzdarmsyndrom oder hypermetaboler Stoffwechsellage wie bei ausgedehnten Verbrennungen),

- eine parenterale Ernährung möglicherweise auch therapeutisch wirksam ist (z. B. initial in der Behandlung eines Schubs einer chronisch-entzündlichen Darmerkrankung) und wenn

- eine exakte Dosierung der Substratzufuhr notwendig ist (z. B. im Rahmen der Behandlung von akuten Stoffwechselentgleisungen wie beim Leberkoma.

(Cave: Stoffwechselentgleisungen sind wegen der gestörten Verwertung von Nährstoffen in der Regel Kontraindikationen für eine vollständige parenterale Ernährung).

Aus ernährungsmedizinischer Sicht können 9 von 10 internistischen, onkologischen, pädiatrischen, neurologischen oder kieferchirurgischen Patienten enteral ernährt werden. Bei der Entscheidung zwischen einer enteralen oder einer parenteralen Ernährung sind neben den klinischen Aspekten die Ernährungsphysiologie, die Verträglichkeit, mögliche Komplikationen und auch die Kosten der jeweiligen Ernährungsform zu berücksichtigen.

Die enterale Ernährung ist die physiologischere Form der künstlichen Ernährung. Nach oraler Aufnahme werden etwa 50\% der Glukose von der Leber aufgenommen und die anderen 50\% extrahepatisch verstoffwechselt. Der Anteil des hepatischen Aminosäurestoffwechsels ist etwas niedriger. Enteral zugeführte Fette werden als LCT-Fette zum großen Teil im Fettgewebe gespeichert und als MCT-Fette überwiegend in der Leber verstoffwechselt. Bei parenteraler Zufuhr werden etwa $80 \%$ der Glukose im Muskel, also extrahepatisch metabolisiert. Gleichzeitig übersteigt der Anteil des hepatischen Aminosäurestoffwechsels $50 \%$ und kann bis zu $75 \%$ des Gesamtkörperaminosäureumsatzes betragen. Parenteral zugeführte Fette werden auch bei hohen Zufuhrraten zu 70\% im Fettgewebe gespeichert. Die parenterale Ernährung ist eine Sonderform der Ernährung. Sie ist "unphysiologisch « und bedarf deshalb einer kritischen Indikationsstellung.

Vorteil der enteralen Ernährung ist der Erhalt der gastrointestinalen Barriere, d.h. der strukturellen und funktionellen Integrität des Dünndarms. Die Komplikationen einer künstlichen Ernährung sind bei enteraler Gabe seltener und weniger schwerwiegend. Die Durchführung einer enteralen Ernährung ist im Vergleich zur parenteralen Ernährung einfach. Enterale Nährlösungen sind vollständig bilanzierte Diäten, denen im Normalfall lediglich Wasser zur Deckung des Flüssigkeitsbedarfs hinzugefügt werden muss. Die Steuerung der Kalorien- und Eiweißzufuhr ist für den einzelnen Patienten einfach zu berechnen. Die Relation der Makronährstoffe und auch die Zufuhr an Mikronährstoffen ist durch die Komposi- tion der Formuladiät fest vorgegeben. Eine parenterale Ernährung muss demgegenüber aus zahlreichen Einzelbausteinen (Glukose-, Fett-, Aminosäurelösungen, Elektrolyt-, Vitamin- und Spurenelementzusätze sowie "freie« Flüssigkeit) zusammengesetzt werden. Dieses bedeutet wesentlich mehr Aufwand. Andererseits können einzelne Nährstoffe gezielt gegeben und exakt dosiert werden. Die Einzelbausteine der parenteralen Ernährung müssen dann unter sterilen Kautelen zu einer Mischlösung, d. h. einem neuen Arzneimittel zusammengesetzt werden. Diese Bedingung gilt auch bereits für den Zusatz einer Elektrolytlösung (z. B. 7,45\% Kaliumchlorid) zu einer Glukoselösung. Alternative zu diesem aufwendigen Vorgehen ist die Verwendung von kommerziell hergestellten Infusionsmischlösung. Diese erlauben aber keine individuelle Behandlung.

Die Sondenzugänge sind bei der enteralen Ernährung einfach zu legen, zu versorgen und evtl. auszutauschen. Auch bei längerer Liegezeit ergeben sich meist keine Probleme. Demgegenüber sind zentralvenöse "Gefäßzugänge" mittel- und langfristig auch bei guter Pflege problematisch und stellen ein hohes Infektionsrisiko für den Betroffenen dar. Die Alternative ist die Implantation von Verweilkathetersystemen (s.unten). Die parenterale Ernährung ist wesentlich aufwendiger als die enterale Ernährung. Die Kosten enteraler Nährlösungen betragen im Vergleich zu den für eine vollständige parenterale Ernährung notwendigen Infusionslösungen und Zusätzen zwischen $5 \%$ bei Verwendung hochmolekularer Standard- (nährstoffdefinierter) Diäten und 20\% bei Verwendung spezieller Nährstoff-definierter Diäten (z. B. zur Immunonutrition) oder bei Gabe von niedermolekularen chemisch-definierten Diäten.

- Abb. 2.29 und - Abb. 2.30 zeigt einen Algorithmus für die Entscheidungsfindung bei künstlicher Ernährung.

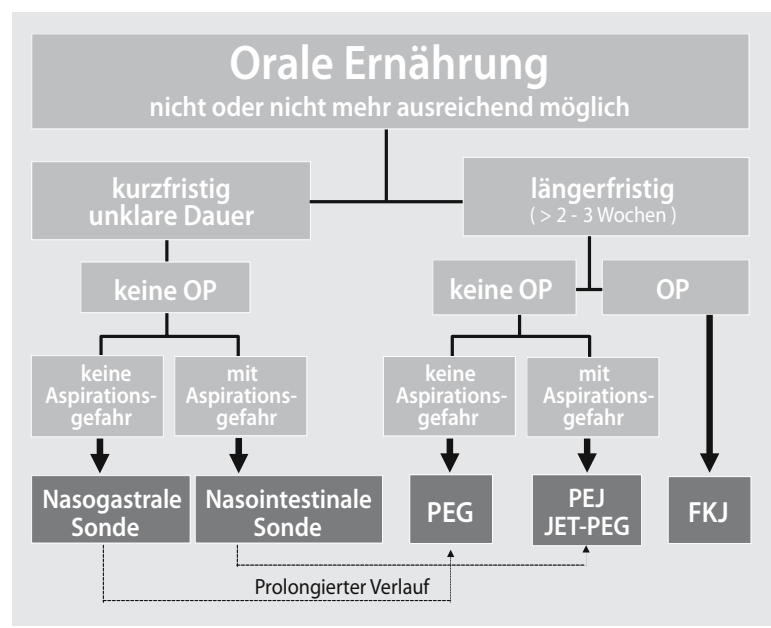

- Abb. 2.29. Algorithmus für die Anlage gastraler und jujunaler Sonden 


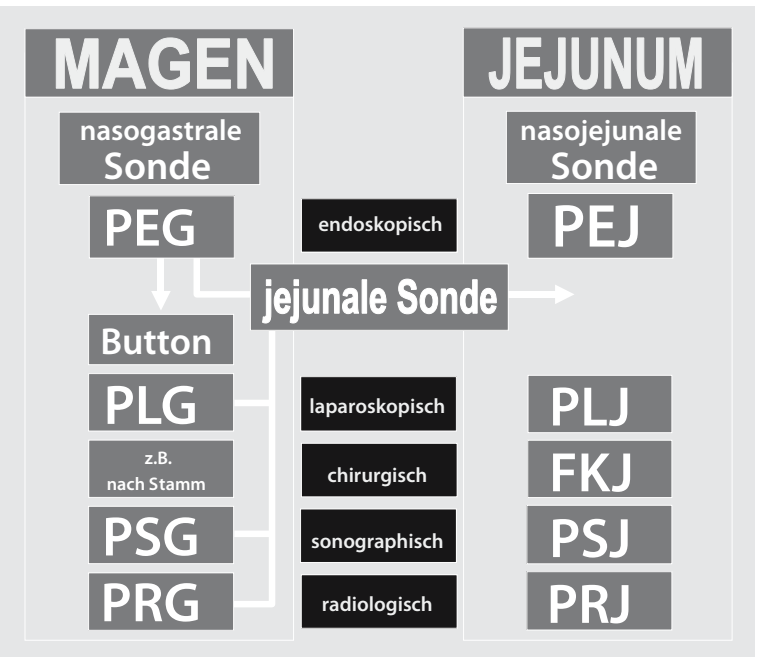

- Abb. 2.30. Sondentechniken

\subsubsection{Enterale Ernährung}

Die »moderne« Form einer enteralen Ernährung geht zurück auf Max Einhorn, der 1910 die Anwendung einer nasoduodenalen Ernährungssonde beschrieb. In den letzten 15 Jahren ist es zu einer beeindruckenden Verbesserung der enteralen Ernährungstechniken und auch unseres Wissens um den Stellenwert der enteralen Ernährung im Gesamtkonzept der Behandlung Schwerkranker gekommen.

Indikation. Die Indikationen für eine enterale Ernährung entsprechen denen für eine künstliche Ernährung. Besteht eine Indikation für eine künstliche Ernährung, muss, sofern keine Einschränkungen oder Kontraindikationen bestehen, in jedem Fall enteral ernährt werden.

Kontraindikationen. Für eine enterale Ernährung sind die folgenden Kontraindikationen zu beachten:

- akutes Abdomen,

- schwere Stoffwechselentgleisungen (wie die diabetische Ketoazidose),

- hämodynamische Instabilität,

- unstillbares Erbrechen (z. B. im Rahmen einer Chemotherapie),

- nicht kompensierbare Malassimilation oder

- Stenosen bzw. hochsitzende Fisteln.

Konzept. Eine enterale Ernährung wird entsprechend dem Energiebedarf unter Berücksichtigung möglicher individueller oder »klinischer" Einschränkungen oder Bedürfnisse berechnet. Der Energiebedarf Erwachsener wird nach den Standardformeln berechnet (s.
- Kap. 1.6.1). Bei Kindern und Jugendlichen sind die Formeln nach Schofield zu verwenden. Bei Säuglingen und Kleinkindern unter $10 \mathrm{~kg} \mathrm{KG}$ wird der Ruheenergieumsatz entsprechend [Körpergewicht $(\mathrm{kg}) \times 0,611] \times$ [Größe $(\mathrm{cm}) \times 0,951 \times 1,99$ ] berechnet. Eine Erweiterung um »Aktivitätsfaktoren « (z. B. für Krankheitsaktivität) ist individuell durchzuführen.

Nährlösungen, Formuladiäten. In der enteralen Ernährung Erwachsener werden verschiedene Formuladiäten verwendet. Man unterscheidet zwischen:

- nährstoffdefinierten Diäten,

- modifiziert nährstoffdefinierten Diäten,

- chemisch-definierten Diäten und

- modifiziert chemisch-definierten Diäten.

Die Formuladiäten sind diätetische Lebensmittel. Ihre Nährstoffzusammensetzung bzw. die täglich zugeführten Mindest- und Höchstmengen werden in $\$ 14 \mathrm{~b}$ sowie Anlage 6 und 7 der DiätVO geregelt (s. - Tab. 2.70). Diese Richtwerte sind entsprechend den Empfehlungen zur Nährstoffzufuhr der DACH formuliert. Sie berücksichtigen nicht den möglicherweise bei kranken Menschen veränderten Nährstoffbedarf. Die Formuladiäten dienen als Trink- oder Sondennahrung.

Entscheidungshilfe bei der Auswahl der jeweiligen Formuladiät ist die Verdauungsfunktion. Liegt diese zwischen 50 und $100 \%$, so ist eine nährstoffdefinierte Diät angezeigt. Bei einer weiteren Einschränkung empfehlen sich modifiziert nährstoffdefinierte Diäten oder auch chemisch-definierte Diäten. Formuladiäten können im Rahmen einer vollständigen enteralen Ernährung oder als Supplement gegeben werden.

Formuladiäten sind wie folgt charakterisiert:

- Nährstoffdefinierte Diät (NDD):

- Enthält komplexe Nährstoffe (Kohlenhydrate, Fett, Eiweiß) wie herkömmliche Nahrung,

- erfordert deshalb weitgehend vollständigegastrointestinale Funktion,

- »deckt« den Bedarf an Vitaminen, Mineralstoffen und Spurenelementen bei einer Menge von ca. $1,8-2,0 \mathrm{l}$,

- die »Kaloriendichte« beträgt in der Regel $1 \mathrm{kcal} / \mathrm{ml}$.

- Sondenkost (SK) ist die geschmacksneutrale Variante einer NDD,

- die Osmolarität ist meist niedrig.

- Modifizierte NDD, Modifikationen können sein:

- Energiereich; Kaloriendichte ca. 1,5 kcal/ml,

- ballaststoffreich,

- Austausch von LCT (langkettige Fettsäuren) gegen MCT (mittelkettige Fettsäuren) bei Malabsorption,

- ballaststoffreich (10 g/100 kcal)und Zuckeraustauschstoffe für Diabetiker,

- hoher Fettanteil (50\% der Energie) bei Ateminsuffizienz, angereichert mit Glutamin, Arginin, Ribo- 
nukleotiden und Omega-3-Fettsäuren als Immunonutrition;

- Veränderung der Kaloriendichte (gesenkt) und der Wassermenge (erhöht) für Kinder.

- Chemisch-definierte Diät (CDD, Oligopeptiddiät, ältere Bezeichnung: Elementardiät)

- Enthalten niedermolekulare Substrate, wie Oligopeptide und Oligosaccharide,
- hoher Kohlenhydratanteil und daher hohe Osmolarität,

- geeignet für Maldigestion und Malabsorption,

- zur oralen Aufnahme meist nicht geeignet, da geschmacklich nicht akzeptabel,

- Sondenzufuhr in jedem Falle empfohlen.

- Modifziert CDD, z. B. verminderter Kaliumgehalt bei Niereninsuffizienz, hoher MCT-Anteil bei Stearrhö.

- Tab. 2.70. Mindest- und Höchstmengen von Nährstoffen in bilanzierten Diäten gemäß der Diätverordnung (DiätVO). Im Vergleich sind die Nährstoffempfehlungen der DACH sowie die toxischen Bereiche gezeigt

\begin{tabular}{|c|c|c|c|c|}
\hline & \multirow[t]{2}{*}{$\mathrm{DACH}$} & \multicolumn{2}{|l|}{ §14b DiätVO Anlage 6} & \multirow[t]{2}{*}{ Toxischer Bereich } \\
\hline & & Mindestmenge pro Tag & Höchstmenge pro Tag & \\
\hline Vitamin A (mg RE) & $0,8-1,0 \mathrm{mg}$ & $0,6 \mathrm{mg}$ & $1,8 \mathrm{mg}$ & $2-15 \mathrm{mg}$ \\
\hline Vitamin $B_{1}$ & $1,0-1,3 \mathrm{mg}$ & $0,8 \mathrm{mg}$ & $2,4 \mathrm{mg}$ & $>400 \mathrm{mg}$ \\
\hline Vitamin $B_{2}$ & $1,2-1,5 \mathrm{mg}$ & $1,0 \mathrm{mg}$ & $3,0 \mathrm{mg}$ & \\
\hline Niacin & $13-17 \mathrm{mg}$ & $10 \mathrm{mg}$ & $30 \mathrm{mg}$ & $500-900 \mathrm{mg}$ \\
\hline Pantothensäure & $(6 \mathrm{mg})^{\mathrm{a}}$ & $6 \mathrm{mg}$ & $18 \mathrm{mg}$ & $>10000 \mathrm{mg}$ \\
\hline Vitamin $\mathrm{B}_{6}$ & $1,2-1,6 \mathrm{mg}$ & $1,0 \mathrm{mg}$ & $3,0 \mathrm{mg}$ & $50-500 \mathrm{mg}$ \\
\hline Vitamin $B_{12}$ & $3,0 \mu \mathrm{g}$ & $3,0 \mu \mathrm{g}$ & $9 \mu \mathrm{g}$ & $200-1000 \mu g$ \\
\hline Folsäure & $400 \mu g$ & $100 \mu g$ & $400 \mu \mathrm{g}$ & $5000-20000 \mu \mathrm{g}$ \\
\hline Biotin & $(30-60 \mu \mathrm{g})^{\mathrm{a}}$ & $75 \mu \mathrm{g}$ & $225 \mu \mathrm{g}$ & $>10000 \mu \mathrm{g}$ \\
\hline Vitamin C & $100 \mathrm{mg}$ & $40 \mathrm{mg}$ & $120 \mathrm{mg}$ & $1000-6000 \mathrm{mg}$ \\
\hline Vitamin $D_{3}$ & $5 \mu \mathrm{g}$ & $2,5 \mu \mathrm{g}$ & $15 \mu \mathrm{g}$ & $50-1250 \mu \mathrm{g}$ \\
\hline Vitamin E (Toc-Äq.) & $12-15 \mathrm{mg}$ & $6 \mathrm{mg}$ & $24 \mathrm{mg}$ & $>700 \mathrm{mg}$ \\
\hline Vitamin $\mathrm{K}_{3}$ & $60-80 \mu \mathrm{g}$ & $30 \mu \mathrm{g}$ & $120 \mu \mathrm{g}$ & \\
\hline Eisen & $10-15 \mathrm{mg}$ & $8 \mathrm{mg}$ & $24 \mathrm{mg}$ & $27 \mathrm{mg}$ \\
\hline Kalzium & $1000-1200 \mathrm{mg}$ & $600 \mathrm{mg}$ & $1000 \mathrm{mg}$ & $>2500 \mathrm{mg}$ \\
\hline Phosphor & $700-1250 \mathrm{mg}$ & $300 \mathrm{mg}$ & $1200 \mathrm{mg}$ & $>4000 \mathrm{mg}$ \\
\hline Kalium & $1000-1900 \mathrm{mg}$ & $1500 \mathrm{mg}$ & $6000 \mathrm{mg}$ & \\
\hline Magnesium & $300-400 \mathrm{mg}$ & $180 \mathrm{mg}$ & $540 \mathrm{mg}$ & $>3000-5000 \mathrm{mg}$ \\
\hline Kupfer & $(0,5-1,5 \mathrm{mg})^{\mathrm{a}}$ & $1,0 \mathrm{mg}$ & $5,0 \mathrm{mg}$ & $10-30 \mathrm{mg}$ \\
\hline Mangan & $(1,0-5,0 \mathrm{mg})^{\mathrm{a}}$ & $2,0 \mathrm{mg}$ & $6,0 \mathrm{mg}$ & $10 \mathrm{mg}$ \\
\hline Zink & $7-10 \mathrm{mg}$ & $5 \mathrm{mg}$ & $25 \mathrm{mg}$ & $20 \mathrm{mg}$ \\
\hline Jod & $180-200 \mu \mathrm{g}$ & $150 \mu \mathrm{g}$ & $300 \mu \mathrm{g}$ & $>1000 \mu \mathrm{g}$ \\
\hline Molybdän & $(25-100 \mu \mathrm{g})^{\mathrm{a}}$ & $60 \mu \mathrm{g}$ & $500 \mu \mathrm{g}$ & $10000 \mu \mathrm{g}$ \\
\hline Fluorid & $2,9-3,8 \mathrm{mg}$ & $1,0 \mathrm{mg}$ & $4,0 \mathrm{mg}$ & $10 \mathrm{mg}$ \\
\hline Selen & $(30-70 \mu \mathrm{g})^{\mathrm{a}}$ & & & $800 \mu \mathrm{g}$ \\
\hline
\end{tabular}


Nährstoffdefinierte Diäten sind hochmolekulare Nährlösungen, welche komplexe Nährstoffe enthalten. Der tägliche Energiebedarf wird bei einer Zufuhrmenge zwischen 1500 und $2500 \mathrm{ml} / \mathrm{Tag}$ "gedeckt «. Diese Standarddiäten enthalten natürliche Fette und intakte Proteine (z. B. Milch- oder Sojaeiweiß). Sie sind gluten-, fruktose-, cholesterin- sowie nahezu allergen- und laktosefrei. Nährstoffdefinierte Diäten sind vollständig bilanzierte Diäten und entsprechen in ihrer Makro- und Mikronährstoffzusammensetzung den Nährstoffempfehlungen der nach DACH für gesunde Menschen. In der angegebenen Zufuhrmenge ist also wahrscheinlich auch der Bedarf an Mikronährstoffen gedeckt. Nährstoffdefinierte Diäten haben die für die Nutzung filiformer Sonden notwendigen Fließeigenschaft. Sie sind in der Standardvariante ballaststoffrei und sowohl für die Sondenernährung als auch als Trinknahrung (z. B. als Supplement zur normalen Ernährung) geeignet. Sondenkost ist geschmacklich neutral. Als Trinknahrung werden viele verschiedene Geschmacksrichtungen (z. B. Kakao, Kaffee, Nuss, Schokolade, Vanille, Himbeer, Pfirsich, Banane, Multifrucht, Gemüse, Tomate) angeboten, so dass die individuellen Wünsche der Patienten weitgehend berücksichtigt werden können. Die verschiedenen nährstoffdefinierten Diäten haben z. T. unterschiedliche Osmolaritäten (von 240-440 mosmol/kg H2O), dies ist beim Kostaufbau oder bei Patienten mit Diarrhoen zu berücksichtigen.

Modifiziert nährstoffdefinierte Diäten entsprechen in ihrer Zusammensetzung den nährstoffdefinierten Diäten. Modifikationen betreffen die Nährstoffdichte, den Austausch von LCT gegen MCT-Fette, den Gehalt an Ballaststoffen, die Verminderung des Gehaltes an leicht aufschließbarer Kohlenhydrate und einen möglichen Anteil an Zuckeraustauschstoffen. Bei Leberkranken mit Zeichen der hepatischen Encephalopathie werden Formuladiäten, welche mit verzweigtkettige Aminosäuren angereichert sind angewendet. Bei starkem Gewichtsverlust, ausgeprägt kataboler Stoffwechsellage und Zeichen der Malnutrition werden NDDs mit einem hohen Anteil von Omega-3-Fettsäuren, Arginin und Nukleotiden und z. T. Glutamin (z. B. zur Immunonutrition im postoperativen Stress oder bei Patienten mit einer HIV-Infektion) empfohlen. Eine Veränderungen der Makronährstoffrelation (fettreiche und kohlenhydratarme Formuladiäten mit einem Fettanteil von etwa 50\%, welche bei Ateminsuffizienz, bei chronisch-obstruktiven Lungenerkrankungen mit einer hohen $\mathrm{CO}_{2}$-Produktion oder während der Entwöhnungsphase vom Beatmungsgerät nach Langzeitbeatmung und auch bei Tumorkachexie zur schnelleren Gewichtszunahme sinnvoll eingesetzt werden können) macht bei Patienten mit entsprechenden Indikationen (Kachexie, hohe endogene Fettoxidation) Sinn. Ein Teil der Diäten ist auch als Trinknahrung (z. B. als Supplement zur normalen Ernährung) geeignet. Die praktische Er- fahrung zeigt jedoch, dass über längeren Zeitraum (z. B. 3 Wochen) im Mittel nur etwa $400 \mathrm{ml}$ eines Supplements pro Tag verzehrt werden. Im Verlauf der Behandlung ist eine abnehmende Akzeptanz auf Seiten der Patienten häufig.

Chemisch-definierte Diäten sind niedermolekular und enthalten Oligo- und Disaccharide, teilhydrolysierte Proteine und Oligopeptide sowie pflanzliche Öle (essentielle Fettsäuren) enthalten. Sie sind in ihrer Nährstoffrelation kohlenhydratreich und fettarm. Chemisch-definierte Diäten haben eine hohe Osmolalität. Sie enthalten keine Ballaststoffe, sind laktose- und glutenfrei sowie naturgemäß allergenarm. Chemisch-definierte Diäten sind bei Verdauungsstörungen (Maldigestion, Malabsorption) und möglicherweise zur initialen Behandlung von Patienten mit chronisch-entzündlichen Darmerkrankungen geeignet. Auf Grund ihres schlechten Geschmacks müssen sie in jedem Fall über eine Sonde appliziert werden. Zur Vermeidung von Nebenwirkungen (Magenretention und Erbrechen, Durchfälle) muss diese Kostform über eine transpylorisch liegende Sonde in das Duodenum (bzw. Jejunum) kontinuierlich mit einer Pumpe und niedriger Infusionsrate infundiert werden.

Modifiziert chemisch-definierte Diäten entsprechen in ihrer Zusammensetzung den chemisch-definierten Diäten. Veränderungen können ein MCT-Anteil oder ein niedriger Kaliumgehalt sein, welcher bei der künstlichen Ernährung von Patienten mit einer Niereninsuffizienz sinnvoll ist.

Die für Erwachsene konzipierten Formuladiäten genügen in ihrer Zusammensetzung nicht dem Bedarf von Säuglingen und Kleinkindern. Bei Säuglingen werden die üblichen Säuglingsformelnahrungen auch zur künstlichen Ernährung verwendet. Daneben gibt es Nährlösungen auf Sojamilchbasis und freie Aminosäuren enthaltende Elementardiäten. Hochmolekulare nährstoffdefinierte Diäten für Kinder sind auf Milcheiweißbasis hergestellt und berücksichtigen den spezifischen Nährstoff- und Flüssigkeitsbedarf dieser Altersgruppe. Diese Diäten haben im Vergleich zu den für Erwachsene geeigneten nährstoffdefinierten Diäten einen höheren Wasser- und einen niedrigeren Eiweißgehalt.

Bei allen Sondenkostformen ist der Wasser- und Elektrolytgehalt zu berücksichtigen. In der Regel entspricht der Wassergehalt $70-85 \%$ der Sondenkostmenge. Er ist bei hochkalorischen Kostformen niedriger. Flüssigkeitsund Nährstoffbedarf müssen für jeden Patienten individuell berechnet werden und können bei Sondenernährung nicht übereinstimmen.

Ein Beispiel: Bei einem $70 \mathrm{~kg}$ schweren Patienten beträgt der Energiebedarf $2100 \mathrm{kcal} /$ Tag. Der Nährstoffbedarf wird durch $2100 \mathrm{ml}$ einer nährstoffdefinierten Diät gedeckt. Dieses bedeutet gleichzeitig eine Flüssigkeitszufuhr von $1680 \mathrm{ml} /$ Tag. Bei einem rechnerischen 
- Tab. 2.71. Kostaufbau bei enteraler Ernährung

Ziel

Kalorienbedarf $2000 \mathrm{kcal} / \mathrm{Tag}(=2000 \mathrm{ml}$ Sondenkost)

Flüssigkeitsbedarf

$2400 \mathrm{ml} / \mathrm{Tag}$ (d.h. $+800 \mathrm{ml} \mathrm{H}_{2} \mathrm{O}$ )
Aufbau

Tag 1

Tag 2

Tag 3

Tag 4

Tag 5
$500 \mathrm{ml}$ Sondenkost $+2000 \mathrm{ml} \mathrm{H} \mathrm{O}(150 \mathrm{ml} / \mathrm{h}$ über $16 \mathrm{~h})$

$1000 \mathrm{ml}$ Sondenkost $+1600 \mathrm{ml} \mathrm{H} \mathrm{O}_{2} \mathrm{O}(175 \mathrm{ml} / \mathrm{h}$ über $16 \mathrm{~h})$

$1500 \mathrm{ml}$ Sondenkost $+1200 \mathrm{ml} \mathrm{H} \mathrm{H}_{2} \mathrm{O}(175 \mathrm{ml} / \mathrm{h}$ über $16 \mathrm{~h})$

$2000 \mathrm{ml}$ Sondenkost $+800 \mathrm{ml} \mathrm{H} 2 \mathrm{O}(175 \mathrm{ml} / \mathrm{h}$ über $16 \mathrm{~h})$

Bei Wunsch schrittweise Verkürzung der Infusionsperiode, maximale Infusionsgeschwindigkeit $250 \mathrm{ml} / \mathrm{h}$
Flüssigkeitsbedarf von $2450 \mathrm{ml} / \mathrm{Tag}$ sind entsprechend zusätzlich $770 \mathrm{ml}$ Flüssigkeit miteinzuberechnen. Zum adaptierten Kostaufbau einer enteralen Ernährung, s.

- Tab. 2.71.

Bei faserreichen Diäten muss mehr Flüssigkeit zugeführt werden. Der Flüssigkeitsbedarf eines normalgewichtigen Erwachsenen schwankt zwischen 2,5 und 4,0 1/Tag. Die Flüssigkeitsbilanz des enteral ernährten Patienten muss deshalb regelmäßig kontrolliert werden.

Selbst zubereitete Sondenkosten sind heute obsolet. In einer den Nährstoffempfehlungen entsprechenden $\mathrm{Zu}$ sammensetzung erfüllen sie nicht die für filiformen Sonden erforderlichen Fließeigenschaften.

\subsubsection{Techniken}

$\mathrm{Zu}$ den verschiedenen Sondentechniken s.

Abb. 2.31

\section{Zugangswege, Sonden, PEG, FKJ}

In der Regel wird eine enterale Ernährung über Sonden durchgeführt. Diese werden durch die Nase in den Magen oder den oberen Dünndarm (i.e. in das Duodenum oder Jejunum eingeführt und dann als nasogastrale, nasoduodenale oder nasojejunale Sonden bezeichnet. Die Ernährungssonden werden durch einen Führungsdraht etwas versteift und können so zielgerecht plaziert werden. Das Einführen wird durch Anfeuchten der Sonde mit einem Gleitmittel sowie lokale Betäubung der Nasenschleimhaut mit Lidocain-Gel für den Patienten erleichtert. Die Sonde wird unter Schlucken vorgeschoben. Fehllagen der Sonde im Bronchialsystem sind selten und werden in der Regel schnell bemerkt. Allerdings ist der Hustenreflex bei alten Menschen gelegentlich schlecht ausgeprägt, so dass es hier zu unbemerkten Fehllagen und unliebsamen Überraschungen kommen kann. Ein Umschlagen der Sonde im Magen ist häufig. Bei gastraler Sondenlage und mehreren frustranen transpylorischen Plazierungsversuchen kann im Einzelfall auf eine spontane Plazierung gewartet werden. Der
Patient wird dazu auf die rechte Seite gelagert und der Draht entfernt. Statt gewaltsamer Versuche empfiehlt es sich bei präpylorischer Lage, eine Spritze mit der Sonde zu konnektieren, 5-10 $\mathrm{ml}$ Wasser zu injizieren und die Sonde vorsichtig zu drehen. Die transpylorische Passage und die Endlage der Sonde müssen radiologisch oder sonographisch kontrolliert durchgeführt und dann dokumentiert werden. Eine auskultatorische Lokalisation der Sondenlage ist fahrlässig, da sie mögliche Fehllagen (z. B. umgeschlagene und mit der Spitze im Ösophagus liegende Sonden) nicht erkennen kann. Andere klinisch gelegentlich praktizierte Lagekontrollen wie die Inspektion (Farbe) und die Bestimmung des pH im Aspirat haben keinen ausreichend prädiktiven Wert und können eine radiologische Lagekontrolle nicht ersetzen.

Ernährungssonden können unter endoskopischer Sicht mit Hilfe eines Führungsdrahtes gezielt im oberen Dünndarm plaziert werden. Eine endoskopische Plazierung ist z. B. bei Stenosen oder großen Hiatushernien sowie Divertikeln der Speiseröhre notwendig. Bei dieser Technik wird zunächst durch das Endoskop ein Führungsdraht vorgeschoben und unter endoskopischer Sicht plaziert. Nach Entfernen des Endoskops erfolgt nachträglich die Umleitung des Führungsdrahtes oronasal mit Hilfe einer Fasszange sowie eines vorübergehend durch die Nase eingeführten Umleitungstubus. Über den Führungsdraht wird dann die Sonde eingeschoben. Die Ernährungssonden sollten in der Regel in das obere Duodenum plaziert werden. Bei gestörter Magenentleerung (z. B. bei Magenausgangsstenose, Gastroparese) und bei erhöhtem Aspirationsrisiko (z. B. bei Patienten mit neurologischen Schluckstörungen) ist eine postpylorische Sondenlage obligat. Allerdings sind auch bei duodenaler Lage ein duodenogastraler Reflux möglich und eine Aspirationsgefahr nicht vollständig auszuschließen. Über längere Perioden (z. B. Wochen/Monate) sind Dislokationen möglich. Bei duodenal plazierten Sonden bleiben letztendlich nur 515\% der Sonden »in Position«.

Die Sonden werden mit einem hautfreundlichen Pflaster an der Nase fixiert. Rigidere Fixationstechniken können im Einzelfall bei nicht kooperativen Patienten 
- Abb. 2.31. Transnasale Sonden: a nasogastral, $b$ nasointestinal (transpylorisch). Transkutane Sonden: $c$ perkutane, endoskopisch unterstützte Gastrostomie (PEG), $d$ PEG mit transpylorischer intestinaler Sonde, e Feinnadelkatheterjejunostomie (FKJ)

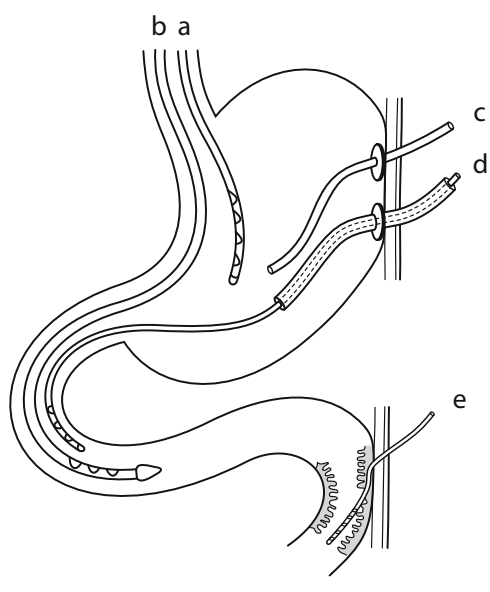

Transnasale Sonden:

a nasogastral

b nasointestinal (transpylorisch)

Transkutane Sonden:

c perkutane, endoskopisch unterstützte Gastrostomie (PEG)

d PEG mit transpylorischer intestinaler Sonde

e Feinnadelkatheterjejunostomie (FKJ) notwendig werden. Sie haben sich aber in der Routine der Krankenversorgung nicht durchgesetzt und erscheinen ethisch zweifelhaft. Bei längerfristiger und auch bei heimenteraler Ernährung ist das Versenken des Sondenendes in die Nase mit Hilfe einer der Naseninnenwand individuell angepassten Nasenolive möglich. Der Patient wird so im Alltag nicht als »Sondenträger« erkannt.

Ernährungssonden sind filiform (Charrieregröße - Ch -7 oder 8, maximal 12 mit einem entsprechenden Außendurchmesser von 2,3-4 mm) und aus modernen Kunststoffen wie Polyurethan oder Silikonkautschuk gefertigt. Sie sind für eine längere Liegedauer geeignet. Demgegenüber sind großlumige PVC- oder auch Latexsonden (Ch 14-18) für die Ernährung obsolet. Sie mögen allenfalls vorübergehend (bis zu maximal 3 Tagen) als sog. Magenablaufsonden noch Verwendung finden. Im Gegensatz zu den schon nach kurzer Liegedauer rigiden PVC-Sonden sind die Polyurethan- und Silikonkautschuk-Sonden weich und geschmeidig. Es besteht keine Gefahr von lokalen Druckulzera oder Komplikationen in Nase, Pharynx und Intestinaltrakt. Ob es bei Ösophagusvarizen oder bereits bestehenden Ulzera der Speiseröhre oder des Magens durch filiforme Ernährungssonden zu Verletzungen kommen kann, ist durch keine Untersuchung belegt. Filiforme Ernährungssonden sind als gastrale Sonden $75-120 \mathrm{~cm}$ und als duodenale Sonden 120-150 cm lang. Endoskopisch zu legende Sonden haben eine Länge von $250 \mathrm{~cm}$. Die Aspiration von Magen- oder Darminhalt und die Applikation von Medikamenten sind über filiforme Sonden nicht möglich. Standardsonden haben 1 Lumen. Spezialsonde sind dreilumig und erlauben die enterale Ernährung (duodenal, jejunal), das Absaugen von gastralem Sekret und die gleichzeitige Belüftung des Magens. Die dreilumige Sonde wird idealerweise endoskopisch (Vorschub- oder Seldinger-Technik) plaziert. Die gastrale Sonde hat Ch 16, die Ernährungssonde Ch 9.
Transnasal gelegte Sonden können wegen des Fremdkörpergefühls, der Entwicklung einer Refluxösophagitis, Druckulzera und einer möglichen Sinusitis nicht für eine längere enterale Ernährung verwendet werden. Bei längerfristiger enteraler Ernährung (>3-4 Wochen), neurogenen Schluckstörungen, inoperablen oder stenosierenden Tumoren des oberen Intestinaltrakts wird eine Enterostomie (Gastrostomie oder Jejunostomie) angelegt. Eine Ernährung über eine Gastrostomie wird vorzugsweise bei bewusstseinsklaren und kooperativen Patienten durchgeführt werden. Gastrostomien sind geeignet für Patienten mit Tumoren der Speiseröhre oder des Pharynx, bei neurologischen Erkrankungen (Schluckstörungen) sowie älteren und pflegebedürftigen Patienten, welche sich nicht bedarfsdeckend ernähren können. Diese direkten Zugänge zum Intestinaltrakt werden unter endoskopischer oder sonographischer Sicht (z. B. als perkutan-endoskopische Gastrostomie=PEG) oder aber im Rahmen einer abdominellen Operation chirurgisch angelegt (i.e. Feinnadelkatheterjejunostomie=FK oder FNK). Bei nicht kooperativen bzw. bewusstlosen Patienten ist eine intestinale Sondenlage zu fordern.

Kontraindikationen gegen die Anlage einer Gastrostomie sind:

- Kontraindikationen gegen eine enterale Ernährung,

- Gerinnungsstörungen,

- Peritonitis,

- Peritonealkarzinose,

- Aszites,

- kurzdauernde künstliche Ernährung,

- eine fehlende "Darstellbarkeit« (Diaphanoskopie) des Magens oder einer oberen Dünndarmschlinge und

- im Einzelfall auch soziale und persönliche Gründe.

Die Methode der Wahl ist heute die perkutan-endoskopische Gastrostomie (PEG). Nach 12-stündiger Nahrungskarenz werden die Haut des Abdomens und des unteren 
Thorax großzügig und gründlich desinfiziert. Der Patient erhält zu seiner Beruhigung 3-5 mg Midazolam (z. B. Dormicum) injiziert. (Cave: Geringere Dosierung bei alten und auch bei kachektischen Patienten).

Nach Vorführen des Endoskops in den Magen wird dieser durch Luftinsufflation gefüllt und die Lichtquelle des Endoskops von außen identifiziert. An der Stelle der größten Helligkeit wird dann die Haut nach Lokalanästhesie (10 ml Lidocain) inzidiert und darauf der Magen unter endoskopischer Sicht von außen punktiert. Über die Punktionskanüle wird bei der sog. Durchzugsmethode ein Faden oder ein flexibler Draht in den Magen geschoben, welcher durch eine über das Endoskop geschobene Fasszange gegriffen und sodann mit dem Endoskop zurückund aus dem Mund herausgeführt wird. An dem Ende des Fadens wird der Gastrostomiekatheter (Ch 9-15) fixiert. Dieser wird mit dem Faden durch die Speiseröhre, den Magen und den Punktionskanal gezogen und schließlich an der Bauchwand mit einer Halteplatte fixiert.

Die Katheterspitze kann bei langen Gastrostomiekathetern mit Hilfe eines Endoskops in dem Dünndarm plaziert werden. Sie wird dann als PEG bezeichnet. Bei axialen Hernien und auch Kardiainsuffizienz sind ein gastraler Reflux und auch eine Aspiration möglich. Bei einem entsprechenden endoskopischen Befund muss eine duodenale Sondenlage angestrebt werden. Diese schließt allerdings einen Reflux von Darminhalt nicht vollständig aus. Es gibt auch mehrlumige PEG-Katheter, welche eine Ernährung über einen duodenal/jejunal liegenden Katheter und eine gleichzeitige gastrale Entlastung über einen gastralen Katheter erlauben. Die Austrittsstelle des Katheters an der Bauchwand wird steril verbunden. Nach Anlage muss der Patient für 4-6h überwacht werden (Herz-Kreislauffunktionen, abdomineller Befund). Er erhält prophylaktisch und für einen Tag ein Breitbandantibiotikum (z. B. 3-mal 2 g Cefotaxim). Ein Kostaufbau kann nach $12 \mathrm{~h}$ begonnen werden. Komplikationen wie Infektionen, Verletzungen, Blutungen, Bauchschmerzen oder Dislokationen kommen bei sachgemäßer Durchführung sehr selten $(<2 \%)$ vor. Auch Risikogruppen (wie z. B. immunsupprimierte HIV-Patienten) haben keine höhere Komplikationsrate. Erfahrungsgemäß verschließt die Fistel schnell.

Eine perkutan-sonographische Gastrostomie (PSG) ist indiziert, wenn eine endoskopische Untersuchung nicht durchgeführt werden kann (z. B. bei fehlender Passage und vollständig stenosierenden Tumoren der Speiseröhre) oder darf (z. B. bei respiratorischer Insuffizienz). Bei dieser Technik wird eine »hohe« Dünndarmschlinge sonographisch dargestellt und unter »Licht« punktiert. Über die Punktionskanüle wird dann ein Draht eingeführt und anschließend der Katheter nach Seldingertechnik plaziert. Es werden spezielle Kathetersysteme verwendet. Bei einer Ernährung in das Jejunum ist das Risiko des Refluxes minimal.
Eine FKJ (oder FNK=Feinnadelkatheter-Junostomie) wird im Rahmen einer Laparatomie (z. B. zur frühzeitigen postoperativen Ernährung) oder laparoskopisch eingelegt. Der Katheterdurchmesser beträgt $4 \mathrm{~mm}$. Der Katheter wird mit einer Naht am Jejunum in der Regel $15-30 \mathrm{~cm}$ distal des Treitz-Bandes bzw. distal einer chirurgischen Anastomose befestigt und findet zusätzlich durch einen etwa $10 \mathrm{~cm}$ langen Tunnel in der Darmwand Halt. Das Jejunum wird an der Austrittsstelle des Katheters an das parietale Bauchfell an der Bauchfellinnenseite angenäht. Eine Indikation zur FKJ besteht bei Patienten nach elektiven chirurgischen Eingriffen (z. B. nach großen Oberbaucheingriffen) und gleichzeitiger Indikation für eine künstliche Ernährung. Typische klinische Indikation sind z. B. Ösophagusresektionen, Gastrektomie oder Pankreatektomie. Die Katheterdislokation und eine Leckage sind die häufigsten der insgesamt aber sehr seltenen Komplikationen ( $<1 \%$ der Fälle). Da der FKJ-Katheter sehr filiform ist, muss er häufig und regelmäßig gespült werden.

Zur Pflege der Gastro- oder Jejunostomie wird jeden 2. Tag der Verband gewechselt sowie der Katheter mehrmals täglich gespült. Im Vergleich zu den nasoenteralen Ernährungssonden sind eine Gastro- und auch eine Jejunostomie nicht nur kosmetisch vorteilhaft. Im Vergleich zu den nasogastralen oder nasoenteralen Sonden finden sich auch weniger Komplikationen. Beide Kathetersysteme können über lange Zeit für die künstliche Ernährung verwendet werden.

Vor Anlage einer Gastrostomie bzw. Jejunostomie müssen die häuslichen und sozialen Verhältnisse des $\mathrm{Pa}$ tienten bekannt und seine weitere Versorgung gesichert sein. Die Kathetersysteme können wieder entfernt werden. Dieser Schritt sollte aber den "versorgenden« Zentren vorbehalten bleiben. Die Betreuung der mit einer Gastrostomie bzw. einer Jejunostomie versorgten Patienten sollte sinnvollerweise durch den Arzt/die Klinik erfolgen, welche(r) das Kathetersystem angelegt hat. Die langfristigen Komplikationsraten von Gastrostomien/Jejunostomien sind bei sachgerechter Anwendung mit $<1 \%$ sehr gering und auch bei immunsupprimierten Patienten nicht erhöht.

\section{Applikation der Sondenkost}

Die Formuladiäten können kontinuierlich oder intermittierend über die Sonde verabreicht werden. Bei gastraler Sondenlage kann die Kost als Mahlzeitenportion (sog. Bolus) oder kontinuierlich über eine Pumpe gegeben werden. Bei Bolusgabe empfiehlt sich ein Bolus von 50-200 $\mathrm{ml}$ über 5-10 Minuten alle 1,5-3 Stunden. Bei duodenaler oder jejunaler Sondenlage ist eine kontinuierliche Infusion obligat. Chemisch-definierte Diäten müssen immer duodenal/jejunal mit einer Pumpe appliziert werden. Nebenwirkungen bei Bolusapplikation sind häufiges Völlegefühl, gastroösophagealer Reflux, Erbrechen, Aspiration, 
aber auch eine schnelle Magenentleerung mit den Symptomen eines "Dumpings « und Diarrhoen. Obwohl die Bolusgabe physiologischer erscheint, wird in der Praxis bevorzugt mit niedrigeren Infusionsraten kontinuierlich ernährt. Dies wird mit der besseren Verträglichkeit und Toleranz erklärt. Auch finden sich bei Schwerkranken und unter hochdosierter Schmerzmedikation häufiger Magenentleerungsstörungen, während die intestinale Motilität meist unbeeinflusst ist.

Bei kontinuierlicher Ernährung mit einer Pumpe ist der zu wählende Infusionszeitraum flexibel an die Gewohnheiten, Möglichkeiten und Toleranz des Patienten anzupassen. Bei einem heimenteral ernährten und berufstätigen Patienten mit einer Gastrostomie ist möglicherweise eine kontinuierliche Ernährung nachts über 8 h geeignet. Ein hospitalisierter Patient (z. B. mit einem floriden Morbus Crohn oder einer Strahlenenteritis) wird dagegen besser über einen längeren Zeitraum in niedrigerer Dosierung pro Zeiteinheit behandelt. Eine Nüchternperiode von mindestens $6 \mathrm{~h}$ ist einzuplanen. Diese erlauben dem Stoffwechsel die Umstellung von anaboler auf katabole Stoffwechselsituation.

Für eine kontinuierliche Sondenernährung werden verschiedene, handliche und technisch ausgereifte Pumpensysteme angeboten. Sie ermöglichen eine kontinuierliche oder stufenweise (z. B. in Schritten von +20 bzw. $+25 \mathrm{ml} / \mathrm{h}$ ) Einstellung der Infusionsgeschwindigkeit. Aufwendigere Pumpensysteme erlauben auch sehr niedrige Förderraten (z. B. 1 oder $5 \mathrm{ml} / \mathrm{h}$ ), welche aber in der Praxis selten benötigt werden. Eine maximale Geschwindigkeit von $200 \mathrm{ml} / \mathrm{h}$ sollte nach klinischer Erfahrung nicht überschritten werden. Die Genauigkeit der enteralen Infusionspumpen ist sehr unterschiedlich. Es empfiehlt sich deshalb, die Pumpen zumindest einmal vor Gebrauch zu »eichen«.

Die Sondenkost wird entweder aus der Flasche, aus Infusionsbeuteln oder aus geeigneten abwaschbaren Containersystemen über ein Infusionssystem infundiert. Eine aseptische Handhabung von Infusionslösungen, -leitung und -beutel ist zumindest bei immunsupprimierten Patienten zu empfehlen. Die »ideale« Temperatur der Formuladiät für die Applikation liegt zwischen 20 und $25^{\circ} \mathrm{C}$. Eine Zwischenlagerung der Nährlösung an einem sauberen und trockenen Ort (z. B. im Kühlschrank) ist möglich, sie sollte nicht in einem Wasserbad oder in einem Mikrowellengerät aufgewärmt werden. Die kontinuierliche Ernährung mit einer Pumpe ist für Patienten und Pflegepersonal nicht aufwendig. Sie wird gut toleriert und hat am wenigsten Nebenwirkungen. Pumpe und Nährlösungen können leicht transportiert werden und erlauben dem enteral ernährten Patienten eine gewisse Freizügigkeit. Die Nährlösungen sollten nicht länger als 4-6h im Beutel bzw. im Container gehalten werden. Die Infusionssysteme werden täglich gewechselt.
Eine kontinuierliche enterale Ernährung wird in Oberkörperhochlage $\left(30^{\circ}\right)$ durchgeführt. Diese Position schützt nicht vollständig vor gastroösophagealem Reflux und einer möglichen Aspiration. Bei gastraler Sondenlage müssen erfahrungsgemäß im Liegen die Linksseiten- und natürlich die Bauchlage gemieden werden, da sie die Magenentleerung behindern. Bei gastraler Ernährung ist der $\mathrm{pH}$ im Magensaft >3-4. Der für die Ernährung berechnete Nährstoff- und Flüssigkeitsbedarf ist das Therapieziel. Eine enterale Ernährung wird (besonders bei duodenaler und jejunaler Sondenlage) vorsichtig aufgebaut, um eine bessere Verträglichkeit zu erreichen und Komplikationen zu vermeiden. - Tab. 2.71 zeigt ein Beispiel eines Kostaufbaus.

\section{Fehler und Probleme}

Häufigste Probleme der enteralen Ernährung sind Erbrechen und Aspiration, Diarrhoen und Obstipation. Komplikationen unter enteraler Ernährung treten in $\mathrm{Zu}$ sammenhang mit der Sonde, der Sondenkost oder als intestinale und metabolische Probleme auf. Die meisten Probleme können gelöst werden und zwingen nicht zum Abbruch der enteralen Ernährung. Häufige Fehler bei enteraler Ernährung sind falsches Sondenmaterial und Sondenfehllagen, mangelhafte Sondenpflege, falscher Applikationsmodus, ungeeignete Sondenkost, fehlender Kostaufbau, zu hochkalorische Ernährung, Nichtbeachtung des Ernährungszustandes und des Energiebedarfs, Nichtberücksichtigung des Flüssigkeits- und Natriumbedarfs und eine für Sondenernährung ungeeignete Begleitmedikation. Für eine erfolgreiche enterale Ernährung gelten die folgenden Empfehlungen: frühzeitig beginnen, möglichst bei stabiler klinischer Situation, gute Überwachung, konsequenter Durchführung (d. h. auch: nicht so schnell aufgeben).

\section{Sondenkostabhängige Probleme}

Sie betreffen eine mögliche bakterielle Kontamination der Kost (z. B. bei längerem Stehen bei Raumtemperatur), die Zusammensetzung (z. B. hohe Osmolalität bei chemischdefinierten Diäten), den niedrigen Natriumgehalt, mögliche Wechselwirkungen mit Medikamenten und deren Zusätzen (z. B. Sorbit bei Säften).

\section{Sondenabhängige Komplikationen}

Fehllagen und Dislokationen, Klinische Indikatoren der gastrointestinalen Funktion (z. B. die Auskultation von Darmgeräuschen, der Abgang von Flatus oder Stuhl sowie ein gastraler Reflux $<1000 \mathrm{ml} / \mathrm{Tag}$ ) sind keine exakten Kenngrößen. Sie erlauben keine sichere Entscheidung für oder gegen eine enterale Ernährung. Verstopfen der Sonde (z. B. bei ungenügendem Spülen, Spülen mit Früchtetees oder Obstsäften, Gabe von gemörserten Tabletten durch die Sonde), Abknicken des Sondenendes, lokale Schleimhautverletzungen und mögliche Materialprobleme. 


\section{Infektionen}

Sie können lokal bei Gastrostomie oder nach Erbrechen und Aspiration als Aspirationspneumonie auftreten.

\section{Metabolische Komplikationen}

Dehydration, Hyper- und Hypoglykämie und Elektrolytentgleisungen (Hyponatriämie, Hyper- und Hypokaliämie, Hypophosphatämie). Bei längerer und hochkalorischer Ernährung können insbesondere bei kachektischen Patienten ein "overloading syndrome" und Ödeme beobachtet werden. Von diesem Syndrom ist das $» T u b e-f e e-$ ding-Syndrom " zu unterscheiden, welches bei hyperosmolarer Sondenkost, osmotischen Diarrhoen und gleichzeitig zu geringer Flüssigkeitszufuhr auftreten kann. Es ist durch die zunehmende und ausgeprägte Exsikkose (bis hin zu einem prärenalen Nierenversagen) charakterisiert. Laborchemisch sind die Natrium-, Harnstoff- und Kreatininspiegel im Serum erhöht. Das Risiko einer Dehydratation ist bei älteren Menschen, intensivmedizinisch behandelten Patienten (Trauma, Sepsis) und bei Patienten mit Gehirntumoren erfahrungsgemäß erhöht. Die Behandlung ist die vorsichtige, bilanzierte und ausreichende Flüssigkeitszufuhr. Die Ernährung wird gleichzeitig mit einer nährstoffdefinierten Diät niedriger Osmolarität fortgesetzt.

\section{Pulmonale Aspiration}

Dieses ist die schwerste Komplikation einer enteralen Ernährung. Ein gastraler Reflux $>1000 \mathrm{ml} / \mathrm{Tag}$ bzw. Erbrechen während einer enteralen Ernährung müssen sehr ernst genommen werden. Eine gute Prävention ist. Keine Ernährung über gastral liegende Sonden bei bewusstseinsgetrübten bzw. nicht kooperativen Patienten durchführen. Bei Verdacht auf eine Sondendislokation ist zunächst radiologisch die Sondenlage zu überprüfen. Diese sollte postpylorisch und sicher duodenal liegen. Bei Fehllage ist eine Lagekorrektur durch Einführen eines Führungsdrahtes anzustreben. Der Oberkörper des Patienten muss während der Ernährung um $30^{\circ}$ hochgelagert werden.

\section{Gastrointestinale Komplikationen}

Diese können sein: Übelkeit, Erbrechen, abdominale Distension, Flatulenz, Bauchkrämpfe, Durchfälle, aber auch Obstipation.

Magenentleerungsstörungen. Bis hin zu einer Gastroparese sind sie bei schwerkranken Patienten (z. B. im Rahmen einer Sepsis, bei Schädel-Hirntrauma, während Stoffwechselkomata, bei hochdosierter Gabe von Opiaten oder Anticholinergika) und insbesondere bei Diabetikern häufig. Der sog. "postoperative Ileus« betrifft meist Magen und Kolon, während eine Atonie des Dünndarms in dieser Situation selten ist. Die Diagnose wird klinisch anhand der schlechteren Toleranz (Erbrechen sowie der spontanen Entleerung von Mageninhalt über die Sonde) ohne gleichzeitig erkennbarer Ursache gestellt. Die gastrale Transitzeit beträgt bei Gesunden für Wasser etwa 20 min und für feste Nahrung ca. 180 min.Bei einer bei Schwerkranken auf einer Intensivstation auftretenden Magenentleerungsstörung ist vorrangig deren Ursache zu klären bzw. zu beseitigen. Zu den unmittelbar ernährungsabhängigen Ursachen gehören Nährlösungen mit hoher Osmolarität oder Formuladiäten mit einem hohen Fettgehalt. Eine Gastroparese kann im Rahmen von Stoffwechselstörungen (z. B. bei Hypokaliämie, Hypomagnesiämie) auftreten. Eine Magenentleerungsstörung kann z. B. auch Folge der künstlichen Beatmung (Beatmungstyp, hoher PEEP, s.u.) sein oder durch die Lagerung des Patienten erklärt werden. Die Notwendigkeit einer Therapie bedrohlicher Organinsuffizienzen (z. B. die Respiratortherapie mit einem hohen PEEP, hohe Katecholamingaben, Sedierung und Analgesierung, Antibiotikatherapie) ist gegenüber dem möglichen Wert der künstliche Ernährung abzuwägen. Da das systemische Krankheitsbild auch von der Ernährung und der Funktion des Gastrointestinaltrakts abhängen (z. B. Mukosatrophie bei enteraler Nahrungskarenz, die enterale Ernährung senkt die bakterielle Translokation im Darm und beeinflusst dadurch auch die Inzidenz der Sepsis und des Multiorganversagen), ist aber die Bedeutung der enteralen Ernährung im Gesamtkonzept der Therapie nicht gering einzuschätzen. Nach Ausschluss einer naheliegenden Ursache ist die Behandlung der gestörten Magenentleerung zu diskutieren. Vor einer möglichen Behandlung sollte überlegt werden, ob wesentliche Vorraussetzungen der gastrointestinalen Motilität (wie z. B. eine adäquate Perfusion im Splanchnikusgebiet, eine intakte Wasser- und Elektrolythomöostase, ein ausgeglichener Säure-Basenhaushalt, ein kolloidosmotischer Druck von $>18 \mathrm{mmHg}$ bzw. ein Serumalbumin $>25 \mathrm{~g} / \mathrm{l}$ ) vorliegen. Eine Dünndarmatonie bei hohem Sympathikotonus, Störungen der Elektrolythomöostase, eine Hypoalbuminämie, eine verminderte oder gestörte Mikrozirkulation in der Mukosa und ein Darmwandödem bei venösem Rückstau (z. B. bei Herzinsuffizienz) bedeuten jeweils eine eingeschränkte Resorption der Nährstoffe im Dünndarm. und schränken die Toleranz gegenüber einer enteralen Ernährung ein.

Dünndarmatonie, lleus. Die Diagnose einer Dünndarmatonie wird in der Praxis klinisch gestellt (Reflux, Meteorismus, Distension, Darmgeräusche, Abwehrspannung, Bauchschmerzen) und durch den Ultraschallbefund (fehlende Darmbewegung, "weit« gestellt Schlingen, freie Spiegel) ergänzt. Eine Magenausgangsstenose muss endoskopisch ausgeschlossen werden. Ein anatomischer Ileus wird radiologisch ausgeschlossen. In jedem Fall muss vor einer Behandlung eine Druckentlastung über eine dicklumige Sonde erfolgen. Eine medikamentöse Behandlung von gastrointestinalen Motilitätsstörungen 
ist möglich. Bei Magenentleerungsstörungen bzw. Gastroparese kann eine medikamentöse Behandlung dem prokinetisch wirksamen Antibiotikum Erythromycin (in niedriger Dosierung, i.e. 3-mal $250 \mathrm{mg} / \mathrm{Tag}$ ) versucht werden. Parasympathikolytisch wirksame Medikamente (wie Neostigmin, z. B. Prostigmin) oder auch Ceruletid (z. B. Takus) sind wegen Nebenwirkungen (z. B. Bradykardie, AV-Knotenblock, Hypotension, Bronchospasmus, Gallenkoliken bei Gallensteinen, Pankreatitis) und Kontraindikationen (Asthma bronchiale, Thyreotoxikose, mechanischer Ileus, Bradykardie, Myokardinfarkt) nur sehr eingeschränkt verwendbar.

Erbrechen. Das Risiko des Erbrechens ist vor Beginn der enteralen Ernährung richtig einzuschätzen. So ist z. B. bei Tumorpatienten, die bestrahlt oder chemotherapeutisch behandelt werden, während der Behandlungszyklen eine enterale Ernährung nur in Ausnahmen möglich. In diesen Fällen ist auf eine optimale antiiemetische Medikation mit Dimenhydrinat, Metoclopramid, Ondansetron zu achten. Metoclopropramid und der Dopaminantagonist Domperidon vermindern die Fundusrelaxation und erhöhen die Antrummotilität. Bei beatmeten Patienten ist auch der Beatmungstyp wichtig. Bei positiv-endexpiratorischem Druck (sog. PEEP-Beatmung) ist die Magenentleerung behindert. In diesem Falle wäre eine "Dekompression" durch Veränderung des Beatmungstyps und nicht eine medikamentöse Behandlung die Therapie der Wahl. Ist nach Erbrechen, gastraler bzw. intestinaler Entlastung und einer Phase der Nahrungskarenz ein erneuter Kostaufbau vorgesehen, sollte zu Beginn das Ausmaß des Refluxes über eine gastral liegende Ablaufsonde überprüft werden. Übersteigt dieser $1000 \mathrm{ml} /$ Tag oder $15 \%$ des pro Stunde infundierten Volumens, ist eine adäquate enterale Ernährung offensichtlich nicht möglich.

Diarrhoen. Sie werden bei $5-30 \%$ der enteral ernährten Patienten beobachtet und führen im klinischen Alltag nahezu regelhaft zu einem Abbruch des Ernährungsregimes. Die Resorption von Nährstoffen ist auch bei intensivmedizinisch behandelten Patienten in der Regel nicht eingeschränkt. Die Diarrhoe ist immer behandelbar. Mögliche Ursachen sind:

- die Sondenlage (gastrale Lage mit sturzartiger Magenentleerung und schneller Dünndarmpassage),

- der Applikationsmodus (Bolusgabe ins Duodenum, großer Bolus, hohe Zufuhrraten),

- fehlender Kostaufbau,

- die hohe Osmolalität der Formuladiät (besonders bei chemisch-definierten Diäten),

- zu kalte Sondenkost (Raumtemperatur wird empfohlen),

- bakterielle Kontamination (Kost nicht länger als $6 \mathrm{~h}$ bei Raumtemperatur stehen lassen, die Infusionssysteme müssen alle $24 \mathrm{~h}$ gewechselt werden),
- die Begleitmedikation (Antazida, $\mathrm{H}_{2}$-Blocker, Atropin, Pirenzipin, Terbutalin, Sorbit, Dopamin in Infusionsraten $>4 \mu \mathrm{g} / \mathrm{kgKG} / \mathrm{min}$ ); hohe Osmolarität flüssiger Arzneimittel; hoher Sorbitgehalt sowie Arzneimittel, welche häufig Diarrhoen verursachen können, wie Alkylantien, Ampicillin, Antacida, Antimetabolite, Clindamycin, Colchicin, Clofibrat, Digitalis, Erythromycin, 5-Fluorouracil, Eisensalze, Magnesiumsalze, Methotrexat, Methyldopa, Kaliumsalze, Propranolol, Chinidin, Tetracycine,

- eine niedrige Natriumzufuhr $(<80-100 \mathrm{mmol} / \mathrm{Tag})$,

- niedrige Albuminspiegel im Serum (bei Werten $<25 \mathrm{~g} /$ 1 besteht häufig ein Ödem der Dünndarmschleimhaut),

- eine Imbalanz zwischen Motilität, Sekretion und Resorption, eine bei langfristiger Nahrungskarenz bzw. parenteraler Ernährung häufige Atrophie der Dünndarmschleimhaut oder auch

- vorher nicht bekannte intestinale Erkrankungen (Nahrungsmittelunverträglichkeiten, Malassimilation, Gallensäureverlustsyndrom, entzündliche Darmerkrankungen, TBC, Antibiotika-assoziierte Durchfälle).

Die Abklärung erfolgt klinisch. Bei unklaren und länger bestehenden Diarrhoen ist eine mikrobiologische $\mathrm{Di}$ agnostik durchzuführen. Eine hohe Stuhlfrequenz bei Entzündungen des Rektums ist abzugrenzen und gezielt therapeutisch anzugehen. Bei Diarrhoen sollte zunächst die enterale Nährstoffzufuhr um $50 \%$ gedrosselt werden. In seltenen Fällen wird sie ganz beendet und vorübergehend parenteral ernährt. Im Anschluss an eine 2- bis 3tägige Unterbrechung wird ein langsamer und stufenweiser Kostaufbau begonnen. Die Sonde sollte sicher duodenal liegen, die Nährstoffzufuhr muss kontinuierlich über $16 \mathrm{~h}$ erfolgen, die Dosierung wird individuell berechnet. Wenn möglich wird eine ballaststoffreiche nährstoffdefinierte Diät gewählt. Bei einer Fettmalassimilation ist eine MCThaltige Sondenkost indiziert. Die meisten Formuladiäten sind natriumarm. Natrium kann deshalb zusätzlich bis zu einer Menge von $50 \mathrm{mmol} / \mathrm{Tag}(1,5 \mathrm{~g} \mathrm{NaCl})$ unter Berücksichtigung der klinischen und metabolischen Situation des Patienten gegeben werden. Bei Hypoalbuminämie, Ödemen und Diarrhoen muss die Albuminsynthese durch eine konsequente und hochkalorische Ernährung stimuliert werden. Die Substitution von Albumin ist fragwürdig. Die Medikation des Patienten ist täglich zu überprüfen. Bei Fortbestand der Diarrhoen ist eine weiterführende Diagnostik anzustreben. Die unter enteraler Ernährung vermuteten osmotischen Diarrhoen werden meist nur innerhalb der 1. Woche nach Beginn des Ernährungsregimes beobachtet und sind bei richtiger Handhabung der Technik selten. Ein vorsichtiger und langsamer Kostaufbau mit Formuladiäten, deren Osmolarität unter $400 \mathrm{mosmol} / \mathrm{l}$ liegt, verhindert das Auftreten osmotischer Diarrhoen. Eine medikamentöse Behandlung der mit der 
enteralen Ernährung gelegentlich assoziierten Diarrhoen ist in der Regel nicht indiziert.

Obstipation. Ein Intervall von mehr als 3 Tagen zwischen 2 Stuhlgängen wird im Vergleich zur Diarrhoe unter enteraler Ernährung selten beobachtet. Tritt eine Obstipation auf, könnte der geringe Ballaststoffgehalt der jeweiligen Sondenkost oder eine zu geringe Flüssigkeitszufuhr Ursache sein. Differentialdiagnostisch sind ein Ileus (Röntgen: Abdomenübersicht) oder eine Dickdarmatonie (z. B. Elektrolytentgleisungen bei Dehydration), intraabdominelle Entzündungen oder hochdosierte Gabe von Schmerzmedikamenten auszuschließen. Therapeutisch sind neben dem Wechsel der Sondenkost (ballaststoffreiche Formuladiät bevorzugen, lösliche Ballaststoffe, Guarpektine) vorrangig die Elektrolyt- und Flüssigkeitsbilanz auszugleichen. Eine vermehrte Flatulenz ist eine Nebenwirkung der hohen Ballaststoffzufuhr. Bei Vorliegen von Darmgeräuschen wird in der Klinik in Einzelfällen eine Stimulation des Darms mit Neostigmin oder Ceruletid versucht. Darüber hinaus sind Einläufe und die Einlage eines Darmrohrs (ohne Blockung) zur Druckentlastung (Lage über $24 \mathrm{~h}$ bedeutet eine mögliche Verletzungsgefahr) wirksam.

\section{Prävention/Vorgehen}

Pulmonale Aspiration. Keine gastrale Ernährung bei bewusstseinsgetrübten, nicht kooperativen Patienten, radiologische Kontrolle der Sondenlage, Ausschluss einer Magenentleerungsstörung, Lagerung des Patienten?,Osmolarität/ Fettgehalt der Nährlösung, Stoffwechselstörungen (Elektrolyte?), Beatmungstyp (hoher PEEP), Analgesie/Sedierung?, Perfusion im Splanchnikusgebiet?

Gastrointestinale Komplikationen. Sondenlage? Applikationsmodus? Kostaufbau? Temperatur der Nährlösung? Kontamination der Kost? Natriumgehalt? Wasserund Elektrolythomoistase? Albuminspiegel? Gastrointestinale Erkrankungen? Osmolarität (bis $450 \mathrm{mosmol} / \mathrm{kg}$ )? Ballaststoffzufuhr? Kolloidosmotischer Druck? Herzinsuffizienz? Dosis? Säure-Basenhaushalt? Katecholamingabe? Verminderung der Infusionsrate, Berechnung des Bedarfs, erneuter Kostaufbau, Flüssigkeitszufuhr neu berechnen, duodenale/jejunale Sondenlagen, Infusion über 16 Stunden, Übergang auf mNDD/CDD, zusätzliche Natriumgabe, (+50 mmol Na oder $1,5 \mathrm{~g} \mathrm{NaCl} /$ d), Ballaststoffzufuhr (=lösliche Ballaststoffe) erhöhen $(+20 \mathrm{~g} / \mathrm{d})$.

\section{Medikation bei enteraler Ernährung}

Probleme bestehen häufig bei der Medikation enteral ernährter Patienten. Diese ergeben sich aus der Darreichungsform und Osmolarität des Medikaments, der intestinalen Lage der Ernährungssonde, deren filiformen Durchmessers sowie dem in der Regel kontinuierlichem
Ernährungsregime. Es sollten kommerziell hergestellte flüssige oder verdünnte und visköse Medikamente oder allenfalls Suspensionen gewählt werden. Diese Darreichungsformen enthalten Zusätze wie Alkohol oder Sorbit. Ihre Osmolalität kann im Einzelfall bis zu 10000 mosmol/kg $\mathrm{H}_{2} \mathrm{O}$ betragen. Dies kann bei intragastraler Bolusgabe zu einem "Dumping ", bei intraduodenaler oder intrajejunaler Applikation zu Diarrhoen führen. Die Kenntnis der Osmolarität eines Medikaments ist bei enteraler Ernährung obligat. Es ist obsolet, gemörserte und mit Flüssigkeit versetzte Tabletten durch filiforme Sonden zu applizieren, da dies regelhaft zu einer Sondenverstopfung führt. Auch das Mischen eines Medikaments mit der Sondenkost führt aufgrund der möglichen Inkompatibilitäten und Wechselwirkungen zu Problemen. Die Stabilität von Medikamenten in Mischung mit Nährstofflösungen ist unterschiedlich und muss beim Hersteller erfragt werden. Schwierigkeiten ergeben sich z. B. bei Elektrolytsupplementen (Ausfällen der Nährlösung), aluminiumhaltigen Antazida und einigen H2-Blockern. Wechselwirkungen bestehen z. B. zwischen Formuladiäten und Warfarin und Phenytoin. Im schlechtesten Fall bedeutet dies eine verminderte $\mathrm{Zu}$ fuhr und Resorption von Medikament und Nährstoffen und damit eine verminderte Medikamentenwirkung bei gleichzeitig unzureichender Ernährung. Die Freisetzung oder Aktivierung des betroffenen Medikaments muss unabhängig von der gastralen Phase sein. Da die Resorption von vielen Medikamenten im Nüchternzustand besser ist, werden sie bei enteral ernährten Patienten vor Beginn oder frühestens $2 \mathrm{~h}$ nach Ende der Ernährungsperiode gegeben. Grundsätzlich ist es möglich, die parenterale Form eines Arzneimittels enteral über eine Ernährungssonde zu geben. In diesem Fall ist sicherzustellen, dass der Wirkstoff gut resorbiert wird, er im sauren Milieu des Magens nicht zerstört wird, das Arzneimittel nicht zu stark sauer oder basisch reagiert, seine Osmolarität tolerierbar ist und es die Schleimhaut im Magen-Darm-Trakt nicht zu stark irritiert. Für die Resorption parenteraler Arzneimittel gilt, dass sie zwar mit ähnlichen oder gleichem Handelsnamen wie die vergleichbare orale Arzneiform auf dem Markt sind, z. T. aber anders dosiert sind und einen anderen Ester oder ein anderes Salz des Arzneistoffes enthalten, welcher unterschiedlich resorbiert werden kann.

\section{Überwachung und Problemlösung}

Künstlich ernährte Patienten müssen intensiv betreut werden. Das Ausmaß der Überwachung ist abhängig von der Grunderkrankung, dem klinischen Bild, dem Ernährungszustand sowie den subjektiven und objektiven Problemen des Patienten. Eine optimale Zusammenarbeit zwischen dem Patienten und seinen Angehörigen auf der einen und der Ernährungsschwester, der Diätassisten- 
tin, dem ernährungsmedizinisch verantwortlichen Arzt (d. h. dem »Ernährungsteam «) und dem Hausarzt auf der anderen Seite ist nur nach einer gründlichen Schulung und Unterweisung des Patienten und seiner Angehörigen (und evtl. auch des Hausarztes) zu Beginn der künstlichen Ernährung möglich. Die obligate Überwachung beinhaltet zunächst die Zuwendung zum Patienten mit der Frage nach dem subjektiven Befinden.

- Wie geht es dem Patienten?

- Wie verbringt der Patient seine Tage?

- Wie ist seine Stimmungslage? Hat er noch andere Probleme?

- Welchen Eindruck haben die Angehörigen?

- Besteht zur Zeit ein Leidensdruck?

- Wird die Grunderkrankung gegenwärtig behandelt?

Kostform, Sondenkostmenge, Flüssigkeitszufuhr, Applikationsmodus, Infusionsdauer, Sondenpflege und Sondenlage sowie der Stuhlgang (Frequenz, Menge, Konsistenz) müssen hinterfragt werden.

Die körperliche Untersuchung erfasst gezielt und differenziert den Hydrations- und Ernährungszustand mit klinischer Untersuchung, Anthropometrie und ggf. bioelektrischer Impedanzmessung. Es schließt sich die Untersuchung der Herz- und Lungenfunktion an. Spezielle ernährungsmedizinische Aspekte sind klinische Zeichen der Fehlernährung (Hautturgor, Schleimhäute, Haare, Nägel, Ödeme, Muskelkraft), eine mögliche abdominelle Distension, Darmgeräusche, Lebergröße, der Lokalbefund im Bereich des Stomas sowie die neurologische und ophthalmologische Untersuchung.

Sinnvolle und regelmäßig durchzuführende Laboruntersuchungen sind die Bestimmung des roten und weißen Blutbildes, die Messungen der Serumelektrolyte, des Serumharnstoffs und -kreatinins, der Plasmaspiegel von Glukose und Triglyzeriden sowie des Albumins und des Bilirubins. Bei unklaren klinischen Problemen wird die Krankheitsaktivität durch die Messung der Blutsenkungsgeschwindigkeit, der Serumeiweißelektrophorese und des C-reaktiven Proteins charakterisiert. Die Messung der Harnstoffausscheidung im 24-h-Urin ist für die Einschätzung der Stoffwechsellage und auch der tatsächlichen Nährstoffzufuhr wichtig. In größeren Abständen müssen die Parameter des Eisenstoffwechsel, der Serumzinkspiegel sowie von Vitamin $B_{12}$ und Folsäure überwacht werden. Ein Spurenelementmangel wird gelegentlich bei der Ernährung mit chemisch-definierten Diäten beobachtet.

Bei Verschluss der Sonde ist zunächst ein vorsichtiges Spülen des Katheters mit Wasser angesagt. Der Versuch ist möglicherweise nach Umlagerung des Patienten zu wiederholen. Bei Erfolglosigkeit ist das Katheterende unter Durchleuchtung zu lokalisieren (ist die Sonde abgeknickt?) und evtl. eine Neuplazierung nach Lagekorrektur und Einführen eines Führungsdrahtes zu versuchen. Im
Zweifelsfall eine Neuanlage der Ernährungssonde durchzuführen.

Im Umgang mit den Patienten müssen unrealistische Erwartungen und ein falsches medizinisches Verständnis vermieden werden. Ernährung dient zunächst dem Erhalt des Ernährungszustands. In diesem Sinne ist Ernährung Teil der supportiven Behandlung (welche z. B. auch die Schmerztherapie und die psychosoziale Unterstützung umfasst). Der Erhalt der Körperzellmasse ist für die in der Regel schwerkranken Patienten realistisch. Die ernährungsmedizinische Überwachung wird zu Beginn einer enteralen Ernährung 2-mal/Woche erfolgen. Bei stabilem Verlauf und auch bei heimenteraler Ernährung sind die Untersuchungen in 4- bis 8-wöchigem Abstand zu wiederholen. Eine umfassende und vollständige Verlaufsdokumentation ist selbstverständlich.

Bei ambulant geführten Patienten kann die Kostenfrage ein Problem darstellen. Die Richtlinien zur Erstattungsfähigkeit enteraler Trink- und Sondennahrung besagen, dass die begründete Verordnung von Diäten entsprechen $\$ 14 \mathrm{~b}$ der Diätverordnung zulässig ist bei Morbus Crohn, Kurzdarmsyndrom, Malnutrition, $\mathrm{Mu}-$ koviszidose, chronisch-terminaler Niereninsuffizienz unter eiweißarmer Ernährung und bei konsumierenden Erkrankungen.

\subsubsection{Parenterale Ernährung}

1967 gelang Dudrick und Mitarbeitern die Zufuhr einer hyperosmolaren Infusionslösung über einen zentralvenösen Katheter. Die parenterale Ernährung hat sich seitdem zu einer sicheren und etablierten Technik entwickelt. Im letzten Jahrzehnt ist häufig die Diskussion »enteral oder parenteral« geführt worden. Konsens besteht heute, dass die enterale Zufuhr, wann immer möglich zu bevorzugen ist. Zum Erreichen einer adäquaten Kalorienzufuhr kann jedoch zumindest vorübergehend die Kombination »enteral und parenteral« sinnvoll sein. Bei einigen Krankheitsbildern wie dem Kurzdarmsyndrom kann die parenterale Ernährung die einzige Möglichkeit der Substratzufuhr darstellen. Zahlen aus den Vereinigten Staaten belegen, dass dort mehr als 10000 Menschen lebenslang ausschließlich parenteral ernährt werden müssen. Eine parenterale Ernährung wird als hypokalorische und "unvollständige" periphervenöse Ernährung (PE) oder als vollständige, bedarfsdeckende und im Einzelfall auch bedarfsüberschreitende vollständige bzw. totale parenterale Ernährung (TPE) durchgeführt. Eine periphervenöse Ernährung ist keine optimale und deshalb nur eine vorübergehende und kurzfristige Ernährungsform (bis zu 3 Tagen). Sie wird vor allem "substitutiv« zum Beispiel in der unmittelbar postoperativen Ernährung zur Minderung der katabolen Stoffwechsellage eingesetzt. 


\section{Techniken \\ Periphervenöse und "zentralvenös« infundierte vollständige parenterale Ernährung}

Ernährung. Die Nährlösung wird unter sterilen Kautelen meist in eine oberflächlichen Armvene (in der Regel die V. basilica in der Ellenbeuge) oder am Handrücken eingeführte Teflonkanüle infundiert. Eine periphervenöse Ernährung wird nur kurzfristig über 3-4 Tage durchgeführt, sie ist hypokalorisch. Aufgrund der Gefahr einer Thrombophlebitis dürfen nur niedrigkonzentrierte Glukose- $(<10 \%)$ und Aminosäurelösungen $(<3,5 \%)$ infundiert werden. Lipidemulsionen sind isoton und werden deshalb problemslos im Rahmen einer periphervenösen Ernährung verwendet. Osmolarität einer Mischlösung darf bei periphervenöser Ernährung 600-800 mosmol/1 nicht überschreiten.Von der Industrie werden verschiedene "Komplettlösungen" angeboten. Ihr Elektrolytgehalt ist am Basisbedarf orientiert. Die periphervenöse Gabe von Elektrolytkonzentraten ist obsolet. Eine vollständig parenterale Ernährung erfolgt über einen zentralvenösen Katheter unter Verwendung hypertoner Infusionslösungen ist nur über einen zentralvenösen Verweilkatheter möglich. Diese Katheter bestehen aus Polyethylen, Polyurethan oder Silikonkautschuk. Sie haben eine hohe Biostabilität und Biokompatibilität. Die Größe liegt zwischen 25 und 14 Gauge bei einem inneren Durchmesser von 0,5-2,0 mm. Die Länge des Katheters ist von der geplanten Einstichstelle abhängig. Die Katheter werden unter streng aseptischen Bedingungen nach Punktion mit einer großlumigen Kanüle durch die Haut in die V. basilica, die V. subclavia oder die V. jugularis eingeführt. Am häufigsten wird für die langzeitparenterale Ernährung die rechte V. subclavia verwendet. Die Katheterspitze wird mit Hilfe eines Führungsdrahts und/oder unter Durchleuchtung mit einem Bildwandler in die obere Hohlvene (V. cava superior) geführt. Man unterscheidet ein- und mehrlumige Katheter. Letzere erlauben die getrennte Infusion von Nährlösung und Medikamenten oder Blutprodukten. Für die längerfristige (>3 Monate) und heimparenterale Ernährung werden implantierbare Verweilkatheter (Portkatheter, HickmanBroviac-Katheter) verwendet. Diese Katheter werden zur Senkung des Infektionsrisikos und der Katheterdislokation über einen längeren subkutanen Tunnel ausgeleitet. Die Katheterspitze wird über die V. cephalica oder die V. jugularis externa in die obere Hohlvene geführt. Ein Port ist ein Reservoir, an welches der eigentliche Katheter angeschlossen ist. Er wird subkutan implantiert und ist durch eine Silikonmembran abgetrennt. Diese Membran kann jeweils zur Infusion mit speziellen Portnadeln (Hoover-Nadeln) punktiert werden. Bei einem Hickman-Broviac-Katheter wird der Katheterschlauch selbst unter der Haut hervorgeleitet (»getunnelt») und kann direkt an das Infusionssystem angeschlossen werden. Die Verweilkathetersysteme werden in der Onkologie im
Rahmen einer »zyklischen" Chemotherapie verwendet. Die verschiedenen Systeme sind sehr sicher und haben eine niedrige Infektions- und Komplikationsrate. Für die heimparenterale Ernähung haben sich HickmanBroviac-Katheter bewährt. Portsysteme sind besonders für die "zyklische« Chemotherapie geeignet. Die bei Anlage zentralvenöser Katheter mögliche Komplikationsrate (Verletzung, fälschliche Punktion einer Arterie, Hämato- und Pneumothorax, Infektionen, Luftembolie, Nervenverletzungen) ist in spezialisierten Zentren sehr niedrig.

Desinfektion. An den Kathetern muss immer aseptisch gearbeitet werden. Vor jeder Applikation werden die Hände des Therapeuten bzw. des Betreuers für 3-5 min mit einer antimikrobiellen Seife gewaschen. Alle zugänglichen Teile des Infusionsystems und insbesondere die Dreiwegehähne werden vor und nach jeder Benutzung mit Isopropylalkohol (70\%) desinfiziert. Da eine direkte Beziehung zwischen der bakteriellen Besiedlung der Haut und des Katheters besteht, ist außerdem eine regelmäßige und gründliche Hautdesinfektion notwendig. Die Einstichstelle wird mit durchsichtigem Pflaster verschlossen. Dieses wird routinemäßig alle 2 Tage gewechselt. Die Infusionskatheter dürfen niemals zur Entnahme von Blutproben benutzt werden. Venenkatheter sollten immer elektiv und unter optimalen Bedingungen gelegt werden.

Infusion. Die einzelnen Nährlösungen oder auch die Infusionsmischlösungen werden kontinuierlich über eine Pumpe infundiert. In der Regel wird als Infusionszeitraum eine Periode von $24 \mathrm{~h}$ eingeplant. Ziel ist die Konstanz der Homöostase. Andererseits ist dieses eine "unphysiologische« Ernährungsweise, so dass einzelne Autoren das Konzept einer »zyklisch«-parenteralen Ernährung propagieren. Bei dieser Ernährungsform wird die Infusionsperiode auf $12-16 \mathrm{~h}$ beschränkt. Es schließt sich eine 8- bis 12-stündige »Ruhephase« an. Die möglichen Vorteile einer »zyklisch«-parenteralen Ernährung sind aber wissenschaftlich nicht geprüft.

\section{Indikation}

Die Indikation zur parenteralen Ernährung ist die Indikation zur künstlichen Ernährung, wenn keine bedarfsdeckende enterale Ernährung möglich ist. Eine parenterale Ernährung erlaubt eine exakte Dosierung der Nährstoffzufuhr, die während instabiler Stoffwechselsituationen notwendig ist. Die parenterale Ernährung ist in speziellen Fällen (z. B. bei akutem Schub einer chronisch-entzündlichen Darmerkrankung) auch therapeutisch wirksam. In den meisten Fällen entspricht die Nährstoffzusammensetzung einer parenteralen Ernährung den für die Ernährung Gesunder bekannten Empfehlungen. Es ist zu 
berücksichtigen, dass es sich bei den verschiedenen Infusionslösungen um Arzneimittel und nicht um Lebensmittel handelt. Dies macht eine exakte Dosisberechnung und eine Rezeptur notwendig. Kontraindikationen gegen eine parenterale Ernährung sind:

- instabile Kreislaufverhältnisse und Schockzustand,

- unzureichende zelluläre Sauerstoffversorgung (schwere Hypoxie, mitochondriale Defekte),

- manifeste Substratverwertungsstörungen

- ausgeprägte Überwässerung (Hyperhydration).

\section{Rezeptur einer parenteralen Ernährung}

Eine parenterale Ernährung wird individuell entsprechend dem Energie- und Nährstoffbedarf unter Berücksichtigung möglicher individueller oder klinischer Einschränkungen oder Bedürfnisse berechnet. Da es sich bei den parenteralen Nährlösungen um Arzneimittel handelt, wird die Zusammensetzung in einer Rezeptur festgelegt (s. - Abb. 2.32). Die folgenden Rechenschritte sind zu beachten:

- Berechnung des Ruheenergiebedarf. Bei Säuglingen und Kleinkindern unter $10 \mathrm{~kg}$ KG wird der Ruheenergieumsatz wie folgt berechnet: Körpergewicht $(\mathrm{kg})$ $\times 0,611 \times$ Größe $(\mathrm{cm}) \times 0,951 \times 1,99$.

- Schätzung des aktuellen Energiebedarfs: 1-1,3bzw.1,5-1,7-fach bei katabolen Patienten).

- Berechnung des Eiweiß-/Aminosäurebedarfs: 1,3 bzw. bei Sepsis 1.5g Aminosäuren/kgKG/Tag; "Leber-adaptiertes Aminosäuremuster nur bei hepatischer Enzephalopathie: abhängig von der sog. »Differenzosmolalität«: >15 keine Aminosäuren, 10-15 Komalösung, <10 leberadapiertes AS-Muster, Dosierung mit 0,6-0,8 g/kg KG/Tag unter Berücksichtigung des Serumharnstoffspiegels und der Nierenfunktion)

- Berechnung der »Nicht-Eiweiß-Kalorien« (»2. minus 3. «)

- Aufteilung der »Nicht-Eiweiß-Kalorien«, z. B. 70\% Glukose, 30\% Fett bzw. 50\% Glukose, 50\% Fett bei kataboler Stoffwechsellage

- Substitution von Elektrolyten, Spurenelementen und Vitaminen (n. Empfehlungen und Nierenfunktion).

- Deckung des Flüssigkeitsbedarfs (in der Regel 30$40 \mathrm{ml} / \mathrm{kg} \mathrm{KG/Tag} \mathrm{bzw.} \mathrm{nach} \mathrm{Bilanz} \mathrm{und} \mathrm{"Klinik«,}$ d. h. unter Berücksichtigung des zentralen Venendrucks (=ZVD) und der Körpertemperatur, d. h. pro Grad Temperaturerhöhung + 500-1000 ml). Der Flüssigkeitsbedarf beträgt bei Neugeborenen während der 1. Lebenswoche 50-140, während des 1 . Lebensjahres 100-140, während des 2. Lebensjahres 80-120, vom 3.-5. Lebensjahr 80-100, vom 6.-10. Lebensjahr 60-80 und vom 10.-14. Lebensjahr 50$70 \mathrm{ml} / \mathrm{kgKG} / \mathrm{Tag}$.

\section{(? Cave}

Vorsicht bei Gerinnungsstörungen (Fette), respiratorische Insuffizienz (Glukose, Insulin), Leberfunktion (Kohlenhydrate), Nierenfunktion (Aminosäuren, Flüssigkeit, Elektrolyte), bei Multiorganversagen (weitere Reduktion der Kalorien und Infusionsrate bzw. Abbruch der künstlichen Ernährung).

Die Vorgehensweise wird in einer Rezeptur festgelegt und dokumentiert (s. - Abb. 2.32). Bei konventioneller parenteraler Ernährung werden 50-55\% des errechneten Energiebedarfs in Form von Glukoselösungen, 30-35\% als Lipidemulsion und 10-15\% als Aminosäurelösung gegeben. Diese Nährstoffrelationen können bei entsprechenden metabolischen Gegebenheiten und Bedürfnissen verändert werden (z. B. bei Tumorkachexie Verminderung des Kohlenhydratanteils bei gleichzeitiger Erhöhung der Fettmenge). Elektrolyte, Vitamine und Spurenelemente werden entsprechend den Empfehlungen von Fachgesellschaften und Expertengremien zum Nährstoffbedarf und unter klinischer bzw. biochemischer Kontrolle substituiert. Die Flüssigkeitszufuhr wird der Flüssigkeitsbilanz, den hämodynamischen Kenngrößen, möglichen unsichtbaren Verlusten (Körpertemperatur, $+10 \%$ pro ${ }^{\circ} \mathrm{C}$ Temperaturerhöhung) und dem klinischen Bild (z. B. vorsichtige Zufuhr bei Hypoalbuminämie) angepasst. Bei beatmeten Patienten ist ein zusätzlicher Flüssigkeitsverlust anzunehmen.

\section{Infusionslösungen, Elektrolyt-, Vitamin- und Spurenelementpräparate}

Für die kurzfristige parenterale Ernährung sind die von der Industrie angebotenen "Zwei«- (Glukose und Aminosäuren) und »Dreikammerbeutel« (Glukose, Aminosäuren und Fette) mit unterschiedlichem Kaloriengehalt nicht zuletzt aus ökonomischen Gründen ausreichend. Für die längerfristige und vollständig bedarfsdeckende, d. h. »totale« parenterale Ernährung hingegen gibt es keine voll bilanzierten, den individuellen Bedarf der Patienten optimal »deckenden« Infusionslösungen. Hier ist es sinnvoll, das Ernährungsregime individuell für jeden Patienten aus Einzelbausteinen zusammenzusetzen. Dies sind Infusionslösungen, welche Kohlenhydrate, Fette, Aminosäuren sowie Vitamin-, Spurenelement- und Elektrolytzusätze enthalten. Die Lösungen und Zusätze sind Medikamente, welche dem Arzneimittelgesetz unterliegen. Die Mischung der Einzelkomponenten muss deshalb einer speziellen Rezeptur folgen. Die Mischung bedeutet nach dem Arzneimittelrecht die Herstellung eines neuen Medikaments, was nur unter besonderen Kautelen (z. B. Sterilität, Kompatibilität der Inhaltsstoffe, exakte Dosierung, Überwachung) erfolgt. Die einzelnen Infusionslösungen und die Zusätze werden mit Hilfe eines automatisierten Pumpensystems unter sterilen Kautelen in einem Mischbeutel zusammengemischt (»all in one«). Die Mischung wird vom Apotheker bzw. unter 


\section{Rezeptur für eine vollständige parenterale Ernährung}

Patientendaten

\section{Name}

Größe (cm)

Diagnose

Eiweißbedarf (g/Tag)

Energieverbracuh (kcal/Tag)

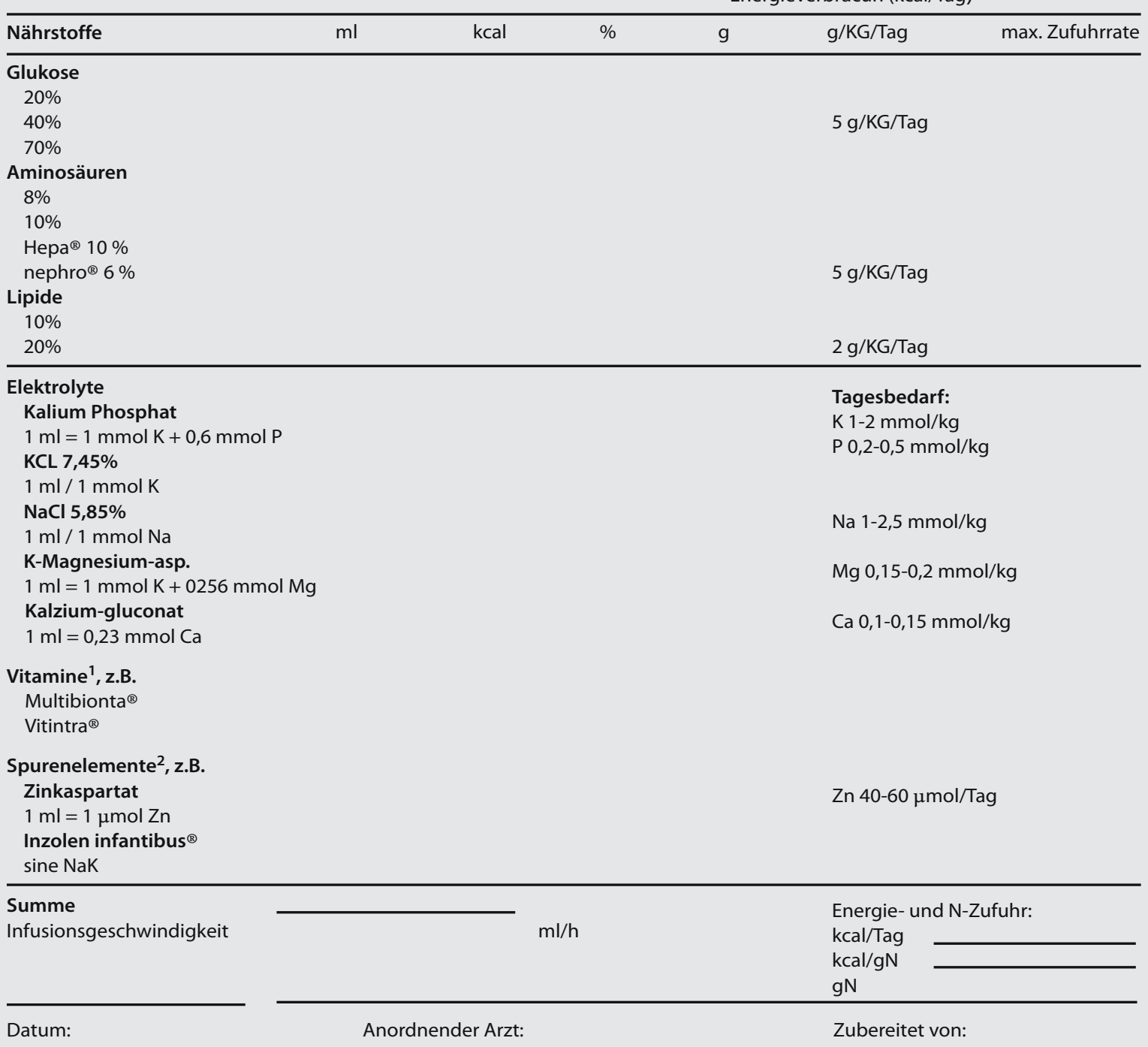

- Abb. 2.32. Rezeptformular für eine parentale Ernährung mit ausgewählten Infusionslösungen, ${ }^{1}$ Tab. $2.75,{ }^{2}$ Tab. 2.78

seiner Überwachung vorgenommen. Mischlösungen dürfen außer den Nährlösungen keine weiteren Medikamente (wie z. B. Insulin oder Albumin) beigemischt werden.

Probleme sind die mögliche Instabilität der Infusionslösung, Aggregation von Lipidpartikeln, und die ungenaue Medikamentendosierung infolge Adsorption an das Kunststoffmaterial. Medikamente sollten deshalb immer im Nebenschluss über das Infusionsystem gegeben werden. Die Mischbeutel fassen z. B. ein Gesamtvolumen von 3,01 . Sie bestehen aus gasdurchlässigem Ethylvinylazetat (EVA). Die Mischlösung wird mittels einer volumetrischen Pumpe kontinuierlich über $24 \mathrm{~h}$ infundiert. Bei richtiger
Berechnung ergeben die verschiedenen Infusionslösungen und Zusätze eine vollständige, d.h. den Nährstoff- und Flüssigkeitsbedarf deckende parenterale Nährlösung. Diese Methode erleichtert die Infusionstechnik, vermindert das Infektionsrisiko, erlaubt eine gleichmäßige Versorgung mit Nährstoffen und senkt das Risiko von Entgleisungen der Stoffwechsel- und Elektrolythomöostase. Nachteile der Technik sind die begrenzte Haltbarkeit der Mischlösungen (in der Regel maximal $24 \mathrm{~h}$ ), die ein tägliches Mischen notwendig machen. Die Stabilität von Einzelbestandteilen wie z. B. einiger Vitamine wie Vitamin $A$ und $B_{2}$ ist aber auch innerhalb dieses kurzen Zeitraums nicht gewährleistet. 


\section{Kohlenhydrate, Glukose}

Glukose ist der wesentliche Energieträger einer parenteralen Ernährung sind Kohlenhydratlösungen. Es handelt sich in der Regel Glukoselösungen, welche in Konzentrationen zwischen 5 und 70\% von der Industrie oder der Apotheke angeboten werden. Bei vollständiger parenteraler Ernährung werden 20, 40 und in Einzelfällen $70 \%$ ige-Lösungen verwendet. Der Kaloriengehalt ist 3,4 kcal/g Glukose. Die Osmolarität der Lösungen beträgt bei einer 10\%-Glukoselösung 505 und bei einer 70\%-Lösung 3535 mosmol/l. Die Dosierung der Glukosemenge richtet sich bei Kindern und Erwachsenen nach dem Ernährungszweck (z. B. Verhütung von Hypoglykämien, Prävention eines gesteigerten Proteinabbaus und Hemmung einer hohen Ketonkörperproduktion; Deckung der Glukoseoxidationsrate; bei depletierten Patienten das Wiederauffüllen der körpereigenen Glykogenspeicher) und berücksichtigt mögliche und in der Klinik häufige Einschränkungen des Glukosestoffwechsels. $100 \mathrm{~g}$ Glukose/Tag reichen aus, um den Abbau körpereigener Eiweiße um die Hälfte zu drosseln. Höhere Dosierungen haben keinen weiteren proteinsparenden Effekt. Zur Vermeidung von Hypoglykämien sind z. B. bei Patienten mit einem Leberversagen 150-175 g Glukose/Tag zu infundieren. Ein maximaler Energiegewinn wird (abhängig von einer adäquaten endogenen Insulinsekretion und -wirkung) bei der Infusion von 4-5 g/kgKG/Tag (oder etwa $280-350 \mathrm{~g} / \mathrm{Tag}$ bei einem $70 \mathrm{~kg}$ schweren Patienten) erreicht. Bei diesen Infusionsraten werden etwa $50 \%$ der Glukose oxidiert. Eine weitere Steigerung der Glukoseverbrennung ist auch durch Insulingabe nicht $\mathrm{zu}$ erreichen. Bei depletierten und kachektischen Patienten können vorübergehend zur Repletion der endogenen Glykogenspeicher größere Mengen von Glukose (z. B. $400 \mathrm{~g} / \mathrm{Tag}$ ) infundiert werden. Die Infusionsdosis richtet sich dann nach der Glukoseoxidationsrate (maximal 2,5 g/kgKG/Tag) und der Speicherkapazität der Leber

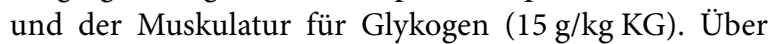
diese Kapazitäten hinausgehende Glukoseinfusionsraten steigern die Lipidsynthese und führen zu Hypertriglyzeridämie, Leberverfettung und Leberschäden. In der klinischen Praxis stehen diesen Empfehlungen häufig Einschränkungen (z. B. hämodynamische Probleme, Glukoseverwertungsstörungen) entgegen. Es ist deshalb sinnvoll, in diesen Situationen vorsichtig mit einer Gesamtmenge von $200 \mathrm{~g}$ Glukose/Tag zu beginnen und die Zufuhrrate ggf. kontrolliert auf Bedarfswerte zu steigern. Der Blutzuckerspiegel sollte unter Infusion bei $180 \mathrm{mg} \%$ (10mmol/l) liegen. Bei Neugeborenen ist der Glukoseverbrauch des Gehirns Orientierung der Dosierung. Die Glukoseinfusionsrate sollte in diesen Fällen mit 6-7 mg/ $\mathrm{kgKG} / \mathrm{min}$ etwas oberhalb des Bedarfs liegen. Bei unreifen Frühgeborenen sind niedrigere Dosierungen zu wählen $(2-4 \mathrm{mg} / \mathrm{kgKG} / \mathrm{min})$ (z. B. Glukose/Xylit-Lösungen im Verhältnis 1:1).

\section{Zuckeraustauschstoffe}

Neben Glukoselösungen werden im deutschsprachigen Raum in der parenteralen Ernährung Erwachsener Zuckeraustauschstoffe (=Nichtglukosekohlenhydrate) verwandt. Es handelt sich hierbei um Fruktose, Sorbit und Xylit. Die früher verwandte Fruktose kommt aufgrund von beobachteten Fällen eines Leberversagens bei nicht bekannter Fruktoseintoleranz nicht mehr zum Einsatz. Dasselbe gilt für Sorbit, welches dieselben Risiken bietet. Xylit wird überwiegend in Form von Mischlösungen mit Glukose angeboten. Die maximalen Dosierungen sind für Xylit bei

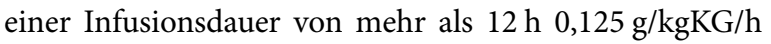
und betragen im Rahmen einer vollständigen parenteralen Ernährung maximal 3 g/kgKG/Tag. Zuckeraustauschstoffe unterscheiden sich in ihrem Stoffwechsel von der Glukose. Im Gegensatz zu i.v. verabreichter Glukose, welche zu etwa $80 \%$ im Muskel verstoffwechselt wird, werden Zuckeraustauschstoffe nach parenteraler Zufuhr insulinunabhängig zu $80 \%$ in der Leber verstoffwechselt. Dies erklärt, warum z. B. Xylit die hepatische Harnstoffproduktionsrate wesentlich besser senkt als äquimolare Mengen von Glukose. Xylit wird im Pentosephosphatzyklus verstoffwechselt. Im Gegensatz zu den Zuckeraustauschstoffen hemmt Glukose die Glukoseproduktion der Leber. Das Enzym Fruktokinase, welches die Phosphorylierung der Fruktose katalysiert, ist in der Leber im Vergleich zu den Aktivitäten von Glukokinase und Hexokinase 10fach aktiver. Da Zuckeraustauschstoffe den ersten geschwindigkeitsbestimmenden Stoffwechselschritt der Glykolyse, die Phosphofruktokinasereaktion, umgehen, werden sie wesentlich schneller verstoffwechselt als Glukose. Intrazellulär kommt es unter hochdosierter Infusion $\mathrm{zu}$ einer Akkumulation von Triosephosphaten und zu einer Depletion energiereicher Adeninnukleotide. Die Aufnahme von Zuckeraustauschstoffen in Muskel- und Fettzelle erfolgt insulinunabhängig und schnell, die Veränderungen der Blutzuckerspiegel sind deshalb unter Zufuhr dieser Substrate gering. Alle weiteren Schritte des Glukoseabbaus sind analog zum Glukosestoffwechsel insulinabhängig. Die Infusion von fruktose- und sorbithaltigen Lösungen ist bei Säuglingen und Kleinkindern zu unterlassen und bei Erwachsenen nur nach anamnestischem Ausschluss einer Fruktoseintoleranz (=Mangel an Fruktose-1-PhosphatAldolase) oder deren Symptomen (Übelkeit, Erbrechen und Hypoglykämie nach Obstverzehr, schwerste klinische Symptomatik wie Azidose, Hypophosphatämie, Hypoglykämie, Hypotonie, Apnoe aufgrund der intrazellularen Akkumulation von Fruktose-1-Phosphat, der ATP-Depletion und der Hemmung von Glykogenabbau und der Glukoneogenese, nach parenteraler Fruktosezufuhr) erlaubt. Bei bewusstlosen Patienten dürfen Fruktose- und Sorbitlösungen nicht gegeben werden, ohne dass vorher ein Fruktosetoleranztest durchgeführt worden ist. In klinisch instabilen Situationen muss eine strenge metabolische Kontrolle insbesondere des Blutzuckers erfolgen. 


\section{Fette}

Lipidemulsionen haben eine hohe Energiedichte. Sie dienen der Energiezufuhr sowie der Substitution essentieller Fettsäuren. Die infundierten Lipide bestimmen die Phospholipidzusammensetzung von Zellmembranen und damit deren Fluidität, die essentiell für die Funktionen Transport und Rezeptorbildung ist. Lipide wirken darüber hinaus immunstimulatorisch, immunneutral und auch immunsuppressiv. Lipidemulsionen werden aus Sojabohnen-, Distel- oder Olivenöl hergestellt und in Konzentrationen von 10, 20 und 30\% angeboten (s. - Tab. 2.72). Eine Emulsion enthält eine physikalische Mischung von LCT- und MCT-Fetten (50\% LCT und 50\% MCT). Die Fettpartikel, auch Lipomikronen genannt, sind 1-2 $\mu$ groß und entsprechen damit den endogen gebildeten Chylomikronen. Unter Infusion von Lipidemulsionen kommt es nur zu geringen Anstiegen der Plasmacholesterinspiegel, gleichzeitig fällt die HDL-Konzentration ab und erscheint Lipoprotein X als pathologisches Lipoprotein. Da der Eilecithingehalt bei 10\%-Emulsionen hoch ist, werden heute nur noch 20\%- Emulsionen empfohlen. Die von der Industrie angebotenen Lipidemulsionen enthalten unterschiedliche Mengen einfach und mehrfach ungesättigter Fettsäuren (Linolsäure, a-Linolensäure). MCT-haltige Emulsionen enthalten Fettsäuren mit Kettenlängen C8 (73\%) bis C10 (25\%). Sie werden aus Palmkern- und Kokosnussöl hergestellt. Der Energiegehalt beträgt $9 \mathrm{kcal} / \mathrm{g}$ bei LCT- oder $8,3 \mathrm{kcal} / \mathrm{g}$ bei MCT-haltigen Emulsionen. Unter Berücksichtigung des in der Emulsion enthaltenden Glyzerinanteils entspricht dies bei einer 10\%-Emulsion $1,1 \mathrm{kcal} / \mathrm{ml}$ und bei einer $20 \%$-Emulsion $2,0 \mathrm{kcal} / \mathrm{ml}$. Die Fettsäurezusammensetzung der in den Emulsionen enthaltenden Triglyzeride hat einen sehr hohen Anteil an essentiellen Fettsäuren (>50\% Linolsäure). Lipidemulsionen sind isoton. Lipidemulsionen enthalten Vitamin $\mathrm{K}$ und Vitamin E. Der Mittlere Gehalt liegt bei einer Fettemulsion auf Sojaölbasis zwischen 25 und $65 \mu \mathrm{g} / \mathrm{mol}$
Vitamin $\mathrm{K}$ und $10 \mathrm{mg} / 100 \mathrm{ml}$ Vitamin-E-Äquivalenten. Diese Mengen sind geeignet den physiologischen $\mathrm{Vi}$ taminbedarf zu decken. Zur Deckung des essentiellen Fettsäurebedarfes müssen 5-10\% der Gesamtkalorien in Form von Fettemulsionen gegeben werden.

In praxi bedeutet dies, dass z. B. 3-mal wöchentlich $250 \mathrm{ml}$ einer 20\%-Emulsion infundiert werden. Ernährungsphysiologisch sinnvoller ist es, 30-35\% der täglichen Gesamtenergien als Fett zu geben. Dieser Anteil kann bei speziellen Indikationen (z. B. bei Patienten mit einer Leberzirrhose) bis auf 50\% gesteigert werden. Die empfohlenen Lipidinfusionsraten variieren zwischen 0,03 und $0,15 \mathrm{~g} / \mathrm{kgKG} / \mathrm{h}$. Lipidemulsionen werden entweder zyklisch (d.h. nur über einen Zeitraum von $8 \mathrm{~h}$ ) oder kontinuierlich (über $24 \mathrm{~h}$ ) in einer Mischinfusion (»all in one«) infundiert. Bei kontinuierlicher Gabe werden eher niedrige Infusionsraten empfohlen. Die maximale tägliche Zufuhrrate beträgt für Erwachsene bei kontinuierlicher Infusion über $24 \mathrm{~h} \mathrm{1,5} \mathrm{g} / \mathrm{kgKG} /$ Tag. Etwa $1 / 3$ der Lipide wird oxidiert, $2 / 3$ werden im Fettgewebe gespeichert. Eine uneingeschränkte Klärung (d. h. Verschwinden der Triglyzeride aus dem Blut) vorausgesetzt nimmt der Anteil der Fettspeicherung bei höheren Infusionsraten disproportional zu. Bei gleichzeitiger Infusion anderer energiereicher Substrate (i.e. Glukose) werden die Fette aufgrund der durch die Glukose verursachten Hyperinsulinämie bevorzugt gespeichert und die Glukose präferentiell verbrannt. Der direkte Energiegewinn aus Lipiden ist also im Rahmen einer vollständigen parenteralen Ernährung begrenzt. Mittelkettige Fettsäuren werden bevorzugt in der Leber verstoffwechselt und dort entweder oxidiert oder zu Ketonkörpern umgewandelt. Die Hydrolyse von MCT ist gegenüber LCT erhöht; sie können im Gegensatz zu den LCT die Blut-Hirn-Schranke passieren. MCT-Fette werden deshalb nur als LCT/MCT-Gemische mit einem MCT-Anteil von maximal 50\% empfohlen. Werden diese Gemische im Rahmen einer vollständigen parenteralen

\begin{tabular}{|c|c|c|c|c|c|c|c|c|c|c|c|c|}
\hline & \multicolumn{2}{|c|}{ Intralipid } & \multicolumn{2}{|c|}{ Lipovenös } & \multicolumn{2}{|c|}{ Lipofundin } & \multicolumn{2}{|c|}{ Abbolipid } & \multicolumn{2}{|c|}{ Salvilipid } & \multicolumn{2}{|c|}{$\begin{array}{l}\text { Lipofundin } \\
\text { MCT }\end{array}$} \\
\hline & $10 \%$ & $20 \%$ & $10 \%$ & $20 \%$ & $10 \%$ & $20 \%$ & $10 \%$ & $20 \%$ & $10 \%$ & $20 \%$ & $10 \%$ & $20 \%$ \\
\hline \multirow{2}{*}{$\begin{array}{l}\text { Neutralfette } \\
(\mathrm{g} / \mathrm{l})\end{array}$} & 100 & 200 & 100 & 200 & 100 & 200 & 100 & 200 & 100 & 200 & 100 & 200 \\
\hline & \multicolumn{2}{|c|}{ Sojabohnenöl } & \multicolumn{2}{|c|}{ Sojabohnenöl } & \multicolumn{2}{|c|}{ Sojabohnenöl } & \multicolumn{2}{|c|}{$\begin{array}{l}\text { 50\% Sojabohnenöl } \\
50 \% \text { Diestelöl }\end{array}$} & \multicolumn{2}{|c|}{ Sojabohnenöl } & \multicolumn{2}{|c|}{$\begin{array}{l}\text { Sojabohnenöl } \\
50 \% \text { LCT } \\
50 \% \text { MCT }\end{array}$} \\
\hline $\begin{array}{l}\text { Phospholipide } \\
\text { (g/l) }\end{array}$ & 6 & 6 & 6 & 12 & 7,5 & 15 & 7,4 & 12 & 12 & 12 & 12 & 12 \\
\hline Glyzerin (g/l) & 22 & 22 & 25 & 25 & 25 & 25 & 25 & 25 & 25 & 25 & 25 & 25 \\
\hline Kcal & 1100 & 2000 & 1080 & 2000 & 1068 & 1035 & 1100 & 2000 & 1100 & 2000 & 1058 & 1908 \\
\hline
\end{tabular}


Ernährung infundiert, sind die möglichen metabolischen Nebenwirkungen gering.

Bei Neugeborenen dürfen maximal $0,15 \mathrm{~g} / \mathrm{kgKG} / \mathrm{h}$ infundiert werden. Der Wert und die Sicherheit MCThaltiger Lipidemulsionen ist für Neugeborene und Kinder nicht belegt. MCT-Emulsionen werden bei Akutkranken nicht besser als LCT-Emulsionen verstoffwechselt. Demgegenüber kann die Hydrolyse der MCT- gegenüber den LCT-Fetten bei chronisch Kranken gesteigert sein.

Sowohl die Infusion von LCT- als auch die von LCT/ MCT-Emulsionen führen zu einer Hemmung der Glukoseoxidation. LCT/MCT-Emulsionen haben möglicherweise einen geringeren proteinsparenden Effekt als LCT-Fette. Anstelle der LCT/MCT-Gemische sind »strukturierte Lipide « Lipidemulsionen mit verschiedenen Triglyzeriden mittel- und/oder langkettiger Fettsäuren. Der »klinische Wert « von "strukturierten Lipide" mit einem Gehalt von über 4\% Omega-3-Fettsäuren ist bisher nicht sicher belegt. Ein Verhältnis der Zufuhr von Omega-6 zu Omega-3 Fettsäuren von etwa 3:1 gilt als »immunneutral«.

Die Lipidemulsionen sind in der Regel in einem Mischbeutel über etwa $24 \mathrm{~h}$ stabil. Durch ihren milchigen Aspekt kaschieren Lipide das mögliche Ausfällen anderer Komponenten in der Mischlösung. Die Stabilität einer Infusionsmischlösung ist deshalb von einem Apotheker zu überprüfen. Werden Mischlösungen z. B. im Rahmen einer heimparenteralen Ernährung über längere Zeit (z. B. über 1 Woche) gelagert, kommt es häufiger zum Ausfällen von Präzipitaten, welche Ausgangspunkt unerwünschter Komplikationen (Katheterverschluss, Embolien) sind. Bei einer längeren Lagerung ist deshalb von der Verwendung lipidhaltiger Mischlösungen abzuraten und die Lipidemulsion getrennt zu infundieren.

Kontraindikationen gegen die Gabe von Lipidemulsionen sind schockbedingte Mikrozirkulationsstörung und Verbrauchskoagulopathie, das "respiratory distress syndrome " (mögliche Verstärkung der Sauerstoffdiffusionstörung) sowie schwereTriglyzeridverwertungsstörungen (Nüchterntriglyzeridspiegel $>5 \mathrm{mmol} / \mathrm{l}$, bzw. unter Infusion Anstieg gegenüber dem Ausgangswert um mehr als das 3-fache). Lipide haben keinen stimulierenden Effekt auf das exokrine Pankreas und sind deshalb auch nicht grundsätzlich bei akuter Pankreatitis kontraindiziert (Ausnahme: Hypertriglyzeridämie bei akuter Pankreatitis). Unverträglichkeiten sind selten und werden nur bei hochdosierter Zufuhr beobachtet. Bei Neugeborenen können sowohl die Klärrate als auch die Oxidation von Fetten eingeschränkt sein.

\section{(? Cave}

\section{Verzicht bei Carnitinmangel.}

Probleme bestehen besonders bei Frühgeborenen. Probleme sind auch bei ikterischen Neugeborenen zu erwarten. Bei ihnen konkurrieren die freien Fettsäuren und das Bilirubin um die Bindungsstellen des Albumins. Das molare
Verhältnis von freien Fettsäuren und Albumin sollte 6:1 nicht überschreiten. Vorsicht ist auch bei gleichzeitiger Gabe von Medikamenten geboten, welche an Albumin gebunden transportiert werden. Kontraindikationen gegen die Gabe von Lipidemulsionen bestehen auch bei Säuglingen mit pulmonaler Hypertension und bronchopulmonaler Hypertension. Aufgrund der genannten Probleme muss die Indikation zur Infusion von Lipidemulsionen sehr sorgfältig und bei Neugeborenen unter Berücksichtigung des Reifungs- und Ernährungszustands (Energiebedarf?, Versorgung mit essentiellen Fettsäuren?), der zugrunde liegenden Erkrankung, der voraussichtlichen Dauer der künstlichen Ernährung und den Plasmaspiegeln der freien Fettsäuren $(0,2 \mathrm{mmol} / \mathrm{l})$ und der Ketonkörper gestellt werden.

Bei eingeschränkter Verstoffwechslung werden Infusionsraten von $<0,5 \mathrm{~g} / \mathrm{kgKG} / \mathrm{Tag}$ und eine Infusion über $24 \mathrm{~h}$ empfohlen. Im Rahmen einer vollständigen parenteralen Ernährung von Erwachsenen mit normalem Fettstoffwechsel wird ein Anteil von 30-35\% an der Gesamtenergiezufuhr eingeplant. Die maximale Infusionsmenge beträgt $1 \mathrm{~g} \mathrm{Fett} / \mathrm{kg} / \mathrm{Tag}$. Die Plasmatriglyzeridspiegel sollten vor Infusion $<5 \mathrm{mmol} / \mathrm{l}$ betragen und unter Infusion das 3-fache des Ausgangswerts, die Spiegel der freien Fettsäuren $1,5 \mathrm{mmol} / \mathrm{l}$ sowie die Ketonkörperkonzentrationen im Plasma 1,0 mmol/l nicht übersteigen.Bei parenteraler Ernährung kommt es innerhalb von 2 Wochen zu einem Abfall der Serumcarnitinspiegel. Bei parenteral ernährten Neugeborenen wird dieser Befund bereits früher beobachtet. Die Veränderungen der Serumcartininspiegel werden mit einer verschlechterten Lipidutilisation und dem Ansteigen der Plasmakonzentrationen der freien Fettsäuren in Beziehung gebracht. Eine niegrig-dosierte Carnitingabe von $10-20 \mathrm{mg} / \mathrm{kgKG} /$ Tag führt zu einer Senkung der Fettsäurespiegel. Ein ernährungsbedingter Carnitinmangel ist am ehesten bei carnitinfrei (z. B. parenteral oder auch mit Sojamilch) ernährten Frühgeborenen zu erwarten. Der Carnitingehalt der Muttermilch beträgt 50-100 $\mu \mathrm{mol} / \mathrm{l}$. Bei angeborenen Carnitinsynthesestörungen sind die Serumfettsäurespiegel hoch, die Bildung von Ketonkörpern (z. B. beim Fasten oder unter Infusionen mit Lipidemulsionen) ist niedrig. Sehr selten werden während der Infusion einer Lipidemulsion eine Urtikaria, makulöse Hautrötungen und Idiosynkrasien mit Tachykardien, Dyspnoe, Übelkeit, Erbrechen, Schwitzen, Erschöpfung und Kopfschmerzen beobachtet. Die Symptome bleiben meist unklar. Als Ursachen werden geringe Proteinverunreinigungen im Rahmen des Herstellungsprozesses angenommen. In frühen Arbeiten wurde auf die möglichen Wirkungen von Lipidemulsionen auf das Immun- und Gerinnungssystem sowie die Leberfunktion hingewiesen. Diese Komplikationen sind bei hohen Lipidinfusionsraten $(>0,1 \mathrm{~g} / \mathrm{kgKG} / \mathrm{h})$ beobachtet worden.

Lipidemulsionen sind ein guter Nährboden für Mikroorganismen und können bei unsachgemäßer Zubereitung 
von Mischlösungen Ursache von systemischen Infektionen sein. Die Häufigkeit von infusionslösungsabhängigen Infektionen an allen unter parenteraler Ernährung beobachteten Infektionen beträgt aber nur $2-4 \%$.

\section{Aminosäuren}

Aminosäurelösungen sind grundlegender Bestandteil einer vollständigen parenteralen Ernährung. Zur Deckung des Stickstoffbedarfs und zur Kompensation von Stickstoffverlusten werden in der parenteralen Ernährung kristalline Aminosäurelösungen verwendet. Die Stickstoffverluste Schwerkranker können bis zu $35 \mathrm{~g} / \mathrm{Tag}$ betragen. Für die parenterale Ernährung werden verschiedene Aminosäurelösungen in Konzentrationen von 3,5-15\% angeboten. Die Osmolarität der Lösungen variiert zwischen 450 und 1450 mosmol/l. Die Aminosäurelösungen enthalten essentielle und nicht essentielle Aminosäuren in unterschiedlichen Absolut- und Relativmengen. Keine der kommerziell erhältlichen Lösungen hat ein vollständiges Aminosäuremuster. Das Fehlen einzelner Aminosäuren wird mit deren schlechter Löslichkeit (z. B. Tyrosin, Cystein) oder ihrer Instabilität in wäßriger Lösung (Glutamin) erklärt. Aus Glutamin entstehen Pyroglutamat und Ammoniak. Geeignete Aminosäurelösungen sollten zumindest alle essentiellen und nicht-essentiellen Aminosäuren enthalten. Dieses kann heute durch Kombination von konventionellen Aminosäurelösungen mit Dipeptidlösungen annähernd erreicht werden.

Den Aminosäurelösungen liegen verschiedene Konzepte zugrunde. Sie orientieren sich entweder an dem Bedarf der einzelnen essentiellen Aminosäuren (»bedarfsadaptiertes « Aminosäuremuster) oder am Stoffwechsel der essentiellen und nicht-essentiellen Aminosäuren (»utilisationsadaptiertes« oder »transferadaptiertes« Muster). Bei einem »bedarfsadaptiertem « Aminosäuremuster werden der Bedarf an essentiellen Aminosäuren gedeckt und zusätzlich Arginin, Histidin und Glycin als Stickstoffquelle supplementiert. Eine spätere Optimierung der Aminosäurelösungen wurde durch eine Adaptation des Aminosäuremusters an die Zusammensetzung biologisch hochwertiger Eiweiße versucht. Neuere Konzepte berücksichtigen die Wechselwirkungen einzelner Aminosäuren, die Veränderungen im Plasmaaminogramm bei verschiedenen Erkrankungen und unter Aminosäureinfusion sowie die Kinetik einzelner Aminosäuren. Grundlage sind Kenntnisse des »Plasmatransfers" und der Verbrauchsraten der Aminosäuren. Die Zusammensetzung der Aminosäurelösungen ist im Hinblick auf den Erhalt des "steady states" (d. h. Ziel sind konstante Plasmaaminosäurespiegel) konzipiert. Die Grenzen dieser Konzepte ergeben sich aus den Unterschieden der freien Aminosäurekonzentrationen im Plasma und im Gewebe (30\% der Plasmaaminosäuren, aber nur 7\% der Muskelaminosäuren sind essentielle Aminosäuren) sowie den Unterschieden zwischen den Konzentrationen der freien Aminosäuren in den einzel- nen Geweben und deren Anteilen in der Zusammensetzung einzelner Proteine.

Eine im Vergleich zu den anderen Makronährstoffen disproportionale Erhöhung der Aminosäurezufuhr führt zu einer Steigerung der endogenen Aminosäureoxidation, der Harnstoffproduktion, der Thermogenese und der Körpertemperatur. Für eine optimale Verstoffwechslung der Aminosäuren ist das Verhältnis zwischen Energieund Stickstoffzufuhr zu beachten. Es liegt bei einer "gesunden" Ernährung zwischen 250 und $300 \mathrm{kcal} / \mathrm{gN}$. Dieses Verhältnis sollte bei parenteraler Ernährung zwischen 100 und $200 \mathrm{kcal} / \mathrm{gN}$ liegen. Zu den Zusammenhängen zwischen Energie- und Aminosäurezufuhr einerseits und der Stickstoffbilanz bei Schwerkranken andererseits (s. - Abb. 2.33). Einzelne Aminosäuren Histidin und Taurin sind bei parenteraler Ernährung essentielle Aminosäuren (z. B. auch bei Kindern und bei Urämie). Cystein kann bei Leberzirrhose nicht ausreichend aus Methionin gebildet und muss deshalb supplementiert werden. Die endogene Bildung von Tyrosin aus Phenylalanin ist auch bei Leberzirrhose und Niereninsuffizienz vermindert. Glutamin ist die im Vergleich zu anderen Aminosäuren höchstkonzentrierte freie Aminosäure im Muskel. Die Gewebekonzentrationen von Glutamin sind bei Schwerkranken regelhaft

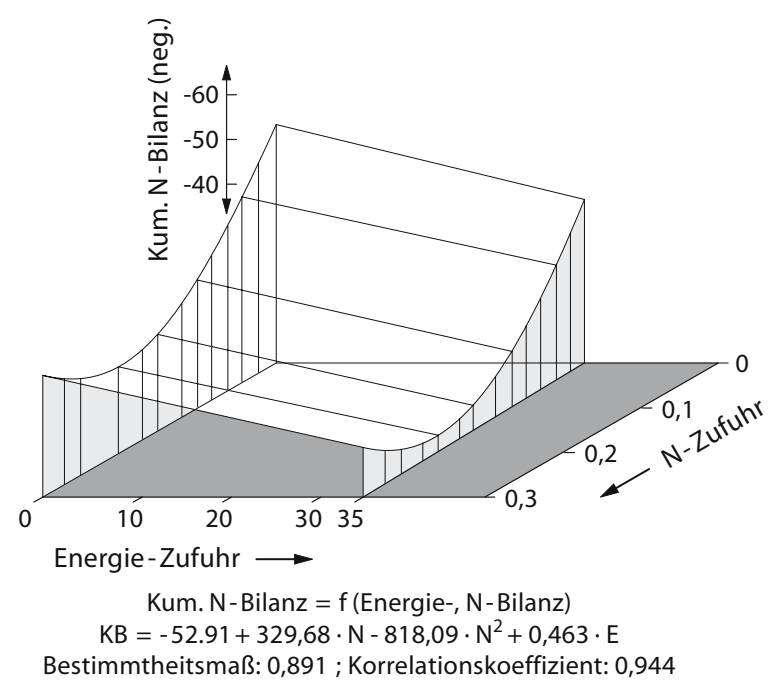

- Abb. 2.33. Stickstoff- und Energiebilanz bei steigenden Aminosäure- und Energiezufuhrraten.

Die Daten zeigen den mathematisch ermittelten Einfluss von $\mathrm{N}$ - und Energiezufuhr auf die kumulative N-Bilanz bei intensivmedizinisch stationierten Patienten während der ersten postoperativen Tage. Der dominierende Einfluss der N-Zufuhr auf die N-Bilanz ist an der starken Neigung der Kurve über der y-Achse erkennbar. Das Optimum der postoperativen $\mathrm{N}$-Zufuhr liegt bei ca $0,2 \mathrm{~g} / \mathrm{kg} \mathrm{KG/Tag}$. Demgegenüber ist der Einfluss der Energiezufuhr auf die N-Bilanz zu schwach. (Reproduktion der Daten von Prof. Dr. Berendt, Klinik für Anästhesiologie der Medizinischen Fakultät der RWTH Aachen, mit freundlicher Genehmigung des Autors) 
um bis zu 50\% erniedrigt. Glutamin gilt deshalb Schwerkranken als »bedingt essentiell«. Glutamin ist nicht hitzestabil und besitzt bei Raumtemperatur nur eine geringe Löslichkeit (etwa $3 \mathrm{~g} / 100 \mathrm{ml}$ ). Das Fehlen von Glutamin in L-Aminosäureinfusionslösungen ist kritisch, da Glutamin das entscheidende Energiesubstrat für den Darm und die immunkompetenten Zellen ist. Glutamin wird endogen durch den Abbau von Muskeleiweiß und von der Lunge bereitgestellt. Bei Kranken beträgt die Glutaminfreisetzung aus dem Muskel zwischen 9 und $13 \mathrm{~g} / \mathrm{Tag}$. Der Verlust an Glutamin geht im Muskel mit einer Drosselung der Proteinsynthese einher. Es liegt daher nahe, Glutamin zumindest bei Schwerkranken in geeigneter Form zu substituieren, um die Glutaminhomöostase des Körpers zu erhalten. $\mathrm{Zu}$ den Standardaminosäurelösungen kann bei Trauma und Sepsis z. B. Glutamin (5-10 g/100 g Aminosäuren) in Form von Dipeptidlösung (z. B. als Alanylglutamin oder Glycylglutamin) supplementiert werden, um den Abbau von Muskelproteinen und die Proteinsynthese des Intestinums, immunkompetenter Zellen und auch des Muskels gezielt zu beeinflussen. Dipeptide mit C-teminalen Glutaminresten haben eine hohe Wasserlöslichkeit. Darüber hinaus sind sie hitzestabil und können auch über längere Zeit gelagert werden. Alanylglutamin wird z.Z. als $20 \%$-Konzentrat angeboten. Die Dosierung beträgt $1,5-$ $2,0 \mathrm{ml} / \mathrm{kgKG} /$ Tag. Maximal können $20 \%$ der insgesamt zugeführten Aminosäuren als Dipeptid gegeben werden (z. B. 1,2 g Aminosäuren+0,3 g Dipeptid/kgKG/Tag). Die Infusion einer mit Alanylglutamin (20 g entsprechen $13 \mathrm{~g}$ Glutamin) supplementierten Aminosäurelösung hat bei Schwerkranken einen im Vergleich zu einer isonitrogenen, konventionellen Aminosäurezufuhr deutlich ausgeprägteren proteinsparenden Effekt. Über die Nahrung werden 3-5 g Glutamin pro Tag aufgenommen. In klinischen Studien sind z. T. weitaus höhere Dosierung (bis zu $40 \mathrm{~g} / \mathrm{Tag}$ ) infundiert worden. Dipeptidlösungen werden rasch verstoffwechselt. Sie werden durch die Niere, aber auch durch endothelassozierte Dipeptidasen gespalten. Das Glutamin steht somit schnell dem Energie- und Proteinstoffwechsel zur Verfügung. Indikationen für die Supplementierung mit Dipeptiden bestehen bei intestinalen Erkrankungen (z. B. bei einer Strahlenenteritis), Immunsuppression (z. B. nach Knochenmarkstransplantation) oder auch schwer katabolen Zuständen (z. B. bei ausgedehnten Verbrennungen).

Bei Neu- und Frühgeborenen ist der Eiweiß- bzw. Aminosäurebedarf erhöht und kann bis zu $4 \mathrm{~g} / \mathrm{kgKG} /$ Tag betragen. Bei Kindern im ersten Lebensjahr beträgt er 1,2-2,5, bis zum 5. Lebensjahr 1,5 und später $1,0 \mathrm{~g} /$ kgKG/Tag. Da der Aminosäurebedarf bei Kindern von dem Erwachsener abweicht (z. B. ist Taurin hier essentiell) und darüber hinaus zahlreiche Erkrankungen mit Störungen des Aminosäurestoffwechsels einhergehen, werden für Kinder und auch für Schwerkranke, Niereninsuffizienz und bei Sepsis und Trauma spezielle Ami- nosäurelösungen angeboten. Diese unterscheiden sich in der Relation von essentiellen und nicht-essentiellen Aminosäuren und auch im Gehalt an verzweigtkettigen Aminosäuren, Phenylalanin, Methionin und Arginin. Infusionslösungen für Neugeborene enthalten insbesondere Cystein und Taurin. Bei Neugeborenen und insbesondere unreifen Säuglingen sind auch die Aminosäuren Taurin, Histidin, Cystein, Tyrosin, Prolin und Arginin essentiell oder semiessentiell. Darüber hinaus ist der Bedarf an essentiellen Aminosäuren bei Neugeborenen höher als bei Erwachsenen. Da die Gewichtsproportionen von Leber, Niere, Gehirn und Muskulatur bei Säuglingen und Erwachsenen unterschiedlich sind, weichen auch der Aminosäurestoffwechsel und -bedarf voneinander ab. Im 1. Lebensjahr werden Zufuhrraten zwischen 1,5-2,5 g/kgKG/Tag, vom 2.-5. Lebensjahr $1,5 \mathrm{~g} / \mathrm{kgKG} / \mathrm{Tag}$ und später $1 \mathrm{~g} / \mathrm{kgKG} / \mathrm{Tag}$ empfohlen. Aminosäuren dürfen maximal bis zu einer Menge von $1,5-(2,0) \mathrm{g} / \mathrm{kgKG} /$ Tag zugeführt werden.

Kontraindikationen gegen die Infusion von Aminosäuren sind angeborene Störungen des Aminosäurestoffwechsels sowie Aminosäureverwertungsstörungen (wie z. B. bei Leber- oder akutem Nierenversagen).

\section{Elektrolyte}

Elektrolyte und Mineralien werden als Konzentrate oder als Teil- (=Halb-) oder Vollelektrolytlösungen substituiert. Halbelektrolytlösungen enthalten Elektrolyte in der Hälfte der Plasmakonzentration, sie sind hypoton. Vollelektrolytlösungen haben eine dem Plasma gleiche Zusammensetzung und dienen auch dem Ersatz von Extrazellulärflüssigkeit. Substratlösungen sind normalerweise elektrolytfrei, es gibt aber auch "teilsupplementierte « Kohlenhydrat- und Aminosäurelösungen. Voll- und Halbelektrolytlösungen enthalten kein Phosphat. Elektrolytkonzentrate sind $\mathrm{Na}-$ triumchlorid, Kaliumchlorid, Kaliumhydrogenphosphat, Glyzerophosphat, Natriumbikarbonat, Kaliumbikarbonat, Natriumlaktat, Kaliumlaktat, Kalziumchlorid, Kalziumglukonat, Magnesiumsulfat, Magnesiumchlorid, Lysinund Argininhydrochlorid. Sie werden in verschiedenen Konzentrationen angeboten. Die Elektrolytkonzentrate erlauben eine individuelle Dosierung und Korrektur bei möglichen Entgleisungen. Zusätzlich gibt es sog. »Korrekturlösungen« wie Natriumbikarbonat, Trispuffer, Lysin- und Argininhydrochlorid, welche bei Störungen des Säure-Basenhaushalts eingesetzt werden. Die Zufuhr der Elektrolyte und Mineralien folgen den Empfehlungen der Fachgesellschaften (s. - Tab. 2.73).

Der Kalium- und auch der Phosphatbedarf sind bei hoher Glukosezufuhrrate erhöht. Verluste durch Sekrete und Ausscheidungen können einen Verlust an Natrium von 60-100 mmol/l, an Kalium von 5-20 mmol/l und Chlorid von $45-100 \mathrm{mmol} / \mathrm{l}$ Sekret betragen. Bei Mischlösungen sind mögliche Inkompatibilitäten zwischen einzelnen Lösungsbestandteilen auszuschließen. Erfahrungsgemäß 
- Tab. 2.73. Empfehlungen für die tägliche parenterale Elektrolytzufuhr

\begin{tabular}{|l|l|}
\hline & $\mathbf{m m o l} / \mathbf{k g ~ K G}$ \\
\hline Natrium & $1,0-1,5$ \\
\hline Kalium & $0,5-1,0$ \\
\hline Phosphat & $0,2-0,3$ \\
\hline Kalzium & $0,1-0,3$ \\
\hline Magnesium & $0,1-0,2$ \\
\hline Chlorid & $1,0-2,0$ \\
\hline
\end{tabular}

sind im Beutel Kalziumkonzentrationen bis $4 \mathrm{mmol} / \mathrm{l}$, Magnesiumkonzentrationen bis $5 \mathrm{mmol} / \mathrm{l}$ und Phosphatspiegel bis $20 \mathrm{mmol} / \mathrm{l}$ ohne Bildung von Präzipitäten möglich. Häufiges Problem von parenteralen Mischlösungen ist das Ausfällen von Kalzium und Phosphat. Dieses wird durch hohe Konzentrationen der beiden Mineralstoffe, einen hohen $\mathrm{pH}$, eine niedrige Aminosäurekonzentration, hohe Umgebungstemperaturen und die Dauer der Infusionsperiode bestimmt. Phosphat sollte in Form organischer Verbindungen (z. B. als Glyzerophosphat, Glukosephosphat) supplementiert werden. Diese Verbindungen werden intrazellulär hydrolysiert, freies Phosphat steht somit direkt dem Energiestoffwechsel zur Verfügung. Bei Bildung von Kalzium- bzw. Magnesiumphosphaten kann es durch die Veränderungen der Ladungen zu Instabilitäten der Fetttröpfchenoberfläche und damit der Lipidemulsion kommen. Ist eine hohe Zufuhrrate erwünscht, müssen Elektrolytkonzentrate langsam und separat von der parenteralen Ernährung infundiert werden. In der klinische Erfahrung bestehen die häufigsten Probleme bei Kindern mit einem hohem Kalzium- und Phosphatbedarf und Patienten mit Flüssigkeitsbeschränkungen (z. B. wegen Herz- oder Niereninsuffizienz).

\section{Vitamine und Spurenelemente}

Vitamine und Spurenelemente sind Teil einer vollständigen parenteralen Ernährung. Der Bedarf an Vitaminen und Spurenelementen weicht bei parenteraler von den für die enterale Ernährung geltenden Empfehlungen ab (s. - Tab. 2.74-2.77). Die Mikronährstoffe werden in Form von Multivitamin- oder Spurenelementpräparaten substituiert. Neben den Kombinationspräparaten werden auch Einzelkomponenten angeboten (s. - Tab. 2.75-2.78). - Tab. 2.76 zeigt die klinischen Symptome eines Vitaminmangels, deren mögliche Toxizität und die Stabilität von Vitaminen in parenteralen Mischlösungen.

Probleme der Mikronährstoffversorgung können z. B. bei Leber- und Niereninsuffizienz durch die Akkumulation einzelner Vitamine und Spurenelemente entstehen.
In diesen Fällen sind spezielle Empfehlungen zu beachten und ist die Substitution vorsichtig unter Kontrolle der Plasmaspiegel durchzuführen. Umgekehrt kann während schwerer kataboler Stoffwechselsituationen (wie z. B. bei Sepsis und Verbrennungen) oder auch Sekretverlusten und schweren Diarrhoen ein erhöhter Bedarf (z. B. an Zink) vorliegen, der eine Dosiserhöhung notwendig macht. Die großen körpereigenen Speicher einzelner Mikronährstoffe stellen die Sinnhaftigkeit einer vollständigen Substitution in Frage. Umgekehrt ist bei Schwerkranken der körpereigene Bestand zu Beginn der künstlichen Ernährung nicht einschätzbar und die Mobilisierung von Vitaminen aus den endogenen Speichern unsicher. Klinische Zeichen eines Vitamin- oder Spurenelementmangels sind Ausdruck einer länger bestehenden Malnutrition. Da der Bedarf an Mikronährstoffen während einer parenteralen Ernährung unverändert oder sogar erhöht ist und die Mikronährstoffe als Koenzyme auch Voraussetzung für eine adäquate Verstoffwechslung der Makronährstoffe sind, erscheint eine zumindest bedarfsdeckende Gabe aus Sicht der Homoeostase sinnvoll.

Vitamine sind in einer parenteralen Mischlösung nur begrenzt stabil. Dieses betrifft insbesondere die fettlöslichen Vitamine, die Vitamine der B-Gruppe und Vitamin C. Die Stabilität von Vitaminen in Mischlösung ist von zahlreichen Faktoren wie pH, Temperatur, Licht, der gleichzeitigen Anwesenheit anderer Vitamine und Makronährstoffen sowie der Lagerungszeit abhängig. Der lichtabhängige Zerfall betrifft besonders Vitamin A und $B_{2}$. Durch Lichtschutz kann der Schaden möglicherweise begrenzt werden. Spurenelemente sind in den angesprochenen Dosierungen in Mischlösungen weitgehend stabil. Umgekehrt scheinen sie nicht die Stabilität der gesamten Mischlösung zu beeinflussen. Unter klinischen Bedingungen ist die Infusion von Vitaminen in parenteralen Mischlösungen in Mischbeuteln mit einem besonderem Lichtschutz über einen Zeitraum von maximal $24 \mathrm{~h}$ üblich. In begrenztem Umfang muss unter diesen Bedingungen mit einem Verlust an Vitaminen gerechnet werden. Alternative zu der Mischinfusion ist die separate Infusion von Vitaminen und einer geeigneten Trägerlösung (z. B. eine 5\%-Glukoselösung) innerhalb einer kürzeren Zeitperiode.

\section{Volumenersatztherapie}

Als Volumenersatz werden in der Intensivmedizin natürliche (Plasmapräparate, Humanalbumin) oder hypertone (Osmolarität $>1000$ mosmol/l) und hypertonhyperonkotische Lösungen verwendet (Osmolarität bis 2400 mosmol/l). Bei einer Hypovolämie dienen sie der Wiederherstellung und Aufrechterhaltung des intravasalen Volumens, der Optimierung der kapillären Perfusion bzw. Reperfusion, der Verbesserung der Sauerstofftransportkapazität, der Verminderung der Mediatoraktivierung, der Verbesserung der Flusseigenschaften sowie indirekt dem Ausgleich des Säure-Basenhaushalts. 
- Tab. 2.74. Empfehlungen verschiedener Fachgesellschaften für die parenterale Vitaminzufuhr

\begin{tabular}{|c|c|c|c|}
\hline & \multirow{2}{*}{$\begin{array}{l}\text { Nach DAKE } \\
\text { Für Erwachsene }\end{array}$} & \multicolumn{2}{|l|}{ Nach $A_{M A} A^{a}$ und $A_{S C N}{ }^{c}$} \\
\hline & & Für Kinder (<11 Jahre) & Für Erwachsene \\
\hline $\begin{array}{l}\text { Wasserlösliche Vitamine } \\
\text { Vitamin } B_{1}(\mathrm{mg}) \\
\text { Vitamin } B_{2}(\mathrm{mg}) \\
\text { Vitamin } B_{6}(\mathrm{mg}) \\
\text { Niacin }(\mathrm{mg}) \\
\text { Pantothensäure }(\mathrm{mg}) \\
\text { Biotin }(\mu \mathrm{g}) \\
\text { Folsäure }(\mu \mathrm{g}) \\
\text { Vitamin } C(\mathrm{mg}) \\
\text { Vitamin } B_{12}(\mu \mathrm{g})\end{array}$ & $\begin{array}{l}3-4 \\
3-5 \\
4-6 \\
40-50 \\
10-20 \\
60-120 \\
160-400 \\
100-300 \\
1000 / 3 \text { Monate }\end{array}$ & $\begin{array}{l}1,2 \\
1,4 \\
1,0 \\
17 \\
5,0 \\
20 \\
140 \\
80 \\
1,0\end{array}$ & $\begin{array}{l}3,0 \\
3.6 \\
4,0 \\
40 \\
15 \\
60 \\
400 \\
100 \\
5,0\end{array}$ \\
\hline $\begin{array}{l}\text { Fettlösliche Vitamine } \\
\text { Vitamin A (Retinolpalmitat9 }(\mu \mathrm{g}) \\
\text { Vitamin E (a-Tocopheroläquivalent) (mg) } \\
\text { Vitamin D }(\mu \mathrm{g}) \\
\text { Viatmin K }(\mu \mathrm{g})\end{array}$ & $\begin{array}{l}1800 \\
20-40 \\
5 \\
100-150\end{array}$ & $\begin{array}{l}690 \\
4,7 \\
10 \\
200\end{array}$ & $\begin{array}{l}990 \\
6,7 \\
5 \\
-\end{array}$ \\
\hline
\end{tabular}

a Deutsche Arbeitsgemeinschaft für Klinische ernährung. Infusionstherapie 17:60-61, 1991

${ }^{\mathrm{b}}$ American Medical Association, Nutrition Advisory Group. J. Parenteral Enteral Nutrition 3:258-262,1979

c American Society for Clinical Nutrition, Subcommittee on Pediatric Parenteral Nutrient Requirements from the Committee of Clinical Practice Issues. Am. J. Clin. Nutr. 48:1324-1342, 1988

- Tab. 2.75. Ausgewählte Medikamente für die parenterale Vitaminzufuhr

\begin{tabular}{|c|c|c|c|c|c|}
\hline & $\begin{array}{l}\text { Multibionta } \mathrm{N} \\
10 \mathrm{ml}\end{array}$ & $\begin{array}{l}\text { Soluvit } \\
1 \text { Injektionsflasche } \\
\text { (163 mg Trocken- } \\
\text { substanz) }\end{array}$ & $\begin{array}{l}\text { Cernevit } \\
1 \text { Injektionsflasche } \\
\text { ( } 750 \mathrm{mg} \text { Trocken- } \\
\text { substanz) }\end{array}$ & $\begin{array}{l}\text { Vitalipid } \\
\text { Adult } \\
110 \mathrm{ml}\end{array}$ & $\begin{array}{l}\text { Vitalipid } \\
\text { Infant } \\
10 \mathrm{ml}\end{array}$ \\
\hline $\begin{array}{l}\text { Wasserlösliche Vitamine } \\
\text { Vitamin } B_{1}(\mathrm{mg}) \\
\text { Vitamin } B_{2} \\
\text { Vitamin } B_{6}(\mathrm{mg}) \\
\text { Niacin }(\mathrm{mg}) \\
\text { Pantothensäure }(\mathrm{mg}) \\
\text { Biotin }(\mu \mathrm{g}) \\
\text { Folsäure }(\mu \mathrm{g}) \\
\text { Vitamin } C(\mathrm{mg}) \\
\text { Vitamin } B_{12}\end{array}$ & $\begin{array}{l}10,0 \\
7,3 \\
15 \\
40 \\
25 \\
- \\
- \\
100 \\
-\end{array}$ & $\begin{array}{l}1,24 \\
1,8 \\
2,4 \\
10 \\
10 \\
300 \\
200 \\
30 \\
2\end{array}$ & $\begin{array}{l}- \\
5,7 \\
5,5 \\
46 \\
16,2 \\
69 \\
414 \\
125 \\
6\end{array}$ & $\begin{array}{l}- \\
- \\
- \\
- \\
- \\
- \\
- \\
- \\
-\end{array}$ & $\begin{array}{l}- \\
- \\
- \\
- \\
- \\
- \\
- \\
- \\
-\end{array}$ \\
\hline $\begin{array}{l}\text { Fettlösliche Vitamine } \\
\text { Vitamin A (Retinolpalmitat) (mg) } \\
\text { Vitamin } E^{1}(\mathrm{mg}) \\
\text { Vitamin } D(\mu \mathrm{g}) \\
\text { Vitamin } \mathrm{K}(\mu \mathrm{g})\end{array}$ & $\begin{array}{l}1,65 \\
5 \\
- \\
-\end{array}$ & $\begin{array}{l}- \\
- \\
- \\
-\end{array}$ & $\begin{array}{l}1,925 \\
10,2 \\
5,5 \\
-\end{array}$ & $\begin{array}{l}1,941 \\
9,1 \\
5 \\
150\end{array}$ & $\begin{array}{l}1,353 \\
6,4 \\
10 \\
200\end{array}$ \\
\hline Empfohlene Dosierung (pro Tag) & $10 \mathrm{ml}$ & 1 Injektionsflasche & $\begin{array}{l}1 \text { Injektionsflasche } \\
\text { Cave: Anwendung } \\
\text { nur bis zu } 7 \text { Tagen }\end{array}$ & $10 \mathrm{ml}$ & $1 \mathrm{ml} / \mathrm{kg}$ \\
\hline Zusätze & $\begin{array}{l}\text { Benzylalkohol } \\
\text { Glyzerin } \\
\text { Polysorbat } \\
\text { Tris(hydroxy- } \\
\text { methyl) } \\
\text {-amionomethan }\end{array}$ & $\begin{array}{l}\text { Glycin } \\
\text { Edetinsäure } \\
\text { Dinatriumsalz } \\
\text { Methyl-4-Hydoxy- } \\
\text { benzoat }\end{array}$ & $\begin{array}{l}\text { Glycin } \\
\text { Glycocholsäure } \\
\text { Phospholipide } \\
\text { Natriumhydroxid }\end{array}$ & \multicolumn{2}{|c|}{$\begin{array}{l}\text { Sojabohnenöl 1g/l g } \\
\text { Phosphatidylcholin } \\
\text { Glycerin } \\
\text { Natriumhydroxid }\end{array}$} \\
\hline
\end{tabular}

\footnotetext{
${ }^{1}$ (a-Tocopheroläquivalent)
} 
- Tab. 2.76. Klinische Manifestation eines Vitaminmangels, mögliche Zeichen der Toxizität und Stabilität von Vitaminen in einer Mischlösung

\begin{tabular}{|c|c|c|c|}
\hline & Klinische Manifestation eines Mangels & Zeichen der Toxizität & Stabilität in Mischlösung (Verluste in\%) \\
\hline Vitamin $B_{1}$ & $\begin{array}{l}\text { Beriberi, Ödeme, Ataxie, Kardiomegalie, } \\
\text { Neuritis }\end{array}$ & $\begin{array}{l}\text { Übelkeit, Anorexie, Lethargie, } \\
\text { Obstipation }\end{array}$ & $\begin{array}{l}6 \% \text { innerhalb } 24 \mathrm{~h} \text { bei Raumtemperatur } \\
\text { instabil mit Bisulfit, abhängig von Konzent- } \\
\text { ration, } \mathrm{pH} \text {, Temperatur }\end{array}$ \\
\hline Vitamin $B_{2}$ & $\begin{array}{l}\text { Konjunktivits, Glossitis, Dermatitis, Kera- } \\
\text { tosis, Cheilitis }\end{array}$ & Nicht bekannt & $\begin{array}{l}85 \% \text { innerhalb } 24 \mathrm{~h} \text { bei Raumtemperatur, } \\
\text { Lichtschutz erhöht die Stabilität }\end{array}$ \\
\hline Nikotinsäure & Pellagra, Dermatitis, Demenz, Durchfälle & $\begin{array}{l}\text { "flush«, Juckreiz, Ulkus?, } \\
\text { Harnsäure } \uparrow\end{array}$ & Stabil \\
\hline Vitamin $B_{6}$ & Anämie, Polyneuritis, Dermatitis & $\begin{array}{l}\text { Periphere Neuropathie, } \\
\text { Krämpfe, Abhängigkeit }\end{array}$ & Stabil bei Lichtschutz \\
\hline Vitamin C & $\begin{array}{l}\text { Skorbut, Zahnfleischbluten, Schwäche, } \\
\text { Petechien, Erregbarkeit }\end{array}$ & Kalziumoxalatsteine & $\begin{array}{l}91 \% \text { innerhalb } 24 \mathrm{~h} \text { bei Raumtemperatur, } \\
\text { Halbwertszeit } 1,1 \mathrm{~h} \text {, Verlust in Gegenwart } \\
\text { von Kupfer höher }\end{array}$ \\
\hline $\begin{array}{l}\text { Pantothen- } \\
\text { säure }\end{array}$ & $\begin{array}{l}\text { Tachykardie, Erregbarkeit, Hypotension, } \\
\text { »Burning feet syndrome» }\end{array}$ & Diarrhoe & Nicht bekannt \\
\hline Biotin & $\begin{array}{l}\text { Dermatitis, Neuritis, Alopezie, } \\
\text { Anorexie, Depression }\end{array}$ & Nicht bekannt & Nicht bekannt \\
\hline Folsäure & Makrozytäre Anämie & Konvulsionen & Stabil innerhalb $24 \mathrm{~h}$ bei Raumtemperatur \\
\hline Vitamin $B_{12}$ & $\begin{array}{l}\text { Perniziosa, Parästhesien, fenikuläre } \\
\text { Spinalerkrankungen, Encephalopathie, } \\
\text { Polyneuropathie }\end{array}$ & Nicht bekannt & Nicht bekannt \\
\hline Vitamin A & $\begin{array}{l}\text { Bitot-Flecken, Xerophthalmie, Nacht- } \\
\text { blindheit, Verhornung }\end{array}$ & $\begin{array}{l}\text { Teratogenese, Übelkeit, Erbre- } \\
\text { chen, Schwindel, Knochen- } \\
\text { schmerzen, Hirndruck } \uparrow, \text { Müdig- } \\
\text { keit, Fieber }\end{array}$ & $\begin{array}{l}75 \% \text { innerhalb von } 24 \mathrm{~h} \text { bei Raumtempera- } \\
\text { tur, adsorbiert an PVC }\end{array}$ \\
\hline Vitamin D & Osteomalazie, Tetanie & Hyperkalzämie & Nicht bekannt, adsorbiert an PVC \\
\hline Vitamin E & $\begin{array}{l}\text { Hämolytische Anämie, Degeneration } \\
\text { von Neuronen }\end{array}$ & Nicht bekannt & Nicht bekannt \\
\hline Vitamin $\mathrm{K}$ & Blutung & Nicht bekannt & Nicht bekannt \\
\hline
\end{tabular}

Tab. 2.77. Empfehlungen für die tägliche parenterale Zufuhr von Spurenelementen

\begin{tabular}{|c|c|c|c|c|c|}
\hline & \multirow{2}{*}{$\begin{array}{l}\text { Nach Bässlera } \\
\text { Für Erwachsene }\end{array}$} & \multicolumn{3}{|l|}{ Nach AMA ${ }^{b}$} & \multirow{2}{*}{$\begin{array}{l}\text { Nach Eliac und } \\
\text { Shenkin }^{d} \\
\text { Für Erwachsene }\end{array}$} \\
\hline & & Für Erwachsene & Für Kinder (pro kg) & Für Neugeborene (pro kg) & \\
\hline Eisen $(\mu \mathrm{mol})$ & $10-75$ & & & & $18-96$ \\
\hline Zink $(\mu \mathrm{mol})$ & $21-75$ & $38-60$ & 1,5 & 4,5 & $38-102$ \\
\hline Mangan ( $\mu \mathrm{mol})$ & $3-14$ & $3-14$ & $0,14-0,2$ & $0,04-0,2$ & $2,8-15$ \\
\hline Kupfer ( $\mu \mathrm{mol})$ & $7-23$ & $7-23$ & 0,28 & 0,28 & $8,4-23,8$ \\
\hline Molypdän ( $\mu \mathrm{mol})$ & 0,2 & & & & $0,17-0,31$ \\
\hline Chrom ( $\mu \mathrm{mol})$ & $0,2-0,2$ & $0,2-0,3$ & $0,003-0,004$ & $0,003-0,0040$ & $0,13-0,4$ \\
\hline Selen ( $\mu \mathrm{mol})$ & $0,25-0,8$ & $0,5-1,0$ & $0,025-0,028$ & $0,025-0,028$ & $0,38-0,75$ \\
\hline $\operatorname{Jod}(\mu \mathrm{mol})$ & $0,8-1,2$ & & & & $0,3-1,6$ \\
\hline Fluor ( $\mu \mathrm{mol})$ & 49 & & & & 51-162 \\
\hline
\end{tabular}

a Nach Bässler 1990, b Nach AMA 1979, c Nach Elia 1990, d Nach Shenkin 1986 
- Tab. 2.78. Ausgewählte Spurenelement- und Elektrolytkonzentrationen in der parenteralen Ernährung (Stand: Rote Liste 1997)

\begin{tabular}{|c|c|c|c|c|c|c|}
\hline & $\begin{array}{l}\text { Inzolen infantibus } \\
\text { (10 ml Ampulle) }\end{array}$ & $\begin{array}{l}\text { Inzolen infantibus } \\
\text { sine NaK } \\
\text { (10 ml Ampulle) }\end{array}$ & $\begin{array}{l}\text { Inzolen KT } \\
\text { infantibus } \\
\text { (10 ml Ampulle) }\end{array}$ & $\begin{array}{l}\text { Addel } \\
\text { (10 ml Ampulle) }\end{array}$ & $\begin{array}{l}\text { Tracitrans } \\
\text { (10 ml Ampulle) }\end{array}$ & $\begin{array}{l}\text { Elotrace } \\
(100 \mathrm{ml})\end{array}$ \\
\hline $\begin{array}{l}\text { Eisen } \\
\text { Zink } \\
\text { Mangan } \\
\text { Kupfer } \\
\text { Molybdän } \\
\text { Chrom } \\
\text { Selen } \\
\text { Kobalt } \\
\text { Fluorid } \\
\text { Jodid }\end{array}$ & $\begin{array}{l}(\mu \mathrm{mol}) \\
16 \\
15 \\
5 \\
5 \\
- \\
1,5 \\
- \\
- \\
- \\
-\end{array}$ & $\begin{array}{l}(\mu \mathrm{mol}) \\
16 \\
15 \\
5 \\
5 \\
- \\
2 \\
- \\
2 \\
- \\
-\end{array}$ & $\begin{array}{l}(\mu \mathrm{mol}) \\
10 \\
12 \\
2 \\
4 \\
- \\
2 \\
- \\
2 \\
- \\
-\end{array}$ & $\begin{array}{l}(\mu \mathrm{mol}) \\
50 \\
20 \\
40 \\
5 \\
- \\
- \\
- \\
- \\
50 \\
1\end{array}$ & $\begin{array}{l}(\mu \mathrm{mol}) \\
50 \\
20 \\
40 \\
5 \\
- \\
- \\
- \\
- \\
50 \\
1\end{array}$ & $\begin{array}{l}(\mu \mathrm{mol}) \\
20 \\
92 \\
5,5 \\
19 \\
0,2 \\
- \\
1,5 \\
- \\
50 \\
1,0\end{array}$ \\
\hline $\begin{array}{l}\text { Natrium } \\
\text { Kalium } \\
\text { Kalzium } \\
\text { Magnesium } \\
\text { Chlorid } \\
\text { Phosphat }\end{array}$ & $\begin{array}{l}(\mathrm{mmol}) \\
5 \\
5 \\
2,5 \\
2 \\
- \\
-\end{array}$ & $\begin{array}{l}(\mathrm{mmol}) \\
- \\
- \\
- \\
2,5 \\
- \\
-\end{array}$ & $\begin{array}{l}(\mathrm{mmol}) \\
0,5 \\
5 \\
- \\
2,5 \\
- \\
0,5\end{array}$ & $\begin{array}{l}\text { (mmol) } \\
- \\
- \\
5 \\
1,5 \\
13 \\
-\end{array}$ & $\begin{array}{l}(\mathrm{mmol}) \\
- \\
- \\
5 \\
1,5 \\
13,3 \\
-\end{array}$ & $\begin{array}{l}(\mathrm{mmol}) \\
70 \\
60 \\
4 \\
5 \\
18 \\
35\end{array}$ \\
\hline Zusätze & & & & Xylit (0,3 g) & Sorbit (3 g) & $\begin{array}{l}\text { Apfelsäure } \\
50 \mathrm{mmol}\end{array}$ \\
\hline
\end{tabular}

Hypertone Lösungen enthalten einen hohen Anteil von osmotisch aktiven Bestandteilen (Natriumchlorid, Glukose, Mannit, Sorbit, Harnstoff), welche zu einer zeitlich begrenzten Erhöhung der Plasmaosmolarität führen. Der durch den Anstieg der Plasmaosmolarität aufgebaute Konzentrationsgradient zwischen dem intravasalen und dem interstitiellen bzw. intrazellulärem Raum bewirkt eine Mobilisierung von Flüssigkeit. Hyperton-hyperonkotische Lösungen enthalten zusätzlich ein Kolloid, welches den Gradienten unterstützt.

Bei den Kolloiden handelt es sich um Gelatine, Dextran, Hydroxyethylstärke=HES oder vernetzte Polypeptide, welche als Infusionslösung in unterschiedlichen Konzentrationen (3-10\%) angeboten werden. Dextrane sind Polysaccharide, welche nach hydrolytischer Spaltung in Fraktionen mit einer mittleren Molekulargewicht von 40000-200000 Da vorliegen. Hinsichtlich der Volumenwirkung bindet $1 \mathrm{~g}$ Dextran ca. $25 \mathrm{ml}$ Wasser. Je nach Molekulargewicht dauert der Effekt des Dextrans 3-6 h an. Gelatine entsteht aus Kollagenmasse und hat ein Molekulargewicht von 30000-35000 Da. Die Wasserbindungskapazität beträgt $14-40 \mathrm{ml} / \mathrm{g}$ Gelatine. Die intravasale Verweildauer der Gelatine beträgt 2-3 h. HES ist ein hochverzweigtes Amylopektin mit einem Molekulargewicht zwischen 200000 und 450000 Da. Die Wasserbindungskapazität beträgt $10-14 \mathrm{ml} / \mathrm{g}$ Stärke. Die Dosis wird maximal auf 1,5 g Kolloid/kgKG/Tag begrenzt.
Hyperton-hyperonkotische Infusionslösungen sind hypertonen Lösungen in der klinischen Situation überlegen. Unter den derzeit zur Verfügung stehenden Volumenersatzmitteln werden heute überwiegend 6-10\%ige Hydroxyethylstärkelösungen verwendet. Im Vergleich zu Plasmapräparaten oder Humanalbuminlösungen stellen hyperton-hyperonkotische Lösungen eine Kostenersparnis und eine Vermeidung einer möglichen Infektionsquelle dar. Wirkmechanismen hypertoner-hyperonkotischer Infusionslösungen sind die rasche Mobilisierung extravasaler Flüssigkeit, der positiv-inotrope Effekt, die Vasodilatation, die Verbesserung der Fluidität, neurale Reflexmechanismen sowie eine Stimulierung des Vasomotorenzentrums. Im Hinblick auf die rasche Stabilisierung der hämodynamischen Verhältnisse (in der Regel nach Infusion von $500-1500 \mathrm{ml}$ ) und dem in der Gesamtbilanz des Infusionsregimes verminderten Flüssigkeitsbedarfs sind hyperton-hyperonkotische Lösungen bei Hypovolämie (z. B. bei Trauma, Verbrennung, Sepsis), Hypotonie, aber auch zu deren Prävention (z. B. im Rahmen einer Periduralanästhesie) geeignet.

Eine Einschränkung der Verwendung hyperton-hyperonkotischer Infusionslösung ergibt sich durch die Verschiebungen der Serumelektrolyte bei schneller Infusionsrate und Störungen der Blut-Hirnschranke und länger erhöhtem intrakranialem Druck (z. B. bei SchädelHirn-Trauma). Andererseits kommt es unter Infusion 
von hyperonkotisch-hyperosmolaren Lösungen zu einer Abnahme des Hirndrucks. Vor Mehrfachapplikationen bei länger bestehender Hirndruckerhöhung wird gewarnt. Bei Blutungen sind hypertone oder hyperton-hyperonkotische Lösungen ein rascher und initialer Flüssigkeitsersatz. Sie überbrücken die Zeit bis zum Einsetzen einer adäquaten Behandlung. Diese ist ein bestimmendes Kriterium für Komplikationen im Rahmen der Intensivbehandlung. Bei unkontrollierten Blutungen (z. B. bei Leber- oder Milzruptur) muss primär die Blutungsquelle gesucht und versorgt werden. In dieser Situation kann es durch Infusion hyperton-hyperonkotischer Lösungen zu einer Verstärkung der Blutung kommen, so dass die Entscheidung zur Volumenersatztherapie auf Grund der klinischen Situation getroffen werden muss. Bis zu einer Infusionsmenge von $8 \mathrm{ml} / \mathrm{kgKG} / \mathrm{Tag}$ wird die Blutgerinnung nicht beeinträchtigt, eine Hämolyse wird nicht beobachtet. Kontraindikationen gegen die Anwendung hypertoner Lösungen sind eine Dehydration, Niereninsuffizienz und ein beginnendes Nierenversagen (Dextrane, Gelatine), geriatrische Patienten und Kinder.

Nachteile der Lösungen sind möglicherweise unerwünschte Beeinflussung des Blutgerinnungssystems, das Auftreten von Unverträglichkeitsreaktionen (»anaphylaktoide« Reaktion bei Dextranen, s. unten), eine vermehrte Histaminfreisetzung (Gelatine) oder die fragliche Ablagerung kolloidaler Makromoleküle im retikuloendothelialen System. Die Häufigkeit von Unverträglichkeitsreaktionen beträgt bei HES bis zu 2,7\%, bei Dextranen bis zu 4.7\% und bei Gelatine bis zu 21,3\%. Die Inzidenz anaphylaktoider Reaktionen auf Dextran konnte durch die Einführung einer Haptenprophylaxe (Abbinden präformierter Antikörper gegen Dextran durch Infusion von $20 \mathrm{ml}$ eines monovalenten Dextrans mit einem Molekulargewicht von 1000 (z. B. Promit) über einen Zeitraum von 3-10 min) deutlich gesenkt werden. Präformierte Antikörper gegen Dextrane können bei bis zu 70\% der Patienten beobachtet werden. Aufgrund der möglichen Gefahren ist der Patient während der ersten $10 \mathrm{~min}$ zu beobachten und die Bereitstellung von Notfallmedikamenten sicherzustellen. Sofortmaßnahmen bei Unverträglichkeit sind die Gabe von Kortikosteroiden (z. B. $100 \mathrm{mg}$ Prednisolon i.v. bei Tachykardie, Übelkeit und Erbrechen bzw. 250-1000 mg und Sauerstoffgabe und evtl. Adrenalin bei Schock und Dyspnoe). Bei den geringsten Auffälligkeiten ist die Infusion sofort zu beenden und ein anderes Volumenersatzmittel zu infundieren.

Die Infusion von Albumin wird bei Patienten mit einer Hypoalbuminämie diskutiert. Erniedrigte Serumalbuminspiegel $(<3,5 \mathrm{~g} / \mathrm{l})$ bedeuten prospektiv für den Krankheitsverlauf eine schlechte Prognose, sie sind mit einer hohen Morbidität und Mortalität assoziiert. Randomisierte Studien belegen, dass die i.v.-Gabe von Albumin zwar den Serumalbuminspiegel verbessert, den klinischen Verlauf aber nicht zu beeinflussen vermag. Da niedrige
Serumalbuminspiegel nicht eindeutig eine Depletion der körpereigenen Proteinspeicher anzeigen, sondern eher Ergebnis der bei schweren Erkrankungen regelhaften Akutphasereaktion sind, ist eine routinemäßige Gabe von Albumin nicht gerechtfertigt. Ein als Zeichen der Fehlernährung erniedrigter Serumalbuminspiegel wird sinnvollerweise durch eine bedarfsdeckende und vorzugsweise enterale Ernährung angehoben. Unter parenteraler Ernährung kommt es zu keiner Veränderung der Albuminkonzentration. Gefahren einer i.v.-Albumingabe sind Koagulopathien, eine gestörte renale Wasser- und Natriumausscheidung, eine Hyperammonämie (Albuminlösungen enthalten Ammoniak) und seltene allergische Reaktionen (1:10000). Humane Albuminpräparate sind teuer.

\section{Probleme}

Mögliche Probleme der parenteralen Ernährung betreffen den Katheter (Komplikationen bei der Punktion (s. oben), Fehllagen, Verschluss und Materialbruch, Katheterembolie, Luftembolie bei undichten Verbindungen oder während eines Katheterwechsels), die Blutgefäße (Thrombosen und Verletzungen in den punktierten Blutgefäßen), lokale und systemische Infektionen (Kathetersepsis), Störungen der Flüssigkeitsbilanz und der Stoffwechselhomöostase (Hyper- und Hypoglykämie, Hypophosphatämie, Elektrolytverschiebungen, hohe Harnstoffproduktion), Lungenfunktionsstörungen (hohe CO2-Produktion und Hyperkapnie bei hoher Kohlenhydratzufuhr) und bei längerem Verlauf Organstörungen (Fettleber, hepato-biliäre Komplikationen, metabolische Knochenerkrankung) und Mangelzustände (Vitamine, Spurenelemente, essentielle Fettsäuren).

\section{Vitaminmangel}

Bei längerfristiger parenteraler Ernährung kann ein Vitaminmangel trotz (nach Berechnung) "vollständiger« parenteraler Substitution auftreten. Innerhalb von 30 Tagen zeigen z. B. bis zu $80 \%$ der Patienten niedrige Plasma-Vitamin C- und $B_{6}$-Spiegel. Etwa $40 \%$ der parenteral ernährten Patienten haben erniedrigte Vitamin A- und E-Konzentrationen. $10 \%$ zeigen einen Thiaminmangel. Bei einer Laktatazidose $(\mathrm{pH}<7,2$, Plasmalaktatspiegel $>8 \mathrm{mmol} / \mathrm{l})$ ist an die Möglichkeit eines Thiaminmangels zu denken. In diesem Fall ist eine hochdosierte Gabe von Vitamin $B_{1}$ (initiale Dosierung 100-400 mg/Tag, dann $50 \mathrm{mg} / \mathrm{Tag}$ bis zur Besserung des klinischen Bildes) indiziert. Ursache des Vitaminmangels sind die unausgewogene und fehlerhafte Komposition der kommerziellen Vitaminpräparate, aber auch deren mangelnde Stabilität in Mischlösungen. Die Gefahr einer Vitaminüberdosierung ist gering. Sie betrifft einzelne Vitamine, z. B. Vitamin A (klinisches Zeichen: toxischer Leberschaden, erhöhter Hirndruck), D (Hyperkalzämie) oder das Niacin (vermehrte Histaminfreisetzung). 


\section{Infektionen}

Bei ungefähr 20\% der parenteral ernährten Patienten werden Infektionen beobachtet. Katheterinfektionen können lokal im Bereich der Einstichstelle und systemisch (auch ohne lokalen Befund) ablaufen. Ausgangspunkte der Infektionen sind eine Kontamination der Infusionssysteme und die lokale Hautbesiedlung. Kontaminationen an Verbindungsstücken und Dreiwegehähnen sind häufig. Die Erreger sind vor allem Staphylococcus epidermidis, Staphylococcus aureus, Escherichia coli und Candida species. In allen bisherigen Untersuchungen korreliert die Häufigkeit der Manipulationen am Kathetersystem mit der von Infektionen. Zur Senkung von Infektionen sind Filtersysteme mit einer Porengröße von $0,22-0,45 \mu$ empfohlen worden, welche täglich gewechselt werden müssen. Diese filtern Mikroorganismen, müssen andererseits dem Druck der Infusionspumpe standhalten. Sie verstopfen bei Infusion von Lipidemulsionen und können im Rahmen einer vollständigen parenteralen Ernährung nicht verwendet werden. Die Verwendung von Filtern wird für die parenterale Ernährung von Patienten mit hohem Infektionsrisiko empfohlen. Bei periphervenöser Ernährung sind lokale Thrombophlebitiden innerhalb der ersten 72 h nach Anlage des Katheters häufig und werden bei bis zu 46\% der Patienten gefunden. Die Gefahr einer lokalen Thrombophlebitis kann durch aseptische Punktionstechnik, Verbände und niedrige Osmolarität der Infusionslösung, gesenkt und damit die »Lebensdauer« der Verweilkanüle verlängert werden.

\section{Metabolische Probleme}

Diese sind substratabhängig (s. - Tab. 2.79). Bei einer hohen Glukosezufuhr erklären die Hyperinsulinämie und die konsekutive periphere Insulinresistenz die Glukoseverwertungsstörung in Muskel und Fettzelle, die gleichzeitige Leberverfettung und die Bildung von Gallensteinen. Bei hoher Fettzufuhr kann es zu Störungen des Lipidtransfers und auch der Plasmaklärrate der Triglyzeride kommen. Diese Störung wird bei Schwerkranken durch Wirkung von Zytokinen (z. B. Tumornekrosefaktor alpha=TNFalpha) erklärt bzw. verstärkt. Die Gabe von Heparin kann zu einer vorübergehenden Verbesserung der Lipidklärrate führen. Der Effekt des Heparins erschöpft sich allerdings innerhalb von 3 Tagen. Bei hohen Zufuhrraten von LCT-Emulsionen sind Überladungen des retikuloendothelialen Systems beschrieben worden. Metabolische Probleme bei Aminosäurezufuhr ergeben sich bei gestörter Aminosäureverwertung (wie z. B. bei dekompensierter Leberzirrhose, Niereninsuffizienz, Sepsis und Multiorganversagen). Hohe Aminosäureinfusionsraten bewirken eine hohe Harnstoffproduktionsrate (vgl. - Abb. 2.33), einen Anstieg der glomerulären Filtrationsrate mit nachfolgender Nierenschädigung und tragen möglicherweise auch zur Entwicklung einer Azotämie bei.

\section{Hepatische Komplikationen}

Sie werden bei etwa $15 \%$ aller parenteral ernährten Patienten beobachtet (s. - Tab. 2.80). Dazu gehören eine Steatosis hepatis, eine Cholestase sowie die Bildung von sludge und Gallensteinen. Leberschäden sind abhängig von der Dauer und dem Konzept der parenteralen Ernährung. Sie sind regelhaft nach ausgedehnten Dünndarmresektionen. Die Komplikationen werden gehäuft bei kohlenhydratreicher/fettarmer parenteraler Ernährung und einer längeren Behandlungsdauer (>1-2 Wochen) beobachtet. Klinisch sind Leberschäden häufiger bei Neugeborenen und Kindern, Patienten mit chronisch-entzündlichen Darmerkrankungen, Erkrankungen oder Resektion des Ileums und auch bei malignen Lebererkrankungen. Ein pathologisch verändertes Enzymmuster, erhöhte Bilirubinspiegel oder auch die Bildung von Gallensteinen haben bei längerfristiger parenteraler Ernährung eine wesentlich höhere Inzidenz von 1-100\%. Eine Cholelithiasis wird bei 10-40\% der länger parenteral ernährten Kinder beobachtet. Leberschäden und Gallensteinbildung treten besonders während einer langfristigen heimparenteralen Ernährung auf. Bei Kindern können sie bereits nach 2-14 Tagen beobachtet werden. Die Leberschäden bis hin zum Leberversagen waren häufig nach Behandlungsperioden von länger als 4 Monaten. Demgegenüber waren Sludge und Gallensteine häufig bereits nach 3 Wochen einer parenteralen Ernährung nachweisbar.

Die pathophysiologischen Ursachen sind vielfältig und nicht eindeutig geklärt. Die Prävention oder Behandlung von Leberschäden bei parenteraler Ernährung besteht in der Bevorzugung der enteralen Ernährung, einer ausgewogenen, bedarfsdeckenden und »zyklischen « parenteralen Ernährung sowie der Gabe »semi-essentieller« Substrate wie L-Glutamin, Carnitin oder Cholin.

Histologische Veränderungen, welche unter parenteraler Ernährung »lebergesunder Patienten« beobachtet wurden, sind eine Leberverfettung, Hepatitis, Leberzellnekrosen, eine zentral-lobuläre Schädigung, intra- und extrahepatische Cholestase, Proliferation von Gallengängen und Kupferzellen und eine Fibrose. Die biochemischen Veränderungen betreffen Anstiege der Serumenzymaktivitäten der Aspartat- und Alaninaminotransferase, der alkalischen Phosphatase, der Gamma-Glutamyltranspeptidase und des Bilirubins. Diese Veränderungen sind 1-4 Wochen nach Beginn einer parenteralen Ernährung nachweisbar. Nach 6 Wochen sind die Laborwerte gegenüber dem Ausgangswert verdoppelt. Die Bedeutung der »biochemischen« Leberschäden ist heute unklar. Die unter parenteralen Ernährung häufigen Abweichungen klinisch-chemischer Befunde zeigen keine Beziehung zu Ergebnissen quantitativer Leberfunktionstests oder zu histologischen Befunden der Leber. Es ist deshalb unsicher, ob tatsächlich unter längerfristig parenteraler Ernährung ein Verlust der syn- 
- Tab. 2.79. Maximale Substratoxidationsraten, Probleme sowie mögliche Komplikationen bei hoher parenteraler Substratzufuhr. Die Angaben gelten für Erwachsene

\begin{tabular}{|c|c|c|c|}
\hline & Kohlenhydrate & Fette & Aminosäuren \\
\hline Maximale Oxidationsraten & $\begin{array}{l}3-4 \text { (g/kg KG/Tag) } \\
210-280 \text { (g/70 kg KG/Tag) }\end{array}$ & $\begin{array}{l}0,5-1,0 \text { (g/kg KG/Tag) } \\
35-70 \text { (g/70 kg KG/Tag) }\end{array}$ & $\begin{array}{l}-1,5(2,0)(\mathrm{g} / \mathrm{kg} \mathrm{KG} / \mathrm{Tag}) \\
-105 \text { (g/70 kg KG/Tag) }\end{array}$ \\
\hline Probleme & $\begin{array}{l}\text { Hyperinsuinämie, } \\
\text { Glukoseintoleranz, } \\
\text { Laktatakkumulation, } \\
\text { Lipogenese, Steatosis, } \\
\text { Ketogenese gehemmt, } \\
\text { BCAA niedrig (Enzephalopathie?), } \\
\text { Hypophosphatämie, } \\
\text { hohe } \mathrm{CO}_{2} \text {-Produktion }\end{array}$ & $\begin{array}{l}\text { Lipidtransfer, } \\
\text { Lipidspeicherung, } \\
\text { »immune response«?, } \\
\text { Leberschaden, RES (LCT)?, } \\
\text { Enzephalopathie (MCT?, } \\
\text { hohe Ketogenese (MCT), } \\
\text { Hypoxämie? }\end{array}$ & $\begin{array}{l}\text { AS-Muster unvollständig, } \\
\text { hohe endogene Oxidation, } \\
\text { Harnstoffproduktion hoch, } \\
\text { metabolische Azidose?, } \\
\text { mögliche Verwertungsstörung, } \\
\text { Organ-selektiver Bedarf }\end{array}$ \\
\hline
\end{tabular}

Tab. 2.80. Pathophysiologie hepatischer Komplikationen bei parenteraler Ernährung

\begin{tabular}{|c|c|c|}
\hline & Prädisponierende Faktoren & Faktoren der parenteralen Ernährung \\
\hline Steatose & Hunger, Fehlernährung, KH-Intoleranz & $\begin{array}{l}\text { Exzessive Kalorienzufuhr, Kalorien-/N- } \\
\text { Verhältnis } \uparrow, \text { Mangel an essentiellen FS, } \\
\text { Carnitinmangel }\end{array}$ \\
\hline $\begin{array}{l}\text { Cholestase } \\
\text { - Bei Kindern }\end{array}$ & $\begin{array}{l}\text { Unreife des bilären Systems, Sepsis, große Operationen, } \\
\text { bakterielle Überbesiedlung des Darms, Hypoxie }\end{array}$ & Exzessive Aminosäurezufuhr, lange Dauer \\
\hline - Bei Erwachsenen & $\begin{array}{l}\text { Nulldiät, Sepsis, Erkrankungen des lleums, entzündliche } \\
\text { Darmerkrankungen, bakterielle Überbesiedlung }\end{array}$ & $\begin{array}{l}\text { Lange Dauer, Kalorien-N-Verhältnis } \downarrow \text {, } \\
\text { kontinuierlicheApplikation, Fehlen se- } \\
\text { miessentieller Nahrungsbestandteile }\end{array}$ \\
\hline Gallensteine & Hunger, Stase/gestörter Gallenfluss & Geringer Gallenfluss, hohe Lithogenität \\
\hline
\end{tabular}

thetischen oder exkretorischen Leistungen der Leber besteht. Deshalb darf ein entsprechender Befund ohne den tatsächlichen Nachweis einer Leberschädigung nicht mit einem signifikanten Verlust der Leberfunktion gleichgesetzt werden. Entsprechend dieser Einschätzung ergeben sich bei auffälligem biochemischem Befund für ursprünglich »lebergesunde« Patienten nicht automatisch diätetische Empfehlungen, welche denen für chronisch Leberkranke mit eingeschränkter Leberfunktion entsprechen. So besteht für diese Situation keine Indikation für die Gabe leberadaptierter Aminosäurelösungen.

Bei sonographischem Nachweis von sludge oder Gallensteinen ist eine frühzeitige enterale Ernährung anzustreben. Therapeutisch kann das Problem durch die Senkung der hohen Cholesterinsättigung der Galleflüssigkeit durch orale Gabe von Ursodesoxycholsäure $(10 \mathrm{mg} /$ kgKG/Tag), oder durch Verminderung der Mukoglukoproteinbildung z. B. durch Azetylsalizylsäure oder nichtsteroidale Antiphlogistika angegangen werden.

\section{Temperaturerhöhung, Sepsis}

Bei unklaren Temperaturen $\left(>38,5^{\circ} \mathrm{C}\right)$ und Verdacht auf Kathetersepsis sollte die Infusion zunächst abgebrochen werden. Blutkulturen müssen gleichzeitig aus dem Infusionskatheter und einer peripheren Vene abgenommen und auf Bakterien und Pilze untersucht werden. Der Katheter und das Infusionssystem werden gewechselt. Sowohl die Katheterspitze als auch die verbliebene Infusionslösung werden zu weiteren bakteriologischen Untersuchungen eingesandt, um einen Keimnachweis zu führen. Sodann wird unter aseptischen Bedingungen ein neuer venöser Zugang gelegt und die parenteraler Ernährung mit neuem Konzept (bei Temperaturerhöhung eher hypokalorisch) fortgeführt. Auch in dieser Situation darf der Katheter ausschließlich für die Infusion von Nährlösungen benutzt werden. Der Nachweis einer Kathetersepsis ergibt sich durch den Vergleich der Ergebnisse der Blutkulturen (die Keimzahl ist in der "Katheterkultur « 5 fach höher als in der peripheren Blutkultur) sowie dem Keimnachweis an 
der Katheterspitze. Bei Fortbestand des Fiebers muss eine umfassende Abklärung angestrebt werden. - Abb.2.34 zeigt einen Entscheidungsalgorithmus bei parenteral ernährten Patienten mit Fieber.

\section{Katheterverschluss}

Ursache eines Katheterverschlusses sind ein Thrombus oder ein Fremdkörper (Präzipitate von Medikamenten). Bei längerer Liegezeit bildet sich um die Katheterspitze herum eine Membran aus Fibrin, Bindegewebe und Immunglobulinen, die bei Aspiration den Katheter vollständig verschließen, gleichzeitig aber noch eine Infusion zulassen kann. Risikofaktoren für die Bildung von Thromben sind die Katheteroberfläche, der Fluß durch den Katheter und um die Katheterspitze sowie mögliche Störungen des Gerinnungssystems. Ein Thrombus kann den Katheter verstopfen und ist gleichzeitig auch ein potentieller Ausgangspunkt für eine Infektion. Bei gewaltsamer Entfernung kann er absiedeln und eine Embolie verursachen. Das regelmäßige Spülen des Katheters mit einer Heparinlösung (5-10 ml einer bei Erwachsenen 100-1000 IE/ml und bei Kindern $10 \mathrm{IE} / \mathrm{ml}$ enthaltenden Lösung) dient der Prävention. Wird die parenterale Ernährung z. B. zur Durchführung von Untersuchungen unterbrochen, so ist der Katheter mit $2 \mathrm{ml}$ einer Heparinlösung (1000 IE/ml; sog. »Heparinschloss«) aufzufüllen. Bei Katheterverschluss durch einen Thrombus ist eine antithrombolytische Behandlung mit Urokinase
(5000-10000 IE) angezeigt. Das Volumen der Urokinaselösung sollte dem Kathetervolumen entsprechen. Die Lösung wird vorsichtig und ohne wesentlichen Druck in den Katheter injiziert, der anschließend für 30-120 min verschlossen wird. Nach Aspiration von Blut wird der Katheter mit einer Heparinlösung gespült. Der Erfolg einer Urokinasebehandlung variiert zwischen 30 und 100\%. Bei Präzipitaten oder Ablagerungen von Lipidemulsionen ist Urokinase nicht hilfreich. Lipidabhängige Katheterverschlüsse erfolgen nicht plötzlich: meist ist der Katheter bereits 3-4 Tage vor dem Verschluss »schwer gängig". Solche Probleme treten häufig unter Infusion von Mischlösungen auf. In diesen Fällen kann versucht werden, den Katheter mit $3 \mathrm{ml}$ einer $70 \%$ Alkohollösung (maximal 0,55 ml/kg KG) zu spülen. Nach Literaturangaben kann der Katheter auch regelmäßig mit einer 10-20\%igen Ethanollösung anstelle von Kochsalz gespült werden. Im Zweifelsfall ist der Katheter zu wechseln. In der Literatur wird auch die Möglichkeit beschrieben, die verschlossenen Katheter mit Salzsäure ( $3 \mathrm{ml} \mathrm{0,1} \mathrm{N}$ $\mathrm{HCl}$, bei Kleinkindern bis zu $3 \mu \mathrm{g} / \mathrm{kg} \mathrm{KG}$, maximal $1 \mathrm{ml}$ ) zu spülen. Ist der Katheter wieder durchgängig, wird ausgiebig mit $0,9 \%-\mathrm{NaCl}$ gespült und ein sog. "Heparinschloss" ( $2 \mathrm{ml}$ Heparinlösung, $1000 \mathrm{IE} / \mathrm{ml}$ ) angelegt. Gelingt dies nicht, wird die jeweilige "Spüllösung" (Urokinase, Alkohol) für 1-2 h im Katheter belassen. Danach wird zunächst aspiriert. Bei Misserfolg sollten die "Spüllösung« oder der Katheter gewechselt werden.

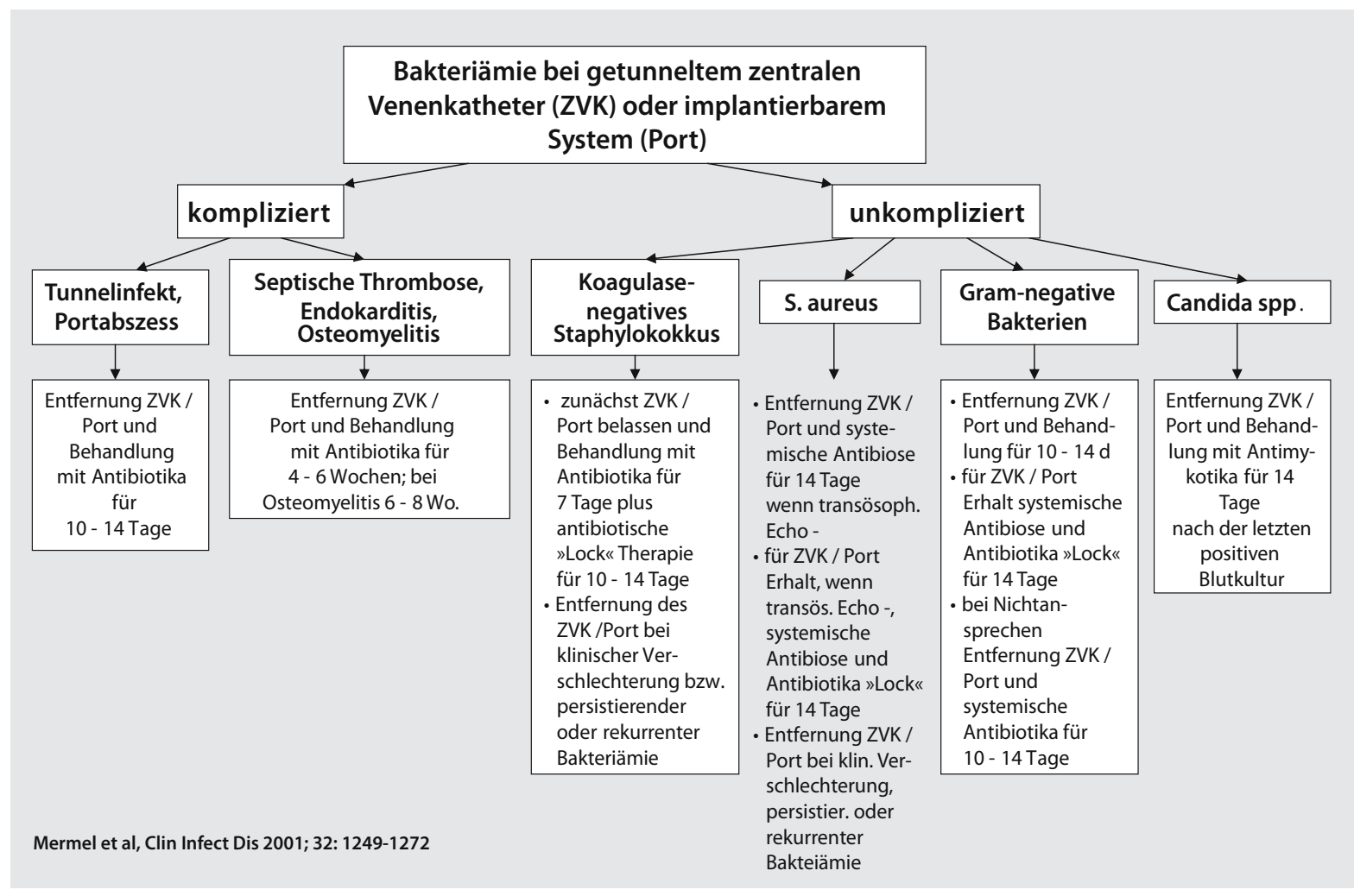

- Abb. 2.34. Port-assoziierte Infektionen. Empfehlung der Infections Disease Society of America 2001 (IDSA) 


\section{Monitoring}

Die Überwachung ist bei parenteraler Ernährung aufwendig und umfassend. Als Teil der Gesamtbehandlung bei Schwerkranken ist das Ernährungskonzept von zahlreichen Parametern (Hämodynamik, Respiration, Körpertemperatur, Ausscheidung und Bilanz, Medikation) abhängig. Subjektives und durch eine klinische Untersuchung objektivierbares Befinden müssen täglich erfasst werden. Die Kathetereinstichstelle wird regelmäßig inspiziert (transparente Pflaster benutzen, Wechsel des Pflasters alle 2 Tage), die Infusionssysteme finden nicht länger als $24 \mathrm{~h}$ Verwendung. Die Katheteranlage sowie die Liegedauer des Katheters werden in der Krankenakte protokolliert. Die Katheterlage wird radiologisch dokumentiert.

\section{Laboruntersuchungen}

Ernährungsmedizinisch wichtige, bei instabilem Verlauf täglich und bei stabilen Verlauf zunächst $2 \mathrm{mal}$ wöchentlich durchzuführende Laboruntersuchungen sind die Bestimmung des roten und weißen Blutbildes, die Kenngrößen der Blutgerinnung (Prothrombinzeit, partielle Thromboplastinzeit, Thrombozytenzahl), die Messungen der Serumelektrolyte (inklusive Kalzium, Magnesium und Phosphat), der Serumosmolalität, des Serumharnstoffs und -kreatinins, der arteriellen Blutgase, der Plasmaspiegel von Glukose, Laktat und Triglyzeriden sowie der Serumelektrophorese, des Bilirubins, der Pankreasenzyme, des Leberenzymmusters (mit Bestimmung der Cholinesterase) und des Ammoniaks. Die Krankheitsaktivität wird durch die Messung der Blutsenkungsgeschwindigkeit, der Serumeiweißelektrophorese und des C-reaktiven Proteins charakterisiert. Die Messung der Harnstoffausscheidung im 24-h-Urin ist für die Einschätzung der Stoffwechsellage und auch der Nährstoffzufuhr wichtig. Die Rate der Harnstoffproduktion kann sowohl bei zu niedriger Energie- und Aminosäurezufuhr als auch bei zu hoher Aminosäuregabe erhöht sein (vgl. - Abb. 2.33). Im EinzelfalI können diese Untersuchungen durch die Messung des Plasmaaminogramms und eine differenzierte Untersuchung der Plasmalipide ergänzt werden. Bei langfristiger parenteraler Ernährung werden alle 2 Monate die Parameter des Eisenstoffwechsel, der Serumzinkspiegel, Vitamin A und E, die alkalische Phosphatase und das Vitamin $\mathrm{D}_{3}$ (zum Ausschluss einer metabolischen Knochenerkrankung) sowie Biotin, Vitamin $\mathrm{C}, \mathrm{B}_{6}$ und $\mathrm{B}_{12}$ sowie Folsäure bestimmt. Zusätzlich zu den klinischen, hämatologischen und biochemischen Untersuchungen wird regelmäßig (d.h. in der Klinik täglich, ambulant 1-mal/Woche) der Ernährungszustand (Körpergewicht, Körperzusammensetzung) bestimmt.

\section{Spezielle Indikationen}

Spezielle Probleme der künstlichen Ernährung ergeben sich bei schweren Erkrankungen und Stoffwechselent- gleisungen. In der Regel erfordern diese Situationen keine besonderen Ernährungsformen, sondern eine kontrollierte und individuell konzipierte Ernährung, welche die besonderen Gegebenheiten und Probleme berücksichtigt. Hierbei kann neben dem Erhalt oder der Verbesserung des Ernährungszustands als »nutritional support " durchaus auch das Ziel bestehen, durch entsprechende Zusammensetzung oder Anreicherung der Ernährung das Krankheitsbild therapeutisch zu beeinflussen. Einzelne Nährstoffe wie Glutamin, Arginin, Omega-3-Fettsäuren oder Antioxidantien ( $\beta$-Carotin, Vitamin E und C sowie Selen) sind auch therapeutisch wirksam, d. h. sie beeinflussen in geeigneter Dosierung auch die Kenngrößen der Krankheitsaktivität. Der mögliche immun-nutritive Effekt einzelner gezielt angereicherter Nährlösungen wird heute diskutiert. Es gibt aber bisher keine abschließende und allgemeingültige Empfehlung zur Gabe speziell angereicherter Infusionslösungen.

\section{Leberzirrhose, Leberversagen}

Zur Einteilung der Krankheitsschwere, Änderungen des Plasmaaminosäuremusters, zum klinischen Bild und zur Ernährungstherapie des Leberversagens wird der sog. Child-score verwendet (s. - Tab. 2.81). Das klinische Bild bestimmt die Behandlung (s. - Tab. 2.82). Patienten mit alkoholischen Lebererkrankungen sind häufig fehlernährt und profitieren nachweislich von einer frühzeitigen ernährungsmedizinischen Intervention. Der Erhalt und/ oder eine Verbesserung des Ernährungszustands sind besonders bei Patienten mit geringen Verlusten möglich, dies gilt weniger bei bereits kachektischen Patienten. Das Ernährungsregime muss vollständig sein und z. B. die Vitamine $B_{1}, B_{2}, B$, A und D sowie Folsäure und Zink berücksichtigen. Bei der Zufuhr von Elektrolyten ist bei gleichzeitiger Gabe von Diuretika Vorsicht geboten (z. B. bei gleichzeitiger Gabe von Kalium und kaliumsparenden Diuretika wie Spironolacton).

Da etwa $70 \%$ der Patienten Kohlenhydratverwertungsstörungen und $40 \%$ einen sekundären Diabetes mellitus aufweisen, ist die Glukosezufuhr auf 200$240 \mathrm{~g} / \mathrm{Tag}$ zu beschränken. Bei Leberausfall werden zur Vermeidung einer Hypoglykämie 150-200 g Glukose/ Tag infundiert. Gegenüber der Kohlenhydratverwertung sind die Fettklärrate und -oxidation bei den meisten Patienten nicht beeinträchtigt. Die Fettzufuhr kann deshalb unter Kontrolle der Plasmatriglyzeridspiegel bis auf 50\% der Energiezufuhr gesteigert werden. In dieser Dosierung bestehen im Rahmen einer vollständigen parenteralen Ernährung keine Kontraindikationen gegen die Gabe von MCT-Fetten. Die Bildung von Ketonkörpern aus den mittelkettigen Fettsäuren ist bei der für Zirrhosekranke regelhaften Hyperinsulinämie und der gleichzeitigen Einschränkung der Leberfunktion gering. 
Klinisch »stabile« Zirrhosepatienten können konventionelle Aminosäurelösungen problemlos verwerten. Liegen klinische Zeichen einer hepatischen Enzephalopathie bzw. Zeichen einer Aminosäureverwertungsstörung vor, kann die Indikation für spezielle Aminosäuregemische mit erhöhter Konzentration verzweigtkettiger Aminosäuren oder aber sogar eine Kontraindikation für die Infusion von Aminosäuren gegeben sein. Der gezielte Einsatz von an das veränderte Plasmaaminogramm adaptierten Aminosäurelösungen oder von lediglich verzweigtkettige Aminosäuren enthaltenden "Komalösungen" ist von dem Nachweis einer Aminosäureverwertungsstörung abhängig. Bei erhöhten Gesamtaminosäurekonzentrationen ( $>3-4 \mathrm{mmol} / \mathrm{l}$ ) oder einer Differenzosmolalität $>15$ mos$\mathrm{mol} / \mathrm{kg}_{2} \mathrm{O}$ sollten kurzfristig entweder keine Aminosäuren oder abhängig vom klinischen Bild vorübergehend (bis $\mathrm{zu} 48 \mathrm{~h}$, maximale Dosierung $40 \mathrm{~g} / \mathrm{Tag}$ ) nur verzweigtkettige Aminosäuren enthaltende Komalösungen infundiert werden. In der kritischen Phase sind die Pa- tienten zumeist überhaupt nicht »ernährbar«. Allenfalls wird eine hypokalorische Energiezufuhr (z. B. $20 \mathrm{kcal} /$ kgKG/Tag) »toleriert«. Sogenannte »Komalösungen« haben keinen nutritiven Wert. Zur Vermeidung einer länger bestehenden katabolen Stoffwechsellage sollte nach Erholung der Leberfunktion baldmöglich eine Lösung mit "vollständigem " Aminosäuremuster verwendet werden. Diese Lösungen werden in einer Dosierung von 0,4-1,0 g Aminosäuren $/ \mathrm{kgKG} / \mathrm{Tag}$ infundiert. Eine ausgeglichen Stickstoffbilanz wird bei klinisch stabilen Patienten erst ab $0,8 \mathrm{~g} / \mathrm{kgKG} / \mathrm{Tag}$ erreicht. Eine sicher positive Bilanz wird ab einer Zufuhrrate von $>1,2 \mathrm{~g} / \mathrm{kgKG} / \mathrm{Tag}$ beobachtet. Gleichzeitig sollte auch wieder mit dem enteralen Kostaufbau begonnen werden.

Der klinische Wert spezieller, für Zirrhosekranke konzipierte Aminosäurelösungen oder auch mit verzweigtkettigen Aminosäuren angereicherter Formuladiäten ist strittig. Die Abgrenzung von Aminosäureverwertungsstörung und Hyperammonämie als mögliche Ursachen

- Tab. 2.81. Berechnung der Child-Klassifikation

\begin{tabular}{|c|c|c|c|}
\hline & \multicolumn{3}{|c|}{ Punkte } \\
\hline & 1 & 2 & 3 \\
\hline Albumin (mg/dl) & $>3,5$ & $2,8-3,5$ & $<2,8$ \\
\hline Bilirubin(mg/dl) & $<2,0$ & $2,0-3,0$ & $>3,0$ \\
\hline Quick (\%) & $>70$ & $40-70$ & $<40$ \\
\hline Aszites & Kein & Mäßig viel & Viel \\
\hline Enzephalopathie & Keine & Grad I-II & $>$ Grad II \\
\hline
\end{tabular}

Auswertung:

Gesamtpunktzahl:

Child A 5-6 Punkte, Child B 7-9 Punkte, Child C 10-15 Punkte

- Tab. 2.82. Klinische Charakterisierung und künstliche Ernährung bei verschiedenen Verlaufsformen eines Leberversagens

\begin{tabular}{|c|c|c|c|}
\hline & Subakut & Akut & Fulminant \\
\hline $\begin{array}{l}\text { Klinik } \\
\text { Enzephalopathie } \\
\text { (Grad III-IV) } \\
\text { Bilirubin } \\
\text { Entwicklung des Ikterus } \\
\text { Zerebrales Ödem } \\
\text { Prothrombinzeit } \\
\text { Prognose }\end{array}$ & $\begin{array}{l}\text { Ja } \\
\text { Deutlich erhöht } \\
\text { 29-72 Tage } \\
\text { Selten } \\
\text { Gering verlängert } \\
\text { Schlecht }\end{array}$ & $\begin{array}{l}\text { Ja } \\
\text { Deutlich erhöht } \\
\text { 8-12 Tage } \\
\text { Häufig } \\
\text { Deutlich verlängert } \\
\text { Schlecht }\end{array}$ & $\begin{array}{l}\text { Ja } \\
\text { Gering erhöht } \\
\text { 0-7 Tage } \\
\text { Häufig } \\
\text { Deutlich verlängert } \\
\text { mäßig }\end{array}$ \\
\hline Ernährungstherapie & $\begin{array}{l}\text { Vollständig parenterale } \\
\text { Ernährung ( } 100 \% \text { des er- } \\
\text { reichten Bedarfs), Wahl der } \\
\text { kompletten Aminosäurelö- } \\
\text { sungnach Differenzosmolali- } \\
\text { tät, Bilanzierung nach Klinik }\end{array}$ & $\begin{array}{l}\text { Vollständig parenterale } \\
\text { Ernährung (60-100\% des } \\
\text { errechneten Bedarfs), } \\
\text { »Komalösung«?, Bilanzierung } \\
\text { nach Klinik }\end{array}$ & $\begin{array}{l}\text { Glukose: } 2 \text { g/kg KG/Tag, } \\
\text { Bilanzierung nach Klinik }\end{array}$ \\
\hline
\end{tabular}


der Enzephalopathie ist wichtig für den sinnvollen Einsatz von verzweigtkettigen Aminosäuren. Bei Hyperammonämie muss vorrangig eine Entgiftung des Darms (mit Laktulose und Neomycin) zur Verminderung der Ammoniakbildung durchgeführt werden. Gleichzeitig kann eine Verbesserung der endogenen Entgiftung durch parenterale Gabe von Ornitinaspartat versucht werden.

\section{Chronische Nierenerkrankungen/Akutes Nierenversagen}

Das Überleben von Patienten mit einem akuten Nierenversagen ist eng mit deren Energie- und Proteinbilanz assoziiert. Früher wurden Patienten mit chronischen Nierenerkrankungen fettfrei mit hypertonen Glukoselösungen und essentiellen Aminosäuren parenteral ernährt. Ein solches Regime ist unausgewogen und führt z. B. zu einer Verarmung an essentiellen Fettsäuren, Vitaminen, Spurenelementen und verschiedenen nicht-essentiellen Aminosäuren (wie Arginin, Ornithin). Ergebnis ist häufig eine niedrige Harnstoffproduktion (erwünscht) bei gleichzeitiger Hyperammonämie (unerwünscht), welche mit der schlechten Entgiftung durch den Harnstoffzyklus erklärt wird.

Patienten mit chronischen Nierenerkrankungen und auch mit akutem Nierenversagen müssen ausgewogen und (wenn aus klinischer Sicht möglich) bedarfsdeckend ernährt werden. Die Indikation für eine künstliche Ernährung besteht bei Malnutrition und/oder Hyperkatabolismus. Die Aminosäurezufuhr sollte bei $0,8 \mathrm{~g} / \mathrm{kgKG} /$ Tag liegen und kann bei einer Nierenersatztherapie auf $1,2 \mathrm{~g} / \mathrm{kg} / \mathrm{Tag}$ gesteigert werden. Eine den Kalorienbedarf deckende enterale Ernährung sollte bei allen Patienten angestrebt werden. Der gastrale Reflux und die bei Nierenkranken häufig gestörte intestinale Peristaltik erschweren eine enterale Ernährung und müssen deshalb besonders überwacht werden. Aufgrund des hohen Eiweiß-, Kaliumund Phosphatgehalts finden Standardformuladiäten häufig keine Anwendung. Es wurden deshalb für Nierenkranke spezielle enterale Sondenkostformen entwickelt, die durch einen niedrigeren Gehalt an hochwertigem Eiweiß in Form von Oligopeptiden und Aminosäuren sowie einer Begrenzung der Elektrolytmenge charakterisiert sind.

Für die parenterale Ernährung des Nierenkranken stehen spezielle Aminosäurelösungen zur Verfügung. Für Patienten an der Dialyse kann der Einsatz dieser Lösungen kritisch diskutiert werden. Entgegen früheren und heute korrigierten Annahmen enthalten die parenteralen Nephrolösungen essentielle und auch nicht-essentielle Aminosäuren. Zusätzlich enthält eine kommerzielle Infusionslösung das Dipeptid Glycyltyrosin, welches eine adäquate Bereitstellung von Tyrosin gewährleistet. Ziele der künstlichen Ernährung sind eine Normalisierung des Plasmaaminosäuremusters, eine verbesserte Stickstoffretention sowie eine Steigerung der Proteinsynthese. Eine Indikation für speziell auf das Nierenversagen abgestimmte Nährlösungen besteht bei einem Abfall der Nierenfunktion auf $<30 \%$ des Normalwerts (i.e. "Kreatininclearance $\ll 30 \mathrm{ml} / \mathrm{min} \times 1,73 \mathrm{~m}^{2}$ oder Serumkreatinin $>2,5-3,0 \mathrm{mg} / 100 \mathrm{ml}$ ).

Da Spurenelemente wie z. B. Chrom, Selen, Zink und Elektrolyte bzw. Mineralien wie Phosphate und Magnesium über die Niere ausgeschieden werden, ist eine vorsichtige und kontrollierte Zufuhr notwendig. Aufgrund der häufig notwendigen Flüssigkeitsbeschränkung werden hochkonzentrierte Lösungen (z. B. 70\%-Glukoselösungen) gegeben. Dieses kann bei Mischinfusionslösungen zu Kompatibilitätsproblemen führen, was vor Infusion ausgeschlossen werden muss. Pragmatisch können 200$350 \mathrm{~g}$ Glukose, $50-100 \mathrm{~g}$ Fette und bis zu $50 \mathrm{~g}$ Aminosäuren infundiert werden. In der Praxis ist diese Menge mit dem rechnerischen Nährstoff-Bedarf zu vergleichen. $\mathrm{Da}$ viele Nierenkranke untergewichtig sind, besteht die Gefahr einer Hyperalimentation, welche in der Akutsituation nicht anzustreben ist.

Bei Patienten mit akutem Nierenversagen unter Dialysetherapie werden täglich die wasserlöslichen Vitamine, jeweils 2-mal/Woche die fettlöslichen Vitamine und Spurenelemente sowie Elektrolyte und Flüssigkeit nach Bedarf bzw. Bilanz substituiert. Eine Hyperalimentierung sollte in jedem Fall vermieden werden.

\section{Störungen der Lungenfunktion}

Spezielle ernährungsmedizinische Probleme Lungenkranker ergeben sich aus dem gestörten Gasaustausch (z. B. bei obstruktiven Lungenerkrankungen), der hohen Atemarbeit bei akuten Erkrankungen, der gestörten Atemmechanik und eingeschränkten Atemarbeit (z. B. bei der bei chronischen Lungenerkrankungen regelhaften Kachexie oder bei neurologischen Erkrankungen wie dem Guillain-Barré-Syndrom). Die alveoläre Ventilation ist $\mathrm{zu}$ der $\mathrm{CO}_{2}$-Produktion proportional. Eine niedrige $\mathrm{CO}_{2}$-Produktion und damit eine Verminderung der Atemarbeit kann durch die Reduktion des Kohlenhydratanteils (auf $<50 \%$ der Kalorien) bei gleichzeitiger Steigerung der Fettzufuhr (auf bis zu 50\% der Kalorien) erreicht werden. Ein entsprechendes Ernährungsregime muss individuell konzipiert und kontrolliert durchgeführt werden. Bei Patienten mit einer $\mathrm{CO}_{2}$-Retention sollte die Ernährung vorzugsweise isokalorisch sein. In der Regel ist auch bei Lungenkranken eine ausgewogene, möglichst enterale Ernährung ggf. mit Anlage einer PEG anzustreben. Die parenterale Ernährung hat ihre Indikation vorwiegend in der Intensivmedizin oder schwerer Infektexazerbation. Der Wert von Fettemulsionen ist aus ernährungsmedizinischer Sicht (d.h. im Hinblick auf den Ernährungszustand und die zu leistende Atemarbeit) unstrittig. Eine Beeinflussung des Gasaustausches ist bei Patienten mit akuten und schweren Lungenerkrankungen (z. B. beim ARDS) durch Einsatz von Omega-3-Fettsäuren) möglich. 


\section{Akute Pankreatitis}

In der Annahme,dass eine enterale Ernährung eine Stimulierung der exkretorischen Pankreasfunktion bedeuten könnte, war eine parenterale Ernährung zur Ruhigstellung des Organs über Jahrzehnte die Praxis. Heute steht auch hier die frühzeitige enterale, unter Umständen auch nur minimale Substratzufuhr zum Erhalt der Darmmukosabarriere und zur Vermeidung einer bakteriellen Translokation im Vordergrund. Die parenterale Ernährung hat ihre Indikation allenfalls in Kombination mit einer enteralen Zufuhr für Patienten mit schweren Verlauf einer nekrotisierenden Pankreatitis. Bei »leichten« und ggf. »mittelschweren« klinischen Verlaufsformen (i.e. maximale initiale Serumamylase $<500 \mathrm{U} / \mathrm{ml}$ ) kann häufig auf eine künstliche Ernährung verzichtet werden. Wichtig ist in jedem Fall eine adäquate Schmerztherapie (z. B. über einen Periduralkatheter). Besondere Ernährungsprobleme ergeben sich bei "produktiven " Fisteln, welche eine exakte Bilanzierung notwendig machen. Es gibt keine Einschränkungen für die Gabe von Lipidemulsionen. Ausnahmen sind Fettverwertungsstörungen, welche durch Plasmatriglyzeridkonzentrationen $>5 \mathrm{mmol} / \mathrm{l}$ und deren Anstieg unter Infusion um mehr als das 3 fache des Ausgangswerts gekennzeichnet sind. Die Gefahr einer hyperlipidämieinduzierten Schädigung der Bauchspeicheldrüse besteht bei Triglyzeridkonzentrationen zwischen 10 und $15 \mathrm{mmol} / \mathrm{l}$.

\section{Chronisch entzündliche Darmerkrankungen}

Der therapeutische Einfluss einer Ernährung ist beim akuten Schub einer chronisch entzündlichen Darmerkrankung der medikamentösen Therapie nachgeordnet. Dennoch kann durch eine kurzfristige parenterale, aber vor allem auch durch eine enterale Ernährung mit Nährstoff- oder ggf. Chemisch-definierten Diäten bei Patienten mit akuten Schub eines Morbus Crohn in $60-80 \%$ der Fälle ohne gleichzeitige medikamentöse Behandlung eine Remission erreicht werden. Im Vergleich zu einer medikamentösen Therapie tritt die Remission unter künstlicher Ernährung aber erst später (>10tägige Behandlung) ein. Eine künstliche, oftmals parenterale Ernährung ist bei produktiven und besonders bei prästenotischen Fisteln aus ernährungsmedizinischer Sicht notwendig, aber therapeutisch ohne Effekt (z. B. auf den Fistelverschluss). Bei schweren Komplikationen (Fisteln, Stenose, Stuhlfrequenz $>10$ Stühle/Tag) und ausgeprägter Malnutrition ist die künstliche Ernährung als kontrollierte heimenterale Ernährung über mindestens 3 Monate zu planen. Der Energiebedarf von Patienten mit einem Morbus Crohn ist nicht wesentlich erhöht, der Eiweißbedarf kann bei schwerer Malassimilation oder auch dem Vorliegen einer exsudativen Enteropathie (ca. 1/3 der Patienten sind betroffen) erhöht sein. Da der Bedarf einzelner Nährstoffe (z. B. Zink, Eisen) bei Patienten mit chronisch-entzündlichen Darmerkrankungen von dem Gesunder abweicht und die kommerziell angebotenen Formuladiäten deshalb teilweise inadäquat sind, muss eine längerfristige enterale oder parenterale Ernährung kontrolliert durchgeführt werden (Cave: Vitamin- und Spurenelementmangel; 1/3 der Patienten entwickeln innerhalb von 4-8 Wochen unter künstlicher Ernährung mit einer Chemisch-definierten Diät einen Eisenmangel).

Der therapeutische Erfolg einer künstlichen Ernährung ist bei Patienten mit einer Colitis ulcerosa nicht belegt, eine parenterale Ernährung lediglich ernährungsmedizinisch indiziert und schwerwiegenden klinischen Situationen (wie z. B. schweren Blutungen und einem toxischen Megacolon) vorbehalten.

Für die Langzeitbehandlung kann bei Patienten mit einem Morbus Crohn und rezidivierenden Schüben im Hinblick auf eine langfristige Remission eine Eliminationsdiät nach Beendigung der künstlichen Ernährung geplant werden. Sie wird über 6 Monate fortgeführt.

\section{Kurzdarmsyndrom}

Die Länge des »normalen « Dünndarms ist unterschiedlich, sie beträgt bei Kindern im Mittel 2,5 m, bei Erwachsenen zwischen 4 und $6 \mathrm{~m}$. Das Ausmaß einer Darmresektion und die Funktion des verbliebenen Restdarms (Ileum, Jejunum oder Duodenum) bestimmen die Notwendigkeit gezielter ernährungsmedizinischer Maßnahmen. Nach Resektion des Duodenums betreffen die Ernährungsdefizite Eisen, Folsäure und Calcium. Sind Jejunum und Ileum betroffen, kommt es zu Verlusten von Eiweiß, Energie, wasserlöslichen Vitaminen, Spurenelementen und Elektrolyten. Nach Resektion des distalen Ileums wird eine Steatorrhoe sowie ein Mangel an Vitamin- $\mathrm{B}_{12}$ und fettlöslichen Vitaminen beobachtet. Nach Colektomie sind massive Verluste von Wasser und Elektrolyten häufig. Per definitionem besteht ein Kurzdarmsyndrom bei einer Dünndarmrestlänge von $<2 \mathrm{~m}$. Eine jejunokolische Anastomose und eine Jejunostomie sind die häufigsten klinischen Situationen, welche eine ernährungsmedizinische Intervention notwendig machen. Im Hinblick auf die Flüssigkeits- und Elektrolytbilanz (aber auch auf das Nierensteinrisiko) ist der Erhalt des Kolons bedeutsam.

Spezielle Probleme ergeben sich bei Patienten mit einer Jejunostomie: Die Verluste über das Stoma können im Extremfall bis zu 81 bzw. kg/Tag betragen; die Na-Konzentrationen betragen $60-120 \mathrm{mmol} / \mathrm{kg}$. Diese Probleme erfordern eine individuelle Bilanzierung von Wasser und Elektrolyten. Unterschreitet die resorptive Kapazität als Kurz- oder Restdarm 2/3 der normalen Verdauungsleistung, so besteht eine in der Regel sichere Indikation für eine längerfristige (heimparenterale) Ernährung. Auch hier ist eine enterale Ernährung anzustreben. Nur bei Unverträglichkeit einer nährstoffdefinierten Diät sollte eine chemisch definierte Diät zum Einsatz kommen. Mit einer länger dauernden Adaptation ist zu rechnen. Häufig muss kombiniert ernährt werden. Obwohl sich eine funktionelle Adaptation in Einzelfällen über einen Zeitraum von 
bis zu 2 Jahren erstrecken kann, muss bei Kindern mit einer Restdarmlänge von $20 \mathrm{~cm}$ bei erhaltener bzw. $40 \mathrm{~cm}$ bei fehlender Ileozökalklappe bzw. bei Erwachsenen mit einem Restdarm von $50 \mathrm{~cm}$ bei erhaltener und $100 \mathrm{~cm}$ bei Verlust der Ileozökalklappe davon ausgegangen werden, dass eine ausreichende enterale Ernährung langfristig nicht möglich ist. Ziele der künstlichen Ernährung sind der Erhalt des Ernährungszustands sowie die Vermeidung von Elektrolytstoffwechselstörungen bzw. einer Dehydration. Die künstliche Ernährung muss exakt berechnet, vollständig (Vitamin $\mathrm{B}_{12}$ !) und bedarfsdeckend sein.

Spezielle Probleme ergeben sich vor allem aus den häufig auftretenden septischen Katheterkomplikationen und der cholestatischen Leberschädigung bis hin zum Leberversagen. Hierbei ist der Verlust von Gallensäuren von Bedeutung. Bei Patienten mit erhaltenenem Kolon ist die Hyperoxalurie mit Bildung von Nierensteinen ein Problem. Bei hoher renaler Oxalsäureausscheidung und Bildung von Oxalsäuresteinen werden eine fett- und oxalsäurearme Ernährung und die orale Gabe von Kalzium empfohlen. Die Serumspiegel von Kalium, Magnesium und Zink werden engmaschig kontrolliert. Wird die spontane orale Ernährung unverändert fortgesetzt, kann es bei vermehrter Bildung von D-Laktat durch bakterielle Fermentation der Kohlenhydrate zu neurologischen Symptomen (verwaschene Sprache, Bewusstseinsveränderungen) kommen. Therapie ist die Verminderung der oralen Kohlenhydratzufuhr und eventuell der Einsatz eines nicht resorbierbaren Antibiotikum (z. B. Metronidazol oder Colistinsulfat).

\section{Aids}

Eine künstliche Ernährung sollte frühzeitig bei Verdacht auf Malnutrition (Anorexie, chronische Diarrhoen, nicht behandelbare Malassimilation, Gewichtsverlust $>5 \%$ in 3 Monaten, BMI $<20 \mathrm{~kg} / \mathrm{m}^{2}$, zerebraler Toxoplasmose und Demenz, Endstadium der Erkrankung) begonnen werden. Die Indikation muss einfühlsam und unter Berücksichtigung der Prognose gestellt werden. Besteht eine Indikation, sollte von vornherein eine längerfristige künstliche Ernährung (PEG-, Portanlage) geplant werden. Die zugrundeliegende Ursache des Gewichtsverlustes muss gesucht werden. Der Nährstoffbedarf (Energie-/Eiweißbedarf) weicht bei Aids-Patienten nicht wesentlich von den Normalwerten ab. Es kann deshalb auch in dieser Patientengruppe mit Standardkonzepten unter Beobachtung des individuellen Verlaufs gearbeitet werden. Die Häufigkeit von Komplikationen ist bei künstlicher Ernährung von HIV-Patienten nicht höher als bei anderen Patienten. Besonderer Wert ist auf Sicherheit, Hygiene (Sondenkost) und Asepsis zu legen. $\mathrm{Zu}$ bevorzugen ist auch hier die enterale Ernährung z. B. über PEG. Sowohl eine heimenterale als auch eine heimparenterale Ernährung sind effiziente Maßnahmen zum Erhalt oder zur Verbesserung des Ernährungszustands und auch der Lebensqualität.

\section{Tumorerkrankungen}

Der Gewichtsverlust des Tumorpatienten hat im Rahmen des »Kachexie-Anorexie-Syndroms« verschiedene Ursachen. Bei kachektischen Tumorpatienten und auch bei nicht-kachektischen Patienten mit epithelialen Tumoren im Gastrointestinaltrakt (=hohes Risiko eines Gewichtsverlustes) sollte eine ernährungsmedizinische Beratung mit einer möglichen zur Indikation einer künstlichen Ernährung Teil des Behandlungskonzepts sein. Dies dient der Prävention und Therapie der Malnutrition Bei bestehender Indikation wird diese vorzugsweise enteral z. B. über eine PEG durchgeführt. Die vorübergehende Gabe von oralen Supplementen ist oftmals wenig effektiv (Anorexie) und kann aus ernährungsmedizinischer Sicht einen Zeitverlust bedeuten.

Gastrointestinale Tumoren führen häufiger als z. B. hämatologische Tumorerkrankungen zu einer Kachexie, so dass bei ihnen bereits ein geringer Gewichtsverlust ( $>5 \%$ des Ausgangsgewichts innerhalb von 3 Monaten), Untergewicht $(<90 \%$ des idealen Körpergewichts) sowie Inappetenz und Anorexie Indikationen für eine künstliche Ernährung darstellen. Mechanische Ernährungsprobleme finden sich häufiger in späten Tumorstadien, können aber auch früh im Verlauf der Erkrankung z. B. Ösophaguskarzinom auftreten. Bei einer frühzeitigen Ernährung sind die Prävention der Malnutrition und auch der Erhalt der körperlichen Leistungsfähigkeit möglich. Bei kachektischen Tumorpatienten kann der Ernährungszustand in der Regel verbessert werden. Ein therapeutischer Effekt oder Einfluss auf Erfolg und Toleranz einer onkologischen Behandlung konnte aber bisher nicht gezeigt werden.

Zur Beeinflussung des Verdauungssystem durch die Chemotherapie und zum Einsatz von Antiemetika (s. - Tab. 2.83 und - Tab. 2.84). Bei allen Tumorpatienten muss eine ausreichende Schmerztherapie (WHO-Stufenschema) gewährleistet sein.

Eine totale parenterale Ernährung ist bei Tumorkranken nur bei fortgeschrittenen Peritonealkarzinose indiziert. Die Häufigkeit von Komplikationen ist bei künstlicher Ernährung von Tumorpatienten nicht höher als bei anderen Patienten. Es gibt bisher keinen klinischen Anhalt dafür, dass durch intensivierte ernährungsmedizinische Maßnahmen das Tumorwachstum oder die Metastasierung des Tumors gefördert werden.

\section{Insulinresistenz, gestörter Glukosestoffwechsel, Diabetes mellitus}

Störungen des Glukosestoffwechsels werden häufig bei Schwerkranken und im Rahmen einer künstlichen Ernährung beobachtet. Sie betreffen nicht nur Patienten mit einem primären Diabetes mellitus. Die Probleme des Kohlenhydratstoffwechsels ergeben sich durch die bei schweren Krankheiten regelhaft vorhandene Insulinresistenz, die "unphysiologische« und kontinuierliche 
- Tab. 2.83. Chemotherapie-induziertes Erbrechen: Wirkungsbeginn und -dauer emetisch wirksamer Zytostatika

\begin{tabular}{|c|c|c|}
\hline Medikament & Wirkungsbeginn (Stunden) & Dauer (Stunden) \\
\hline \multicolumn{3}{|c|}{ 1. Sehr hohe emetische Aktivität } \\
\hline Cisplatin & $1-6$ & $4-48$ \\
\hline Dacarbacin & $1-3$ & $1-12$ \\
\hline Nitrogenmustard & $0,5-2$ & $8-24$ \\
\hline Streptozotocin & $1-4$ & $12-25$ \\
\hline \multicolumn{3}{|c|}{ 2. Hohe emetische Aktivität } \\
\hline Nitrosoharnstoffe & $2-6$ & $4-24$ \\
\hline Cyclophosphamid (i.v.) & $4-12$ & $4-10$ \\
\hline Actinomycin-D & $2-6$ & $12-24$ \\
\hline Procarbazin & $24-27$ & $6-12$ \\
\hline \multicolumn{3}{|c|}{ 3. Mittlere emetische Aktivität } \\
\hline Adriamycin & $4-6$ & $6-12$ \\
\hline Daunorubicin & $2-6$ & u. 24 \\
\hline Hexamethylmelamin & $3-6$ & $?$ \\
\hline Asparaginase & $1-3$ & $6-24$ \\
\hline Mitomycin-C & $1-4$ & $48-72$ \\
\hline Ifosfamid & $1-2$ & -24 \\
\hline Cytosin-Arabinosid & $6-12$ & $3-5$ \\
\hline \multicolumn{3}{|c|}{ 4. Schwache emetische Aktivität } \\
\hline Etoposid & $3-8$ & -12 \\
\hline Hydroxyurea & $6-12$ & $?$ \\
\hline Methotrexat & $4-12$ & $3-12$ \\
\hline 5-Fluorouracil & $3-6$ & $?$ \\
\hline Vinblastin & $4-8$ & $?$ \\
\hline Bleomycin & $3-6$ & $?$ \\
\hline \multicolumn{3}{|l|}{ Cyclophosphamid (p.o.) } \\
\hline \multicolumn{3}{|l|}{ Busulfan } \\
\hline \multicolumn{3}{|l|}{ Chlorambucil } \\
\hline \multicolumn{3}{|l|}{ Melphalan } \\
\hline \multicolumn{3}{|l|}{ Vindesin } \\
\hline Vincristin & & \\
\hline
\end{tabular}

- Tab. 2.84. Ausgewählte Antiemetika und ihre Dosierung

\begin{tabular}{|l|l|l}
\hline Substanzgruppe & Freiname & Dosierung (mg) \\
\hline 1. Antihistaminika & $\begin{array}{l}\text { Dimenhydrat } \\
\text { Meclozin }\end{array}$ & $\begin{array}{l}50-100 \\
25-50\end{array}$ \\
\hline 2. Phenothiazine & Triflupromazin & $10-20$ \\
\hline $\begin{array}{l}\text { 3. Benzamid- und Benzimidazolon- } \\
\text { Derivate }\end{array}$ & $\begin{array}{l}\text { Metroclopramid } \\
\text { Domperidon }\end{array}$ & $10-20$ \\
\hline $\begin{array}{l}\text { 4. 5-HTa }{ }_{3} \text {-Antagonisten } \\
\text { 5. Verschiedene: Kortikosteroide }\end{array}$ & $\begin{array}{l}\text { Ondansetron } \\
\text { Transquillizer }\end{array}$ & \begin{tabular}{l}
$10-20$ \\
\hline
\end{tabular} \\
\hline
\end{tabular}

${ }^{\mathrm{a}} 5-\mathrm{HT}=5-$ Hydroxytryptamin 
Nahrungszufuhr (=Hyperinsulinämie), den im Krankheitsverlauf wechselnden Insulinbedarf, insulinantagonistisch wirksame Faktoren (z. B. Stresshormone, Zytokine), die Insulingabe selbst (Zeitpunkt und Ort der Applikation, Dosis, Wirkprofil) und dem bei Stoffwechselproblemen häufigen Wechsel des therapeutischen Konzepts.

Gefahren der Insulintherapie sind rasch und drastisch wechselnde Blutglukosespiegel, Hyper- und auch Hypoglykämien. Die Einstellung des Blutzuckers ist bei einer künstlichen Ernährung gelegentlich problematisch. Da eine kontinuierliche Ernährung einer »kontinuierlich" postprandialen Situation entspricht, sind Glukosekonzentrationen zwischen 8 und $10 \mathrm{mmol} / \mathrm{l}$ (bis zu 180 mg\%) akzeptabel, der Blutzuckerwert darf unter künstlicher Ernährung nicht $>10 \mathrm{mmol} / \mathrm{l}$ liegen. Als unterer Grenzwert gelten 5,6 mmol/l (100 mg\%). Das Hypoglykämierisiko steigt drastisch bei Blutzuckerspiegeln $<5,6 \mathrm{mmol} / \mathrm{l}$.

Bei mit Insulin behandelten Diabetikern ist die Insulindosis bekannt. Obwohl sich der Insulinbedarf im Krankenhaus von dem zu Hause sowohl bei Typ-Ials auch bei Typ-II-Diabetikern unterscheidet, bietet die vorher bekannte Insulindosis einen Richtwert. Bei künstlicher Ernährung kann dann der approximative Insulinbedarf durch den Vergleich zwischen der Diät und der aktuell zugeführten Glukose-/Kohlenhydratmengen errechnet werden. Bei zunehmender Insulinresistenz, wie sie z. B. bei einer Sepsis beobachtet wird, ist ein höherer Insulinbedarf anzunehmen.

Zur Bestimmung des initialen oder endgültigen Insulinbedarfs sind für parenteral ernährte Patienten verschiedene Algorithmen empfohlen worden. Beim Gesunden besteht eine basale Insulinsekretion (ca. 1 IE/Stunde). Bei normalglykämischen Diabetikern beträgt der Insulinbedarf pro BE bzw. KHE $(=10-12 \mathrm{~g}$ Kohlenhydrate bzw. Glukose) 1-2 IE. Inter- und auch intraindividuell (z. B. tageszeitenabhängig) können allerdings große Schwankungen beobachtet werden. So kann bei Diabetikern der Insulinbedarf während einer Infektion $1 \mathrm{IE} / 3-5 \mathrm{~g}$ infundierter Glukose betragen.

Bei adäquater Stoffwechsellage und fehlenden Spätfolgen des Diabetes mellitus müssen die perioperative Mortalität und Morbidität von Diabetikern nicht erhöht sein. Dennoch ist eine intensive peri- und postoperative Überwachung notwendig. Da während einer Vollnarkose und auch postoperativ eine Aktivierung der Stresshormone (Katecholamine, Kortisol, Glukagon) und auch des Immunsystems besteht, kann bei Diabetikern eine Regional- oder Epiduralanästhesie bevorzugt werden. Diese Techniken sind bei Vorliegen einer autonomen Neuropathie obsolet. Für die perioperative Stoffwechselführung gelten folgende Regeln:

- Diabetiker sollten mindestens 2 Tage vor größeren Eingriffen stationär aufgenommen werden;
- Durchführung des Eingriffs nur nach Stabilisierung der Stoffwechsellage, des Elektrolythaushalts und des Hydrationszustands;

- Biguanide mehrere Tage vor der OP absetzen;

- Diabetiker möglichst früh morgens als erste operieren.

Die perioperative Behandlung des Diabetikers ist in - Tab. 2.85 dargestellt. Der Stoffwechsel ist peri- (30- bis 60-minütig) und postoperativ (2-stündlich) engmaschig zu kontrollieren. Die "Zielwerte« der Blutkonzentrationen liegen zwischen 100 und $180 \mathrm{mg} \%$. Postoperativ ist der Insulinbedarf erhöht. Pragmatisch wird die Insulindosis um 30\% erhöht. Die verschiedenen Algorithmen zur Insulindosisfindung sind im Rahmen einer künstlichen Ernährung begrenzt hilfreich. Zum praktischen Vorgehen sind zunächst die klinische Situation und die Medikation des Patienten im Hinblick auf die gestörte Glukosetoleranz einzuschätzen (Katecholamine, Steroide, Diuretika).

Für die künstliche Ernährung gilt: Vor einem enteralen oder parenteralen Kostaufbau muss immer erst eine möglichst optimale Stoffwechseleinstellung erfolgen. Die Flüssigkeitszufuhr wird exakt und bedarfsgerecht berechnet. Der Kostaufbau erfolgt nach Einstellung des basalen Glukosestoffwechsels bis hin zu dem errechneten Kalorienbedarf. Die Insulindosis wird parallel zum Kostaufbau gesteigert. Beim Typ-1-Diabetes wird die Insulindosis mit dem vor der akuten Krankheit bestehenden basalen Insulinbedarf verglichen. Beim Typ-II-Diabetes, unbekannter Restsekretion und nahezu normaler Insulinwirksamkeit sollte die Insulindosierung mit $60 \%$ des errechneten Bedarfs begonnen werden.

Bei kontinuierlicher, über $24 \mathrm{~h}$ konzipierter künstlicher Ernährung werden NPH-Insuline mit einem Wirkprofil von $8-12 \mathrm{~h} 2-\mathrm{mal} / \mathrm{Tag}$ s.c. injiziert. Insuline mit einem längeren Wirkprofil sind im Rahmen einer künstlichen Ernährung obsolet. Bei der Injektion von NPH-Insulinen ist zur Vermeidung von Hypoglykämien auf eine gleichzeitig kontinuierliche Infusion der Nährlösung zu achten. Das Wirkprofil der Altinsuline ist bei s.c.-Gabe der konstanten und kontinuierlichen Nährstoffinfusion nicht angepasst. In dieser Situation bewirken Altinsuline starke Blutzuckerschwankungen und eine instabilen Stoffwechsellage, welche erfahrungsgemäß schwer zu steuern ist. Eine kontinuierliche i.v.-Infusion von Altinsulin oder die Gabe einer fixen Insulindosis in den Infusionsmischbeutel ist möglichst zu vermeiden: Sie bewirkt eine zunehmende Insulinresistenz und macht mittelfristig eine Dosissteigerung notwendig.

Demgegenüber ist bei Stoffwechselentgleisung (z. B. bei Diabetischer Ketoazidose) eine exakte und flexible Dosierung von Insulin notwendig. Bei Blutzuckerentgleisungen müssen im Zweifelsfall die Glukose- und Energiezufuhr reduziert, die Flüssigkeitszufuhr gesteigert und die Elektrolytbilanz ausgeglichen werden. Nur in dieser Situation ist die kontinuierliche Infusion von Altinsulin 
über einen Perfusor indiziert. Der Zusatz von Altinsulin zu den Mischlösungen ist obsolet (unklare "Dosierung " wegen Adhäsion an der Mischbeutelwand, mangelnde Flexibilität in der Dosisanpassung, unklare Stabilität und Kompatibilität).

Bei einem Insulinbedarf $>100-120$ IE/Tag liegt eine schwere Insulinresistenz vor. In diesem Fall sollte die Insulindosis nicht weiter gesteigert, sondern eine Klärung der Ursachen (Kathetersepsis, Flüssigkeitszufuhr) und eine vorübergehende Reduktion der Glukosezufuhr mit erneutem Kostaufbau oder ein Wechsel des Ernährungsregimes (z. B. zyklische anstatt kontinuierliche Ernährung) durchgeführt werden. Bei primärem und sekundärem Diabetes ist während einer künstlichen Ernährung eine engmaschige Überwachung der Homöostase notwendig. Sie umfasst die Messung des Blutzuckers (4- bis 6-mal täglich), der Plasmalaktat-, -harnstoff- und -kreatininspiegel (einmal täglich), der Serumelektrolyte, inklusive Phosphat die Beurteilung des Säure-Basenhaushalts (Blutgasanalyse 2- bis 3mal täglich) und die tägliche Erfassung der Flüssigkeitsbilanz.

Die Stoffwechselprobleme des Zuckerkranken betreffen nicht nur den Kohlenhydratstoffwechsel, so dass bei der Planung einer künstlichen Ernährung auch der Aminosäure- (Azotämie, hohe Harnstoffproduktionsrate und verschlechterte Nierenfunktion bei hoher Infusionsrate) und Fettstoffwechsel (verschlechterte Triglyzeridklärrate, mögliche Ketonämie) zu beachten sind.

Schwere Blutzuckerentgleisungen/Diabetische Ketoazidose. Eine diabetische Ketoazidose ist eine Kontraindikation für eine künstliche Ernährung. Bei Blut- zuckerentgleisung werden die Glukose- und Energiezufuhr reduziert, die Flüssigkeitszufuhr gesteigert und die Elektrolytgabe (Kalium, Phosphat) kontrolliert. In dieser Situation ist die kontinuierliche Infusion von Altinsulin über einen Perfusor indiziert. Bei schweren Dekompensationen (eine Ketoazidose liegt vor, wenn der Blutzuckerspiegel $>350 \mathrm{mg} \%$ und der Ketonkörpernachweis im Urin $++/+++$ ist) wird unter Kontrolle der Blutzucker- und Serumelektrolytspiegel, der Flüssigkeitsbilanz sowie der Kenngrößen des Säure-Basenhaushalts Insulin in einer Dosierung von $0,05-0,10 \mathrm{IE} / \mathrm{kgKG} / \mathrm{h}$ infundiert. Ziel der Behandlung ist eine langsame Normalisierung der Homöostase. Eine rasche oder gar abrupte Normalisierung der Blutzuckerspiegel ist unerwünscht und für den Patienten schädlich. Durch die intensivierte und kontrollierte Behandlung mit Insulin werden eine optimale Einstellung des Stoffwechsels und damit ein erneuter Kostaufbau möglich. Für die Behandlung der diabetischen Ketoazidose gelten die folgenden Regeln:

- initiale Einschätzung des Ausmaßes von Dehydration (z. B. klinisch oder anhand des Vergleichs zwischen Ist- und vorher stabilem Körpergewicht), Hyperosmolarität und Azidose. Die Serumosmolarität ist bei diabetischer Stoffwechselentgleisung erhöht, ein Koma tritt bei Werten $>340$ mosm/l auf. Zur Abgrenzung von einer diabetischem Ketozidose werden bei einem hyperosmolaren Koma Blutzuckerspiegel von $>600 \mathrm{mg} / \mathrm{dl}$ und keine Azidose beobachtet. Bestimmung des Serumkaliums und der Nierenfunktion sind notwendig. Ausschluss einer Sepsis oder anderer gleichzeitig bestehender schwerer Erkrankungen.

- Tab. 2.85. Entscheidungsalgorithmus für die perioperative Behandlung bei Patienten mit Diabetes mellitus

\begin{tabular}{|c|c|c|c|}
\hline & \multirow[t]{2}{*}{ Diätisch } & \multicolumn{2}{|l|}{ Behandlungsregime } \\
\hline & & Medikamentös & Mit Insulin \\
\hline Leichte OP & Stoffwechselkontrolle & $\begin{array}{l}\text { Medikamente absetzen, } \\
\text { Wiederbeginn der Behand- } \\
\text { lung vor der 1. Mahlzeit, Stoff- } \\
\text { wechselkontrolle }\end{array}$ & $\begin{array}{l}\text { Nüchtern, Insulindosis 50\% (NPH), } \\
\text { Stoffwechselkontrolle }\end{array}$ \\
\hline Mittelschwere OP & Stoffwechselkontrolle & $\begin{array}{l}\text { Medikamente absetzen, Stoff- } \\
\text { wechselkontrolle, } \\
\text { evtl. Insulingaben, } \\
\text { Wiederbeginn der Behand- } \\
\text { lung vor der 1. Mahlzeit }\end{array}$ & $\begin{array}{l}\text { Nüchtern, Insulindosis 50\% (NPH), } \\
\text { Stoffwechselkontrolle, } \\
10 \% \text { Glukose i.v. (100-150 g/Tag) }\end{array}$ \\
\hline Schwere OP & $\begin{array}{l}\text { Stoffwechselkontrolle, } \\
\text { evtl. Insulingabe, } \\
10 \% \text { Glukose i.v. } \\
(100-150 \mathrm{~g} / \mathrm{Tag})\end{array}$ & $\begin{array}{l}\text { Präoperative Umstellung auf } \\
\text { Insulin, Stoffwechselkontrolle, } \\
10 \% \text { Glikose i.v. (100-150 g/ } \\
\text { Tag) }\end{array}$ & $\begin{array}{l}\text { Am Abend vor OP Insulindosis } 50 \% \text { (NPH), } \\
\text { am OP-Tag: Umstellung auf i.v. Insulinthe- } \\
\text { rapie, } \\
\text { Perfusor: } 50 \text { IE Alt-Insulin auf } 50 \mathrm{ml} \text { und } \\
0,9 \% \mathrm{NaCl} \text { ( } 1 \mathrm{ml}=1 \mathrm{IE}) \text {, Stoffwechselkontrol- } \\
\text { le, Umstellung auf s.c.-Insulintherapie bei } \\
\text { Beginn der oralen Ernährung }\end{array}$ \\
\hline
\end{tabular}


- Flüssigkeitssubstitution (Cave: Blutdruck, Urinausscheidung), in der 1 . Stunde $500-1000 \mathrm{ml}$.

- Insulingabe, 5-10 IE i.v., danach 0,1 IE/kg/Std. Die Insulindosis wird nach dem Abfall der Blutzuckerspiegel (nicht unter $15 \mathrm{mmol} / \mathrm{l}$ ) sowie dem Anstieg des $\mathrm{pH}$ Werts angepasst (Cave Hypokaliämie).

- Kaliumgabe (5-20 mmol/h i.v.) bei einem Serumkalium $<5 \mathrm{mmol} / \mathrm{l}$ und einer ausreichenden Nierenfunktion.

- Infusion von Natriumbikarbonat (1molare Lösung, 4\%) nur bei schwerer Azidose ( $\mathrm{pH}<7,0)$, z. B. 50-100 mmol über 30-60 min. Kontrolle des »base excess $«=\mathrm{BE}$. Genaue Dosierung nach der Formel: $\mathrm{ml}$ Natriumbikarbonatlösung $=\mathrm{BE} \times \mathrm{kgKG} \times 0,3$. Der Plasmabikarbonatspiegel liegt bei einer diabetischen Ketoazidose und einem $\mathrm{pH}<7,2$ unter $10 \mathrm{mmol} / \mathrm{l}$. Evtl. gleichzeitige Kaliumgabe wegen der Gefahr einer Hypokaliämie.

- Die Flüssigkeitssubstitution wird während der 2. Stunde fortgesetzt $(500 \mathrm{ml} / \mathrm{h})$. Die Osmolarität sollte während der ersten $24 \mathrm{~h}>285 \mathrm{mosmol} / \mathrm{l}$ liegen. Bei Natriumspiegeln $>150$ mmol kann die Gabe einer hypoosmolaren Lösung (z. B. $0,45 \% \mathrm{NaCl}$ ) erwogen werden.

- Nach Erreichen der Zielblutzuckerspiegel (10$15 \mathrm{mmol} / \mathrm{l}$ ) wird die i.v.-Insulingabe bis zur Normalisierung des Blut-pH $(>7,35)$ und einem negativen Ketonkörpernachweis fortgeführt. Bei Phosphatspiegeln $<2,0$ mg/dl kann Phosphat substituiert werden. Nach Normalisierung der Parameter und stabiler Hämodynamik wird eine s.c.-Insulinbehandlung eingeleitet.

\section{Intensivmedizin (Trauma, Verbrennungen, SIRS, Sepsis)}

Ziele sind die Behandlung einer bereits bestehenden Malnutrition, die Verminderung von kataboler Stoffwechsellage und Verlust an Körperzellmasse, der Erhalt des zellulären Hydrationszustands unter Minimierung der Expansion der ECM, die Stabilisierung und Optimierung des metabolischen Milieus und die Senkung der Morbidität und Letalität sowie die Verkürzung der Erholungsphase (Rekonvaleszenz). Eine vorsichtige die metabolischen Grenzen berücksichtigende und sorgfältig überwachte künstliche Ernährung kann für das »outcome« günstig sein. Der Wert einer kurzfristigen künstlichen Ernährung ( $<3$ Tage) ist fraglich. Eine hochdosierte (sog. »aggressive«) künstliche Ernährung ist eher schädlich.

Die Indikation sollte bei mangelernährten Patienten und bei den Patienten gestellt werden, bei denen nicht innerhalb von 7 Tagen mit einer oralen Kostzufuhr begonnen bzw. über 14 Tage keine adäquate orale Energiezufuhr zu erwarten ist. Eine frühzeitige enterale Ernährung ist einer parenteralen Ernährung vorzuziehen. Da die Motilität des Dünndarms auch in kritischen Situationen meist nicht beeinträchtigt ist, wird die enterale Ernährung über eine tief duodenal, möglichst jejunal platzierte Sonde durchgeführt. Die jejunale Platzierung der Sonde gelingt häufig nur endoskopisch. Problem sind dann die häufig auftretende Dislokationen. Bei längerfristig durchgeführter enteraler Ernährung wird eine PEG angelegt. Sofern das Krankheitsbild die Durchführung einer Laparotomie erfordert, ist zur Implantation einer Feinnadelkatheterjejunostomie zu raten.

Auslöser einer Sepsis sind grampositive Bakterien (wie Staphylokokkus aureus), gramnegative Bakterien (wie E. Coli), Pilze (wie Candida albicans) und selten Parasiten und Viren. Das »systemische Entzündungssyndrom" (=»systemic inflammatory response syndrome $=$ SIRS) ist ein Zustand, welcher durch eine überschießende systemische Antwort auf infektiöse und nicht-infektöse Schädigungen (z. B. Trauma, Pankreatitis, Verbrennungen) charakterisiert ist. Bei einem SIRS müssen 2 oder mehr der folgenden Zeichen erfüllt sein: Es ist die Körpertemperatur erhöht $\left(>38,5^{\circ} \mathrm{C}\right)$ oder erniedrigt $\left(<35,0^{\circ} \mathrm{C}\right)$, die Herzfrequenz beträgt mehr als 90 Schläge/ Minute, die Atemfrequenz liegt $>20$ Atemzüge/Minute bei einer arteriellen $\mathrm{CO}_{2}$-Spannung $<32 \mathrm{mmHg}$ (oder es besteht die Indikation für eine künstliche Beatmung), Leukozyten $>12000$ oder $<4000 / \mathrm{mm}^{3}$ oder unreife Formen $>10 \%$.

Eine Sepsis ist als systemische Antwort auf eine Infektion ein komplexes intensivmedizinisches Problem. Zusätzlich zum SIRS gibt es bei Sepsis den Erregernachweis oder einen Infektionsfocus (z. B. eine Perforation mit eitriger Peritonitis-Abzess). Bei einer schweren Sepsis besteht zusätzlich zu den Zeichen der Sepsis wenigstens ein Zeichen einer verminderten Organdurchblutung (z. B. Urinausscheidung $<0,5 \mathrm{ml} / \mathrm{kg}$ Körpergewicht und Stunde oder Nierenersatztherapie wie Dialyse), Plasmalaktatspiegel $>2 \mathrm{mmol} / \mathrm{l}$, Thrombozytenzahl < $100000 / \mathrm{ml}$, Herz- bzw. Lungenversagen). Ein septischer Schock ist definiert durch den Abfall des mittleren Blutdruckes $<60 \mathrm{mmHg}$ unter Infusion von Volumen (z. B. $40-60 \mathrm{ml} / \mathrm{kg}$ Körpergewicht 0,9\% NaCl-Lösung) oder einem pulminalem Verschlussdruck zwischen 12 und $20 \mathrm{mmHg}$. Gleichzeitig besteht die Notwendigkeit einer den Kreislauf stabilisierenden Medikation (z. B. Dopamin als Infusion, $<5 \mu \mathrm{g} / \mathrm{kg} \times$ min oder Adrenalin $<0,25 \mu \mathrm{g} / \mathrm{kg} \times \mathrm{min}$ ) zur Aufrechterhaltung des Blutdruckes von $60 \mathrm{mmHg}$ (oder $80 \mathrm{mmHg}$ bei vorher bestehendem Bluthochdruck. Bei refraktärem Schock sind höhere Dosierungen von Katecholaminen notwendig. Das häufig resultierende Multiorganversagen ist die häufigste Todesursache.

Die pathophysioogischen Konzepte gehen von einer zentralen Rolle des Darms bei der Entstehung der Sepsis ("gut derived sepsis") und des Multiorganversagens (»motor of multiple organ failure«) aus. Deshalb sollte im Sinne eines »minimal enteral supports« die enterale 
Ernährung (wenn auch geringdosiert, z. B. $20-25 \mathrm{ml} / \mathrm{h}$ einer nährstoffdefinierten Diät) immer versucht werden. Bei jejunaler Sondenlage werden die geringen Mengen in der Regel auch von Schwerstkranken vertragen und haben sich trotz der geringen Dosierung in einzelnen Studien als günstig erwiesen.

Ernährung ist keine Behandlung einer schweren Allgemeinerkrankung. Während der Stress- bzw. Akutphasenreaktion (erhöhte Serumspiegel des C-reaktiven Proteins) und bei hämodynamischer Instabilität ist eine optimale Verwertung der Nährstoffe nicht möglich.

Klinische Untersuchungen zeigen regelhaft, dass unter bedarfsgerechter und auch bei hochkalorischer (i.e. $>40$ $\mathrm{kcal} / \mathrm{kgKG} / \mathrm{Tag}$ ) parenteraler Ernährung Schwerstkranker keine erfolgreiche Kompensation oder gar Besserung des Ernährungszustands möglich ist. Der krankheitsabhängige Verlust körpereigener Proteine kann durch Ernährung nicht aufgehalten werden. Die Verluste an BCM betragen dann bis zu 20\%. Bei Gabe von Wachstumsfaktoren (i.e. Wachstumshormon) besteht eine erhöhte Letalität und ist so keine Alternative.

Bei künstlich ernährten Schwerkranken kommt es unter künstlicher Ernährung häufig zu einer Zunahme der Extrazellulär- und auch der Fettmasse. Eine vollständige künstliche Ernährung ist bei hämodynamischer Instabilität und Sepsis kontraindiziert da eine Verwertung der zugeführten Nährstoffe in diesen Situationen sehr eingeschränkt oder unmöglich ist. Hier wird die Kalorienzufuhr gedrosselt, um metabolische und intestinale Komplikationen zu vermeiden. Unter klinischer Überwachung und Laborkontrollen kann die Energie- und Substratzufuhr im Verlauf vorsichtig gesteigert werden.

Entgegen früherer Annahmen ist der Energieverbrauch septischer Patienten nicht wesentlich erhöht. Die Verteilung der Makronährstoffe sollte gleichmäßig sein (50\% Glukose, 50\% Lipide), deren intrazelluläre Verwertung bleibt in der klinischen Situation meist unklar. Das klinisch-chemische "Monitoring " erfasst neben den Plasmaspiegel der Substrate vor allem Elektrolyte und Laktat.

Glukosezufuhr. Abhängig vom Behandlungsziel sollte die infundierte Glukosemenge nie oberhalb der maximalen Glukoseoxidationsrate liegen und etwa 3-4 g/kgKG/ Tag betragen. Die Kohlenhydratverwertung kann anhand der Plasmaspiegel von Glukose und Laktat sowie der $\mathrm{CO}_{2}$-Produktionsrate beurteilt werden. Angesichts der respiratorischen Probleme muss die Glukosezufuhr genau festgelegt werden. Besteht eine Hyperglykämie bei primär nicht diabetischen Patienten, sollte die Gabe von Insulin zunächst vermieden und die Glukoseinfusionsrate gesenkt werden. Bei Insulinbehandlung ist eine Insulindosis $>100$ IE/Tag möglichst zu vermeiden. Der prognostische Einfluss einer "strengen" Glukoseeinstellung (110mg\%) ist belegt.
Aminosäurezufuhr. Sie liegt abhängig vom Ernährungszustand und dem Ausmaß der katabolen Stoffwechsellage zwischen 1,0 und maximal 2,0 $\mathrm{g} / \mathrm{kgKG} / \mathrm{Tag}$ und wird vorsichtig an Hand der Harnstoffproduktionsrate und unter Kontrolle der Kenngrößen des Säure-Basenhaushalts (Cave: Azotämie) »titriert«. Bei Patienten nach schwerem Operationstrauma wird eine "optimale" Stickstoffbilanz bei einer Aminosäurezufuhr von $1,25 \mathrm{~g} / \mathrm{kgKG} / \mathrm{Tag}$ erreicht. Hohe Aminosäureinfusionsraten bedeuten nicht automatisch eine entsprechend hohe Stickstoffretention, da sie die Harnstoffproduktionsrate und die Stickstoffausscheidung steigern (s. - Abb. 2.33). Im Gegensatz zu dieser Beziehung ist die Korrelation zwischen Energiezufuhr und Stickstoffbilanz bei den verschiedenen Aminosäureinfusionsraten linear. Beim Multiorganversagen bestimmt das schwere Leberversagen aufgrund der Aminosäureverwertungsstörung und/oder Hyperammonämie die Zusammensetzung des Ernährungsregimes.

Immunmodulierende Substanzen. Der Stellenwert spezieller immunmodulierender Substrate (Glutamin, Omega-3-Fettsäuren, Arginin oder Nukleotide, vgl. - Tab. 2.86) und Antioxidanzien (Vitamine C und E, Selen) in der künstlichen Ernährung Schwerkranker wird intensiv diskutiert. Ziele sind bei dem Erhalt der intestinalen Barriere immunstimulierende und antiinflammatorische Effekte im Rahmen der Akutphasenreaktion der krankheitsbedingten Immunsuppression. Nach einer aktuellen Metaanalyse kann die Supplementierung der parenteralen Ernährung für ausschließlich parenteral ernährte Patienten empfohlen werden. Ob dies auch für enteral ernährte Patienten gilt, ist offen. Auch die Vorteile einer enteralen Glutaminzufuhr müssen durch weitere Studien bestätigt werden. Die enterale Gabe einer Kombination von Arginin, Omega-3-Fettsäuren und Ribonukleotiden kann derzeit für den kritisch Kranken nicht allgemein empfohlen werden, da hier in einigen Studien eine erhöhte Letalität beobachtet worden ist. Diskutiert werden hierbei schädliche Auswirkungen der durch Arginin vermehrten NOBildung sowie eine Verstärkung der Immunsuppression durch die antiinflammatorischen Omega-3-Fettsäuren. Im ARDS hat sich die Zufuhr von Omega-3-Fettsäuren als vorteilhaft für die Erholung der Lungenfunktion erwiesen. Günstige Daten für eine hochdosierte Vitamin C und und E Zufuhr bedürfen der weiteren Verstärkung. Für die Selenzufuhr hat eine Metaanalyse bereits günstige Auswirkungen gezeigt.

\section{Perioperative Ernährung}

Der Wert einer prä- und kurzfristig postoperativen Ernährung ist unsicher und ohne Einfluss auf die Morbidität und Letalität. Demgegenüber ist möglicherweise die Zahl der Infektionen in der parenteral ernährten Gruppe höher als bei enteraler Ernährung. Ziel ist heute der frühest mögliche orale bzw. enterale Kostaufbau. Eine 
- Tab. 2.86. Bedeutung immunonutritiv und pharmakologisch wirksamer Nährstoffe

\begin{tabular}{|c|c|c|}
\hline & Funktion & Klinische Wirkung/Indikation \\
\hline L-Glutamin & $\begin{array}{l}\text { Energiesubstrat für schnell replizierende } \\
\text { Zellen (z. B. Enterozyten); Austausch von } \\
\text { Stickstoff zwischen verschiedenden Organen } \\
\text { (Muskel, Darm, Immunsystem); verbesserte } \\
\text { Stickstoffbilanz }\end{array}$ & $\begin{array}{l}\text { Prävention der Muskelatropie; Senkung des } \\
\text { Risikos einer bakteriellen Translokation; } \\
\text { Prävention der Leberverfettung (??); Möglicher } \\
\text { Wert nach KM-Transplantation }\end{array}$ \\
\hline Arginin & $\begin{array}{l}\text { Stimuliert T-Lymphozyten; verbesserte } \\
\text { Stickstoffbilanz; hämodynamischer Effekt }\end{array}$ & $\begin{array}{l}\text { Immunsupprimierte Patienten; Patienten mit } \\
\text { hohem Infektionsrisiko }\end{array}$ \\
\hline Kurzkettige Fettsäuren & $\begin{array}{l}\text { Energiesubstrat der Colonozyten; Stimulie- } \\
\text { rung der } \mathrm{Na}^{+} \text {-Resorption im Kolon; Antimito- } \\
\text { gen antiproliferativ }\end{array}$ & $\begin{array}{l}\text { Als Einlauf bei Diversionskolitis oder distalen } \\
\text { Kolitiden (bis } 50 \mathrm{~cm} \text { abanal) }\end{array}$ \\
\hline$\omega_{3}$-Fettsäuren & $\begin{array}{l}\text { Immunmodulation: Verminderung der } \\
\text { Prostaglandinsynthese }\end{array}$ & $\begin{array}{l}\text { Sepsis, HIV-Infektion?, Colitis ulcerosa?, } \\
\text { Rheumatische Erkrankungen }\end{array}$ \\
\hline Antioxidantien & Schutz vor Schädigung durch Radikale & $\begin{array}{l}\text { Prävention einer ischämischen Organschädi- } \\
\text { gung; Prävention der Athrosklerose (Vitamin E) }\end{array}$ \\
\hline
\end{tabular}

präoperative parenterale Ernährung kann nur für Patienten mit schwerer Mangelernährung empfohlen werden, die enteral nicht »ernährbar" sind. Eine postoperative künstliche Ernährung, welche möglichst enteral erfolgen sollte, ist bei mangelernährten Patienten immer, ansonsten nur bei absehbar längerer Verzögerung eines oralen Kostaufbaus ( $>7$ Tage) indiziert. Metaanalysen belegen den Wert einer frühzeitigen postoperativen enteralen Ernährung mit speziellen immunmodulatorisch wirksamen Diäten, welche Arginin, Omega-3-Fettsäuren, Glutamin und Nukleotide enthalten. Vorteile sind besonders für mangelernährte Patienten nach großen Oberbaucheingriffen (Gastrektomie, Ösophagus- und Pankreasresektion) gezeigt worden.

\section{Schwangerschaft}

Eine Indikation für eine künstliche Ernährung während einer Schwangerschaft ist selten. Diese können sich bei unstillbarem Erbrechen (Hyperemesis gravidarum) oder vorher bestehenden Erkrankungen (z. B. chronisch-entzündliche Darmerkrankungen) zum Erhalt oder zur Verbesserung des Ernährungszustands und der Entwicklung von Mutter und Fetus ergeben. Die Ernährung sollte vorzugsweise enteral bzw. heimenteral unter engmaschiger Überwachung der Mutter und des Fetus (Ultraschall) durchgeführt werden.

\section{Neurologische Patienten}

Bei neurologischen Patienten werden eine Reihe von metabolischen und endokrinen Störungen beobachtet. Dieses gilt besonders für Patienten mit Erkrankungen im Bereich des Zentralnervensystems (z. B. bei Hirntumoren oder nach schweren Schädel-Hirn-Traumen). Flüssigkeitsbilanz und Stoffwechsellage müssen engmaschig kontrolliert werden. Die Ernährung wird vorzugsweise enteral durchgeführt. Aufgrund der Aspirationsgefahr sollte die Ernährung in $30^{\circ}$ Lagerung erfolgen. Das Aspirationsrisiko sollte als typische Komplikation besonders kritisch gegenüber dem therapeutischen Nutzen abgewogen werden. Bei Schluckbeschwerden muss die Ernährungssonde tief duodenal, möglichst jejunal plaziert werden. In der Regel ist eine Indikation für eine Gastrostomie mit jeunaler Sondenlage (PEJ) gegeben.

\section{Alte Menschen}

Die Indikation für eine künstliche Ernährung alter Menschen ist von medizinischen Kriterien, dem Willen des Patienten und auch von ethischen Gesichtspunkten abhängig. Pflegebedürftige und auch altersdemente Patienten verweigern häufig die Nahrungsaufnahme, lehnen auch das Legen einer Sonde ab oder entfernen diese nach Anlage. Als Konsequenz werden die Patienten dann meist fixiert. Diese Situation ist für den Patienten entwürdigend und für Arzt und Pflegepersonal ausgesprochen unbefriedigend. Die künstliche Ernährung wird meist abgebrochen und den Patienten eine notwendige Behandlung vorenthalten. Hier sollte durch intensive Gespräche ggf. unter Einbeziehung eines lokalen Ethikkomitees ein Konsens gefunden werden. Bei dementen, nicht ein einwilligungsfähigen Patienten ist unbedingt die Bestellung eine Betreuers anzustreben, damit eine juristisch verbindliche Einwilligung zu invasiven Maßnahmen PEG-, Portimplantation und zur künstlichen Ernährung selbst gegeben werden kann. 
In lebensbedrohlichen Notfällen kann eine künstliche Ernährung bei nicht willensfähigen Patienten unter dem Gesichtspunkt der "Geschäftsführung ohne Auftrag “ oder unter dem Gesichtspunkt des »rechtfertigenden Notstands« durchgeführt werden, wobei die künstliche Ernährung gegenüber der Fixation das höherwertige Rechtsgut darstellt.Spezielle ernährungsmedizinische Probleme alter Menschen ergeben sich regelhaft aus gleichzeitig vorbestehenden chronischen Erkrankungen, dem häufig schlechten Ernährungszustand und der Dehydratation. Die künstliche Ernährung sollte möglichst enteral durchgeführt werden und den Nährstoff- und Flüssigkeitsbedarf unter Berücksichtigung der bestehenden Erkrankungen decken. Bei der Berechnung sind alterskorrigierte Referenzwerte zu berücksichtigen. Wenn keine Kontraindikationen bestehen, wird eine ballaststoffreiche Formuladiät bei gleichzeitig ausreichender Flüssigkeitszufuhr gewählt. Dabei sind die möglichen Wechselwirkungen mit Medikamenten zu beachten. Eine frühzeitige Entlassung des Patienten wird angestrebt. Die künstliche Ernährung wird unter Berücksichtigung der Gesamtsituation des Patienten und seiner individuellen Wünsche als heimenterale Ernährung geplant und durchgeführt. Bei den im Alter häufig eingeschränkten Kompensationsmechanismen ist eine engmaschige Überwachung des Patienten durch den Klinikarzt sowie später durch den Hausarzt obligat.

\section{Anorexia nervosa}

Eine zwingende Indikation für eine vollständige künstliche Ernährung besteht bei einem Körpergewicht $<40 \mathrm{~kg}$ oder einem BMI $<16 \mathrm{~kg} / \mathrm{m}^{2}$. Obwohl Digestion und Absorption meist nicht beeinträchtigt sind, muss zur Adaptation und im Hinblick auf die Vermeidung eines "overloading" und "refeeding syndroms" vorsichtig und stufenweise aufbauend ernährt werden (beginnend mit etwa 50\% des errechneten Energiebedarfs). Bei einem Körpergewicht von $<40 \mathrm{~kg}$ wird je eine Hälfte des errechneten Nährstoff- und Flüssigkeitsbedarfs parenteral und die andere Hälfte enteral verabreicht, liegt das Gewicht $>40 \mathrm{~kg}$ wird ausschließlich enteral ernährt. Der Patient wird zusätzlich ermuntert, spontan zu essen. Ab Körpergewichten zwischen 45 und $50 \mathrm{~kg}$ ist eine psychiatrische Intervention möglich. Diese Zahlen werden von einigen Psychiatern flexibel betrachtet. Aus medizinischen Gründen ist die künstliche Ernährung von Patienten mit Essstörungen engmaschig zu überwachen (täglich Gewicht, Elektrolyte, wöchentlich Körperzusammensetzung, Harnstoffproduktion, Albumin, Ketonkörper im Urin), auch um mögliche Täuschungen der Patienten zu erkennen.

\section{Pädiatrische Probleme}

Frühgeborene und Kinder mit einem zu niedrigen Geburtsgewicht. Diese sind die häufigste Indikation für eine künstliche Ernährung in der Pädiatrie. Die Notwen- digkeit ergibt sich aus dem begrenzten körpereigenen Energiespeicher dieser Säuglinge, weshalb eine frühzeitige (innerhalb der ersten $48 \mathrm{~h}$ ) künstliche Ernährung anzustreben ist. Die funktionelle Entwicklung des Darms ist in der 26. Schwangerschaftswoche abgeschlossen. Bei vorhandenen gastrointestinalen Funktionen wird enteral ernährt. Für die enterale Ernährung von Frühgeborenen werden konzentrierte und angereicherte Milchprodukte verwandt. Das Stillen sollte wann immer möglich fortgeführt werden.

Eine parenterale Ernährung unterliegt einer sehr strengen Indikationsstellung. Hierbei werden im Hinblick auf eine normale Entwicklung und ein normales Wachstum $3 \mathrm{~g}$ Aminosäuren/kgKG/Tag infundiert. Bei Frühgeborenen sind Cystein, Taurin, Tyrosin und Histidin essentielle Aminosäuren. Bei hohen Aminosäureinfusionsraten besteht die Gefahr einer Hyperammonämie. Glukose wird unter Kontrolle der Blutzuckerspiegel in einer Dosierung von $20-25$ g/kgKG/Tag gegeben. Die Verstoffwechslung von Lipiden ist abhängig von Alter und Entwicklung. Die Dosierung muss vorsichtig von 0,25 bis auf maximal 3,0 g/kgKG/Tag unter Berücksichtigung des Plasmatriglyzeridspiegels und des Energiebedarfs gesteigert werden. Der Bedarf an essentiellen Fettsäuren wird bei einer Infusionsrate von $0,5 \mathrm{~g} / \mathrm{kgKG} /$ Tag gedeckt. Bei den betroffenen Kindern ist ein Ikterus häufig, welcher möglicherweise durch die freien Fettsäuren verstärkt wird (Kompetition zwischen Bilirubin und freien Fettsäuren um die Bindungsstellen des Albumins), sofern der molare Quotient zwischen den freien Fettsäuren und dem Albumin 6 übersteigt. Eine Hyperbilirubinämie ist keine absolute Kontraindikation gegen die parenterale Gabe von Lipidemulsionen.

Frühgeborene haben funktionell unreife Nieren, so dass ein hoher Wasser- und Natriumverlust durch die künstliche Ernährung kompensiert werden muss.

Bei Durchfällen, Erbrechen und gastrointestinalen Fisteln ist der Kaliumverlust zu korrigieren. Bei verschiedenen Erkrankungen (z. B. bei biliärer Atresie oder einem DiGeorge-Syndrom) sowie bei Patienten unter Diuretikatherapie sind die Serumspiegel des Magnesiums erniedrigt. Bei hohen Lipidinfusionsraten ist der Bedarf an Vitamin A erhöht. Bei Patienten mit Diarrhoen, Drainagen und Stomata müssen der Zink-, Selen- und Molybdänbedarf beachtet werden.

Die parenterale Ernährung kann über eine Nabelvene, peripher- oder zentralvenös durchgeführt werden.

Die häufigste Komplikation der parenteralen Ernährung ist bei dieser Patientengruppe die Cholestase (postmikrosomal oder posthepatisch mit erhöhten Spiegeln des konjugierten oder direkten Bilirubins). Diese Komplikation begünstigende Faktoren sind die Dauer der parenteralen Ernährung, eine hohe Glukose- und Aminosäurezufuhr sowie eine Sepsis. Ziele der künstlichen Ernährung von Kindern und Jugendlichen sind neben der 
Prävention und Behandlung der Malnutrition ein normales Wachstum und eine normale Entwicklung. Dies wird durch eine optimale und ausgewogene Versorgung mit Nährstoffen erreicht.

Spezielle Probleme ergeben sich bei Tumorerkrankungen, Störungen des Immunsystems, zystischer Fibrose und eingeschränkter Lungenfunktion, gastroenterologischen und neurologischen Krankheitsbildern. Der positive Effekt einer künstlichen Ernährung auf die Parameter des Ernährungszustands und der Immunfunktion ist für Kinder gut belegt. Die künstliche Ernährung muss in jedem Fall frühzeitig begonnen werden, die Effizienz der Maßnahme überwiegt eindeutig deren Risiko. Dieses ist z. B. für Jugendliche mit einem $M$. Crohn eindrücklich belegt. Spezielle Gesichtspunkte der verschiedenen Erkrankungen entsprechen den für Erwachsene dargestellten Problemen.

Bei Kindern mit angeborenen Stoffwechselerkrankungen müssen die spezielle metabolische Einschränkung, alternative Substrate und Stoffwechselwege, essentielle Nährstoffe und Kofaktoren im Hinblick auf eine Minimierung der Komplikation diskutiert werden. Die ernährungsmedizinische Intervention stellt jedoch die einzige Behandlungsmöglichkeit dar. Für zahlreiche angeborene Stoffwechselerkrankungen gibt es spezielle und chemisch-definierte Diäten, welche akut und kurzfristig die metabolische Situation der betroffenen Kinder bessern. Langfristige Untersuchungen liegen nicht vor.

\subsubsection{Heimenterale und heimparenterale Ernährung}

Sowohl eine enterale als auch eine parenterale Ernährung können zuhause als sog. Heimernährung fortgeführt werden. Da der Erfolg einer künstlichen Ernährung wesentlich durch deren Dauer bestimmt wird, ist bei gegebener Indikation (z. B. bei deutlichem Untergewicht) aus ernährungsmedizinischer Sicht ein langfristige Behandlung anzustreben. Dieses bedeutet für den betroffenen Patienten in der Regel einen wesentlichen Zugewinn an Lebensqualität. Grundvoraussetzung für eine Heimernährung ist eine stabile Krankheitsphase. Gerade in dieser Situation darf die Ernährung nicht vergessen werden, sichert sie doch den Erhalt der Ernährungszustands und erlaubt eine vollständige Rehabilitation des Patienten. Der bloße Hinweis, »der Patient isst doch «, ist bei Schwerkranken und chronisch Kranken häufig trügerisch. Ernährungsmedizinisch ist bei schwerer Malnutriton und chronischen Erkrankungen eine lange Behandlungsdauer zur Verbesserung des Ernährungszustands notwendig.

Entscheidend für eine erfolgreiche Durchführung ist die frühzeitige Information des Patienten, seiner Angehörigen und auch der behandelnden Ärzte über die Mög- lichkeiten der Heimernährung. Bei der Entscheidung sind die Prognose der Grunderkrankung bzw. Lebenserwartung zu berücksichtigen. Die Vorgehensweise muss von dem Patienten und seinen Angehörigen akzeptiert werden. Das soziale Umfeld sollte intakt sein. Insgesamt muss durch die Heimernährung eine verbesserte Lebensqualität zu erwarten sein. Technische Voraussetzung ist, dass die Anlage eines Verweilkathetersystems (Gastrostomie bzw. Hickman-Broviac-Katheter) und ein sachgerechter Umgang mit dem Kathetersystem möglich ist. Eine künstliche Heimernährung ist bei chronisch Kranken immer Teil eines Gesamtkonzepts (»supportive care"), welches die Behandlung der Grunderkrankung und der mit ihr verbundenen Probleme wie Schmerzen, Übelkeit, Trauer und Depression mitumfassen muss.

Indikationen für eine heimenterale und heimparenterale Ernährung sind:

- längerfristige künstliche Ernährung notwendig und geplant,

- schlechter Ernährungszustand,

- spontane Nahrungsaufnahme ist für den Erhalt des Ernährungszustands nicht ausreichend

- klinisch und metabolisch stabiler Zustand des Patienten,

- verbesserte Lebensqualität zu erwarten,

- Anlage einer Gastrostomie oder eines Verweilkathersystems möglich,

- Akzeptanz und Wunsch des/der Patienten/in und der Angehörigen,

- Kooperation möglich, intaktes soziales Umfeld.

Durchführung. Koordination, Patientenschulung und Planung einer künstlichen Heimernährung obliegen dem Ernährungsteam. Zur praktischen Vorgehensweise muss nach der medizinischen Indikationsstellung und der Organisation in der Klinik die Frage geklärt werden, wer die Versorgung des Patienten übernimmt (der Patient selbst, Verwandte oder Lebenspartner, ambulante Krankenpflege, Sozialstation oder Pflegeheim)? Desweiteren müssen die häusliche Versorgung mit den Nährlösungen in Absprache mit der Industrie oder einer Apotheke sowie die Finanzierung durch die Krankenkasse gesichert sein. Der Hausarzt sollte ausführlich informiert werden. Der Patient und seine Angehörigen müssen vor Entlassung aus dem Krankenhaus im Hinblick auf die Durchführung der künstlichen Heimernährung sehr gut geschult werden. Die künstliche Heimernährung sollte soweit wie möglich an den Lebensstil des Betroffenen angepasst werden. Schulungsinhalte sind zum Beispiel der Verbandswechsel, der Umgang mit den Katheter- und Pumpensystemen, Dosierung von Sondenkost und Flüssigkeit, Verhalten bei Problemen wie Durst, Völlegefühl, Diarrhoen, Fieber oder technischen Schwierigkeiten. In jedem Fall muss der Patient jederzeit Kontakt mit der Klinik aufnehmen können. 
Soweit (z. B. aus Sicht der Erkrankung) zumutbar wird er sich zu Beginn im Abstand von 1-2 Wochen, bei stabilem Verlauf alle 4-8 Wochen in einer Spezialambulanz für heimenterale und heimparenterale Ernährung vorstellen. Hier werden die Stimmungslage der Betroffenen und mögliche praktische Probleme erörtert sowie gründliche ernährungsmedizinische und klinische Untersuchungen durchgeführt. Bei dieser Gelegenheit müssen auch die Zielsetzung und Indikation neu überdacht werden, ggf. wird die Rezeptur der Ernährung verändert.

Langfristige Nebeneffekte und Komplikationen einer heimparenteralen Ernährung ergeben sich aus dem »unphysiologischen « Applikationsmodus der »Umgehung" des Dünndarms und gefäßabhängigen Problemen, metabolischen Instabilitäten, Leberschäden, Venenthrombose und Sepsis. Eine Kathetersepsis ist die häufigste Komplikation einer heimparenteralen Ernährung. Häufige Infektionen finden sich bei jüngeren Patienten (Alter <45 Jahre), Patienten mit Morbus Crohn, nach Jejunostomie, dehydrierten Patienten, Rauchern, während schlechter "Katheterpflege« und im Verlauf nach einer Cavathrombose.

Etwa 50\% der Fälle einer Kathetersepsis bei heimparenteraler Ernährung werden durch grampositive Organismen (koagulasenegative Staphylokokken, Staphylococcus aureus), 30\% durch gramnegative Bakterien (Klebsiellen, Escherichia coli, Pseudomonas) und 15\% durch Pilze (Candida albicans) verursacht. Das Risiko einer Kathetersepsis betrifft nicht alle heimparenteral ernährten Patienten gleichmäßig, sondern wird wesentlich durch patientenspezifische (z. B. Morbus Crohn, Cavathrombose) und Umgebungsfaktoren (Katheterpflege) bestimmt. Die betroffenen Patienten erleiden häufig rezidivierende Infektionen, welche zu mehrfacher Entfernung und Neuanlage der Kathetersysteme führen. Präventive Maßnahmen sind ein adäquater Hydrationszustand des Patienten, eine langfristige Antikoagulation sowie eine regelmäßige Desinfektion des Katheteransatzes und -schlosses sowie die Hautdesinfektion mit Wasserstoffperoxid und Povidon-Jod mindestens jeden 3. Tag. Hat ein heimparenteral ernährter Patient Fieber, so ist dem in - Abb. 2.34 vorgeschlagenen Algorithmus zu folgen.

\subsubsection{Ethische Probleme}

Bei der Entscheidung über Einsatz oder Abbruch einer künstlichen Ernährung muss neben der ernährungsmedizinischen Indikation der Nutzen für den Patienten kritisch und ernsthaft erwogen werden. Grundsätzlich gelten dieselben Argumente, welche z. B. im Hinblick auf eine medikamentöse Behandlung mit Antibiotika oder Chemotherapeutika diskutiert werden. Bei schweren Erkrankungen sind Erfolg oder Misserfolg einer Behandlung häufig nicht vorhersehbar. In unklaren Situationen ist es deshalb gerechtfertigt, mit den Betroffenen zusammen eine zeitlich begrenzte Entscheidung zu fällen, um den möglichen subjektiven (i.e. aus Sicht des Patienten) und objektiven (i.e. aus Sicht des Arztes) Nutzen der künstlichen Ernährung festzustellen.

Die Frage der im Einzelfall angemessenen Behandlung ist nicht allgemein zu beantworten. Wenn für den Patienten keinerlei Behandlungsperspektive besteht (z. B. bei Komapatienten, die das Bewusstsein aller Voraussicht nach nicht wiedererlangen werden) und auch die übrige Therapie mit Ausnahme der Sauerstoffgabe, der Schmerzmedikation und der Flüssigkeitszufuhr abgebrochen wird, muss auch die künstliche Ernährung beendet werden. Künstliche Ernährung ist keine symbolische Behandlung bei infauster Prognose einer schweren Grunderkrankung.

Eine künstliche Ernährung ist eher der Therapie zuzuordnen und der medikamentösen Behandlung vergleichbar. Wird die Ernährung andererseits zu den Grundbedürfnissen eines Menschen gerechnet, wäre eine künstliche Ernährung auch Teil der Grundpflege, welche die Sauerstoffgabe, die Analgesie und die Flüssigkeitszufuhr umfasst. Nach dieser Einschätzung müsste die künstliche Ernährung auch bei infauster Prognose bis zum Lebensende fortgeführt werden.

Der Nutzen einer Behandlung ist eine wichtige Entscheidungshilfe. Lebenserhalt, Schmerztherapie und Behandlung der Grunderkrankung nutzen dem Patienten. Demgegenüber müssen jedweder Schaden und mögliche Komplikationen vermieden oder abgewendet werden. Ein weiteres wichtiges Entscheidungskriterium ist der Erhalt der Autonomie. Der Patient und/oder seine Angehörigen müssen vollständig informiert und in die Entscheidung über die künstliche Ernährung miteingebunden werden. Das Wissen um die Würde und die Integrität des Menschen sowie die Liebe zum Menschen sind die Basis der Entscheidung. Lehnt der Patient aus subjektiven Gründen eine medizinisch indizierte künstliche Ernährung ab, so ist der "Nicht-Einsatz« oder Abbruch gerechtfertigt.

In lebensbedrohlichen Situationen (einem Körpergewicht unter $40 \mathrm{~kg}$ z. B. bei Patienten mit Anorexia nervosa oder während eines z. B. politisch motivierten Hungerstreiks) muss der Arzt die Entscheidung für eine künstliche Ernährung im Sinne des Lebenserhalts und der Abwendung weiteren Schadens für den Patienten treffen. Lehnt umgekehrt der Arzt eine von dem Patienten gewünschte künstliche Ernährung ab, so sollte er den Patienten umgehend einem anderen Arzt vorstellen. Wenn der Patient seinen Willen nicht äußern kann, er keine entsprechenden Verhaltensmaßregeln vor seiner Erkrankung festgelegt hat und auch keine Angehörigen oder Freunde vorhanden oder erreichbar sind, ist die Entscheidung nach bestem Wissen und Gewissen im Sinne des Patienten zu 
treffen. Diese Entscheidung muss von den Krankenhausärzten zusammen mit dem Hausarzt, einem Juristen und in Absprache mit der lokalen Ethikkommission getroffen werden. Die Ethikkommission sollte bereits grundsätzlich mit dem Problem befasst sein. Es dürfen allerdings keine grundsätzlichen und einseitigen Entscheidungen präjudiziert werden.

Die Entscheidung für oder gegen eine künstliche Ernährung berücksichtgt auch die Lebensqualität des Patienten. Es ist deshalb notwendig, die Ernährungserhebung durch ein längeres ärztliches Gespräch und gezielte Fragen zur Lebensweise und nach der gesundheitsbezogenen Lebensqualität des Patienten zu ergänzen. Die Untersuchung der gesundheitsbezogenen Lebensqualität berücksichtigt den Einfluss subjektiver Faktoren auf Gesundheit und Krankheit (z. B. das emotionale Wohlbefinden, die geistige Gesundheit). Der Wert einzelner Aussagen wird dabei wesentlich durch die Erfahrung und das Einfühlungsvermögen des Untersuchers bestimmt. Die subjektive Wirklichkeit des Patienten kann qualitativ (z. B. durch ein strukturiertes Gespräch) oder quantitativ (z. B. durch einen standardisierten oder auch psychometrischen Fragebogen) erfasst werden. Die Tests haben verschiedene Subskalen wie z. B. körperliche Leistungsfähigkeit, psychische Verfassung, Energie/Erschöpfung, geistige Leistungsfähigkeit, soziale Kontakte oder berufliche Tätigkeit. Im Rahmen wissenschaftlicher Untersuchungen werden z. B. bei chronisch kranken Menschen standardisierte Fragebögen zur gesundheitsbezogenen Lebensqualität (z. B. der SF30-Fragebogen) verwendet. Im Rahmen von Rehabilitationsmaßnahmen werden pragmatische Einteilungen verwendet. So werden die Patienten im englischen Sprachraum in 4 Gruppen eingeteilt: Gruppe 1: vollbeschäftigt, eigenständiges Führen des Haushalts; Gruppe 2: Teilzeitarbeit und Haushaltshilfe; Gruppe 3: arbeitsunfähig, Haushaltshilfe, verlässt das Haus nur mit Unterstützung; Gruppe 4: an das Haus gebunden, Pflegefall.

Der Erhalt bzw. die Wiedererlangung von Lebensqualität ist eine zentrale Maxime des ärztlichen Handelns. Neuere klinische Studien belegen, dass gerade die Ernährungsmedizin durch Aufrechterhaltung bzw. Wiedererlangung eines guten Ernährungszustandes hier einen wesentlichen Beitrag leisten kann. Sowohl bei chronischen gutartigen Erkrankungen als auch bei progredienten malignen Grunderkrankungen hat der Ernährungszustand einen unmittelbaren Einfluss auf die Befindlichkeit und die Lebensqualität des betroffenen Patienten. Allein aus dieser Erkenntnis erwächst aus ärztlicher Sicht die Notwendigkeit, parallel zu anderen notwendigen therapeutischen Maßnahmen den Erhalt bzw. die Wiedererlangung eines guten Ernährungszustandes als wesentliche integrative Aufgabe ärztlichen Handelns und einer patientenorientierten Betreuung zu verstehen. Prospektive klinische Studien belegen, dass auch eine gezielte künstliche enterale Ernährung über eine perkutane endoskopische Gastrostomie (PEG-Sonde) signifikant nicht nur den Ernährungszustand sondern konsekutiv auch die Lebensqualität von Patienten positiv beeinflusst. Die vorliegenden klinischen Studien zeigen auch, dass dieser positive Einfluss einer gezielten enteralen Ernährung über ein PEG-Sondensystem die Lebensqualität sowohl bei Patienten mit (I.) benignen sowie malignen Grunderkrankungen, bei (II.) kooperativen sowie nicht-kooperativen komatösen Patienten und (III.) auch bei Patienten mit Tumorkachexie signifikant verbessern kann.

\subsection{Ernährungstherapie}

Die Zufuhr von Nährstoffen dient in erster Linie der Deckung des Nährstoffbedarfs und dem Erhalt bzw. der Verbesserung des Ernährungszustands. In diesem Sinne entspricht Ernährung dem Konzept des »nutritional support«. Darüber hinaus sind einzelne Nährstoffe (wie Glutamin, Arginin, Omega-3-Fettsäuren, Antioxidantien ( $\beta$-Carotin, Vitamin $E$ und $C$ sowie Selen) und auch Ernährungskonzepte (z. B. die künstliche Ernährung bei Patienten mit einem Morbus Crohn) auch therapeutisch wirksam, d.h. sie beeinflussen in geeigneter Dosierung auch die Kenngrößen der Krankheitsaktivität. - Tab. 2.86 stellt die gegenwärtigen Konzepte der Ernährungstherapie dar. Sie erscheint heute gegenüber den medikamentösen Behandlungsregimen eher unterlegen. Die Grenzen der Ernährungstherapie werden derzeit am Beispiel des Einsatzes von verzweigtkettigen Aminosäuren bei Patienten mit einer hepatischen Enzephalopathie und auch am Beispiel der künstlichen Ernährung in der Behandlung von Patienten mit einem akuten Schub eines Morbus Crohn offensichtlich. Mögliche ernährungstherapeutische Optionen sollten unter kontrollierten Bedingungen zum Wohle des Patienten genutzt werden.

\subsection{Wechselwirkungen zwischen Medikamenten und Ernährung}

Die möglichen Wechselwirkungen zwischen Medikamenten und Ernährung (Appetit, Resorption, Mangel) sind in den - Tab. 2.87-2.89 dargestellt. Auf die mögliche Änderung der Wirksamkeit einzelner Medikamente wird hier nicht eingegangen. Die Tabellen sollen als Orientierungshilfen zu einer weiterführenden Klärung der klinischenund ernährungsmedizinischen Probleme anregen. 
- Tab. 2.87. Medikamente, die den Appetit beeinflussen

\begin{tabular}{|l|l|l|}
\hline Appetitminderung & Appetitsteigerung & Geschmacksveränderung \\
\hline Amphetamine & Anabolika & Zytostatika \\
\hline Zytostatika & Benzodiazepine & Captopril \\
Clonidin & Cyproheptadin & Clofibrat \\
Colchicin & Glukokortikoide & Lithium \\
Digitalis & Insulin & Metronidazol \\
Furosemid & Orale Kontrazeptiva & Penicillamin \\
Hydralazin & Phenothiazin & Phenytoin \\
Isoniazid & Sulfonylharnstoffe & \\
Östrogene & Trizyklische Antidepressiva & \\
Penicillamin & & \\
Spironolacton & & \\
Sulfasalazin & & \\
Thiazide & & \\
\hline
\end{tabular}

Tab. 2.88. Medikamente, die einen Nährstoffmangel erzeugen können

\begin{tabular}{|c|c|c|}
\hline Medikament & Wirkstoff & Nährstoffmangel \\
\hline Antazida & Aluminiumhydroxid & Folsäure, Vitamin $B_{2}$, Kalzium, Kupfer \\
\hline $\mathrm{H}_{2}$-Rezeptorantagonisten & $\begin{array}{l}\text { Cimetidin } \\
\text { Ranitidin }\end{array}$ & Vitamin $B_{12}$ \\
\hline Laxantien & $\begin{array}{l}\text { Phenolphthalein } \\
\text { Senna }\end{array}$ & $\begin{array}{l}\text { Kalium } \\
\text { Kalzium, Fett }\end{array}$ \\
\hline Analgetika/Antirheumatika & $\begin{array}{l}\text { Azetylsalizylsäure } \\
\text { Sulfasalazin } \\
\text { Colchicin } \\
\text { Prednison }\end{array}$ & $\begin{array}{l}\text { Vitamin C, Folsäure, } \\
\text { Eisen } \\
\text { Folsäure } \\
\text { Fett, Vitamin } \mathrm{B}_{12} \\
\text { Kalzium }\end{array}$ \\
\hline Antibiotika & $\begin{array}{l}\text { Tetrazyklin } \\
\text { Gentamycin } \\
\text { Neomycin } \\
\text { Trimethoprim } \\
\text { Isoniazid }\end{array}$ & $\begin{array}{l}\text { Kalzium } \\
\text { Kalzium, Magnesium } \\
\text { Fett, Stickstoff } \\
\text { Folsäure } \\
\text { Vitamin } B_{6} \text {, Niacin, Vitamin D }\end{array}$ \\
\hline Malariamittel & Pyrimethamin & Folsäure \\
\hline Antihypertensiva & Hydralazin & Vitamin $B_{6}$ \\
\hline Diuretika & $\begin{array}{l}\text { Thiazide } \\
\text { Furosemid } \\
\text { Triamteren }\end{array}$ & $\begin{array}{l}\text { Kalium } \\
\text { Kalium, Kalzium, Magnesium } \\
\text { Folsäure }\end{array}$ \\
\hline Lipidsenker & $\begin{array}{l}\text { Cholestyramin } \\
\text { Colestipol }\end{array}$ & $\begin{array}{l}\text { Fett } \\
\text { Vitamin A, Vitamin K, Vitamin } B_{12} \text {, Folsäure }\end{array}$ \\
\hline Antikonvulsiva & $\begin{array}{l}\text { Phenytoin } \\
\text { Phenobarbitursäure } \\
\text { Valproinsäure }\end{array}$ & Vitamin D, Vitamin K \\
\hline Tranquilizer & Chlorpromazin & Vitamin $B_{2}$ \\
\hline Zytostatika & $\begin{array}{l}\text { Methotrexat } \\
\text { Cisplatin }\end{array}$ & $\begin{array}{l}\text { Folsäure, Kalzium } \\
\text { Magnesium }\end{array}$ \\
\hline Antikoagulantien & Warfarin & Vitamin $\mathrm{K}$ \\
\hline Orale Kontrazeptiva & & Vitamin $B_{6}$, Vitamin $C$, Folsäure \\
\hline
\end{tabular}


- Tab. 2.89. Einfluss der Ernährung auf die Resorption von Arzeimitteln

\begin{tabular}{|l|l|l|}
\hline Wirkstoff & Resorption verzögert & \\
\hline Resorption vermindert & Azetylsalizylsäure & Carbamazepin \\
\hline Amoxicillin & Amoxicillin & Diazepam \\
\hline Ampicillin & Cephalexin & Hydralazin \\
\hline Azetylsalizylsäure & Chinidin & Lithium \\
\hline Atenolol & Cimetidin & Metoprolol \\
Captopril & Diclofenac & Nikotinsäure \\
Cephalexin & Digoxin & Phenytoin \\
\hline Folsäure & Furosemid & Propranolol \\
\hline Eisen & Glipizid & Riboflavin \\
\hline Isoniazid & Metronidazol & Thiazide \\
\hline Ketoconazol & Paracetamol & \\
Levodopa & Phenytoin & \\
Lincomycin & Sulfonamide & \\
\hline Tetrazyklin & Theophyllin & \\
Penicillin & & \\
\hline Penytoin & & \\
Rifampicin & & \\
Sotalol & & \\
Theophyllin & & \\
\hline
\end{tabular}

\section{Literatur}

Board of Directors: Guidelines for the use of parenteral and enteral nutrition (1994) J. Parenteral and Enteral Nutrition 17 (Supp): 1-52

Böhles HJ (1991) Ernährungsstörungen im Kindesalter, Pathophysiologie und Leitlinien der Flüssigkeits-, Elektrolyt- und Ernährungstherapie für Studium und Praxis. Wissenschaftliche Verlagsgesellschaft, Stuttgart

Dach (2000) (2000) Referenzwerte für die Nährstoffzufuhr. 1. Aufl. Umschau/Braus, Frankfurt

Derksen-Lubsen G, Steensel-Moll van HA, Visser HKA (eds) (1994) Compendium Kindergeneeskunde. Diagnostiek en Behandeling. Bohn Staffleu Van Loghum, Houten

DGEM Leitlinie Enterale Ernährung (2003), Teil 1 und 2, Akt. Ernährungsmedizin 28:1-120

DGEM Leitlinie Enterale Ernährung, Teil 1 und 2 (2004) Akt. Ernährungsmedizin 29: 189-230

Elwyn DH (1993) Protein and energy requirementt: effect of clinical state. Clin Nutrition 12 (Suppl. 1): 44-51

Fernandes J, Saudubray J-M, Tada K (eds) (1990) Inborn metabolic diseases. Diagnosis and treatment. Springer, Berlin Heidelberg New York

Frankel DA (1992), Supplementation of trace elements in parenteral nutrition. Nutrition Research 13:583-596

Gibney M et al (2004) Clinical Nutrition, The Human Nutrition Textbook Series, Blackwell Science, Oxford 2004

Hartig W et al.(2004) Ernährungs- und Infusionstherapie. Thieme, Stuttgart

Huth K, Kluthe R (1995) Lehrbuch der Ernährungstherapie. Thieme, Stuttgart

Koch et al. (2003) The Maternal Phenylketonuria International Study. Pediatrics 112, Suppl.

Löser Chr., Keymling M (2001) Praxis der enteralen Ernährung. Thieme, Stuttgart
Marginson G, Hark L (1999) Medical Nutrition Disease. Blackwell Science, London

Nelson JK, Moxness KE, Jensen MD, Gastineau CF (1994) Mayo Clinic diet manual, A handbook of nutrition practices, 7 th edn. Auflage, Mosby, St. Louis

Rote Liste 2006

Shaw V, Lawson M (2001) Clinical Pediatric Diatetics, $2^{\text {nd }}$ Ed. Blackwell Science, London

Shils ME, Shike M, Ross AC, Caballero B, Cousins RI (2006) Modern Nutrition in Health and Disease. Lippincott, Williams \& Williams, Philadelphia

Stein J, Jouch K (2004) Praxisbuch klinische Ernährung und Infusionstherapie. Springer, Heidelberg

Stump SE (1998) Nutrition and diagnose-related care, $4^{\text {th }}$ Ed., Lippincott, Williams \& Wilkins, Baltimore

The British Dietetic Association (2001) Manual of dietetic practise, 3rd Ed. Blackwell Science, London

Toeller M (2002) Erbährung bei Diabetes mellitus. Akt. Ern. Med. 27:101-107 